Sahar Moradi Karkaj

\title{
Die Staatenhaftung im Völkerrecht
}

am Beispiel von informationstechnischen Gefährdungslagen 
Beiträge zum

ausländischen öffentlichen Recht und Völkerrecht

Herausgegeben von

der Max-Planck-Gesellschaft

zur Förderung der Wissenschaften e.V.,

vertreten durch Prof. Dr. Armin von Bogdandy

und Prof. Dr. Anne Peters

Band 309 
Sahar Moradi Karkaj

\section{Die Staatenhaftung im Völkerrecht}

am Beispiel von informationstechnischen Gefährdungslagen 
Die Deutsche Nationalbibliothek verzeichnet diese Publikation in der Deutschen Nationalbibliografie; detaillierte bibliografische Daten sind im Internet über http://dnb.d-nb.de abrufbar.

Zugl.: Frankfurt am Main, Univ., Diss., 2020

1. Auflage 2021

(c) Sahar Moradi Karkaj

Publiziert von

Nomos Verlagsgesellschaft $\mathrm{mbH} \& \mathrm{Co}$. KG

Waldseestraße 3-5 | 76530 Baden-Baden

www.nomos.de

Gesamtherstellung:

Nomos Verlagsgesellschaft mbH \& Co. KG

Waldseestraße 3-5 | 76530 Baden-Baden

ISBN (Print): 978-3-8487-7216-2

ISBN (ePDF): 978-3-7489-1228-6

DOI: https://doi.org/10.5771/9783748912286

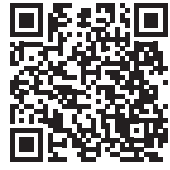

Onlineversion Nomos eLibrary

D30

\section{(c) $(1) \Theta \Theta$}

Dieses Werk ist lizenziert unter einer Creative Commons Namensnennung

- Nicht kommerziell - Keine Bearbeitungen 4.0 International Lizenz. 
Beiträge zum

ausländischen öffentlichen Recht und Völkerrecht

Herausgegeben von

der Max-Planck-Gesellschaft

zur Förderung der Wissenschaften e.V., vertreten durch Prof. Dr. Armin von Bogdandy

und Prof. Dr. Anne Peters

Band 309 
Sahar Moradi Karkaj

\section{Die Staatenhaftung im Völkerrecht}

am Beispiel von informationstechnischen Gefährdungslagen 
Die Deutsche Nationalbibliothek verzeichnet diese Publikation in der Deutschen Nationalbibliografie; detaillierte bibliografische Daten sind im Internet über http://dnb.d-nb.de abrufbar.

Zugl.: Frankfurt am Main, Univ., Diss., 2020

1. Auflage 2021

(c) Sahar Moradi Karkaj

Publiziert von

Nomos Verlagsgesellschaft $\mathrm{mbH} \& \mathrm{Co}$. KG

Waldseestraße 3-5 | 76530 Baden-Baden

www.nomos.de

Gesamtherstellung:

Nomos Verlagsgesellschaft $\mathrm{mbH} \& \mathrm{Co}$. KG

Waldseestraße 3-5 | 76530 Baden-Baden

ISBN (Print): 978-3-8487-7216-2

ISBN (ePDF): 978-3-7489-1228-6

DOI: https://doi.org/10.5771/9783748912286

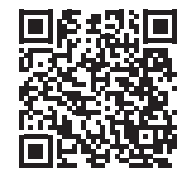

Onlineversion

Nomos elibrary

D30

\section{(c) (i) $(\mathrm{BY}$}

Dieses Werk ist lizenziert unter einer Creative Commons Namensnennung

- Nicht kommerziell - Keine Bearbeitungen 4.0 International Lizenz. 


\section{Vorwort}

Die vorliegende Arbeit wurde im Sommersemester 2020 von der rechtswissenschaftlichen Fakultät der Goethe-Universität Frankfurt am Main als Dissertation angenommen. Rechtsprechung und Literatur konnten im Wesentlichen bis zu diesem Zeitpunkt Berücksichtigung finden.

An dieser Stelle gilt mein aufrichtiger Dank allen, die zum Gelingen meiner Arbeit beigetragen haben.

Zuvorderst gebührt mein besonderer Dank dem Betreuer der Arbeit, Herrn Professor Dr. Stefan Kadelbach, LL.M. Meine Begeisterung für das internationale Recht ist im Grunde auf seine Person und sein Engagement zurückzuführen. Er gab mir wichtige Anregungen, stand mir als Gesprächspartner stets zur Verfügung und gewährte mir zugleich die notwendigen Freiräume für die Promotion. Ferner danke ich Herrn Professor Dr. Dr. Rainer Hofmann für die Übernahme und zügige Erstellung des Zweitgutachtens und Herrn Professor Dr. Alexander Peukert für die Übernahme des Vorsitzes im Rahmen der Disputation.

Für die Aufnahme in die Schriftenreihe des Max-Planck-Instituts für ausländisches öffentliches Recht und Völkerrecht bin ich den Direktoren am Institut, Herrn Professor Dr. Armin von Bogdandy und Frau Professorin Dr. Anne Peters, LL.M., zu Dank verpflichtet.

Die Dissertation wurde mit dem Baker McKenzie-Preis 2020 ausgezeichnet. Diese wertvolle Auszeichnung sowie die finanzielle Unterstützung durch das Bundesministerium des Innern, für Bau und Heimat ermöglichten die Drucklegung. Für diese besondere Ehre bedanke ich mich.

Aufgrund ihrer Freundschaft und des Rückhalts während des Entstehens dieser Arbeit bin ich Özlem Erhalac, Dr. Adele Kirschner, Deniz Kurt, Fardin Pardis und Sofia Stankova sehr verbunden. Herrn Dr. Klaus Kupka danke ich ebenfalls recht herzlich für seinen Rat und Zuspruch im richtigen Moment.

Meiner Schwester, Dr. Sanaz Moradi Karkaj, danke ich von Herzen für das Mitleiden, das Mitfreuen und die Unterstützung in jeder Phase der Promotion. Sie hat mich (nicht nur) während dieser Zeit gestärkt und motiviert. 
Nicht in Worte fassen lässt sich meine Dankbarkeit gegenüber meinen Eltern, Soheila Moradi Karkaj und Mohammad Moradi Karkaj, die mich zeitlebens bedingungslos unterstützt haben. Ihre liebevolle und selbstlose Fürsorge ebnete mir meinen Weg und ermöglichte überhaupt erst die Verwirklichung meines Promotionsvorhabens. Ihnen ist diese Arbeit gewidmet.

Frankfurt am Main (Deutschland)

Sahar Moradi Karkaj 


\section{Inhaltsverzeichnis}

Abkürzungsverzeichnis

Gegenstand und Gang der Untersuchung

1. Kapitel: Staatenhaftung in der Systematik des Völkerrechts

A. Die Arbeiten der Völkerrechtskommission der Vereinten

Nationen

I. Staatenverantwortlichkeit 26

II. Staatenhaftung 28

1. Staatenhaftung - Kartografie der Völkerrechtskommission

a) Anwendungsbereich gemäß den AIL von 1996

b) Schadensvermeidung gemäß den AIL von 1996

c) Wiedergutmachung gemäß den AIL von $1996 \quad 40$

2. Staatenhaftung - ,a case of misplaced emphasis“? 42

a) Anwendungsbereich der Haftungsstudie 44

b) Prävention grenzüberschreitender Schäden durch gefährliche Aktivitäten

c) Verlustzuweisung bei grenzüberschreitenden Schäden durch gefährliche Aktivitäten 49

III. Kodifikation oder Weiterentwicklung von Völkerrecht 51

1. Staatenverantwortlichkeit 52

2. Schadensprävention und Verlustzuweisung 53

3. Staatenhaftung 56

B. Rechtsprechung 58

I. Schadensvermeidungspflicht 59

1. Verhaltensbezogene und ergebnisbezogene Pflichten 59

2. Verfahrensbezogene und materielle Pflichten 65

3. Staatenverantwortlichkeit oder doch Staatenhaftung? 69

a) Haftungsart und -standard 70

b) Schnittstelle zwischen Schadensvermeidungspflicht und Verhinderungspflicht $\quad 78$

II. Verhinderungs- und Schutzpflichten $\quad 82$

1. Völkerrechtlich verbotene Handlungen 82 
2. Nicht-staatliche Verbrechen 88

3. Menschenrechtsverletzungen 90

III. Ergebnis 94

C. Völkergewohnheitsrecht 95

I. Staatenhaftung unter dem Deckmantel von ex gratiaZahlungen

1. Gut Dam-Fall 98

2. Fluss Mur-Fall 99

3. Juliana-Fall 100

4. Cosmos 954-Fall 100

5. Fukuyu Maru-Fall 101

6. Tschernobyl-Fall 102

7. Aerial Herbicide Spraying-Fall 104

8. Ergebnis 105

II. Wandel von Staatenhaftung zur privaten Haftung 106

1. Cherry Point-Fall 106

2. Sandoz Rhein-Fall 108

3. Baia Mare-Fall 109

4. Montara Oil Spill-Fall 110

5. Deepwater Horizon-Fall 111

6. Ergebnis 112

D. Völkervertragsrecht 113

I. Originäre Staatenhaftung 116

1. Weltraumhaftung 116

a) Verschuldensabhängige Haftung 118

b) Verschuldensunabhängige Haftung 120

2. Anlage VI zum Umweltschutzprotokoll zum Antarktisvertrag 121

3. VN-Wasserlaufkonvention 124

4. Wirtschaftsvölkerrecht 128

a) Allgemeines Zoll- und Handelsabkommen 129

b) Übereinkommen über Subventionen und Ausgleichsmaßnahmen 130

c) Internationale Investitionsschutzverträge 131

II. Ergänzende Staatenhaftung 135

1. Zivilrechtliche Haftung nach den Nuklearhaftungsabkommen

2. Staatliche Haftung nach den Nuklearhaftungsabkommen 
III. Subsidiäre Staatenhaftung

1. Subsidiäre Haftung nach den

Nuklearhaftungsabkommen

2. Subsidiäre Haftung im antarktischen Haftungssystem 143

IV. Modellcharakter zivilrechtlicher Haftungssysteme 145

1. Kausaler Schaden durch besonders gefährliche Aktivität 145

2. Haftungsstandard 146

3. Haftungsträger 147

a) Schadensverursacher 148

b) Entschädigungsfonds bei Überschreiten der Haftungshöchstgrenze 148

c) Entschädigungsfonds bei Nachweisproblemen $\quad 150$

V. Ergebnis

I. Grundsatz der guten Nachbarschaft 159

II. Schädigungsverbot 162

1. Grundsatz der Schadensprävention 166

2. Grundsatz angemessener Nutzung 170

3. Vorsorgegrundsatz 172

III. Verursachergrundsatz 176

IV. Haftungsgrundsätze in den nationalen Rechtsordnungen 182

V. Ergebnis 188

F. Zusammenfassung 191

2. Kapitel: Konstitutive Elemente der staatlichen Einstandspflichten 193

A. Konstitutive Elemente der Staatenverantwortlichkeit 193

I. Völkerrechtswidrigkeit 193

1. Verletzung negativer Verpflichtungen 194

2. Verletzung positiver Verpflichtungen 196

a) Territorium bzw. Hoheitsgewalt 197

b) Reale Handlungsmöglichkeit 198

c) Kenntnis 200

II. Konsequenzen der Staatenverantwortlichkeit 201

B. Konstitutive Elemente der Staatenhaftung 203

I. Anwendungsbereich 203

1. Schaden 203

2. (Hoch-)gefährliche Aktivität 206 
3. Kausalität 209

II. Schadensvermeidung und Schadenskompensation 211

III. Gebotene Sorgfalt 213

IV. Haftungsart und -standard 218

$\begin{array}{ll}\text { C. Zusammenfassung } 220 & 218\end{array}$

3. Kapitel: Staatenverantwortlichkeit für informationstechnische $\begin{array}{ll}\text { Systeme } & 221\end{array}$

A. Informationsoperationen 221

I. Informationsangriffe 222

II. Informationsausbeutung 224

B. Informationsoperationen in der internationalen Praxis 225

I. Informationsangriffe auf Estland und Georgien 226

II. Informationsangriff durch Schadprogramm „Stuxnet“ 229

III. Informationsausbeutung durch Schadprogramme „Duqu“ und "Flame“ 230

IV. Informationsausbeutung durch Geheimdienste „Five Eyes“ 231

V. Informationsausbeutung durch Hackergruppe „Guardians of Peace“

VI. Informationsausbeutung und -angriff auf das „Democratic National Committee“ 233

C. Völkerrechtswidrigkeit 234

I. Informationskrieg und Informationskriegsführung 236

1. Informationsangriff als Gewalt im Sinne der VN-Charta 236

2. Informationsangriff als Angriff im Sinne des humanitären Völkerrechts 240

3. Informationskrieg und Informationskriegsführung in der internationalen Praxis 242

II. Informationsintervention 244

1. Informationstechnische Systeme als domaine réservé 245

2. Informationsangriff als unerlaubter Zwang 246

3. Informationseinmischung als Souveränitätsverletzung $\quad 247$

4. Informationsintervention in der internationalen Praxis 249

III. Informationsspionage 252

1. Völkerrechtliche Verbote zur Informationsspionage $\quad 252$

2. Informationsspionage in der internationalen Praxis 255

D. Zurechnung 256

I. Zurechnungsregeln 256 
II. Zurechnung in der internationalen Praxis 264

E. Verletzung positiver Verpflichtungen 268

I. Territorium bzw. Hoheitsgewalt 272

II. Reale Handlungsmöglichkeit 274

III. Kenntnis 277

IV. Positive Verpflichtungen in der internationalen Praxis $\quad 280$

F. Zusammenfassung 282

4. Kapitel: Staatenhaftung für informationstechnische Systeme 285

A. Anwendbarkeit von Umweltvölkerrecht in der virtuellen Welt 285

I. Analogiebasis 286

II. Bedürfnis der Übertragbarkeit 289

III. Vergleichbarkeit 291

IV. Ergebnis 294

B. Umsetzung der Staatenhaftung für informationstechnische Systeme 294

I. Anwendungsbereich 295

1. Schaden 295

2. (Hoch-)Gefährliche Aktivität 299

3. Kausalität 301

II. Schadensvermeidung und Schadenskompensation 304

1. Schadensprävention 305

2. Schadensvorsorge 307

a) Kooperation 308

b) Risikoanalyse 309

c) Beste Schutzpraktiken 309

3. Schadensminderung 311

4. Schadenskompensation 313

III. Gebotene Sorgfalt 313

IV. Haftungsart und -standard 316

1. Haftungsart 316

a) Primäre Staatenhaftung für staatliche und staatlich befürwortete Informationsoperationen 317

b) Subsidiäre und ergänzende Haftung des Ursprungsstaates für nicht-staatliche Informationsoperationen 
Inhaltsverzeichnis

c) Errichtung eines Entschädigungsfonds für eine subsidiäre Haftung bei nicht feststellbarem Ursprung der Informationsoperation und für eine ergänzende Haftung bei begrenzter Einstandspflicht des Ursprungsstaates

2. Haftungsstandard

Schlussbetrachtung

Bibliografie 


\section{Abkürzungsverzeichnis}

AEUV

AF Law Review

AIL

AJIL

Anlage VI

APuZ

Asia Pac. J. Envtl. L.

ASIL Proc.

ASLJ

ASR

Austral. YBIL

AVR

AYIL

Baltic YBIL

BGBl.

BJIL

BSDR

BULR

BYBIL

BZÜ

Cal. LR

Can. YBIL

Case Western Reserve JIL

CDR

Chi. JIL
Vertrag über die Arbeitsweise der Europäischen Union

Air Force Law Review

Draft Articles on international liability for injurious consequences arising out of acts not prohibited by international law

American Journal of International Law

Anlage VI des Antarktisumweltschutzprotokolls von 2005 über die Haftung bei umweltgefährdenden Notfällen

Aus Politik und Zeitgeschichte

Asia Pacific Journal of Environmental Law

American Society of International Law Proceedings

Arizona State Law Journal

Articles on the Responsibility of States for Internationally Wrongful Acts

Australian Yearbook of International Law

Archiv des Völkerrechts

Asian Yearbook of International Law

Baltic Yearbook of International Law

Bundesgesetzblatt

Berkeley Journal of International Law

Baltic Security \& Defence Review

Boston University Law Review

British Yearbook of International Law

Brüsseler Zusatzübereinkommen von 1963

California Law Review

Canadian Yearbook of International Law

Case Western Reserve Journal of International

Law

The Cyber Defense Review

Chicago Journal of International Law 
Chinese JIL

CJICL

CLJ

CLR

CLSR

Col. JTL

Colum. J. Envtl. L.

Cornell ILJ

CRAMRA

Denver JILP

DÜ

DVBl.

EG

EJIL

EJIL: Talk!

ELQ

ESIL Reflections

EU

EuG

EuGH

EuR

Fondsübereinkommen 1992

Fordham ILJ

GATT

GEI

Geo. JIL

GGE
Chinese Journal of International Law

Cambridge Journal of International and Comparative Law

Cambridge Law Journal

Cornell Law Review

Computer Law \& Security Review

Columbia Journal of Transnational Law

Columbia Journal of Environmental Law

Cornell International Law Journal

Übereinkommen zur Regelung der Tätigkeiten

im Zusammenhang mit mineralischen Ressourcen der Antarktis 1988

Denver Journal of International Law and Policy

Übereinkommen zur Durchführung des Teiles XI

des Seerechtsübereinkommens der Vereinten von 1994

Deutsches Verwaltungsblatt

Europäische Gemeinschaft

European Journal of International Law

Blog of the European Journal of International Law

Ecology Law Quarterly

European Society of International Law Reflections

Europäische Union

Gericht der Europäischen Union

Gerichtshof der Europäischen Union

Europarecht

Internationales Übereinkommen von 1992 über

die Errichtung eines Internationalen Fonds zur

Entschädigung für Ölverschmutzungsschäden

Fordham International Law Journal

General Agreement on Tariffs and Trade

Gericht Erster Instanz

Georgetown Journal of International Law

Groups of Governmental Experts on Developments in the Field of Information and Telecommunications in the Context of International Security 
GIELR

GILR

GOJIL

GP

GV Resolution

GWLR

GYIL

Haftungsprinzipien

Harv. ILJ

Harv. JOLT

Harv. NSJ

HIIG

HNS-Übereinkommen 2010

IACtHR

IBA

ICJ Reports

ICLQ

ICON

ICRC

IDI

IDI-Resolution

IGH

IGH-Statut

IJCIP

IJICL
Georgetown International Environmental Law Review

Georgetown International Law Review

Goettingen Journal of International Law

Gemeinsames Protokoll zur Anwendung des Wiener und Pariser Abkommens von 1988

Resolution der Generalversammlung der Vereinten Nationen

The George Washington Law Review

German Yearbook of International Law

Draft principles on the allocation of loss in the case of transboundary harm arising out of hazardous activities

Harvard International Law Journal

Harvard Journal of Law and Technology

Harvard National Security Journal

Alexander von Humboldt Institut für Internet und Gesellschaft

Internationales Übereinkommen über Haftung und Entschädigung für Schäden bei der Beförderung schädlicher und gefährlicher Stoffe auf See Interamerikanischer Gerichtshof für Menschenrechte

International Bar Association

International Court of Justice Reports

International and Comparative Law Quarterly

International Journal of Constitutional Law

International Committee of the Red Cross

Institut de Droit International

Institut de Droit International, Responsibility and Liability under International Law for Environmental Damage, Resolution of 4 September 1997

Internationaler Gerichtshof

Statut des Internationalen Gerichtshofs

International Journal of Critical Infrastructure Protection

Indonesian Journal of International \& Comparative Law 
ILA

ILC

ILM

ILSA J. Intl. \& Comp. L.

Ind. GLSJ

IStGHJ

ITLOS-Meeresbodenkammer

IWF-Übereinkommen

J. Envtl. L.

J. Marshall J. Computer \& Info. L.

JCSL

JIEL

JNSLP

JSS

$\mathrm{JuS}$

LCLR

LJIL

Loy. L.A. ICLR

McGill Int'l J. Sust. Dev. L. \& Pol'y

McGill LJ

Mich. JIL

Minn. JIL

MLR

MPEPIL

NATO

NATO CCD COE

NILR

NStZ
International Law Association

International Law Commission

International Legal Materials

ILSA Journal of International and Comparative Law

Indiana Journal of Global Legal Studies

Internationalen Strafgerichtshofs für das ehemalige Jugoslawien

Kammer für Meeresbodenstreitigkeiten des Internationalen Seegerichtshofs

Übereinkommen über den Internationalen Währungsfonds

Journal of Environmental Law

The John Marshall Journal of Information Technology \& Privacy Law

Journal of Conflict \& Security Law

Journal of International Economic Law

Journal of National Security Law and Policy

Journal of Strategic Security

Juristische Schulung

Lewis \& Clark Law Review

Leiden Journal of International Law

Loyola of Los Angeles International and Comparative Law Review

McGill International Journal of Sustainable Development Law and Policy

McGill Law Journal

Michigan Journal of International Law

Minnesota Journal of International Law

Mexican Law Review

Max Planck Encyclopedia of Public International Law

North Atlantic Treaty Organization

North Atlantic Treaty Organization Cooperative

Cyber Defence Centre of Excellence

Netherlands International Law Review

Neue Zeitschrift für Strafrecht 
Nw. JILB

NYIL

Ölhaftungsübereinkommen 1992

OLR

ÖZöRV

Pace Int'l L. Rev. Online

Companion

Präventionsartikel

PÜ

RdC

RECIEL

Rio Deklaration

SCM-Übereinkommen

Slg.

SLPR

SRÜ

StIGH

Stockholm Deklaration

SWP

SZR

TDM

Tex. ILJ

Tex. LR
Northwestern Journal of International Law and Business

Netherlands Yearbook of International Law

Internationales Übereinkommen von 1992 über die zivilrechtliche Haftung für Ölverschmutzungsschäden

Oregon Law Review

Österreichische Zeitschrift für öffentliches Recht und Völkerrecht

Pace International Law Review Online Companion

Draft articles on prevention of transboundary harm from hazardous activities

Pariser Übereinkommen über die Haftung gegenüber Dritten auf dem Gebiet der Kernenergie von 1960

Recueil des Cours. Académie de Droit International. Collected Courses of the Hague Academy of International Law

Review of European, Comparative and International Environmental Law

Rio Deklaration über Umwelt und Entwicklung von 1992

Agreement on Subsidies and Countervailing Measures

Sammlung der Rechtsprechung des Gerichtshofes und des Gerichts Erster Instanz

Stanford Law \& Policy Review

Seerechtsübereinkommen der Vereinten Nationen

Ständiger Internationaler Gerichtshof

Stockholm Deklaration der Konferenz der Vereinten Nationen über die Umwelt des Menschen von 1972

Stiftung Wissenschaft und Politik

Sonderziehungsrechten

Transnational Dispute Management

Texas International Law Journal

Texas Law Review 
TRIPS

ÜEE

ULR

UN Doc.

UNRIAA

UNTS

UPR

USP

Va JIL

$\mathrm{VN}$

VN-Charta

VN-Generalversammlung

VN-Wasserlaufkonvention

Weltraumhaftungsübereinkommen

Weltraumvertrag

WLR

Wm \& Mary LR

WTO

WÜ

WÜ 1997

Yale LJ

Yale LJ F

YBILC

YIEL

YIL
Agreement on Trade-Related Aspects of International Property Rights

Übereinkommen über ergänzende Entschädigung bei Nuklearschäden von 1997

Utrecht Law Review

United Nations Document

United Nations Reports of International Arbitral Awards

United Nations Treaty Series

Umwelt- und Planungsrecht

Umweltschutzprotokoll zum Antarktisvertrag 1991

Virginia Journal of International Law

Vereinte Nationen

Charta der Vereinten Nationen

Generalversammlung der Vereinten Nationen

Übereinkommen über das Recht der nichtschiff-

fahrtlichen Nutzung internationaler Wasserläufe von 1997

Übereinkommen über die völkerrechtliche Haftung für Schäden durch Weltraumgegenstände von 1972

Vertrag über die Grundsätze zur Regelung der Tätigkeiten von Staaten bei der Erforschung und Nutzung des Weltraums einschließlich des Mondes und anderer Himmelskörper von 1967

Washington Law Review

William \& Mary Law Review

World Trade Organization

Wiener Übereinkommen über die zivilrechtliche Haftung für nukleare Schäden von 1963

Wiener Übereinkommen über die zivilrechtliche Haftung für nukleare Schäden von 1997

Yale Law Journal

Yale Law Journal Forum

Yearbook of the International Law Commission

Yearbook of International Environmental

Yale Journal of International Law 
YIL Online

ZaöRV

ZfU

ZöR

ZUR

Zusatzfonds 2003
Yale Journal of International Law Online

Zeitschrift für ausländisches öffentliches Recht und Völkerrecht

Zeitschrift für Umweltpolitik \& Umweltrecht

Zeitschrift für Öffentliches Recht

Zeitschrift für Umweltrecht

Internationaler Zusatzentschädigungsfonds von 2003 für Ölverschmutzungsschäden 


\section{Gegenstand und Gang der Untersuchung}

Die einem Staat zurechenbare Verletzung seiner völkerrechtlichen Pflicht begründet als Konsequenz dessen Pflicht zur Beseitigung des völkerrechtswidrig verursachten Zustands und zur Wiedergutmachung. Diese Einstandspflicht wird als „Staatenverantwortlichkeit“ bezeichnet. ${ }^{1}$ Welche völkerrechtlichen Konsequenzen haben aber nachteilige Auswirkungen, die auf keiner Pflichtverletzung, also auf völkerrechtlich nicht verbotenen Aktivitäten beruhen? Die Arbeit thematisiert diese Fragestellung unter dem Begriff „Staatenhaftung“.

Die Notwendigkeit einer staatlichen Haftung für Aktivitäten, die etwa aus politischen, gesellschaftlichen, wirtschaftlichen oder wissenschaftlichen Gründen erlaubt sind, bei denen aber die Möglichkeit des Schadenseintritts auch bei Anwendung der gebotenen Sorgfalt nicht auszuschlieBen ist, zeigt sich in den verschiedensten Bereichen. ${ }^{2}$ Negative Auswirkungen von Atomunfällen oder Explosionen von Ölplattformen sowie von überriskanten Spekulationen an den Finanzmärkten, aber auch Gefahren durch Geoengineering, Gentechnologie und künstliche Intelligenz stellen die internationale Gemeinschaft vor Herausforderungen. Obwohl sich die Möglichkeiten zwischenstaatlicher Beeinträchtigungen aufgrund des technischen Fortschritts und der zunehmenden Globalisierung vervielfältigen, ${ }^{3}$ wird die Frage der Reglementierung von grenzüberschreitenden Schäden durch völkerrechtlich nicht verbotene Aktivitäten vornehmlich im Umweltkontext diskutiert. ${ }^{4}$ Das Bedürfnis nach einer völkerrechtlichen Schadensreglementierung wird aber auch und gerade bei Aktivitäten in der virtuellen Welt virulent. Informationstechnischen Systemen ist ein neuartiges Risiko immanent. Sie sind nicht nur Motor und Katalysator für gesellschaftliche und wirtschaftliche Entwicklungen, sondern bedeuten

1 Siehe hierzu J. Crawford, Brownlie's Principles of Public International Law, 2019, S. $523 \mathrm{ff}$.

2 L. F. E. Goldie, Liability for Damage and the Progressive Development of International Law, ICLQ 14 (1965), S. 1189 (1998 f.); G. Handl, Liability as an obligation established by a primary rule of international law, NYIL 16 (1985), S. 49 (64).

3 R. Higgins, International Law in a Changing International System, CLJ 58 (1999), S. 78 (82).

4 R. Lefeber, Transboundary Environmental Interference and the Origin of State Liability, 1996, S. 53. 
eine zunehmende Bedrohung für den weltweiten Frieden und die internationale Sicherheit sowie für eine freie und offene Gesellschaft. ${ }^{5}$ Die steigende staatliche, wirtschaftliche, industrielle und soziale Abhängigkeit von informationstechnischen Systemen bietet eine große Angriffsfläche. So sind sowohl private als auch staatliche Informationsinfrastrukturen schädigenden Aktivitäten ausgesetzt. ${ }^{6}$ Krieg, Terrorismus, Spionage und ähnliche konfliktbegründende Aktivitäten werden durch vernetzte informationstechnische Systeme in eine neue Dimension verlagert. ${ }^{7}$

Demzufolge stellt sich die zentrale Frage, ob die bestehenden völkerrechtlichen Regeln ausreichen, um auftretende Konflikte durch die Nutzung informationstechnischer Systeme zu begrenzen und der friedensstiftenden Funktion des Völkerrechts gerecht zu werden.

Vor diesem Hintergrund untersucht diese Arbeit im 1. Kapitel zunächst völkerrechtliche Rechtserkenntnisquellen und Rechtsquellen auf existente staatliche Einstandspflichten. Dabei ist die Unterscheidung zwischen dem Regime der Staatenverantwortlichkeit und dem Konzept der Staatenhaftung wesentlich. Die Herausbildung des Rechts der Staatenverantwortlichkeit hat gezeigt, dass verschiedene Konfliktlagen aufgrund völkerrechtswidrigen Verhaltens durch ein einheitliches Regelwerk reguliert werden können. Das Regime der Staatenverantwortlichkeit hat damit einen großen Anteil an einer strukturierten Völkerrechtsordnung. ${ }^{8}$ Es ist zu beleuchten, inwiefern auch das Konzept der Staatenhaftung für völkerrechtlich nicht verbotene Aktivitäten in dieser Ordnung verankert ist und zu deren Vereinheitlichung beiträgt.

Hierauf basierend wird sodann im 2. Kapitel ein allgemeingültiges Lösungsmodell mit klaren Parametern entwickelt, um grenzüberschreitende Schäden durch völkerrechtlich nicht verbotene Aktivitäten zu reglementieren. Das Konzept der Staatenhaftung muss dabei einen orientierenden Blick auf das Regime der Staatenverantwortlichkeit werfen, denn Staatenverantwortlichkeit und Staatenhaftung komplementieren sich.

Auf der Grundlage der gewonnen Erkenntnisse widmet sich die Arbeit im 3. Kapitel den völkerrechtlichen Pflichten von Staaten in der virtuellen Welt. Im Völkerrecht mangelt es derzeit an effektiven Regeln für die ge-

5 L. Crespo/B. Wanner/S. Ghernaouti, Cybersecurity Capacity Building, in: M. Bartsch/S. Frey (Hg.), Cybersecurity Best Practices, 2018, S. 525 (526).

6 D. B. Hollis, An e-SOS for Cyberspace, Harv. ILJ 52 (2011), S. 373 (375 f.).

7 T. Stein/T. Marauhn, Völkerrechtliche Aspekte von Informationsoperationen, ZaöRV 60 (2000), S. 1 (1 ff.).

8 C. Binder, Die Grenzen der Vertragstreue im Völkerrecht, 2013, S. 317. 
genwärtige Gefahr durch informationstechnische Systeme. Die internationale Debatte befasst sich insoweit mit völkerrechtlichen Verboten von Angriffen auf Informationsinfrastrukturen und Zurechnungsfragen. Damit rückt das Regime der Staatenverantwortlichkeit für völkerrechtswidriges Verhalten in der virtuellen Welt in den Fokus der internationalen Gemeinschaft. Zahlreiche virtuelle Konfliktlagen vergegenwärtigen aber, dass das Regime der Staatenverantwortlichkeit allein nicht geeignet ist, um grenzüberschreitenden negativen Auswirkungen durch informationstechnische Systeme zu begegnen. ${ }^{9}$

Daher legt die Arbeit schließlich im 4. Kapitel den Fokus auf eine risikoorientierte Haftung von Staaten, die informationstechnische Systeme ohne hinreichende Sicherung nutzbar und für Aktivitäten mit grenzüberschreitenden negativen Auswirkungen zugänglich machen. ${ }^{10}$ Schäden durch völkerrechtlich nicht verbotene, aber gefährliche Aktivitäten dürfen nicht zulasten der Opfer gehen, sondern müssen vielmehr demjenigen zugeschrieben werden, der das Risiko negativer Auswirkungen begründet und ein gewisses Maß an Kontrolle über diese Aktivitäten ausüben kann. ${ }^{11}$

Das Ziel dieser Arbeit ist, anhand der Problemstellungen in der virtuellen Welt nachzuweisen, dass das Konzept der Staatenhaftung durchaus völkerrechtliche Geltungsfähigkeit entfaltet und eine allgemeingültige Antwort auf internationale Gefährdungslagen durch völkerrechtlich nicht verbotene Aktivitäten bietet.

9 S.-H. Schulze, Cyber-,War“ - Testfall der Staatenverantwortlichkeit, 2015, S. 148 f.

10 Vgl. H. Krieger, Krieg gegen anonymous, AVR 50 (2012), S. 1 (7).

11 L. A. de la Fayette, International liability for damage to the environment, in: M. Fitzmaurice/D. M. Ong/P. Merkouris (Hg.), Research Handbook on International Environmental Law, 2010, S. 320 (327). 


\section{Kapitel: Staatenhaftung in der Systematik des Völkerrechts}

Die systematische Aufarbeitung der völkerrechtlichen Grundlagen staatlicher Einstandspflichten ist unerlässlich, um die Notwendigkeit der Staatenhaftung für neuartige Gefährdungslagen - wie sie in der virtuellen Welt bestehen - aufzeigen und deren Voraussetzungen definieren zu können. Ausgangspunkt der Untersuchung ist daher die Frage, ob das Konzept der Staatenhaftung völkerrechtlich verankert ist und inwiefern es sich vom Regime der Staatenverantwortlichkeit unterscheidet. Um eine Antwort auf diese Frage zu finden, sind die wichtigsten Rechtsquellen des Völkerrechts zu beleuchten. Das Statut des Internationalen Gerichtshofs (IGH-Statut) enthält in seinem Art. 38 Abs. 1 lit. a-c eine Aufzählung von im Völkerrecht bindenden Quellen. ${ }^{1}$ Die Haftung von Staaten kann demnach durch Völkervertragsrecht vorgegeben, in Völkergewohnheitsrecht erwachsen oder als allgemeiner Rechtsgrundsatz anerkannt sein. Der Nachweis des Bestehens eines völkerrechtlichen Haftungskonzepts unter Bezugnahme auf diese völkerrechtlichen Rechtsquellen gelingt mithilfe von Rechtserkenntnisquellen im Sinne des Art. 38 Abs. 1 lit.d IGH-Statut. Wissenschaftliche Arbeiten und Gerichtsentscheidungen reflektieren völkerrechtliche Vorgänge und legen ein grundlegendes Verständnis über spezifische Rechtsfragen dar. ${ }^{2}$ Dementsprechend werden zunächst Lehrmeinungen (A.) und richterliche Entscheidungen (B.) zu internationalen Einstandspflichten besprochen. Dieser Ansatz ermöglicht es ferner, das Konzept der Staatenhaftung von der Staatenverantwortlichkeit abzugrenzen sowie aus einem logischen Verständnis der sich überschneidenden Rechtskreise zu betrachten. Staatenpraxis und opinio iuris (C.) illustrieren zum einen die praktische Relevanz und Notwendigkeit eines allgemeinen völkerrechtlichen Regimes der Staatenhaftung und zum anderen dessen völkerrechtli-

1 Zur Frage, ob die Aufzählung von Rechtsquellen in Art.38 IGH-Statut abschließend oder etwa zu weitreichend ist, siehe G. Fitzmaurice, Some Problems Regarding the Formal Sources of International Law, in: M. Koskenniemi (Hg.), Sources of International Law, 2000, S. 57 (57 ff.); P. Malanczuk, Akehurst`s Modern Introduction to International Law, 1997, S. 36, $52 \mathrm{ff}$.

2 Vgl. A. Z. Borda, A Formal Approach to Article 38(1)(d) of the ICJ Statute from the Perspective of the International Criminal Courts and Tribunals, EJIL 24 (2013), S. 649 (654 ff.); A. E. Roberts, Traditional and modern approaches to customary international law: A reconciliation, AJIL 95 (2001), S. 757 (774 f.). 
che Bestandskraft. Aus der Untersuchung internationaler Übereinkünfte zu Einstandspflichten in grenzüberschreitenden Schadensfällen (D.) lassen sich Kernelemente internationaler Haftungssysteme abstrahieren. Schließlich sind die allgemeinen Rechtsgrundsätze (E.) geeignet, ein Fundament für ein völkerrechtliches Haftungsregime darzutun und dessen Komponenten universelle Geltung zu verleihen.

\section{A. Die Arbeiten der Völkerrechtskommission der Vereinten Nationen}

Die Völkerrechtskommission ist eine von den Vereinten Nationen im Jahre 1947 eingerichtete Kommission mit dem Auftrag zur Kodifizierung und Fortentwicklung des Völkerrechts. ${ }^{3}$ Die Kommission besteht aus internationalen Rechtsexperten, deren Arbeiten zu den Lehrmeinungen der fähigsten Völkerrechtler im Sinne des Art. 38 Abs. 1 lit. d IGH-Statut zählen ${ }^{4}$ oder auch analog zu Art. 38 Abs. 1 lit.d IGH-Statut als zusätzliches Instrument zur Bestimmung von völkerrechtlichen Regelungen angesehen werden. ${ }^{5}$ Außerdem helfen Überlegungen der Völkerrechtskommission zu zukünftigem Völkerrecht, ein Rechtsgebiet zu formen. Entsprechende Arbeiten der Kommission erwachsen nicht selten in geltendes Recht und werden von internationalen Gerichten zur Rechtsfindung herangezogen. ${ }^{6}$ In jedem Fall liefern die völkerrechtlichen Kodifikations- und Weiterentwicklungsbestrebungen der Völkerrechtskommission elementare Erkennt-

3 Durch GV Resolution (Resolution der Generalversammlung der Vereinten Nationen) 174 (II) vom 21.11.1947 wurde die Völkerrechtskommission (International Law Commission, ILC) als Nebenorgan der Generalversammlung der Vereinten Nationen gegründet und mit eigenem Statut versehen. Die Aufgaben der Völkerrechtskommission sind in Art. 1 Abs. 1 und Art. 15 ihres Statuts festgelegt. Näheres zur Funktion und Arbeitsweise der Kommission unter: http://legal.un.org/ilc/.

4 F. L. Bordin, Reflections of Customary International Law, ICLQ 63 (2014), S. 535 (537); S. Sivakumaran, The Influence of Teachings of Publicists on the Development of International Law, ICLQ 66 (2017), S. 1 (3 f.); M. Wood, Teachings of the Most Highly Qualified Publicists (Art. 38 (1) ICJ Statute), in: R. Wolfrum (Hg.), MPEPIL 2017, http://www.mpepil.com, Rn. 11.

5 A. Z. Borda, A Formal Approach to Article 38(1)(d) of the ICJ Statute from the Perspective of the International Criminal Courts and Tribunals, EJIL 24 (2013), S. 649 (656); M. Peil, Scholarly Writings as a Source of Law, CJICL 1 (2012), S. 136 (148).

6 F. L. Bordin, Reflections of Customary International Law, ICLQ 63 (2014), S. 535 (545); M. Peil, Scholarly Writings as a Source of Law, CJICL 1 (2012), S. 136 (146); M. Wood, Teachings of the Most Highly Qualified Publicists (Art. 38 (1) ICJ Statute), in: R. Wolfrum (Hg.), MPEPIL 2017, http://www.mpepil.com, Rn. 13. 


\section{Kapitel: Staatenhaftung in der Systematik des Völkerrechts}

nisse über ein Rechtsgebiet und sind mithin für die Fragestellung dieser Arbeit von essenzieller Bedeutung. ${ }^{7}$

Daher ist anhand der Arbeiten der Völkerrechtskommission der Vereinten Nationen und ihrer Sonderberichterstatter zu diesem Themenkomplex zu eruieren, ob ein Konzept der Staatenhaftung für völkerrechtlich nicht verbotene Aktivitäten im Völkerrecht Anerkennung findet. Die Problematik, die eine allgemeingültige Regulierung von risikobehafteten Aktivitäten umgibt, und die Schwierigkeiten einer Abgrenzung zwischen Staatenverantwortlichkeit für völkerrechtswidriges Verhalten und Staatenhaftung für völkerrechtlich nicht verbotene Aktivitäten spiegeln sich in den Diskussionen und Vorbereitungsarbeiten der Völkerrechtskommission der Vereinten Nationen zur Frage staatlicher Einstandspflichten wider. ${ }^{8}$

\section{Staatenverantwortlichkeit}

Im Jahr 1953 beauftragte die Generalversammlung der Vereinten Nationen (VN-Generalversammlung) die Völkerrechtskommission mit der Ausarbeitung eines Vertragsentwurfes zur Staatenverantwortlichkeit. ${ }^{9}$ Bereits zu Anbeginn legte die Kommission fest, dass das Projekt ausschließlich die Konsequenzen völkerrechtswidrigen Verhaltens behandeln wird, ohne dabei die Frage nach einer Verantwortlichkeit für rechtmäßige Aktivitäten

7 Die Völkerrechtskommission selbst betont die herausragende Bedeutung, die ihrer Arbeit etwa bei der Bestimmung von Völkergewohnheitsrecht zukommt. ILC, Report of the International Law Commission on the work of its seventieth session, UN Doc. A/73/10, 2018, S. 142 f., Rn. $1 \mathrm{f}$.

8 Neben der Völkerrechtskommission beschäftigen sich auch andere Expertengremien mit staatlichen Einstandspflichten für grenzüberschreitende Schäden. Siehe etwa Institut de Droit International (IDI), Eighth Commission, Francisco Orrego Vicuña (Rapporteur), Session of Strasbourg, 1997 - Second Part, Responsibility and Liability under International Law for Environmental Damage, Resolution of 4 September 1997, IDI-Yearbook 1998-67/II, S. 487-513 (im Folgenden: IDI-Resolution); Experts Group on Environmental Law of the World Commission on Environment and Development, R. D. Munro (Chairman)/J. G. Lammers (Rapporteur), Environmental Protection and Sustainable Development: Legal Principles and Recommendations, 1987; International Law Association (ILA), Dietrich Rauschning (Rapporteur), Montreal Rules of International Law Applicable to Transfrontier Pollution, UPR 1 (1983), S. 21-22. Im Folgenden wird auf die Arbeiten dieser Expertengremien nur insoweit verwiesen, wie sich aus den Regelungsvorschlägen Besonderheiten für das Konzept der Staatenhaftung ergeben.

9 GV Resolution 799 (VIII) vom 07.12.1953. 
vorwegzunehmen. ${ }^{10}$ Dieser Ansatz fand ebenso im Sechsten Ausschuss der VN-Generalversammlung breite Zustimmung. ${ }^{11}$

Nach jahrzehntelangen Arbeiten und unzähligen Debatten unter der Leitung verschiedener Sonderberichterstatter formulierte die Kommission schließlich 59 Artikel zur „Staatenverantwortlichkeit für völkerrechtswidrige Handlungen“, die im Jahr 2001 von der VN-Generalversammlung angenommen wurden. ${ }^{12}$ Der Anwendungsbereich der "Articles on the Responsibility of States for Internationally Wrongful Acts“ (ASR) ist bei Verletzung einer Primärnorm, das heißt bei Missachtung einer völkerrechtlichen Handlungs- oder Unterlassungspflicht, eröffnet. Das Regelwerk selbst besteht aus Sekundärnormen, die Prämissen für das einem Staat zurechenbare völkerrechtswidrige Verhalten sowie dessen mögliche Rechtfertigungsgründe und Rechtsfolgen festlegen. ${ }^{13}$

Auch wenn die ASR die Verantwortlichkeit für völkerrechtswidrige Handlungen regeln, betrifft Kapitel V der ASR Aspekte staatlicher Einstandspflichten für rechtmäßiges Verhalten. In diesem Kapitel sind verschiedene Unrechtsausschließungsgründe geregelt ${ }^{14}$ und Art. 27 lit. b ASR bestimmt, dass die Geltendmachung eines Umstandes, der die Rechtswidrigkeit nach Maßgabe von Kapitel V der ASR ausschließt, nicht die Frage des Schadensersatzes für materielle Verluste dem Grunde nach berührt. So entfällt zwar ein Völkerrechtsverstoß; der in die Rechte eines anderen

10 ILC, Report of the International Law Commission on the work of its thirtieth session, YBILC 1978-II/2, UN Doc. A/33/10, S. 149, Rn. 172.

11 Ibid.

12 Der Entwurf wurde durch die GV Resolution 56/83 vom 12.12.2001 angenommen und im Anhang zur Resolution abgedruckt. Der Kommentar der Völkerrechtskommission zu den Artikeln findet sich in ILC, Report of the International Law Commission on the work of its fifty-third session, Draft articles on responsibility of States for internationally wrongful acts, Text of the draft articles with commentaries thereto, YBILC 2001-II/2, UN Doc. A 56/10, S. 30 ff., Rn. 77 (im Folgenden: ASR).

13 Ausführlich zu den ASR siehe J. Crawford, The International Law Commission's Articles on State Responsibility, 2005.

14 Die Rechtswidrigkeit ist ausgeschlossen, wenn die Maßnahme als rechtmäßiger Notwehrakt angesehen werden kann (Art. 21 ASR), die Handlung eine völkerrechtskonforme Gegenmaßnahme darstellt (Art. 22 ASR) oder aufgrund höherer Gewalt notwendig wird (Art. 23 ASR). Ferner kann der Notlage der verantwortlichen Person (Art. 24 ASR) oder dem sogenannten Staatsnotstand (Art. 25 ASR) rechtfertigende Wirkung zukommen. Ein Verhalten kann auch gerechtfertigt sein, wenn es von zwingenden Völkerrechtsnormen gefordert wird (Art. 26 ASR). Von Bedeutung ist auch die Einwilligung des verletzten Staates als Rechtfertigungsgrund (Art. 20 ASR). 
Staates eingreifende Staat ist aber dennoch zum Ersatz der entstandenen Schäden verpflichtet.

\section{Staatenhaftung}

Die Notwendigkeit, zwischenstaatliche Schäden, die durch völkerrechtmäßige Aktivitäten entstehen, zu kompensieren, führte bereits im Rahmen der Arbeiten zur Staatenverantwortlichkeit zu Diskussionen innerhalb der Völkerrechtskommission. ${ }^{15}$ Die Kommission befand, dass die Verantwortlichkeit für unerlaubte Handlungen einerseits und die Verantwortlichkeit für Risiken durch erlaubte Aktivitäten andererseits auf grundverschiedenen Hypothesen beruhen und daher in separaten Projekten bearbeitet werden sollten. ${ }^{16}$

Die Thematik eines Interessenausgleiches für Schäden durch rechtmäßige risikobehaftete Aktivitäten wurde schließlich im Jahr 1973 unter der Überschrift „Internationale Haftung für schadhafte Folgen durch völkerrechtlich nicht verbotene Handlungen" auf die Agenda der Völkerrechtskommission gesetzt. ${ }^{17}$ Entscheidende Unterstützung erfuhr das Projekt insbesondere durch Entwicklungsländer, die aufgrund ihrer begrenzten industriellen Kapazitäten kaum von risikobehafteten Technologien profi-

15 ILC, Summary records of the twenty-fifth session, 1204th meeting, YBILC 1973-I, UN Doc. A/CN.4/217 and Add.1; A/CN.4/233; A/CN.4/246 and Add.1 to 3; A/ CN.4/264 and Add.1, S. 14. Ausführlich zu den Kontroversen innerhalb der Völkerrechtskommission siehe J. Barboza, The Environment, Risk and Liability in International Law, 2011, S. 73 ff.; A. E. Boyle, State Responsibility and International Liability for Injurious Consequences of Acts Not Prohibited by International Law, ICLQ 39 (1990), S. 1 (2).

16 ILC, Report of the International Law Commission on the work of its twenty-first session, YBILC 1969-II, UN Doc. A/7610/Rev.1, S. 233, Rn. 83; ILC, Report of the International Law Commission on the work of its twenty-fifth session, YBILC 1973-II, UN Doc. A/9010/Rev.1, S. 169, Rn. 38; ILC, Report of the International Law Commission on the work of its thirtieth session, YBILC 1978-II/2, UN Doc. A/33/10, S. 149, Rn. 172; Sonderberichterstatter R. Q. Quentin-Baxter, Preliminary report on international liability for injurious consequences of acts not prohibited by international law, YBILC 1980-II/1, UN Doc. A/CN.4/334 and Add.l and 2, S. 253, Rn. 19.

17 ILC, Report of the International Law Commission on the work of its twentysixth session, YBILC 1974-II/1, UN Doc. A/9610/Rev.1, S.305, Rn. 163, International liability for injurious consequences arising out of the performance of activities other than internationally wrongful acts (im Folgenden: Haftungsstudie). 
tieren, aber oftmals zum Opfer grenzüberschreitender Schäden durch derartige Aktivitäten werden. ${ }^{18}$

Auf Veranlassung der VN-Generalversammlung ${ }^{19}$ gründete die Kommission im Jahr 1978 eine entsprechende Arbeitsgruppe und benannte Robert Q. Quentin-Baxter zum ersten Sonderberichterstatter. ${ }^{20}$ Sinn und Zweck der Haftungsstudie ergeben sich laut Quentin-Baxter aus der Tatsache, dass gerade nicht alle grenzüberschreitenden Schäden völkerrechtswidrig sind. ${ }^{21}$

Die Arbeitsgruppe von 1978 bekräftigte, dass Staatenhaftung einerseits und Staatenverantwortlichkeit andererseits in unterschiedlichen Regelungsbereichen zu verorten sind. ${ }^{22}$ Die Staatenverantwortlichkeit entwickelte sich ursprünglich aus der Notwendigkeit heraus, Ausländer vor Rechtsverletzungen auf fremdstaatlichem Hoheitsgebiet zu schützen. ${ }^{23}$ Die Staatenhaftung hatte ihren Ursprung hingegen in der Regelungsbedürftigkeit moderner risikobehafteter Aktivitäten. ${ }^{24}$ Entsprechend zog die Ar-

18 J. Barboza, The Environment, Risk and Liability in International Law, 2011, S. 116, 117; P. Stubbe, State Accountability for Space Debris, 2018, S. 107 f.

19 GV Resolution 32/151 vom 19.12.1977, S. 214 f., Rn. 7.

20 ILC, Report of the International Law Commission on the work of its thirtieth session, YBILC 1978-II/2, UN Doc. A/33/10, S. $149 \mathrm{ff} .$, Rn. $170 \mathrm{ff}$.

21 Sonderberichterstatter R. Q. Quentin-Baxter stellte zutreffend fest: „If all transboundary harm were wrongful, there would be no need for this topic. Every activity that caused or threatened such harm would be prohibited, except with the consent of the States whose interest was affected." Sonderberichterstatter $R$. Q. Quentin-Baxter, Second report on international liability for injurious consequences arising out of acts not prohibited by international law, YBILC 1981-II/1, UN Doc. A/CN.4/346 and Add.1 and 2, S. 122, Rn. 85. Vgl. auch die Resolution der IDI, in der zwischen Staatenverantwortlichkeit aufgrund einer Pflichtverletzung (Art. 1 Abs. 1 IDI-Resolution) und Einstandspflichten allein aufgrund von Schäden (Art. 1 Abs. 2 IDI-Resolution) differenziert wird.

22 ILC Working Group, Report of the Working Group on international liability for injurious consequence arising out of acts not prohibited by international law, UN Doc. A/CN.4/L.284 and Corr 1., in: ILC, Report of the International Law Commission on the work of its thirtieth session, YBILC 1978-II/2, UN Doc. A/33/10, Annex, S. 151, Rn. 15, 24. Die Arbeitsgruppe setzte sich zusammen aus Robert Q. Quentin-Baxter (Vorsitzender), Roberto Ago, Jorge Castañeda und Frank X. J. C. Njenga. ILC, Report of the International Law Commission on the work of its thirtieth session, YBILC 1978-II/2, UN Doc. A/33/10, S. 149, Rn. 171.

23 Y. Matsui, The Transformation of the Law of State Responsibility, in: R. Provost (Hg.), State Responsibility in International Law, 2002, S. 17.

24 ILC Working Group, Report of the Working Group on international liability for injurious consequence arising out of acts not prohibited by international law, UN Doc. A/CN.4/L.284 and Corr 1., in: ILC, Report of the International 


\section{Kapitel: Staatenhaftung in der Systematik des Völkerrechts}

beitsgruppe diesbezüglich Vergleiche zu Technologien der Nuklearindustrie, zu Aktivitäten im Weltraum, zur Nutzung gemeinsamer Ressourcen, zum Erdöltransport zur See sowie zur Meeresverschmutzung ${ }^{25}$ und zu Nachteilen, die Anrainerstaaten bezüglich ihrer Wasserläufe erleiden. ${ }^{26}$ Die Arbeitsgruppe betonte zudem, dass die Staatenhaftung - im Unterschied zur Staatenverantwortlichkeit - keine Sekundärnormen, sondern Primärnormen zum Gegenstand hat. ${ }^{27}$

Seit die Staatenhaftung im Jahr 1978 in das Arbeitsprogramm der Völkerrechtskommission aufgenommen wurde, entstanden zahlreiche Berichte und Regelungsentwürfe zu diesem Themenkomplex. ${ }^{28}$ Der erste Sonderberichterstatter Robert Q. Quentin-Baxter entwarf im Rahmen von fünf Berichten fünf Artikel zur Staatenhaftung und in seiner Schematic Outline eine Übersicht der Grundpfeiler eines Staatenhaftungskonzeptes. Diese Entwürfe wurden allerdings nicht dem Redaktionsausschuss der Kommission übergeben. Im Jahr 1985 ernannte die Kommission Julio Barboza dann zum neuen Sonderberichterstatter für die Haftungsstudie. Barboza erarbeitete bis zum Jahr 1996 insgesamt zwölf Berichte. Die Kommission nahm sukzessive in den Jahren 1994 und 1995 einige Artikelentwürfe vorläufig an. Die Arbeitsgruppe revidierte schließlich im Jahr 1996 die bisherigen Arbeiten und übergab einen überarbeiteten Entwurf an die Generalversammlung zur Kommentierung (1. Staatenhaftung - Kartografie der Völkerrechtskommission).

Trotz der vorläufigen Annahme der Artikelentwürfe entschied die Kommission im Jahr 1997, die komplette Thematik von Neuem zu überarbeiten. Die Studie wurde aufgeteilt in "Prävention grenzüberschreitender Schäden aus gefährlichen Aktivitäten“ sowie „Internationale Haftung im Schadensfall durch grenzüberschreitende Schäden aus gefährlichen Aktivi-

Law Commission on the work of its thirtieth session, YBILC 1978-II/2, UN Doc. A/33/10, Annex, S. 151, Rn. 10.

25 Id., S. 150, Rn. 12.

26 Id., S. 151, Rn. 19.

27 Id., S. 151, Rn. 24.

28 Siehe Analytical Guide to the Work of the International Law Commission, International liability for injurious consequence arising out of acts not prohibited by international law, abrufbar unter: http://legal.un.org/ilc/guide/9.shtml (geprüft am 15.05.2020). 
täten "29 und Pemmaraju Sreenivasa Rao wurde zum neuen Sonderberichterstatter ernannt ${ }^{30}$ (2. Staatenhaftung - „a case of misplaced emphasis“?).

Diese Kehrtwende erscheint umso erstaunlicher, als ein Jahr zuvor eine andere Arbeitsgruppe das Werk der Kommission und ihrer Sonderberichterstatter bereits revidiert und einen Entwurf von Artikeln an die VN-Generalversammlung zur Kommentierung übersandt hatte. Jedenfalls spiegeln diese Entwicklungen in der Völkerrechtskommission sehr eindrücklich die Kontroversen dieser Thematik. ${ }^{31}$ So negierte eine beachtliche Anzahl an Kommissionsmitgliedern und gewichtigen Stimmen von Staatenvertretern aus dem Sechsten Ausschuss der VN-Generalversammlung die Existenz einer völkerrechtlichen Staatenhaftung und lehnte eine Einschränkung staatlicher Souveränität aufgrund völkerrechtlich nicht verbotener Aktivitäten entschieden ab. ${ }^{32}$ Gleichwohl befürwortete die VN-Generalversammlung im Ergebnis mehrheitlich die Fortführung der Arbeiten an der Haftungsstudie. ${ }^{33}$

\section{Staatenhaftung - Kartografie der Völkerrechtskommission}

Das ursprüngliche Ziel des Projektes war eine Art Vorbild und Anstoß für Abkommen zu schaffen, die einen gerechten Interessenausgleich zwischen Staaten im Bereich spezifischer erlaubter Aktivitäten mit möglichen schä-

29 Prevention of transboundary damage from hazardous activities and international liability in case of loss from transboundary harm arising out of hazardous activities, ILC, Report of the International Law Commission on the work of its forty-ninth session, YBILC 1997-II/2, UN Doc. A/52/10, S. 59, Rn. $165 \mathrm{ff}$. bezugnehmend auf ILC Working Group, Report of the Working Group on international liability for injurious consequences arising out of acts not prohibited by international law, 1997, UN Doc. A/CN.4/L.536, S. 2, Rn. 3.

30 ILC, Report of the International Law Commission on the work of its forty-ninth session, YBILC 1997-II/2, UN Doc. A/52/10, S. 9, Rn. 17.

31 A. E. Boyle bezeichnet das Projekt der Völkerrechtskommission gar als "one of the most laboured and confusing studies the ILC has ever undertaken." $A$. E. Boyle, Liability for injurious consequence arising out of acts not prohibited by international law, in: J. Crawford/A. Pellet/S. Olleson (Hg.), The Law of International Responsibility, 2010, S. 95.

32 Siehe J. Barboza, The Environment, Risk and Liability in International Law, 2011, S.77; A. E. Boyle, Liability for injurious consequence arising out of acts not prohibited by international law, in: J. Crawford/A. Pellet/S. Olleson (Hg.), The Law of International Responsibility, 2010, S. 98.

33 Siehe J. Barboza, The Environment, Risk and Liability in International Law, 2011, S. 77. 


\section{Kapitel: Staatenhaftung in der Systematik des Völkerrechts}

digenden Auswirkungen erzielen. ${ }^{34}$ Gleichzeitig sollte das zu schaffende Regelwerk subsidiär gelten und damit nur Konstellationen erfassen, die unter kein spezielles Abkommen fallen. ${ }^{35}$

Das Grundgerüst des Projektes stellte der erste Sonderberichterstatter Quentin-Baxter in der Schematic Outline ${ }^{36}$ vor und definierte die sogenannte zusammengesetzte Primärpflicht (compound primary obligation) als Kern der Staatenhaftung. ${ }^{37}$ Die Pflichten zur Schadensprävention, Schadensminderung und Schadenskompensation stellen nach dieser Konzeption des ersten Sonderberichterstatters eine Einheit von verhaltensbezogenen Primärpflichten der Staatenhaftung dar. ${ }^{38}$ Dabei sei Wiedergutmachung gleichbedeutend mit Schadensvermeidung, denn „[r]eparation has always the purpose of restoring as fully as possible a pre-existing situation; and in the context of the present topic, it may often amount to prevention after the event. "'39 Wesentlich für die Konzeption von Quentin-Baxter ist, dass die Verletzung von Pflichten zur Schadensvermeidung nicht zu Konsequenzen nach dem Regime der Staatenverantwortlichkeit führt. ${ }^{40} \mathrm{Im}$ Gegenteil greift der Wiedergutmachungsmechanismus der Staatenhaftung, und zwar sowohl bei Beachtung als auch bei Verletzung der Schadensvermeidungs-

34 Siehe S. Erichsen, Das Liability-Projekt der ILC, ZaöRV 51 (1991), S. 94 (100); T. Gehring/M. Jachtenfuchs, Liability for Transboundary Environmental Damage Towards a General Liability Regime?, EJIL 4 (1993), S. 92 (95).

35 Siehe J. Barboza, The Environment, Risk and Liability in International Law, 2011, S. 106; S. Erichsen, Das Liability-Projekt der ILC, ZaöRV 51 (1991), S. 94 (100).

36 Sonderberichterstatter R. Q. Quentin-Baxter, Third report on international liability for injurious consequences arising out of acts not prohibited by international law, YBILC 1982-II/1, UN Doc. A/CN.4/360, S. 62, Rn. 53.

37 Ders., Fourth report on international liability for injurious consequences arising out of acts not prohibited by international law, YBILC 1983-II/1, UN Doc. A/ CN.4/373, S. 213, Rn. 40.

38 Ibid.; R. Lefeber bekräftigt das Bestehen einer einzigen zusammengesetzten Pflicht der völkerrechtlich haftenden Staaten „to take all procedural and substantive measures that may be necessary to avoid, minimize and repair environmental interference causing significant harm during the life cycle of an activity." $R$. Lefeber, Transboundary Environmental Interference and the Origin of State Liability, 1996, S. 34; siehe auch D. Magraw, Transboundary Harm, AJIL 80 (1986), S. 305 (311).

39 Sonderberichterstatter R. Q. Quentin-Baxter, Fourth report on international liability for injurious consequences arising out of acts not prohibited by international law, YBILC 1983-II/1, UN Doc. A/CN.4/373, S. 214, Rn. 47.

40 An diesem Aspekt wird bemängelt, er führe zu konzeptionellen Schwierigkeiten und hindere die Umsetzbarkeit der Staatenhaftung. So etwa J. Barboza, Liability, Chinese JIL 1 (2002), S. 499 (501); L. de la Fayette, The ILC and International Liability, RECIEL 6 (1997), S. 322 (323). 
pflichten. Quentin-Baxter konzipierte also eine verschuldensunabhängige Haftung von Staaten. ${ }^{41}$ Die Verletzung der Schadensvermeidungspflichten stellt zwar keine Völkerrechtswidrigkeit dar, ${ }^{42}$ kann aber beispielsweise zu einer erhöhten Kompensationspflicht führen oder Beweiserleichterungen bei der Ermittlung der Höhe des Schadensausgleiches für den betroffenen Staat bedeuten. ${ }^{43}$

Erst die Verletzung der letzten in dem Haftungskonzept verankerten Pflicht - die Verletzung der Wiedergutmachungspflicht - führt zur Bejahung der Völkerrechtswidrigkeit und damit zum Greifen der Staatenverantwortlichkeit. ${ }^{44}$ In dem Entwurf zur Staatenhaftung des ersten Sonderberichterstatters selbst sind demnach keine Elemente staatlicher Einstandspflichten für völkerrechtswidriges Verhalten enthalten. ${ }^{45}$ Dementsprechend können die staatlichen Einstandspflichten aus Staatenverant-

41 Dieses Konzept wird dahingehend kritisiert, dass es die Pflichten zur Schadensvermeidung verhaltensbezogen ausgestaltet (obligation of conduct), gleichzeitig aber eine ergebnisbezogene Wiedergutmachungspflicht (obligation of result) begründet. J. Barboza etwa moniert: „Besides, a liability system imposing due diligence obligations of prevention and, whether or not they are satisfied, compensation if damage occurs may be contradictory: it would mean establishing obligations of a dual nature-i.e., of due diligence and of result at the same time and on the same subject." J. Barboza, The Environment, Risk and Liability in International Law, 2011, S. 81. So auch L. de la Fayette, The ILC and International Liability, RECIEL 6 (1997), S. 322 (327). Diese Kritik verkennt allerdings, dass die Verletzung der gebotenen Sorgfalt im Ergebnis durch das Ausmaß der Schadenskompensation sanktioniert wird und damit erst eine Differenzierung zwischen verschuldensabhängiger und verschuldensunabhängiger Haftung - wie sie etwa im Regime der Weltraumhaftung vorgesehen ist - ermöglicht. Vgl. P. Stubbe, State Accountability for Space Debris, 2018, S. $391 \mathrm{ff}$.

42 Vgl. auch Art. 4 Abs. 2 IDI-Resolution, der klarstellt, dass das Versäumnis von Staaten, geeignete Regeln und Kontrollmechanismen zur Implementierung von Umweltregimen einzuführen, nicht notwendigerweise eine Pflichtverletzung bedeutet.

43 Schematic Outline, Section 2, Sonderberichterstatter R. Q. Quentin-Baxter, Third report on international liability for injurious consequences arising out of acts not prohibited by international law, YBILC 1982-II/1, UN Doc. A/CN.4/360, S. 62 ff., Rn. 53; J. Barboza, The Environment, Risk and Liability in International Law, 2011, S. 108.

44 Sonderberichterstatter R. Q. Quentin-Baxter, Third report on international liability for injurious consequences arising out of acts not prohibited by international law, YBILC 1982-II/1, UN Doc. A/CN.4/360, S. 53, Rn. 10.

45 Schematic Outline, Section 2, paragraph 8, Sonderberichterstatter R. Q. QuentinBaxter, Third report on international liability for injurious consequences arising out of acts not prohibited by international law, YBILC 1982-II/1, UN Doc. A/ CN.4/360, S. 62 ff., Rn. 53. 


\section{Kapitel: Staatenhaftung in der Systematik des Völkerrechts}

wortlichkeit und Staatenhaftung nebeneinander bestehen und schließen sich nicht gegenseitig aus. ${ }^{46}$ Nach Quentin-Baxters Regelungsmethode lässt sich dogmatisch eine klare Trennung zwischen Staatenhaftung und Staatenverantwortlichkeit vornehmen. ${ }^{47}$

Die Präventionskomponente wurde allerdings als "alien element“ in der Haftungsthematik bezeichnet. ${ }^{48}$ Kritiker des Konzepts sehen hierin einen Fremdkörper im System der Staatenhaftung, da die Verletzung entsprechender Pflichten zur Staatenverantwortlichkeit für völkerrechtswidriges Verhalten führen müsse, ${ }^{49}$ während die Staatenhaftung ohne Völkerrechtsverletzung zur Anwendung gelange. Entsprechend fänden sich in Haftungssystemen für erlaubte Tätigkeiten in der Regel keine Präventionspflichten..$^{50}$ Es bestehe kein Anreiz für Staaten, Präventionspflichten zu erfüllen, wenn in jedem Fall eine Schadensausgleichspflicht greife. ${ }^{51}$ Dies verkennt jedoch, dass das System von Quentin-Baxter die Missachtung von Präventionspflichten auf Ebene der Wiedergutmachungspflicht berücksichtigt. Denn die (Kompensations-)Pflichten, die den Staat, auf dessen Hoheitsgebiet oder unter dessen Hoheitsgewalt die schadensauslösende Aktivität stattfindet (Ursprungsstaat), treffen, müssen nach Treu und Glau-

46 Sonderberichterstatter R. Q. Quentin-Baxter, Second report on international liability for injurious consequences arising out of acts not prohibited by international law, YBILC 1981-II/1, UN Doc. A/CN.4/346 and Add.1 and 2, S. 105, Rn.9, S. 107, Rn. 17; A. Randelzhofer, Probleme der völkerrechtlichen Gefährdungshaftung, in: D. Rauschning/ders. (Hg.), Staatenverantwortlichkeit, 1984, S. 61.

47 M. B. Akehurst, International Liability for Injurious Consequences out of Acts not Prohibited by International Law, NYIL 16 (1985), S. 3 (14 f.); S. Erichsen, Das Liability-Projekt der ILC: Fortentwicklung des allgemeinen Umweltrechts oder Kodifizierung einer Haftung für besonders gefährliche Aktivitäten?, ZaöRV 51 (1991), S. 94 (105); D. B. Magraw, Transboundary Harm, AJIL 80 (1986), S. 305 (317).

48 J. Barboza, Liability, Chinese JIL 1 (2002), S. 499 (500).

$49 \mathrm{~J}$. Barboza kritisiert die Idee einer zusammengesetzten Primärpflicht, da „[t]he idea of a compound primary norm, with due respect, does not seem correct. The breach of prevention rules leads to the realm of responsibility and therefore to secondary norms imposing obligations of a secondary nature." J. Barboza, The Environment, Risk and Liability in International Law, 2011, S. 79. So auch L. de la Fayette, The ILC and International Liability, RECIEL 6 (1997), S. 322 (323).

50 Sonderberichterstatter $J$. Barboza, Tenth report on international liability for injurious consequences arising out of acts not prohibited by international law, YBILC 1994-II/1, UN Doc. A/CN.4/459, S. 134, Rn. 25; J. Barboza, Liability, Chinese JIL 1 (2002), S. 499 (500); ders., The Environment, Risk and Liability in International Law, 2011, S. 81.

51 J. Barboza, Liability, Chinese JIL 1 (2002), S. 499 (500); L. de la Fayette, The ILC and International Liability, RECIEL 6 (1997), S. 322 (327). 
ben unter Berücksichtigung der sogenannten gemeinsamen Erwartungen ausgehandelt werden. ${ }^{52}$ Dabei stellen gerade auch die vom Ursprungsstaat ergriffenen Präventionsmaßnahmen einen wesentlichen Faktor dar. ${ }^{53}$

Der zweite Sonderberichterstatter Julio Barboza folgte zunächst dem Konzept von Quentin-Baxter. ${ }^{54}$ So bestätigte Barboza in seinen ersten fünf Berichten, dass der Staat für Schäden durch völkerrechtlich nicht verbotene Aktivitäten völkerrechtlich zu haften hat. ${ }^{55}$ In seinem sechsten Bericht leitete Barboza dann einen Richtungswechsel ein, indem er erstmals privatrechtliche Haftungsvorschriften in die Haftungsstudie einbrachte. ${ }^{56}$ Auch wenn Artikel 9 im sechsten Bericht immer noch eine staatliche

52 Schematic Outline, Section 4, 6, Sonderberichterstatter R. Q. Quentin-Baxter, Third report on international liability for injurious consequences arising out of acts not prohibited by international law, YBILC 1982-II/1, UN Doc. A/CN.4/360, S. 62 ff., Rn. 53; J. Barboza, The Environment, Risk and Liability in International Law, 2011, S. $108 \mathrm{f}$.

53 Schematic Outline, Section 2, 3, Sonderberichterstatter R. Q. Quentin-Baxter, Third report on international liability for injurious consequences arising out of acts not prohibited by international law, YBILC 1982-II/1, UN Doc. A/CN.4/360, S. 62 ff., Rn. 53; vgl. S. Erichsen, Das Liability-Projekt der ILC, ZaöRV 51 (1991), S. 94 (106f.).

54 Sonderberichterstatter J. Barboza, Preliminary report on international liability for injurious consequences arising out of acts not prohibited by international law, YBILC 1985-II/1, UN Doc. A/CN.4/394, S. 99 f., Rn.13f. Die Diskussionen im Rahmen der ersten fünf Berichte des zweiten Sonderberichterstatters zu ergänzenden Bestimmungen im Konzept der Staatenhaftung stellten keine wesentliche Abkehr vom Konzept des ersten Sonderberichterstatters dar. So forderte J. Barboza unter Bezugnahme auf die Korfu Kanal-Entscheidung etwa, dass (hypothetische) Kenntnis von der risikobehafteten Aktivität auf staatseigenem Territorium gegeben sein muss, damit eine staatliche Haftung für zwischenstaatliche Schäden greife. Die Klausel solle Entwicklungsländer angesichts ihrer mangelnden technischen Kapazitäten vor zu hohen Anforderungen schützen. Siehe Art. 4, Sonderberichterstatter J. Barboza, Third report on international liability for injurious consequences arising out of acts not prohibited by international law, YBILC 1987-II/1, UN Doc. A/CN.4/405, S. 55 f., Rn. 65 ff. Diese Voraussetzung wurde richtigerweise in späteren Berichten aufgegeben. Denn, entgegen der Auffassung des zweiten Sonderberichterstatters, ist Kenntnis Voraussetzung für die Staatenverantwortlichkeit aufgrund der Verletzung positiver Handlungspflichten und nicht für die Staatenhaftung, die allenfalls Vorhersehbarkeit einer Schädigung erfordert.

55 J. Barboza, The Environment, Risk and Liability in International Law, 2011, S. 103.

56 Sonderberichterstatter J. Barboza, Sixth report on international liability for injurious consequences arising out of acts not prohibited by international law, YBILC 1990-II/1, UN Doc. A/CN.4/428 and Add.1, S. 98 f., Rn. 62. 
Einstandspflicht begründet, ${ }^{57}$ gewann in dieser Phase somit auch eine private Haftung an Bedeutung. Neben den zwischenstaatlichen Verhandlungen zum Schadensausgleich sollte zusätzlich eine effektive Möglichkeit für Opfer grenzüberschreitender Schädigungen geschaffen werden, den privaten innerstaatlichen Rechtsweg zu wählen. ${ }^{58}$ In seinem zehnten Bericht vollzog Barboza dann einen umfassenden Paradigmenwechsel. ${ }^{59}$ Der Bericht charakterisiert die Präventionspflichten als rechtsverbindliche Vorschriften (,hard“ preventive obligations), deren Verletzung zu Konsequenzen nach dem Recht der Staatenverantwortlichkeit führt. ${ }^{60}$ Darüber hinaus ersetzt der Bericht die Idee einer Staatenhaftung komplett durch eine verschuldensunabhängige Haftung Privater für Schäden durch risikobehaftete nicht verbotene Aktivitäten. ${ }^{61}$ Laut Barboza selbst erfuhr der zehnte Bericht allerdings keine weitere Beachtung durch die Kommission, ${ }^{62}$ so dass dieser für das Staatenhaftungskonzept der Völkerrechtskommission zumindest in dieser Phase - eine untergeordnete Rolle einnimmt.

Die Arbeitsgruppe aus dem Jahr 1996 konsolidierte schließlich die bisherigen Arbeiten der Völkerrechtskommission und entwarf einen Kodifizierungsvorschlag unter Berücksichtigung vorläufig genehmigter Regelungsentwürfe, der Berichte der beiden Sonderberichterstatter sowie dem Diskussionsstand im Rahmen der Kommission und der VN-Generalversammlung zu diesem Themenkomplex. ${ }^{63}$ Der Entwurf zur „Internationalen Haftung für schadhafte Folgen durch völkerrechtlich nicht verbotene Handlungen“ aus dem Jahr 1996 enthält im Ergebnis 22 Artikel mit entsprechenden Kommentaren zum Themenkomplex Schadensvermeidung (Prevention) und Wiedergutmachung (Compensation or Other Relief). Die

Id., S. 91, Rn. 28.

58 Id., S. 109, Rn. 63.

59 Sonderberichterstatter J. Barboza, Tenth report on international liability for injurious consequences arising out of acts not prohibited by international law, YBILC 1994-II/1, UN Doc. A/CN.4/459, S. 129 ff.; J. Barboza, The Environment, Risk and Liability in International Law, 2011, S. 83.

60 Sonderberichterstatter J. Barboza, Tenth report on international liability for injurious consequences arising out of acts not prohibited by international law, YBILC 1994-II/1, UN Doc. A/CN.4/459, S. 136, Rn. 30 ff.; J. Barboza, The Environment, Risk and Liability in International Law, 2011, S. 83.

61 Sonderberichterstatter Barboza, Tenth report on international liability for injurious consequences arising out of acts not prohibited by international law, YBILC 1994-II/1, UN Doc. A/CN.4/459, S. 138, Rn. 42.

62 J. Barboza, The Environment, Risk and Liability in International Law, 2011, S. 83, Fn. 29.

63 Id., S. 109. 
„Draft Articles on international liability for injurious consequences arising out of acts not prohibited by international law" (AIL) ${ }^{64}$ basieren auf dem Grundgedanken, dass Staaten grundsätzlich frei sind, risikobehaftete Aktivitäten auf ihrem Hoheitsgebiet bzw. unter ihrer Hoheitsgewalt zu erlauben; diese souveräne Freiheit unterliegt allerdings gewissen Grenzen, sofern andere Staaten betroffen sind (vgl. Art. 3 AIL). ${ }^{65}$ Dabei orientieren sich die AIL an dem Leitsatz, dass das Opfer nicht den gesamten Schaden tragen darf (Art. 19 und 21 AIL), und sind unter dem Blickwinkel eines gerechten Interessenausgleiches ausgestaltet. ${ }^{66}$

\section{a) Anwendungsbereich gemäß den AIL von 1996}

Der Anwendungsbereich der AIL bezieht sich gemäß seines Art. 1 lit. a auf Aktivitäten, die völkerrechtlich nicht verboten sind und denen das Risiko eines erheblichen grenzüberschreitenden Schadens immanent ist.

Unrechtmäßiges Verhalten im Sinne der ASR fällt aus dem Anwendungsbereich der AIL heraus. Andernfalls müsste die Aktivität gemäß Art. 30 ASR beendet und nicht lediglich Wiedergutmachung im Sinne der AIL gewährt werden. Dies wäre mit Blick auf Aktivitäten, die nicht völkerrechtlich verboten sind, unangemessen und in Anbetracht der Vorteile, die aus solchen Aktivitäten regelmäßig hervorgehen, auch nicht praktikabel. Sonderberichterstatter Quentin-Baxter erklärte, dass der Vorwurf der

64 ILC Working Group, Report of the Working Group on international liability for injurious consequence arising out of acts not prohibited by international law, Draft Articles on international liability for injurious consequences arising out of acts not prohibited by international law with Commentaries, UN Doc. A/CN.4/ L.533 and Add.1, in: ILC, Report of the International Law Commission on the work of its forty-eighth session, YBILC 1996-II/2, UN Doc. A/51/10, Annex I, S. 100-132, Rn. 6 (im Folgenden: AIL).

65 Vgl. auch Experts Group on Environmental Law of the World Commission on Environment and Development, R. D. Munro (Chairman)/J. G. Lammers (Rapporteur), Environmental Protection and Sustainable Development: Legal Principles and Recommendations, 1987, S. $80 \mathrm{ff}$.

66 Damit folgen die AIL Prinzip 21 der Stockholm Deklaration der UN-Konferenz über die menschliche Umwelt vom 16. Juni 1972: „States have, in accordance with the Charter of the United Nations and the principles of international law, the sovereign right to exploit their own resources pursuant to their own environmental policies, and the responsibility to ensure that activities within their jurisdiction or control do not cause damage to the environment of other States or of areas beyond the limits of national jurisdiction. "UN Doc. A/CONF.48/14 (1972), S. 5 . 


\section{Kapitel: Staatenhaftung in der Systematik des Völkerrechts}

Völkerrechtswidrigkeit keine zweckmäßige Antwort auf neue Gefahrenlagen, etwa durch moderne Technologien, bietet. Aktivitäten, die der Gesellschaft hauptsächlich nutzen, seien trotz schädigender Nebenwirkungen nicht zu beschränken, sondern zu regulieren. ${ }^{67}$

Staaten können aber nur bei vorhersehbarer Gefährdung einen Regelungsbedarf erkennen. ${ }^{68}$ Dies ist mit Blick auf alle möglichen schädigenden Einzelakte (acts) nicht der Fall. Daher bezieht sich der Anwendungsbereich der Haftungsstudie auf Aktivitäten (activities), denn bei diesen ist die Wahrscheinlichkeit des Schadenseintritts kalkulierbar. ${ }^{69}$ Die Überschrift der Haftungsstudie „Liability (...) out of acts (...)“ ist demnach irreführend. Die Formulierung sollte lediglich die intendierte Abgrenzung zur Staatenverantwortlichkeit für völkerrechtswidriges Verhalten verdeutlichen. ${ }^{70}$ Die Völkerrechtskommission einigte sich jedenfalls darauf, dass sich die Studie auf Aktivitäten bezieht. ${ }^{71}$

Es bestand unterdessen Uneinigkeit hinsichtlich anderer völkerrechtlich nicht verbotener Aktivitäten, denen kein Risiko im Sinne des Art. 1 lit. a AIL inhärent ist, die aber dennoch schädliche Auswirkungen haben kön-

67 Sonderberichterstatter R. Q. Quentin-Baxter, Third report on international liability for injurious consequences arising out of acts not prohibited by international law, YBILC 1982-II/1, UN Doc. A/CN.4/360, S. 59 f., Rn. 36 ff.

68 Dies entbindet die Staaten im Übrigen nicht von der kontinuierlichen Pflicht, gefährliche Aktivitäten auf ihrem Hoheitsgebiet bzw. unter ihrer Hoheitsgewalt zu identifizieren. AIL, Kommentar zu Art. 4 AIL, S. 110, Abs. 2.

69 Sonderberichterstatter R. Q. Quentin-Baxter erklärt diesbezüglich: „In this way, account can be taken of the probability of accidents which are not foreseeable individually but are highly predictable as a class." (Hervorhebung hinzugefügt) Sonderberichterstatter R. Q. Quentin-Baxter, Third report on international liability for injurious consequences arising out of acts not prohibited by international law, YBILC 1982-II/1, UN Doc. A/CN.4/360, S. 60, Rn. 39; siehe auch S. Erichsen, Das Liability-Projekt der ILC, ZaöRV 51 (1991), S. 94 (109 f.).

70 Sonderberichterstatter R. Q. Quentin-Baxter, Third report on international liability for injurious consequences arising out of acts not prohibited by international law, YBILC 1982-II/1, UN Doc. A/CN.4/360, S. 59 f., Rn. 36 ff.; S. Erichsen, Das Liability-Projekt der ILC, ZaöRV 51 (1991), S. 94 (109); L. de la Fayette, The ILC and International Liability, RECIEL 6 (1997), S. 322 (327).

71 In der französischen Version heißt es im Übrigen seit Anbeginn der Haftungsstudie „activités“ (Aktivitäten) statt „actes“ (Handlungen). ILC, Report of the International Law Commission on the work of its thirty-eighth session, YBILC 1986-II/2, UN Doc. A/41/10, S. 58, Rn. 216; ILC, Report of the International Law Commission on the work of its forty-fourth session, YBILC 1992-II/2, UN Doc. A/47/10, S. 51, Rn. 348; Sonderberichterstatter J. Barboza, Eighth report on international liability for injurious consequences arising out of acts not prohibited by international law, YBILC 1992-II/1, UN Doc. A/CN.4/443, S. 63, Fn. 22. 
nen; dementsprechend wurde Art. 1 lit.b AIL, der den Anwendungsbereich auf diese Aktivitäten ausweiten würde, in Klammern gesetzt und später aus dem Anwendungsbereich der Haftungsstudie gestrichen. ${ }^{72}$

Schließlich fallen nur erhebliche Schädigungen in den Anwendungsbereich der Haftungsstudie, die kausal auf einer risikobehafteten Aktivität beruhen, ${ }^{73}$ die unter der Hoheitsgewalt oder Kontrolle eines Staates bzw. auf dessen Hoheitsgebiet stattfinden. ${ }^{74}$

\section{b) Schadensvermeidung gemäß den AIL von 1996}

Art. 4 AIL fordert, dass der Ursprungsstaat alle angemessenen Maßnahmen zur Schadensvermeidung sowohl vor als auch nach Eintritt des schadensauslösenden Ereignisses ergreift. ${ }^{75} \mathrm{Zu}$ diesem Zweck sollen die Staaten nach Art. 6 AIL miteinander kooperieren.

Wesentlich für die Komponente der Schadensvermeidung im Entwurf der Völkerrechtskommission aus dem Jahr 1996 ist zum einen, dass der Schaden nicht zu verhindern ist; es ist im Rahmen der Schadensvermeidung lediglich die gebotene Sorgfalt geschuldet. ${ }^{76}$ Zum anderen sind die verhaltensbezogenen Pflichten zur Schadensvermeidung nicht rechtsverbindlich. ${ }^{77}$ Dies bedeutet, dass eine Verletzung dieser Pflichten ausdrücklich kein völkerrechtswidriges Verhalten im Sinne der ASR darstellt ${ }^{78}$ und somit im Vergleich zu den Rechtsfolgen der Staatenverantwortlichkeit auch weniger weitreichende Konsequenzen folgen. ${ }^{79}$

72 Vgl. auch Art. 4 Abs. 1 IDI-Resolution, wonach die verschuldensunabhängige Einstandspflicht insbesondere bei „ultra-hazardous activities and activities entailing risk or having other similar characteristics" greifen soll.

73 Art. 2 lit. a AIL. Eine Begrenzung der Haftungsstudie auf besonders gefährliche Aktivitäten, das heißt solche Aktivitäten, denen nicht nur das hohe Risiko erheblicher Schadensfolgen, sondern das geringe Risiko katastrophaler Schadensfolgen immanent ist, lehnte die Mehrheit der Mitglieder der Arbeitsgruppe ab. J. Barbo$z a$, The Environment, Risk and Liability in International Law, 2011, S. 110.

74 AIL, Kommentar zu Art. 1 AIL, S. 104, Abs. 5.

75 In Kapitel II der AIL werden spezifische Aspekte der Schadensvermeidung weiter ausgeführt.

76 AIL, Kommentar zu Art. 4 AIL, S. 110 f., Abs. 4 ff.

77 AIL, Kommentar zu Art. 22 AIL, S. 131, Abs. 2.

78 Ibid.

79 Vgl. AIL, Kommentar zu Art. 21 AIL, S. 130 f. Als Wiedergutmachung kann zwar ausnahmsweise Wiederherstellung gefordert werden, aber eine Unterlassungspflicht trifft den Ursprungsstaat grundsätzlich nicht. 


\section{Kapitel: Staatenhaftung in der Systematik des Völkerrechts}

c) Wiedergutmachung gemäß den AIL von 1996

Der Kern der Haftungsstudie ist in Art. 5 AIL zusammengefasst, wonach erhebliche grenzüberschreitende Schäden durch völkerrechtlich nicht verbotene Aktivitäten Wiedergutmachungsmachungspflichten bedingen. Dabei weist der Kommentar zu Art. 5 AIL auf das Zusammenspiel von Schadensvermeidung und Wiedergutmachung hin. Art. 5 AIL komplementiere Art. 4 AIL und sei dessen logische Folge. ${ }^{80}$ Vermeidung und Kompensation von erheblichen grenzüberschreitenden Schäden stellen demnach ein Kontinuum ${ }^{81}$ der Staatenhaftung dar und müssen somit in einem Rechtsrahmen Beachtung finden. ${ }^{82}$

Die AIL bestimmen nicht explizit, ob vorrangig der Ursprungsstaat oder der private Schadensverursacher Wiedergutmachung leisten muss. ${ }^{83}$ In Anbetracht der Tatsache, dass die Haftungsstudie ursprünglich eine völkerrechtliche Haftung regeln sollte und die Entwürfe und Berichte der Völkerrechtskommission zunächst ausschließlich eine Wiedergutmachungspflicht der Ursprungsstaaten besprachen, ist an dieser Stelle keinesfalls von einem Vorrang der privaten Haftung auszugehen. ${ }^{84}$ Außerdem adressiert der Entwurf von 1996 im Rahmen der vorgegebenen Schadensvermeidungspflichten ausschließlich Staaten, so dass ein veränderter Fokus auf eine private Haftung dem gesamten Gefüge der Haftungsstudie widersprechen würde. ${ }^{85}$ Das Kontinuum aus Schadensvermeidung und Wiedergutmachung, welches in den AIL zusammengefasst ist, deutet insgesamt auf eine primäre Staatenhaftung hin. ${ }^{86}$ Der Entwurf eröffnet dem Opfer eine Wahlmöglichkeit zwischen nationalen und internationalen Verfahren, um

80 AIL, Kommentar zu Art. 5 AIL, S. 112, Abs. 5.

81 So schon Sonderberichterstatter R. Q. Quentin-Baxter, Fourth report on international liability for injurious consequences arising out of acts not prohibited by international law, YBILC 1983-II/1, UN Doc. A/CN.4/373, S. 212.

82 Vgl. J. Barboza, The Environment, Risk and Liability in International Law, 2011, S. 116.

83 Zur Diskussion über die Bestimmung der haftenden Partei siehe ILC, Report of the International Law Commission on the work of its forty-second session, YBILC 1990-II/2, UN Doc. A/45/10, S. 110, Rn. 509.

84 S. Erichsen, Das Liability-Projekt der ILC, ZaöRV 51 (1991), S. 94 (125).

85 Ibid.

86 Es handelt sich demnach nicht lediglich um eine subsidiäre Staatenhaftung. Die private Haftung kann allenfalls auf dieselbe Ebene mit der Staatenhaftung gestellt werden, um den Geschädigten eine Wahlmöglichkeit zu bieten. So auch S. Erichsen, Das Liability-Projekt der ILC, ZaöRV 51 (1991), S. 94 (125). Vgl. auch L. de la Fayette, The ILC and International Liability, RECIEL 6 (1997), S. 322 
seinen Anspruch auf Wiedergutmachung geltend zu machen. ${ }^{87}$ Dabei indiziert der Kommentar zu den AIL, dass in vielen Konstellationen zwischenstaatliche Verhandlungen sachgerechter und zielführender sind als der innerstaatliche private Rechtsweg. ${ }^{88}$ Für die zwischenstaatlichen Verhandlungen zur Wiedergutmachung bestimmt Art. 22 AIL entsprechende Faktoren, die Art und Ausmaß der Kompensationspflicht oder anderer Abhilfemaßnahmen beeinflussen. Einzubeziehen sind unter anderem die Beachtung bzw. Missachtung von Präventionspflichten sowie die Sorgfalt, die der Ursprungsstaat bei der Schadensvermeidung hat walten lassen. ${ }^{89}$ Wenn der Schaden unvermeidbar war oder er trotz Anwendung der gebotenen Sorgfalt eingetreten ist, scheidet die Wiedergutmachungspflicht zwar nicht aus, das Ausmaß der Kompensationspflicht ist allerdings im Sinne eines gerechten Interessenausgleiches zu begrenzen..${ }^{90}$ Umgekehrt begründet die Verletzung von Präventionspflichten bzw. die Missachtung der gebotenen Sorgfalt zwar keine Völkerrechtswidrigkeit, sie wird aber entsprechend der ursprünglichen Konzeption von Quentin-Baxter durch eine weitreichendere Wiedergutmachungspflicht sanktioniert. ${ }^{91}$ Die Tatsache, dass die Wiedergutmachungspflicht selbst dann greift, wenn die Schadensvermeidungspflichten beachtet wurden, belegt, dass der Entwurf aus dem Jahr 1996 eine verschuldensunabhängige Staatenhaftung für erhebliche grenzüberschreitende Schäden durch risikobehaftete völkerrechtlich nicht verbotene Aktivitäten etabliert. ${ }^{92}$

(329f.); S. Suchartikul, State Responsibility and International Liability under International Law, Loy. L.A. ICLR 18 (1996), S. 821 (831).

87 AIL, Kommentar zu Art. 5 AIL, S. 112, Abs. 7.

88 AIL, Kommentar zu Art. 21 AIL, S. 130, Abs. 1.

89 Art. 22 lit. a und b AIL.

90 AIL, Kommentar zu Art. 21 AIL, S. 131, Abs. 3.

91 AIL, Kommentar zu Art. 21 AIL, S. 131, Abs. 2.

92 Soweit ausweislich der AIL-Kommentare eine verschuldensunabhängige Haftung (strict or absolute liability) nicht Gegenstand des Entwurfes sein soll (AIL, Kommentar zu Art. 5 AIL, S. 112, Rn. 3 und Kommentar zu Kapitel III, S. 128, Rn. 1), ist dies im Ergebnis nicht überzeugend, da nach den AIL in jedem Fall Wiedergutmachung zu leisten ist. So auch $L$. de la Fayette, der ,the ostensible (but not real) rejection of strict liability as the basis for compensation" bemängelt. $L$. de la Fayette, The ILC and International Liability, RECIEL 6 (1997), S. 322 (323). Vgl. auch Art. 4 Abs. 1 IDI-Resolution, der eine „strict responsibility of the State on the basis of harm or injury alone" vorsieht. 


\section{Kapitel: Staatenhaftung in der Systematik des Völkerrechts}

\section{Staatenhaftung - „a case of misplaced emphasis“?}

Von Anbeginn der Haftungsstudie äußerten Mitglieder der Völkerrechtskommission Bedenken gegen eine gesonderte Regelungsbedürftigkeit einer staatlichen Haftung. Diese sei vielmehr Bestandteil der Staatenverantwortlichkeit. ${ }^{93}$ Unter der Überschrift "The Attack on Liability“ beschreibt Barboza Bestrebungen von Mitgliedern der Völkerrechtskommission, die Haftungsstudie auf die Ausgestaltung von Vorschriften zur Prävention zu beschränken. ${ }^{94}$ Die im Jahr 1997 neu eingesetzte Arbeitsgruppe lehnte den AIL-Entwurf mit dem Hinweis ab, dass die Studie hinsichtlich Inhalt, Reichweite sowie Titel mit theoretischen und konzeptionellen Schwierigkeiten behaftet und das Verhältnis zum Regime der Staatenverantwortlichkeit klärungsbedürftig sei. ${ }^{95}$ Außerdem wurde die gemeinsame Regelung von Prävention und Kompensation kritisiert, die zu einer Vermischung von Primär- und Sekundärnormen führe. ${ }^{96}$ Ablehnung erfuhren insbesondere die Regelungen zur verschuldensunabhängigen Staatenhaftung, die keine Basis in der Staatenpraxis und Rechtsüberzeugung der Staatengemeinschaft hätten und damit weder als Kodifizierung noch als Weiterentwicklung von Recht Bestand haben könnten. ${ }^{97}$

93 Siehe hierzu J. Barboza, The Environment, Risk and Liability in International Law, 2011, S. 126.

94 J. Barboza, Liability, Chinese JIL 1 (2002), S. 499 (505); ders., The Environment, Risk and Liability in International Law, 2011, S. 125 ff.; siehe auch L. de la Fayette, The ILC and International Liability, RECIEL 6 (1997), S. 322 (330).

95 ILC, Report of the International Law Commission on the work of its fortyninth session, YBILC 1997-II/2, UN Doc. A/52/10, S. 59, Rn. 165; ILC Working Group, Report of the Working Group on international liability for injurious consequences arising out of acts not prohibited by international law, 1997, UN Doc. A/CN.4/L.536, S. 2, Rn. 3.

96 ILC, Report of the International Law Commission on the work of its forty-ninth session, 1997, Topical summary of debate in the Sixth Committee, UN Doc. A/CN.4/483, S. 16, Rn. 11; siehe auch L. de la Fayette, The ILC and International Liability, RECIEL 6 (1997), S. 322 (323); G. Handl, Liability as an obligation established by a primary rule of international law, NYIL 16 (1985), S. 49 (55). Die Kritiker verkennen allerdings, dass es sich sowohl bei den Präventions- als auch Wiedergutmachungspflichten um Primärnormen handelt. Erst die Verletzung dieser „zusammengesetzten Primärnorm“ führt zu den Sekundärnormen der Staatenverantwortlichkeit.

97 Sonderberichterstatter $P$. S. Rao, First report on the legal regime for allocation of loss in case of transboundary harm arising out of hazardous activities, YBILC 2003-II/1, UN Doc. A/CN.4/531, S. 80, Rn. 22; ILC, Report of the International Law Commission on the work of its fifty-fifth session, YBILC 2003-II/2, UN 
Die Völkerrechtskommission entschied schließlich, die Themen Schadensprävention (prevention) und Haftung (international liability) in getrennten Projekten zu behandeln. ${ }^{98}$ Pemmaraju Sreenivasa Rao wurde zum Sonderberichterstatter für beide Themenbereiche ernannt. ${ }^{99}$ Der Fokus seiner Arbeiten zu den Präventions- und Schadensausgleichspflichten lag auf Sekundär- und Nebenpflichten und Schadensberechnungsmethoden. ${ }^{100}$

Im Jahr 2001 legte die Kommission die „Draft articles on prevention of transboundary harm from hazardous activities" (Präventionsartikel) ${ }^{101}$ vor und schloss die Haftungsstudie im Jahr 2006 mit den „Draft principles on the allocation of loss in the case of transboundary harm arising out of hazardous activities" (Haftungsprinzipien)102 ab. Die einzige Verbindung zwischen den Entwürfen bildet ihr Anwendungsbereich. ${ }^{103}$ Indes bedeutet die Aufteilung in zwei Teilkomplexe eine wesentliche Abkehr von der Idee einer Staatenhaftung im Sinne einer „zusammengesetzten Primärnorm". 104

Doc. A/58/10, S. 51, Rn. 228; siehe auch J. Barboza, The Environment, Risk and Liability in International Law, 2011, S. $128 \mathrm{f}$.

98 ILC, Report of the International Law Commission on the work of its fortyninth session, YBILC 1997-II/2, UN Doc. A/52/10, S. 59, Rn. 165, 168; ILC Working Group, Report of the Working Group on international liability for injurious consequences arising out of acts not prohibited by international law, 1997, UN Doc. A/CN.4/L.536, S. 2, Rn. 3.

99 ILC, Report of the International Law Commission on the work of its fortyninth session, YBILC 1997-II/2, UN Doc. A/52/10, S. 9, Rn. 17.

100 J. Bäumler, Das Schädigungsverbot im Völkerrecht, 2017, S. 67.

101 ILC, Report of the International Law Commission on the work of its fifty-third session, Draft articles on prevention of transboundary harm from hazardous activities, with commentaries, YBILC 2001-II/2, UN Doc. A/56/10, S. 148 ff., Rn. 98 (im Folgenden: Präventionsartikel).

102 ILC, Report of the International Law Commission on the work of its fiftyeighth session, Draft principles on the allocation of loss in the case of transboundary harm arising out of hazardous activities, with commentaries, YBILC 2006-II/2, UN Doc. A/61/10, S. 59 ff., Rn. 67 (im Folgenden: Haftungsprinzipien).

103 Haftungsprinzipien, Kommentar zu Prinzip 1, S. 61 ff., Abs. 1 ff. und Kommentar zu Prinzip 2, S. 56, Abs. 2.

104 Haftungsprinzipien, Kommentar zu Prinzip 1, S. 62, Abs. 1: „The interrelated nature of the concepts of ,prevention' and ,liability' needs no particular emphasis in the context of the work of the Commission." 
a) Anwendungsbereich der Haftungsstudie

Die kontroversen Interessen, welche die Mitglieder der Völkerrechtskommission und die Mitglieder des Sechsten Ausschusses der VN-Generalversammlung verfolgten, kamen überdies auch im Rahmen der Festlegung des Anwendungsbereiches der Haftungsstudie zum Tragen. ${ }^{105}$ Zunächst wurde die Präferenz deutlich, eine Haftung für einen breiten Anwendungsbereich zu konzipieren. ${ }^{106}$ Allerdings regte sich gegen diesen Ansatz von Anbeginn der Haftungsstudie Widerstand. Damit das Thema praktikabel bleibe ${ }^{107}$ und aufgrund angeblich fehlender Staatenpraxis in anderen Bereichen $^{108}$, solle kein grundlegendes Haftungssystem für alle Bereiche des Völkerrechts geschaffen werden. Nur Aktivitäten, welche die Nutzung und Verwaltung der Umwelt beträfen, sollten erfasst werden. Im Ergebnis

105 Auch wenn die Mitglieder der Kommission unabhängige Experten und damit losgelöst von den Interessen ihrer Herkunftsstaaten die Ausgestaltung vornahmen, reflektieren die vertretenen Ansichten im Ergebnis offenkundig die staatlichen Interessen der politischen Landschaft. J. Barboza, Liability, Chinese JIL 1 (2002), S. 499 (504). Zu der Kontroverse innerhalb der Kommission und dem Ausschuss siehe J. Bäumler, Das Schädigungsverbot im Völkerrecht, 2017, S. 64 ff.; A. E. Boyle, State Responsibility and International Liability for Injurious Consequences of Acts Not Prohibited by International Law, ICLQ 39 (1990), S. 1 (3 f.); D. B. Magraw, Transboundary Harm, AJIL 80 (1986), S. 305 (323 ff.).

106 Vgl. ILC Secretariat, Topical summary of the discussion on the report of the International Law Commission on the work of its thirty-second session (1980) in the Sixth Committee during the thirty-fifth session of the General Assembly, UN Doc. A/CN.4/L.326, 1981, S. 95, Rn.339, S. 99, Rn. 349; ILC, Report of the International Law Commission on the work of its thirty-second session, YBILC 1980-II/2, UN Doc. A/35/10, S. 160, Rn. 139.

107 Sonderberichterstatter R. Q. Quentin-Baxter, Fourth Report on international liability for injurious consequences arising out of acts not prohibited by international law, YBILC 1983-II/1, UN Doc. A/CN.4/373, S. 205, Rn. 14 f. Vgl. auch $J$. Barboza, der auf die Erfolglosigkeit eines solchen, der Konvention des Europarates über die zivilrechtliche Haftung für Schäden aus umweltgefährdenden Tätigkeiten vom 21. Juni 1993 (Lugano-Konvention) zugrundeliegenden, allgemeingültigen Ansatzes hinweist. J. Barboza, The Environment, Risk and Liability in International Law, 2011, S. 129.

108 Sonderberichterstatter R. Q. Quentin-Baxter, Preliminary report on international liability for injurious consequences arising out of acts not prohibited by international law, YBILC 1980-II/1, UN Doc. A/CN.4/334 and Add.1 and 2, S. 265, Rn.66; ILC Secretariat, Survey of liability regimes relevant to the topic of international liability for injurious consequences arising out of acts not prohibited by international law, (international liability in case of loss from transboundary harm arising out of hazardous activities), YBILC 1995-II/1, UN Doc. A/CN.4/471, S. $62 \mathrm{ff}$. 
führte der politisch bedingte Einwand der Gegner der Haftungsthematik zu einer Kompromisslösung, wonach lediglich Aktivitäten mit negativen physischen Auswirkungen auf Menschen, Eigentum oder Umwelt dem Anwendungsbereich der Haftungsstudie unterfallen. Zwischenstaatliche Interessenkonflikte, etwa in wirtschaftlichen, monetären, sozio-ökonomischen oder ähnlichen Bereichen ${ }^{109}$, und Schäden an Gemeinschaftsgütern $^{110}$ bleiben damit außerhalb des Anwendungsbereiches der Präventionsartikel und Haftungsprinzipien.

Die Entwürfe betreffen ferner nur Schäden, die grenzüberschreitend und erheblich sind. Eine Grenzüberschreitung ist zu bejahen, wenn Aktivitäten auf dem Hoheitsgebiet oder unter der Hoheitsgewalt oder Kontrolle eines Staates stattfinden und zu Schäden auf staatsfremdem Hoheitsgebiet oder an Orten unter staatsfremder Hoheitsgewalt oder Kontrolle führen. ${ }^{111}$ Auch wenn eine gemeinsame Grenze nicht notwendig ist, sind demnach nur zwischenstaatliche Schäden von den Entwürfen erfasst. Das Erheblichkeitskriterium ist in dem Kommentar der Völkerrechtskommission nur sehr vage definiert: „It is to be understood that, significant" is something more than, detectable "but need not be at the level of, serious' or , substantial." "112 Jedenfalls sei eine Einzelfallbetrachtung für die Beurteilung der Erheblichkeit des Schadens notwendig. ${ }^{113}$

Der Anwendungsbereich der Entwürfe erfasst schließlich völkerrechtlich nicht verbotene Aktivitäten. Mit dieser Voraussetzung soll die Abgrenzung der Thematik von der Staatenverantwortlichkeit bekräftigt werden. ${ }^{114}$ Daneben geht es nur noch um risikobehaftete Aktivitäten. ${ }^{115}$ Maßgeblich ist dabei das Verhältnis von Eintrittswahrscheinlichkeit und Umfang des zu erwartenden Schadens. Demnach sind ausschließlich ge-

109 Haftungsprinzipien, Kommentar zu Prinzip 1, S. 64, Abs. 12.

110 Sonderberichterstatter P. S. Rao, First report on prevention of transboundary damage from hazardous activities, YBILC 1998-II/1, UN Doc. A/CN.4/487 and Add.1, S. 198, Rn. 107 ff.; ILC, Report of the International Law Commission on the work of its fifty-fourth session, YBILC 2002-II/2, UN. Doc. A/57/10, S. 91, Rn. 447.

111 Besondere Bedeutung verleiht die Kommission in diesem Zusammenhang dem Territorialitätsprinzip. Siehe Präventionsartikel, Kommentar zu Art. 1, S. 150, Abs. 7.

112 Präventionsartikel, Kommentar zu Art. 2, S. 152, Abs. 4.

113 Präventionsartikel, Kommentar zu Art. 2, S. 153, Abs. 7.

114 Haftungsprinzipien, Kommentar zu Prinzip 1, S. 62, Abs. 5.

115 Art. 1 lit. b AIL wurde gestrichen. 


\section{Kapitel: Staatenhaftung in der Systematik des Völkerrechts}

fährliche und hochgefährliche Aktivitäten zu reglementieren. ${ }^{116}$ Auf eine Auflistung von bestimmten Aktivitäten, die unter das Regime fallen, wurde mit Hinweis auf den stetigen wissenschaftlichen und technologischen Fortschritt verzichtet. ${ }^{117}$

Im Ergebnis reduzierte die Kommission die Relevanz des neuen Entwurfs implizit auf eine begrenzte Anzahl von Ressourcen- und Energieprojekten, die vornehmlich dem Umweltkontext entstammen. ${ }^{118}$

b) Prävention grenzüberschreitender Schäden durch gefährliche Aktivitäten

Aufgrund der größeren inhaltlichen Fortschritte bei der Bestimmung von Präventionspflichten widmete sich die Kommission zunächst der Ausgestaltung dieser Thematik ${ }^{119}$ und schloss diesen Teilkomplex im Jahr 2001 mit einem Entwurf von 19 Artikeln zur Prävention ab (Präventionsartikel). Die finale Ausgestaltung der Präventionspflichten unterscheidet sich

116 Bei gefährlichen Aktivitäten besteht die hohe Wahrscheinlichkeit erheblicher Schadensfolgen und bei hochgefährlichen Aktivitäten die geringe Wahrscheinlichkeit katastrophaler Schadensfolgen. Art. 2 lit. a Präventionsartikel; Präventionsartikel, Kommentar zu Art. 2 lit. a, S. 152, Abs. 1 f.

117 Die Kommission benannte aber exemplarisch Aktivitäten, die nicht unter das Regime fallen, nämlich „activities which cause harm in their normal operation; harm caused by creeping pollution, that is, harm caused over a period of time, harm caused by a combination of effects from multiple sources, activities which do not have a physical quality and whose consequences flow from an intervening policy decision relating to monitoring, socio-economic or similar fields; harm caused on the environment in general, or global commons in particular". Sonderberichterstatter $P$. S. Rao, Third report on international liability for injurious consequences arising out of acts not prohibited by international law (prevention of transboundary damage from hazardous activities), YBILC 2000-II/1, UN Doc. A/CN.4/510, S. 118, Rn. 15.

118 J. Bäumler stellt zutreffend fest: „Wenn man berücksichtigt, dass zur Zeit der Diskussion um die materielle Reichweite der Liability-Studie Zweifel an der Existenz des Schädigungsverbots in weiteren Feldern des Völkerrechts den Ausschlag gaben, bedarf diese Einschränkung mehr als 40 Jahre später der Überprüfung. Denn letztlich waren es wohl vor allem politische und Gründe der Praktikabilität, die zu der einschränkenden Sichtweise geführt haben." J. Bäumler, Das Schädigungsverbot im Völkerrecht, 2017, S. 69.

119 ILC, Report of the International Law Commission on the work of its fortyninth session, YBILC 1997-II/2, UN Doc. A/52/10, S. 59, Rn. 165 ff., 168. 
nur unwesentlich von vorherigen Entwürfen. ${ }^{120}$ Die Präventionsartikel dienen der Regulierung von völkerrechtlich nicht verbotenen Aktivitäten, denen das Risiko erheblicher grenzüberschreitender Schäden immanent ist. Auch der neue Entwurf zielt dabei auf einen gerechten Interessenausgleich (Präventionsartikel 9 Abs. 2 und Präventionsartikel 10). Allerdings verfolgt er eine präventive Schutzrichtung, betrifft also staatliche Pflichten vor dem Eintritt des schadensauslösenden Ereignisses. ${ }^{121}$

Präventionsartikel 3 bildet den Kern der staatlichen Pflichten zur Schadensvermeidung, indem er bestimmt, dass Staaten alle angemessenen Maßnahmen zur Prävention von erheblichen grenzüberschreitenden Schäden ergreifen oder zumindest deren Risiko minimieren sollten. Diese Pflicht greift zwar nicht bei unvorhersehbaren Schadensfällen, erfordert aber entsprechende Vorsorge bei wissenschaftlich ungewissen, das heißt nicht auszuschließenden Gefahren von erheblichen und irreversiblen Schäden. ${ }^{122}$ Eine Vorsorge ist etwa durch Pläne zum Umgang mit Notfällen zu gewährleisten (Präventionsartikel 10). Ferner sind die notwendigen administrativen und finanziellen Mittel sowie Regulierungsmechanismen zur Schadensprävention durch Staaten bereitzustellen. ${ }^{123}$ Im Rahmen der Prävention bestehen neben der materiellen Pflicht zur Schadensvermeidung auch verfahrensbezogene Pflichten, ${ }^{124}$ einschließlich einer allgemeinen Kooperationspflicht (Präventionsartikel 4), Informations- und Benachrichtigungspflicht (Präventionsartikel 8), Rücksprachepflicht (Präventionsartikel 9) sowie der Pflicht zu einer vorherigen Risikoanalyse, etwa in Gestalt einer Umweltverträglichkeitsprüfung, (Präventionsartikel 7). Schließlich soll Personen, die dem Risiko eines erheblichen grenzüberschreitenden Schadens ausgesetzt sind oder sein könnten, der gleichberechtigte (non-discrimination) Rechtsweg zu den Gerichten im Ursprungsstaat offenstehen (Präventionsartikel 15).

Die Präventionspflichten sind verhaltensbezogen (obligation of conduct), das heißt ein Verstoß gegen die Präventionsartikel ist nicht schon aufgrund des Eintritts eines erheblichen grenzüberschreitenden Schadens zu bejahen. ${ }^{125}$ Die Staaten sind lediglich aufgefordert, im Rahmen der

120 J. Barboza, The Environment, Risk and Liability in International Law, 2011, S. 120.

121 Präventionsartikel, Allgemeiner Kommentar, S. 148, Abs. $1 \mathrm{f}$.

122 Präventionsartikel, Kommentar zu Art.3, S. 155, Abs.14; Präventionsartikel, Kommentar zu Art. 10, S. 162, Abs. 5.

123 Präventionsartikel, Kommentar zu Art. 3, S. 155, Abs. 15.

124 Präventionsartikel, Kommentar zu Art. 3, S. 154, Abs. 10.

125 Präventionsartikel, Kommentar zu Art. 3, S. 154, Abs. 7. 


\section{Kapitel: Staatenhaftung in der Systematik des Völkerrechts}

Schadensprävention die gebotene Sorgfalt walten zu lassen. Der Sorgfaltsstandard bemisst sich nach dem, was im Verhältnis zum Risiko eines grenzüberschreitenden Schadens in der konkreten Situation allgemein als angemessen gilt. ${ }^{126}$ Der Maßstab ist nicht statisch, sondern orientiert sich am jeweiligen Stand der Technik. Außerdem findet der Entwicklungsstand des jeweiligen Staates Berücksichtigung. ${ }^{127}$

Die Missachtung der gebotenen Sorgfalt, und damit die Verletzung der Präventionspflichten, führt zu Konsequenzen nach der Staatenverantwortlichkeit, ohne dass dabei die Aktivität selbst als völkerrechtswidrig zu qualifizieren ist. ${ }^{128}$ Die Kommission räumt aber gleichwohl ein, dass ein Schadensrisiko unter Umständen nicht vorhersehbar sein kann oder Schäden auch bei Beachtung der gebotenen Sorgfalt nicht verhindert werden können. ${ }^{129}$

Der Entwurf zur Prävention lässt aber offen, welche Konsequenzen der Staat zu tragen hat, wenn trotz Einhaltung der Präventionspflichten grenzüberschreitende Schäden durch risikobehaftete Aktivitäten entstehen. ${ }^{130}$

126 Präventionsartikel, Kommentar zu Art. 3, S. 154, Abs. 11. Der Sorgfaltsmaßstab ist allerdings nicht ohne Weiteres zu bestimmen und belastet den geschädigten Staat mit Schwierigkeiten im Rahmen der Beweisführung. A. E. Boyle, Globalising Environmental Liability, J. Envtl. L. 17 (2005), S. 3 (7).

127 Präventionsartikel, Kommentar zu Art. 3, S. 155, Abs. 17. In diesem Zusammenhang werden die Staaten dazu angehalten, zum beiderseitigen Vorteil Systeme zum Kapazitätenaufbau, zum Technologietransfer und zur Finanzausstattung zu etablieren. Präventionsartikel, Kommentar zu Art. 3, S. 155 Abs. 16.

128 Präventionsartikel, Kommentar zu Art.1, S. 150, Abs. 6; Haftungsprinzipien, Allgemeiner Kommentar, S. 59 f., Abs. 6.

129 Haftungsprinzipien, Kommentar zu Prinzip 1, S. 63, Abs. 7.

130 A. E. Boyle stellt fest: „The one condition missing from the 2001 articles is that states make provision for liability or compensation in the event of foreseeable transboundary damage. If the present draft principles on liability are adopted as drafted, states will be free to engage in harmful activities without any obligation to provide effective remedies or redress for transboundary damage unless they are themselves at fault. That is exactly the defect which the Commission was invited to rectify when it began work on the topic in 1978." A. E. Boyle, Globalising Environmental Liability, J. Envtl. L. 17 (2005), S. 3 (26). 
c) Verlustzuweisung bei grenzüberschreitenden Schäden durch gefährliche Aktivitäten

Trotz des beschriebenen Widerstandes gegen die Haftungsthematik ${ }^{131}$ forderte die Generalversammlung im Jahr 2002 die Völkerrechtskommission auf, ihre Erwägungen zum Haftungsaspekt wieder aufzunehmen und dabei die Verflechtung von Prävention und Haftung zu berücksichtigen. ${ }^{132}$ Die Kommission klammerte jedoch eine Haftung von Staaten aus und konzentrierte sich im Rahmen des neuen Entwurfs auf eine Verlustzuweisung zwischen den Akteuren risikobehafteter Aktivitäten. Im Jahr 2006 erstellte die Kommission einen Entwurf von acht Prinzipien zur Verlustzuweisung im Schadensfall (Haftungsprinzipien).

Sinn und Zweck des Entwurfs sind in Haftungsprinzip 3 verankert. Der Entwurf zielt auf eine unverzügliche und angemessene Entschädigung für Opfer grenzüberschreitender Schäden (Haftungsprinzip 3 lit.a). Das Kriterium der Unverzüglichkeit bezieht sich auf prozessrechtliche Aspekte, die den Zugang zu nationalen und internationalen Rechtsmitteln regeln und den Zeitrahmen für die Entscheidungsfindung über Entschädigungszahlungen vorgeben. ${ }^{133}$ Haftungsprinzip 6 spezifiziert, dass der Zugang zu effektiven Rechtsbehelfen für in- und ausländische Kläger gleichberechtigt (non-discriminatory) sein sollte. Das Erfordernis der Angemessenheit einer Entschädigung ist schon dann erfüllt, wenn diese zum erlittenen Schaden im Verhältnis steht. Somit gewährleistet der Entwurf keine volle Kompensation für Geschädigte. ${ }^{134}$

Das Ziel einer unverzüglichen und angemessenen Entschädigung werde gemäß Haftungsprinzip 4 in erster Linie durch eine verschuldensun-

131 Im Sechsten Ausschuss der VN-Generalversammlung sprachen sich die Vertreter von potenziellen Ursprungsstaaten grenzüberschreitender Schäden, also insbesondere Industriestaaten, gegen die Thematik aus. Sie wandten ein, das Regime sei ein Novum und die Folgen einer solchen Haftung seien unkalkulierbar. Dabei fällt auf, dass derartige Haftungssysteme für gefährliche erlaubte Aktivitäten Bestandteil der innerstaatlichen Rechtsordnung gerade dieser Staaten sind. Zudem drängten insbesondere Vertreter von Staaten, die den grenzüberschreitenden Schäden potenziell ausgesetzt sein werden, das heißt insbesondere Entwicklungsländer, auf die Ausarbeitung eines internationalen Haftungsregimes. J. Barboza, Liability, Chinese JIL 1 (2002), S. 499 (504).

132 GV Resolution 56/82 vom 18.12.2002, S. 2, Rn. 3.

133 Haftungsprinzipien, Kommentar zu Prinzip 4, S. 77, Abs. 7.

134 Haftungsprinzipien, Kommentar zu Prinzip 4, S. 78, Abs. 8. 
abhängige Haftung der privaten Betreiber, ${ }^{135}$ das heißt derjenigen, die den Nutzen, die Kontrolle oder Leitung über die risikobehaftete Aktivität haben, ${ }^{136}$ erreicht. Die Ausgestaltung der Haftungsprinzipien unterliegt der Annahme, dass Staaten ihren Pflichten unter den Präventionsartikeln nachgekommen sind. ${ }^{137}$ Die Rolle der Staaten beschränkt sich im Rahmen der Haftungsprinzipien demnach auf die Gestaltung von funktionsfähigen Mechanismen und Gesetzen, die eine unverzügliche und angemessene Entschädigung möglich machen (Haftungsprinzip 4 Abs. 1). ${ }^{138}$ Dafür sollten Staaten eine verschuldensunabhängige Haftung der privaten Betreiber oder anderer haftbarer Personen bzw. Institutionen sicherstellen (Haftungsprinzip 4 Abs. 2) $)^{139}$ und eine Versicherungs- oder Rücklagenpflicht vorschreiben (Haftungsprinzip 4 Abs. 3) sowie in angebrachten Fällen die Pflicht zu Einrichtung industrieweiter Fonds aufstellen (Haftungsprinzip 4 Abs. 4). Nur sofern sich diese Maßnahmen mit Blick auf eine angemessene Entschädigung als ungenügend erweisen, sollten Ursprungsstaaten zusätzliche finanzielle Mittel bereitstellen (Haftungsprinzip 4 Abs. 5). ${ }^{140}$ Doch selbst diese subsidiäre staatliche Einstandspflicht ist lediglich als Empfehlung formuliert und daher von der ursprünglichen Vision einer völkerrechtlichen Staatenhaftung weit entfernt. ${ }^{141}$ Gemäß Haftungsprinzip

135 Vgl. auch Art. 5 und Art. 6 Abs. 1 IDI-Resolution, wonach die primäre verschuldensunabhängige Umwelthaftung grundsätzlich den Betreiber treffen sollte.

136 Haftungsprinzipien, Kommentar zu Prinzip 4, S. 78, Abs. 10.

137 Haftungsprinzipien, Kommentar zu Prinzip 1, S. 63, Abs. 8; Haftungsprinzipien, Kommentar zu Prinzip 4, S. 77, Abs. 2.

138 Haftungsprinzipien, Kommentar zu Prinzip 4, S. 76 f., Abs. 1.

139 Staatenverantwortlichkeit und private Haftung schließen sich nicht gegenseitig aus. Die Präventionsartikel sollen komplementär zu den Haftungsprinzipien wirken. Haftungsprinzipien, Allgemeiner Kommentar, S. 60, Abs. 9; Haftungsprinzipien, Kommentar zu Prinzip 1, S. 62 f., Abs. 6.

140 Vgl. auch Art. 6 Abs. 2 IDI-Resolution, der besagt: „international responsibility (...) may be incurred for failure of the State to comply with the obligation to establish and implement civil liability mechanisms under national law, including insurance schemes, compensation funds and other remedies and safeguards, as provided for under such regimes."

141 Bereits in seinem zweiten Bericht drängte Sonderberichterstatter P. S. Rao erfolgreich darauf, den Fokus auf eine Betreiberhaftung zu legen und die Staatenhaftung aus dem Anwendungsbereich der Haftungsstudie herausrauszunehmen. Dementsprechend lag der neue Schwerpunkt der Ausarbeitung auf gegen Betreiber gerichtete Entschädigungsverfahren, während zwischenstaatliche Verhandlungen an Bedeutung verloren. Sonderberichterstatter P. S. Rao, Second report on the legal regime for the allocation of loss in case of transboundary harm arising out of hazardous activities, YBILC 2004-II/1, UN Doc. A/CN.4/540, Rn. 36, S. 72, Abs. 5; Rn. 38, S. 81 ff., Entwurfsvorschlag Prinzip 8. 
7 sollten auch Fonds durch globale, regionale oder bilaterale Abkommen eingerichtet werden, die bei Zahlungsunfähigkeit der privaten Betreiber subsidiär Kompensation gewähren. ${ }^{142}$

Haftungsprinzip 5 beschreibt Maßnahmen, die als Reaktion auf das schädigende Ereignis zu ergreifen sind. Die betroffenen Staaten sollen alle realisierbaren Maßnahmen ergreifen, um die Auswirkungen des Schadens zu mindern oder wenn möglich zu beseitigen. Hier ist zu beachten, dass der neue Ansatz im Gegensatz zu einem ersten Entwurf aus dem Jahr 2004 den Reaktionsmaßnahmen des Haftungsprinzips 5 einen obligatorischen Charakter verleiht, indem dort die Begrifflichkeit „sollte“ (should) durch ein verbindlicheres „soll“ (shall) ersetzt wurde. ${ }^{143}$ Dies ist damit zu erklären, dass es sich im Wesentlichen um Maßnahmen zur Schadensprävention handelt, die denjenigen der Präventionsartikel gleichen und damit als rechtsverbindlich qualifiziert werden können. ${ }^{144}$ In jedem Fall macht der Kommentar zu den Haftungsprinzipien deutlich, dass der private Betreiber auch mit Blick auf Reaktionsmaßnahmen primär in der Verantwortung steht. ${ }^{145}$ Die Kosten der Abhilfemaßnahmen sind als Teil der Kompensationspflicht von der haftenden Person bzw. Institution zu tragen. ${ }^{146}$

\section{Kodifikation oder Weiterentwicklung von Völkerrecht}

Von besonderer Bedeutung im Kontext staatlicher Einstandspflichten ist, dass sich die Kommission im Gegensatz zu ihren bisherigen Arbeiten nicht mehr primär mit der Kodifizierung von Völkergewohnheitsrecht befasste, sondern sich auch ihrer Aufgabe zur Entwicklung von Rechtsnormen widmete. ${ }^{147}$ Damit ist die Frage nach der völkerrechtlichen Verbindlichkeit dieser Regelwerke der Völkerrechtskommission aufgeworfen.

142 Staaten könnten hier als Beitragsleistende herangezogen werden. Vgl. Art. 6 Abs. 2 i.V.m. Art. 8 IDI-Resolution; Art. 12 IDI-Resolution.

143 Haftungsprinzip 6 Abs. 1, das sich mit effektiven Rechtsbehelfen befasst, und Haftungsprinzip 8 Abs. 2, das Nichtdiskriminierung aufgrund von Nationalität, Wohnsitz oder Aufenthaltsort zum Gegenstand hat, enthalten ebenfalls ein verbindliches ,soll“.

144 J. Barboza, The Environment, Risk and Liability in International Law, 2011, S. 150.

145 Haftungsprinzipien, Kommentar zu Prinzip 5, S. 84, Abs. 8.

146 Vgl. Haftungsprinzip 2 lit. a sublit. iv; J. Barboza, The Environment, Risk and Liability in International Law, 2011, S. $147 \mathrm{f}$.

147 Vgl. C. Tomuschat, The International Law Commission, GYIL 49 (2006), S. 77 (104). 


\section{Kapitel: Staatenhaftung in der Systematik des Völkerrechts}

\section{Staatenverantwortlichkeit}

Die VN-Generalversammlung beauftragte die Völkerrechtskommission ursprünglich mit der Ausarbeitung eines Vertragsentwurfes zur Staatenverantwortlichkeit. ${ }^{148}$ Auf Anraten der Kommission ${ }^{149}$ wurde der finale ASREntwurf im Jahr 2001 zunächst als Anlage in eine Resolution der VNGeneralversammlung aufgenommen. ${ }^{150}$ Resolutionen der VN-Generalversammlung entfalten als Empfehlung gemäß Art. 13 Abs. 1 Charta der Vereinten Nationen (VN-Charta) keine rechtsverbindliche Wirkung. Dieser Ansatz ist dem Umstand geschuldet, dass eine völkervertragliche Kodifizierung der Staatenverantwortlichkeit Anlass zu Diskussionen um das von der Völkerrechtskommission ausgeklügelte System im Ganzen und Raum für Vorbehalte gegen einzelne Bestimmungen bieten würde. ${ }^{151}$ Die divergierenden Interessen in der politischen Landschaft und der Widerstand von Staaten gegen ein bindendes Instrument zur Staatenverantwortlichkeit $^{152}$ würden die Wirksamkeit der Vorschriften im zwischenstaatlichen Gefüge hemmen. ${ }^{153}$

Der akademische Völkerrechtsdiskurs, die internationale Rechtsprechung und das Verhalten von Staaten basierten dagegen bereits vor Abschluss des Projektes durch die Völkerrechtskommission und auch danach zunehmend auf dem konsolidierten Regelwerk der Völkerrechtskommission und trugen auf diese Weise stetig zur steigenden Bedeutung der ASR in der Völkerrechtsordnung bei. ${ }^{154}$ Insbesondere die Bezugnahme auf das Regime durch internationale Spruchkörper ${ }^{155}$ zeigt, dass die ASR mittler-

148 GV Resolution 799 (VIII) vom 07.12.1953.

149 ILC, Report of the International Law Commission on the work of its fifty-third session, YBILC 2001-II/2, UN Doc. A/56/10, S. 25, Rn. 72.

150 GV Resolution 56/83 vom 12.12.2001.

151 A. Pellet, The ILC's Articles on State Responsibility for Internationally Wrongful Acts and Related Texts, in: J. Crawford/ders./S. Olleson (Hg.), The Law of International Responsibility, 2010, S. 75 (87); C. Tomuschat, The International Law Commission, GYIL 49 (2006), S. 77 (96 ff.).

152 Siehe zum Beispiel Report of the Secretary-General, Responsibility of States for internationally wrongful acts, Comments and information received from Governments, UN Doc. A/65/96 vom 14.05.2010.

153 C. Tomuschat, The International Law Commission, GYIL 49 (2006), S. 77 (97 f.).

154 D. D. Caron, The ILC Articles on State Responsibility, AJIL 96 (2002), S. 857 (858, 873); R. Rosenstock, The ILC and State Responsibility, AJIL 96 (2002), S. 792 (792, 796 f.).

155 Siehe Report of the Secretary-General, Responsibility of States for internationally wrongful acts Compilation of decisions of international courts, tribunals and 
weile einen Kernbereich des allgemeinen Völkerrechts bilden. So sind die Artikel der Völkerrechtskommission über die Verantwortlichkeit von Staaten für völkerrechtswidriges Verhalten mittlerweile zum größten Teil ${ }^{156}$ als Völkergewohnheitsrecht anerkannt. ${ }^{157}$ Paradoxerweise entfaltet dieses Instrument der Völkerrechtskommission als bloße Empfehlung größere völkerrechtliche Wirkung als es möglicherweise in Form einer überarbeiteten Konvention getan hätte. ${ }^{158}$

\section{Schadensprävention und Verlustzuweisung}

Die Präventionsartikel sind als Grundlage für die Ausarbeitung eines internationalen Abkommens gedacht, ${ }^{159}$ während die Haftungsprinzipien den Staaten als Richtlinie bei der Implementierung einer Haftung in grenzüberschreitenden Schadensfällen dienen sollen. ${ }^{160}$ Die VN-Generalversammlung hat die Entwürfe lediglich der Aufmerksamkeit der Regierungen empfohlen. ${ }^{161} \mathrm{Ob}$ die Regelwerke trotz dieses geringen formalen

other bodies, Addendum, UN Doc. A/71/80Add.1 vom 20.06.2017; Report of the Secretary-General, Responsibility of States for internationally wrongful acts Compilation of decisions of international courts, tribunals and other bodies, UN Doc. A/74/83 vom 23.04.2019.

156 Streitig ist insbesondere der völkergewohnheitsrechtliche Status von Art. 40, 46, 50 ASR. J. Crawford, State Responsibility, in: R. Wolfrum (Hg.), MPEPIL 2006, http://www.mpepil.com, Rn. 65.

157 O. Dörr, Völkerrechtliche Verantwortlichkeit, in: K. Ipsen, Völkerrecht, 2018, $\$ 29$, Rn. 2 m.w.N.; S. Kadelbach, The Concept of State Responsibility for Private Acts under General International Law, in: S. Hobe/S. Freeland/B. Schmidt-Tedd (Hg.), In Heaven as on Earth?, 2013, S. 105 (105).

158 D. D. Caron, The ILC Articles on State Responsibility, AJIL 96 (2002), S. 857 $(858,866)$; C. Tomuschat, The International Law Commission, GYIL 49 (2006), S. 77 (97).

159 ILC, Report of the International Law Commission on the work of its fifty-third session, International liability for injurious consequences arising out of acts not prohibited by international law (international liability in case of loss from transboundary harm arising out of hazardous activities), YBILC 2001-II/2, UN. Doc. A/56/10 and Corr. 1., S. 145, Rn. 94.

160 ILC, Report of the International Law Commission on the work of its fiftyeighth session, International liability for injurious consequences arising out of acts not prohibited by international law (international liability in case of loss from transboundary harm arising out of hazardous activities), YBILC 2006-II/2, UN. Doc. A/61/10, S. 57, Rn. 63.

161 Die Regierungen wurden hinsichtlich beider Instrumente um Stellungnahmen zu weitergehenden Maßnahmen ersucht. GV Resolution 62/68 vom 08.01.2008, 


\section{Kapitel: Staatenhaftung in der Systematik des Völkerrechts}

Status Einfluss auf die Völkerrechtsordnung haben, ist differenziert zu beantworten.

Die folgende Rechtsquellenanalyse wird zeigen, dass die Präventionsartikel im Wesentlichen als Kodifikation bestehenden Völkerrechts anzusehen sind. ${ }^{162}$ Solche Maßnahmen, die von Staaten zur Regulierung gefährlicher Aktivitäten zu ergreifen sind, finden sich in diversen völkerrechtlichen Abkommen zu spezifischen grenzüberschreitenden Gefährdungslagen und sind auch völkergewohnheitsrechtlich verankert. Die Präventionsartikel präzisieren diese Pflichten für einen breiten Anwendungsbereich; sie werden bereits bei zwischenstaatlichen Konfliktsituationen in Bezug genommen und im Rahmen der Entscheidungsfindung durch internationale Spruchkörper besprochen. ${ }^{163}$

Nicht so eindeutig ist die völkerrechtliche Einordnung der Haftungsprinzipien. ${ }^{164}$ Auch wenn verbindliche Bestimmungen in den Haftungsprinzipien enthalten sind, ist - unüblich für die Arbeiten der Kommission

S. 2, Rn. 3 ff.; GV Resolution 65/28 vom 10.01.2011, S. 1 f., Rn. 1 ff.; GV Resolution 68/114 vom 18.12.2013, S. 1 f., Rn. 1 ff.; GV Resolution 71/143 vom 20.12.2016, S. 1 f., Rn. 1 ff.; GV Resolution 74/189 vom 18.12.2019, S. 2, Rn. 1 ff.

162 Siehe infra 1. Kapitel C.-E.

163 Report of the Secretary General, Consideration of prevention of transboundary harm from hazardous activities and allocation of loss in the case of such harm: Compilation of decisions of international courts, tribunals and other bodies, UN Doc. A/68/94 vom 13.06.2013; Report of the Secretary General, Consideration of prevention of transboundary harm from hazardous activities and allocation of loss in the case of such harm: Compilation of decisions of international courts, tribunals and other bodies, UN Doc. A/71/98 vom 23.06.2016; Report of the Secretary General, Consideration of prevention of transboundary harm from hazardous activities and allocation of loss in the case of such harm: Compilation of decisions of international courts, tribunals and other bodies, UN Doc. A/74/132 vom 04.07.2019. Auch die Völkerrechtskommission bestätigte, dass - in Abgrenzung zu den Haftungsprinzipien - die Präventionsartikel aufgrund vorhandener Staatenpraxis, reif für eine Kodifikation und Weiterentwicklung in Gestalt eines entsprechenden Abkommens sind. ILC, Report of the International Law Commission on the work of its fifty-eighth session, International liability for injurious consequences arising out of acts not prohibited by international law (international liability in case of loss from transboundary harm arising out of hazardous activities), YBILC 2006-II/2, UN. Doc. A/61/10, S. 57, Rn. 63.

164 D. K. Anton, The Principle of Residual Liability in the Seabed Disputes Chamber of the International Tribunal for the Law of the Sea, McGill Int'l J. Sust. Dev. L. \& Pol'y 7 (2011-2012), S. 242 (254). 
- auch der finale Entwurf überwiegend als Richtlinie ausformuliert ${ }^{165}$ und begründet mithin keinen rechtsverbindlichen Charakter. ${ }^{166}$ Die zentrale Komponente des Entwurfs, nach der Staaten eine unverzügliche und angemessene Entschädigung durch Maßnahmen nach Haftungsprinzip 4 gewährleisten sollten, stellt lediglich eine Empfehlung dar und unterscheidet sich grundsätzlich von einer völkerrechtlichen Verpflichtung des Ursprungsstaates, Wiedergutmachung für grenzüberschreitende Schäden sicherzustellen. Das ursprüngliche Ziel der Haftungsstudie, derartige Schäden, die nicht nach den Regeln der Staatenverantwortlichkeit zu kompensieren sind, durch eine völkerrechtliche Haftung aufzufangen, ist somit auch nach jahrelangen Verhandlungen und völkerrechtlichen Diskursen nicht erreicht. Im Gegenteil bleibt es dabei, dass nur Staaten, welche ihre völkerrechtlichen (Präventions-)Pflichten verletzen, verantwortlich sind. ${ }^{167}$ Aufgrund der regelmäßig fehlenden Vorhersehbarkeit der Schadensverursachung durch moderne Technologien kann eine Staatenverantwortlichkeit aber oftmals nicht konstituiert werden. Ferner erforderte es die gebotene Sorgfalt bei einigen besonders gefährlichen Aktivitäten, etwa beim Betrieb von Kernkraftwerken oder bei technischen Eingriffen in (bio-)geochemische Kreisläufe der Erde, diese Aktivitäten gänzlich zu verbieten. ${ }^{168}$ Das ist aber gerade aufgrund der dann wiederum entstehenden Nachteile

165 Siehe Haftungsprinzip 4, Haftungsprinzip 5 lit.c und e, Haftungsprinzip 6 Abs. 2, Haftungsprinzip 7 sowie Haftungsprinzip 8 Abs. 1 und 3. Dies sollte eine breitere Akzeptanz der substanziellen Verpflichtungen erreichen und mögliche Hindernisse einer zwingenden Harmonisierung nationaler Gesetze und Rechtssysteme vermeiden. Haftungsprinzipien, Allgemeiner Kommentar, S. 60, Abs. 12.

166 A. E. Boyle, Globalising Environmental Liability, J. Envtl. L. 17 (2005), S. 3 (17).

167 A. E. Boyle kritisiert: „If the present draft principles on liability are adopted as drafted, states will be free to engage in harmful activities without any obligation to provide effective remedies or redress for transboundary damage unless they are themselves at fault. That is exactly the defect which the Commission was invited to rectify when it began work on the topic in 1978." A. E. Boyle, Globalising Environmental Liability, J. Envtl. L. 17 (2005), S. 3 (25).

168 Vgl. A. Randelzhofer/B. Simma, Das Kernkraftwerk an der Grenze, in: D. Blumenwitz (Hg.), Festschrift für Friedrich Berber, 1973, S. 389 (414 ff., 422); B. Saxler/J. Siegfried/A. Proelss, International liability for transboundary damage arising from stratospheric aerosol injections, Law, Innovation and Technology 7 (2015), S. 112 (123); C. G. Weeramantry, Nukleare Reaktorkatastrophe in Japan, Ein offener Brief an die Umweltminister aller Staaten, 14. März 2011, abrufbar unter: https://www.ialana.de/files/pdf/veröffentlichungen/Offener_Brief_von_C_Weeramantry_Fukushima.pdf (geprüft am 15.05.2020). 


\section{Kapitel: Staatenhaftung in der Systematik des Völkerrechts}

meist nicht realisierbar. ${ }^{169}$ Die Frage nach einer Schadenskompensation in derartigen Konstellationen bleibt daher durch das Recht der Staatenverantwortlichkeit unbeantwortet. Dementsprechend reicht die Komplementärfunktion der Präventionsartikel nicht aus, um dem grundlegenden Gerechtigkeitsgedanken der Haftungsstudie, nämlich dem Schutz unbeteiligter Opfer risikobehafteter Aktivitäten, gerecht zu werden. ${ }^{170}$

Die finale Ausgestaltung der Prinzipien entzieht dem Projekt der Völkerrechtskommission ihre Wirkung und Kraft und stellt gar einen Rückschritt zu bestehendem Völkerrecht dar. ${ }^{171}$ Die Präambel der Haftungsprinzipien spricht von dem Ziel, einen Beitrag zur Entwicklung des internationalen Rechts zu leisten, allerdings sind bisher keine substanziellen Fortschritte durch diesen Entwurf für den Bereich grenzüberschreitender Schäden durch gefährliche Aktivitäten zu verzeichnen. ${ }^{172}$ Demnach ist eine Entwicklung, wie sie bei den ASR erfolgte, in diesem Bereich nicht zu erwarten. ${ }^{173}$

\section{Staatenhaftung}

Der letzte Sonderberichterstatter für die Haftungsstudie, Rao, erklärte: „State liability for transboundary damage for either ultrahazardous activities or hazardous activities does not appear to have support even as a measure of progressive development of law.“174 Die Staatenhaftung sei „a

169 Vgl. J. Barboza, The Environment, Risk and Liability in International Law, 2011, S. 1,3 .

170 Id., S. $155 \mathrm{f}$.

171 Id., S. 146 f., 160; A. E. Boyle, Globalising Environmental Liability, J. Envtl. L. 17 (2005), S. 3 (20).

$172 \mathrm{~J}$. Barboza bestätigt, dass „the principles (...) should have merited a more positive pronouncement from the ILC in order to comply with its mission of developing international law in the field“. J. Barboza, The Environment, Risk and Liability in International Law, 2011, S. 146.

173 P. Stubbe, State Accountability for Space Debris, 2018, S. 71 ff., 118; G. Handl charakterisiert das Resultat der Haftungsstudie zutreffend als „hugely disappointing (...) represent[ing] a triumph of political expediency over pain-staking, education-intensive efforts at progressive development." G. Handl, International Accountability for Transboundary Environmental Harm Revisited, Environmental Policy and Law 37 (2007), S. 116 (122).

174 Sonderberichterstatter $P$. S. Rao, Third report on the legal regime for allocation of loss in case of transboundary harm arising out of hazardous activities, YBILC 2006-II/1, UN Doc. A/CN.4/566, S. 83, Rn. 31. 
case of misplaced emphasis“. ${ }^{175}$ Der Fokus auf eine private Haftung für grenzüberschreitende Schäden widerspricht allerdings der altbewährten Konzeption des Völkerrechts, die den Staat als Nukleus der internationalen Ordnung anerkennt. ${ }^{176}$

Fraglich ist daher, ob der Entwurf von 1996 in der Tat lediglich eine Übung im Recht darstellt oder ob die jahrelangen Arbeiten der Kommission nicht doch fundierte Aussagen zum völkerrechtlichen Stellenwert der Staatenhaftung liefern oder als Blaupause für ein solches Konzept dienen können. Immerhin hatte die Arbeitsgruppe von 1996 den Entwurf zur Staatenhaftung bereits überarbeitet, vorläufig angenommen und an die Generalversammlung der Vereinten Nationen zur Kommentierung übersandt. Dass die im Jahr 1997 neu eingesetzte Arbeitsgruppe dem Entwurf trotzdem eine Absage erteilte und unter dem neuen Berichterstatter schließlich die originäre Staatenhaftung gänzlich ausklammerte, scheint zwar angesichts des Widerstandes in der Staatengemeinschaft, die einer staatlichen Einstandspflicht für grenzüberschreitende Schäden durch die rechtmäßige Ausübung ihrer souveränen Rechte kritisch gegenübersteht, nachvollziehbar. Allerdings ging es der Völkerrechtskommission bei der Haftungsstudie gerade darum, das Völkerrecht in diesem Bereich weiterzuentwickeln. ${ }^{177}$ Der Entwurf aus dem Jahr 1996 wäre dazu geeignet, im Laufe der Zeit eine mit den ASR vergleichbare Wirkung zu entfalten. ${ }^{178}$ Zudem führte die Völkerrechtskommission selbst seit Beginn der Haftungsstudie immer wieder Argumente zugunsten eines verbindlichen Stellenwertes der Staatenhaftung an. ${ }^{179}$ In einem Bericht aus dem Jahr

175 Ders., First report on the legal regime for allocation of loss in case of transboundary harm arising out of hazardous activities, YBILC 2003-II/1, UN Doc. A/CN.4/531, S. 79.

176 C. Foster, The ILC Draft Principles on the Allocation of Loss in the Case of Transboundary Harm Arising out of Hazardous Activities, RECIEL 14 (2005), S. 265 (272); C. W. Jenks, Liability for Ultra-Hazardous Activities in International Law, RdC 117 (1966-I), S. 99 (180).

177 Vgl. Haftungsprinzipien, Präambel.

178 Vgl. J. Barboza: „We all know the place that custom occupies in the international community nowadays and how it develops with relative rapidity from texts through the general acceptance by the States."J. Barboza, Liability, Chinese JIL 1 (2002), S. 499 (524).

179 Siehe zum Beispiel AIL, Kommentar zu Art. 5 AIL, S. 113, Abs. 10: „In including this article within the set of fundamental principles of the topic, the Commission takes careful note of principle 22 of the Stockholm Declaration ${ }^{53}$ and principle 13 of the Rio Declaration ${ }^{54}$ in which States are encouraged to cooperate in developing further international law regarding liability and compensation for environmental damage caused by activities within their jurisdiction or con- 


\section{Kapitel: Staatenhaftung in der Systematik des Völkerrechts}

2004 etwa dokumentiert sie historische Staatenpraxis als Basis für ein völkergewohnheitsrechtlich verankertes Konzept der Staatenhaftung. ${ }^{180}$ Auch im Rahmen der VN-Generalversammlung bekundeten einige Staatenvertreter ein Interesse an der Ausarbeitung einer internationalen Haftung und zeigten, dass ein Konzept der Staatenhaftung durchaus Anklang in der Staatengemeinschaft findet. ${ }^{181}$ Ferner existieren bereits völkerrechtliche Haftungsnormen für bestimmte gefährliche Aktivitäten mit potenziell grenzüberschreitenden Auswirkungen, so dass die Staatenhaftung keineswegs einem unbestellten Feld entspringt. ${ }^{182}$

Im Folgenden wird die Untersuchung von Rechtsprechung, Staatenpraxis und opinio iuris, Haftungsabkommen sowie die Betrachtung allgemeiner Rechtsgrundsätze zeigen, dass die Aussage des Sonderberichterstatters Rao, die Staatenhaftung sei „a case of misplaced emphasis“ ${ }^{183}$, verfehlt ist und dass sehr wohl eine völkerrechtliche Basis für eine Haftung der Staaten für nicht verbotene Aktivitäten besteht.

\section{B. Rechtsprechung}

Das Konzept der Staatenhaftung, wie es ursprünglich von der Völkerrechtskommission gezeichnet wurde, bildet sich in der Rechtsprechung zu staatlichen Einstandspflichten ab. Die Entscheidungen sind allerdings unterschiedlichen Interpretationen zugänglich und verdeutlichen die Schwierigkeiten, die bei der Abgrenzung von Staatenverantwortlichkeit und Staatenhaftung bestehen. Es ist unabdingbar, zwischen staatlichen Schadensvermeidungspflichten (I.) und Verhinderungs- bzw. Schutzpflichten (II.) zu unterscheiden, damit die Trennlinie zwischen staatlichen Einstands-

trol to areas beyond their national jurisdiction. These principles demonstrate the aspirations and preferences of the international community."

180 ILC Secretariat, Survey of liability regimes relevant to the topic of international liability for injurious consequences arising out of acts not prohibited by international law (international liability in case of loss from transboundary harm arising out of hazardous activities), YBILC 2004-II/1, UN Doc. A/CN.4/543, S. $128 \mathrm{ff} .$, Rn. $215 \mathrm{ff}$.

181 GV Resolution 32/151 vom 19.12.1977, S. 214 f., Rn. 7; J. Barboza, The Environment, Risk and Liability in International Law, 2011, S. $116 \mathrm{f}$.

182 S. Erichsen, Das Liability-Projekt der ILC, ZaöRV 51 (1991), S. 94 (104).

183 Sonderberichterstatter $P$. S. Rao, First report on the legal regime for allocation of loss in case of transboundary harm arising out of hazardous activities, YBILC 2003-II/1, UN Doc. A/CN.4/531, S. 79. 
pflichten für völkerrechtlich nicht verbotene Aktivitäten einerseits und solchen für völkerrechtswidriges Verhalten andererseits nicht verwischt.

\section{Schadensvermeidungspflicht}

Die Schadensvermeidungspflicht ist Gegenstand der Staatenhaftung für völkerrechtlich nicht verbotene Aktivitäten. Sie besteht aus verfahrensbezogenen und materiellen Pflichten, die vornehmlich dem Umweltkontext entstammen. Diese staatlichen Schadensvermeidungspflichten können mit anderen völkerrechtlichen Handlungspflichten korrelieren.

\section{Verhaltensbezogene und ergebnisbezogene Pflichten}

Die wegweisenden Entscheidungen des Schiedsgerichts im Trail SmelterFall ${ }^{184}$ aus den Jahren 1938 und 1941 bestimmen den völkerrechtlichen Diskurs um staatliche Einstandspflichten für grenzüberschreitende Schäden. ${ }^{185}$ Die Konkretisierung des Bedeutungsgehaltes der schiedsgerichtlichen Ausführungen unterliegt dabei kontroversen Lesearten. ${ }^{186}$ Erst eine eingehende Analyse des Falles kann aufzeigen, dass das Schiedsgericht die Verletzung einer staatlichen Schadensvermeidungspflicht besprach und Kriterien der Staatenhaftung für völkerrechtlich nicht verbotene Aktivitäten definierte.

184 Trail Smelter Arbitration (United States v. Canada), Award of 16 April 1938 and 11 March 1941, 3 UNRIAA (1941), S. 1905 (1905 ff.).

185 Bemerkenswert ist, dass die schiedsgerichtlichen Entscheidungen nicht nur von den beiden Parteien des Rechtsstreites - den USA Opferstaat und Kanada als Schädigerstaat - akzeptiert und anerkannt wurden, sondern seit jeher auch in der internationalen Gemeinschaft als Anknüpfungspunkt für völkerrechtliche Diskussionen um einen vernünftigen und gerechten Interessenausgleich zwischen Staaten dienen. Siehe etwa J. Bäumler, Das Schädigungsverbot im Völkerrecht, 2017, S. 75 ff.; K. Mickelson, Rereading Trail Smelter, Can. YBIL 31 (1993), S. 219 (219 ff.); A. L. Parrish, Trail Smelter Déjà Vu, BULR 85 (2005), S. 363 (363 ff.); J. E. Read, The Trail Smelter Dispute, Can. YBIL 1 (1963), S. 213 (213 ff.); A. P. Rubin, Pollution by Analogy, OLR 50 (1971), S. 259 (272).

186 K. A. Brent, The Certain Activities case, Asia Pac. J. Envtl. L. 20 (2017), S. 28 (33 f.); J. Ellis, Has International Law Outgrown Trail Smelter?, in: R. M. Bratspies/R. A. Miller (Hg.), Transboundary Harm in International Law, 2006, S. 56 (56); K. Mickelson, Rereading Trail Smelter, Can. YBIL 31 (1993), S. 219 (219 f.). 


\section{Kapitel: Staatenhaftung in der Systematik des Völkerrechts}

Im Trail Smelter-Fall ging es um den Betrieb einer Blei- und Zinkschmelze im kanadischen British Columbia, der zu grenzüberschreitenden Schäden im benachbarten US-amerikanischen Bundesstaat Washington führte. Windströme trugen die Schwefeldioxid-Emissionen der Blei- und Zinkschmelze über die Grenze und verschmutzten unter anderem anliegende Wälder und landwirtschaftlich genutzte Felder. ${ }^{187}$ Die aus dem privaten Betrieb der Schmelze resultierenden Schäden an Grundstücken und Verluste durch verminderte Ernteerträge betrafen zwar auch natürliche Personen, allerdings waren ausschließlich zwischenstaatliche Ansprüche und Pflichten Gegenstand des Schiedsverfahrens. ${ }^{188}$ Die Regierungen der USA und Kanadas ratifizierten eine Schiedsvereinbarung, wonach Völkerrecht und einschlägiges amerikanisches Recht maßgeblich für die Entscheidungsfindung sein sollten. Die Frage nach staatlichen Pflichten zur Wiedergutmachung für entstandenen Schaden und Prävention zukünftiger Schädigungen sollte dabei im Sinne eines gerechten Interessenausgleiches beantwortet werden. ${ }^{189}$

Das Schiedsgericht verpflichtete Kanada zur Zahlung von Schadensersatz ${ }^{190}$ sowie dazu, gegen das schädigende Verhalten Maßnahmen zu ergreifen. ${ }^{191}$ Die Feststellung der Schiedsrichter, „under the principles of international law (...) no State has the right to use or permit the use of its territory in such a manner as to cause injury (...) in or to the territory of another or the properties or persons therein, when the case is of serious consequence and the injury is established by clear and convincing evidence ${ }^{\text {“192}}$, ist Gegenstand divergierender Interpretationsansätze. ${ }^{193}$ Die

187 Trail Smelter Arbitration (United States v. Canada), Award of 16 April 1938 and 11 March 1941, 3 UNRIAA (1941), S. 1905 (1907 ff.).

188 Id., S. 1905 (1913).

189 Art. IV Convention for Settlement of Difficulties Arising From Operation of Smelter at Trail, B.C., 15 April 1935, abgedruckt in: AJIL 30 (1936), S. 163-167.

190 Trail Smelter Arbitration (United States v. Canada), Award of 16 April 1938 and 11 March 1941, 3 UNRIAA (1941), S. 1905 (1933).

191 Die Schiedsrichter entnahmen diese Pflicht der Feststellung des Völkerrechtlers Clyde Eagleton: „A State owes at all times a duty to protect other States against injurious acts by individuals from within its jurisdiction." (C. Eagleton, Responsibility of States in International Law, 1928, S. 80). Sie untermauerten diese Aussage unter Bezugnahme auf andere internationale Streitverfahren und nationale Entscheidungen. Trail Smelter Arbitration (United States v. Canada), Award of 16 April 1938 and 11 March 1941, 3 UNRIAA (1941), S. 1905 (1938 f., 1963, 1966).

192 Id., S. 1905 (1965).

193 J. Ellis, Has International Law Outgrown Trail Smelter?, in: R. M. Bratspies/R. A. Miller (Hg.), Transboundary Harm in International Law, 2006, S. 56 (59 ff.). 
Ausführungen des Schiedsgerichtes werden dahingehend kritisiert, dass sie klare Haftungsmaßstäbe vermissen lassen und unklar bleibe, ob Schadensvermeidung Gegenstand einer ergebnisbezogenen (obligation of result) oder aber verhaltensbezogenen (obligation of conduct) Pflicht ist. ${ }^{194}$ Im Rahmen ergebnisbezogener Pflichten ist ein bestimmter Erfolg geschuldet, während verhaltensbezogene Pflichten lediglich die besten Bemühungen um einen Erfolg fordern. ${ }^{195}$

Zunächst stellte das Gericht eine Einstandspflicht für die entstandenen Schäden fest, ohne ein etwaiges Verschulden seitens Kanadas zu thematisieren. ${ }^{196}$ Maßgeblich war nur die Beweislage, nach der die Schäden von kanadischem Territorium ausgingen. ${ }^{197}$ Das Schiedsgericht bestimmte ferner eine staatliche Einstandspflicht für Schäden, die zukünftig durch den Betrieb der Zinkschmelze entstehen könnten. ${ }^{198}$ Von besonderer Bedeutung ist in diesem Zusammenhang, dass diese Einstandspflicht auch dann greifen soll, wenn die erforderlichen Maßnahmen zur Schadensvermeidung ergriffen worden sind. ${ }^{199}$ Diese schiedsrichterlichen Ausführun-

194 J. Bäumler, Das Schädigungsverbot im Völkerrecht, 2017, S. 82; K. A. Brent, The Certain Activities case, Asia Pac. J. Envtl. L. 20 (2017), S. 28 (33 f.); L. F. E. Goldie, Liability for Damage and the Progressive Development of International Law, ICLQ 14 (1965), S. 1189 (1227, 1230); G. Handl, International Accountability for Transboundary Environmental Harm Revisited, Environmental Policy and Law 37 (2007), S. 116 (119); P. Sands/J. Peel, Principles of International Environmental Law, 2018, S. 746.

195 Sonderberichterstatter J. Crawford stellte zutreffend fest, dass „obligations of result involve in some measure a guarantee of the outcome, whereas obligations of conduct are in the nature of best efforts obligations, obligations to do all in one's power to achieve a result, but without ultimate commitment." Sonderberichterstatter J. Crawford, Second report on State responsibility, YBILC 1999II/1, UN Doc. A/CN.4/498 and Add.1 - 4, S.21, Rn. 57. Die Terminologien "obligation of result" und „obligation of conduct" stammen ursprünglich aus dem europäischen Rechtssystem. Teilweise wird vertreten, dass sich die Bedeutung dieser Begrifflichkeiten im internationalen Kontext umgekehrt habe. So sind nach Sonderberichterstatter $R$. Ago Schadensvermeidungspflichten ergebnisbezogen, da es den Staaten überlassen sei, welche Maßnahmen sie ergreifen, um einen bestimmten Erfolg zu erreichen. Hierzu ausführlich P.-M. Dupuy, Reviewing the Difficulties of Codification, EJIL 10 (1999), S. 371 (371 ff.).

196 L. F. E. Goldie, Liability for Damage and the Progressive Development of International Law, ICLQ 14 (1965), S. 1189 (1230 f.).

197 Trail Smelter Arbitration (United States v. Canada), Award of 16 April 1938 and 11 March 1941, 3 UNRIAA (1941), S. 1905 (1923, 1933).

198 Id., S. 1905 (1966).

199 Id., S. 1905 (1980). 


\section{Kapitel: Staatenhaftung in der Systematik des Völkerrechts}

gen werden als Indikator für eine ergebnisbezogene Pflicht begriffen. ${ }^{200}$ Allein die unterlassene Verhinderung des grenzüberschreitenden Schadens begründe ein völkerrechtswidriges Verhalten und führe zu Rechtsfolgen der Staatenverantwortlichkeit. ${ }^{201}$

Andererseits diskutierte das Gericht aber ausführlich die zulässigen Schadstoffemissionswerte und stellte ein System zur Kontrolle der Emissionen für die Blei- und Zinkschmelze auf, um zukünftige grenzüberschreitende Schäden zu vermeiden. ${ }^{202}$ Dies wird dahingehend interpretiert, dass die Schiedsrichter eine verhaltensbezogene Pflicht beschrieben. ${ }^{203}$ So seien lediglich Maßnahmen zur Schadensvermeidung unter Beachtung der gebotenen Sorgfalt zu ergreifen. Die Missachtung der einschlägigen Sorgfaltsstandards begründe ein völkerrechtliches Delikt, welches zu den Rechtsfolgen der Staatenverantwortlichkeit führe. Der Anwendungsbereich der Staatenverantwortlichkeit sei mithin (unabhängig vom Schadenseintritt) zu bejahen, wenn die geforderten Maßnahmen zur Schadensvermeidung unterblieben. ${ }^{204}$

Beide Ansätze kämpfen um die Einordnung der Pflicht zur Schadensvermeidung in eine der beiden Kategorien, weil sie den Trail Smelter-Fall in den Kontext der Staatenverantwortlichkeit für grenzüberschreitende Schä-

200 K. A. Brent, The Certain Activities case, Asia Pac. J. Envtl. L. 20 (2017), S. 28 (33); L. F. E. Goldie, Liability for Damage and the Progressive Development of International Law, ICLQ 14 (1965), S. 1189 (1230 f.).

201 Vgl. J. Bäumler, Das Schädigungsverbot im Völkerrecht, 2017, S.7; M. J. L. Hardy, International Protection Against Nuclear Risks, ICLQ 10 (1961), S. 739 (751 ff.); J. Peavey-Joanis, A Pyrrhic Victory, in: R. M. Bratspies/R. A. Miller (Hg.), Transboundary Harm in International Law, 2006, S.254 (260); A. P. Rubin, Pollution by Analogy, OLR 50 (1971), S. 259 (262, 272 ff.); R. Verheyen, Climate Change Damage and International Law, 2005, S. 152.

202 Trail Smelter Arbitration (United States v. Canada), Award of 16 April 1938 and 11 March 1941, 3 UNRIAA (1941), S. 1905 (1966).

203 P.-M. Dupuy/C. Hoss, Trail Smelter and Terrorism, in: R. M. Bratspies/R. A. Miller (Hg.), Transboundary Harm in International Law, 2006, S. 225 (226 ff.); T. Stephens, International courts and environmental protection, 2009, S. 158.

204 P. Birnie/A. E. Boyle/C. Redgwell, International Law and the Environment, 2009, S. 147 f., 151; A. E. Boyle, Transboundary air pollution, in: S. Jayakumar/T. Koh/R. Beckman/H. D. Phan (Hg.), Transboundary Pollution, 2015, S. 233 (237); P.-M. Dupuy/C. Hoss, Trail Smelter and Terrorism, in: R. M. Bratspies/R. A. Miller (Hg.), Transboundary Harm in International Law, 2006, S. 225 (227); G. Handl, Trail Smelter in Contemporary International Environmental Law, in: R. M. Bratspies/R. A. Miller (Hg.), Transboundary Harm in International Law, 2006, S. 125 (132 f.). 
den stellen. ${ }^{205}$ Die Kategorisierung ist insofern unmöglich, als Staatenverantwortlichkeit den Verstoß gegen einen anerkannten Rechtssatz (Völkerrechtswidrigkeit) voraussetzt. Die grenzüberschreitende Schädigung konstituiert zwar eine Beeinträchtigung der fremdstaatlichen Rechtsgüter und Interessen, allerdings ist weder der Schaden für sich genommen völkerrechtswidrig, ${ }^{206}$ noch unterliegt die Handlung, das Betreiben der Zinkund Bleischmelze, einem völkerrechtlichen Verbot, so dass für sich genommen keine Normverletzung vorliegt. ${ }^{207}$

Damit geht es letztlich nicht um die Rechtsfolgen der Staatenverantwortlichkeit, sondern vielmehr um zwei Seiten einer Medaille - der Staatenhaftung. ${ }^{208}$ Die eine Seite betrifft die verhaltensbezogene Pflicht zur Vermeidung grenzüberschreitender Schädigungen, die andere Seite die ergebnisbezogene Pflicht, keinen grenzüberschreitenden Schaden (durch eine völkerrechtlich nicht verbotene Aktivität) zu verursachen. ${ }^{209}$ Die Tatsache, dass unabhängig von der Beachtung der ersten Pflicht die zweite Pflicht greift, verdeutlicht, dass es hier nicht um die sekundären Pflichten der Staatenverantwortlichkeit geht, sondern um primäre Pflichten, die sich aus dem Konzept der Staatenhaftung für völkerrechtlich nicht verbotene

205 P.-M. Dupuy/C. Hoss, Trail Smelter and Terrorism, in: R. M. Bratspies/R. A. Miller (Hg.), Transboundary Harm in International Law, 2006, S. 225 (227); L. de la Fayette, The ILC and International Liability, RECIEL 6 (1997), S. 322 (326); G. Handl, Trail Smelter in Contemporary International Environmental Law, in: R. M. Bratspies/R. A. Miller (Hg.), Transboundary Harm in International Law, 2006, S. 125 (130); A. P. Rubin, Pollution by Analogy, OLR 50 (1971), S. 259 (272 ff.); P. Zumbansen, The Conundrum of Corporate Social Responsibility, in: R. M. Bratspies/R. A. Miller (Hg.), Transboundary Harm in International Law, 2006, S. 240 (243).

206 T. Stephens, International Courts and Environmental Protection, 2009, S. 158; $R$. Verheyen, Climate Change Damage and International Law, 2005, S. 152 f. mit dem Hinweis, dass dem Trail Smelter-Fall allerdings keine eindeutige Aussage hierzu entnommen werden könne; vgl. auch K. Bannelier-Christakis, Cyber Diligence, Baltic YBIL 14 (2014), S. 23 (26).

207 J. Bäumler, Das Schädigungsverbot im Völkerrecht, 2017, S. 17.

208 Im völkerrechtlichen Diskurs wird das Konzept der Staatenhaftung zwar mit den Trail Smelter-Entscheidungen in Verbindung gebracht. Im Ergebnis sind die Ausführungen hierzu aber nicht stringent, da das Konzept regelmäßig mit dem Regime der Staatenverantwortlichkeit vermischt wird. Vgl. etwa M. A. Drumbl, Trail Smelter and the International Law Commission 's Work on State Responsibility for Internationally Wrongful Acts and State Liability, in: R. M. Bratspies/R. A. Miller (Hg.), Transboundary Harm in International Law, 2006, S. 85 (91 ff., 98).

209 Vgl. L. de la Fayette, The ILC and International Liability, RECIEL 6 (1997), S. 322 (325 f.). 
Aktivitäten oder - in den Worten des ersten Sonderberichterstatters der Völkerrechtskommission für die Haftungsstudie, Quentin-Baxter, - aus der sogenannten compound primary obligation ergeben. ${ }^{210}$

Rosalyn Higgins stellt zutreffend fest: „Cases like Trail Smelter-which we had all in our youth thought was something to do with international responsibility for harm to your neighbour (and a clear example of the absence of need of malice, or culpa)-are not now questions of state responsibility but are put into another category [- the category of State liability].“211

Im Ergebnis lassen sich dem Trail Smelter-Fall folgende für das Konzept der Staatenhaftung relevante Schlussfolgerungen entnehmen: Nicht nur staatliche Aktivitäten, sondern auch private Aktivitäten auf staatlichem Territorium können die Staatenhaftung begründen. Die Staatenhaftung bezieht sich auf Aktivitäten, die nicht rechtswidrig sind, sondern lediglich aufgrund möglicher schädigender Auswirkungen Einschränkungen unterworfen werden. Die gebotene Sorgfalt definiert diese Einschränkungen, wobei deren Beachtung die staatliche Einstandspflicht nicht entbehrlich macht. Die Staatenhaftung ist verhaltensbezogen und ergebnisbezogen zugleich. ${ }^{212}$ Es besteht die verhaltensbezogene Pflicht, unter Beachtung der gebotenen Sorgfalt Maßnahmen zur Schadensvermeidung zu ergreifen, sowie die ergebnisbezogene Pflicht, keinen grenzüberschreitenden Schaden zu verursachen. Im Ergebnis sind eingetretene Schäden in jedem Fall zu kompensieren. Des Weiteren entspringt die Pflicht zur Schadensvermeidung einem bereits bestehenden Grundsatz des Völkerrechts und betrifft auch Schädigungsquellen, die nicht dem Umweltkontext entspringen. ${ }^{213}$ Allerdings fallen nur erhebliche grenzüberschreitende Schäden in bzw. an staatsfremdem Territorium, Menschen oder Eigentum unter den Anwendungsbereich der Staatenhaftung. Erforderlich ist außerdem, dass diese

210 Sonderberichterstatter R. Q. Quentin-Baxter, Fourth report on international liability for injurious consequences arising out of acts not prohibited by international law, YBILC 1983-II/1, UN Doc. A/CN.4/373, S. 213, Rn. 40.

211 R. Higgins, Problems and Process, 1995, S. 164.

212 Vgl. U. Beyerlin/T. Marauhn, International Environmental Law, 2011, S. 40 f.; A. Epiney, Das „Verbot erheblicher grenzüberschreitender Umweltbeeinträchtigungen“, AVR 33 (1995), S. 309 (329); P. Stubbe, State Accountability for Space Debris, 2018, S. $189 \mathrm{ff}$.

213 J. Bäumler, Das Schädigungsverbot im Völkerrecht, 2017, S. 83. 
Schäden durch eindeutige und überzeugende Beweise belegt sind, um die staatliche Einstandspflicht auszulösen. ${ }^{214}$

\section{Verfahrensbezogene und materielle Pflichten}

Zuletzt bestätigte der Internationale Gerichtshof (IGH) im Jahr 2015 das Konzept der Staatenhaftung in seiner Entscheidung zum Grenzstreit zwischen Nicaragua und Costa Rica ${ }^{215}$, in dem es unter anderem um die Zulässigkeit eines Straßenbauprojektes Costa Ricas an der Grenze zu Nicaragua ging. Der Gerichtshof stellte mit Blick auf den Grenzstreit fest, dass "the claimed right to be free from transboundary harm is the principal right underpinning Nicaragua's request and is derived from the right of a State to sovereignty and territorial integrity " 216 und befasste sich daraufhin mit der Pflicht von Staaten, erhebliche grenzüberschreitende Schäden zu vermeiden.

Der IGH hatte zu entscheiden, ob Costa Rica aufgrund des Projektes verfahrensbezogene Pflichten (procedural obligations) und/oder materielle Pflichten (substantive obligations) des Umweltvölkerrechts verletzt hat. ${ }^{217}$ Verfahrensbezogene Bestimmungen betreffen die Pflichten, die sich ausschließlich auf das zu durchlaufende Verfahren bei der Durchführung eines Vorhabens beziehen. ${ }^{218}$ Dazu zählen Pflichten zur Benachrichtigung, Information, Warnung, Rücksprache und Durchführung einer Umwelt-

214 Trail Smelter Arbitration (United States v. Canada), Award of 16 April 1938 and 11 March 1941, 3 UNRIAA (1941), S. 1905 (1965).

215 IGH, Certain activities carried out by Nicaragua in the Border Area (Costa Rica v. Nicaragua) and Construction of a Road in Costa Rica along the San Juan River (Nicaragua v. Costa Rica), Judgment of 16 December 2015, ICJ Reports 2015, S. 665 (665 ff.).

216 IGH, Construction of a Road in Costa Rica along the San Juan River (Nicaragua v. Costa Rica); Certain Activities Carried Out by Nicaragua in the Border Area (Costa Rica v. Nicaragua), Provisional Measures, Order of 13 December 2013, ICJ Reports 2013, S. 398 (403 Rn. 19).

217 IGH, Certain activities carried out by Nicaragua in the Border Area (Costa Rica v. Nicaragua) and Construction of a Road in Costa Rica along the San Juan River (Nicaragua v. Costa Rica), Judgment of 16 December 2015, ICJ Reports 2015, S. 665 (705 ff. Rn. 100 ff.).

218 A. Epiney, Umweltvölkerrechtliche Rahmenbedingungen für Entwicklungsprojekte, in: W. Meng/U. Magnus/S. Schlemmer-Schulte/T. Cottier/P.-T. Stoll/dies. (Hg.), Das internationale Recht im Nord-Süd-Verhältnis, 2005, S. 329 (334). 


\section{Kapitel: Staatenhaftung in der Systematik des Völkerrechts}

verträglichkeitsprüfung. ${ }^{219}$ Die materiellen Anforderungen an das zu realisierende Projekt werden durch materiell-rechtliche Bestimmungen aufgestellt. ${ }^{220}$ Die Pflicht, erhebliche grenzüberschreitende Schäden zu vermeiden, stellt eine solche Bestimmung dar. ${ }^{221}$ Die verfahrensbezogenen Pflichten zeichnen sich durch die Besonderheit aus, dass sie Bestandteil der materiellen Pflicht zur Schadensvermeidung sind und gleichzeitig unabhängig von dieser Pflicht bestehen. ${ }^{222}$

Der Gerichtshof bejahte zunächst die Verletzung der verfahrensbezogenen Pflicht zur Vornahme einer Umweltverträglichkeitsprüfung, ${ }^{223}$ um in einem nächsten Schritt die Verletzung der materiellen Pflicht zur Schadensvermeidung mit dem bloßen Hinweis darauf, dass kein erheblicher grenzüberschreitender Schaden verursacht wurde, abzulehnen. ${ }^{224}$ Die Entscheidung wird dahingehend kritisiert, dass eine ergebnisbezogene Pflicht zur Schadensvermeidung keinen Rückhalt in der aktuellen Staatenpraxis hat und mit dem überwiegenden Verständnis einer verhaltensbezogenen Pflicht konfligiert. ${ }^{225}$ Die Verletzung der verfahrensbezogenen Pflicht, eine Umweltverträglichkeitsprüfung durchzuführen, führe unabhängig vom Schadenseintritt zur Verletzung der materiellen Schadensver-

219 IGH, Pulp Mills on the River Uruguay (Argentina v. Uruguay), Judgment of 20 April 2010, ICJ Reports 2010, S. 14 (14ff.); J. Brunnée, Procedure and Substance in International Environmental Law, ESIL Reflections 5 (2016), abrufbar unter: https://esil-sedi.eu/post_name-123/ (geprüft am 15.05.2020).

220 A. Epiney, Umweltvölkerrechtliche Rahmenbedingungen für Entwicklungsprojekte, in: W. Meng/U. Magnus/S. Schlemmer-Schulte/T. Cottier/P.-T. Stoll/dies. (Hg.), Das internationale Recht im Nord-Süd-Verhältnis, 2005, S. 329 (341).

221 J. Brunnée, Procedure and Substance in International Environmental Law, ESIL Reflections 5 (2016), abrufbar unter: https://esil-sedi.eu/post_name-123/ (geprüft am 15.05.2020); A. Epiney, Umweltvölkerrechtliche Rahmenbedingungen für Entwicklungsprojekte, in: W. Meng/U. Magnus/S. Schlemmer-Schulte/T. Cottier/P.-T. Stoll/dies. (Hg.), Das internationale Recht im Nord-Süd-Verhältnis, 2005, S. 329 (341).

222 J. Brunnée, Procedure and Substance in International Environmental Law, ESIL Reflections 5 (2016), abrufbar unter: https://esil-sedi.eu/post_name-123/ (geprüft am 15.05.2020).

223 IGH, Certain activities carried out by Nicaragua in the Border Area (Costa Rica v. Nicaragua) and Construction of a Road in Costa Rica along the San Juan River (Nicaragua v. Costa Rica), Judgment of 16 December 2015, ICJ Reports 2015, S. 665 (723 Rn. 162).

224 Id., S. 665 (737 Rn. 217).

225 K. A. Brent, The Certain Activities case: what implications for the no-harm rule?, Asia Pac. J. Envtl. L. 20 (2017), S. 28 (54 f.); J. Brunnée, Procedure and Substance in International Environmental Law, ESIL Reflections 5 (2016), abrufbar unter: https://esil-sedi.eu/post_name-123/ (geprüft am 15.05.2020). 
meidungspflicht, da sie Bestandteil letzterer sei und begründe damit ein völkerrechtswidriges Verhalten. ${ }^{226}$

Die materielle Bestimmung zur Schadensvermeidung ist aber - wie gezeigt - Wesen der Staatenhaftung; diese enthält eine verhaltensbezogene Komponente, die einen Sorgfaltsstandard aufstellt, und auch eine ergebnisbezogene Komponente, nach der kein Schaden verursacht werden darf. Demnach geht es in der Entscheidung zum Grenzstreit zwischen Nicaragua und Costa Rica vielmehr darum, ob die staatliche Einstandspflicht aufgrund der Verletzung der verfahrensbezogenen Pflichten der Schadensvermeidung greift. Dies ist richtigerweise wegen des fehlenden Schadenseintritts zu verneinen. ${ }^{227}$

Gestützt wird dies durch die bereits im Jahr 2010 ergangene Entscheidung des IGH in der Rechtssache Pulp Mills.228 Der Fall betraf den Bau und Betrieb von Zellstofffabriken in Uruguay. Argentinien befürchtete eine Fluss- und Umweltverschmutzung, die als Verletzung eines bilateralen Abkommens zwischen Argentinien und Uruguay zu werten gewesen wäre. Im Ergebnis ging es zwar um die Frage einer staatlichen Einstandspflicht für eine Völkerrechtsverletzung, gleichwohl nahm der IGH in diesem Zusammenhang die Möglichkeit wahr und bestätigte unter Bezugnahme auf seine vorhergehende Rechtsprechung, dass Staaten (umwelt-)völ-

226 P. Birnie/A. E. Boyle/C. Redgwell, International Law and the Environment, 2009, S. 143; J. Brunnée, Procedure and Substance in International Environmental Law, ESIL Reflections 5 (2016), abrufbar unter: https://esil-sedi.eu/post_name-1 23/ (geprüft am 15.05.2020).

227 J. Brunnée kritisiert den innovativen Aussagegehalt dieser Entscheidung, denn „it appears as if the ICJ distinguishes between the duty to take diligent steps to prevent significant transboundary harm, which it then deals with under the rubric of separate procedural obligations, and the duty to take diligent steps not to cause harm, which it considers cannot be violated simply by a failure to act diligently“ und schlussfolgert aus der Entscheidung zutreffend, dass „[u]ntil such time as harm may materialise, states would have limited recourse under the no-harm rule if another state fails to take adequate measures to prevent a risky activity under its jurisdiction or control from causing significant transboundary harm (ie enact relevant domestic law rules and/or enforce them). It may still be possible to hold the source state to account for not conducting an EIA or for failing to notify and consult with potentially affected states. But it would only be possible to invoke the substantive obligation once harm has been caused." J. Brunnée, Procedure and Substance in International Environmental Law, ESIL Reflections 5 (2016), abrufbar unter: https://esil-sedi.eu/post_name-1 23/ (geprüft am 15.05.2020).

228 IGH, Pulp Mills on the River Uruguay (Argentina v. Uruguay), Judgment of 20 April 2010, ICJ Reports 2010, S. 14 (14 ff.). 


\section{Kapitel: Staatenhaftung in der Systematik des Völkerrechts}

kergewohnheitsrechtlich eine verhaltensbezogene Pflicht trifft, grenzüberschreitende Schäden zu vermeiden, und dass die Staaten zugleich die ergebnisbezogene Pflicht haben, keinen grenzüberschreitenden Schaden zu verursachen. ${ }^{229}$ Der IGH prüfte entsprechend in einem ersten Schritt, ob Uruguay seiner verhaltensbezogenen Schadensvermeidungspflicht nachgekommen war. Der Gerichtshof führte aus, dass die gebotene Sorgfalt im Rahmen der verhaltensbezogenen Pflicht zum einen erfordert, geeignete Vorschriften und Maßnahmen zu erlassen ${ }^{230}$ sowie ein gewisses Maß an „Wachsamkeit" bei deren Durchsetzung walten zu lassen und entsprechende Kontrollmechanismen zu implementieren.231 Die Maßnahmen haben außerdem dem Stand der Technik zu entsprechen.232 Der IGH erläuterte weiter, dass die gebotene Sorgfalt zum anderen verfahrensbezogene Maßnahmen, also Benachrichtigung, Information, Warnung, Rücksprache und Umweltverträglichkeitsprüfung erfordert. ${ }^{233}$ Die Verletzung der verfahrensbezogenen Pflichten allein genügte jedoch im zugrunde liegenden Fall nicht, um die Verletzung der materiellen Pflicht im Gesamten zu bejahen. ${ }^{234}$ So verneinte der Gerichtshof die Verletzung der Schadensvermeidungspflicht durch Uruguay und prüfte in einem nächsten Schritt, ob ein grenzüberschreitender Schaden durch den Betrieb der Zellstofffabrik verursacht worden war. Dies lässt den Schluss zu, dass der Gerichthof hier das Konzept der Staatenhaftung zur Grundlage seiner Entscheidung machte, ${ }^{235}$ da für die Staatenverantwortlichkeit neben dem Rechtsbruch gerade kein Schadenseintritt erforderlich ist. Im Ergebnis befand der Gerichtshof, dass Argentinien einen grenzüberschreitenden Schaden durch

229 Id., S. 14 (55 f. Rn. 101, 82 f. Rn. 204); siehe auch IGH, Legality of the Threat or Use of Nuclear Weapons, Advisory Opinion of 8 July 1996, ICJ Reports 1996, S. 226 (241 f. Rn. 29); IGH, Gabcíkovo-Nagymaros Projekt (Hungary v. Slovakia), Judgment of 25 September 1997, ICJ Reports 1997, S. 7 (41 Rn. 53).

230 IGH, Pulp Mills on the River Uruguay (Argentina v. Uruguay), Judgment of 20 April 2010, ICJ Reports 2010, S. 14 (77 Rn. 187).

231 Id., S. 14 (79 Rn. 197).

232 Id., S. 14 (88 f. Rn. 223).

233 J. Brunnée, Procedure and Substance in International Environmental Law, ESIL Reflections 5 (2016), abrufbar unter: https://esil-sedi.eu/post_name-123/ (geprüft am 15.05.2020).

234 So lehnte der IGH das Argument Argentiniens ab, dass die Missachtung verfahrensbezogener Pflichten eine Verletzung der materiellen Schadensvermeidungspflicht bedeutet. IGH, Pulp Mills on the River Uruguay (Argentina v. Uruguay), Judgment of 20 April 2010, ICJ Reports 2010, S. 14 (48 Rn. 72 ff., 49 Rn. 78).

235 D. M. Schmitt, Staatenverantwortlichkeit für Schäden an der biologischen Vielfalt, 2018, S. 338. 
die Zellstofffabrik nicht nachgewiesen hat und lehnte in diesem Zusammenhang auch eine Beweislastumkehr zulasten Uruguays auf Grundlage des Vorsorgegebotes ab. 236

Die beiden Entscheidungen verdeutlichen, dass die verhaltensbezogene Pflicht zur Schadensvermeidung durch die ergebnisbezogene Pflicht, keinen grenzüberschreitenden Schaden zu verursachen, ergänzt und vervollständigt wird. Es handelt sich dabei um primäre Pflichten der Staatenhaftung, wobei der Schadenseintritt konstitutiv für die Begründung der staatlichen Einstandspflicht ist. ${ }^{237}$ Diese Interpretation der Urteile wird durch den Umstand gestützt, dass es vorliegend um völkerrechtlich nicht verbotene Aktivitäten ging und nicht um ein völkerrechtswidriges Verhalten.

\section{Staatenverantwortlichkeit oder doch Staatenhaftung?}

Die Konkretisierung konventionsrechtlicher (Schadensvermeidungs-)Pflichten durch Gutachtenerstattung zählt ebenfalls zu den Aufgaben internationaler Spruchkörper. Zu diesem Zweck nehmen die richterlichen Ausführungen auch auf außervertragliche völkerrechtliche Bestimmungen Bezug und tragen mithin zum Verständnis staatlicher Pflichten in international relevanten Schadensfällen bei. Aufschlussreich sind insbesondere die folgenden Ausführungen zu Haftungsart und -standard. Internationale Spruchkörper, die staatliche Einstandspflichten nur unter engen Voraussetzungen bejahen, begünstigen Haftungslücken, die ohne alternative Lösungen zulasten der Opfer gehen. Die Analyse der einschlägigen Gutachten bestätigt zudem, dass das Erfordernis eines Schadenseintritts notwendige Voraussetzung für das Eingreifen einer Einstandspflicht nach dem Konzept der Staatenhaftung ist. Dies unterscheidet die Staatenhaftung von der Staatenverantwortlichkeit, die grundsätzlich bereits bei Verletzung einer Verhinderungspflicht, das heißt auch ohne Schadenseintritt greift.

236 IGH, Pulp Mills on the River Uruguay (Argentina v. Uruguay), Judgment of 20 April 2010, ICJ Reports 2010, S. 14 (98 f. Rn. 257).

237 Im Gegensatz zu den selbstständigen verfahrensbezogenen Pflichten, die unabhängig von einem Schadenseintritt verletzt sein können. IGH, Pulp Mills on the River Uruguay (Argentina v. Uruguay), Judgment of 20 April 2010, ICJ Reports 2010, S. 14 (49 Rn. 78 f.). 
a) Haftungsart und -standard

Die Kammer für Meeresbodenstreitigkeiten des Internationalen Seegerichtshofs (ITLOS-Meeresbodenkammer) befasste sich in dem Gutachten „Verantwortlichkeiten und Pflichten der Staaten, die Personen und andere Rechtsträger in Bezug auf Tätigkeiten im internationalen Meeresbodengebiet befürworten "238 aus dem Jahr 2011 mit der Frage der völkerrechtlichen Schadensvermeidungspflichten. Anlass des Gutachtens war die Frage nach den Pflichten von Vertragsstaaten des Seerechtsübereinkommens der Vereinten Nationen aus dem Jahr 1982 (SRÜ) ${ }^{239}$ in Bezug auf privatwirtschaftliche Forschungs- und Abbauvorhaben von mineralischen Ressourcen im internationalisierten Gebiet ${ }^{240}$ der Tiefsee nach Teil XI SRÜ i.V.m. dem Übereinkommen zur Durchführung des Teiles XI SRÜ von 1994 $(D U ̈)^{241}$. Private Wirtschaftsunternehmen dürfen nur im Gebiet tätig werden, sofern sie von einem Vertragsstaat oder seinen Staatsangehörigen befürwortet (sponsored) und kontrolliert werden (Art. 153 Abs. 2 SRÜ). Den befürwortenden Vertragsstaat (sponsoring State) ${ }^{242}$ treffen mit Blick auf die potenziell schadensauslösenden Tätigkeiten der befürworteten privaten Vertragsnehmer (contractors) Schadensvermeidungs- und Einstandspflichten, welche von der ITLOS-Meeresbodenkammer unter Bezugnahme auf außervertragliche völkerrechtliche Bestimmungen erörtert wurden. ${ }^{243}$

238 ITLOS, Responsibilities and obligations of states sponsoring persons and entities with respect to activities in the Area, Advisory Opinion of 1 February 2011, ITLOS Case No. 17, ITLOS Reports 2011, S. 10 (10 ff.).

239 Seerechtsübereinkommen der Vereinten Nationen vom 10. Dezember 1982, in Kraft getreten am 16. November 1994, BGBl. 1994 II S. 1798, $1799 \mathrm{ff}$.

240 "Gebiet" im Sinne des SRÜ umfasst den Meeresboden und den Meeresuntergrund jenseits der Grenzen des Bereichs nationaler Hoheitsbefugnisse (Art. 1 Abs. 1 Nr. 1 SRÜ).

241 Teil XI SRÜ befasst sich mit der Erforschung und Ausbeutung der Ressourcen des Meeresbodens und des Meeresuntergrundes jenseits der Grenzen nationaler Hoheitsbefugnisse. Durch das Übereinkommen zur Durchführung des Teiles XI SRÜ vom 28. Juli 1994, BGBl. 1994 II S. 2566, (DÜ) erfolgte eine umfassende Änderung von Teil XI SRÜ, wobei nach Art. 2 DÜ das DÜ und Teil XI SRÜ zusammen als eine Übereinkunft auszulegen sind und im Falle eines Widerspruchs zwischen beiden Instrumenten das DÜ vorgeht.

242 Siehe zum Begriff des „sponsoring State“ $R$. Wolfrum, Hohe See und Tiefseeboden (Gebiet), in: W. Graf Vitzthum (Hg.), Handbuch des Seerechts, 2006, Kapitel 4, S. 287 (335 ff. Rn. 140 ff., Rn. 154).

243 ITLOS, Responsibilities and obligations of states sponsoring persons and entities with respect to activities in the Area, Advisory Opinion of 1 February 2011, ITLOS Case No. 17, ITLOS Reports 2011, S. 10 (57 Rn. 171). 
Das Gutachten widmet sich zunächst den Schadensvermeidungspflichten, die sich aus Art. 139 Abs. 1, Art. 153 Abs. 4 und Art. 4 Abs. 4 Anhang III SRÜ ergeben. ${ }^{244}$ Befürwortende Vertragsstaaten müssen alle notwendigen und angemessenen Maßnahmen ergreifen, um mögliche schädliche Umweltauswirkungen auf den Tiefseeboden durch Aktivitäten befürworteter privater Vertragsnehmer im Gebiet zu vermeiden. ${ }^{245}$ Die ITLOS-Meeresbodenkammer erläuterte - unter Bezugnahme auf die Pulp Mills-Entscheidung des IGH und die Präventionsartikel der Völkerrechtskommission -, dass Kontroll- und Überwachungsmechanismen einzusetzen, nationale Vorschriften zu erlassen und Verwaltungsmaßnahmen zu ergreifen sind, damit Forschungs- und Abbauprojekte in Übereinstimmung mit den Vorgaben des Tiefseebodenregimes durchgeführt werden können. ${ }^{246}$ Das Gutachten bestimmt ferner, dass die Schadensvermeidungspflicht des befürwortenden Vertragsstaates verhaltensbezogen ist ${ }^{247}$ und nach Maßgabe eines objektiven Sorgfaltsmaßstabes, das heißt unabhängig von unterschiedlichen Kapazitäten oder dem volkswirtschaftlichen Entwicklungsstand der jeweiligen Vertragsstaaten, besteht. ${ }^{248}$ Das Gutachten analysiert außerdem umweltvölkerrechtliche Schadensvermeidungspflichten, welche durch tiefseevölkerrechtliches Sekundärrecht, die sogenannten Mining Codes, präzisiert werden. ${ }^{249}$ Darunter fallen die Pflicht zur Durchführung einer Umweltverträglichkeitsprüfung und die Einhaltung des Vorsorgegrundsatzes sowie die Berücksichtigung von sogenannten besten Umwelt-

244 Id., S. 10 (38 Rn. 99).

245 Siehe auch Art. 194 Abs. 2 SRÜ, wonach Staaten alle notwendigen Maßnahmen ergreifen müssen, um sicherzustellen, dass Aktivitäten unter ihrer Hoheitsgewalt oder Kontrolle fremden Staaten und deren Umwelt keine Schäden durch Umweltverschmutzung zufügen.

246 ITLOS, Responsibilities and obligations of states sponsoring persons and entities with respect to activities in the Area, Advisory Opinion of 1 February 2011, ITLOS Case No. 17, ITLOS Reports 2011, S. 10 (40 f. Rn. 108, 42 Rn. 115 f.).

247 Id., S. 10 (41 Rn. 110).

248 Id., S. 10 (53 f. Rn. 158). Der - etwa in der Rechtsordnung der WTO bekannte - Maßstab eines „special and differential treatment for developing and least developed countries“ soll demnach im Rahmen des SRÜ nicht zur Anwendung gelangen. H. Jessen, Staatenverantwortlichkeit und seevölkerrechtliche Haftungsgrundsätze für Umweltschäden durch Tiefseebodenbergbau, ZUR 2 (2012), S. 71 (78).

249 H. Jessen, Staatenverantwortlichkeit und seevölkerrechtliche Haftungsgrundsätze für Umweltschäden durch Tiefseebodenbergbau, ZUR 2 (2012), S. 71 (73). 


\section{Kapitel: Staatenhaftung in der Systematik des Völkerrechts}

schutzpraktiken (best environmental practices). ${ }^{250}$ Die Erörterungen zu diesen drei Aspekten der Schadensvermeidung unterliegen einer progressiven Sichtweise der ITLOS-Meeresbodenkammer. ${ }^{251}$ Diese sprach nämlich der Pflicht zur Durchführung einer Umweltverträglichkeitsprüfung einen völkergewohnheitsrechtlichen Verbindlichkeitsstatus $\mathrm{zu}^{252}$ und erläuterte die Entwicklung zur völkergewohnheitsrechtlichen Geltung des Vorsorgegrundsatzes. ${ }^{253}$ Außerdem ergänzte die ITLOS-Meeresbodenkammer das im Umweltkontext grundsätzlich geltende Erfordernis der Nutzung der „besten verfügbaren Technik“. 254 Das umfassendere Konzept der „besten Umweltschutzpraktiken" - als spezielle Ausformung des Vorsorgegrundsatzes - verdichtet sich demnach zum anwendbaren Maßstab. ${ }^{255}$ Zur Schadensvorsorge in der marinen Umwelt der Tiefsee ist demzufolge nicht nur das nach aktuellem Erkenntnisstand beste Einzelmittel sondern die beste Kombination an Einsatzmitteln, etwa umweltbezogene Kontrollmaßnahmen und weitergehende Strategieentwicklungen, anzuwenden. ${ }^{256}$ Diese drei Leitprinzipien des (fortschrittlich interpretierten) Umweltvölkerrechts ${ }^{257}$ sind zum einen Teil der gebotenen Sorgfalt im Rahmen der

250 ITLOS, Responsibilities and obligations of states sponsoring persons and entities with respect to activities in the Area, Advisory Opinion of 1 February 2011, ITLOS Case No. 17, ITLOS Reports 2011, S. 10 (44 Rn. 122, 45 ff., Rn. 125 ff.).

251 H. Jessen, Staatenverantwortlichkeit und seevölkerrechtliche Haftungsgrundsätze für Umweltschäden durch Tiefseebodenbergbau, ZUR 2 (2012), S. 71 (77 f.).

252 ITLOS, Responsibilities and obligations of states sponsoring persons and entities with respect to activities in the Area, Advisory Opinion of 1 February 2011, ITLOS Case No. 17, ITLOS Reports 2011, S. 10 (50 Rn. 145).

253 Id., S. 10 (47 Rn. 135).

254 Id., S. 10 (48 Rn. 136).

255 D. K. Anton/R. A. Makgill/C. R. Payne, Seabed Mining, Environmental Policy and Law 41 (2011), S. 60 (63); H. Jessen, Staatenverantwortlichkeit und seevölkerrechtliche Haftungsgrundsätze für Umweltschäden durch Tiefseebodenbergbau, ZUR 2 (2012), S. 71 (77).

256 Vgl. United Nations Environment Programme, Expert Group on Best Available Techniques and Best Environmental Practices, Note by Secretariat 29 January 2003, Development of Guidelines on Best Available Techniques and Provisional Guidance on Best Environmental Practices Relevant to Art. 5 and Annex C of the Stockholm Convention on Persistent Organic Pollutants: Use of the Terms Best Available Techniques, Best Environmental Practices and Related Concepts in International Environmental Instruments, UNEP/POPS/EGB.1/INF/3, Annex, abrufbar unter: http://chm.pops.int/Portals/0/docs/from_old_website/d ocuments/meetings/bat_bep/1st_session/EGB1_INF3_terms3.p.pdf (geprüft am 15.05.2020).

257 H. Jessen, Staatenverantwortlichkeit und seevölkerrechtliche Haftungsgrundsätze für Umweltschäden durch Tiefseebodenbergbau, ZUR 2 (2012), S. 71 (77). 
staatlichen Pflicht, die Einhaltung der völkerrechtlichen Vorgaben durch nicht-staatliche Akteure sicherzustellen (obligation to ensure) ${ }^{258}$, und zum anderen unabhängige unmittelbare Pflichten (direct obligations) ${ }^{259}$ des Staates. ${ }^{260}$

Ferner befasst sich das Gutachten ausgiebig mit der Haftungsart und dem Haftungsstandard für Schäden durch privatwirtschaftliche Forschungs- und Abbauprojekte im Gebiet. ${ }^{261}$ Die ITLOS-Meeresbodenkammer legte dafür die Vorschriften des SRÜ aus und konstatierte, dass sich die Bestimmungen zur Kompensationspflicht (liability) auf die sekundären Rechtsfolgen für die Verletzung einer primären Verpflichtung (responsibility) beziehen ${ }^{262}$ und es mithin um eine staatliche Einstandspflicht im Sinne der Staatenverantwortlichkeit für völkerrechtswidriges Verhalten gehe. ${ }^{263}$ Allerdings bestätigte die Kammer zugleich, dass die Einstandspflicht der befürwortenden Vertragsstaaten gemäß Art. 139 Abs. 2 S. 2 SRÜ ${ }^{264}$ von dem Schaden, der durch die privatwirtschaftlichen Forschungs- oder Abbauaktivitäten der befürworteten Vertragsnehmer entsteht, abhängig ist. ${ }^{265}$ Wollte man aber wie die ITLOS-Meeresbodenkammer davon ausgehen, dass die Verletzung der Schadensvermeidungspflicht des befürwortenden Vertragsstaates hinsichtlich privatwirtschaftlicher Abbau- und Forschungsprojekte - in anderen Worten der Pflicht sicherzustellen, dass die Aktivitä-

258 ITLOS, Responsibilities and obligations of states sponsoring persons and entities with respect to activities in the Area, Advisory Opinion of 1 February 2011, ITLOS Case No. 17, ITLOS Reports 2011, S. 10 (40 f. Rn. 108).

259 Id., S. 10 (44 Rn. 121).

260 Id., S. 10 (44 Rn. 122 f.).

261 In Rahmen der staatlichen Einstandspflicht ist entsprechend Art.139 SRÜ zwischen einer Verletzung von unmittelbaren Pflichten der Vertragsstaaten einerseits und der Verletzung von Schadensvermeidungspflichten der befürwortenden Vertragsstaaten hinsichtlich der Aktivitäten von befürworteten Vertragsnehmern andererseits zu unterscheiden, wobei der Schwerpunkt des Gutachtens auf letzterer Pflicht liegt. ITLOS, Responsibilities and obligations of states sponsoring persons and entities with respect to activities in the Area, Advisory Opinion of 1 February 2011, ITLOS Case No. 17, ITLOS Reports 2011, S. 10 (58 Rn. 177).

262 Id., S. 10 (30 Rn. 65 f., 31 Rn. 69, Rn. 71).

263 Id., S. 10 (31 Rn. 70, 57 Rn. 172).

264 Die staatliche Einstandspflicht für die Verletzung unmittelbarer Pflichten des Vertragsstaates wird allein durch Art. 139 Abs. 2 S. 1 SRÜ bestimmt. ITLOS, Responsibilities and obligations of states sponsoring persons and entities with respect to activities in the Area, Advisory Opinion of 1 February 2011, ITLOS Case No. 17, ITLOS Reports 2011, S. 10 (58 Rn. 177).

265 Id., S. 10 (57 Rn. 173). 


\section{Kapitel: Staatenhaftung in der Systematik des Völkerrechts}

ten im Gebiet durch befürwortete Vertragsnehmer in Übereinstimmung und unter Beachtung der Vorgaben des Tiefseebodenregimes erfolgen ( $o b$ ligation to ensure $)^{266}$ - die Verletzung einer völkerrechtlichen Primärnorm bedeutet, ${ }^{267}$ ist nicht ersichtlich, warum zusätzlich das Erfordernis eines Schadens zur Begründung der Verantwortlichkeit zu fordern ist. ${ }^{268}$ Diese Tatbestandsvoraussetzung ist für das Recht der Staatenverantwortlichkeit atypisch, da diese grundsätzlich nur die Verletzung einer Primärnorm voraussetzt. ${ }^{269}$ Die Meeresbodenkammer kategorisierte diese Voraussetzung als seevölkerrechtliche Ausnahme zu den völkergewohnheitsrechtlichen Voraussetzungen der Staatenverantwortlichkeit. ${ }^{270}$ Die Differenzierung der ITLOS-Meeresbodenkammer, wonach die staatliche Verantwortlichkeit nach dem SRÜ bei Schadensverursachung greife, während in Konstellationen bloßer Verletzung von Schadensvermeidungspflichten - ohne Schadenseintritt - das völkergewohnheitsrechtliche Regime der Staatenverantwortlichkeit zur Anwendung gelange, ${ }^{271}$ ist wenig überzeugend. Das Schadenserfordernis stellt vielmehr ein Indiz für eine staatliche Einstandspflicht im Sinne der Staatenhaftung für völkerrechtlich nicht verbotene Aktivitäten dar. Die Schadensvermeidungspflicht und die Kompensationspflicht sind im SRÜ in einem Artikel zusammengefasst (Art.139 SRÜ). Nur sofern diese zusammengesetzte Primärnorm verletzt ist, ist eine völkerrechtswidrige Vertragsverletzung zu bejahen. Die Haftungsbestim-

266 In diesem Zusammenhang nimmt das Gutachten insbesondere auf die Kernbestimmungen Art. 139 Abs. 1, Art. 153 Abs. 4 und Art. 4 Abs. 4 Anhang III SRÜ Bezug. ITLOS, Responsibilities and obligations of states sponsoring persons and entities with respect to activities in the Area, Advisory Opinion of 1 February 2011, ITLOS Case No. 17, ITLOS Reports 2011, S. 10 (40 Rn. 103).

267 Id., S. 10 (41 Rn. 109).

268 Id., S. 10 (58 Rn. 176).

269 Die ITLOS-Meeresbodenkammer selbst bestätigt unter Bezugnahme auf die Entscheidung im Rainbow Warrior-Fall (Case concerning the difference between New Zealand and France concerning the interpretation or application of two agreements, concluded on 9 July 1986 between the two States and which related to the problems arising from the Rainbow Warrior Affair, UNRIAA (1990) Vol. XX, S. 215 (267 Rn. 110)) und den Kommentar der Völkerrechtskommission zu den ASR (Kommentar zu Art. 2 ASR, S. 36, Rn. 9), dass Staatenverantwortlichkeit grundsätzlich allein durch völkerrechtswidriges Verhalten und unabhängig von einem etwaigen (materiellen) Schaden begründet wird. ITLOS, Responsibilities and obligations of states sponsoring persons and entities with respect to activities in the Area, Advisory Opinion of 1 February 2011, ITLOS Case No. 17, ITLOS Reports 2011, S. 10 (58 Rn. 178).

Id., S. 10 (58 Rn. 178).

271 Id., S. 10 (66 Rn. 210). 
mungen des SRÜ greifen unbeschadet anderer völkerrechtlicher Bestimmungen (Art. 139 Abs. 2 SRÜ), so dass eine völkerrechtswidrige Vertragsverletzung dann durch die völkergewohnheitsrechtlichen Rechtsfolgen der Staatenverantwortlichkeit geahndet werden kann. ${ }^{272}$ Im Übrigen ist dem Seevölkerrecht eine staatliche Einstandspflicht für erlaubte Aktivitäten auch nicht fremd. Gemäß Art. 110 SRÜ ist es einem Kriegsschiff ausdrücklich erlaubt, ein fremdes Schiff, das den Verdachtstatbeständen des Abs. 1 unterfällt, anzuhalten. Es besteht allerdings gemäß Abs. 3 eine Ersatzpflicht für entstandene Schäden oder Verluste, sofern sich der Verdacht als unbegründet erweist und das angehaltene Schiff keine den Verdacht rechtfertigende Handlung begangen hat. Dementsprechend überrascht die Vehemenz, mit der die ITLOS-Meeresbodenkammer die Existenz einer völkerrechtlichen Staatenhaftung unter Hinweis auf die diesbezüglichen Bestrebungen der Völkerrechtskommission verneint hat. ${ }^{273}$

Des Weiteren sind in diesem Kontext die Diskussionen zum Haftungsstandard für Schäden am Gebiet durch privatwirtschaftliche Abbau- und Forschungsprojekte interessant. Art. 139 SRÜ bestimmt, dass Staaten nicht für privatwirtschaftliche Forschungs- und Abbauprojekte durch befürwortete Vertragsnehmer haften, „sofern der Vertragsstaat alle notwendigen und angemessenen Maßnahmen ergriffen hat, um die wirksame Einhaltung nach Artikel 153 Absatz 4 und Anlage III Artikel 4 Absatz 4 zu

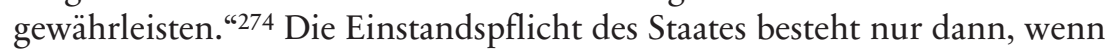
der Schaden in kausaler Verbindung zur vertragsstaatlichen Sorgfaltswidrigkeit steht. ${ }^{275}$ Das Fehlverhalten des befürworteten Vertragsnehmers wird dem Staat hingegen nicht zugerechnet. ${ }^{276}$ Demnach konstituiert Art.139 SRÜ nur eine verschuldensabhängige Einstandspflicht. Das SRÜ selbst enthält zwar keine expliziten Bestimmungen zu einer verschuldensunabhängigen Einstandspflicht der befürwortenden Vertragsstaaten, eine

272 Art. 235 SRÜ bestimmt allgemein, dass die Vertragsstaaten zum Schutz und zur Bewahrung der Meeresumwelt verpflichtet sind und in Übereinstimmung mit dem Völkerrecht haften.

273 ITLOS, Responsibilities and obligations of states sponsoring persons and entities with respect to activities in the Area, Advisory Opinion of 1 February 2011, ITLOS Case No. 17, ITLOS Reports 2011, S. 10 (65 f. Rn. 209).

274 Diese Haftungsausnahme gilt allerdings nicht für unmittelbare Pflichten des Vertragsstaates. ITLOS, Responsibilities and obligations of states sponsoring persons and entities with respect to activities in the Area, Advisory Opinion of 1 February 2011, ITLOS Case No. 17, ITLOS Reports 2011, S. 10 (65 Rn. 206 f.).

275 Id., S. 10 (59 Rn. 181).

276 Id., S. 10 (59 f. Rn. 182). 


\section{Kapitel: Staatenhaftung in der Systematik des Völkerrechts}

derartige Haftung ist nichtsdestoweniger möglich, wenn sich eine entsprechende Haftungsregel seit der Verabschiedung des SRÜ im Jahr 1982 entwickelt und etabliert hat. ${ }^{277} \mathrm{Im}$ Rahmen der mündlichen Verhandlung argumentierte der mexikanische Botschafter Joel Hernandez, dass in die staatliche Pflicht zur Schadensvermeidung eine subsidiäre Haftung der befürwortenden Staaten hineinzulesen sei. ${ }^{278}$ Außerdem wurde diese Interpretation des SRÜ durch die Stellungnahmen von International Union for the Conservation of Nature und Stichting Greenpeace Council gemeinsam mit World Wide Fund for Nature unterstützt. ${ }^{279}$ Die Stellungnahmen gründen auf die sich im Völkerrecht abzeichnende Entwicklung einer subsidiären Staatenhaftung und verweisen auf die Haftungsprinzipien der Völkerrechtskommission, die zwar eine primäre Haftung der privaten Betreiber etablieren, aber auch eine subsidiäre Haftung von Staaten vorsehen, wenn eine unverzügliche und angemessene Entschädigung durch den privaten Betreiber ausbleibt. Das Übereinkommen dürfe nicht nur nach Sinn und Zweck, sondern müsse auch im Lichte der gegenwärtigen Gegebenheiten und normativen Entwicklungen interpretiert werden. $\mathrm{Zu}$ den notwendigen und angemessenen Maßnahmen, die vom befürwortenden Staat zur Beachtung des Übereinkommens zu ergreifen seien, zähle letztendlich auch die Pflicht, Finanzmittel verfügbar zu machen, um Schäden zu beheben. Demnach ende eine Haftung für Schäden nach dem SRÜ nicht mit einer etwaigen Insolvenz oder einem sonstigen Haftungsausfall des befürworteten Vertragsnehmers. Im Gegenteil greife in dieser Konstellation

277 D. K. Anton, The Principle of Residual Liability in the Seabed Disputes Chamber of the International Tribunal for the Law of the Sea, McGill Int'l J. Sust. Dev. L. \& Pol'y 7 (2012), S. 241 (253).

278 Dies entspricht dem Verständnis Quentin-Baxters, wonach Schadenskompensation mit Schadensprävention nach dem schadensauslösenden Ereignis gleichzusetzen ist. Ambassador Joel Hernandez (Legal Adviser of the Mexican Ministry of Foreign Affairs of Mexico), ITLOS Case No. 17, Responsibilities and obligations of States sponsoring persons and entities with respect to activities in the Area, Public sitting (15 September 2010), Verbatim Record, ITLOS/PV.2010/2/Rev.2, abrufbar unter: https:/www.itlos.org/fileadmin/itlos/documents/cases/case_no_ 17/C17_PV_2010_2_Rev2_E.pdf (geprüft am 15.05.2020), S. 46 f.

279 Beide Stellungnahmen der Nichtregierungsorganisationen sind bemerkenswerterweise auf der ITLOS-Internetseite verlinkt, so dass den Aussagen besonderes Gewicht beizumessen ist. ITLOS, Case No. 7, Responsibilities and obligations of States sponsoring persons and entities with respect to activities in the Area, abrufbar unter: https://www.itlos.org/cases/list-of-cases/case-no-17/\#c584 (geprüft am 15.05.2020). 
eine subsidiäre Haftung des befürwortenden Staates. ${ }^{280}$ Die ITLOS-Meeresbodenkammer war allerdings nicht gewillt, eine verschuldensunabhängige Haftung anzuerkennen. So sei ein Rückgriff auf den befürwortenden Staaten auch dann ausgeschlossen, wenn der Vertragsnehmer unfähig sei, die volle Schadenssumme zu begleichen. ${ }^{281}$ Das Gutachten der ITLOS-Meeresbodenkammer begrenzt damit die Haftung für Schäden durch Abbauund Forschungsprojekte im Gebiet auf einen verschuldensabhängigen Haftungsstandard. ${ }^{282}$

Wie die ITLOS-Meeresbodenkammer selbst einräumte, führt ihr Verständnis vom seevölkerrechtlichen Haftungsregime und den ergänzenden völkerrechtlichen Bestimmungen zu Haftungslücken. ${ }^{283}$ Mit der Absage an eine subsidiäre Staatenhaftung bleibt eine Haftung aus, wenn der Vertragsnehmer zwar schuldhaft handelt, jedoch unfähig ist, die volle Schadenssumme zu begleichen oder sein Vermögen außerhalb eines möglichen internationalen Vollstreckungszugriffes des befürwortenden Vertragsstaates liegt. Ferner führt die Ablehnung eines verschuldensunabhängigen Haftungsstandards dazu, dass Schäden nicht zu kompensieren sind, wenn der befürwortende Staat die geforderten notwendigen und geeigneten Maßnahmen zur Schadensvermeidung ergriffen hat und das schadensauslösende Verhalten des befürworteten Vertragsnehmers ebenso unter Beachtung der vertraglichen Vorgaben erfolgt. Schließlich begründet die Negierung einer völkerrechtlichen Staatenhaftung für erlaubte Aktivitäten eine Haftungslücke, sofern der befürwortende Vertragsstaat es zwar unterlassen

280 International Union for the Conservation of Nature and Natural Resources, Commission on Environmental Law, Oceans, Coastal and Coral Reefs Specialist Group, Written Statement, 19 August 2010, ITLOS Case No. 7, Responsibilities and obligations of States sponsoring persons and entities with respect to activities in the Area, abrufbar unter: https:/www.itlos.org/fileadmin/itlos/docum ents/cases/case_no_17/StatementIUCN.pdf (geprüft am 15.05.2020), S. $29 \mathrm{ff}$., Rn. 98 ff.; Stichting Greenpeace Council (Greenpeace International)/The World Wide Fund For Nature, Memorial, 13 August 2010, ITLOS Case No. 7, Responsibilities and obligations of States sponsoring persons and entities with respect to activities in the Area, abrufbar unter: https://www.itlos.org/fileadmin/itlos /documents/cases/case_no_17/Statement_Greenpeace_WWF.pdf (geprüft am 15.05.2020), S. $19 \mathrm{ff}$.

281 ITLOS, Responsibilities and obligations of states sponsoring persons and entities with respect to activities in the Area, Advisory Opinion of 1 February 2011, ITLOS Case No. 17, ITLOS Reports 2011, S. 10 (64 f. Rn. 204).

282 Id., S. 10 (61 Rn. 189).

283 Id., S. 10 (64 Rn. 203). 


\section{Kapitel: Staatenhaftung in der Systematik des Völkerrechts}

hat, alle notwendigen und geeigneten Maßnahmen zu ergreifen, diese Sorgfaltswidrigkeit aber nicht in kausaler Verbindung zum Schaden steht.

Um den benannten Haftungslücken zu begegnen, empfahl die ITLOSMeeresbodenkammer, vorerst das Einrichten eines Fonds zur Schadensdeckung in Betracht zu ziehen. ${ }^{284}$ Dieser Ansatz entspricht der Leitidee der Art. $136 \mathrm{ff}$. SRÜ, nämlich der Anerkennung und Bewahrung eines "Gemeinsamen Erbes der Menschheit" (common heritage) ${ }^{285}$, die eine Art „Tiefseeboden-Solidargemeinschaft“ ${ }^{\text {“286 }}$ begründet. ${ }^{287}$ So könnte etwa über die Mining Codes eine ergänzende und verschuldensunabhängige Staatenhaftung etabliert werden. ${ }^{288}$ Unter Bezugnahme auf Art. 304 SRÜ betonte die ITLOS-Meeresbodenkammer, dass das Haftungssystem nicht „statisch“ und mithin geeignet ist, völkervertragliche und völkergewohnheitsrechtliche Entwicklungen einzubeziehen. ${ }^{289}$ Damit hat die ITLOS-Meeresbodenkammer zumindest die Tür für das Konzept einer Staatenhaftung offen gelassen.

b) Schnittstelle zwischen Schadensvermeidungspflicht und Verhinderungspflicht

Staatliche Schadensvermeidungspflichten waren auch Gegenstand des Gutachtens des Interamerikanischen Gerichtshofes für Menschenrechte (IACtHR) aus dem Jahr 2017. ${ }^{290}$ Anlass des Gutachtens war eine Vorlagefrage

284 Id., S. 10 (65 Rn. 205).

285 Siehe weiterführend zum „Gemeinsamen Erbe der Menschheit“ $R$. Wolfrum, The Principle of the Common Heritage of Mankind, ZaöRV (43) 1983, S. $312 \mathrm{ff}$.

286 H. Jessen, Staatenverantwortlichkeit und seevölkerrechtliche Haftungsgrundsätze für Umweltschäden durch Tiefseebodenbergbau, ZUR 2 (2012), S. 71 (73).

287 M. Hipp, The International Sea Bed Authority, in: P. Ehlers/R. Lagoni (Hg.), International Maritime Organisations and Their Contribution Towards a Sustainable Marine Development, 2006, S. 135 (135 ff.); H. Jessen, Staatenverantwortlichkeit und seevölkerrechtliche Haftungsgrundsätze für Umweltschäden durch Tiefseebodenbergbau, ZUR 2 (2012), S. 71 (73); R. Wolfrum, Hohe See und Tiefseeboden (Gebiet), in: W. Graf Vitzthum (Hg.), Handbuch des Seerechts 2006, Kapitel 4, S. 287 (338 Rn. 155).

288 H. Jessen, Staatenverantwortlichkeit und seevölkerrechtliche Haftungsgrundsätze für Umweltschäden durch Tiefseebodenbergbau, ZUR 2 (2012), S. 71 (79).

289 ITLOS, Responsibilities and obligations of states sponsoring persons and entities with respect to activities in the Area, Advisory Opinion of 1 February 2011, ITLOS Case No. 17, ITLOS Reports 2011, S. 10 (66 Rn. 211).

290 IACtHR, The Environment and Human Rights (State obligations in relation to the environment in the context of the protection and guarantee of the rights 
Kolumbiens zur Interpretation der Amerikanischen Menschenrechtskonvention mit Blick auf legale Infrastrukturarbeiten, denen das Risiko eines erheblichen Schadens an der Meeresumwelt im breiteren karibischen Raum inhärent war. Das Gutachten thematisiert Umweltverpflichtungen, die den völkerrechtlichen Menschenrechtsschutz aller Mitglieder der Organisation der Amerikanischen Staaten betreffen und entfaltet damit weitreichende Wirkung. ${ }^{291}$

Der IACtHR bestätigte in seinem Gutachten unter Bezugnahme auf Entscheidungen des IGH, die Erklärungen von Stockholm und Rio sowie das Seerechtsübereinkommen der Vereinten Nationen, dass Staaten verpflichtet sind, sicherzustellen, dass Aktivitäten auf ihrem Territorium der Umwelt anderer Staaten und Gebieten außerhalb ihrer territorial begrenzten Kontrolle keinen erheblichen Schaden zufügen. ${ }^{292}$ Staaten müssen Aktivitäten mit potenziell schädigenden Auswirkungen regulieren, beaufsichtigen und kontrollieren. Die Durchführung einer Umweltverträglichkeitsprüfung und das Erstellen von Notfallplänen zählen ebenfalls zu den erforderlichen Schadensvermeidungsmaßnahmen. ${ }^{293}$ Ferner müssen Staaten mit potenziell betroffenen Staaten kooperieren, das heißt diese über mögliche umweltschädigende Auswirkungen informieren, Rücksprache halten und nach Treu und Glauben verhandeln. ${ }^{294}$ Außerdem bekräftigte der IACtHR die Bedeutung des Vorsorgegrundsatzes nach dem Verständnis der Erklärung von Rio. Danach hat Schadensvermeidung bereits zu erfolgen, wenn zwar keine wissenschaftliche Gewissheit aber eine plausible Annahme dafür besteht, dass eine Aktivität ernsthafte und irreversible Umweltschäden verursachen kann. ${ }^{295}$ In diesem Zusammenhang verweist das Gutachten auf die Pulp Mills-Entscheidung des IGH ${ }^{296}$, in der dem Vorsorgegrundsatz zur Lösung des Streitfalles eine gewisse Bedeutung zugestanden wird, sowie auf das Gutachten der ITLOS-Meeresbodenkam-

to life and to personal integrity - interpretation and scope of Articles 4(1) and 5(1) of the American Convention on Human Rights). Advisory Opinion OC-23/17 of 15 November 2017. Series A No. 23, requested by the Republic of Colombia (im Folgenden: IACtHR, Advisory Opinion on Environment and Human Rights) abrufbar unter: http://www.corteidh.or.cr/docs/opiniones/seriea 23_ing.pdf (geprüft am 15.05.2020).

291 I d., S. 15, Rn. 29-31, S. 17, Rn. 35.

$292 I d .$, S. 52 f., Rn. 128 f.

293 Id., S. 56 ff., Rn. $141 \mathrm{ff}$.

$294 I d .$, S. 72 ff., Rn. $181 \mathrm{ff}$.

295 Id., S. 69 ff., Rn. 175 ff.

296 IGH, Pulp Mills on the River Uruguay (Argentina v. Uruguay), Judgment of 20 April 2010, ICJ Reports 2010, S. 14 (71 Rn. 164). 


\section{Kapitel: Staatenhaftung in der Systematik des Völkerrechts}

mer zu den Verantwortlichkeiten und Pflichten derjenigen Staaten, die Personen und andere Rechtsträger in Bezug auf Tätigkeiten im internationalen Meeresbodengebiet befürworten ${ }^{297}$, wonach der Vorsorgegrundsatz die Tendenz aufweist, in Völkergewohnheitsrecht zu erwachsen. ${ }^{298}$

Von besonderer Bedeutung ist die Feststellung des IACtHR, dass Staaten nicht nur zur Schadensvermeidung verpflichtet sind, sondern auch zur unverzüglichen, angemessenen und effektiven Wiedergutmachung für grenzüberschreitende Schäden durch Aktivitäten auf ihrem Territorium oder unter ihrer Hoheitsgewalt. ${ }^{299}$ Der Gerichtshof betonte wiederholt, dass diese staatlichen umweltvölkerrechtlichen Pflichten unabhängig von der Recht- oder Unrechtmäßigkeit der schadensauslösenden Aktivität greifen und bestärkte damit das Konzept der Staatenhaftung. ${ }^{300}$ Der IACtHR belegte die staatliche Einstandspflicht mit einem Verweis auf die Präventionsartikel der Völkerrechtskommission, welche staatliche Pflichten für völkerrechtlich nicht verbotene Aktivitäten wiedergeben. ${ }^{301}$

Der IACtHR forderte ferner einen kausalen Zusammenhang zwischen Schäden, die Menschen entstehen, und dem Handeln oder Unterlassen des Ursprungsstaates bezüglich der schadensauslösenden Aktivität. ${ }^{302}$ Das Kausalitätserfordernis formulierte der IACtHR in Anlehnung an das Gutachten der ITLOS-Meeresbodenkammer zu den Verantwortlichkeiten und Pflichten der Staaten, die Personen und andere Rechtsträger in Bezug auf Tätigkeiten im internationalen Meeresbodengebiet befürworten. ${ }^{303}$ Die ITLOS-Meeresbodenkammer betonte allerdings an diversen Stellen im fraglichen Gutachten, dass eine staatliche Einstandspflicht nach dem

297 ITLOS, Responsibilities and obligations of states sponsoring persons and entities with respect to activities in the Area, Advisory Opinion of 1 February 2011, ITLOS Case No. 17, ITLOS Reports 2011, S. 10 (47 Rn. 135).

298 IACtHR, Advisory Opinion on Environment and Human Rights, S. 71, Rn. 177, S. 72, Rn. 180.

299 Id., S. 43 f., Rn. 103.

300 Ibid.

301 Id., S. 43, Fn. 196.

302 Id., S. 43 f., Rn. 103. Die Aussage des Gerichtshofes, dass eine staatliche Einstandspflicht unabhängig von der Völkerrechtmäßigkeit oder Völkerrechtswidrigkeit der schädigenden Aktivität entsteht, wird der Bedeutung der völkerrechtlichen Qualität der zugrunde liegenden schadensauslösenden Aktivität für eine Unterscheidung zwischen Staatenverantwortlichkeit und Staatenhaftung nicht gerecht. Allerdings würde bei völkerrechtswidrigem Verhalten naturgemäß Staatenverantwortlichkeit greifen. Die Passage bringt damit lediglich zum Ausdruck, dass auch eine völkerrechtmäßige Aktivität staatliche Einstandspflichten begründen kann. 
Seerechtsübereinkommen nur für völkerrechtswidriges Verhalten in Betracht komme. ${ }^{304}$ Nach Auffassung der ITLOS-Meeresbodenkammer seien die Bestrebungen der Völkerrechtskommission, staatliche Einstandspflichten für völkerrechtlich nicht verbotene Aktivitäten zu etablieren, noch nicht in eine völkerrechtliche Regel erwachsen. ${ }^{305}$ Der Verweis auf das Gutachten ITLOS-Meeresbodenkammer enthält aber das Präfix „Similarly, see“306, so dass das Kausalitätserfordernis mit dem Verständnis der ITLOSMeeresbodenkammer lediglich vergleichbar sein soll. Es besteht folglich kein Widerspruch zu einer staatlichen Einstandspflicht ohne Völkerrechtsverstoß, wie es zuvor von dem Gerichtshof bekräftigt wurde. ${ }^{307}$ Das Kausalitätserfordernis ist schließlich mit Blick auf den dem Gutachten des IACtHR zugrunde liegenden Sachverhalt zu interpretieren. Es bezieht sich auf den ursächlichen Zusammenhang zwischen dem Verhalten des

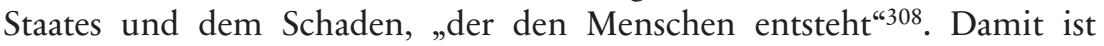
die grenzüberschreitende Verletzung von Menschenrechten und nicht der grenzüberschreitende Umweltschaden für sich gemeint. Nach Auffassung des IACtHR können extraterritoriale Menschenrechtsverletzungen infolge von grenzüberschreitenden Umweltschäden zur Staatenverantwortlichkeit des Ursprungsstaates führen. ${ }^{309}$ Diese Verantwortlichkeit entsteht aber unbeschadet der Tatsache, dass die staatlichen Umweltpflichten zur Schadensvermeidung und Wiedergutmachung dem System der Staatenhaftung für völkerrechtmäßige Aktivtäten entspringen. ${ }^{310}$

304 ITLOS, Responsibilities and obligations of states sponsoring persons and entities with respect to activities in the Area, Advisory Opinion of 1 February 2011, ITLOS Case No. 17, ITLOS Reports 2011, S. 10 (59 Rn. 181).

305 Id., S. 10 (65 f. Rn. 209).

306 IACtHR, Advisory Opinion on Environment and Human Rights, S. 43 f., Fn. 197.

307 Kritisiert wird in diesem Zusammenhang der angebliche „lack of legal rigour" hingegen von G. Vega-Barbosa/L. Aboagye, A Commentary on the Advisory Opinion of the Inter-American Court of Human Rights on the Environment and Human Rights, Note e commenti, DPCE Online 34 (2018), abrufbar unter: http://www.dpceonline.it/index.php/dpceonline/article/view/506 (geprüft am 15.05.2020), Rn. 291 (296).

308 Das Gutachten besagt: „States may be held responsible for any significant damage caused to persons" (Hervorhebung hinzugefügt), IACtHR, Advisory Opinion on Environment and Human Rights, S. 43 f., Rn. 103.

309 Id., S. 43, Rn. 101. Das innovative System eines extraterritorialen Menschenrechtsschutzes basiert auf staatlichen Schutzpflichten und wird unter Punkt II. besprochen.

310 Id., S. 43 f., Rn. 103. 


\section{Kapitel: Staatenhaftung in der Systematik des Völkerrechts}

II. Verhinderungs- und Schutzpflichten

Wie gezeigt, lösen nicht-staatliche Aktivitäten, die negative Auswirkungen haben können, staatliche Handlungspflichten aus. Es ist zu unterscheiden, ob ein Unterlassen zur Staatenverantwortlichkeit führt oder lediglich Wiedergutmachungspflichten der Staatenhaftung bedingt. Dabei ist das Wesensmerkmal des zugrunde liegenden Verhaltens für die Einordnung der staatlichen Handlungspflicht zu betrachten. Die Schadensvermeidungspflicht greift bei völkerrechtlich nicht verbotenen Aktivitäten und ist Gegenstand der Staatenhaftung. Verhinderungspflichten betreffen hingegen Handlungen, die völkerrechtlich verboten sind, sofern sie von Staaten vorgenommen würden, und staatliche Schutzpflichten beziehen sich auf kriminelle bzw. menschrechtsverletzende Handlungen. Die Verletzung von Verhinderungs- bzw. Schutzpflichten führt zu Staatenverantwortlichkeit.

\section{Völkerrechtlich verbotene Handlungen}

Die Korfu Kanal-Entscheidung des IGH aus dem Jahr $1949^{311}$ bestätigt die Unsicherheiten, die bei einer Abgrenzung zwischen Staatenverantwortlichkeit und Staatenhaftung bestehen und verdeutlicht gleichzeitig den Unterschied zwischen Schadensvermeidungspflichten und Verhinderungspflichten. Hintergrund der Entscheidung waren Minen im Kanal vor der Küste Albaniens, die Personenschäden und Sachschäden an Kriegsschiffen des Vereinigten Königreichs verursachten, die sich auf friedlicher Durchfahrt im Kanal befanden. ${ }^{312}$ Der Ursprung der Minen konnte nicht geklärt werden. Insbesondere blieb unklar, ob die albanische Regierung selbst die Minen gelegt oder den Vorgang zumindest geduldet hatte. ${ }^{313}$ Die Pflicht Albaniens zur Warnung vor den Minen leitete der IGH aus allgemeinen Grundsätzen her, nämlich „elementary considerations of humanity, even more exacting in peace than in war; the principle of the freedom of maritime commination; and every State's obligation not to allow knowingly its territory to be used for acts contrary to the rights of other States. "314 Eine klare Trennung zwischen Schadensvermeidungspflichten der Staaten-

311 IGH, Corfu Channel Case (United Kingdom of Great Britain and Northern Ireland v. Albania), Judgment of 9 April 1949, ICJ Reports 1949, S. 4 (4 ff.).

312 Id., S. 4 (5).

313 Id., S. 4 (16 ff.).

314 Id., S. 4 (22). 
haftung und Verhinderungspflichten, die bei Verletzung zur Staatenverantwortlichkeit führen, bietet diese Auswahl an Grundsätzen allerdings nicht. ${ }^{315}$ Insbesondere der Umstand, dass der Gerichtshof nicht näher definiert, was mit „Handlungen entgegen fremdstaatlichen Rechten“ gemeint ist und unter welchen Umständen sich Staaten hierauf berufen können, führt zu konfusen Interpretationen der Entscheidung. So wird die Korfu Kanal-Entscheidung in aller Regel in einem Atemzug mit dem Trail Smelter-Schiedsspruch genannt. Die Korfu Kanal-Entscheidung erweitere die Anwendbarkeit der Schadensvermeidungspflicht aus dem Trail Smelter-Verfahren auf Bereiche außerhalb des Umweltkontextes. Obwohl der IGH die schiedsgerichtlichen Entscheidungen im Trail Smelter-Fall nicht explizit in Bezug nahm, erfährt diese Interpretation der Korfu Kanal-Entscheidung in der Völkerrechtslehre breite Zustimmung. ${ }^{316}$ Die Schadensverursachung im Trail Smelter-Fall sei gleichbedeutend mit der Verletzung fremdstaatlicher Rechte im Korfu Kanal-Fall. Das staatliche Unterlassen in beiden Fällen sei völkerrechtswidrig und führe zur Staatenverantwortlichkeit. ${ }^{317}$ Theoretisch könnte demnach jede schädigende (nicht-staatliche)

315 Vgl. ILC Secretariat, Survey of liability regimes relevant to the topic of international liability for injurious consequences arising out of acts not prohibited by international law (international liability in case of loss from transboundary harm arising out of hazardous activities), YBILC 2004-II/1, UN Doc. A/ CN.4/543, S. 129, Rn. 226.

316 J. Bäumler, Das Schädigungsverbot im Völkerrecht, 2017, S. 3; K. A. Brent, The Certain Activities case, Asia Pac. J. Envtl. L. 20 (2017), S. 28 (34); R. Crootof, International Cybertorts, CLR 103 (2018), S. 565 (601); P.-M. Dupuy/C. Hoss, Trail Smelter and Terrorism, in: R. M. Bratspies/R. A. Miller (Hg.), Transboundary Harm in International Law, 2006, S. 225 (226); J. D. Jolley, Attribution, State Responsibility, and the Duty to Prevent Malicious Cyber-Attacks in International Law, 2017, abrufbar unter: http://theses.gla.ac.uk/id/eprint/8452 (geprüft am 15.05.2020), S. 191 ff.; V. P. Nanda/G. (R.) Pring, International Environmental Law and Policy for the 21st Century, 2013, S. 82; P. N. Okowa, State Responsibility for Transboundary Air Pollution in International Law, 2000, S. 68; J. E. Viñuales, The Contribution of the International Court of Justice to the Development of International Environmental Law, Fordham ILJ 32 (2008), S. 232 (238); B. A. Walton, Duties Owed, Yale LJ 126 (2017), S. 1460 (1488). Nichtsdestoweniger wird der unterschiedliche Kontext der Entscheidungen hervorgehoben. Siehe etwa P. Birnie/A. E. Boyle/C. Redgwell, International Law and the Environment, 2009, S. 144.

317 Siehe zum Beispiel J. D. Jolley, Attribution, State Responsibility, and the Duty to Prevent Malicious Cyber-Attacks in International Law, 2017, abrufbar unter: http://theses.gla.ac.uk/id/eprint/8452 (geprüft am 15.05.2020), S. 191 ff.; M. Rau, IGH v. 9.4.1949 - Korfu-Kanal, Souveränität verpflichtet! Unterlassenshaftung und Beweiserleichterung im Recht der Staatenverantwortlichkeit, in: J. Men- 


\section{Kapitel: Staatenhaftung in der Systematik des Völkerrechts}

Handlung eine Verletzung fremdstaatlicher Rechte bedeuten und damit eine Völkerrechtswidrigkeit begründen. Dies käme allerdings einer Umgehung der völkerrechtlichen Grundregel, nach der ein Staat nicht für privates Verhalten verantwortlich ist ${ }^{318}$, gleich und führte gleichzeitig zu einer ausufernden Anwendung des Regimes der Staatenverantwortlichkeit mit dessen weitreichenden Konsequenzen. ${ }^{319}$ Dem begegnen Interpretationsansätze aus jüngerer Zeit mit der Idee, die benannten staatlichen Handlungspflichten mit Blick auf Aktivitäten, die negative Auswirkungen auf fremde Staaten haben können, unter das Konzept der Staatenhaftung zu subsumieren. ${ }^{320}$ Doch dies führt ebenso zu einer Vermischung von Verhinderungspflichten im Sinne der Korfu Kanal-Entscheidung und Schadensvermeidungspflichten, die Gegenstand der Trail Smelter-Entscheidungen waren. ${ }^{321}$

Der Korfu Kanal-Fall unterscheidet sich in einem wesentlichen Punkt vom Trail Smelter-Fall. Die Verletzung fremdstaatlicher Interessen erfolgt hier aufgrund einer Handlung, die, sofern von Staaten vorgenommen, völkerrechtswidrig wäre. Das Minenlegen in internationalen Meerengen und damit die Einschränkung des Rechts der friedlichen Durchfahrt ist völkerrechtswidrig. ${ }^{322}$ Die staatliche Einstandspflicht kann demnach nicht

zel/J. Hoffmann/T. Pierlings (Hg.), Völkerrechtsprechung, 2005, S. 756 (758f.); R. Rivera, U.S. State Responsibility á la Trail Smelter, MLR 5 (2012), S. 3 (16); J. E. Vinuales, The Contribution of the International Court of Justice to the Development of International Environmental Law, Fordham ILJ 32 (2008), S. 232 (237 ff.).

318 S. Kadelbach, The Concept of State Responsibility for Private Acts under General International Law, in: S. Hobe/S. Freeland/B. Schmidt-Tedd (Hg.), In Heaven as on Earth?, 2013, S. 105 (106); R. Lefeber, Transboundary Environmental Interference and the Origin of State Liability, 1996, S. 56; A. Seibert-Fohr, Die völkerrechtliche Verantwortung des Staats für das Handeln von Privaten, ZaöRV 73 (2013), S. 37 (43).

319 Vgl. A. Epiney, Das „Verbot erheblicher grenzüberschreitender Umweltbeeinträchtigungen“, AVR 33 (1995), S. 309 (356f.).

320 B. A. Walton, Duties Owed, Yale LJ 126 (2017), S. 1460 (1488); R. Crootof, International Cybertorts, CLR 103 (2018), S. 565 (601); J. E. Messerschmidt, Hackback, Col. JTL 52 (2013), S. 275 (311).

321 Vgl.J. Barboza, The Environment, Risk and Liability in International Law, 2011, S. 46.

322 Das Recht auf friedliche Durchfahrt durch Meerengen wurde durch den IGH expressis verbis als Bestandteil des geltenden Völkerrechts anerkannt. L. Arimat$s u$, International Law Applicable to Naval Mines, Chatham House, International Security Department Workshop Summary, October 2014, abrufbar unter: https:/www.chathamhouse.org/sites/files/chathamhouse/field/field_docu ment/20140226NavalMines.pdf (geprüft am 15.05.2020), S. 4 f.; R. Lapidoth, 
dem Konzept der Staatenhaftung für Aktivitäten, die völkerrechtlich nicht verboten sind, entspringen. Diese Schlussfolgerung wird durch die Besonderheit gestützt, dass das Minenlegen eine kriegerische Handlung darstellt, die nicht mit wirtschaftlichen Tätigkeiten vergleichbar ist, bei denen der Schaden eine gänzlich unbeabsichtigte Nebenfolge darstellt. ${ }^{323}$ Das Minenlegen und mithin die Behinderung der friedlichen Durchfahrt unterscheiden sich grundlegend vom Betreiben einer Zink- und Bleischmelze. Der Grund für eine mögliche Einstandspflicht mit Blick auf erstere Handlung beruht auf einem völkerrechtlichen Verbotstatbestand, ${ }^{324}$ während bei letzterer Aktivität der Gefährdungstatbestand ausschlaggebend ist. ${ }^{325}$

Fraglich ist allerdings, ob dies im Umkehrschluss bedeutet, dass eine unterlassene Verhinderung der Beeinträchtigung fremdstaatlicher Interessen durch eine an sich völkerrechtswidrige Handlung denknotwendig zur Staatenverantwortlichkeit führt. Fest steht, dass eine Staatenverantwortlichkeit nur dann bejaht werden kann, wenn der Staat selbst eine völkerrechtliche Primärpflicht verletzt. Im Rahmen nicht-staatlicher Handlungen ist also das staatliche Fehlverhalten näher zu spezifizieren. Betrachtet man die Ausführungen des Gerichtshofes, fließt die Handlungspflicht Albaniens aus dem Kennenmüssen bzw. der unterstellten Kenntnisnahme

International Straits, in: R. Wolfrum (Hg.), MPEPIL 2018, http://www.mpepil. com, Rn. 3 f.; M. Rau, IGH v. 9.4.1949 - Korfu-Kanal, Souveränität verpflichtet! Unterlassenshaftung und Beweiserleichterung im Recht der Staatenverantwortlichkeit, in: J. Menzel/J. Hoffmann/T. Pierlings (Hg.), Völkerrechtsprechung, 2005, S. 756 (758); M. Waibel, Corfu Channel Case, in: R. Wolfrum (Hg.), MPEPIL 2013, http://www.mpepil.com, Rn. 12.

323 Auch wenn der IGH die Verhinderungspflicht in seiner Korfu Kanal-Entscheidung nicht auf einen bestimmten Sachzusammenhang beschränkt, liegt der Schluss nahe, dass der Gerichtshof eine staatliche Einstandspflicht für militärähnliche Handlungen außerhalb des Krieges aufstellt. So sei die betreffende Handlungspflicht „even more exacting in peace than in war.“ IGH, Corfu Channel Case (United Kingdom of Great Britain and Northern Ireland v. Albania), Judgment of 9 April 1949, ICJ Reports 1949, S. 4 (22).

324 In diesem Zusammenhang ist zu betonen, dass der Völkerrechtsverstoß nicht durch das (nicht-staatliche) Minenlegen, sondern durch die unterlassene Warnung durch Albanien begründet wird. Allerdings besteht diese Handlungspflicht zur Warnung aufgrund des völkerrechtlich missbilligten Verhaltens dem Minenlegen.

325 Vgl. J. Barboza, The Environment, Risk and Liability in International Law, 2011, S. 91: „The contingent character of the damage consequent to an inherent risk of the activity prevents its being prohibited, but only if its usefulness is considerably higher than the harm it threats with and a legal regime of prevention/compensation sits on the other plate of the scale." 


\section{Kapitel: Staatenhaftung in der Systematik des Völkerrechts}

vom Minenlegen in staatseigenen Gewässern. ${ }^{326}$ Es ist allgemein schwierig, einem Unterlassen eine rechtliche Wirkung zuzuschreiben. ${ }^{327}$ Während völkervertraglich normierte Schutzpflichten, etwa in Konventionen zum Menschenrechtsschutz, die Völkerrechtswidrigkeit des staatlichen Unterlassens vorgeben, ist die rechtliche Wirkung des Unterlassens in der vorliegenden Situation klärungsbedürftig. In Anlehnung an das Konzept der Staatenhaftung muss daher die völkerrechtliche Qualität des schädigenden Verhaltens maßgeblich sein. ${ }^{328}$ Mangels explizit normierter Handlungspflichten hilft das Qualitäts-Kriterium, eine allgemeine völkerrechtliche Verhinderungspflicht von einer staatlichen Schadensvermeidungspflicht abzugrenzen. 329 Das Minenlegen bzw. die Beeinträchtigung der friedli-

326 IGH, Corfu Channel Case (United Kingdom of Great Britain and Northern Ireland v. Albania), Judgment of 9 April 1949, ICJ Reports 1949, S. 4 (22); Summary S. 6 f.: „As regards the obligations resulting for her [Albania] from this knowledge, they are not disputed. It was her duty to notify shipping and especially to warn the ships proceeding through the Strait on October 22nd of the danger to which they were exposed. In fact, nothing was attempted by Albania to prevent the disaster, and these grave omissions involve her international responsibility."

327 O. Dörr, Weitere Rechtsquellen des Völkerrechts, in: K. Ipsen, Völkerrecht, 2018, $\mathbb{1 9}$, Rn. 8.

$328 \mathrm{~J}$. Barboza differenziert indes zwischen gefährlichen (hazardous) Aktivitäten, bei denen Staatenhaftung greifen soll, und schädlichen (noxious) Aktivitäten, die dem Anwendungsbereich der Staatenverantwortlichkeit unterfallen sollen. Im Trail Smelter-Fall transformiere sich die schädliche Aktivität durch das aufgestellte Emissionsmanagementregime in eine gefährliche Aktivität. So sei zunächst - wie auch im Rahmen des Korfu Kanal-Falles - Staatenverantwortlichkeit angezeigt und später greife für die nunmehr gefährliche Aktivität die Staatenhaftung. J. Barboza, The Environment, Risk and Liability in International Law, 2011, S. $46 \mathrm{ff}$., 51 f. Allerdings soll sich die Qualität der Aktivität allein durch die Kenntnis von deren schädigenden Wirkung wandeln. Es ist nicht überzeugend, dass allein diese Kenntnis zu unterschiedlichen Einstandspflichten für ein und dieselbe Aktivität führen soll. Zudem kann „schädlich“ nicht mit „völkerrechtswidrig“ gleichgesetzt werden. Eine grundsätzliche Unterscheidung von Aktivitäten nach an sich völkerrechtswidrig einerseits und völkerrechtlich erlaubt andererseits ist daher der Vorzug zu geben.

329 Vgl. id., S. 71. Die völkerrechtliche Qualität der zugrundeliegenden Handlung ist auch deswegen maßgeblich, weil andernfalls die Beeinträchtigung fremdstaatlicher Interessen im Sinne der Korfu Kanal-Entscheidung nur zu verhindern ist, wenn sie von nicht-staatlichen Akteuren vorgenommen wird. Den Staaten selbst wäre es entsprechend der Aussage "not to allow knowingly its territory to be used" (Hervorhebung hinzugefügt) erlaubt, fremdstaatliche Interessen zu beeinträchtigen, wenn diese nicht zugleich einem völkerrechtlichen Verbotstatbestand unterfielen. Dieser Ansatz würde zu paradoxen Ergebnissen führen. 
chen Durchfahrt stellt ein Verhalten dar, welches Staaten selbst völkerrechtlich untersagt ist. In Anbetracht dieser Tatsache müssen Staaten bei Kenntnis bzw. Kennenmüssen ${ }^{330}$ handeln, um ein entsprechendes nichtstaatliches Verhalten und deren Auswirkungen zu verhindern. ${ }^{331}$ An dieser Stelle sei nochmals darauf hingewiesen, dass es hier nicht um den Vorwurf einer Komplizenschaft mit den Tätern, sondern um eine eigene staatliche Verantwortlichkeit geht. Gleichwohl kann die staatliche Handlungspflicht nicht völlig losgelöst von dem nicht-staatlichen Verhalten beurteilt werden. Dies bedeutet im Umkehrschluss, dass die staatliche Verhinderungspflicht greift, wenn nicht-staatliche Handlungen völkerrechtlich missbilligt sind. Der IGH postulierte in seiner Korfu Kanal-Entscheidung eine Staatenverantwortlichkeit für die Verletzung einer allgemeinen Verhinderungspflicht von völkerrechtlich verbotenen Handlungen und deren Auswirkungen. Die Verantwortlichkeit entsteht nur dann, wenn die Handlung auf dem staatseigenen Territorium in Kenntnis des Staates erfolgt oder dieser hiervon Kenntnis haben müsste.

Im Ergebnis führt im Rahmen der Verhinderungspflicht allein die unterlassene Verhinderung der Beeinträchtigung fremdstaatlicher Interessen zur Staatenverantwortlichkeit, weil die staatliche Handlungspflicht ein Verhalten betrifft, das völkerrechtlich verboten ist. Im Rahmen der Staatenhaftung hingegen ist die unterlassene Schadensvermeidung nur im $\mathrm{Zu}-$ sammenspiel mit unterlassener Wiedergutmachung völkerrechtswidrig, ${ }^{332}$ da die Vermeidungspflicht sich auf den Schaden bezieht, während die

Demnach sind „acts contrary to the right of other States“ Handlungen, die völkerrechtlich verboten sind. Im Rahmen der Trail Smelter-Entscheidungen ist dagegen die Klarstellung "to use or permit the use of its territory" (Hervorhebung hinzugefügt) notwendig, weil es hier um völkerrechtlich erlaubte Aktivitäten geht.

330 Die Staatenhaftung hingegen erfordert keine Kenntnis von der Aktivität, sondern nur, dass der Schaden durch die fragliche Aktivität vorhersehbar ist. Vgl. id., S. 90. Kenntnis ist im Rahmen der Staatenhaftung nur insofern relevant, als gegebenenfalls striktere Sorgfaltsanforderungen gelten (vgl. Art. 22 lit. c AIL).

331 IGH, Corfu Channel Case (United Kingdom of Great Britain and Northern Ireland v. Albania), Judgment of 9 April 1949, ICJ Reports 1949, S. 4 (22).

332 Dies entspricht auch der ursprünglichen Konzeption der Staatenhaftung der Völkerrechtskommission aus dem Jahr 1996. Vgl. AIL, Kommentar zu Art. 5 AIL, S. 112, Abs. 5: "The article thus rejects a regime which would permit the conduct of activities hazardous to other States without any form of compensation or other relief when harm occurs." (Hervorhebung hinzugefügt). 
schadensauslösende Tätigkeit selbst nicht verhindert werden muss, weil sie keinem völkerrechtlichen Verbot unterliegt. ${ }^{333}$

\section{Nicht-staatliche Verbrechen}

Staatliche Handlungspflichten ergeben sich auch, wenn fremde Staatsorgane oder Staatsangehörige zu Opfern von Verbrechen durch Private auf staatseigenem Hoheitsgebiet werden. Die Staatenverantwortlichkeit entsteht, wenn der Staat es unterlässt, geeignete Maßnahmen zur Verhinderung dieser Taten zu ergreifen sowie in Fällen von Straffreiheit für diese Verbrechen oder staatlichem Unwillen zu deren Verfolgung. ${ }^{334}$ Auch wenn der Staat die kriminelle Handlung nicht befürwortet, kann die unterlassene verantwortungsbewusste Erfüllung staatlicher Funktionen ein völkerrechtswidriges Verhalten darstellen. ${ }^{335}$ Schutzpflichten sind mit Schadensvermeidungspflichten vergleichbar, da auch sie aus mehreren Komponenten, das heißt Verhinderung und Verfolgung der privaten Taten sowie Wiedergutmachung, bestehen. ${ }^{336}$ Der Unterschied zur Schadensvermeidungspflicht liegt darin, dass die zugrunde liegende Aktivität einen Unrechtstatbestand erfüllt, ${ }^{337}$ der die staatliche Handlungspflicht bedingt. Es geht mithin nicht um das Risiko durch eine erlaubte Tätigkeit, die zur Staatenhaftung führt, sondern um die unterlassene Ausübung staatlicher

333 J. Barboza, The Environment, Risk and Liability in International Law, 2011, S. 46.

334 Ständiger Internationaler Gerichtshof (StIGH), S.S. Lotus (France v. Turkey), Judgment of 7 September 1927, PCIJ Series A, No. 10, Dissenting Opinion of Judge Moore, S. 88: „It is well settled that a State is bound to use due diligence to prevent the commission within its dominions of criminal acts against another nation or its people.“; M. N. Schmitt, Preemptive Strategies in International Law, Mich. JIL 24 (2003), S. 513 (540 f.); A. Seibert-Fohr, Die völkerrechtliche Verantwortung des Staats für das Handeln von Privaten, ZaöRV 73 (2013), S. 37 (43 f.); M. J. Sklerov, Solving the Dilemma of State Response to Cyberattacks, Military Law Review 201 (2009), S. 1 (12f.).

335 S. Kadelbach, The Concept of State Responsibility for Private Acts under General International Law, in: S. Hobe/S. Freeland/B. Schmidt-Tedd (Hg.), In Heaven as on Earth?, 2013, S. 105 (107).

336 A. Seibert-Fohr, Die völkerrechtliche Verantwortung des Staats für das Handeln von Privaten, ZaöRV 73 (2013), S. 37 (43 Fn. 17).

337 Privates illegales Verhalten hat einen völkerrechtlich missbilligten Inhalt. Vgl. O. Kimminich, Völkerrechtliche Haftung für das Handeln Privater im Bereich des internationalen Umweltschutzes, AVR 22 (1984), S. 241 (263). 
Schutzfunktionen mit Blick auf private Verbrechen. Dieses staatliche Fehlverhalten begründet Staatenverantwortlichkeit.

Die Entscheidung des IGH zum Teheraner Geisel-Fall ${ }^{338}$ aus dem Jahr 1980 beschreibt, unter welchen Voraussetzungen Staatenverantwortlichkeit wegen der Verletzung von staatlichen Handlungspflichten entsteht. In dem Fall ging es um Schutzpflichten des Iran im Bereich des Diplomatenund Konsularrechts. Demonstranten erstürmten die in Teheran gelegene US-amerikanische Botschaft und hielten Botschaftsangehörige gefangen. Im Folgenden kam es zu weiteren Überfällen auf die amerikanischen Konsulate in Tabriz und Schiraz. Die US-Regierung bat die iranische Regierung wiederholt um Hilfe, um die Erstürmung der Botschaft und der Konsulate sowie die Geiselnahme des Botschaftspersonals zu verhindern und später zumindest zu beenden. Gleichwohl ergriffen die iranischen Behörden keine Maßnahmen, um dem Unrecht zu begegnen. ${ }^{339}$ Der IGH untersuchte zunächst, ob ein tatsächliches Handeln des Staates oder ein Auftrag an die militante Gruppe durch staatliche Stellen gegeben war, was eine Zurechnung der betreffenden Handlungen bedeutet hätte. Die Besetzung und der Beginn der Geiselnahme ließen sich nach der Beweislage nicht dem Staat zurechnen und waren demnach nicht als Handlung des Staates zu qualifizieren. Die Geschehnisse betrafen für sich genommen völkerrechtlich unerhebliches Verhalten von Privatpersonen. ${ }^{340}$ Allerdings ergab sich aus dem Wiener Übereinkommen über diplomatische Beziehungen und dem Wiener Übereinkommen über konsularische Beziehungen sowie aus den gleichlautenden Verpflichtungen des allgemeinen Völkerrechts die Pflicht der iranischen Regierung zur Ergreifung geeigneter Maßnahmen zum Schutz der Gebäude und des Stabs der amerikanischen Botschaft und der Konsulate. ${ }^{341}$ Der IGH bejahte daher die Staatenverantwortlichkeit des Iran aufgrund der vollständigen Untätigkeit der iranischen Regie-

338 IGH, United States Diplomatic and Consular Staff in Tehran (USA v. Iran), Judgment of 24 May 1980, ICJ Reports 1980, S. 3 (3 ff.).

339 Id., S. 3 ( 31 f. Rn. 63 ff.). Der IGH unterteilte die Geschehnisse zur Beantwortung der Frage nach der Verantwortlichkeit des Iran in zwei Phasen (S. 28 f. Rn. 56). Die erste Phase betraf den bewaffneten Überfall auf die Botschaft in Teheran, die Gefangennahme der Botschaftsinsassen als Geiseln sowie die Überfälle auf die Konsulate in Tabriz und Shiraz (S.30 Rn.60). Die zweite Phase bezog sich auf die Fortdauer der Geiselnahme bis zum Tag der Freilassung der Geiseln (S. 33 Rn. 69).

340 Id., S. 3 (28 ff. Rn. 56 ff.).

341 Id., S. 3 (32 Rn. 67). 


\section{Kapitel: Staatenhaftung in der Systematik des Völkerrechts}

rung $^{342}$ und verurteilte den Iran, unverzüglich alle notwendigen Maßnahmen zur Beendigung der Geiselnahme zu ergreifen, Wiedergutmachung zu leisten sowie Entschädigung für das erlittene Unrecht zu zahlen. ${ }^{343}$

\section{Menschenrechtsverletzungen}

Insbesondere im Bereich des Menschenrechtsschutzes sind Schutzpflichten völkervertraglich etabliert. Die Menschenrechtsabkommen verpflichten die Vertragsparteien, die darin verankerten Rechte nicht nur zu achten, sondern auch deren Ausübung sicherzustellen. ${ }^{344}$

Wie das im Rahmen der Schadensvermeidungspflichten besprochene Gutachten des IACtHR aus dem Jahr 2007 verdeutlicht, ergeben sich Besonderheiten für Schutzpflichten im Rahmen grenzüberschreitender Menschenrechtsverletzungen durch umweltschädigende Aktivitäten. Hier überschneiden sich die Rechtskreise der Staatenhaftung und Staatenverantwortlichkeit, weil die zugrunde liegende Aktivität für sich genommen völkerrechtlich nicht verboten ist aber gleichzeitig mit völkerrechtswidrigem Verhalten von Staaten korreliert. Führt die Aktivität nämlich zu grenzüberschreitenden Umweltschäden und gleichzeitig zu extraterritorialen Menschrechtsverletzungen, bedingt die Aktivität sowohl staatliche Pflichten zur Schadensvermeidung und Wiedergutmachung als auch staatliche Pflichten zum Schutz von Menschenrechten aufgrund der völkervertraglichen Vorgaben.

342 Zudem bejahte der IGH die Staatenverantwortlichkeit des Iran sowohl für die weiteren Überfälle auf die amerikanischen Konsulate in Tabriz und Schiraz als auch für das ungehinderte Zuendeführen der Geiselnahme in Teheran, weil die iranische Regierung das fragliche Verhalten als sein eigenes anerkannt und angenommen habe. Der Gerichtshof stellte fest: „The approval given to [the seizure] by the Ayatollah Khomeini and other organs of the Iranian State, and the decision to perpetuate them, translated the continuing occupation of the Embassy and detention of the hostages into acts of that State" (Hervorhebung hinzugefügt). Id., S. 3 (35 Rn. 74).

343 Id., S. 3 (44 f. Rn. 95).

344 Siehe Art. 1 Amerikanische Menschenrechtskonvention; Art. 1 Europäische Menschenrechtskonvention; Art. 2 Abs. 1 Internationaler Pakt über bürgerliche und politische Rechte; Art. 2 Übereinkommen der Vereinten Nationen gegen Folter und andere grausame, unmenschliche oder erniedrigende Behandlung oder Strafe. Auch im humanitären Völkerrecht findet sich im gemeinsamen Art. 1 der Genfer Konventionen eine Basis für staatliche Schutzpflichten. 
Das Gutachten bespricht die positiven und negativen Pflichten von Staaten zur Achtung konventionsrechtlich verankerter Menschenrechte. Staaten haben nicht nur die negative Pflicht, keine Beeinträchtigung von menschenwürdigem Leben durch umweltverschmutzendes Verhalten zu verursachen, ${ }^{345}$ sondern auch positive Pflichten, alle angemessenen zur Verfügung stehenden Maßnahmen zu ergreifen, um den Risiken für das Leben einer Person oder bestimmten Personengruppe durch Umweltschäden zu begegnen. ${ }^{346}$ Diese Pflicht setzt - wie auch bei der Verhinderungspflicht - staatliche Kenntnis bzw. staatliches Kennenmüssen voraus. 347

Positive Schutzpflichten können nach den maßgeblichen Verträgen zum Menschenrechtsschutz aber grundsätzlich nur begründet werden, wenn die Hoheitsgewalt des jeweiligen Vertragsstaates betroffen ist. Die Hoheitsgewalt ist betroffen, wenn die Rechtsverletzung auf nationalem Territorium erfolgt. ${ }^{348}$ Der IACtHR hat für die Amerikanische Menschenrechtskonvention extraterritoriale Schutzpflichten im Zusammenhang mit grenzüberschreitenden Umweltschäden aufgestellt. Sofern eine kausale Beziehung zwischen den Aktivitäten auf dem Territorium des Ursprungsstaates und den extraterritorialen Menschenrechtsverletzungen besteht, ist die Hoheitsgewalt des Ursprungsstaates über die betroffenen Personen anzunehmen. Der Ursprungsstaat ist demnach verantwortlich, wenn die Menschrechtsverletzung infolge der grenzüberschreitenden Umweltschädigung kausal auf der Verletzung von dessen Schutzpflichten beruht. ${ }^{349}$

345 IACtHR, Advisory Opinion on Environment and Human Rights, S. 48 f., Rn. 117.

346 Die staatliche Pflicht zum Schutz vor Rechtsverletzungen infolge von Umweltschäden betreffe nicht nur die Rechte auf Leben und körperliche Unversehrtheit, sondern auch eine Vielzahl anderer Rechte, wie etwa wirtschaftliche, soziale und kulturelle Rechte sowie Umweltrechte. Id., S. 21, Rn. 47.

347 Diese Pflicht setzt nicht nur staatliche Kenntnis bzw. Kennenmüssen von der Lebensgefährdung, sondern auch eine kausale Verbindung zwischen dem Umweltschaden und der Menschrechtsverletzung voraus. Id., S. 49 f., Rn. 118 ff.

348 In der authentischen englischen Fassung der Vertragstexte wird für Hoheitsgewalt der Begriff „jurisdiction“ verwandt. Aufgrund der Weite des Begriffes ,jurisdiction" gibt es keine einheitliche Übersetzung in das Deutsche. Je nach Zusammenhang bedeutet ,jurisdiction“ Hoheitsgewalt, Herrschaftsgewalt, Kompetenz oder auch Zuständigkeit. W. Kälin/J. Künzli, Universeller Menschenrechtsschutz, 2019, S. 136 ff.; H. Krieger, Die Verantwortlichkeit Deutschlands nach der EMRK für seine Streitkräfte im Auslandseinsatz, ZaöRV 62 (2002), S. 669 (670 f.) m.w.N.

349 IACtHR, Advisory Opinion on Environment and Human Rights, S. 43, Rn. $101 \mathrm{f}$. 


\section{Kapitel: Staatenhaftung in der Systematik des Völkerrechts}

Da die Menschenrechtsverletzungen auf Umweltschäden basieren, erfordert die Schutzpflicht in dieser Konstellation dieselben Maßnahmen, die auch zur Beachtung der Schadensvermeidungspflicht zu ergreifen sind. Nichtsdestoweniger besteht die Schutzpflicht aufgrund einer Aktivität, die Menschenrechte verletzt, während die Schadensvermeidungspflicht sich immer noch auf die an sich völkerrechtlich nicht verbotene Aktivität bezieht. ${ }^{350}$ Die wegweisende Entscheidung wird zwar vorwiegend in den Kontext des extraterritorialen Menschenrechtsschutzes gestellt, ${ }^{351}$ allerdings muss die implizite Bestätigung einer Staatenhaftung für grenzüberschreitende Umweltschäden durch völkerrechtlich nicht verbotene Aktivitäten ebenso Beachtung finden. Das Gutachten verdeutlicht sehr schön den Unterschied der beiden sich überschneidenden Rechtskreise, nämlich einer Staatenverantwortlichkeit für die Verletzung von Menschenrechten einerseits und einer Staatenhaftung für grenzüberschreitende Umweltschäden andererseits. ${ }^{352}$

Eine zweite Besonderheit im Bereich der Menschenrechte ist, dass auch eigene Staatsangehörige vom Schutzkreis umfasst sind - ungeachtet auf welchem Territorium sie sich befinden. ${ }^{353}$ Die Entscheidung des IACtHR zum Velásquez Rodríguez-Fall ${ }^{354}$ aus dem Jahr 1988 zeigt, dass die Staatsangehörigkeit eine rechtliche Beziehung zwischen dem Staat und dem Opfer von Menschrechtsverletzungen schafft. Der Heimatstaat verfügt hinsichtlich der eigenen Staatsangehörigen über rechtliche und tatsächliche Handlungsmöglichkeiten, die dessen Hoheitsgewalt begründen und

350 So betont der IACtHR auch, dass nicht jede grenzüberschreitende Schädigung die Verletzung von Menschenrechten zur Folge hat. Id., S. 43, Rn. 102.

351 Siehe beispielsweise A. Berkes, A New Extraterritorial Jurisdictional Link Recognised by the IACtHR, EJIL: Talk!, 28 March 2018, abrufbar unter: https:/www .ejiltalk.org/a-new-extraterritorial-jurisdictional-link-recognised-by-the-iacthr/ (geprüft am 15.05.2020); M. Feria-Tinta/S. C. Milnes, The Rise of Environmental Law in International Dispute Resolution, YIEL 27 (2016), S. 64 (64 ff.).

352 Vgl. A. Douban, Liability for Environmental Damage, in: R. Wolfrum (Hg.), MPEPIL 2013, http://www.mpepil.com, Rn. 26: „As noted above, liability for environmental damage could also be invoked in the case of a breach of other international legal norms, although the mechanism of compensation is related to the concept of State responsibility, which requires compensation for any material or moral injuries caused as a result of the breach (Art. 31 Draft Articles on Responsibility of States for Internationally Wrongful Acts).“

353 A. Seibert-Fohr, Die völkerrechtliche Verantwortung des Staats für das Handeln von Privaten, ZaöRV 73 (2013), S. 37 (45 Fn. 26).

354 IACtHR, Velásquez Rodríguez v. Honduras, Judgment of 29 July 1988, IACtHR Series C, No. 4 (1988), abrufbar unter: https://www.refworld.org/cases,IACRTH R,40279a9e4.html (geprüft am 15.05.2020). 
Schutzpflichten auslösen. ${ }^{355}$ Der Velásquez Rodríguez-Fall steht im Kontext mit dem Verschwinden von Oppositionellen in Honduras zur damaligen Zeit. ${ }^{356}$ Ein Student wurde von unbekannten Personen entführt und kam vermutlich ums Leben. Die honduranischen Behörden ergriffen weder Maßnahmen zur Verhinderung oder Verfolgung der Menschenrechtsverletzung noch zu deren Entschädigung. ${ }^{357}$ Der Gerichtshof betonte in diesem Zusammenhang, dass eine illegale Handlung, die Menschenrechte verletzt und dem Staat nicht zurechenbar ist, weil diese etwa durch private oder unbekannte Personen erfolgt, trotzdem zur Staatenverantwortlichkeit führen kann; nicht wegen der Handlung selbst, sondern wegen der Missachtung der gebotenen Sorgfalt, die der Staat aufgrund seiner konventionsrechtlich verankerten Schutzpflicht hätte walten lassen müssen. ${ }^{358}$ Die Entscheidung bekräftigt mit dem Hinweis auf die gebotene Sorgfalt, dass es sich bei der Schutzpflicht um eine verhaltensbezogene Pflicht handelt. ${ }^{359}$

Dies bestätigte auch der IGH in seiner Entscheidung zum Bosnischen Völkermord-Fall aus dem Jahr 2007. ${ }^{360}$ Es ging um das unterlassene Einschreiten Serbiens gegen den Völkermord in Srebrenica. Die entsprechende Schutzpflicht, welche sich aus Art. 1 der Völkermordkonvention ergibt, erstreckt sich auch auf Gebiete außerhalb des staatseigenen Territoriums. Der IGH bestätigte, dass die Schutzpflicht verhaltensbezogen ist, denn „it is clear that the obligation in question is one of conduct and not one of result, in the sense that a State cannot be under an obligation to succeed, whatever the circumstances, in preventing the commission of genocide: the obligation of States parties is rather to employ all means reasonably available to them, so as to prevent genocide as far as possible. " ${ }^{361}$ Demnach muss der Staat eine reale Handlungsmöglichkeit haben, mit der gebotenen Sorgfalt die Rechtsverletzung zu verhindern. Sofern eine Rechtsverletzung

355 A. Seibert-Fohr, Die völkerrechtliche Verantwortung des Staats für das Handeln von Privaten, ZaöRV 73 (2013), S. 37 (45).

356 Id., S. 37 (45 f.).

357 Im Ergebnis war daher Honduras für die unzureichende Gewährleistung des Rechts auf Leben, persönliche Freiheit und menschliche Behandlung verantwortlich. IACtHR, Velásquez Rodríguez v. Honduras, Judgment of 29 July 1988, IACtHR Series C, No. 4 (1988), S. 36, Rn. 194.

358 Id., S. 32, Rn. 172.

359 Id., S. 31, Rn. $166 \mathrm{f}$.

360 IGH, Application of the Convention on the Prevention and Punishment of the Crime of Genocide (Bosnia and Herzegovina v. Serbia and Montenegro), Judgment of 26 February 2007, ICJ Reports 2007, S. 43 (43 ff.).

361 Id., S. 43 (221 Rn. 430). 
eintritt, obwohl der Staat alles ihm Mögliche zur Verhinderung unternommen hat, ist keine Pflichtverletzung gegeben. ${ }^{362}$

\section{Ergebnis}

Die Rechtsprechungsanalyse illustriert, dass das Konzept der Staatenhaftung aus zwei Komponenten besteht: der verhaltensbezogenen Pflicht, unter Beachtung der gebotenen Sorgfalt Maßnahmen zur Schadensvermeidung zu ergreifen, und der ergebnisbezogenen Pflicht, keinen grenzüberschreitenden Schaden zu verursachen. Zudem wird zwischen verfahrensbezogenen und materiellen Schadensvermeidungspflichten differenziert. Die verfahrensbezogenen Pflichten, das heißt Benachrichtigung, Information, Warnung, Rücksprache und Durchführung einer Umweltverträglichkeitsprüfung, sind dabei zugleich Bestandteil der materiellen Pflicht zur Schadensvermeidung. Die materielle Schadensvermeidungspflicht erfordert außerdem den Erlass geeigneter Vorschriften und Maßnahmen zur Regulierung von grenzüberschreitenden Aktivitäten sowie die effektive Kontrolle und Durchsetzung der maßgebenden Vorgaben. Dabei gilt der Maßstab des Standes der Technik. Schließlich gewinnt auch Schadensvorsorge als Element der Schadensvermeidung an Bedeutung.

Angesichts der diskussionswürdigen Ergebnisse der Rechtsprechung zur Unterscheidung zwischen verfahrensbezogenen und materiellen Pflichten muss letztlich gelten, dass weder die Verletzung der verfahrensbezogenen Pflichten allein noch die Verletzung der materiellen Schadensvermeidungspflicht im Gesamten zur staatlichen Einstandspflicht für völkerrechtlich nicht verbotene Aktivitäten führt. Denn erst die Verletzung der ergebnisbezogenen Pflicht, mithin der Schadenseintritt, begründet die Staaten-

362 Der IGH betonte den verhaltensbezogenen Charakter von völkerrechtlichen Handlungspflichten bereits im Rahmen des Nicaragua-Falles aus dem Jahr 1986. Der Gerichtshof prüfte, ob Nicaragua die Pflicht zur Verhinderung des Waffenhandels verletzt hatte und verneinte dies unter Bezugnahme auf die gebotene Sorgfalt, die von Nicaragua in der konkreten Situation erwartet werden konnte. In diesem Zusammenhang deutete der Gerichtshof an, dass für Entwicklungsländer, wie Nicaragua, nicht dieselben Anforderungen wie für entwickelte Länder gelten könnten und stellte damit einen subjektiven Standard auf. IGH, Military and Paramilitary Activities in and against Nicaragua (Nicaragua v. United States of America), Merits, Judgment of 27 June 1986, ICJ Reports 1986, S. 14 (85 Rn. 157). 
haftung. Dies ist auch der wesentliche Unterschied zur Staatenverantwortlichkeit.

Die staatliche Einstandspflicht für völkerrechtlich nicht verbotene Aktivitäten greift bei einem verschuldensabhängigen Haftungsstandard indes nur dann, wenn die gebotene Sorgfalt zur Schadensvermeidung missachtet wurde. Dieser Sorgfaltsverstoß und damit das staatliche Verschulden sind jedoch nicht mit Völkerrechtswidrigkeit gleichzusetzen, so dass es hier nicht um Staatenverantwortlichkeit geht. In dieser Konstellation ist vielmehr die Staatenhaftung das richtige Regularium. Würde man dem nicht folgen, könnte es in Fällen fehlenden staatlichen Verschuldens schon grundsätzlich keine (subsidiäre) Einstandspflicht für Schäden durch nicht-staatliche Aktivitäten geben. Die Staatenhaftung kennt aber eben auch einen verschuldensunabhängigen Haftungsstandard, demzufolge der Ursprungsstaat für Schäden durch nicht-staatliche Aktivitäten einstehen muss, ohne dass es auf ein Fehlverhalten ankommt.

Die Staatenverantwortlichkeit ist hingegen geprägt von einem Unrechtsbewusstsein der internationalen Gemeinschaft und kommt zum Tragen, wenn Handlungen der Völkerrechtsordnung entgegenstehen. Im internationalen Gefüge ist die verantwortungsbewusste Erfüllung staatlicher Funktionen unerlässlich. Besonders deutlich wird dies im Rahmen völkervertraglich verankerter Schutzpflichten. Die staatlichen Verhinderungsbzw. Schutzpflichten knüpfen, wie aus der Rechtsprechungsanalyse hervorgeht, an Hoheitsgebiet bzw. Hoheitsgewalt, Kenntnis und reale Handlungsmöglichkeit der pflichtigen Staaten an.

\section{Völkergewohnheitsrecht}

Die Rechtsprechungsanalyse hat gezeigt, dass das Konzept der Staatenhaftung bereits zur Lösung von internationalen Rechtsstreitigkeiten zur Anwendung gelangt. Allerdings werden internationale Schadensfälle durch völkerrechtlich nicht verbotene Aktivitäten regelmäßig zwischen den betroffenen Staaten auf diplomatischer Ebene im internationalen Verkehr und damit außergerichtlich gelöst, da ihnen nicht dasselbe Konfliktpotenzial innewohnt wie völkerrechtswidrigem Verhalten. Es ist also naheliegend, dass das Konzept der Staatenhaftung eine als Recht angesehene allgemeine Übung darstellt. Eine solche völkergewohnheitsrechtliche Geltung des Konzepts ist anzunehmen, wenn der Nachweis eines entsprechenden beständigen Verhaltens der Völkerrechtssubjekte, das zugleich von einer 


\section{Kapitel: Staatenhaftung in der Systematik des Völkerrechts}

Rechtsüberzeugung (opinio iuris) getragen ist, gelingt. ${ }^{363}$ Während sich die geforderte allgemeine Übung etwa durch tatsächliche Handlungen auf Ebene der internationalen Beziehungen oder durch Akte internationaler Institutionen auf vielfältige Weise belegen lässt, ist die Feststellung einer übereinstimmenden Rechtsüberzeugung aufgrund des subjektiven Charakters naturgemäß schwierig. ${ }^{364}$ Die Entstehung von Völkergewohnheitsrecht kann somit von der Staatengemeinschaft durch die Negierung einer rechtlichen Verpflichtung verhindert werden. Opinio iuris ist nämlich zu verneinen, wenn die Übung lediglich aus Gefälligkeit befolgt wird oder wenn Staaten sich vorbehalten, jederzeit wieder davon abzuweichen. ${ }^{365}$

Der völkergewohnheitsrechtliche Stellenwert der Staatenhaftung unterscheidet sich im Hinblick auf die Komponenten der Schadensvermeidung und Wiedergutmachung. Wie gezeigt, bestätigen die Arbeiten der Völkerrechtskommission und die Rechtsprechung als Rechtserkenntnisquellen den (umwelt-)völkergewohnheitsrechtlichen Stellenwert der staatlichen Pflicht zur Schadensvermeidung. Dieser wird in der Völkerrechtsgemeinschaft auch kaum mehr in Abrede gestellt. ${ }^{366}$ Gleichzeitig verdeutlicht die

363 Vgl. A. Epiney/M. Scheyli, Strukturprinzipien des Umweltvölkerrechts, 1998, S. $98 \mathrm{f}$.; S. Kadelbach/T. Kleinlein, International Law - a Constitution of Mankind?, GYIL 50 (2007), S. 303 (338); E. Kadens/E. A. Young, How customary is customary international law?, Wm \& Mary LR 54 (2013), S. 885 (888); W. Weiß, Allgemeine Rechtsgrundsätze des Völkerrechts, AVR 39 (2001), S. 394 (396f.).

364 A. Epiney/M. Scheyli, Strukturprinzipien des Umweltvölkerrechts, 1998, S. 99 f.; J. Kammerhofer, Uncertainty in the Formal Sources of International Law, EJIL 15 (2004), S. 523 (532 ff.).

$365 \mathrm{~J}$. Wolf, Die gegenwärtige Entwicklung der Lehre über die völkerrechtliche Verantwortlichkeit der Staaten, ZaöRV 31 (1983), S. 481 (506); ausführlich zu den Problematiken bei der Feststellung von Völkergewohnheitsrecht siehe $J$. Kammerhofer, Uncertainty in the Formal Sources of International Law, EJIL 15 (2004), S. 523 (523 ff.).

366 Seit Anbeginn der Haftungsstudie war sich die Völkerrechtskommission bezüglich der Präventionskomponente im Wesentlichen einig. Sie belegte deren völkergewohnheitsrechtliche Geltung mit ihrer Übersicht zu existenten Haftungsregimen und Haftungsfällen aus der Praxis. ILC Secretariat, Survey of liability regimes relevant to the topic of international liability for injurious consequences arising out of acts not prohibited by international law (international liability in case of loss from transboundary harm arising out of hazardous activities), YBILC 2004-II/1, UN Doc. A/CN.4/543, S. 111, Rn. 113 ff.; ILC Secretariat, Survey of practice relevant to international liability for injurious consequences arising out of acts not prohibited by international law, YBILC 1985II/1, UN Doc. A/CN.4/384, S. 1 ff.; ILC Secretariat, Survey of liability regimes relevant to the topic of international liability for injurious consequences arising 
Analyse der Rechtserkenntnisquellen aber, dass Uneinigkeit besteht, ob die staatliche Pflicht zur Wiedergutmachung von grenzüberschreitenden Schäden nur bei völkerrechtswidrigem Verhalten oder auch bei völkerrechtlich nicht verbotenen Aktivitäten greift.

Zwar kommen zahlreiche Analysen zur Staatenpraxis und opinio iuris bezüglich einer Staatenhaftung in der Völkerrechtsliteratur zu dem Ergebnis, dass die Staatenhaftung (noch) keinen völkergewohnheitsrechtlichen Stellenwert hat. ${ }^{367}$ Anhand von prägnanten Beispielen wird im Folgenden aber aufgezeigt, dass das Konzept der Staatenhaftung vielen Fällen zugrunde liegt, auch wenn die Staaten sich meist weigern, deren völkergewohnheitsrechtliche Ausformung anzuerkennen.

\section{Staatenhaftung unter dem Deckmantel von ex gratia-Zahlungen}

Die nachfolgenden Fälle zeigen, inwieweit im internationalen Kontext Schadensersatzansprüche gegenüber Staaten, von deren Territorium oder unter deren Hoheitsgewalt grenzüberschreitende Schädigungen ausgingen (Ursprungsstaaten), geltend gemacht wurden und diese dementsprechend auch Entschädigungen für grenzüberschreitende Schäden geleistet haben,

out of acts not prohibited by international law, (international liability in case of loss from transboundary harm arising out of hazardous activities), YBILC 1995-II/1, UN Doc. A/CN.4/471, S. 62 ff.; siehe auch G. Handl, Transboundary Impacts, in: D. Bodansky/J. Brunnée/E. Hey (Hg.), The Oxford Handbook of International Environmental Law, 2007, S. 531 (534).

367 Siehe etwa J. Barboza, The Environment, Risk and Liability in International Law, 2011, S. 45 ff.; G. Bornheim, Haftung für grenzüberschreitende Umweltbeeinträchtigungen im Völkerrecht und im Internationalen Privatrecht, 1995, S. 153 f.; A. E. Boyle, State Responsibility and International Liability for Injurious Consequences of Acts Not Prohibited by International Law, ICLQ 39 (1990), S. 1 (15) m.w.N.; S. Erichsen, Der ökologische Schaden im internationalen Umwelthaftungsrecht, 1993, S. 77 ff.; S. Förster, Internationale Haftungsregeln für schädliche Folgewirkungen gentechnisch veränderter Organismen, 2007, S. 192 ff.; G. Handl, State Liability for Accidental Transnational Environmental Damage by Private Persons, AJIL 74 (1980), S. 525 (535 ff.). Es finden sich allerdings auch Stimmen, nach denen sich eine Gefährdungshaftung der Staaten im Umweltbereich zumindest für besonders gefährliche Aktivitäten etabliert habe. Siehe etwa L. F. E. Goldie, Liability for Damage and the Progressive Development of International Law, ICLQ 14 (1965), S. 1189 (1262); J. M. Kelson, State Responsibility and the Abnormally Dangerous Activity, Harv. ILJ 13 (1972) S. 197 (243); J. G. Lammers, Pollution of the International Watercourses, 1984, S. $632 \mathrm{ff}$. 


\section{Kapitel: Staatenhaftung in der Systematik des Völkerrechts}

ohne dass den Entschädigungsansprüchen Völkerrechtsverstöße zugrunde lagen. Für die Beurteilung des völkergewohnheitsrechtlichen Stellenwertes der staatlichen Wiedergutmachungspflicht ist dabei entscheidend, ob die Entschädigungszahlung von opinio iuris getragen ist oder lediglich ex gratia, das heißt ohne Anerkennung einer Rechtspflicht, erfolgte.

\section{Gut Dam-Fall}

Im Gut Dam-Fall ging es um die Frage, inwieweit Kanada Schäden am Eigentum von US-amerikanischen Bürgern im Zusammenhang mit dem Bau und Betrieb eines Staudammes im St. Lawrence Fluss zwischen kanadischem und US-amerikanischem Territorium zu ersetzen hatte. ${ }^{368}$ Die USA stimmten dem kanadischen Projekt im Jahr 1903 unter der Prämisse der Schadloshaltung von US-amerikanischen Bürgern $\mathrm{zu}^{369}$ und Kanada akzeptierte die Verpflichtung zu Kompensationsleistungen für grenzüberschreitende Schäden durch den Gut Dam in einer diplomatischen Note aus dem Jahr 1952 sowie in einer Pressemitteilung, von deren Inhalt das amerikanische Außenministerium vor Veröffentlichung im Jahr 1953 unterrichtet wurde. ${ }^{370}$ Die beiden Regierungen einigten sich schließlich darauf, dass Kanada einen pauschalen Betrag zur Entschädigung für die geltend gemachten Schäden leistet. ${ }^{371}$ Der rechtsetzende Charakter von Übereinkünften zu pauschalen Entschädigungsleistungen wird indes infrage gestellt. Diese gewährten keinen Nachweis von opinio iuris zu einer entsprechenden Einstandspflicht. Im Gegensatz zu vollen Entschädigungsleistungen stellten pauschale Entschädigungsleistungen lediglich eine zwischenstaatliche Kompromisslösung dar; ihnen lägen damit außerrechtliche Zahlungsgründe zugrunde. ${ }^{372}$ Die Staatenhaftung ist aber gerade dadurch

368 Siehe Report of the Agent of the United States before the Lake Ontario Claims Tribunal, ILM 8 (1969), S.118; Agreement between the Government of the United States of America and the Government of Canada concerning the Establishment of an International Arbitral Tribunal to Dispose of United States Claims relating to Gut Dam, 25. März 1965 (in Kraft seit 11. Oktober 1966), ILM 4 (1965), S. 468.

369 Report of the Agent of the United States before the Lake Ontario Claims Tribunal, ILM 8 (1969), S. 120.

370 Id., S. $138 \mathrm{f}$.

371 Id., S. 141.

372 R. Lefeber, Transboundary Environmental Interference and the Origin of State Liability, 1996, S. 141 f., 177. 
gekennzeichnet, dass nicht der komplette Schaden zu ersetzen ist, sondern lediglich ein gerechter Interessenausgleich zu erfolgen hat. ${ }^{373}$ Allerdings entspringt die Zahlungsverpflichtung im vorliegenden Fall einem Vertrag zwischen den beiden Staaten und ist ferner durch die Abhängigkeit Kanadas von der Einwilligung der USA zum Projekt gekennzeichnet, so dass der Fall keine eindeutige Aussage über den gewohnheitsrechtlichen Status der Staatenhaftung geben kann. ${ }^{374}$ Nichtsdestoweniger bietet der Fall einen Hinweis auf die Auffassung der Parteien darüber, wie Schadensfälle aufgrund gefährlicher Aktivitäten zu regeln sind. ${ }^{375}$ Die bilaterale Übereinkunft zwischen Kanada und den USA reflektiert deren Rechtsüberzeugung von einer staatlichen Einstandspflicht für grenzüberschreitende Schäden. 376

\section{Fluss Mur-Fall}

Im Jahr 1956 verschmutzten österreichische Wasserkraftanalagen den Fluss Mur, indem die privaten Betreiber der Kraftwerke Sedimente und Schlamm in den Fluss ablaufen ließen, um Überschwemmungen zu verhindern. Dies führte zu unerwarteten Schäden an der Flusslandschaft sowie zu Beschädigungen von Papierfabriken auf der zur ehemaligen Republik Jugoslawien gehörenden Seite des Flusses. Die ehemalige Republik Jugoslawien verlangte Kompensationsleistungen für die wirtschaftlichen Verluste, die die zwei Papierwerke erlitten, und für Schäden der Fischerei. Österreich willigte in die Kompensationsforderungen ein und ersetzte

373 S. Erichsen, Das Liability-Projekt der ILC, ZaöRV 51 (1991), S. 94 (100); T. Gebring/M. Jachtenfuchs, Liability for Transboundary Environmental Damage Towards a General Liability Regime?, EJIL 4 (1993), S. 92 (95).

374 Die Aussagekraft des Gut Dam-Falles zum allgemeinen Konzept einer staatlichen Einstandspflicht im Völkerrecht relativieren beispielsweise J. Barboza, The Environment, Risk and Liability in International Law, 2011, S. 53 f.; G. Handl, State Liability for Accidental Transnational Environmental Damage by Private Persons, AJIL 74 (1980), S. 525 (538f.), S. Mahmoudi, Gut Dam Claims, in: R. Wolfrum (Hg.), MPEPIL 2007, http://www.mpepil.com, Rn. 14.

375 J. Barboza, The Environment, Risk and Liability in International Law, 2011, S. 54.

376 Eine entsprechende Rechtsüberzeugung liegt beispielsweise auch dem River Plate Treaty zugrunde. In dem Übereinkommen verpflichteten sich Uruguay und Argentinien zu einer verschuldensunabhängigen Staatenhaftung für Schäden im Territorium des jeweils anderen Staates. Artikel 51 Treaty of the La Plata River and its Maritime Limits, 13. November 1973, ILM 13 (1974), S. $251 \mathrm{ff}$. 
die Schäden nicht nur monetär, sondern auch durch die Lieferung von $\mathrm{Pa}$ pier. ${ }^{377}$ Damit lässt sich sehr eindrücklich eine Rechtsüberzeugung der beiden Parteien von einer staatlichen Einstandspflicht für grenzüberschreitende Schäden durch private Aktivitäten belegen. ${ }^{378}$

\section{Juliana-Fall}

Im Jahr 1975 verschmutzte der unter liberianischer Flagge fahrende Tanker Juliana unfallbedingt japanische Gewässer mit Öl. Dies führte zu immensen Schäden der Fischerei. Obwohl Liberia keinerlei Fehlverhalten vorgeworfen wurde, kompensierte die liberianische Regierung im Folgenden die Einnahmeverluste der japanischen Fischer. ${ }^{379}$ Mangels hinreichender Informationen über den Zahlungsgrund ${ }^{380}$ wird in der Völkerrechtsliteratur eingewandt, dass nicht notwendigerweise die Anerkennung einer Rechtspflicht hinter der Zahlungsbereitschaft gestanden habe. ${ }^{381}$ Dennoch ist dem Juliana-Fall eine besondere Relevanz zuzugestehen, da er aufzeigt, dass Staaten für extraterritoriale Schäden durch private Aktivitäten aufkommen, ohne dass eine Völkerrechtsverletzung vorliegt. ${ }^{382}$

\section{Cosmos 954-Fall}

Durch den Absturz des sowjetischen nuklear angetriebenen Satelliten Cosmos $954 \mathrm{im} \mathrm{Jahr} 1978$ gingen radioaktive Bruchteile in Kanada nieder. Neben Verstößen gegen völkervertragliches Weltraumrecht berief sich Kanada auf allgemeine Grundsätze des Völkerrechts, um seine Kompensati-

377 Siehe zum Fall G. Handl, State Liability for Accidental Transnational Environmental Damage by Private Persons, AJIL 74 (1980), S. 525 (545 f.) unter Bezugnahme auf das Protokoll über die außerordentliche Tagung der gemischten Murkommission vom 15. April 1959, Bundeskanzleramt GZ 106.454 - 2a/59, Bundesministerium für Land- und Forstwirtschaft ZI. 46.709-IV9/59.

378 Id., S. 525 (546).

379 Siehe zum Fall id., S. 525 (546f.).

380 R. Lefeber, Transboundary Environmental Interference and the Origin of State Liability, 1996, S. 176.

381 G. Handl, State Liability for Accidental Transnational Environmental Damage by Private Persons, AJIL 74 (1980), S. 525 (546f.); R. Lefeber, Transboundary Environmental Interference and the Origin of State Liability, 1996, S. 176. Ibid. 
onsforderungen gegen die Sowjetunion zu begründen. ${ }^{383}$ Die staatliche Pflicht zur Kompensation für die Beeinträchtigung der territorialen Souveränität durch den radioaktiven Weltraummüll leitete Kanada aus einem verschuldensunabhängigen Haftungsstandard her, welcher für besonders gefährliche Aktivitäten völkerrechtlich anerkannt sei. ${ }^{384}$ Ein Völkerrechtsverstoß lag der Forderung nicht zugrunde. ${ }^{385}$ Die Staaten einigten sich nach Verhandlungen auf diplomatischer Ebene darauf, dass die Ansprüche Kanadas mit einer Kompensationsleistung durch die Sowjetunion vollständig und endgültig abgegolten werden. ${ }^{386}$ Auch wenn die Sowjetunion eine Entschädigungsverpflichtung nicht ausdrücklich anerkannte und die Vergleichsvereinbarung überdies keinen Zahlungsgrund benennt, bezieht sich die Vereinbarung explizit auf Kanadas Forderung und erlaubt die Schlussfolgerung, dass die rechtlichen Argumente Kanadas die Grundlage für die Vereinbarung bildeten. ${ }^{387}$ Folglich bestärkt der Fall die völkergewohnheitsrechtliche Geltung der Staatenhaftung.

\section{Fukuyu Maru-Fall}

In der Vergangenheit waren Atomversuche mit Schadensfolgen Anlass für Entschädigungszahlungen. Zwar betonten die Staaten, wie etwa die USA und Großbritannien, wiederholt die Rechtmäßigkeit von Atomversuchen, erklärten sich aber trotzdem bereit, für potenzielle Schäden einzustehen. ${ }^{388}$ Deutlich wird diese Haltung am Beispiel des amerikanischen

383 Claim against the Union of Soviet Socialist Republics for Damage caused by Soviet Cosmos 954, 13. Januar 1979, ILM 18 (1979), S. 899-901 und additional Annex A, ILM 18 (1979), S.902-908; siehe auch S. Erichsen, Der ökologische Schaden im internationalen Umwelthaftungsrecht, 1993, S. 77; T. Gehring/M. Jachtenfuchs, Haftung und Umwelt, 1988, S. 107 f.; P. Sands/J. Peel, Principles of International Environmental Law, 2018, S. 763.

384 Claim against the Union of Soviet Socialist Republics for Damage caused by Soviet Cosmos 954 und additional Annex A, 13. Januar 1979, ILM 18 (1979), S. 908.

385 J. Barboza, The Environment, Risk and Liability in International Law, 2011, S. 55 .

386 Art. I und II, Protocol between Canada and the Soviet Union, 2. April 1981, ILM 20 (1981), S. 689.

387 P. Sands/J. Peel, Principles of International Environmental Law, 2018, S. 763.

388 Siehe hierzu J. Barboza, The Environment, Risk and Liability in International Law, 2011, S. 55 ff.; J. G. Lammers, Pollution of the International Watercourses, 1984, S. $319 \mathrm{ff}$. 


\section{Kapitel: Staatenhaftung in der Systematik des Völkerrechts}

Atomversuchs nahe den Marshall-Inseln. Im Jahr 1947 führte die Detonation einer Wasserstoffbombe zu einem radioaktiven Niederschlag, der weit über die festgelegte Gefahrenzone hinausreichte. Die USA hatten zuvor Schadensvermeidungsmaßnahmen ergriffen, dennoch wurde aufgrund von unvorhersehbaren Umständen, wie etwa dem Wind, das japanische Fischerreifahrzeug Fukuyu Maru außerhalb der Gefahrenzone dem Niederschlag ausgesetzt. Die japanische Regierung forderte daraufhin Entschädigung für die Verletzung der Besatzung der Fukuyu Maru und für die Kontamination der Umwelt sowie Fischbestände, welche zu erheblichen wirtschaftlichen Verlusten der japanischen Fischerei führte. Die US-amerikanische Regierung erklärte sich im Jahr 1955 bereit, Entschädigung für die Schäden durch den Atomversuch zu leisten. ${ }^{389}$ Zwar ist auch hier einzuräumen, dass die Zahlung ohne Anerkennung einer Rechtspflicht erfolgte. Dennoch signalisiert die grundsätzliche Bereitschaft zur Kompensationsleitung für Schäden durch die als rechtmäßig angesehenen Atomversuche eine gewisse rechtliche Verbindlichkeit. ${ }^{390}$

\section{Tschernobyl-Fall}

Schließlich behielten sich in der Vergangenheit zahlreiche Staaten, deren Territorium durch völkerrechtlich nicht verbotene Aktivitäten beeinträchtigt wurde, das Recht vor, Entschädigungsansprüche gegen den Ursprungsstaat geltend zu machen. ${ }^{391}$ So wird der Reaktorunfall im Kernkraftwerk von Tschernobyl im Jahr 1986 mit seinen weitreichenden negativen Folgen fälschlicherweise als Beleg dafür herangezogen, dass Entschädigungen in der internationalen Praxis weder gefordert noch geleistet wurden. ${ }^{392}$ Es

389 J. Barboza, The Environment, Risk and Liability in International Law, 2011, S. 56 f.; M. McDougal/N. A. Schlei, The Hydrogen Bomb Tests in Perspective, Yale LJ 64 (1955), S. 648 (652); M. M. Whiteman, Digest of international law, Band 4, 1965, S. $565 \mathrm{ff}$.

390 J. Barboza, The Environment, Risk and Liability in International Law, 2011, S. 63; G. Handl, International Accountability for Transboundary Environmental Harm Revisited, Environmental Policy and Law 37 (2007), S. 116 (124 Fn. 80); J. G. Lammers, Pollution of the International Watercourses, 1984, S. $320 \mathrm{f}$.

391 J. Barboza, The Environment, Risk and Liability in International Law, 2011, S. $58 \mathrm{ff}$. mit weiteren Beispielen.

392 Vgl. P. Birnie/A. E. Boyle/C. Redgwell, International Law and the Environment, 2009, S. 518 f.; S. Förster, Internationale Haftungsregeln für schädliche Folgewirkungen gentechnisch veränderter Organismen, 2007, S. 195, Fn. 476; J. Mellor, Radioactive Waste and Russia's Northern Fleet, Denver JILP 28 (1999), 
ist zunächst zu bedenken, dass sich einige Staaten aber das Recht, Entschädigungsklagen gegen die Sowjetunion einzureichen, vorbehielten. ${ }^{393}$ Die Zurückhaltung lag nur darin begründet, dass der Erfolg einer Klage nicht absehbar war $^{394}$ und die Staaten, die selbst Atomkraftwerke betrieben, keinen Präzedenzfall schaffen wollten, der gegebenenfalls auf sie selbst hätte zurückfallen können. ${ }^{395}$ Interessant ist in diesem Zusammenhang auch, dass Deutschland, Großbritannien und Schweden in der Folgezeit Entschädigung an Personen, die von dem radioaktiven Niederschlag auf ihrem Hoheitsgebiet betroffen waren, leisteten und viele westliche Staaten der Ukraine finanzielle Unterstützung für die Stilllegung und Entsorgung von Kernmaterial gewährten. ${ }^{396}$ Darin kann eine Art Solidarhaftung der Staaten gesehen werden, die Schäden durch völkerrechtlich nicht verbotene Aktivitäten auffangen. ${ }^{397}$ Schließlich ist nicht außer Acht zulassen, dass die ehemalige Sowjetunion - und nach deren Zerfall die Russische Föderation als Rechtsnachfolgerin - die Pflicht zur Kompensation zumindest für Opfer in den in unmittelbarer Nähe zum Unglücksort gelegenen und am direktesten betroffenen (neuen) Staaten tatsächlich anerkannte und zum Teil auch umsetzte. ${ }^{398}$

S. 51 (65 f.); V. P. Nanda/G. (R.) Pring, International Environmental Law \& Policy for the 21st Century, 2013, S. 61, Fn. 205.

393 Schweden erklärte beispielsweise, dass „customary international law (...) principles exist which might be invoked to support a claim against the USSR". Siehe P. Sands/J. Peel, Principles of International Environmental Law, 2018, S. $752 \mathrm{f}$. bezugnehmend auf Korrespondenz mit der schwedischen Botschaft in London, 10. Dezember 1987.

394 Die Sowjetunion war nicht Partei von entsprechenden Übereinkommen zur Haftung gegenüber Dritten auf dem Gebiet der Kernenergie. So mangelte es an klaren Rechtsgrundlagen und Verfahren zur Geltendmachung der Ansprüche. P. J. Sands, The Environment, Community and International Law, Harv. ILJ 30 (1989), S. 393 (406f.).

395 P. Birnie/A. E. Boyle/C. Redgwell, International Law and the Environment, 2009, S. 518; L. A. Malone, The Chernobyl Accident, Colum. J. Envtl. L. 12 (1987), S. 203 (207); P. J. Sands, The Environment, Community and International Law, Harv. ILJ (1989), S. 393 (401 ff.); P. Sands/J. Peel, Principles of International Environmental Law, 2018, S. 753.

396 J. Mellor, Radioactive Waste and Russia's Northern Fleet, Denver JILP 28 (1999), S. 51 (65 f.); P. Sands/J. Peel, Principles of International Environmental Law, 2018, S. 753; J. M. Van Dyke, Liability and Compensation for Harm Caused by Nuclear Activities, Denver JILP 35 (2006), S. 13 (29f.).

397 Vgl. J. Mellor, Radioactive Waste and Russia's Northern Fleet, Denver JILP 28 (1999), S. 51 (65 f.).

398 J. M. Van Dyke, Liability and Compensation for Harm Caused by Nuclear Activities, Denver JILP 35 (2006), S. 13 (30, 33). 


\section{Aerial Herbicide Spraying-Fall}

Das Streitverfahren zwischen Ecuador und Kolumbien, das dem IGH im Jahre 2008 vorgelegt und 2013 von der Liste der anhängigen Verfahren vor dem IGH aufgrund einer einvernehmlichen Streitbeilegung gestrichen wurde, verleiht ex gratia-Zahlungen im Kontext einer völkergewohnheitsrechtlichen Einordnung der Staatenhaftung Gewicht. ${ }^{399}$ Gegenstand des Streites war der völkerrechtlich nicht verbotene Einsatz von Herbiziden, die von Kolumbien zur Vernichtung einer illegalen Kokain- und Mohnplantage genutzt wurden. Ecuador beklagte, dass die Herbizide zu erheblichen Schäden an Menschen, Tieren und der Umwelt in der ecuadorianischen Grenzregion geführt hätten. ${ }^{400}$ Die Einigung zwischen den Parteien führte schließlich zu einer Entschädigungszahlung Kolumbiens, aber ohne offizielle Anerkennung einer entsprechenden Einstandspflicht. ${ }^{401}$ Eine Entscheidung des IGH in dieser Konstellation hätte erfordert, dass der Gerichtshof zu der Frage eines gerechten Interessenausgleiches in grenzüberschreitenden Schadensfällen durch gefährliche völkerrechtlich nicht verbotene Aktivitäten Stellung nimmt ${ }^{402}$ und möglicherweise rechtliche Kompensationsansprüche Ecuadors für Umweltschäden durch den rechtmäßigen Herbizideinsatz Kolumbiens begründet; ${ }^{403}$ mithin das Konzept der Staatenhaftung bestärkt.

399 IGH, Aerial Herbicide Spraying (Ecuador v. Colombia), Order of 13 September 2013, ICJ Reports 2013, S. 278 (278 f.).

400 Letter from the Ambassador of Ecuador (appointed) to the Kingdom of the Netherlands to the Registrar of the International Court of Justice, 31 March 2008, abrufbar unter: http:/www.icj-cij.org/docket/files/138/14474.pdf (geprüft am 15.05.2020).

401 Agreement between the Republic of Ecuador and the Republic of Colombia, 9 September 2013, abrufbar unter: http://cdn.ipsnoticias.net/wp-content/uploads/ 2013/10/Acuerdo-glifosato-Ecuador-Colombia.pdf (geprüft am 15.05.2020).

402 R. Esposito, The ICJ and the Future of Transboundary Harm Disputes, Pace Int'l L. Rev. Online Companion 2 (2010), abrufbar unter: https://digitalcommons.pa ce.edu/cgi/viewcontent.cgi? referer=\&httpsredir=1\&article=1014\&context=pilro nline (geprüft am 15.05.2020), S. 1 (52).

403 Vgl. J. Bäumler, Das Schädigungsverbot im Völkerrecht, 2017, S. 86; R. Esposito, The ICJ and the Future of Transboundary Harm Disputes, Pace Int'l L. Rev. Online Companion 2 (2010), abrufbar unter: https://digitalcommons.pace.edu /cgi/viewcontent.cgi? referer=\&httpsredir=1\&article=1014\&context=pilronline (geprüft am 15.05.2020), S. 1 (31 ff.). 


\section{Ergebnis}

Wenngleich die Staatengemeinschaft einer Staatenhaftung für Schäden durch völkerrechtlich nicht verbotene Aktivitäten ablehnend gegenübersteht, zeigt die Kasuistik, dass Kompensationsleistungen von Ursprungsstaaten gefordert und auch von diesen geleistet wurden. Zwar mag gegen die Aussagekraft dieser Fälle angeführt werden, dass die Entschädigungsleistungen grundsätzlich ohne Anerkennung einer Rechtspflicht geleistet wurden und als ex gratia-Zahlungen allenfalls einem moralischen Pflichtbewusstsein und nicht etwa einer Rechtsüberzeugung der Staaten entspringen. ${ }^{404}$ Die Einordnung als ex gratia-Zahlungen ist in Anbetracht der Beständigkeit im internationalen Verkehr aber mittlerweile fragwürdig, 405 denn je einheitlicher und länger sich die internationale Praxis hinsichtlich einer Regel verhält, desto stärker ist grundsätzlich die Vermutung der Anerkennung dieses Verhaltens als Recht. ${ }^{406}$ Diesen Fällen lässt sich jedenfalls ein Muster entnehmen, das auf die rechtliche Bedeutung der staatlichen Einstandspflicht für Schäden durch völkerrechtlich nicht verbotene Aktivitäten hindeutet. ${ }^{407}$ Alfred Rubin stellt zutreffend fest, dass , $[\mathrm{n}] \mathrm{o}$ argument that the sum paid in settlement was given ex gratia can wholly overcome the implication, except perhaps in some isolated cases, that the settlement reflected an opinio iuris shared by both the claimant and the respondent states that the settlement was legally compelled." ${ }^{408}$ Angesichts der Völkerrechtmäßigkeit der schadensauslösenden Aktivität ist zwar regelmäßig eine einvernehmliche Streitbeilegung möglich. Dies steht aber der Annahme einer rechtlichen Überzeugung von einer Entschädigungsverpflichtung nicht entgegen. Die zwischenstaatlichen Vergleichsverhandlungen führen nämlich regelmäßig zu Entschädigungsvereinbarungen, die auf einer berechtigten Erwartungshaltung des geschädigten Staates und

404 AIL, Kommentar zu Art. 5 AIL, S. 116, Rn. 32; R. Lefeber, Transboundary environmental interference and the origin of state liability, 1996, S. 177.

405 G. Handl, International Accountability for Transboundary Environmental Harm Revisited, Environmental Policy and Law, 37 (2007), S. 116 (124 Fn. 80).

406 A. Epiney/M. Scheyli, Strukturprinzipien des Umweltvölkerrechts, 1998, S. 100.

407 J. Barboza, Liability, Chinese JIL 1 (2002) S. 499 (520); ders., The Environment, Risk and Liability in International Law, 2011, S. 63; G. Handl, International Accountability for Transboundary Environmental Harm Revisited, Environmental Policy and Law, 37 (2007), S. 116 (120, 124 Fn. 80); N. L. J. T. Horbach, Liability Versus Responsibility Under International Law, 1996, S. 435 f.

408 A. P. Rubin, Pollution by Analogy: The Trail Smelter Arbitration, OLR 50 (1971), S. 259 (279). 


\section{Kapitel: Staatenhaftung in der Systematik des Völkerrechts}

einer Einschätzung des Urheberstaates über die Gebotenheit von Kompensationsleistungen beruhen. ${ }^{409}$ Die Untersuchung von Staatenpraxis und opinio iuris bestätigt schließlich, dass sich die Staaten zwar weigern ihre Einstandspflicht als rechtlich verbindlich zu beschreiben, gleichwohl haben sie sich im Ergebnis jedoch so verhalten, als akzeptierten sie eine Haftung für staatliche, aber auch private Aktivitäten auf ihrem Territorium. ${ }^{410}$

\section{Wandel von Staatenhaftung zur privaten Haftung}

Neben diesen staatlichen ex gratia-Zahlungen haben sich auch Fälle ereignet, in denen anfänglich Entschädigungen von Staaten verlangt und/oder angeboten wurden, die aber schließlich durch die privaten Schadensverursacher geleistet wurden. Diese Fälle verdeutlichen, welche Rolle dem Ursprungsstaat zukommt und können als völkergewohnheitsrechtlicher Indikator herangezogen werden.

\section{Cherry Point-Fall}

Im Jahr 1971 kam es beim Entladen eines mit Rohöl beladenen liberianischen Tankers an der Cherry Point Raffinerie in den USA zu einem Unfall. Das Auslaufen von Öl führte zu erheblichen Verschmutzungen der kanadischen Küste. Daraufhin verlangte Kanada in einer an das US-Außenministerium gerichteten diplomatischen Note finanzielle Entschädigung. ${ }^{411}$ Von besonderer Bedeutung ist die Bezugnahme der kanadischen Regierung auf die Trail Smelter-Entscheidung zur Begründung des Kompensationsanspruches. Die Anerkennung der staatlichen Kompensationspflicht durch Kanada im Rahmen des Trail Smelter-Falles begründete die Erwartung, dass die dort entwickelten Grundsätze nun auch von den USA eingehalten wür-

409 C. Foster, The ILC Draft Principles on the Allocation of Loss in the Case of Transboundary Harm Arising out of Hazardous Activities, RECIEL 14 (2005), S. 265 (279).

410 So stellt auch J. Barboza zutreffend fest, dass „in cases where no convention exists to assign responsibility to States or operators, States usually act as if they had some sort of liability." J. Barboza, The Environment, Risk and Liability in International Law, 2011, S. 42.

411 Siehe A. L. C. de Maestral (Hg.), Canadian Practice in International Law during 1972 as Reflected in Resolutions of the House of Commons and in Government Statements in the House of Commons, Can. YBIL 11 (1973), S. 314 (333 f.). 
den. ${ }^{412}$ Die Hoheitsgewalt der USA über die schadensauslösende Aktivität reichte nach kanadischem Verständnis aus, um eine verschuldensunabhängige Haftung der Vereinigten Staaten für grenzüberschreitende Schäden durch private Aktivitäten zu begründen. ${ }^{413}$

Trotz dieser eindeutigen Rechtsüberzeugung von einem völkerrechtlichen Konzept der Staatenhaftung kam es jedoch nicht zu einer Kompensationsleistung der USA, da der private Betreiber der Raffinerie (ARCO) die Entschädigungszahlungen übernahm und Kanada die Ansprüche gegen die US-Regierung fallen ließ und lediglich signalisierte, dass die Haftungsverpflichtung der USA zu greifen habe, wenn es ihr misslinge, die Entschädigung durch ARCO sicherzustellen. ${ }^{414}$ Letztlich mussten die USA zum Aspekt der Staatenhaftung nach der Übernahme der Wiederherstellungskosten durch ARCO keine Stellung mehr nehmen. ${ }^{415}$ Der Fall illustriert aber sehr gut den Wandel von einer originären Staatenhaftung zur privaten Haftung und allenfalls subsidiären Staatenhaftung, sobald der private Betreiber die Entschädigungskosten übernimmt.

412 Siehe id., S. 314 (334). Der kanadische Staatssekretär für auswärtige Angelegenheiten Hon. Mitchell Sharp erklärte in diesem Zusammenhang: „We are especially concerned to ensure observance of the principle established in the 1938 Trail smelter arbitration between Canada and the United States. This has established that one country may not permit the use of its territory in such a manner as to cause injury to the territory of another and shall be responsible to pay compensation for any injury so suffered. Canada accepted this responsibility in the Trail smelter case and we would expect that the same principle would be implemented in the present situation. Indeed, this principle has already received acceptance by a considerable number of states and hopefully it will be adopted at the Stockholm conference as a fundamental rule of international environmental law.“

413 Siehe ibid; hierzu auch J. Barboza, The Environment, Risk and Liability in International Law, 2011, S. 54; G. Handl, State Liability for Accidental Transnational Environmental Damage by Private Persons, AJIL 74 (1980), S. 525 (545); X. Hanqin, Transboundary Damage in International Law, 2003, S. 79.

414 ILC Secretariat, Survey of State practice relevant to international liability for injurious consequences arising out of acts not prohibited by international law, YBILC 1985-II/1, UN Doc. A/CN.4/384, S. 100 f., Rn. 479 ff.; X. Hanqin, Transboundary Damage in International Law, 2003, S. 79.

415 ILC Secretariat, Survey of State practice relevant to international liability for injurious consequences arising out of acts not prohibited by international law, YBILC 1985-II/1, UN Doc. A/CN.4/384, S. 101, Rn. 481; J. Barboza, The Environment, Risk and Liability in International Law, 2011, S. 54. 


\section{Sandoz Rhein-Fall}

Im Jahr 1986 brach in einer Lagerhalle des Chemieunternehmens San$d o z$ in der Schweiz ein Großfeuer aus. Hochgiftige Substanzen gelangten über das Löschwasser in den Rhein und lösten ein großes Fischsterben aus. ${ }^{416}$ Die betroffenen Staaten Frankreich und Deutschland intendierten zunächst, Entschädigungen von der Schweiz zu fordern. ${ }^{417}$ Auch erklärte sich die Schweiz hierzu bereit. ${ }^{418}$ Die Schweiz räumte in diesem Zusammenhang ein, dass sie striktere Sorgfaltsstandards zur Regulierung der schweizerischen Pharmaindustrie hätte etablieren müssen. ${ }^{419}$ Demnach erfolgte die Anerkenntnis der Entschädigungspflicht wohl aufgrund des Eingeständnisses eines staatlichen Fehlverhaltens und begründet mithin die Akzeptanz einer verschuldensabhängigen Staatenhaftung. ${ }^{420}$ Indes kommt es auch in diesem Fall zu einer Umwandlung der Ansprüche gegen den Staat zu Ansprüchen gegen den privaten Verursacher, ${ }^{421}$ da sich die Sandoz AG zu Entschädigungsleistungen bereiterklärte - vermutlich um den Ruf des Unternehmens zu rehabilitieren. ${ }^{422}$ Die betroffenen Staaten verfolgten ihre Ansprüche gegen die Schweiz auch deswegen nicht, weil dies zu politisch unerwünschten Maßnahmen gegen die Industrie im eigenen Land und damit zu negativen Auswirkungen auf die Wirtschaft hätte führen können. ${ }^{423}$ Zudem hatten die betroffenen Staaten selbst in der Vergangenheit zur Verschmutzung des Rheins beigetragen und hätten sich im Rahmen eines zwischenstaatlichen Rechtsstreites zwangsläufig internationaler

416 Siehe zum Fall A. Schwabach, The Sandoz Spill, ELQ 16 (1989), S. 443 (443 ff.).

417 Id., S. 443 (469).

418 ILC Secretariat, Survey of liability regimes relevant to the topic of international liability for injurious consequences arising out of acts not prohibited by international law (international liability in case of loss from transboundary harm arising out of hazardous activities), YBILC 2004-II/1, UN Doc. A/CN.4/543, S. 160, Rn. 430; A. Schwabach, The Sandoz Spill, ELQ 16 (1989), S. 443 (452 f.).

419 A. Schwabach, The Sandoz Spill, ELQ 16 (1989), S. 443 (453).

420 Vgl.J. Barboza, The Environment, Risk and Liability in International Law, 2011, S. 52; A. Schwabach, The Sandoz Spill, ELQ 16 (1989), S. 443 (466 f.).

421 Siehe H. U. J. d'Oliveira, The Sandoz Blaze, in: F. Francioni/T. Scovazzi (Hg.), International Responsibility for Environmental Harm, 1991, S. 429 (440); A. Schwabach, The Sandoz Spill, ELQ 16 (1989), S. 443 (453 f.).

422 Vgl. G. Handl, International Accountability for Transboundary Environmental Harm Revisited, Environmental Policy and Law, 37 (2007), S. 116 (119); A. Schwabach, The Sandoz Spill, ELQ 16 (1989), S. 443 (469).

423 A. Schwabach, The Sandoz Spill, ELQ 16 (1989), S. 443 (469 f.). 
Kritik wegen eigener Umweltverschmutzungen ausgesetzt. ${ }^{424}$ Immerhin erklärte sich die Schweiz bereit, die Sandoz AG bei der Regulierung der Schäden zu unterstützen, was wiederum der unentbehrlichen Rolle von Staaten in internationalen Schadensfällen Rechnung trägt, ${ }^{425}$ denn gerade bei grenzüberschreitenden Schadensersatzansprüchen ist eine rasche Streitbeilegung durch eine zivilrechtlich Haftung nur mittels einer direkten oder indirekten Beteiligung der betroffenen Staaten möglich. ${ }^{426}$

\section{Baia Mare-Fall}

Bei einer Goldmine in Baia Mare in Rumänien brach im Jahr 2000 der Damm eines Abwasserbeckens und austretende Zyanidlauge sowie schwermetallhaltiger Schlamm verseuchten die Flüsse Theiss und Donau. ${ }^{427}$ Trotz einschlägiger Präventionsregelungen und effektiver Abhilfemaßnahmen kam es zu erheblichen Umweltschäden. ${ }^{428}$ Ungarn und Serbien forderten zunächst Schadensersatzleistungen von Rumänien für die Umweltverschmutzung und schließlich klagte Ungarn gegen den privaten Betreiber der Goldmine. ${ }^{429}$ Die Betreiberfirma ging allerdings in Konkurs und die Nachfolgefirma übernahm keine Haftung für den Schaden. ${ }^{430}$ Rumänien betrachtete indes den privaten Betreiber der Goldmine als Verursacher

$424 I d .$, S. 443 (470).

425 Vgl. J. Barboza, The Environment, Risk and Liability in International Law, 2011, S. 52; G. Handl, International Accountability for Transboundary Environmental Harm Revisited, Environmental Policy and Law, 37 (2007), S. 116 (119, 123 Fn. 28).

426 H. U. J. d'Oliveira, The Sandoz Blaze, in: F. Francioni/T. Scovazzi (Hg.), International Responsibility for Environmental Harm, 1991, S. 429 (440); G. Handl, International Accountability for Transboundary Environmental Harm Revisited, Environmental Policy and Law, 37 (2007), S. 116 (119); S. C. McCaffrey, Expediting the Provision of Compensation to Accident Victims, in: G. Handl/R. E. Lutz (Hg.), Transferring Hazardous Technologies and Substances, 1989, S. 199 (199 ff., 239).

427 Siehe zum Fall A. L. Springer, Cases of Conflict, 2016, S. $84 \mathrm{ff}$.

428 A. Tanzi, Liability for Lawful Acts, in: R. Wolfrum (Hg.), MPEPIL 2013, http:// www.mpepil.com, Rn. 24.

429 Siehe G. Handl, International Accountability for Transboundary Environmental Harm Revisited, Environmental Policy and Law, 37 (2007), S. 116 (123 Fn. 29).

430 Siehe H. Jeppesen, Haftung verschoben auf irgendwann, Deutsche Welle Online, 25. Februar 2013, abrufbar unter: https://www.dw.com/de/haftung-verschoben-a uf-irgendwann/a-16623112 (geprüft am 15.05.2020). 
und sah sich nicht in der Verantwortung. ${ }^{431}$ Der Fall steht exemplarisch für die Ineffizienz von zivilrechtlich ausgestalteten Haftungssystemen in transnationalen Schadensfällen. ${ }^{432}$ So kritisierte der ungarische Delegierte im Sechsten Ausschuss der VN-Generalversammlung im Jahr 2004, dass trotz aller Bemühungen um eine außergerichtliche Einigung und Einleitung eines Gerichtsverfahrens die Geschädigten bis dato keine Entschädigung erhalten haben. ${ }^{433}$

\section{Montara Oil Spill-Fall}

Nach einem Unfall auf der Ölbohrplattform Montara in Australien sickerten im Jahr 2009 Unmengen von Öl unkontrolliert in die Timorsee, was zur Kontamination der Gewässer sowie zu einem Fischsterben führte. Die indonesische Regierung strebte eine Entschädigungsvereinbarung mit Australien an und forderte schließlich vom Eigentümer der Plattform, dem thailändischen Ölkonzern PTTEP Australasia, Ersatz für die grenzüberschreitenden Schäden. ${ }^{434}$ In Anbetracht der Schwierigkeiten, die Ansprüche durchzusetzen, erklärte Indonesien, dass ein zwingender Bedarf für ein internationales Haftungsregime für grenzüberschreitende Schäden durch Ölbohrungen bestehe. ${ }^{435}$ Osttimor, das ebenfalls von der Ölverseuchung betroffen war, forderte sowohl von PTTEP als auch von Australien

431 A. L. Springer, Cases of Conflict, 2016, S. $92 \mathrm{f}$.

432 G. Handl, International Accountability for Transboundary Environmental Harm Revisited, Environmental Policy and Law, 37 (2007), S. 116 (119); vgl. auch S. C. McCaffrey, Expediting the Provision of Compensation to Accident Victims, in: G. Handl/R. E. Lutz (Hg.), Transferring Hazardous Technologies and Substances, 1989, S. 199 (199 ff.) in Bezug auf den Bhopal-Fall, bei dem im Jahr 1984 der Tank einer Pestizid-Fabrik explodierte und zu einer Chemiekatastrophe in Indien führte.

433 GA Sixth Committee, Summary record of the 20th meeting, 3 November 2004, UN General Assembly Official Records, UN Doc. A/C.6/59/SR.20, S. 6, Rn. 30.

434 Siehe G. Handl, Allocating transboundary loss from offshore oil and gas accidents, in: ders./K. Svendsen, Kristoffer (Hg.), Managing the Risk of Offshore Oil and Gas Accidents, 2019, S. 253 (253 ff.) m.w.N.

435 Siehe ders., International Law and the Liability for Catastrophic Environmental Damage, Remarks, ASIL Proc. 105 (2011), S. 427 (428). 
Schadensersatz und bestärkte durch diese Forderung nach einer originären Haftung die Rolle des Staates in grenzüberschreitenden Schadensfällen. 436

\section{Deepwater Horizon-Fall}

Schließlich dient der Deepwater Horizon-Fall aus dem Jahr 2010 als Negativbeispiel dafür, dass im internationalen Verkehr die Rolle des Staates und damit einhergehende staatliche Einstandspflichten für grenzüberschreitende Schäden nicht durch eine private Haftung des Schadensverursachers gewohnheitsrechtlich untergraben werden sollten. Die Explosion der Ölbohrplattform Deepwater Horizon des britischen Energiekonzerns BP führte zu einer Umweltkatastrophe im Golf von Mexico. Während die US-Regierung umgehend Schritte einleitete, um das Unternehmen zur Verantwortung zu ziehen, und es daraufhin zu umfangreichen Säuberungsmaßnahmen und Entschädigungen für Betroffene an der Küste der USA kam, kämpfen mexikanische Fischer und andere betroffene Bürger Mexikos bis heute für eine Entschädigung für die erlittenen Verluste. 437 Dies liegt zunächst an der fehlenden Unterstützung der mexikanischen Regierung, die weder angemessene Untersuchungen zum Nachweis der negativen Auswirkungen auf die mexikanische Küstenregion unternommen hat, noch rechtlich Entschädigung für die Betroffenen einforderte. Die mexikanische Regierung strengte zwar 2013 eine Klage gegen BP an, allerdings kam es 2018 zu einer vertraulichen Vergleichsvereinbarung zwischen der mexikanischen Regierung und BP, die den Konzern aus jeglicher Verantwortung entließ, wobei keinerlei Entschädigung an betroffene Mexikaner geleistet wurde. ${ }^{438}$ Unterdessen wurden Klagen betroffener mexikanischer Bundesstaaten vor US-Gerichten mit der Begründung abgelehnt, dass die mögli-

436 Vgl. S. Bosma/J. Underwood, Compensation claims arising from the Montara Oil Spill, in: G. Handl/K. Svendsen, Kristoffer (Hg.), Managing the Risk of Offshore Oil and Gas Accidents, 2019, S. 425 (430) m.w.N.

437 Siehe V. Mercado/L. A. Soto, Fischer gegen Ölmulti, Entwicklung und Zusammenarbeit Online, 19. März 2017, abrufbar unter: https:/www.dandc.eu/de/arti cle/sieben-jahre-nach-dem-untergang-der-oelbohrplattform-deepwater-horizon-k aempft-mexiko-noch, (geprüft am 15.05.2020).

438 N. Janowitz, Revealed: Documents Show BP Quietly Paid Just \$25 Million to Mexico After The Worst Oil Spill Of The Century, BuzzFeedNews, 28 September 2018, abrufbar unter: https://www.buzzfeednews.com/article/nathanieljano witz/bp-mexico-deepwater-horizon-oil (geprüft am 15.05.2020). 


\section{Kapitel: Staatenhaftung in der Systematik des Völkerrechts}

cherweise geschädigten Gebiete in die Verantwortung der mexikanischen Regierung fielen. ${ }^{439}$

Der Fall verdeutlicht, dass zivilrechtliche Lösungen in internationalen Schadensfällen nicht immer befriedigend sind und Staaten einschreiten müssen, um eine unverzügliche und angemessene Entschädigung der Betroffenen zu gewährleisten. Die mexikanische Regierung hätte die Staatenhaftung der USA einfordern müssen. Allerdings ist die Untätigkeit Mexikos, eine entsprechende Forderungen gegen die USA durchzusetzen, wohl dem Umstand geschuldet, dass sich Mexiko im Jahr 1979 selbst geweigert hatte, für Umweltschäden in den USA zu haften, die durch eine Explosion der Ölbohrplattform IXTOC I in mexikanischen Gewässern im Jahr 1979 entstanden waren. ${ }^{440}$ Demnach lässt sich der Deepwater Horizon-Fall nicht für eine Rechtsüberzeugung anführen, dient aber nachhaltig als Beispiel für die Notwendigkeit einer Staatenhaftung im internationalen Verkehr.

\section{Ergebnis}

Die Ausführungen zeigen, dass regelmäßig Schadensersatzforderungen zunächst auf internationaler Ebene gegen den Ursprungsstaat geltend gemacht und erst fallen gelassen wurden, nachdem der private Verursacher eintrat. Die subsidiäre private Haftung stützt damit das Konzept einer primären Staatenhaftung. Dabei wird nicht verkannt, dass sich anhand dieser Fälle eine Kohärenz von tatsächlichem staatlichem Verhalten und Rechtsüberzeugung nicht ohne Weiteres nachweisen lässt. ${ }^{441}$ In der internationalen Praxis werden zahlreiche grenzüberschreitende Schadensfälle

439 V. Mercado/L. A. Soto, Fischer gegen Ölmulti, Entwicklung und Zusammenarbeit Online, 19. März 2017, abrufbar unter: https://www.dandc.eu/de/article/sie ben-jahre-nach-dem-untergang-der-oelbohrplattform-deepwater-horizon-kaempf t-mexiko-noch, (geprüft am 15.05.2020).

440 Vgl.J.-E. Vinnem/W. Røed, Offshore Risk Assessment Band 1, 2020, S. $107 \mathrm{ff}$.

441 Die Expertengruppe zum Umweltrecht der Weltkommission für Umwelt und Entwicklung gibt zu bedenken: „International State practice en dehors treaties shows a very varied picture and it is not yet possible to draw definite conclusions from that practice as to the (non) existence of a rule of customary international law or a principle of international law imposing strict liability under international law upon States on account of ultrahazardous activities." Experts Group on Environmental Law of the World Commission on Environment and Development, R. D. Munro (Chairman)/J. G. Lammers (Rapporteur), Environmental Protection and Sustainable Development: Legal Principles and Recommendations, 1987, S. 82. 
durch eine zivilrechtliche Haftung des privaten Verursachers gelöst, was die Entwicklung zu einer völkergewohnheitsrechtlichen Staatenhaftung hemmt. Zivilprozesse in internationalen Streitfällen zwischen Geschädigtem und privatem Verursacher sind jedoch umständlich, zeitaufwendig sowie kostenintensiv. ${ }^{442}$ Wie gezeigt, sollte das Konzept der Staatenhaftung im Sinne eines gerechten Opferschutzes nicht völkergewohnheitsrechtlich unterlaufen werden.

Der Staat hat im Rahmen von Einstandspflichten für grenzüberschreitende Schäden immer eine wichtige Funktion, wenn nicht sogar eine Schlüsselfunktion. Die dargestellten Fälle zeigen mithin die Notwendigkeit auf, dass der Staat als eine Art Garant fungiert, der zumindest ergänzend oder bei Haftungsausfall des privaten Verursachers subsidiär eintreten muss.

\section{Völkervertragsrecht}

Das Völkervertragsrecht basiert auf dem ausdrücklichen Konsens der Vertragsstaaten und stellt staatliche Willensbekundungen dar, „that makes law positive rather than hypothetical“. ${ }^{443}$ Damit ist diese Quelle des Völkerrechts das richtige Instrument, um die Schwächen bezüglich der völkergewohnheitsrechtlichen Anerkennung der Staatenhaftung auszugleichen. ${ }^{444}$

Die abstrakte Pflicht der Staaten, Rücksicht auf die Interessen anderer Staaten zu nehmen, ist bereits durch diverse Abkommen in konkrete Pflichten und Rechte erwachsen. So haben Staaten in Ermangelung einer allgemeingültigen Kodifizierung der Staatenhaftung bereits eine Reihe von Haftungsübereinkommen ${ }^{45}$ zum Zweck eines gerechten Interessen-

442 G. Handl, International Accountability for Transboundary Environmental Harm Revisited, Environmental Policy and Law, 37 (2007), S. 116 (119).

443 Vgl. J. Kammerhofer, Uncertainty in the Formal Sources of International Law, EJIL 15 (2004), S. 523 (536).

444 S. Förster, Internationale Haftungsregeln für schädliche Folgewirkungen gentechnisch veränderter Organismen, 2007, S. 95.

445 Eine Übersicht von Haftungskonvention findet sich in dem Dokument des ILC Secretariat, Survey of liability regimes relevant to the topic of international liability for injurious consequences arising out of acts not prohibited by international law (international liability in case of loss from transboundary harm arising out of hazardous activities), YBILC 2004-II/1, UN Doc. A/CN.4/543, S. 112 ff., Rn. 117 ff. Ferner befassen sich unter anderem die Arbeiten C. Langenfeld/P. Minnerop, Environmental Liability Provisions in International Law, in: Umweltbundesamt (Hg.), Environmental Liability in International Law, 


\section{Kapitel: Staatenhaftung in der Systematik des Völkerrechts}

ausgleiches unterzeichnet und teilweise auch ratifiziert, um zwischenstaatliche Schädigungen zu reglementieren ohne das betreffende Verhalten im Vorhinein zu verbieten. ${ }^{446}$

Das Völkervertragsrecht kennt im Wesentlichen drei Haftungssysteme, in denen Staaten Einstandspflichten für völkerrechtlich nicht verbotene Aktivitäten treffen:

Völkerrechtliche Verträge, die eine selbstständige primäre Einstandspflicht von Staaten für völkerrechtlich nicht verbotene Aktivitäten normieren, finden sich für unterschiedliche Regelungsbereiche (I. Originäre Staatenhaftung). Allerdings gestalten die meisten Konventionen in erster Linie Vorgaben für eine zivilrechtliche Haftung des privaten Betreibers, ${ }^{447}$ um potenziellen Opfern bei einem Schadensereignis mit grenzüberschreitender Wirkung möglichst weitgehende und ohne Beweisschwierigkeiten durchsetzbare Ausgleichsansprüche zu gewähren. ${ }^{448}$ Gleichzeitig ist es im staatlichen Interesse, nützliche Technologien und die Funktionsfähigkeit der jeweiligen Industrien zu fördern. Zu diesem Zweck sehen diese Haftungssysteme in der Regel Haftungsobergrenzen vor. ${ }^{449}$ Staatliche Einstandspflichten können greifen, wenn die privaten Betreiber zwar die Summe bis zur geforderten Haftungshöchstgrenze aufbringen, aber darüber hinausgehende Schäden entstehen oder wenn Staaten im Rahmen von zwischenstaatlichen kollektiven Finanzierungmechanismen eine Bei-

Berichte des Umweltbundesamtes, Band 02/05, 2005, Teil 2, und P. Sands/J. Peel, Principles of International Environmental Law, 2018, Teil II mit der geschichtlichen Entwicklung, Regelungssystematik und Reichweite der einzelnen Übereinkommen zur Haftungsthematik.

446 Im Sinne von Prinzip 22 der Erklärung von Stockholm von 1972, der die Staaten zur weiteren Entwicklung von Haftungs- und Kompensationsmechanismen aufruft, verhandeln Staaten zunehmend über den Abschluss von Haftungsabkommen. Den internationalen Haftungsübereinkommen liegen der Gedanke eines gerechten Schadensausgleiches durch angemessene Kompensationsregelungen und zunehmend auch eine präventive Schutzrichtung zugrunde. So $S$. Förster, Internationale Haftungsregeln für schädliche Folgewirkungen gentechnisch veränderter Organismen, 2007, S. 131.

447 Staaten haften nach diesen Haftungsvorgaben, wenn sie wie Privatrechtssubjekte agieren. S. Förster, Internationale Haftungsregeln für schädliche Folgewirkungen gentechnisch veränderter Organismen, 2007, S. 91.

448 J. Barboza, The Environment, Risk and Liability in International Law, 2011, S. 32; T. Gehring/M. Jachtenfuchs, Haftung und Umwelt, 1988, S. 52.

449 J. Barboza, The Environment, Risk and Liability in International Law, 2011, S. 36; S. Förster, Internationale Haftungsregeln für schädliche Folgewirkungen gentechnisch veränderter Organismen, 2007, S. 102 f. zum Pariser Übereinkommen und Brüsseler Zusatzübereinkommen. 
tragspflicht trifft (II. Ergänzende Staatenhaftung). ${ }^{450}$ Schließlich können Haftungsübereinkommen eine staatliche Ausfallhaftung begründen, wenn private Betreiber schon ihren grundsätzlichen Entschädigungspflichten bis zur Haftungsgrenze nicht nachkommen (III. Subsidiäre Staatenhaftung). 451

In den Haftungssystemen findet sich wiederum eine Differenzierung zwischen einer verschuldensabhängigen Haftung (fault liability) und einer verschuldensunabhängigen Haftung (no-fault liability). Die verschuldensabhängige Haftung setzt Vorsatz oder Fahrlässigkeit voraus, ${ }^{452}$ damit eine Einstandspflicht für Schäden begründet werden kann. Die verschuldensunabhängige Haftung ist hingegen schon dann gegeben, wenn ein grenzüberschreitender Schaden durch die völkervertraglich reglementierte völkerrechtlich nicht verbotene Aktivität entsteht. Die Abkommen sehen regelmäßig Haftungsbefreiungen und Haftungsausschlussgründe - etwa bei Naturkatastrophen, bestimmten Konflikten oder Verschulden des Geschädigten - vor und normieren damit eine beschränkte Haftung (strict liability), die sich dahingehend von einer unbeschränkten Haftung (absolute liability) unterscheidet. 453

Die Haftungssysteme beruhen auf unterschiedlichen dogmatischen Grundlagen. ${ }^{454}$ Allerdings können aus den Systemen normative Lösungs-

450 R. Lefeber, Transboundary Environmental Interference and the Origin of State Liability, 1996, S. $305 \mathrm{ff}$.

451 Id., S. $301 \mathrm{ff}$; siehe auch S. Förster, Internationale Haftungsregeln für schädliche Folgewirkungen gentechnisch veränderter Organismen, 2007, S. 103 zum Pariser Übereinkommen und Brüsseler Zusatzübereinkommen.

452 Der Begriff „Verschulden“ hat im Völkerrecht nicht nur die Bedeutung von Vorsatz und Fahrlässigkeit, sondern kann auch die Verletzung von Primärnormen bezeichnen. Diese Doppeldeutigkeit führt zu Schwierigkeiten bei der Abgrenzung der staatlichen Einstandspflichten. Siehe hierzu J. Barboza, The Environment, Risk and Liability in International Law, 2011, S. $24 \mathrm{f}$.

453 Vgl. F. Francioni, Liability for Damage to the Antarctic Environment, in: ders./T. Scovazzi (Hg.), International Law for Antarctica, 1996, S. 581 (590 f.); S. D. Murphy, Prospective Liability Regimes for the Transboundary Movement of Hazardous Wastes, AJIL 88 (1994), S. 24 (49); P. J. Sands, The Environment, Community and International Law, Harv. ILJ 30 (1989), S. 393 (405); R. Wolfrum/C. Langenfeld, Umweltschutz durch internationales Haftungsrecht, Heidelberg 1999, S. 73.

454 So stellt etwa S. Förster klar: „Nur bei der selbständigen primären Haftung der Staaten auf vertraglicher Grundlage handelt es sich um eine Haftung der Staaten als Völkerrechtssubjekte. Bei der subsidiären Staatenhaftung basiert die staatliche Einstandspflicht dagegen auf der zivilrechtlichen Haftung des primär Verpflichteten. Die Haftung selbst bleibt daher trotz staatlicher Beteiligung 


\section{Kapitel: Staatenhaftung in der Systematik des Völkerrechts}

modelle für staatliche Einstandspflichten herausgefiltert werden. Übereinstimmungen im Rahmen der vertraglichen Regelungen sind zudem Indikatoren für die völkerrechtliche Verbindlichkeit der Elemente der Staatenhaftung. ${ }^{455}$ Diese Funktion des Völkervertragsrechtes lässt sich der Aussage des IGH zum Gulf of Maine-Fall entnehmen: „it is in codifying conventions that principles and rules of general application can be identified." 456 Die folgenden Haftungsabkommen zeigen sehr prägnant die anerkannten Grundsätze und aktuellen Entwicklungstendenzen im internationalen Haftungsrecht auf.

\section{Originäre Staatenhaftung}

Die originäre Haftung der Staaten als Völkerrechtssubjekte ist bereits für verschiedene gefährliche Aktivitäten vertraglich normiert.

\section{Weltraumhaftung}

Das Weltraumrecht enthält ein völkerrechtliches Haftungssystem, das eine originäre Staatenhaftung für völkerrechtlich nicht verbotene gefährliche Aktivitäten begründet. ${ }^{457}$

zivilrechtlicher Natur.“ S. Förster, Internationale Haftungsregeln für schädliche Folgewirkungen gentechnisch veränderter Organismen, 2007, S. 91.

$455 \mathrm{Vgl}$. in Bezug auf die UN-Wasserlaufkonvention F. R. Loures/A. Rieu-Clarke/J. W. Dellapenna/J. Lammers, The authority and function of the UN Watercourses Convention, in: F. R. Loures/A. Rieu-Clarke (Hg.), The UN Watercourses Convention in Force, 2013, S. 49 (52 f.).

456 IGH, Case Concerning Delimitation of the Maritime Boundary in the Gulf of Maine Area (Canada v. United States of America), Judgment of 12 October 1984, ICJ Reports 1984, S. 246 (290 f. Rn. 83).

457 Sonderberichterstatter R. Q. Quentin-Baxter, Preliminary report on international liability for injurious consequences of acts not prohibited by international law, YBILC 1980-II/1, UN Doc. A/CN.4/334 and Add.1 and 2, S. 263, Rn. 56; L. Condorelli, La réparation des dommages catastrophiques causés par les activités spatiales, in: Bibliothèque de la Faculté de Droit de l'Université Catholique de Louvain (Hg.), La réparation des dommages catastrophiques: Les risques technologiques majeurs en droit international et en droit communautaire, 1990, S. 263 (278f.); S. Kadelbach, The Concept of State Responsibility for Private Acts under General International Law, in: S. Hobe/S. Freeland/B. Schmidt-Tedd (Hg.), In Heaven as on Earth?, 2013, S. 105 (109). 
Der Vertrag über die Grundsätze zur Regelung der Tätigkeiten von Staaten bei der Erforschung und Nutzung des Weltraums einschließlich des Mondes und anderer Himmelskörper von 1967 (Weltraumvertrag) 458 enthält staatliche Pflichten zur Prävention von Umweltschäden im Kontext von nationalen Weltraumaktivitäten durch staatliche sowie nicht-staatliche Stellen. So haben Vertragsstaaten nach Maßgabe von Art. IX Weltraumvertrag die verhaltensbezogene Pflicht, angemessene Maßnahmen zu ergreifen, damit nationale Weltraumaktivitäten unter gebührender Rücksichtnahme auf die Interessen aller anderen Vertragsstaaten erfolgen, sowie die ergebnisbezogene Pflicht, keine schädlichen Verunreinigungen zu verursachen. ${ }^{459}$ Besonderes Gewicht liegt dabei auf zwischenstaatlichen Kooperationspflichten. Der Weltraumvertrag bestimmt ferner, dass die Vertragsstaaten für nicht-staatliche Weltraumaktivitäten verantwortlich sind und dass derartige Aktivitäten der Genehmigung und der ständigen Kontrolle durch die Vertragsstaaten bedürfen (Art. VI Weltraumvertrag). Des Weiteren begründet der Weltraumvertrag eine völkerrechtliche Haftung der Vertragsstaaten für zwischenstaatliche Schäden durch gestartete Gegenstände oder deren Bestandteile auf der Erde, im Luftraum oder im Weltraum (Art. VII Weltraumvertrag). Das Übereinkommen über die völkerrechtliche Haftung für Schäden durch Weltraumgegenstände von 1972 (Weltraumhaftungsübereinkommen ${ }^{460}$ konkretisiert diesen allgemein gehaltenen Haftungsgrundsatz. Das Übereinkommen bestärkt in seiner Präambel, dass für den Start von Weltraumgegenständen Vorsichtsmaßnahmen zu treffen sind sowie vollständige und gerechte Entschädigungsleistungen unverzüglich an Geschädigte zu erfolgen haben. Anschließend normiert das Weltraumhaftungsübereinkommen zwei Haftungsstandards für zwischenstaatliche Schäden durch Weltraumgegenstände. Das Übereinkommen bestimmt zudem, dass Schadensersatzansprüche gegen den haftenden Staat gemäß Art. VIII und IX Weltraumhaftungsübereinkommen

458 Vertrag über die Grundsätze zur Regelung der Tätigkeiten von Staaten bei der Erforschung und Nutzung des Weltraums einschließlich des Mondes und anderer Himmelskörper vom 27. Januar 1967, in Kraft seit dem 10. Oktober 1967, BGBl. 1969 II S. 1967, 1968 ff., sowie die dieses Abkommen ergänzenden Bestimmungen des neuen Mondvertrages vom 14. Dezember 1979, ILM 1979, S. $1434 \mathrm{ff}$.

459 C. Kypraios/E. Carpanelli, Space Debris, in: R. Wolfrum (Hg.), MPEPIL 2018, http://www.mpepil.com, Rn. 15.

460 Übereinkommen über die völkerrechtliche Haftung für Schäden durch Weltraumgegenstände vom 29. März 1972 (Weltraumhaftungsübereinkommen), BGBl. 1975 II S. 1209, $1210 \mathrm{ff}$. 


\section{Kapitel: Staatenhaftung in der Systematik des Völkerrechts}

auf diplomatischem Wege geltend gemacht werden und sieht keinen privaten Rechtsweg vor. Dadurch wird deutlich, dass es sich um eine völkerrechtliche - und nicht um eine völkerrechtlich geregelte zivilrechtliche Haftung handelt. ${ }^{461}$

\section{a) Verschuldensabhängige Haftung}

Gemäß Art. III Weltraumhaftungsübereinkommen haftet der Startstaat nur, sofern Verschulden seitens des Startstaates oder einer Person, für die der Startstaat verantwortlich ist, nachgewiesen werden kann. ${ }^{462}$ Unter den Begriff Startstaat fallen nach Art. I lit.c Weltraumhaftungsübereinkommen Staaten, die einen Weltraumgegenstand starten oder dessen Start durchführen lassen, sowie Staaten, von deren Hoheitsgebiet oder Anlagen ein Weltraumgegenstand gestartet wird. Die Haftung betrifft Schäden, die ein Weltraumgegenstand an einem Weltraumgegenstand eines anderen Startstaates oder an Personen oder Sachen an Bord eines solchen Weltraumgegenstands verursacht hat. Der Grund für das Verschuldenserfordernis ergibt sich aus dem Umstand, dass die Schäden in dieser Konstellation diejenigen treffen, die durch das Entsenden eigener Weltraumobjekte bewusst ein Risiko eingegangen sind und daher nicht den Schutz einer verschuldensunabhängigen Haftung bedürfen. ${ }^{463}$ Auch hier kommt der Gerechtigkeitsgedanke des Haftungsrechtes zum Tragen.

Das Weltraumhaftungsübereinkommen definiert aber weder, was mit Verschulden gemeint ist, noch unter welchen Voraussetzungen ein Fehlverhalten dem Staat zuzurechnen ist. Zur Konkretisierung kann auf Diskussionen zu Verschuldenserfordernis und Zurechnungsmechanismen im Kontext der Staatenverantwortlichkeit zurückgegriffen werden. ${ }^{464}$

461 S. Förster, Internationale Haftungsregeln für schädliche Folgewirkungen gentechnisch veränderter Organismen, 2007, S. 92, Fn. 3.

462 Art. IV Abs. 1 lit. b und Abs. 2 Weltraumhaftungsübereinkommen stellt eine gesamtschuldnerische verschuldensabhängige Haftung für den Fall der Schadensverursachung durch zwei oder mehr Startstaaten auf.

463 P. Stubbe, State Accountability for Space Debris, 2018, S. 379; E. Wins, Weltraumhaftung im Völkerrecht, 2000, S. 76.

464 Für die Staatenverantwortlichkeit wird das Verschuldenserfordernis überwiegend verneint. Dennoch können aus den Diskussionen Anhaltspunkte zur Spezifizierung des Verschuldenserfordernisses entnommen werden. P. Stubbe, State Accountability for Space Debris, 2018, S. 380 ff.; E. Wins, Weltraumhaftung im Völkerrecht, 2000, S. $71 \mathrm{f}$. 
Unstreitig ist ein Verschulden zu bejahen, wenn ein Verhalten darauf abzielt ein bestimmtes Ergebnis, etwa Schadensverursachung durch ein Weltraumobjekt, zu erreichen. Schwieriger ist die Feststellung fahrlässigen Verhaltens, welche grundsätzlich den Nachweis der Verletzung von Sorgfaltsstandards erfordert. Im Weltraumhaftungsübereinkommen finden sich allerdings keine Indikatoren, an denen die angewandte Sorgfalt zu messen wäre. Die Maßstäbe müssen vielmehr extern ermittelt werden. ${ }^{465}$ Die gebotene Sorgfalt ist nicht notwendigerweise in verbindlichen internationalen Verpflichtungen verankert, sondern beispielsweise durch Soft Law-Instrumente, wie etwa durch die Richtlinien zur Verringerung von Weltraummüll bzw. zum Verhalten im Weltraum, vorgegeben. ${ }^{466}$ Dementsprechend ist zu beachten, dass Verschulden nicht mit der Verübung eines völkerrechtswidrigen Aktes gleichzusetzen ist. ${ }^{467}$

Gemäß Art. III Weltraumhaftungsübereinkommen begründet nicht nur das Verschulden des Startstaates selbst dessen Haftung, sondern auch das Fehlverhalten von nicht-staatlichen Akteuren, für die der Startstaat verantwortlich ist. In Anlehnung an das Recht der Staatenverantwortlichkeit ist der Startstaat für nicht-staatliche Akteure „verantwortlich“, wenn diese in Ausübung von staatlichen Aufgaben (Art. 4, 5, 9 ASR) oder unter staatlicher Kontrolle (Art. 8 ASR) agieren oder eine nachträgliche Anerkennung durch den Staat erfolgt (Art. 11 ASR). Darüber hinaus begründet das Weltraumrecht eine Ausnahme von dem Grundsatz, dass reines Privathandeln keinen zurechnungsrelevanten Zusammenhang bietet und somit nicht als Handlung des Staates qualifiziert werden kann. ${ }^{468}$ Nach Art. VI S. 1 des

465 P. Stubbe, State Accountability for Space Debris, 2018, S. 382 f.

466 C. Kypraios/E. Carpanelli, Space Debris, in: R. Wolfrum (Hg.), MPEPIL 2018, http://www.mpepil.com, Rn. 13; I. Marboe, The Importance of Guidelines and Codes of Conduct for Liability of States and Private Actors, in: dies. (Hg.), Soft Law in Outer Space, 2012, S. 119 (134 f.).

467 P. Stubbe, State Accountability for Space Debris, 2018, S. 383. Gleichzeitig ist jedwede Verletzung von Primärnormen a maiore ad minus als fahrlässiges Verhalten zu qualifizieren und begründet mithin Verschulden im Sinne des Artikel III Weltraumhaftungsübereinkommen. So I. Marboe, The Importance of Guidelines and Codes of Conduct for Liability of States and Private Actors, in: dies. (Hg.), Soft Law in Outer Space, 2012, S. 119 (135); P. Stubbe, State Accountability for Space Debris, 2018, S.382; E. Wins, Weltraumhaftung im Völkerrecht, 2000, S. $74 \mathrm{f}$.

468 R. Lefeber, Transboundary Environmental Interference and the Origin of State Liability, 1996, S. 161; M. Pedrazzi, Outer Space, Liability for Damage, in: R. Wolfrum (Hg.), MPEPIL 2008, http://www.mpepil.com, Rn. 2; P. Stubbe, State Accountability for Space Debris, 2018, S. 96, 383 f.; E. Wins, Weltraumhaftung im Völkerrecht, 2000, S. 79 f., 340 f. 


\section{Kapitel: Staatenhaftung in der Systematik des Völkerrechts}

Weltraumvertrages sind Vertragsstaaten für nationale Weltraumaktivitäten - sei es von staatlichen oder nicht-staatlichen Akteuren - verantwortlich. Dabei fallen unter nationale Aktivitäten solche, die ihren Ausgangspunkt auf staatlichem Hoheitsgebiet haben, aber auch Aktivitäten, die von Staatsangehörigen oder nationalen Unternehmen durchgeführt werden. ${ }^{469}$ Dieser spezialgesetzliche Zurechnungsmechanismus des Weltraumvertrages schließt die gewohnheitsrechtlichen Zurechnungskriterien der Staatenverantwortlichkeit nicht etwa aus, sondern ergänzt sie. ${ }^{470}$ Diese Kriterien sind auf die Zurechnung von Vorsatz und Fahrlässigkeit übertragbar und begründen eine Verschuldenshaftung des Startstaates für staatliche bzw. nicht-staatliche Aktivitäten. ${ }^{471}$

\section{b) Verschuldensunabhängige Haftung}

Noch umfangreicher ist die Haftung gemäß Art. II Weltraumhaftungsübereinkommen. Ein Startstaat haftet danach unbedingt für Schäden, die durch einen seiner Weltraumgegenstände auf der Erdoberfläche oder an Luftfahrzeugen im Flug verursacht wurden. Der Startstaat haftet selbst dann, wenn er angemessene Maßnahmen zur Schadensprävention ergriffen hat. Der verschuldensunabhängige Haftungsstandard fließt aus den Gerechtigkeitserwägungen und dem Gedanken, dass die unbeteiligten Opfer nicht die Schäden zu tragen haben. ${ }^{472}$ Sofern zwei oder mehr Staaten gemeinsam einen Weltraumgegenstand starten, haften diese gesamtschuldnerisch für auftretende Schäden (Art. IV Abs. 1 lit. a Weltraumhaftungsübereinkommen). Vorsatz oder grobe Fahrlässigkeit hinsichtlich des entstandenen Schadens seitens einer anderen Partei führt zur Entlastung des Startstaates, es sei denn der Startstaat hat den Schaden im Zusammenhang mit einer völkerrechtswidrigen Aktivität verursacht (Art. VI Weltraumhaftungsübereinkommen). Im Ergebnis statuiert das Weltraumhaftungsüber-

469 P. Stubbe, State Accountability for Space Debris, 2018, S. 260 ff., 383 f.

470 Siehe hierzu id., S. 383, Fn. 70.

471 Id., S. 383 ff.; vgl. auch S. Förster, Internationale Haftungsregeln für schädliche Folgewirkungen gentechnisch veränderter Organismen, 2007, S. 96.

472 P. Stubbe, State Accountability for Space Debris, 2018, S. 391. Nach S. Förster bestand wegen der Schwierigkeiten eines Verschuldensnachweises nach dem Absturz eines Flugkörpers relativ früh Einigkeit über die Einführung einer Gefährdungshaftung für Schäden auf der Erdoberfläche. S. Förster, Internationale Haftungsregeln für schädliche Folgewirkungen gentechnisch veränderter Organismen, 2007, S. 97. 
einkommen damit eine verschuldensunabhängige Haftung des Startstaates für Schäden durch seine Weltraumgegenstände, ungeachtet dessen, von wem diese Aktivität durchgeführt wird. 473

\section{Anlage VI zum Umweltschutzprotokoll zum Antarktisvertrag}

Das Umweltschutzprotokoll zum Antarktisvertrag (USP) ${ }^{474}$ aus dem Jahr 1991 fordert die Vertragsstaaten auf, die Haftung für Schäden durch Aktivitäten im Gebiet des Antarktisvertrages zu reglementieren (Art. 16 USP). Bestrebungen, entsprechende Haftungsbestimmungen auszuarbeiten, führten nach langjährigen Verhandlungen im Jahr 2005 teilweise zum Erfolg. Die 28 Konsultativstaaten, welche Forschung in der Antarktis durchführen und auf jährlichen Konferenzen (Antarctic Treaty Consultative Meetings) die Grundsätze und Ziele des Antarktisvertrages überarbeiten und ergänzen, verabschiedeten im Konsens die gesondert zu ratifizierende Anlage $\mathrm{VI}^{475}$ zum USP. ${ }^{476}$ Die Bestimmungen der Anlage VI zur Haftung für umweltgefährdende Notfälle greifen jedoch das vom USP vorgesehene Haftungssystem nur teilweise auf. Insbesondere die Haftungsbeschränkung auf umweltgefährdende Notfälle im Vertragsgebiet des Antarktisvertrages (Art. 1 Anlage VI), das heißt auf Unfallereignisse, die zu erheblichen nachteiligen Auswirkungen auf die antarktische Umwelt führen oder unmittelbar zu führen drohen (Art. 2 lit. b Anlage VI), begründet Haftungslücken etwa in Bezug auf Schäden an mit der Antarktis verbundenen Ökosystemen. ${ }^{477}$ Aus diesem Grund einigten sich die Staatenvertreter über die Notwendigkeit zur Wiederaufnahme von Verhandlungen zur Ausarbeitung weiterer notwendiger Regeln und Maßnahmen. Gleichwohl wurde die

473 Auch die GV Resolution 47/68 vom 14.12.1992 proklamiert unter der Überschrift "Principles relevant to the Use of Nuclear Power Sources in Outer Space“ eine verschuldensunabhängige Haftung, wonach „each State which launches or procures the launching of a space object and each State from whose territory or facility a space object is launched is internationally liable for damage caused by such space objects or their component parts." (Prinzip 9 Abs. 1).

474 Umweltschutzprotokoll zum Antarktisvertrag vom 4. Oktober 1991, in Kraft seit dem 14. Januar 1998, BGBl. 1994 II S. 2478, 2479 ff.

475 Anlage VI des Antarktisumweltschutzprotokolls vom 14. Juni 2005 über die Haftung bei umweltgefährdenden Notfällen, BGBl. 2017 II S. 722, 723 ff. (noch nicht in Kraft getreten).

476 K. Schmalenbach, Verantwortlichkeit und Haftung, in: A. Proelß (Hg.), Internationales Umweltrecht, 2017, S. 211 (232 f. Rn. 36).

477 A. D. Hemmings, Liability postponed, The Polar Journal 8 (2018), S. 315 (323 f.). 


\section{Kapitel: Staatenhaftung in der Systematik des Völkerrechts}

Entscheidung über weitere Verhandlungen immer wieder verschoben und auch die Anlage selbst ist bisher noch nicht in Kraft getreten. ${ }^{478}$

Die Anlage VI sieht Präventions- und Einstandspflichten vor. Parteien der Anlage VI (Vertragsstaaten) müssen zunächst von „ihren Betreibern“ die Ergreifung vernünftiger Vorsorge- und Gegenmaßnahmen fordern. „Betreiber der Vertragspartei“ ist ein staatlicher oder nicht-staatlicher Betreiber, der vom Hoheitsgebiet dieser Vertragspartei aus im Gebiet des Antarktis-Vertrags durchzuführende Tätigkeiten organisiert, sofern es sich um eine Tätigkeit handelt, die einer Genehmigung oder eines vergleichbaren behördlichen Verfahrens im jeweiligen Vertragsstaat bedarf (Art. 2 lit. d Anlage VI). Die Betreiber sind zur Vornahme angemessener Präventionsmaßnahmen (Art.3 Anlage VI), zum Erstellen von Einsatzplänen (Art. 4 Anlage VI) und zur Durchführung umgehender und wirksamer Gegenmaßnahmen (Art. 5 Anlage VI) hinsichtlich umweltgefährdender Notfälle zu verpflichten. Sofern die erforderlichen Gegenmaßnahmen durch den Betreiber ausbleiben, werden in erster Linie die Staaten des Betreibers aber auch andere Vertragsstaaten „ermutigt“, diese Maßnahmen zu ergreifen (Art. 5 Abs. 2 und Abs. 3 Anlage VI). Damit wird zwar keine staatliche Einstandspflicht normiert, doch kommt in dieser Befugnis zur Ersatzvornahme die zentrale Rolle von Staaten in internationalen Schadensfällen zum Ausdruck. ${ }^{479}$

Daneben enthält die Anlage VI auch ausdrückliche Bestimmungen zur staatlichen Einstandspflicht. Sofern es ein (staatlicher) Betreiber unterlassen hat, Gegenmaßnahmen in umweltgefährdenden Notfällen zu ergreifen, soll dieser denjenigen Vertragsstaaten, die Gegenmaßnahmen nach Art. 5 Abs. 2 Anlage VI ergriffen haben, die Kosten der Ersatzvornahme ersetzen. Staatliche Betreiber haben überdies in Fällen, in denen kein Vertragsstaat Gegenmaßnahmen ergriffen hat, einen Betrag, der den Kosten der Gegenmaßnahmen entsprechen würde, in einen Fonds einzuzahlen (Art. 6 Abs. 2 lit. a Anlage VI). In Anbetracht der Tatsache, dass viele Tätigkeiten in der Antarktis im Rahmen nationaler Antarktisprogramme erfol-

478 Antarctic Treaty Consultative Meetings, Decision 5 (2015) - ATCM XXXVIII CEP XVIII, Sofia, Liability arising from Environmental Emergencies, abrufbar unter: https://ats.aq/devAS/Meetings/Measure/610 (geprüft am 15.05.2020).

479 N. Chua Abdullah/R. M. Shah/Z. H. Husin/H. A. Rahman, Antarctic Tourism, Procedia - Social and Behavioral Sciences 202 (2015), S. 227 (230 f.); R. Wolfrum, Liability for Environmental Damage in Antarctica, in: I. Buffard/J. Crawford/A. Pellet/S. Wittich (Hg.), International Law Between Universalism and Fragmentation: Festschrift in Honour of Gerard Hafner, 2008, S. 817 (823 f.). 
gen ${ }^{480}$ begründet die Anlage VI damit eine verschuldensunabhängige (vgl. Art. 6 Abs. 3 Anlage VI) ${ }^{481}$ Einstandspflicht der Staaten für völkerrechtlich erlaubte Aktivitäten. Soweit die Vertragsstaaten es versäumt haben, die Einhaltung der Bestimmungen der Anlage sicherzustellen, greift darüber hinaus eine staatliche Einstandspflicht für Schäden durch Aktivitäten nicht-staatlicher Betreiber (Art. 10 Anlage VI) ${ }^{482}$ Es besteht allerdings Uneinigkeit darüber, ob die Verletzung staatlicher Überwachungs- und Kontrollpflichten in Bezug auf nicht-staatliche Aktivitäten bereits zu einer Haftung ex delicto - im Sinne der Staatenverantwortlichkeit für völkerrechtswidriges Verhalten - führt oder lediglich eine Haftung sine delicto - im Sinne einer Staatenhaftung für völkerrechtlich nicht verbotene Aktivitäten - auslöst. Denn Haftungsübereinkommen, die eine zivilrechtliche Haftung des Betreibers begründen, verpflichten die Vertragsstaaten zumeist, dafür Sorge zu tragen, dass nicht-staatliche Akteure bei dem Betrieb einer risikobehafteten Aktivität auf ihrem Hoheitsgebiet bzw. unter ihrer Hoheitsgewalt die konventionsrechtlichen Vorgaben beachten und finanzielle Ressourcen bereithalten, um im Schadensfall den (gebietsfremden) Opfern eine unverzügliche und angemessene Entschädigung zu bieten. ${ }^{483}$ Eine Verletzung dieser staatlichen Überwachungs- und Kontrollpflichten stellt - entgegen anderer Stimmen in der Völkerrechtsliteratur ${ }^{484}$ - kein völkerrechtliches Delikt dar. Eine Haftung ex delicto entsteht nur dann, wenn

480 A. D. Hemmings, Liability postponed, The Polar Journal 8 (2018), S. 315 (322).

481 In diesem Kontext wird „strict“ im französischen Text mit „absolue“ und im deutschen Text mit „verschuldensunabhängig“ übersetzt (BGBl. 2017 II S. 722, 728).

482 Diese Haftungsnorm stimmt wesentlich mit Art. 139 SRÜ überein. Siehe hierzu 1. Kapitel B. I. 3. a). Entsprechend finden sich auch hinsichtlich Art. 10 Anlage VI Stimmen in der Völkerrechtsliteratur, die in der Vorschrift eine Verantwortlichkeitsregel im Sinne der Staatenverantwortlichkeit für völkerrechtswidriges Verhalten - und keine Haftungsregel für völkerrechtlich nicht verbotene Aktivitäten - sehen. Siehe etwa K. Bastmeijer, Antarctica in: A. Nollkaemper/I. Plakokefalos (Hg.), The Practice of Shared Responsibility in International Law, 2017, S. 399 (418); K. Schmalenbach, Verantwortlichkeit und Haftung, in: A. Proelß (Hg.), Internationales Umweltrecht, 2017, S. 211 (238 Rn. 50).

483 L. A. de la Fayette, International liability for damage to the environment, in: M. Fitzmaurice/D. M. Ong/P. Merkouris (Hg.), Research Handbook on International Environmental Law, 2010, S.320 (329); X. Hanqin, Transboundary Damage in International Law, 2003, S. 77.

484 J. Barboza, The Environment, Risk and Liability in International Law, 2011, S. 38 f. unter Verweis auf Art. 8 Abs. 3 lit. a Übereinkommen zur Regelung der Tätigkeiten im Zusammenhang mit mineralischen Ressourcen der Antarktis (CRAMRA) und Art. 139 SRÜ. 


\section{Kapitel: Staatenhaftung in der Systematik des Völkerrechts}

der Staat seine vertraglich vorgegebene finanzielle Einstandspflicht nicht erfüllt. ${ }^{485}$ Schadensvermeidung und Schadenskompensation sind nämlich als zusammengesetzte Primärpflicht zu betrachten, die in der Anlage VI für das antarktische Haftungssystem vertraglich normiert ist. Die staatliche Einstandspflicht, welche bei Missachtung staatlicher Überwachungs- und Kontrollpflichten greift, stellt demnach eine verschuldensabhängige Haftung sine delicto dar. ${ }^{486}$

\section{VN-Wasserlaufkonvention}

Auch das Übereinkommen über das Recht der nichtschifffahrtlichen Nutzung internationaler Wasserläufe von 1997 (VN-Wasserlaufkonvention) $)^{487}$ konstituiert eine originäre Staatenhaftung, bestehend aus den Komponenten der Prävention und Kompensation. Die VN-Wasserlaufkonvention basiert auf Artikelentwürfen und dazugehörigen Kommentaren der Völ-

485 Vgl. auch die Ausführungen von R. Lefeber, denen allerdings eine andere Perspektive zugrunde liegt. Seiner Auffassung nach handelt es sich bei der vertraglichen Haftung aufgrund der Verletzung staatlicher Überwachungspflichten grundsätzlich um eine subsidiäre staatliche Haftung ex delicto. Er differenziert indessen anhand der Formulierung im Vertragstext. Wenn der Staat dazu aufgefordert wird, angemessene Maßnahmen zur Einhaltung der vertraglichen Vorgaben durch den privaten Betreiber zu ergreifen, sei eine staatliche Haftung ex delicto lediglich bei Verletzung dieser verhaltensbezogenen Pflicht gegeben. Sofern der Vertragstext die Staaten auffordert, sicherzustellen, dass eine Entschädigungszahlung erfolgt, könne der Staat einer ex delicto Haftung hingegen nur entgehen, indem er anstelle des privaten Betreibers finanziell für den Schaden einstehe. R. Lefeber, Transboundary Environmental Interference and the Origin of State Liability, 1996, S. 302 f.; vgl. auch L. A. de la Fayette, International liability for damage to the environment, in: M. Fitzmaurice/D. M. Ong/P. Merkouris (Hg.), Research Handbook on International Environmental Law, 2010, S. 320 (329f.).

486 Vgl. hierzu die Ausführungen im Rahmen der Rechtsprechungsanalyse zu Art. 139 SRÜ im 1. Kapitel B. I. 3. a). Siehe außerdem Art. 263 SRÜ, der zum einen bestimmt, dass Staaten sicherstellen müssen, dass nicht nur staatliche, sondern auch „in ihrem Namen“ durchgeführte wissenschaftliche Meeresforschung den Vorgaben des SRÜ entspricht, und zum anderen, dass Staaten für aus derartiger Meeresforschung resultierende Schäden der Meeresumwelt haften. Demnach begründet auch nicht-staatliche Meeresforschung, die staatlicherseits genehmigt bzw. überwacht und kontrolliert wird, eine Staatenhaftung. Vgl. X. Hanqin, Transboundary Damage in International Law, 2003, S. 77.

487 Übereinkommen über das Recht der nichtschifffahrtlichen Nutzung internationaler Wasserläufe vom 21. Mai 1997, BGBl. 2006 II S. 742, 743 ff. 
kerrechtskommission. ${ }^{488}$ Trotz des langwierigen Prozesses bis zum Inkrafttreten im Jahr 2014, spielt die Konvention eine bedeutende Rolle in der Kodifikation der völkergewohnheitsrechtlichen Grundsätze des internationalen Wasserrechts. ${ }^{489}$ So stützte sich der IGH bereits vier Monate nach der Annahme der Konvention in seiner Entscheidung zum Gabč́kovo-Nagymaros Project-Fall auf deren Regelungen ${ }^{490}$ und bestätigte auch in weiteren Entscheidungen, dass das Regelwerk den Stand des Völkerrechts für diesen Bereich wiedergibt. ${ }^{491}$ Insbesondere kodifiziert die VN-Wasserlaufkonvention in ihren grundlegenden Bestimmungen zur ausgewogenen und angemessenen Nutzung (Art. 5 und $6 \mathrm{VN}$-Wasserlaufkonvention) und zum Schädigungsverbot (Art. 7 VN-Wasserlaufkonvention) Völkergewohnheitsrecht. ${ }^{492}$ Die Konvention stellt das Recht auf eine ausgewogene und

488 ILC, Report of the International Law Commission on the work of its forty-sixth session, Draft articles on the law of the non-navigational uses of international watercourses and commentaries thereto and resolution on transboundary confined groundwater, YBILC 1994-II/2, UN Doc. A/49/10, S. 89 ff., Rn. 222 (im Folgenden: Artikelentwürfe zur nichtschifffahrtlichen Nutzung internationaler Wasserläufe). Die Bestimmungen der Konvention entsprechen größtenteils dem Text der Artikelentwürfe. Ferner ist die Kommentierung der Völkerrechtskommission für die Auslegung der Bestimmungen maßgeblich, weil die Arbeitsgruppe, welche die Ausgestaltung der Konvention vornahm, sich explizit auf diese bezieht (UN Doc. A/51/869, S.6, Rn. 8). Siehe auch S. C. McCaffrey, International Watercourses, Environmental Protection, in: R. Wolfrum (Hg.), MPEPIL 2011, http://www.mpepil.com, Rn. 6.

489 E. Hey, The Watercourse Convention, RECIEL 7 (1998), S. 291 (291 f.); S. C. McCaffrey, International Watercourses, Environmental Protection, in: R. Wolfrum (Hg.), MPEPIL 2011, http://www.mpepil.com, Rn. 6; P. Wehling, Wasserrechte am Nil, 2018, S. 70 ff.

490 IGH, Gabcíkovo-Nagymaros Projekt (Hungary v. Slovakia), Judgment of 25 September 1997, ICJ Reports 1997, S. 7 (56 Rn. 85).

491 Siehe L. del Castillo-Laborde, Case Law on International Watercourses, in: J. W. Dellapenna/J. Gupta (Hg.), The Evolution of the Law and Politics of Water, 2009, S. 319 (320 ff.); U. Mager, Die Entwicklung des Wasserwirtschaftsrechts, ZaöRV 70 (2010), S. 789 (813).

492 S. C. McCaffrey, The 1997 U.N. Watercourses Convention, Global Business \& Development Law Journal 21 (2008), S. 165 (170); H. Türk, Water in the Contemporary world, in: H. P. Hestermeyer/D. König/N. Matz-Lück/V. Röben/A. Seibert-Fohr/P.-T. Stoll/S. Vöneky (Hg.), Coexistence, Cooperation and Solidarity, 2012, S. 1037 (1049). Der Entwurf der Völkerrechtskommission für das Recht der grenzüberschreitenden Grundgewässer (ILC, Report of the International Law Commission on the work of its sixtieth session, Draft Articles on the Law of Transboundary Aquifers, YBILC 2008-II/2, UN Doc. A/63/10, S. 19 ff., Rn. 53 f.) bestätigt ebenso das Recht zur angemessenen und vernünftigen Nutzung (Art. 4) und die Pflicht zur Schadensvermeidung (Art. 6). Darüber hinaus 


\section{Kapitel: Staatenhaftung in der Systematik des Völkerrechts}

angemessene Nutzung internationaler Wasserläufe der diesbezüglichen Schadensvermeidungspflicht gegenüber und impliziert damit, dass ein Ausgleich zwischen der territorialen Souveränität und der territorialen Integrität von Staaten zu erfolgen hat. ${ }^{493}$ Dabei räumt die VN-Wasserlaufkonvention keinem der beiden Aspekte einen Vorrang ein, sondern überlässt den Ausgleich den zwischenstaatlichen Verhandlungen im konkreten Einzelfall. 494

Im Rahmen dieser Betrachtung ist insbesondere Art. 7 VN-Wasserlaufkonvention von Bedeutung, der eine verhaltensbezogene ${ }^{495}$ Schadensvermeidungspflicht aufstellt, welche sich sowohl auf die staatliche als auch die nicht-staatliche Nutzung von internationalen Wasserläufen bezieht. ${ }^{496}$ Art. 7 Abs. 1 VN-Wasserlaufkonvention bestimmt, dass Wasserlaufstaaten bei der Nutzung eines internationalen Wasserlaufs in ihrem Hoheitsgebiet alle geeigneten Maßnahmen ergreifen müssen, um erhebliche Schäden für andere Wasserlaufstaaten zu vermeiden. ${ }^{497}$ Diese Pflicht betrifft nicht nur Umweltschäden, sondern jede qualitative aber auch quantitative Veränderung des Wassers mit nachteiligen Auswirkungen für betroffene Staaten. ${ }^{498}$

Nach Art. 7 Abs. 2 VN-Wasserlaufkonvention sind Abhilfemaßnahmen und Wiedergutmachung bei Schadenseintritt auch bei Beachtung der Pflicht zur Schadensprävention des Art. 7 Abs. 1 VN-Wasserlaufkonventi-

statuiert der Entwurf die Pflichten zur Beachtung des Vorsorgegebots (Art. 12) sowie zur Überwachung (Art. 13) und Planung (Art. 14).

493 Vgl. U. Mager, Die Entwicklung des Wasserwirtschaftsrechts, ZaöRV 70 (2010), S. $789(811)$.

494 Ibid.; A. Tanzi, The UN Convention on International Watercourses as a Framework for the Avoidance and Settlement of Waterlaw Disputes, LJIL 11 (1998), S. 441 (453 ff.); S. Yasubiro, Some Reflections on the Relationship between the Principle of Equitable Utilization of International Watercourses and the Obligation not to Cause Transfrontier Pollution Harm, AYIL 9 (2000), S. 147 (156ff.).

495 Vgl. Artikelentwürfe zur nichtschifffahrtlichen Nutzung internationaler Wasserläufe, Kommentar zu Art. 7, S. 103, Abs. 3 f.; E. Hey, The Watercourse Convention, RECIEL 7 (1998), S. 291 (294).

496 Artikelentwürfe zur nichtschifffahrtlichen Nutzung internationaler Wasserläufe, Kommentar zu Art. 7, S. 103, Abs. 4.

497 Art. 21 VN-Wasserlaufkonvention verpflichtet die Wasserlaufstaaten zudem zur Verhütung, Verringerung und Bekämpfung der Verschmutzung internationaler Wasserläufe, die anderen Wasserlaufstaaten oder deren Umwelt beträchtlichen Schaden zufügen könnte.

498 U. Mager, Die Entwicklung des Wasserwirtschaftsrechts, ZaöRV 70 (2010), S. 789 (811). 
on zu ergreifen. ${ }^{499}$ Die staatliche Entschädigungspflicht orientiert sich an einem gerechten Interessenausgleich, ${ }^{500}$ wobei auch die Maßnahmen, die ein Wasserlaufstaat zur Schadensprävention ergriffen hat, zu berücksichtigen sind. ${ }^{501}$ Dieser Aspekt lässt Raum dafür, die Frage einer Entschädigung zu erörtern - wie es in Art. 7 Abs. 2 VN-Wasserlaufkonvention heißt. Von besonderer Bedeutung ist, dass der Schädigerstaat danach nicht etwa verpflichtet ist, das schädigende Verhalten zu unterbinden; er muss lediglich mit dem geschädigten Staat Rücksprache halten, die negativen Auswirkungen so möglichst begrenzen und gegebenenfalls Entschädigung leisten. ${ }^{502}$

Die materielle Schadensvermeidungspflicht des Art. 7 VN-Wasserlaufkonvention wird durch verfahrensbezogene Kooperationspflichten wie Informationsaustauch, Konsultation, Notifikation und Verhandlungen (Teil III VN-Wasserlaufkonvention) ergänzt.

Die Bestimmungen der VN-Wasserlaufkonvention dienen insgesamt als Leitlinie für die Ausarbeitung von spezifischen Wasserlaufübereinkünften (vgl. Art. 3 VN-Wasserlaufkonvention). ${ }^{503}$ Die Konvention fungiert also, wie das allgemeine Konzept der Staatenhaftung auch, ${ }^{504}$ als Anstoß sowie Rahmen für die Ausarbeitung spezifischer Abkommen und bietet gleichzeitig einen Auffangmechanismus für ungeregelte Bereiche. ${ }^{505}$ Das Inkrafttreten der VN-Wasserlaufkonvention ist ein Meilenstein für die Idee der Staatenhaftung, da die Konvention Bestimmungen mit allgemeiner Geltung aufstellt. ${ }^{506}$

499 Vgl. auch Artikelentwürfe zur nichtschifffahrtlichen Nutzung internationaler Wasserläufe, Kommentar zu Art. 7, S. 104, Abs. 10.

500 Vgl. auch Artikelentwürfe zur nichtschifffahrtlichen Nutzung internationaler Wasserläufe, Kommentar zu Art. 7, S. 105, Abs. 19.

501 Vgl. Artikelentwürfe zur nichtschifffahrtlichen Nutzung internationaler Wasserläufe, Kommentar zu Art. 5, S. 105, Abs. 18 i.V.m. Kommentar zu Art. 6 Abs. 1 lit. f, S. 101, Abs. 4.

502 So U. Mager, Die Entwicklung des Wasserwirtschaftsrechts, ZaöRV 70 (2010), S. 789 (811).

503 P. Wehling, Wasserrechte am Nil, 2018, S. 79.

504 Vgl.J. Barboza, The Environment, Risk and Liability in International Law, 2011, S. 106; S. Erichsen, Das Liability-Projekt der ILC, ZaöRV 51 (1991), S. 94 (100).

505 U. Mager, Die Entwicklung des Wasserwirtschaftsrechts, ZaöRV 70 (2010), S. 789 (809); K. P. Scanlan, The International Law Commission's First Ten Draft Articles on the Law of the Non-Navigational Uses of International Watercourses, Fordham ILJ 19 (1996), S. 2179 (2223).

506 Vgl. P. Sands/J. Peel, Principles of International Environmental Law, 2018, S. 362 . 


\section{Kapitel: Staatenhaftung in der Systematik des Völkerrechts}

\section{Wirtschaftsvölkerrecht}

Die Haftung für Schäden durch völkerrechtlich nicht verbotene Tätigkeiten wird zwar vorrangig im Umweltvölkerrecht verortet, das Konzept der Staatenhaftung findet sich aber auch im Wirtschaftsvölkerrecht. ${ }^{507}$ Die Weltwirtschaftsordnung ist mit der Umwelt und auch mit Informationsinfrastrukturen insoweit vergleichbar, als sie einer erhöhten Störanfälligkeit und Schädigungsmöglichkeit im zwischenstaatlichen Bereich ausgesetzt sind. ${ }^{508}$ Internationale Kooperation, Rücksichtnahme sowie Schadensvermeidung repräsentieren die Säulen eines gerechten Interessenausgleiches und lassen sich aus der Charta der wirtschaftlichen Rechte und Pflichten von Staaten ableiten. ${ }^{509}$ Bei der Charta handelt es sich um kein rechtsverbindliches Dokument, gleichwohl findet der in ihr zum Ausdruck kommende Gerechtigkeitsgedanke in Teilbereichen des Wirtschaftsvölkerrechtes konkrete Ausgestaltung. ${ }^{510}$ Anhand des Rechtsrahmens der Welthandelsorganisation (WTO) ${ }^{511}$ wird deutlich, dass die originäre Haftung von Staaten für völkerrechtlich nicht verbotene Aktivitäten auch hier zur Anwendung gelangt. ${ }^{512}$ Die Staaten bekräftigen das Konzept der Staaten-

507 Eingehend hierzu J. Bäumler, Das Schädigungsverbot im Völkerrecht, 2017, Kapitel 6.

508 Vgl. id., S. 119.

509 Charter of Economic Rights and Duties of States, UN Doc. A/Res/29/3281 (1974), bestimmt in Art. 30, dass alle Staaten die Pflicht haben, sicherzustellen, dass Aktivitäten unter ihrer Hoheitsgewalt oder Kontrolle der Umwelt anderer Staaten oder den hoheitsfreien Gebieten keinen Schaden zufügen. Art. 12 und Art. 14 bestärken die Pflicht zur gegenseitigen Rücksichtnahme und Kooperation. Vgl. auch R. Schütz, Solidarität im Wirtschaftsvölkerrecht, 1994, S. 67 ff.

510 Ausführlich hierzu J. Bäumler, Das Schädigungsverbot im Völkerrecht, 2017, Kapitel 6; vgl. auch C. Carmody, A Theory of WTO Law, JIEL 11 (2008), S. 527 (532); G. Schwarzenberger, The Principles and Standards of International Economic Law, RdC 117 (1966), S. 1 (88).

511 Mit derzeit 164 Mitgliedstaaten stellt die WTO eine globale Organisation für internationale Handelsfragen dar. (Stand: 15.05.2020) siehe https://www.wto.or g/english/thewto_e/whatis_e/tif_e/org6_e.htm.

512 C. Carmody stellt in diesem Sinne fest: „At the same time, a second type of justice is at work in WTO law and is corrective in the sense that it aims to repair harm done. The harm occurs because countries exercise their individual rights to take action that, strictly speaking, may disappoint expectations." C. Carmody, A Theory of WTO Law, JIEL 11 (2008), S. 527 (532); vgl. auch J. Bäumler, Das Schädigungsverbot im Völkerrecht, 2017, S. 208 f., 236; G. Schwarzenberger, The Principles and Standards of International Economic Law, RdC 117 (1966-I), S. 1 (88). 
haftung zunehmend in bi- und multilateralen Abkommen, insbesondere auch im internationalen Investitionsrecht.

\section{a) Allgemeines Zoll- und Handelsabkommen}

Mit der Nichtverletzungsklage (non-violation complaint), die durch das Allgemeine Zoll- und Handelsabkommen (GATT 47) ) $^{513}$ eingeführt und auch in das Nachfolgeabkommen der Welthandelsorganisation aus dem Jahr 1994 (GATT 94) ${ }^{514}$ aufgenommen wurde, können Entschädigungsansprüche wegen handelspolitischer Maßnahmen eines Mitgliedstaates, die zwar nicht gegen eine Vertragsbestimmung verstoßen, aber dennoch negative Beeinträchtigung eines anderen Mitgliedstaates verursachen, geltend gemacht werden. ${ }^{515}$ So bestimmt Art. XXIII GATT 94, dass ein Mitgliedstaat ein Streitverfahren einleiten darf, wenn eine Maßnahme eines anderen Mitgliedstaates vertraglich begründete Handelsvorteile zunichtemacht oder schmälert. ${ }^{516}$ Die Nichtverletzungsklage ist in diversen Bereichen des Wirtschaftsvölkerrechtes von Bedeutung. Durch den Verweis auf Art. XXIII GATT 94 eröffnen nämlich die meisten WTO-Abkommen die Möglichkeit, eine Entschädigung für die Beeinträchtigung wirtschaftlicher Interessen durch zulässige Handelspolitiken zu fordern. ${ }^{517}$

513 General Agreement on Tariffs and Trade 1947, 55 UNTS 194.

514 General Agreement on Tariffs and Trade 1994, 1867 UNTS 187.

515 R. W. Staiger/A. O. Sykes, How Important Can the Non-Violation Clause Be for the GATT/WTO?, American Economic Journal: Microeconomics 9 (2017), S. 149 (149).

516 Art. XXIII GATT 94: „1. If any contracting party should consider that any benefit accruing to it directly or indirectly under this Agreement is being nullified or impaired or that the attainment of any objective of the Agreement is being impeded as the result of (...) (b) the application by another contracting party of any measure, whether or not it conflicts with the provisions of this Agreement (...).“ (Hervorhebung hinzugefügt). Weiterführend zur Haftung auf Grundlage der Nichtverletzungsklage D.-W. Kim, Non-violation complaints in WTO law, 2006, S. $231 \mathrm{ff}$.

517 Siehe etwa Art. 14 Agreement on Technical Barriers to Trade, 1868 UNTS 120; Art. 8 Agreement on Trade-Related Investment Measures, 1868 UNTS 186; Art. 11 Agreement on the Application of Sanitary and Phytosanitary Measures, 1868 UNTS 493. Eine Ausnahme findet sich beispielsweise im Agreement on Trade-Related Aspects of Intellectual Property Rights (TRIPS), 1869 UNTS 299. Art. 64 Abs. 2 TRIPS unterstellt die Nichtverletzungsklage im Rahmen des TRIPS-Abkommens einem fünfjährigen Moratorium, welches seither durch Ministerentscheidung der WTO verlängert wurde. 


\section{Kapitel: Staatenhaftung in der Systematik des Völkerrechts}

Die Besonderheit der Staatenhaftung kommt auch bei dieser wirtschaftsvölkerrechtlichen Klausel zum Vorschein, denn die WTO-konforme Maßnahme muss nicht zurückgenommen werden, es müssen lediglich Anpassungen, etwa in Gestalt von Entschädigungszahlungen, erfolgen. ${ }^{518}$ Auf diese Weise erfolgt ein gerechter Interessenausgleich zwischen den WTOMitgliedstaaten für zwischenstaatliche Schäden durch rechtmäßige Maßnahmen.

\section{b) Übereinkommen über Subventionen und Ausgleichsmaßnahmen}

Das Übereinkommen über Subventionen und Ausgleichsmaßnahmen aus dem Jahr 1994 (SCM-Übereinkommen) ${ }^{519}$ reglementiert die Zulässigkeit von Subventionen im Rahmen des WTO-Rechts. Es erkennt Subventionen als legitimes Marktregulierungsinstrument an und trägt gleichzeitig deren potenziell schädigenden Auswirkungen auf wirtschaftliche Interessen Rechnung. ${ }^{520}$ Das SCM-Übereinkommen differenziert zwischen verbotenen Export- und Importsubstituierungsmaßnahmen einerseits und internen Stützungsmaßnahmen, die nicht per se rechtswidrig aber angreifbar sind, andererseits. Die meisten Subventionen sind der letzten Kategorie zuzuordnen. ${ }^{521}$ Die Gewährleistung derartiger Subventionen ist grundsätzlich zulässig, allerdings können diese Subventionen angegriffen werden, wenn sie nachteilige Auswirkungen auf die Interessen anderer Mitgliedstaaten begründen. Dies ist dann der Fall, wenn die Schädigung eines nationalen Wirtschaftszweiges, das Zunichtemachen der mittelbaren und unmittelbaren GATT-Vorteile oder die ernsthafte Schädigung der Interessen eines anderen WTO-Mitglieds (Art. 5 und Art. 6 SCM-Übereinkommen) kausal auf der mitgliedstaatlichen Subventionsmaßnahme beruht. ${ }^{522}$

Ist ein Staat der Meinung, dass ihn durch die Subventionsmaßnahmen eines anderen Staates negative Auswirkungen treffen, dann hat er zunächst

518 R. W. Staiger/A. O. Sykes, How Important Can the Non-Violation Clause Be for the GATT/WTO?, American Economic Journal: Microeconomics 9 (2017), S. 149 (155).

519 Agreement on Subsidies and Countervailing Measures, 1867 UNTS 14.

520 J. Bäumler, Das Schädigungsverbot im Völkerrecht, 2017, S. 135 f.

521 Ausführlich hierzu M. Matsushita/T. J. Schoenbaum/P. C. Mavroidis/M. Hahn, The World Trade Organization, 2015, S. $299 \mathrm{ff}$.

522 Die bisherige Rechtsprechung präzisiert die Voraussetzungen der Angreifbarkeit von grundsätzlich zulässigen Subventionen. Vgl. J. Bäumler, Das Schädigungsverbot im Völkerrecht, 2017, S. $136 \mathrm{ff}$. 
die Möglichkeit zur Rücksprache mit dem subventionierenden Staat, um eine Vereinbarung bezüglich der Behebung dieser Auswirkungen, etwa in Form von Kompensationsleistungen, zu treffen (Art.7.1 SCM-Übereinkommen). Wenn keine Einigung möglich ist, kann der Streitbelegungsmechanismus angerufen werden (Art.7.4 SCM-Übereinkommen). Sofern nachteilige Auswirkungen auf die Interessen anderer Mitgliedstaaten nachgewiesen werden, muss der subventionierende Staat die streitige Subvention entweder zurücknehmen oder geeignete Maßnahmen ergreifen, um diese Nachteile zu beseitigen (Art. 7.8 SCM-Übereinkommen). Es geht um die „Neutralisierung“ der nachteiligen Auswirkungen. Demnach muss ein Ausgleich der verursachten Schädigung bzw. der entgangenen Vorteile vorgenommen werden, welcher durch Schadensersatz erreicht werden kann. ${ }^{523}$ Führt auch der Streitbeilegungsmechanismus nicht zum Erfolg und zieht der subventionierende Staat die schädigende Subvention nicht zurück oder ergreift keine geeigneten Maßnahmen zur Beseitigung der negativen Auswirkungen, erhält der benachteiligte Staat grundsätzlich die Berechtigung, Gegenmaßnahmen zu ergreifen (Art. 7.9 SCM-Übereinkommen). ${ }^{524}$ Die Bestimmungen spiegeln das Konzept der Staatenhaftung wider, nach dem Schäden durch rechtmäßige Aktivitäten zunächst zu kompensieren sind und nur wenn eine solche Kompensation ausbleibt, Konsequenzen der Staatenverantwortlichkeit folgen. ${ }^{525}$

c) Internationale Investitionsschutzverträge

Dem Völkerrecht wohnt der Grundsatz inne, dass Enteignungen völkerrechtlich nicht verboten sind. ${ }^{526}$ Die staatliche Einstandspflicht, die aufgrund zulässiger Enteignung von ausländischem Eigentum greift, hat

523 M. Mrozek, Europäisches Marktordnungsrecht, die WTO und die Osterweiterung, 2003, S. 164.

524 Hierzu ausführlich N. E. Nedzel, Antidumping and Cotton Subsidies, Nw. JILB 28 (2008), S. 215 (215 ff.).

525 Ähnliche Regelungsmechanismen liegen auch dem Währungsrecht und IWFÜbereinkommen zugrunde. Auch gibt es bereits Bestrebungen zu multilateralen Regulierungen im Steuerrecht. Siehe J. Bäumler, Das Schädigungsverbot im Völkerrecht, 2017, Kapitel 7 und 8.

526 C. H. Schreuer, The Concept of Expropriation under the ETC and other Investment Protection Treaties, TDM 5 (2005), S. 1 (1). 


\section{Kapitel: Staatenhaftung in der Systematik des Völkerrechts}

ihre Wurzeln im Fremdenrecht, ${ }^{527}$ ist allerdings in jüngerer Zeit vornehmlich im Kontext des internationalen Wirtschaftsrechts zu verorten. ${ }^{528}$ So adressiert das internationale Investitionsschutzrecht das Spannungsverhältnis zwischen staatlicher Souveränität und dem Schutz wirtschaftsvölkerrechtlicher Interessen in multilateralen und in zahlreichen bilateralen Investitionsschutzverträgen zwischen Staaten oder zwischen Staaten und nicht-staatlichen Investorunternehmen. ${ }^{529}$ Die Verträge kodifizieren den völkergewohnheitsrechtlich anerkannten Eigentumsschutz. Dieser Eigentumsschutz illustriert sehr eindrücklich das Konzept der Staatenhaftung, denn „Eigentumsschutz ist in wesentlichen Zügen kein Schutz vor Enteignung, sondern wird erst (...) durch Enteignung aktiviert" ${ }^{\text {530 }}$. Der Eigentumsschutz statuiert zum einen verfahrensbezogene Pflichten, wonach die Enteignung im öffentlichen Interesse, diskriminierungsfrei und in einem rechtsstaatlichen Verfahren zu erfolgen hat, sowie zum anderen die materielle Pflicht zur unverzüglichen, adäquaten und effektiven Entschädi-

527 R. Dolzer/C. Schreuer, Principles of International Investment Law, 2014, S. 136; R. Hofmann, Modernes Investitionsschutzrecht, in: S. Kadelbach/K. Günther (Hg.), Recht ohne Staat?, 2011, S. 119 (123 f.); J. A. Kämmerer, Der Schutz des Eigentums im Völkerrecht, in: 42. Bitburger Gespräche: Eigentum - Ordnungsidee, Zustand und Entwicklungen, Jahrbuch 2004/I, S. 143 (144). So sind auch die Enteignung von Ausländern oder die Zerstörung von neutralem Eigentum im Krieg nicht völkerrechtswidrig, wenn eine angemessene Entschädigung dafür erbracht wird. Siehe M. B. Akehurst, International Liability for injurious consequences arising out of acts not prohibited by international law, NYIL 16 (1985), S. 3 (10 f.).

528 R. Hofmann, Modernes Investitionsschutzrecht, in: S. Kadelbach/K. Günther (Hg.), Recht ohne Staat?, 2011, S. 119 (132f.); J. A. Kämmerer, Der Schutz des Eigentums im Völkerrecht, in: 42. Bitburger Gespräche: Eigentum - Ordnungsidee, Zustand und Entwicklungen, Jahrbuch 2004/I, S. 143 (147); S. H. Nikièma, Compensation for Expropriation, International Institute for Sustainable Development, Best Practices Series March 2013, abrufbar unter: https://www.iisd.o $\mathrm{rg} / \mathrm{pdf} / 2013 /$ best_practice_compensation_expropriation_en.pdf (geprüft am 15.05.2020), S. $2 \mathrm{f}$.

529 M. Krajewski, Wirtschaftsvölkerrecht, 2017, S. 179, Rn. 543.

530 J. A. Kämmerer, Der Schutz des Eigentums im Völkerrecht, in: 42. Bitburger Gespräche: Eigentum - Ordnungsidee, Zustand und Entwicklungen, Jahrbuch 2004/I, S. 143 (156). 
gung. ${ }^{531}$ Nur sofern der Staat diesen primären Pflichten nicht nachkommt, greifen die sekundären Folgen der Staatenverantwortlichkeit. ${ }^{532}$

Es ist zu betonen, dass der Schadensausgleich nicht nur in internationalen Investitionsverträgen seinen Niederschlag gefunden hat, sondern auch außerhalb dieser Übereinkünfte aufgrund einer gemeinsamen völkerrechtlichen Überzeugung erfolgt. ${ }^{533}$ Das internationale Investitionsrecht stärkt aber die Rechtsposition von Investoren, indem es den völkergewohnheitsrechtlichen Eigentumsschutz vertraglich normiert und eine Präzisierung der Voraussetzungen durch eine zunehmende Juridifizierung bewirkt. ${ }^{534}$

Die internationalen Streitbeilegungsinstanzen differenzieren in grenzüberschreitenden Investitionsstreitigkeiten regelmäßig im Sinne der Chorzów Factory-Entscheidung des Ständigen Internationalen Gerichtsho-

531 R. Dolzer/C. Schreuer, Principles of International Investment Law, 2014, S. 137; A. de Nanteuil, Droit international de l'investissement, 2014, S. 342 ff.; $S$. $R$. Ratner, Compensation for Expropriations in a World of Investment Treaties: Beyond the Lawful/Unlawful Distinction, AJIL 111 (2017), S. 7 (7); C. H. Schreuer, The Concept of Expropriation under the ETC and other Investment Protection Treaties, TDM 5 (2005), S. 1 (2).

532 R. Dolzer/C. Schreuer, Principles of International Investment Law, 2014, S. 138; A. de Nanteuil, Droit international de l'investissement, 2014, S. $346 \mathrm{f}$.

533 Vgl. R. Dolzer, Eigentum, Enteignung und Entschädigung im geltenden Völkerrecht, 1985, S. 54 ff.; J. A. Kämmerer, Der Schutz des Eigentums im Völkerrecht, in: 42. Bitburger Gespräche: Eigentum - Ordnungsidee, Zustand und Entwicklungen, Jahrbuch 2004/I, S. 143 (154); M. Krajewski, Wirtschaftsvölkerrecht, 2017, S. 180, Rn. 546; S. H. Nikièma, Compensation for Expropriation, International Institute for Sustainable Development, Best Practices Series, March 2013, abrufbar unter: https://www.iisd.org/pdf/2013/best_practice_compensation_exp ropriation_en.pdf (geprüft am 15.05.2020), S. 2 ff.

534 R. Hofmann, Modernes Investitionsschutzrecht, in: S. Kadelbach/K. Günther (Hg.), Recht ohne Staat?, 2011, S. 119 (133 f.). Der Investor ist, sofern er aufgrund der Verträge in prozessualer und materieller Hinsicht seine Ansprüche selbst gegen den Staat gelten machen kann und die internationalen Streitbeilegungsinstanzen nach völkerrechtlichen Maßstäben urteilen, als Inhaber eines völkerrechtlichen Anspruchs als partielles Völkerrechtssubjekt anzusehen. $R$. Hofmann, Modernes Investitionsschutzrecht, in: S. Kadelbach/K. Günther (Hg.), Recht ohne Staat?, 2011, S. 119 (135); ders., Modern International Investment Law as an Example of Extra-territorial Law-making and Law-enforcement, in: G. Handl/Joachim Zekoll/P. Zumbaansen (Hg.), Beyond Territoriality, 2012, S. 439 (439); J. A. Kämmerer, Der Schutz des Eigentums im Völkerrecht, in: 42. Bitburger Gespräche: Eigentum - Ordnungsidee, Zustand und Entwicklungen, Jahrbuch 2004/I, S. 143 (154); M. Krajewski, Wirtschaftsvölkerrecht, 2017, S. 179 f., Rn. 544 f. 


\section{Kapitel: Staatenhaftung in der Systematik des Völkerrechts}

fes (StIGH) aus dem Jahr $1928^{535}$ zwischen rechtmäßigen und rechtswidrigen Enteignungen. ${ }^{536}$ Im Chorzów Factory-Fall bejahte der Gerichtshof die Völkerrechtswidrigkeit der Enteignung einer deutschen Nitratanlage in Oberschlesien durch Polen. Die streitgegenständliche Enteignung verletze Bestimmungen des Deutsch-Polnischen Abkommens über Oberschlesien von 1922. ${ }^{537}$ Diese unterscheide sich von einer völkerrechtlich nicht verbotenen Enteignung, für deren Rechtmäßigkeit lediglich die Zahlung einer gerechten Entschädigung notwendig sei. ${ }^{538}$ Der Gerichtshof führte ferner aus, dass der Staat für rechtswidrige Enteignungen umfassendere Wiedergutmachung zu leisten habe als für rechtmäßige Enteignungen. Demnach besteht im ersten Fall nicht nur ein Anspruch auf Ersatz eingetretener Schäden, sondern auch auf entgangenen Gewinn (lucrum cessans). Im zweiten Fall hingegen, beschränkt sich die Kompensationspflicht auf den Ersatz positiver Schäden, das heißt der Minderung oder Zerstörung vorhandenen Vermögensgutes (damnum emergens). ${ }^{539}$ Diese Unterscheidung zum Umfang der Entschädigungspflicht kommt nach überwiegender Auffassung bei Investitionsstreitigkeiten zum Einsatz. ${ }^{540}$

Der völkerrechtliche Eigentumsschutz bietet Anknüpfungspunkte für eine originäre Haftung von Staaten für grenzüberschreitende Schäden durch völkerrechtmäßige gefährliche Aktivitäten, denn in der Verwirklichung eines Risikos kann auch eine Form der Aneignung fremden Staatsgebietes gesehen werden, die einen Ausgleich für diese Inanspruchnahme in Form eines Bereicherungsausgleiches erfordert. ${ }^{541}$

535 StIGH, Factory at Chorzów (Germany v. Poland), Judgment of 13 September 1928 (Merits), PCIJ Series A, No. 17.

536 S. H. Nikièma, Compensation for Expropriation, International Institute for Sustainable Development, Best Practices Series, March 2013, abrufbar unter: https:/ /www.iisd.org/pdf/2013/best_practice_compensation_expropriation_en.pdf (geprüft am 15.05.2020), S. 2 f.; S. R. Ratner, Compensation for Expropriations in a World of Investment Treaties, AJIL 111 (2017), S. 7 (8, 20).

537 StIGH, Factory at Chorzów (Germany v. Poland), Judgment of 13 September 1928 (Merits), PCIJ Series A, No. 17, S. 46 (unter Bezugnahme auf seine vorangegangene Entscheidung StIGH, Factory at Chorzów (Germany v. Poland), Judgment of 26 July 1927 (Jurisdiction), PCIJ Series A, No. 9, S. 30).

538 Ibid.

539 Id., S. 47, $51 \mathrm{f}$.

540 A. de Nanteuil, Droit international de l'investissement, 2014, S. 347 f.; S. Ripins$\mathrm{ky} / \mathrm{K}$. Williams, Damages in International Investment Law, 2008, S. 65 f., 85 ff.

541 So L. F. E. Goldie, Concepts of strict and absolute liability and the ranking of liability in terms of relative exposure to risk, NYIL 16 (1985), S. 175 (206 ff.). 


\section{Ergänzende Staatenhaftung}

Die ergänzende Staatenhaftung steht selbstständig neben der Einstandspflicht des privaten Betreibers der völkerrechtlich nicht verbotenen Aktivität. Die Schadenssumme entscheidet, ob diese verschuldensunabhängige Einstandspflicht von Staaten greift.

Das Zusammenspiel von privater Betreiberhaftung und ergänzender Staatenhaftung wird besonders im Bereich der Nuklearkonventionen deutlich.

\section{Zivilrechtliche Haftung nach den Nuklearhaftungsabkommen}

Das Pariser Übereinkommen über die Haftung gegenüber Dritten auf dem Gebiet der Kernenergie von 1960 (PÜ) ${ }^{542}$, welches durch das streng akzessorische Brüsseler Zusatzübereinkommen von 1963 (BZÜ) ${ }^{543}$ komple-

542 Pariser Übereinkommen über die Haftung gegenüber Dritten auf dem Gebiet der Kernenergie vom 29. Juli 1960, BGBl. 1975 II S.957, $959 \mathrm{ff}$. Das Pariser Übereinkommen wurde durch die Protokolle aus den Jahren 1964, 1982 und 2004 überabeitet. Die Protokolle aus den Jahren 1964 und 1982 sind bereits in Kraft getreten, Übereinkommen vom 29. Juli 1960 über die Haftung gegenüber Dritten auf dem Gebiet der Kernenergie in der Fassung des Zusatzprotokolls vom 28. Januar 1964 (Bekanntmachung der Neufassung vom 5. Februar 1976, BGBl. 1976 II S. 310, 311 ff.) und des Protokolls vom 16. November 1982 (Bekanntmachung der Neufassung vom 15. Juli 1982, BGBl. 1985 II S. 963, 964 ff.). Das Protokoll aus dem Jahr 2004 (Protokoll zur Änderung des Übereinkommens vom 29. Juli 1960 über die Haftung gegenüber Dritten auf dem Gebiet der Kernenergie in der Fassung des Zusatzprotokolls vom 28. Januar 1964 und des Protokolls vom 16. November 1982, BGBl. 2008 II S. 902, 904 ff.) ist bisher noch nicht in Kraft getreten (Stand: 15.05.2020), siehe: http://www.oe cd-nea.org/law/paris-convention-ratification.html.

543 Brüsseler Zusatzübereinkommen vom 31. Januar 1963 zum Pariser Übereinkommen vom 29. Juli 1960, BGBl. 1975 II S. 957, 992 ff. Das Brüsseler Zusatzübereinkommen wurde durch die Protokolle aus den Jahren 1964, 1982 und 2004 überarbeitet. Die Protokolle aus den Jahren 1964 und 1982 sind bereits in Kraft getreten, Zusatzübereinkommen zum Pariser Übereinkommen vom 31. Januar 1963 in der Fassung des Zusatzprotokolls vom 28. Januar 1964 (Bekanntmachung der Neufassung vom 5. Februar 1976, BGBl. 1976 II S. 310, 318 ff.) und des Protokolls vom 16. November 1982 (Bekanntmachung der Neufassung vom 15. Juli 1982, BGBl. 1985 II S. 963, 970 ff.). Das Protokoll aus dem Jahr 2004 (Protokoll zur Änderung des Zusatzübereinkommens vom 31. Januar 1963 über die Haftung gegenüber Dritten auf dem Gebiet der Kernenergie in der Fassung des Zusatzprotokolls vom 28. Januar 1964 und des Protokolls vom 


\section{Kapitel: Staatenhaftung in der Systematik des Völkerrechts}

mentiert wird, und das Wiener Übereinkommen über die zivilrechtliche Haftung für nukleare Schäden von 1963 (WÜ) ${ }^{544}$ adressieren die Fragen einer internationalen Haftung für atomare Unfälle. Durch das Gemeinsame Protokoll zur Anwendung des Wiener und Pariser Abkommens von $1988(\mathrm{GP})^{545}$ greifen die Bestimmungen der jeweiligen Übereinkommen auch im Vertragsgebiet des jeweils anderen Übereinkommens. Damit wird insbesondere berücksichtigt, dass nukleare Unfälle zu grenzüberschreitenden Schäden führen können. ${ }^{546}$

Die Übereinkommen begründen eine verschuldensunabhängige Haftung der Inhaber ${ }^{547}$ bzw. Betreiber ${ }^{548}$ von Kernkraftwerken für Sach- und Personenschäden, die kausal auf dem nuklearen Ereignis beruhen (Art. 3 lit. a PÜ; Art. II Abs. 1 i.V.m. Art. I Abs. 1 lit. k WÜ). ${ }^{549}$ Der Ersatzanspruch kann ausschließlich gegen den Inhaber bzw. Betreiber des Kernkraftwerkes geltend gemacht werden (Art. 6 lit. a PÜ; Art. II Abs. 5 WÜ). Der Geschädigte muss nachweisen, dass der Schaden durch das nukleare Ereignis verursacht wurde (Art. 3 lit. a PÜ; Art. IV Abs. 1 WÜ), was die Durchsetzung eines Schadensersatzanspruches bei nuklearen Beeinträchtigungen erheblich erschwert. ${ }^{550}$

16. November 1982, BGBl. 2008 II S. 902, 920 ff.) ist bisher noch nicht in Kraft getreten (Stand: 15.05.2020), siehe: http://www.oecd-nea.org/law/brussels-supple mentary-convention.html.

544 Wiener Übereinkommen über die zivilrechtliche Haftung für nukleare Schäden vom 21. Mai 1963, in Kraft seit dem 12. November 1977, BGBl. 2001 II S. 202, $207 \mathrm{ff}$.

545 Gemeinsames Protokoll über die Anwendung des Wiener Übereinkommens und des Pariser Übereinkommens vom 21. September 1988, in Kraft seit dem 27. April 1992, BGBl. 2001 II S. 202, $203 \mathrm{ff}$.

546 Sind bei grenzüberschreitenden Schäden durch nukleare Ereignisse die involvierten Staaten nicht Vertragsstaaten des PÜ, des WÜ oder des GP, werden weder Einstandspflichten nach dem PÜ noch nach dem WÜ begründet.

547 Inhaber einer Kernanlage ist gemäß Art. 1 lit. a sublit. vi PÜ derjenige, der von der zuständigen Behörde als Inhaber einer solchen bezeichnet oder angesehen wird.

548 Betreiber einer Kernanlage ist gemäß Art. 1 lit. c WÜ derjenige, der vom Anlagenstaat als Betreiber einer solchen bezeichnet oder angesehen wird.

549 Staaten haften für Schäden nach diesen Bestimmungen nur dann, wenn sie selbst Inhaber bzw. Betreiber eines Kernkraftwerkes sind. Mit Inkrafttreten des PÜ 2004 wird der ersatzfähige Schaden um Kosten für Maßnahmen zur Wiederherstellung geschädigter Umwelt erweitert (Art. 1 lit. a sublit. vii und viii PÜ 2004).

550 H. Gaßner/G. Buchholz, Atomhaftung in Europa und Deutschland - Defizite und Empfehlungen zur Fortentwicklung, Gutachten im Auftrag der Bundestagsfraktion Bündnis 90/Die Grünen, 14. März 2013, abrufbar unter: 
Die zivilrechtliche Haftung ist in der Höhe begrenzt. Nach dem PÜ haftet der Inhaber des Kernkraftwerks maximal bis zu einem Betrag von 15 Mio. Sonderziehungsrechten (SZR $)^{551}$, wobei es den Vertragsstaaten obliegt, höhere oder niedrigere Höchstgrenzen festzulegen. In jedem Fall muss die Haftung aber mindestens 5 Mio. SZR betragen (Art. 7 lit. b PÜ). ${ }^{552}$ Das WÜ hingegen setzt keine Haftungshöchstgrenze fest und überlässt es den Vertragsstaaten diese zu bestimmten, wobei eine staatlich bestimmte Haftungsbegrenzung mindestens 5 Mio. US-Dollar betragen muss (Art. V Abs. 1 WÜ).

Die Haftung der Inhaber bzw. Betreiber der Kernkraftwerke ist nach anderen Rechtsgrundlagen ausgeschlossen, ${ }^{553}$ so dass die Haftungsbegrenzungen den für eine Gefährdungshaftung maßgeblichen Gedanken des Opferschutzes beeinträchtigen. Denn nukleare Großschäden überschreiten in aller Regel die vorgegebene Haftungshöchstgrenze ${ }^{554}$ und müssen mit-

https://www.zeit-lu-pe.com/mediapool/132/1322391/data/Gutachten_Atomhaft ung_B90_Gruene_Maerz_2013_.pdf (geprüft am 15.05.2020), S. 14.

551 SZR ist die Recheneinheit des Internationalen Währungsfonds, die dieser für seine eigenen Geschäfte und Transaktionen nutzt. Der Wert eines SZR beträgt derzeit 1,26 Euro (Stand: 14.05.2020), siehe: https://www.imf.org/external/np/fi $\mathrm{n} /$ data/rms_five.aspx\#fn1.

552 Mit Inkrafttreten des PÜ 2004 wird die Haftungshöchstgrenze auf mindestens 700 Mio. € festgelegt (Art. 7 lit. a PÜ 2004). Niedrigere Haftungshöchstgrenzen werden nur unter Berücksichtigung der Risiken unterschiedlicher Kernkraftwerke bis zu einem Mindestbetrag von 70 Mio. $€$ zulässig sein (Art. 7 lit. b PÜ 2004).

553 Nach Art. 6 lit. c sublit. ii PÜ ist eine Haftung nach anderen Vorschriften ausgeschlossen. Das WÜ selbst enthält keine Ausschlussregel. Nichtsdestotrotz wird ein Ausschluss der Haftung nach anderen rechtlichen Grundlagen auch für das WÜ angenommen. So H. Gaßner/G. Buchholz, Atomhaftung in Europa und Deutschland - Defizite und Empfehlungen zur Fortentwicklung, Gutachten im Auftrag der Bundestagsfraktion Bündnis 90/Die Grünen, 14. März 2013, abrufbar unter: https://www.zeit-lu-pe.com/mediapool/132/1322391/data/Gutachte n_Atomhaftung_B90_Gruene_Maerz_2013_.pdf (geprüft am 15.05.2020), S.9, 12, 25; M. Hinteregger, Atomhaftung in Europa, in: P. Apathy/R. Bollenberger/P. Bydlinski/G. Iro/E. Karner/M. Karollus (Hg.), Festschrift für Helmut Koziol, 2010, S. 667 (671).

554 Nach H. Gaßner/G. Buchholz, wurde der Schaden durch das Unglück von Fukushima durch den Betreiber Tepco auf etwa $100 \mathrm{Mrd}$. $€$ geschätzt, so dass selbst die angehobene Haftungshöchstgrenze des PÜ 2004 in Höhe von 700 Mio. $€$ in vergleichbaren Fällen nicht einmal $1 \%$ der Schäden abdecken würde. H. Gaßner/G. Buchbolz, Atomhaftung in Europa und Deutschland - Defizite und Empfehlungen zur Fortentwicklung, Gutachten im Auftrag der Bundestagsfraktion Bündnis 90/Die Grünen, 14. März 2013, abrufbar un- 
hin von der Allgemeinheit aufgefangen werden. ${ }^{555}$ Die Übereinkommen - insbesondere das PÜ - begründen damit faktisch eine Staatenhaftung, denn die vorgegebene Haftungshöchstgrenze ist viel zu niedrig bemessen, um der finanziellen Dimension der Schäden durch nukleare Unfälle zu begegnen. Der Unfall im Kernkraftwerk von Tschernobyl aus dem Jahr 1986 verursachte beispielsweise Schäden in Höhe von etwa $372 \mathrm{Mrd}$. US-Dollar. Die negativen Auswirkungen dieses nuklearen Ereignisses verdeutlichten die Notwendigkeit für eine Überarbeitung der Haftungssysteme. ${ }^{556}$

Im Jahre 1997 wurde deswegen das WÜ durch ein Änderungsprotokoll vollständig revidiert. ${ }^{557}$ Es trägt in seiner überarbeiteten Version die Bezeichnung Wiener Übereinkommen über die zivilrechtliche Haftung für nukleare Schäden 1997 (WÜ 1997).558 Die Änderungen durch das Protokoll von 1997 wurden als Vertrag ausgestaltet und sind mithin nur für dessen Vertragsparteien bindend, so dass teilweise die veralteten Haftungsbestimmungen nach dem WÜ aus dem Jahr 1963 fortgelten. Daneben wurden die Haftungssysteme für nukleare Unfälle durch das Übereinkommen über ergänzende Entschädigung bei Nuklearschäden von 1997 (ÜEE) ${ }^{55}$ erweitert. Das WÜ 1997 und das ÜEE normieren progressive Haftungsregelungen. Die Haftungsvorschriften des WÜ 1997 gelangen nun auch bei Schäden auf hoheitsfreien Gebieten oder auf dem Territorium von Nichtvertragsstaaten zur Anwendung (Art. I A Abs. 1 WÜ 1997). Ferner sind nach dem WÜ 1997 und dem ÜEE nunmehr neben

ter: https://www.zeit-lu-pe.com/mediapool/132/1322391/data/Gutachten_Atom haftung_B90_Gruene_Maerz_2013_.pdf (geprüft am 15.05.2020), S. 12 f.

555 J. Diekmann, Verstärkte Haftung und Deckungsvorsorge für Schäden nuklearer Unfälle, ZfU 34 (2001), S. 119 (121, 127).

556 Ibid.

557 Das Zusatzprotokoll zum Wiener Übereinkommen von 1963 wurde am 12. September 1997 angenommen und ist am 4. Oktober 2003 in Kraft getreten, abrufbar unter: https://www.iaea.org/sites/default/files/infcirc566.pdf (geprüft am 15.05.2020).

558 Siehe Art. 18 Nr. 2 Zusatzprotokoll zum Wiener Übereinkommen von 1963. Das Wiener Übereinkommen 1997 erweitert den Anwendungsbereich des Wiener Übereinkommens von 1963. Die Haftungsvorschriften sollen auch dann eingreifen, wenn der Schaden auf hoheitsfreien Gebieten oder auf dem Territorium von Nichtvertragsstaaten eintritt (Art. I A Nr. 1 Wiener Übereinkommen 1997).

559 Übereinkommen über ergänzende Entschädigung bei Nuklearschäden von 1997 wurde am 12. September 1997 angenommen und ist am 15. April 2015 in Kraft getreten, abrufbar unter: https://www.iaea.org/topics/nuclear-liability-conven tions/convention-supplementary-compensation-nuclear-damage (geprüft am 15.05.2020). 
Personen- und Sachschäden auch erhebliche Umweltschäden, Kosten für angemessene Wiederherstellungsmaßnahmen und damit einhergehende wirtschaftliche Schadensposten von den Haftungsbestimmungen erfasst. Von besonderer Bedeutung ist, dass die Übereinkommen eine präventive Schutzrichtung erhalten, da nach dem neuen Schadensbegriff außerdem die Kosten für Maßnahmen zur Schadensvermeidung ersatzfähig sind (Art. I Abs. 1 lit.k sublit. vi WÜ 1997, Art. I lit.f sublit. vi ÜEE). Schließlich wurden die vertraglich vorgegebenen Haftungsbegrenzungen angepasst und Bestimmungen aufgenommen, nach denen die Kompensation von Schäden durch nukleare Ereignisse bis zu 300 Mio. SZR gewährleistet ist (Art. V Abs. 1 WÜ 1997) und auch darüber hinausgehende Schäden aufgefangen werden (Art. III Abs. 1 ÜEE).

\section{Staatliche Haftung nach den Nuklearhaftungsabkommen}

Die Haftungssysteme sehen neben der zivilrechtlichen Haftung des Inhabers bzw. Betreibers des Kernkraftwerkes auch staatliche Einstandspflichten vor, die verschuldensunabhängig und allein von der Schadenssumme abhängig sind.

Das BZÜ von 1963 statuiert neben der zivilrechtlichen Haftung des PÜ, zwei weitere Kompensationsstufen, die staatliche Einstandspflichten begründen. Es handelt sich demnach um ein dreistufiges Haftungssystem für nukleare Schäden, wobei finanzielle Mittel bis zu einem Betrag von 300 Mio. SZR verfügbar sein müssen (Art. 3 lit. a BZÜ). ${ }^{560}$ Zunächst haben die Vertragsstaaten im Wege der nationalen Gesetzgebung Sorge dafür zu tragen, dass der Inhaber des Kernkraftwerks finanzielle Mittel in Höhe von mindestens 5 Mio. SZR bereitstellt. Für Schäden, die diese Einstandspflicht des privaten Anlageninhabers übersteigen, muss der Staat, in dem sich das betreffende Kernkraftwerk befindet (Anlagenstaat), ergänzende Entschädigung durch öffentliche Mittel leisten (Art. 3 lit. c BZÜ). Diese ergänzende Staatenhaftung der Anlagenstaaten ist auf 175 Mio. SZR beschränkt. Schadenssummen, die darüber hinausgehen, müssen schließlich von allen Vertragsstaaten durch zusätzliche staatliche Mittel bis zu

560 Mit Inkrafttreten des Revisionsprotokolles aus dem Jahr 2004 zum PÜ, welches durch das BZÜ komplementiert wird, erhöht sich der Entschädigungsbetrag auf 1,5 Mrd. EUR. Die Grenze der ersten Kompensationsstufe liegt dann bei 700 Mio. EUR, für die zweite Kompensationsstufe bei 500 Mio. EUR und für die dritte Kompensationsstufe bei 300 Mio. EUR. 


\section{Kapitel: Staatenhaftung in der Systematik des Völkerrechts}

300 Mio. SZR aufgefangen werden (Art. 3 lit. a und b BZÜ). Die Beitragshöhe je Vertragsstaat richtet sich nach dem Bruttosozialprodukt und nach der Leistung nuklearer Reaktoren der jeweiligen Staaten (Art.12 BZÜ). Dies führt zu der Besonderheit, dass der tatsächliche Beitrag zur Gefährdungslage für die Hälfte der zu leistenden staatlichen Mittel völlig unerheblich ist. So werden sogar Vertragsstaaten, die eine Nutzung von Kernenergie aufgrund der damit verbundenen Risiken auf ihrem Hoheitsgebiet untersagen, zu Entschädigungsleistungen verpflichtet. ${ }^{561}$ Das BZÜ begründet mithin eine solidarische ergänzende Staatenhaftung. Die Verpflichtung zur Bereitstellung von Mitteln gilt allerdings nur für Schäden, die im Hoheitsgebiet eines Vertragsstaates entstanden sind (Art. 3 lit.c i.V.m. Art. 2 lit. a BZÜ). 562

Auch das WÜ 1997 hat das Element einer ergänzenden Staatenhaftung in das neue Haftungssystem integriert. Die Vertragsstaaten haben die Möglichkeit, die Haftung des privaten Kernkraftwerkbetreibers auf 150 Mio. SZR (Art. V Abs. 1 WÜ 1997) und für Kernkraftwerke mit geringerem Risikopotenzial auf 5 Mio. SZR (Art. V Abs. 2 WÜ 1997) zu begrenzen. Allerdings nur unter der Voraussetzung, dass der Anlagenstaat öffentliche Mittel zur Kompensation von Schäden, die darüber hinausgehen, bis zu einem Betrag von 300 Mio. SZR zur Verfügung stellt. Diese zusätzlichen öffentlichen Mittel decken nicht die Haftung des Betreibers, sondern begründen eine selbstständige staatliche Einstandspflicht. ${ }^{563}$

Schließlich verpflichtet das ÜEE die Vertragsstaaten, Schäden, die den Betrag von 300 Mio. SZR überschreiten, durch gemeinsame Mittel zu kompensieren (Art. III Abs. 1 ÜEE). Dieses zwischenstaatliche kollektive

561 H. Gaßner/G. Buchholz, Atomhaftung in Europa und Deutschland - Defizite und Empfehlungen zur Fortentwicklung, Gutachten im Auftrag der Bundestagsfraktion Bündnis 90/Die Grünen, 14. März 2013, abrufbar unter: https://ww w.zeit-lu-pe.com/mediapool/132/1322391/data/Gutachten_Atomhaftung_B90_G ruene_Maerz_2013_.pdf (geprüft am 15.05.2020), S. 59.

562 Siehe auch $\bar{M}$. Hinteregger, Atomhaftung in Europa, in: P. Apathy/R. Bollenberger/P. Bydlinski/G. Iro/E. Karner/M. Karollus (Hg.), Festschrift für Helmut Koziol, 2010, S. 667 (673).

563 International Atomic Energy Agency, International Expert Group on Nuclear Liability, The 1997 Vienna Convention on Civil Liability for Nuclear Damage and the 1997 Convention on Supplementary Compensation for Nuclear Damage - Explanatory Texts, IAEA International Law Series No. 3 (Revised), 2017, S. 44 . 
Finanzierungssystem ${ }^{564}$ funktioniert nach einem vorgegebenen Verteilerschlüssel, der sich an dem Ausmaß der Kernenergienutzung durch die jeweiligen Staaten orientiert (Art. III Abs. 1 lit. b i.V.m. Art. IV ÜEE), und begründet damit ebenfalls eine ergänzende Staatenhaftung aller Vertragsstaaten.

An dieser Stelle ist hervorzuheben, dass die Übereinkommen die Existenz des Konzepts der Staatenhaftung im allgemeinen Völkerrecht nicht in Abrede stellen, denn die Bestimmungen der Übereinkommen „shall not affect the rights and obligations of a Contracting Party under the general rules of public international law" (Art. XVIII WÜ 1997; Art. XV ÜEE). 565

Auch wenn die Bestimmungen des internationalen Haftungssystems für atomare Unfälle die Haftung auf den Inhaber bzw. Betreiber einer Kernkraftanlage konzentrieren, sind Elemente einer verschuldensunabhängigen Staatenhaftung für Schäden, die aus der Nutzung von Kernkraftwerken resultieren, vorgesehen.

\section{Subsidiäre Staatenhaftung}

Im Rahmen von konventionsrechtlichen Haftungssystemen finden sich auch Vorgaben für eine subsidiäre Einstandspflicht des Ursprungsstaates, sofern der Betreiber der schadensauslösenden Aktivität die finanziellen Mittel zur Deckung der Haftungssumme nicht aufbringt. ${ }^{566}$ Auch in diesem Zusammenhang sei darauf hingewiesen, dass sich diese subsidiäre Staatenhaftung von der Staatenverantwortlichkeit für die Verletzung vertraglicher Pflichten unterscheidet. Die subsidiäre staatliche Haftung, die bei Ausfall der Haftung des primär Verpflichteten greift, stellt eine Haftung sine delicto dar. Nur wenn der Staat der vertraglich vorgegebenen Ausfallhaftung nicht nachkommt, folgt aufgrund der Vertragsverletzung eine Haftung ex delicto.

564 In vielen zivilrechtlich ausgestalteten Haftungsübereinkommen findet sich ein Finanzierungssystem, welches von den betroffenen Industriegruppen zu tragen ist und eine staatliche Haftung komplett ausklammert.

565 Vgl. International Atomic Energy Agency, International Expert Group on $\mathrm{Nu}$ clear Liability, The 1997 Vienna Convention on Civil Liability for Nuclear Damage and the 1997 Convention on Supplementary Compensation for Nuclear Damage - Explanatory Texts, IAEA International Law Series No. 3 (Revised), 2017, S. $24 \mathrm{f}$.

566 R. Lefeber, Transboundary Environmental Interference and the Origin of State Liability, 1996, S. 301. 


\section{Kapitel: Staatenhaftung in der Systematik des Völkerrechts}

1. Subsidiäre Haftung nach den Nuklearhaftungsabkommen

Die Haftungssysteme für atomare Unfälle beinhalten auch die Pflicht der Inhaber bzw. Betreiber eines Kernkraftwerks, eine Versicherung abzuschließen oder sonstige finanzielle Sicherheit vorzuhalten, um den Schadensersatzverpflichtungen im Schadensfall nachkommen zu können.

Nach dem PÜ sind Inhaber von Kernkraftwerken gehalten, eine Deckungsvorsorge einzugehen und aufrechtzuerhalten, die der Höhe des jeweiligen Haftungshöchstbetrages der Inhaber des Kernkraftwerkes in Höhe von mindestens 5 Mio. SZR entspricht (Art. 10 lit. a i.V.m. Art. 7 PÜ). Das BZÜ unterstreicht, dass die Vertragsstaaten durch ihre nationale Gesetzgebung sicherstellen müssen, dass der Inhaber des Kernkraftwerkes eine der festgelegten Haftungsbegrenzung entsprechende Deckungsvorsorge vorhält (Art. 3 lit. b sublit. i BZÜ). Im Rahmen der Vorbesprechungen zum PÜ diskutierten die Verhandlungspartner, ob Staaten eine Einstandspflicht treffen soll, wenn die Entschädigung durch die privaten Betreiber gänzlich oder teilweise ausbleibt. Im Ergebnis wurde die Aufnahme einer subsidiären Haftung der Staaten jedoch abgelehnt. ${ }^{567}$ Das Haftungssystem regelt mit Blick auf staatliche Einstandspflichten demnach nur die ergänzende Staatenhaftung nach dem BZÜ.

Das WÜ und das WÜ 1997 hingegen überlassen es zum einen den Vertragsstaaten, den Umfang der Deckungsvorsorge im Rahmen nationaler Regelungen vorzugeben, so dass die nationalen Bestimmungen zum Betrag der Deckungsvorsorge nicht mit der für die zivilrechtliche Haftung des Betreibers festgelegten Haftungshöchstgrenze übereinstimmen müssen, ${ }^{568}$ und zum anderen verpflichten sie die Anlagenstaaten, Schadenskompensation bis zu der festgelegten Haftungshöchstgrenze durch öffentliche Mittel sicherzustellen. Demnach trifft den Anlagenstaat eine subsidiäre Haftung, wenn der private Betreiber nicht verpflichtet oder unfähig ist, seinen Schadensersatzpflichten bis zur Haftungshöchstgrenze nachzukommen (Art. VII Abs. 1 WÜ, Art. VII Abs. 1 WÜ 1997). Damit be-

567 S. Förster, Internationale Haftungsregeln für schädliche Folgewirkungen gentechnisch veränderter Organismen, 2007, S. 103.

568 Bei einer unbegrenzten Haftung muss die Deckungsvorsorge des haftenden Inhabers des Kernkraftwerkes nach dem WÜ 1997 aber mindestens 300 Mio. SZR betragen. Wenn die finanzielle Vorsorge nicht ausreicht, muss der Anlagenstaat bis zu dieser Höhe eintreten (Art. VII Abs. 1 lit. a WÜ 1997). 
gründen die Übereinkommen einer verschuldensunabhängige subsidiäre Staatenhaftung. ${ }^{569}$

\section{Subsidiäre Haftung im antarktischen Haftungssystem}

Das Übereinkommen zur Regelung der Tätigkeiten im Zusammenhang mit mineralischen Ressourcen der Antarktis (CRAMRA) ${ }^{570}$ aus dem Jahr 1988 bestimmt Reaktionsmaßnahmen, die zur Prävention, Eindämmung und Beseitigung von Schäden an der Antarktis und damit verbundenen Ökosystemen zu ergreifen sind (Art. 8 Abs. 1 CRAMRA). Außerdem enthält das Übereinkommen Haftungsregeln für Schäden, die durch die Erschließung, Erforschung und das Aufsuchen mineralischer Ressourcen in der Antarktis entstehen. Art. 8 Abs. 2 CRAMRA begründet eine verschuldensunabhängige Haftung der Betreiber von derartigen Tätigkeiten. Daneben trifft den befürwortenden Vertragsstaat ${ }^{571}$ nach Art. 8 Abs. 3 lit. a CRAMRA eine Einstandspflicht für Schäden, die durch befürwortete Betreiber verursacht werden. Der Haftungsstandard ist mit Art. 139 SRÜ vergleichbar, der eine verschuldensabhängige Haftung begründet. Auch im Rahmen von Art. 8 Abs. 3 lit. a CRAMRA haftet der befürwortende Staat nur, wenn er seinen vertraglichen Pflichten bezüglich der von ihm

569 In der Formulierung aus Art. VII Abs. 1 WÜ „The Installation State shall ensure the payment of claims for compensation (...) established against the operator" erkennt $R$. Lefeber eine absolute Pflicht der Staaten, sicherzustellen, dass Ansprüche für nukleare Schäden durch den privaten Betreiber beglichen werden. Sofern der Betreiber die Haftungssumme nicht aufbringt, trifft den Staat nach $R$. Lefeber eine Haftung ex delicto, da er seiner vertraglichen Überwachungspflicht nicht nachgekommen sei. Dieser Staatenverantwortlichkeit könne der Anlagenstaat durch das Bereitstellen finanzieller Mittel zur Deckung der Haftungshöchstgrenze entgehen. R. Lefeber, Transboundary Environmental Interference and the Origin of State Liability, 1996. S. 302 f. Eine umgekehrte Betrachtung ist hier überzeugender: Erst wenn der Staat seiner Haftung sine delicto, das heißt der Pflicht zur Sicherstellung der Kompensationszahlung „by providing the necessary funds“ (Art VII Abs. 1 WÜ, Hervorhebung hinzugefügt) nicht nachkommt, greift eine ex delicto Haftung für die Verletzung vertraglichen Pflichten des Staates.

570 Übereinkommen zur Regelung der Tätigkeiten im Zusammenhang mit mineralischen Ressourcen der Antarktis vom 2. Juni 1988, ILM 27 (1988), S. 859 (nicht in Kraft getreten).

571 Art. 1 Abs. 12 CRAMRA definiert die tatsächliche und wesentliche Verbindung, welche zwischen einem Vertragsstaat und dem Betreiber bestehen muss, damit der Vertragsstaat als „befürwortender Staat“ qualifiziert werden kann. 


\section{Kapitel: Staatenhaftung in der Systematik des Völkerrechts}

befürworteten Betreiber nicht nachgekommen ist und der Schaden kausal auf dieser Pflichtverletzung beruht. Es handelt sich demnach um eine verschuldensabhängige Staatenhaftung. ${ }^{572} \mathrm{Zudem}$ greift die verschuldensabhängige Haftung aber nur insoweit, als der nach Art. 8 Abs. 2 CRAMRA primär haftende befürwortete Betreiber seiner Schadensersatzpflicht nicht oder nur teilweise nachgekommen ist. CRAMRA begründet damit eine subsidiäre verschuldensabhängige Staatenhaftung. ${ }^{573}$ Das Übereinkommen wurde zwar von einem Großteil der damaligen Parteien unterzeichnet, allerdings ist es niemals in Kraft getreten. Es wurde faktisch im Jahr 2005 durch Anlage VI zum Umweltschutzprotokoll zum Antarktisvertrag aus dem Jahr 1991 ersetzt. Dennoch kommt CRAMRA eine Vorbildfunktion für die Weiterentwicklung von umweltvölkerrechtlichen Haftungsregeln $\mathrm{zu}^{574}$

Außerdem finden sich in Anlage VI zum USP Bestimmungen, die eine Art Ausfallhaftung vorsehen. Die Anlage VI bestimmt Haftungshöchstgrenzen (Art. 9 Anlage VI) und verpflichtet die Betreiber, ausreichende finanzielle Mittel vorzuhalten (Art. 11 Anlage VI). Eine verschuldensunabhängige subsidiäre Einstandspflicht von Staaten für Fälle, in denen nicht-staatliche Betreiber zahlungsunfähig oder -unwillig sind, greift zwar nicht. Allerdings ist die Einrichtung eines Haftungsfonds vorgesehen, um unter anderem Staaten die Kosten einer Ersatzvornahme im Sinne des Art. 5 Abs. 2 Anlage VI zu erstatten (Art. 12 Abs. 1 Anlage VI) und mithin die Vornahme von Gegenmaßnahmen bei umweltgefährdenden Notfällen durch (unbeteiligte) Staaten zu fördern. Für die Genehmigung einer Kostenerstattung durch den Fonds sind bestimmte Faktoren zu berücksichtigen. So greift der Fonds bei Ausfall der finanziellen Sicherung durch den Betreiber oder der Nichtidentifizierbarkeit der Betreiber. ${ }^{575}$ In die Genehmigungserwägungen ist ferner einzubeziehen, ob der Antrag auf Kostenerstattung durch einen Vertragsstaat erfolgt, dessen Betreiber den Schaden verursacht hat (Art. 12 Abs. 3 Anlage VI). Dies impliziert wiederum, dass Staaten für Schäden durch „ihre Betreiber“ in gewisser Weise einstehen sollen. Die Finanzierung des Fonds soll durch freiwillige Beiträ-

572 Nach R. Lefeber hingegen begründe Art. 8 Abs. 3 lit. a CRAMRA eine ex delicto Haftung. R. Lefeber, Transboundary Environmental Interference and the Origin of State Liability, 1996, S. 302.

573 K. Schmalenbach, Verantwortlichkeit und Haftung, in: A. Proelß (Hg.), Internationales Umweltrecht, 2017, S. 211 (232 f. Rn. 36).

574 P. Sands/J. Peel, Principles of International Environmental Law, 2018, S. $637 \mathrm{f}$.

575 S. Vöneky/S. Addison-Agyei, Antarctica, in: R. Wolfrum (Hg.), MPEPIL 2011, http://www.mpepil.com, Rn. 86. 
ge von Staaten oder Personen erfolgen (Art. 12 Abs. 4 Anlage VI). Es ist zweifelhaft, ob eine derartige Obliegenheit für Haftungsfälle ausreicht und ob das antarktische Haftungssystem im Gesamten überhaupt zur Anwendung gelangen, geschweige denn ausgeweitet werden wird. ${ }^{576}$ Bis dahin müssen jedenfalls andere Haftungsmechanismen zum Tragen kommen, wenn Schäden in der Antarktis vermieden, gemindert oder kompensiert werden sollen.

IV. Modellcharakter zivilrechtlicher Haftungssysteme

Haftungsübereinkommen konzentrieren sich zunehmend auf eine zivilrechtliche Haftung, so dass die originäre völkerrechtliche Staatenhaftung aus dem Blickfeld gerät. Gleichwohl lässt sich diesen Haftungssystemen ein Muster entnehmen, welches als Modell für das Konzept der Staatenhaftung fungieren kann. Die meisten Haftungsübereinkommen begründen Präventions- und Wiedergutmachungspflichten für Schäden durch besonders gefährliche Aktivitäten (1.). Sie konstituieren eine verschuldensunabhängige Haftung (2.), die in der Regel den privaten Schadensverursacher trifft und durch zusätzliche kollektive Kompensationsmechanismen ergänzt wird (3.).

1. Kausaler Schaden durch besonders gefährliche Aktivität

Die Haftungsübereinkommen reglementieren grundsätzlich besonders gefährliche Aktivitäten. ${ }^{577}$ Die Notwendigkeit für völkervertraglich vorgegebene Wiedergutmachungspflichten trotz geringer Wahrscheinlichkeit eines Schadenseintritts ist durch das Risiko katastrophaler Schadensfolgen aufgrund bestimmter Aktivitäten begründet. ${ }^{578}$ Die Haftungsübereinkommen fassen unter die Schäden stets Personen- und Sachschäden und zumeist auch Vermögenseinbußen und Einkommensverluste sowie die Kosten für Präventions- und Wiederherstellungsmaßnahmen bei Umwelt-

576 A. D. Hemmings, Liability postponed, The Polar Journal 8 (2018), S. 315 (330).

577 P. Sands/J. Peel, Principles of International Environmental Law, 2018, S. 771.

578 C. W. Jenks, Liability for Ultra-Hazardous Activities in International Law, RdC 117 (1966-1), S. 99 (107). 
schäden. ${ }^{579}$ Die Haftung greift grundsätzlich nur, sofern eine kausale Verbindung zwischen dem reglementierten Verhalten und dem Schaden besteht. ${ }^{580}$

\section{Haftungsstandard}

Die Haftungsübereinkommen etablieren grundsätzlich eine beschränkte ${ }^{581}$ verschuldensunabhängige Haftung. ${ }^{582}$ Die Abkommen reflektieren damit die wachsende Bedeutung, die dem Risikopotenzial einer Aktivität als Auslöser einer Haftung zukommt, und gleichzeitig die schwindende Rolle der gebotenen Sorgfalt bei der Schadensvermeidung für die Begründung von Wiedergutmachungspflichten in grenzüberschreitenden Schadensfällen. ${ }^{583}$

Verschulden spielt im Rahmen der Haftungssysteme allenfalls mit Blick auf den Umfang der Kompensationspflicht eine Rolle. Dies entspricht dem Konzept der Haftung für völkerrechtlich nicht verbotene Aktivitäten, bei dem die Missachtung der gebotenen Sorgfalt im Rahmen der Schadensvermeidungspflichten lediglich durch eine umfassende Kompensationspflicht sanktioniert wird. Einige Übereinkommen konstruieren ein duales Haftungssystem, wonach eine begrenzte Kompensationspflicht folgt, wenn der Schaden ohne Verschulden des Haftenden eintritt, und eine unbegrenzte Kompensationspflicht greift, wenn der Schaden auf einem Verschulden des Haftenden basiert. ${ }^{584}$ Daneben sehen einige Systeme eine ver-

579 Siehe B. Saxler/J. Siegfried/A. Proelss, International liability for transboundary damage arising from stratospheric aerosol injections, Law, Innovation and Technology 7 (2015), S. 112 (141 f.) m.w.N.

$580 \mathrm{~J}$. Barboza, The Environment, Risk and Liability in International Law, 2011, S. 33 .

581 Die Ausnahme- und Befreiungstatbestände im Rahmen der beschränkten Haftung sind begrenzter als im Rahmen der Staatenverantwortlichkeit, denn "they are not causes of exemptions of wrongfulness, since liability is not based on wrongful acts, but simple exceptions consequent to certain conducts precluding liability." So id., S. 40, Fn. 31.

582 A. Douhan, Liability for Environmental Damage, in: R. Wolfrum (Hg.), MPEPIL 2013, http://www.mpepil.com, Rn. $20 \mathrm{ff}$.

583 J. Barboza, The Environment, Risk and Liability in International Law, 2011, S. 35 .

584 Siehe zum Beispiel Art. V Abs. 1 und 2 Internationales Übereinkommen von 1992 über die zivilrechtliche Haftung für Ölverschmutzungsschäden vom 27. November 1992 (Ölhaftungsübereinkommen 1992, Protokoll zur Änderung des Internationalen Übereinkommens von 1969, BGBl. 1994 II S. 1150, 1152 ff., Bekanntmachung der Neufassung des Internationalen Übereinkommens von 
schuldensunabhängige Einstandspflicht des Betreibers und zugleich eine Einstandspflicht von anderen Parteien, die den Schaden durch Fehlverhalten verursacht oder zum Schadenseintritt beigetragen haben, vor. Auch diese Systeme etablieren zwei Haftungsstandards, so dass der Umfang der verschuldensbasierten Einstandspflicht unbegrenzt ist, während die Kompensationspflicht ohne Verschulden nur in begrenztem Umfang zu erfolgen hat. ${ }^{585}$ Damit stellen die Übereinkommen eine Verbindung zwischen den normierten Präventionspflichten und den Wiedergutmachungspflichten her.

\section{Haftungsträger}

Die Haftung trifft zunächst den privaten Schadensverursacher. Allerdings greifen zusätzlich Entschädigungsfonds zur Deckung von Schäden, die nicht mehr vom Schadensverursacher zu tragen sind, und auch dann, wenn es zu Nachweisproblemen kommt. Die kollektiven Entschädigungsmechanismen ermöglichen damit einen umfassenderen Opferschutz.

1969, BGBl. 1996 II S. 670, 671 ff., geändert durch Verordnung vom 22. März 2002, BGBl. 2002 II S. 943, 944 ff.); Art. 9 Abs. 1 und 2 und Art. 10 Abs. 1 Übereinkommen über die zivilrechtliche Haftung für Schäden bei der Beförderung gefährlicher Güter auf der Straße, auf der Schiene und mit Binnenschiffen (CRTD 1989, ECE/TRANS/79, noch nicht in Kraft getreten); Art. 9 Abs. 1 und 2 Internationales Übereinkommen über Haftung und Entschädigung für Schäden bei der Beförderung schädlicher und gefährlicher Stoffe auf See (HNS-Übereinkommen 2010, noch nicht in Kraft getreten, abrufbar unter: https://www.hnsco nvention.org/wp-content/uploads/2018/08/2010-HNS-Convention-Consolidated -text_e.pdf).

585 Siehe zum Beispiel Art. 4 und Art. 5 i.V.m. Art. 12 Abs. 1 und 2 Basler Protokoll zur Haftung und Entschädigung für Schäden durch grenzüberschreitende Verbringung gefährlicher Abfälle und ihrer Entsorgung angenommen am 10. Dezember 1999 (Basler Protokoll 1999, UN Doc. UNEP/CHW.1/WG/1/9/2, noch nicht in Kraft getreten); Art. 4 und Art. 5 i.V.m. Art. 9 Abs. 1 und 3 Protokoll zur zivilrechtlichen Haftung und zum Ersatz bei Schäden, die durch grenzüberschreitende Auswirkungen von Industrieunfällen auf grenzüberschreitende Gewässer verursacht sind vom 21. Mai 2003 (Kiew Protokoll 2003, UN Doc. ECE/ MP.WAT/11-ECE/CP.TEIA/9., noch nicht in Kraft getreten). 
a) Schadensverursacher

Die Übereinkommen „kanalisieren“ die Haftung grundsätzlich auf den privaten Schadensverursacher, das heißt denjenigen, der Kontrolle über die Aktivität zum Zeitpunkt des schädigenden Ereignisses ausübt. Das ist nach den Haftungsübereinkommen der private Betreiber bzw. die Person, die im öffentlichen Register des Ursprungsstaates als Eigentümer (der Anlage oder des Schiffes) eingetragen ist und, sofern kein solches Register vorhanden ist, der Besitzer. ${ }^{586}$ Zudem normieren einige Übereinkommen, eine gesamtschuldnerische Haftung oder eine sukzessive Haftung der am Betrieb beteiligten Personen. ${ }^{587}$

\section{b) Entschädigungsfonds bei Überschreiten der Haftungshöchstgrenze}

Die Übereinkommen sehen in aller Regel Haftungshöchstgrenzen vor. ${ }^{588}$ Damit ist keine vollständige Entschädigung für Geschädigte, wie es üblicherweise im Rahmen von verantwortlichkeitsbegründenden Tatbeständen vorgesehen ist, garantiert. Allerdings sorgen zusätzliche kollektive Kompensationsmechanismen zur Deckung von darüber hinausgehenden Schäden für einen Interessenausgleich zwischen den Vorteilen, die der Aktivität entspringen, und den möglichen Schäden. ${ }^{589}$ Die Bedeutung der Kanalisierung der Haftung auf den Schadensverursacher wird demnach

586 Siehe zum Beispiel Art. III Abs. 1 i.V.m. Art. I Abs. 3 Ölhaftungsübereinkommen 1992, wonach auch bei Staatsschiffen in jedem Fall die als Ausrüster registrierte Gesellschaft haftet; ebenso Art. 7 Abs. 1 i.V.m. Art. 1 Abs. 3 HNSÜbereinkommen 2010; siehe auch Art. 5 i.V.m. Art. 1 Abs. 8 CRTD 1989; Art. 6 Abs. 1 und Art. 7 Abs. 1 Europäisches Übereinkommen über die zivilrechtliche Haftung für Schäden durch umweltgefährdende Tätigkeiten vom 21. Juni 1993 (Lugano Übereinkommen 1993, ILM 32 (1993), S. 1228 ff., nicht in Kraft getreten).

587 Siehe zum Beispiel Art. 3 Abs. 1 und 2 i.V.m. Art. 1 Nr. 3 Internationales Übereinkommen über die zivilrechtliche Haftung für Schäden durch Bunkerölverschmutzung vom 23. März 2001 (Bunkeröl-Übereinkommen 2001, BGBl. 2006 II S. 578, 579 ff.); Art. 6 Lugano Übereinkommen 1993; Art. 4 Abs. 1 Basler Protokoll 1999.

588 Siehe zum Beispiel Art. V Abs. 1 Ölhaftungsübereinkommen 1992; Art. 9 Abs. 1 HNS-Übereinkommen 2010; Art. 9 CRTD 1989; Art. 12 Abs. 1 i.V.m. Annex B Basler Protokoll 1999; Art. 9 i.V.m. Annex II Kiew Protokoll 2003.

589 J. Barboza, The Environment, Risk and Liability in International Law, 2011, S.36. Auch die Ausnahme- und Befreiungstatbestände im Rahmen der beschränkten Betreiberhaftung führen unweigerlich zu Haftungslücken, so dass 
durch den Umstand relativiert, dass diese Haftungssysteme nur funktionieren, weil sie durch weitere Haftungsstufen ergänzt werden, in denen zumeist die betroffenen Industrien und teilweise auch Staaten einspringen müssen, um Schäden zu kompensieren. Ein prägnantes Beispiel bildet das Internationale Übereinkommen von 1992 über die zivilrechtliche Haftung für Ölverschmutzungsschäden (Ölhaftungsübereinkommen 1992) ${ }^{590}$, dessen Vertragsstaaten zugleich Vertragsparteien des Internationalen Übereinkommens von 1992 über die Errichtung eines Internationalen Fonds zur Entschädigung für Ölverschmutzungsschäden (Fondsübereinkommen 1992) $)^{591}$ sind. Der Fonds 1992 wurde errichtet, um Opfer von Ölverschmutzungsschäden zu entschädigen, soweit sich die Entschädigung nach dem Ölhaftungsübereinkommen 1992 als inadäquat erweist. Darüber hinaus wurde im Jahr 2003 der Internationale Zusatzentschädigungsfonds von 2003 für Ölverschmutzungsschäden (Zusatzfonds 2003) ) $^{592}$ eingerichtet, um zusätzliche Mittel für die Entschädigung bereitzustellen. Ausweislich der Präambel ist es erklärtes Ziel, mit dem Zusatzfonds die Schwierigkeiten für die Betroffenen in denjenigen Fällen zu mildern, in denen die Gefahr besteht, dass der nach dem Ölhaftungsübereinkommen 1992 und dem Fondsübereinkommen 1992 verfügbare Entschädigungsbetrag nicht ausreicht, um festgestellte Ansprüche in voller Höhe zu befriedigen. Bisher sind allerdings nur wenige Vertragsstaaten dem Zusatzfonds 2003 beigetreten. Die Fonds werden gespeist aus Pflichtbeiträgen der Empfänger von zur See transportiertem Rohöl, gestaffelt nach der Menge des Lieferumfangs (Art. 10 Fondsübereinkommen 1992, Art. 10 Zusatzfonds 2003). Zusätzlich können Staaten die Beitragspflicht von denjenigen, die Öl in deren Hoheitsgebiet empfangen haben, freiwillig übernehmen (Art. 14 Abs. 1 Fondsübereinkommen 1992). Für den Zusatzfonds 2003 sind außerdem staatliche Beiträge vorgesehen, wenn die Beiträge der Schiffseigner eines

die Geschädigten in bestimmten Konstellationen die Schadenslast tragen, sofern kein kollektiver Entschädigungsmechanismus greift.

590 Protokoll zur Änderung des Internationalen Übereinkommens von 1969 BGBl. 1994 II S. 1150, 1152 ff.; Bekanntmachung der Neufassung des Internationalen Übereinkommens von 1969 BGBl. 1996 II S. 670, 671 ff. geändert durch Verordnung vom 22. März 2002 BGBl. 2002 II S. 943, $944 \mathrm{ff.}$

591 Protokoll zur Änderung des Internationalen Übereinkommens von 1971 BGBl. 1994 II S. 1150, 1169 ff.; Bekanntmachung der Neufassung des Internationalen Übereinkommens von 1971 BGBl. 1996 II S. 685, 686 ff. geändert durch Verordnung vom 22. März 2002 BGBl. 2002 II S. 943, 947 ff.

592 Protokoll von 2003 zum Internationalen Übereinkommen von 1992 über die Errichtung eines Internationalen Fonds zur Entschädigung für Ölverschmutzungsschäden BGBl. 2004 II S. 1290, $1291 \mathrm{ff}$. 
Staates nicht die Beitragshöhe für 1 Mio. Tonnen Öl erreichen (Art. 14 Abs. 2 Zusatzfonds 2003). Nach dem Vorbild des Ölhaftungsübereinkommens 1992 und des Fondsübereinkommens 1992 sieht auch das Internationale Übereinkommen über Haftung und Entschädigung für Schäden bei der Beförderung schädlicher und gefährlicher Stoffe auf See (HNS-Übereinkommen 2010) die Errichtung eines Fonds vor (Art. 13 ff. HNS-Übereinkommen 2010), der von den Empfängern von HNS-Fracht finanziert wird (Art. $16 \mathrm{ff}$. HNS-Übereinkommen 2010). ${ }^{593}$ Auch hier können die Staaten die Beitragsleistungen übernehmen (Art. 23 Abs. 1 HNS-Übereinkommen 2010).

\section{c) Entschädigungsfonds bei Nachweisproblemen}

Probleme ergeben sich des Öfteren beim Nachweis von Ursache und Wirkung umweltrelevanter Tätigkeiten. Komplexe Verursachungsstrukturen, Summierungseffekte und Langzeitschäden erschweren die Beweislast des Opfers. ${ }^{594}$ Zivilrechtliche Haftungssysteme lösen diese Nachweisprobleme zum einen durch laxere Nachweisvoraussetzungen oder durch eine Beweislastumkehr zulasten des Betreibers, der von der gefährlichen Aktivität profitiert, oder des Entscheidungsträgers, der die gefährliche Aktivität akzeptiert. ${ }^{595}$ Zum anderen greifen Entschädigungsfonds, wenn der Schadensverursacher nicht festgestellt werden kann. ${ }^{596}$ Auch in diesem Zusammenhang veranschaulicht das Haftungssystem für Ölverschmutzungsschäden die Funktion von Entschädigungsfonds. Art. 4 Abs. 1 lit. b Fondsübereinkommen 1992 besagt, dass der Fonds 1992 auch dann greift, wenn es

593 Vgl. auch das Finanzierungssystem nach Art. III Abs. 1 lit. b i.V.m. Art. IV ÜEE für Nuklearschäden, welches sich an dem Ausmaß der Kernenergienutzung durch die jeweiligen Staaten orientiert.

594 European Commission, Green paper on remedying environmental damage, Document COM (93) 47 final, Brussels, 14 May 1993, abrufbar unter: http://aei.pitt .edu/950/ (geprüft am 15.05.2020), S. 10 f., Ziffer 2.1.8.; J. Barboza, The Environment, Risk and Liability in International Law, 2011, S. 33 ff.; A. Epiney, Das „Verbot erheblicher grenzüberschreitender Umweltbeeinträchtigungen“, AVR 33 (1995), S. 309 (313) m.w.N.

595 Vgl. Art. 3 PÜ; Art. VI Abs. 4 WÜ; Art. 6 Abs. 3 und Art. 11 Lugano Übereinkommen 1993; Art. IV Abs. 2 Weltraumhaftungsübereinkommen; International Oil Pollution Compensation Funds, Claims Manual 2019 Edition, Rn. 1.5.1; J. Barboza, The Environment, Risk and Liability in International Law, 2011, S. 35.

596 S. Förster, Internationale Haftungsregeln für schädliche Folgewirkungen gentechnisch veränderter Organismen, 2007, S. 366. 
dem Geschädigten, nachdem er alle zumutbaren Maßnahmen im Hinblick auf die ihm zur Verfügung stehenden Rechtsbehelfe getroffen hat, nicht möglich war, den vollen ihm nach dem Ölhaftungsübereinkommen 1992 zustehenden Entschädigungsbetrag zu erlangen. Eine Entschädigung ist etwa dann nicht erhältlich, wenn es dem Geschädigten nicht möglich ist, das spezifische Schiff, von dem das Öl ausgetreten ist oder abgelassen wurde, zu ermitteln. In diesem Sinne ist gemäß Art. 4 Abs. 2 lit. b Fondsübereinkommen 1992 eine Entschädigung aus dem Fonds 1992 nur dann ausgeschlossen, wenn nicht nachgewiesen wird, dass ein Schadensereignis durch geladenes Öl eines Schiffes verursacht worden ist. Eine Verbindung zu einem bestimmten Schiff muss hingegen nicht nachgewiesen werden. 597 Das HNS-Übereinkommen 2010 enthält in Art. 14 Abs. 1 lit. b und Abs. 3 lit. b. gleichlautende Bestimmungen für Schäden durch HNS-Fracht. ${ }^{598}$

Schließlich bestehen auch auf europäischer Ebene Bestrebungen, Umweltschäden durch Fonds aufzufangen. Das EU-Parlament fordert in seiner Entschließung zur Durchführung der Umwelthaftungsrichtlinie 2004/35/EG die EU-Kommission auf, „die Möglichkeit der Einrichtung eines europäischen Fonds zum Schutz der Umwelt vor Schäden, die durch unter die Umwelthaftungsrichtlinie fallende industrielle Tätigkeiten verursacht werden, zu untersuchen“. Es betont dabei, dass „durch diesen Fonds jedoch nicht das Verursacherprinzip untergraben werden darf, sodass er für das Insolvenzrisiko und nur in Fällen, in denen die Märkte für Deckungsvorsorge versagen, greifen sollte“. Das EU-Parlament bekräftigt außerdem die Auffassung, „dass dasselbe auch für schwere Unfälle gelten sollte, in denen der für die Schäden verantwortliche Betreiber nicht ermittelt werden kann." ${ }^{\text {" } 99}$

597 Ibid.

598 Wie unter D. III. 2. gezeigt, sieht die Anlage VI des Antarktisumweltschutzprotokolls über die Haftung bei umweltgefährdenden Notfällen ebenfalls die Errichtung eines Haftungsfonds vor, der gemäß Art. 12 Abs. 3 Anlage VI auch dann greifen soll, wenn der für den umweltgefährdenden Notfall verantwortliche Betreiber nicht identifiziert werden kann. Vgl. auch zur Idee eines „seabed sustainability fund" mit gezielten Aufgaben der Meeresumweltforschung oder der Technologieentwicklung für Entwicklungsländer S. Christiansen/A. Jaeckel/K. Houghton, Ecological safeguards for deep seabed mining, German Environment Agency Final Report 113/2019, abrufbar unter: https://www.umw eltbundesamt.de/sites/default/files/medien/1410/publikationen/2019-07-11_texte _113-2019_deep-seabed-mining.pdf (geprüft am 15.05.2020), S. $152 \mathrm{ff}$.

599 Entschließung des Europäischen Parlaments vom 26. Oktober 2017 zu der Anwendung der Richtlinie 2004/35/EG des Europäischen Parlaments und des Rates vom 21. April 2004 über Umwelthaftung zur Vermeidung und Sanierung 
V. Ergebnis

In der Literatur wird geschlussfolgert, dass die Haftungsübereinkommen keine Verpflichtungen auf völkerrechtlicher Ebene begründen, da die konventionsrechtlichen Pflichten den privaten Personen und Institutionen durch innerstaatliches Recht auferlegt würden. So erschöpfe sich die staatliche Pflicht mit Blick auf Schadenskompensation darin, gebietsfremden Geschädigten eine unverzügliche und angemessene Entschädigung zu ermöglichen. 600

Dieser Ansatz lässt außer Acht, dass einige Haftungsübereinkommen in unterschiedlichen Bereichen des Völkerrechts, in denen die Staaten (typischerweise) selbst die Akteure sind oder die schadensauslösenden Aktivitäten befürworten (sponsoring), eine Haftung für völkerrechtmäßige Aktivitäten und mithin staatliche Einstandspflichten begründen. Auch sehen Konventionen im Rahmen der Verlustzurechnung staatliche Einstandspflichten vor, indem Staaten ergänzende Entschädigungszahlungen oder Beiträge zu zwischenstaatlichen Kompensationsmechanismen leisten. Dies „may be considered a form of subsidiary liability, not just a gracious contribution of industry or State“. ${ }^{601}$ Außerdem ist auch die subsidiäre Haftung von Staaten von Belang, wenn die privaten Personen oder Institutionen ihrer Zahlungsverpflichtung nicht nachkommen (können). Demnach haften Staaten durchaus aufgrund der Haftungsübereinkommen auf internationaler Ebene. ${ }^{602}$

Dennoch bestehen Haftungslücken für internationale Schadensfälle. So erfassen die Haftungsübereinkommen nicht alle gefährlichen Aktivitäten, unterliegen unter Umständen konfligierenden Schadensverständnis-

von Umweltschäden („Umwelthaftungsrichtlinie“) (2016/2251(INI)), in: ABl. C 346 vom 27. September 2018, S. 184, Rn. 29. Zur Möglichkeit, einen solchen Fonds zu etablieren, siehe das von der EU-Kommission am 17. April 2013 veröffentlichte Dokument mit dem Titel „Study to explore the feasibility of creating a fund to cover environmental liability and losses occurring from industrial accidents“, abrufbar unter: ec.europa.eu/environment/archives/liability/eld/e ldfund/pdf/Final\%20report\%20ELD\%20Fund\%20BIO\%20for\%20web2.pdf (geprüft am 15.05.2020).

600 E. J. de Aréchaga, International law in the past third of a century, RdC 159 (1978I), S. 1 (272); J. Barboza, The Environment, Risk and Liability in International Law, 2011, S. 41; P.-M. Dupuy, Droit International public, 2000, S. 444.

$601 \mathrm{~J}$. Barboza, The Environment, Risk and Liability in International Law, 2011, S. 42. Aus dem Zusammenhang ergibt sich, dass hier keine subsidiäre (subsidia$r y$ ), sondern eine ergänzende (supplementary) Haftung gemeint sein müsste. 
sen und auch die geografische Beschränkung der Reichweite der Instrumente führt zu Haftungslücken. ${ }^{603}$ Außerdem sind die völkervertraglich zulässigen Haftungsbegrenzungen regelmäßig zu niedrig angesetzt, um der Dimension finanzieller Schäden durch besonders gefährliche Aktivitäten zu begegnen, und können zudem unweigerlich eine Subvention von derartigen Aktivitäten bewirken. Schließlich ist die Schadenslast insbesondere in Konstellationen, in denen der Schadensverursacher nicht bekannt ist oder nach den Haftungsübereinkommen nicht haften muss, vom Geschädigten zu tragen. Die existenten kollektiven Entschädigungsmechanismen müssten ergänzt und ausgeweitet werden, um derartige Haftungslücken zu beseitigen. ${ }^{604}$ Die Staaten sind indes bestrebt, einer Einstandspflicht für Schäden durch völkerrechtlich nicht verbotene Aktivitäten zu entgehen. ${ }^{605}$

Die Defizite im Rahmen der völkervertraglichen Haftungssysteme führen zu einem paradoxen Ergebnis: „The various uncertainties in primary

603 Bisher wurde nur mit dem Europäischen Übereinkommen über die zivilrechtliche Haftung für Schäden durch umweltgefährdende Tätigkeiten vom 21. Juni 1993 (Lugano Übereinkommen 1993, ILM 32 (1993), S. 1230 ff.) der Versuch unternommen, ein Haftungssystem für Umweltschäden durch gefährliche Aktivitäten im Allgemeinen zu etablieren. Das Lugano Übereinkommen 1993 ist allerdings bisher nicht in Kraft getreten und auch zukünftig werden wohl nicht genügend Staaten das Übereinkommen ratifizieren. P. Sands/J. Peel, Principles of International Environmental Law, 2018, S. 772.

604 Id., S. 804.

605 Die Sorge der Staaten, versehentlich eine völkervertraglich normierte Staatenhaftung zu etablieren, spiegelt sich beispielsweise in einer Fußnote zu Art. 8 des Übereinkommens über weiträumige grenzüberschreitende Luftverunreinigung vom 13. November 1979 (BGBl. 1982 II S. 373, 374 ff.). Dort wird hervorgehoben, dass das Übereinkommen keine Bestimmung zu einer Staathaftung für Schäden enthält. Hierzu P. Sands/J. Peel, Principles of International Environmental Law, 2018, S. 764. Auch im Rahmen des Klimaschutzes sind die Staaten bemüht, Einstandspflichten zu entgehen. Während die Vertragsstaaten des Pariser Übereinkommen von 2015 sich darauf einigten, dass Verluste und Schäden im Zusammenhang mit den nachteiligen Auswirkungen des Klimawandels verhindert, minimiert und gegebenenfalls ausgeglichen werden müssen, wurde auf der Konferenz, die zum Abschluss des Abkommens führte, zugleich betont, dass das Übereinkommen keine Grundlage für eine Haftung oder Entschädigung bieten soll (UNFCCC, Dec. 21/1, Adoption of the Paris Agreement, FCCC/CP/ 2015/L.9/Rev.1, Rn. 52). Eine finanzielle Einstandspflicht ergibt sich im Rahmen des Klimaschutzes allenfalls für entwickelte Staaten, welche Entwicklungsstaaten bei den Kosten mit Blick auf die Klimawandelanpassung unterstützen sollen (Art. 4 Abs. 4 Rahmenübereinkommen der Vereinten Nationen über Klimaänderungen 1992, Art. 2 Abs. 3 Kyoto Protokoll 1997, BGBl. 2002 II S.966, 967 ff.). P. Sands/J. Peel, Principles of International Environmental Law, 2018, S. $768 \mathrm{f}$. 


\section{Kapitel: Staatenhaftung in der Systematik des Völkerrechts}

norms make it difficult to deploy them for State responsibility purposes. In turn, it seems that States are not anxious to resolve the ambiguities, as they serve as a convenient buffer against State responsibility.“606

\section{E. Allgemeine Rechtsgrundsätze}

Die Staatenhaftung, welche die Völkerrechtskommission der Vereinten Nationen im Jahr 1996 konzipiert hat und die in der Rechtsprechung vielfach zur Anwendung gelangt ist, findet nicht nur in der Staatenpraxis und in zahlreichen völkerrechtlichen Haftungsübereinkommen ihre Bedeutung, sondern gründet auch auf allgemeinen Rechtsgrundsätzen. Die zuvor besprochenen Rechtsquellen basieren auf einem vertraglichen bzw. gewohnheitsrechtlichen Konsens der Staaten zu konkreten Regeln. ${ }^{607}$ Während diese zur Fragmentierung des Völkerrechts beitragen, ${ }^{608}$ werden allgemeine Rechtsgrundsätze aus bestehenden Regeln abstrahiert ${ }^{609}$ und sorgen für eine rechtssystematische Einheitlichkeit im Völkerrecht. ${ }^{610}$ Sie spiegeln das Völkerrecht als Werteordnung und vervollständigen damit den Konsensgrundsatz, auf dem die Rechtsquellen des Völkerrechts basieren. ${ }^{611}$

606 J. Brunnée, Of Sense and Sensibility, ICLQ 53 (2004), S. 352 (354).

$607 \mathrm{Vgl}$. M. Bos, Prolegomena to the Identification of Custom in International Law, in: T. M. C. Asser Instituut (Hg.), Essays on International \& Comparative Law in Honour of Judge Erades, 1983, S. 1 (3, 12); T. Rensmann, Wertordnung und Verfassung, 2007, S. $385 \mathrm{f}$.

608 H.-W. Jung, Rechtserkenntnis und Rechtsfortbildung im Völkergewohnheitsrecht, 2012, S. 22.

609 Vgl. E. Menzel, Grundprobleme der Ermittlung, Anwendung und Geltung von völkerrechtlichen Normen, JuS 1963, S. 41 (45).

610 Vgl. K. F. Gärditz, Ungeschriebenes Völkerrecht durch Systembildung, AVR 45 (2007), S. 1 (1); H.-W. Jung, Rechtserkenntnis und Rechtsfortbildung im Völkergewohnheitsrecht, 2012, S. 22; S. Kadelbach/T. Kleinlein, Überstaatliches Verfassungsrecht, AVR 44 (2006), S. 235 (262); E. Menzel, Grundprobleme der Ermittlung, Anwendung und Geltung von völkerrechtlichen Normen, JuS 1963, S. 41 (45); R. Wolfrum, General International Law (Principles, Rules, and Standards), in: ders. (Hg.), MPEPIL 2010, http://www.mpepil.com, Rn. 63.

611 Vgl. M. C. Bassiouni, A Functional Approach to "General Principles of International Law", Mich. JIL 11 (1990), S. 768 (786); B. Simma/P. Alston, The Sources of Human Rights Law, Austral. YBIL 12 (1992) S. 82 (105); K. F. Gärditz, Ungeschriebenes Völkerrecht durch Systembildung, AVR 45 (2007), S. 1 (1); H.W. Jung, Rechtserkenntnis und Rechtsfortbildung im Völkergewohnheitsrecht, 2012, S. 23 f.; T. Rensmann, Wertordnung und Verfassung, 2007, S. 386; C. Voigt, 
Dabei ist zu beachten, dass in der Völkerrechtsordnung unterschieden wird zwischen Grundsätzen, die eine Rechtsquelle im Sinne des Art. 38 Abs. 1 lit. c IGH-Statut darstellen, und Grundsätzen, die der Systematisierung, der Auslegung oder der fortschrittlichen Weiterentwicklung des Völkerrechts dienen. ${ }^{612}$ Allerdings ist eine eindeutige Abgrenzung dieser Kategorien oft nicht möglich, weil die dogmatische Herleitung beider aus bestehenden Normen oder bestimmten Rechtssystemen erfolgt ${ }^{613}$ und sich auch ihr Anwendungsbereich in völkerrechtlichen Streitfällen kaum bis gar nicht unterscheidet. ${ }^{614}$ Demnach sind beide Kategorien in die folgenden Erörterungen miteinzubeziehen.

Allgemeine Rechtsgrundsätze werden aus innerstaatlichen Rechtsordnungen im Wege des Vergleichs nationaler Rechtsordnungen bzw. der Hauptrechtssysteme der Welt entnommen. ${ }^{615}$ Sofern die im Kern übereinstimmenden Rechtssätze der verschiedenen Rechtsordnungen bzw. -systeme einen universellen Regelungscharakter aufweisen, können sie auf die völkerrechtliche Ebene gehoben werden. ${ }^{616}$

Allgemeine Rechtsgrundsätze im Sinne des Art. 38 Abs. 1 lit. c IGH-Statut können nach überwiegender Auffassung nicht nur den nationalen Rechtsordnungen, sondern auch den internationalen Beziehungen selbst entnommen werden. ${ }^{617}$ Demnach lassen sich allgemeine Rechtsgrundsätze

The Role of General Principles in International Law and their Relationship to Treaty Law, Retfærd Årgang 31 (2008) Nr. 2/121, S. 3 (5).

612 R. Wolfrum, General International Law (Principles, Rules, and Standards), in: ders. (Hg.), MPEPIL 2010, http://www.mpepil.com, Rn. 20.

613 Ibid.

614 Id., Rn. 21, 60; vgl. auch M. C. Bassiouni, A Functional Approach to "General Principles of International Law", Mich. JIL 11 (1990), S. 768-818 (775 ff.).

615 M. Bothe, Die Bedeutung der Rechtsvergleichung in der Praxis internationaler Gerichte, ZaöRV 36 (1976), S. 280 (292); A. Bleckmann, Grundprobleme und Methoden des Völkerrechts, 1982, S. 140; S. Kadelbach/T. Kleinlein, Überstaatliches Verfassungsrecht, AVR 44 (2006), S. 235 (257); dies., International Law a Constitution of Mankind?, GYIL 50 (2007), S. 303 (339); R. Wolfrum, General International Law (Principles, Rules, and Standards), in: ders. (Hg.), MPEPIL 2010, http://www.mpepil.com, Rn. 31.

616 R. Wolfrum, General International Law (Principles, Rules, and Standards), in: ders. (Hg.), MPEPIL 2010, http://www.mpepil.com, Rn. 55.

617 S. Kadelbach, Zwingendes Völkerrecht, 1992, S. 102; ders./T. Kleinlein, International Law - a Constitution of Mankind?, GYIL 50 (2007), S. 303 (339); B. Simma/P. Alston, The Sources of Human Rights Law, Austral. YBIL 12 (1992), S. 82 (102); W. Weiß, Allgemeine Rechtsgrundsätze des Völkerrechts, AVR 39 (2001), S. 394 (398 f.); R. Wolfrum, General International Law (Principles, Rules, and Standards), in: ders. (Hg.), MPEPIL 2010, http://www.mpepil.com, Rn. 28 f. 


\section{Kapitel: Staatenhaftung in der Systematik des Völkerrechts}

unter anderem aus übereinstimmenden Rechtssätzen in spezifischen Völkerrechtsregimen und internationalen Richtlinien sowie aus der Rechtslogik, die einer normativen Aussage im internationalen Kontext zugrunde liegt, herleiten. ${ }^{618}$ Sofern Rechtsgrundsätze, die dem Völkervertragsrecht oder Völkergewohnheitsrecht entnommen sind, unabhängig von diesen Rechtsquellen neue Rechte und Pflichten begründen, bilden sie eine selbstständige Rechtsquelle. ${ }^{619}$ Es ist jedoch schwierig darzulegen, unter welchen Voraussetzungen sich ein bestimmter Grundsatz von einer Grundregel eines spezifischen Völkerrechtsregimes in einen Grundsatz mit einem allgemeinen Anwendungsbereich wandelt und damit eine selbstständige Rechtsquelle formt. ${ }^{620}$ Zudem können allgemeine Rechtsgrundsätze die Ausgestaltung von völkerrechtlichen Übereinkommen leiten und sich auf diese Weise zu völkervertraglichen Bestimmungen entwickeln. ${ }^{621}$ Schließlich lassen sich allgemeine Rechtsgrundsätze nicht nur aus Völkergewohnheitsrecht herleiten, sondern können umgekehrt in Völkergewohnheitsrecht erwachsen, wenn eine entsprechende Übung der Staaten

Nach anderer Auffassung können sich allgemeine Rechtsgrundsätze lediglich aus nationalem Recht heraus entwickeln. B. Cheng, General Principles of Law, 1987, S. 390.

618 H.-W. Jung, Rechtserkenntnis und Rechtsfortbildung im Völkergewohnheitsrecht, 2012, S. 51 f.; H. Mosler, General Principles of Law, in: R. Bernhardt (Hg.), Encyclopedia of Public International Law, Band II, 1995, S. 511 (511f.); M. V. Soto, General Principles of International Environmental Law, ILSA J. Intl. \& Comp. L. 3 (1996), S. 193 (193); C. Voigt, The Role of General Principles in International Law and their Relationship to Treaty Law, Retfærd Årgang 31 (2008) Nr. 2/121, S. 3 (7f.); R. Wolfrum, General International Law (Principles, Rules, and Standards), in: ders. (Hg.), MPEPIL 2010, http://www.mpepil.com, Rn. 29; K. Ziolkowski, General Principles of International Law as Applicable in Cyberspace, in: dies. (Hg.), Peacetime Regime for State Activities in Cyberspace, 2013, S. 135 (167).

619 W. Weiß, Allgemeine Rechtsgrundsätze des Völkerrechts, AVR 39 (2001), S. 394 (409f.); R. Wolfrum, General International Law (Principles, Rules, and Standards), in: ders. (Hg.), MPEPIL 2010, http://www.mpepil.com, Rn. 34.

620 R. Wolfrum, General International Law (Principles, Rules, and Standards), in: ders. (Hg.), MPEPIL 2010, http://www.mpepil.com, Rn. 41. Eine zwischen Rechtsgrundsätzen und allgemeinen Rechtsgrundsätzen des Völkerrechts differenzierende Betrachtung findet sich bei M. Virally, The Sources of International Law, in: M. Sørensen (Hg.), Manual of Public International Law, 1968, S. 116 (143).

621 C. Voigt, The Role of General Principles in International Law and their Relationship to Treaty Law, Retfærd Årgang 31 (2008) Nr. 2/121, S. 3 (12). 
hinzukommt. ${ }^{622}$ Dementsprechend verwischt hier die Grenze zwischen den Rechtsquellen. ${ }^{623}$

Die benannten Unsicherheiten bei der Herleitung von allgemeinen Rechtsgrundsätzen relativieren sich aber durch das entscheidende Kriterium der Anerkennung durch die Kulturvölker nach Art. 38 lit. c IGHStatut. ${ }^{62}$ Eine derartige Anerkennung basiert auf einem "gemeinsamen juristischen Bewusstsein" (opinio iuris communis) ${ }^{625}$ der internationalen Gemeinschaft und kommt etwa dann zum Ausdruck, wenn ein Grundsatz Anknüpfungspunkte für die internationale Rechtsprechung bietet, ${ }^{626}$ in Resolutionen internationaler Organisationen in Bezug genommen wird oder im Rahmen politischer Erklärungen auf internationalen Konferenzen Erwähnung findet. ${ }^{627}$ Grundsätze zeichnen sich dadurch aus, dass sie zum einen dynamisch sind, und damit gesellschaftlichen, politischen sowie technischen Wandel zu erfassen vermögen, und zum anderen aufgrund ihrer Offenheit verschiedene Gestalt annehmen und bereichsübergreifend zum Einsatz kommen können. ${ }^{628}$ In diesem Zusammenhang steht Rechtsprechungsorganen ein weites Ermessen bei der Anerkennung

622 W. Weiß, Allgemeine Rechtsgrundsätze des Völkerrechts, AVR 39 (2001), S. 394 (404f.).

623 Nach W. Weiß gilt die Konkretisierung eines Rechtssatzes als Anhaltspunkt für dessen völkergewohnheitsrechtliche Geltung. Id., S. 394 (405).

624 B. Simma/P. Alston, The Sources of Human Rights Law, Austral. YBIL 12 (1992), S. 82 (102); W. Weiß, Allgemeine Rechtsgrundsätze des Völkerrechts, AVR 39 (2001), S. 394 (397).

625 G. Herczegh, General principles of international law and the international legal order, 1969, S. 36; C. Voigt, The Role of General Principles in International Law and their Relationship to Treaty Law, Retfærd Årgang 31 (2008) Nr. 2/121, S. 3 (8).

626 Nach S. Kadelbach/T. Kleinlein bezieht sich Art. 38 lit. c IGH-Statut primär auf die gerichtliche Praxis, da die Anerkennung allgemeiner Rechtsgrundsätze sonst sehr langwierig sei. S. Kadelbach/T. Kleinlein, International Law - a Constitution of Mankind?, GYIL 50 (2007), S. 303 (339).

627 S. Kadelbach/T. Kleinlein, Überstaatliches Verfassungsrecht, AVR 44 (2006), S. 235 (259 f.); P. Stubbe, State Accountability for Space Debris, 2018, S. 219; C. Voigt, The Role of General Principles in International Law and their Relationship to Treaty Law, Retfærd Årgang 31 (2008) Nr. 2/121, S. 3 (8); R. Wolfrum, General International Law (Principles, Rules, and Standards), in: ders. (Hg.), MPEPIL 2010, http://www.mpepil.com, Rn. 42, 55.

628 Vgl. C. Voigt, The Role of General Principles in International Law and their Relationship to Treaty Law, Retfærd Årgang 31 (2008) Nr. 2/121, S. 3 (9 ff.); $R$. Wolfrum, General International Law (Principles, Rules, and Standards), in: ders. (Hg.), MPEPIL 2010, http://www.mpepil.com, Rn. 6, 60. Zum Schädigungsverbot J. Bäumler, Das Schädigungsverbot im Völkerrecht, 2017, S. 265 ff. 


\section{Kapitel: Staatenhaftung in der Systematik des Völkerrechts}

von allgemeinen Rechtsgrundsätzen zu. ${ }^{629}$ Denn für Rechtsstreitigkeiten, bei denen keine völkergewohnheitsrechtliche oder völkervertragliche Regelung einschlägig ist, „bieten im Zweifel die allgemeinen Rechtsgrundsätze den methodischen »Notausgang « für die Vermeidung einer non liquet-Situation. “630 Insbesondere die Bestimmung und Anwendung allgemeiner Rechtsgrundsätze durch die Richter des IGH erfährt autoritative Wirkung, da diese bei ihrer Entscheidungsfindung grundsätzlich alle wesentlichen Rechtssysteme der Welt und die dem Völkerrecht inhärente Werteordnung heranziehen. ${ }^{631}$

Sofern also die Grundsätzlichkeit und Konkretisierungsfähigkeit eines Grundsatzes festgestellt ist, kann dieser auch in unreglementierten Bereichen in spezifische Rechte und Pflichten erwachsen und mithin Lücken füllen, wenn die anzuwendenden Rechtsvorschriften unvollständig erscheinen. ${ }^{632}$ Allgemeine Rechtsgrundsätze formen auf diese Weise eine Verbindung zwischen geltendem und zukünftigem Recht. ${ }^{633}$ Dies ist insbesondere mit Blick auf neuere Technologien von Bedeutung, zu deren Reglementierung Staaten noch keine vertraglichen Abmachungen getroffen haben und sich völkergewohnheitsrechtliche Grundsätze in der Entwicklung befinden. Dementsprechend zeichnen allgemeine Rechtsgrundsätze auch den Rahmen für eine Staatenhaftung für informationstechnische Systeme an der Schnittstelle zwischen lex lata und lex ferenda. ${ }^{634}$ Vor diesem Hintergrund sind die Grundsätze des Umweltvölkerrechts, in denen die Komponenten der Schadensvermeidung und Schadenskom-

629 V. Bruns, Völkerrecht als Rechtsordnung, ZaöRV 1 (1929), S. 1 (29); H.-W. Jung, Rechtserkenntnis und Rechtsfortbildung im Völkergewohnheitsrecht, 2012, S. 62 f.; C. Voigt, The Role of General Principles in International Law and their Relationship to Treaty Law, Retfærd Årgang 31 (2008) Nr. 2/121, S. 3 (10).

630 H.-W. Jung, Rechtserkenntnis und Rechtsfortbildung im Völkergewohnheitsrecht, 2012, S. 62.

631 W. Weiß, Allgemeine Rechtsgrundsätze des Völkerrechts, AVR 39 (2001), S. 394 (406).

632 C. Voigt, The Role of General Principles in International Law and their Relationship to Treaty Law, Retfærd Årgang 31 (2008) Nr.2/121, S. 3 (9f.); $R$. Wolfrum, General International Law (Principles, Rules, and Standards), in: ders. (Hg.), MPEPIL 2010, http://www.mpepil.com, Rn. 60.

633 R. Kolb, Principles as sources of international law (with special reference to good faith), NILR 53 (2006), S. 1 (9); C. Voigt, The Role of General Principles in International Law and their Relationship to Treaty Law, Retfærd Årgang 31 (2008) Nr. 2/121, S. 3 (4).

634 Vgl. K. Ziolkowski, General Principles of International Law as Applicable in Cyberspace, in: dies. (Hg.), Peacetime Regime for State Activities in Cyberspace, 2013, S. 135 (155). 
pensation vornehmlich verankert sind, Gegenstand der folgenden Untersuchung.

\section{Grundsatz der guten Nachbarschaft}

Im Privatrechtsverkehr verwurzelt ist die Maxime, dass das eigene Eigentum in einer Weise zu nutzen ist, in der fremdes Eigentum nicht geschädigt wird (sic utere tuo ut alienum non laedas). ${ }^{635}$ Es geht dabei traditionell um die Frage, ob und inwieweit die Interessen des Nachbarn bei der Nutzung des eigenen Grundstücks zu berücksichtigen sind. ${ }^{636}$ Zum einen ist die gebotene Sorgfalt auszuüben, um Schäden an benachbarten Grundstücken zu vermeiden, ${ }^{637}$ und zum anderen löst eine Beeinträchtigung eine verschuldensunabhängige Haftung aus. ${ }^{638}$ Diese Regel wird in der Literatur im Zusammenspiel mit der Lehre vom Rechtsmissbrauch, welche den übermäßigen Gebrauch oder eine zweckwidrige Inanspruchnahme der eigenen Rechte beschreibt, thematisiert. ${ }^{639}$ Verwandte Konzepte sind im römischen Recht, in islamischen und jüdischen Rechtstraditionen zu finden und gelangen in gegenwärtigen nationalen Civil Law- und Common Law-Systemen zur Anwendung. ${ }^{640}$

635 L. Boisson de Chazounes/D. Campanelli, Neighbour States, in: R. Wolfrum (Hg.), MPEPIL 2006, http://www.mpepil.com, Rn. 10; J. Brunnée, Sic utere tuo ut alienum non laedas, in: R. Wolfrum (Hg.), MPEPIL 2010, http://www.mpepil. com, Rn. 1 f.; C. Hinds, Das Prinzip „sic utere tuo ut alienum non laedas“ und seine Bedeutung im internationalen Umweltrecht, AVR 30 (1992), S. 298 (300).

636 J. Brunnée, Sic utere tuo ut alienum non laedas, in: R. Wolfrum (Hg.), MPEPIL 2010, http://www.mpepil.com, Rn. 8; C. Hinds, Das Prinzip „sic utere tuo ut alienum non laedas“ und seine Bedeutung im internationalen Umweltrecht, AVR 30 (1992), S. 298 (301).

637 Fleming v. Lockwood Montana Supreme Court (28 December 1907) (1908) 92 Pacific Reporter 962 (963).

638 Rylands v. Fletcher United Kingdom House of Lords (17 July 1868) (1868) LR 3 HL 330 (341).

639 C. Hinds, Das Prinzip „sic utere tuo ut alienum non laedas“ und seine Bedeutung im internationalen Umweltrecht, AVR 30 (1992), S. 298 (298) m.w.N. $\mathrm{Zu}$ diesem Rechtsinstitut ausführlich M. Byers, Abuse of Rights, McGill LJ 47 (2002), S. 389 (389 ff.).

640 J. Brunnée, Sic utere tuo ut alienum non laedas, in: R. Wolfrum (Hg.), MPEPIL 2010, http://www.mpepil.com, Rn. 2; C. Hinds, Das Prinzip „sic utere tuo ut alienum non laedas" und seine Bedeutung im internationalen Umweltrecht, AVR 30 (1992), S. 298 (300 f.). 


\section{Kapitel: Staatenhaftung in der Systematik des Völkerrechts}

Der Gedanke, der den Konzepten zugrunde liegt, gewinnt auf der völkerrechtlichen Ebene durch den Grundsatz der guten Nachbarschaft (good neighbourliness) an Bedeutung, ${ }^{641}$ der einen Ausgleich konkurrierender souveräner Rechte bedeutet. ${ }^{642}$ Ursprünglich wurde der Grundsatz auf unmittelbare geografische Nachbarstaaten begrenzt, mittlerweile wurde dieser Nachbarschaftsbegriff aber auf alle von Umweltbeeinträchtigungen betroffenen Staaten ausgeweitet. ${ }^{643}$ So stellte der Schiedsrichter Max Huber im Island of Palmas-Fall aus dem Jahr 1928, in dem es um einen Konflikt zwischen den Niederlanden und den Vereinigten Staaten über die Hoheitsrechte über die Insel Palmas ging, fest, dass „, [t]erritorial sovereignty $(\ldots)$ has as corollary a duty: the obligation to protect within the territory the rights of other States, in particular their right to integrity and inviolability". ${ }^{644}$ Unter Bezugnahme auf diese Aussage folgerte die Völkerrechtskommission im Rahmen ihrer Haftungsstudie, dass das Konzept der Staatenhaftung dem Grundsatz der territorialen Souveränität entspringt. ${ }^{645}$ Demnach sind Staaten zwar grundsätzlich berechtigt, Aktivitäten auf ihrem Staatsgebiet auszuüben und zu gestatten, allerdings steht dieser territorialen Souveränität das korrelate Recht von Staaten, dass die Nutzung ihres Staatsgebietes nicht durch fremde Staaten beeinträchtigt

641 A. E. Boyle, State Responsibility and International Liability for Injurious Consequences of Acts Not Prohibited by International Law, ICLQ 39 (1990), S. 1 (13 f.); C. Hinds, Das Prinzip „sic utere tuo ut alienum non laedas“ und seine Bedeutung im internationalen Umweltrecht, AVR 30 (1992), S. 298 (311); M. V. Soto, General Principles of International Environmental Law, ILSA J. Intl. \& Comp. L. 3 (1996), S. 193 (197).

642 G. Handl, Territorial Sovereignty and the Problem of Transnational Pollution, AJIL 69 (1975), S. 50 (56); C. Hinds, Das Prinzip „sic utere tuo ut alienum non laedas" und seine Bedeutung im internationalen Umweltrecht, AVR 30 (1992), S. 298 (308 f.); E. Klein, Umweltschutz im völkerrechtlichen Nachbarrecht, 1976, S. 117.

643 Die VN-Generalversammlung betont, dass der Grundsatz der guten Nachbarschaft auch für Staaten gelten soll, die sich keine Grenze teilen, denn „great changes of political, economic and social nature, as well as the scientific and technological advances that have taken place in the world and led to unprecedented interdependence of nations, have given new dimensions to good-neighbourliness“. GV Resolution 46/62 vom 9.12.1991. Siehe auch C. Hinds, Das Prinzip „sic utere tuo ut alienum non laedas“ und seine Bedeutung im internationalen Umweltrecht, AVR 30 (1992), S. 298 (310 f.).

644 Island of Palmas Case (Netherlands vs. United States of America), Award of 4 April 1928, 2 UNRIAA (1928), S. 829 (839).

645 AIL, Kommentar zu Art. 1 AIL, S. 104, Abs. 6 f. 
wird, gegenüber. ${ }^{646}$ Der Grundsatz der guten Nachbarschaft zielt auf einen gerechten Interessenausgleich zwischen territorialer Souveränität und Integrität und begründet zwischenstaatliche Kooperationspflichten. ${ }^{647}$ Dies ist explizit in Art. $74 \mathrm{VN}-\mathrm{Charta}$ in Bezug auf soziale, wirtschaftliche, handelsbezogene Angelegenheiten ausformuliert ${ }^{648}$ und wird auch durch die Erklärung über Grundsätze des Völkerrechts betreffend freundschaftliche Beziehungen und Zusammenarbeit zwischen den Staaten im Einklang mit der Charta der Vereinten Nationen von 1970 („Friendly Relations-Deklaration") statuiert ${ }^{649}$. Der Grundsatz findet auch auf grenzüberschreitende Umweltschäden Anwendung und konkretisiert dort spezifische Rechte und Pflichten. ${ }^{650}$ So fließen aus dem Grundsatz der guten Nachbarschaft Informations-, Benachrichtigungs-, Warn- und Rücksprachepflichten, ${ }^{651}$ wie zahlreiche völkervertraglichen Übereinkommen, die völkerrechtliche Praxis und Entscheidungen internationaler Gerichte zeigen. ${ }^{652}$

646 J. Barboza, The Environment, Risk and Liability in International Law, 2011, S. 121; C. Hinds, Das Prinzip „sic utere tuo ut alienum non laedas" und seine Bedeutung im internationalen Umweltrecht, AVR 30 (1992), S. 298 (299); D. B. Magraw, The International Law Commission's Study of International Liability for Nonprohibited Acts as it Relates to Developing States, WLR 61 (1986), S. 1041 (1043).

647 P. Sands/J. Peel, Principles of International Environmental Law, 2018, S. 213.

648 Art. 74 VN-Charta: „Die Mitglieder der Vereinten Nationen sind sich ferner darin einig, dass die Politik, die sie für die unter dieses Kapitel fallenden Hoheitsgebiete verfolgen, nicht minder auf dem allgemeinen Grundsatz der guten Nachbarschaft in sozialen, wirtschaftlichen und Handelsangelegenheiten beruhen muß als die Politik, die sie für ihr Mutterland verfolgen; hierbei sind die Interessen und das Wohl der übrigen Welt gebührend zu berücksichtigen."

649 GV Resolution 2625 (XXV) vom 24.10.1970 hebt hervor, „that the peoples of the United Nations are determined to practise tolerance and live together in peace with one another as good neighbours“ und bekräftigt „[t]he duty of State to co-operate with one another in accordance with the Charter".

650 Vgl. A. von Arnauld, Völkerrechtliche Informationspflichten bei Naturkatastrophen, AVR 43 (2005), S. 279 (283 f.); P. Sands/J. Peel, Principles of International Environmental Law, 2018, S. $213 \mathrm{ff}$.

651 P. Sands/J. Peel, Principles of International Environmental Law, 2018, S. 215 f.; M. V. Soto, General Principles of International Environmental Law, ILSA J. Intl. \& Comp. L. 3 (1996), S. 193 (198 f.); K. Ziolkowski, General Principles of International Law as Applicable in Cyberspace, in: dies. (Hg.), Peacetime Regime for State Activities in Cyberspace, 2013, S. 135 (171). Der Technologietransfer wird ebenfalls als Element der Kooperationspflicht angesehen. P. Sands/J. Peel, Principles of International Environmental Law, 2018, S. $720 \mathrm{ff}$.

652 P. Sands/J. Peel, Principles of International Environmental Law, 2018, S. $213 \mathrm{ff.}$ 


\section{Kapitel: Staatenhaftung in der Systematik des Völkerrechts}

Dem Grundsatz der guten Nachbarschaft wird völkergewohnheitsrechtlicher Stellenwert zugeschrieben, ${ }^{653}$ er wird aber verschiedentlich auch als allgemeiner Rechtsgrundsatz eingestuft, denn er hat seinen Ursprung in einer weitverbreiteten Basis nationaler Rechtsordnungen ${ }^{654}$ und hat sich durch die internationale Rechtsprechung sowie durch internationale Übereinkünfte und Erklärungen zu einer selbstständigen Rechtsquelle entwickelt, die in unterschiedlichen Regelungsbereichen herangezogen wird. ${ }^{655}$

\section{Schädigungsverbot}

Das Schädigungsverbot (no harm principle) besagt, dass Staaten auf ihrem Staatsgebiet keine Aktivitäten durchführen oder gestatten dürfen, die zu erheblichen grenzüberschreitenden Schäden führen. ${ }^{656}$ Neben dem Grundsatz der guten Nachbarschaft betrifft also auch das Schädigungsverbot die für die Völkerrechtsordnung fundamentalen Konzepte der territorialen Souveränität und territorialen Integrität. ${ }^{657}$ In diesem Zusammenhang werden zwei Ansätze zur theoretischen Grundlage und dogmatischen Einordnung des Schädigungsverbotes vertreten. ${ }^{658}$ Zum einen wird das Schädigungsverbot als absolutes Verbot der Beeinträchtigung territorialer Integrität fremder Staaten verstanden. Demnach seien territoriale Souveränität und territoriale Integrität sich ergänzende Konzepte, und

$653 \mathrm{~J}$. Healey/H. Pitts, Applying International Environmental Legal Norms to Cyber Statecraft, I/S: A Journal of Law and Policy for the Information Society 8 (2012), S. 356 (373); C. Hinds, Das Prinzip „sic utere tuo ut alienum non laedas“ und seine Bedeutung im internationalen Umweltrecht, AVR 30 (1992), S. 298 (324).

654 Vgl.J. Bäumler, Das Schädigungsverbot im Völkerrecht, 2017, S. 48.

655 R. Wolfrum, General International Law (Principles, Rules, and Standards), in: ders. (Hg.), MPEPIL 2010, http://www.mpepil.com, Rn. 51, 55. M. V. Soto beschreibt den Grundsatz als allgemeinen Rechtsgrundsatz des Umweltvölkerrechts. M. V. Soto, General Principles of International Environmental Law, ILSA J. Intl. \& Comp. L. 3 (1996), S. 193 (197 ff.). Ebenso D. B. Magraw, The International Law Commission's Study of International Liability for Nonprohibited Acts as it relates to Developing States, WLR 61 (1986), S. 1041 (1043).

656 Ausführlich zum Schädigungsverbot siehe J. Bäumler, Das Schädigungsverbot im Völkerrecht, 2017.

657 J. Bäumler, Das Schädigungsverbot im Völkerrecht, 2017, S. 49 f.; A. Epiney, Das „Verbot erheblicher grenzüberschreitender Umweltbeeinträchtigungen“, AVR 33 (1995), S. 309 (319).

658 Zum Meinungsstand siehe A. Epiney, Das „Verbot erheblicher grenzüberschreitender Umweltbeeinträchtigungen“, AVR 33 (1995), S. 309 (319f.). 
die sich aus der territorialen Souveränität ergebenden Rechte seien mithin durch die territoriale Integrität begrenzt. ${ }^{659}$ Damit aber nicht jedwede grenzüberschreitende Schädigung als völkerrechtswidrig eingeordnet wird, soll das Erfordernis der Erheblichkeit der grenzüberschreitenden Schädigung so zu verstehen sein, dass unerhebliche Beeinträchtigungen als Ausnahme vom absoluten Verbot grenzüberschreitender Schädigungen aufgrund völkergewohnheitsrechtlicher Akzeptanz in der Staatengemeinschaft gestattet seien. ${ }^{660}$ Nach einem anderen Ansatz sind territoriale Souveränität und territoriale Integrität gleichrangige Konzepte, so dass die konkurrierenden Rechte unter Verhältnismäßigkeitsgesichtspunkten in Ausgleich zu bringen seien. ${ }^{661}$ Das Erheblichkeitserfordernis der grenzüberschreitenden Schädigung verkörpere diesen Ausgleich. ${ }^{62}$ Dieser Ansatz weist eine gewisse Nähe zu der sogenannten Harmon-Doktrin auf, wonach die Souveränität von Staaten zur unbeschränkten Entfaltung von Aktivitäten auf dem eigenen Staatsgebiet berechtigt. ${ }^{663}$

Keinem der - als gegensätzlich begriffenen ${ }^{664}$ - Ansätze kommt isoliert der Vorrang zu. Vielmehr sind die zugrunde liegenden Rechtsgedanken komplementär zu verstehen. Mit Blick auf völkerrechtlich verbotenes Verhalten bedeutet dies, dass die territoriale Souveränität von vornherein nur Aktivitäten erlaubt, welche die territoriale Integrität anderer Staaten

659 U. Beyerlin, Grenzüberschreitender Umweltschutz und allgemeines Völkerrecht, in: K. Hailbronner/G. Ress/T. Stein (Hg.), Staat und Völkerrechtsordnung: Festschrift für Karl Doehring, 1989, S. 37 (39 ff., 45); B.-O. Bryde, Umweltschutz durch allgemeines Völkerrecht?, AVR 31 (1993), S. 1 (2ff.); A. Epiney, Das „Verbot erheblicher grenzüberschreitender Umweltbeeinträchtigungen“, AVR 33 (1995), S. 309 (322 f.).

660 B.-O. Bryde, Umweltschutz durch allgemeines Völkerrecht?, AVR 31 (1993), S. 1 (1, 6f.); A. Epiney, Das „Verbot erheblicher grenzüberschreitender Umweltbeeinträchtigungen“, AVR 33 (1995), S. 309 (323).

661 R. Hach, Völkerrechtliche Pflichten zur Verminderung grenzüberschreitender Luftverschmutzung in Europa, 1993, S. 38 ff.; C. Hinds, Das Prinzip „sic utere tuo ut alienum non laedas" und seine Bedeutung im internationalen Umweltrecht, AVR 30 (1992), S. 298 (311 ff.); D. Rauschning, Allgemeine Völkerrechtsregeln zum Schutz gegen Umweltbeeinträchtigungen, in: I. von Münch (Hg.), Staatsrecht, Völkerrecht, Europarecht: Festschrift für Hans-Jürgen Schlochauer, 1981, S. 557 (560 ff.); R. Wolfrum, Purposes and Principles of International Environmental Law, GYIL 33 (1990), S. 308 (310 f.).

662 C. Hinds, Das Prinzip „sic utere tuo ut alienum non laedas“ und seine Bedeutung im internationalen Umweltrecht, AVR 30 (1992), S. 298 (324).

663 So A. Epiney, Das „Verbot erheblicher grenzüberschreitender Umweltbeeinträchtigungen“, AVR 33 (1995), S. 309 (322 Fn. 56).

664 Id., S. 309 (319). 


\section{Kapitel: Staatenhaftung in der Systematik des Völkerrechts}

nicht berührt. Die Erheblichkeit der grenzüberschreitenden Schädigung wird hier durch die Völkerrechtswidrigkeit der Aktivität indiziert. Wenn die Aktivität auf dem Staatsgebiet hingegen keinem Verbotstatbestand unterfällt, aber dennoch die territoriale Integrität eines anderen Staates beeinträchtigt, ist lediglich ein Schadensausgleich erforderlich. Dies hat zur Folge, dass Staaten im Falle völkerrechtlich nicht verbotener Aktivitäten negative Auswirkungen auf ihrem Territorium, die durch die Ausübung der souveränen Rechte anderer Staaten bedingt sind, bei Gewährung eines Schadensausgleiches hinnehmen müssen. Da völkerrechtlich nicht verbotene Aktivitäten aufgrund der Globalisierung und Vernetzung der Staaten regelmäßig grenzüberschreitende Auswirkungen haben, ist ein Ausgleichsanspruch aber nur bei Erheblichkeit des Schadens begründet. ${ }^{665}$ Denn die Geltendmachung von sogenannten de minimis Schäden ist in Anbetracht der unter Umständen kosten- und zeitintensiven Streitbeilegungsmechanismen und der damit einhergehenden zwischenstaatlichen Spannungen nicht gerechtfertigt. ${ }^{666}$

Das Schädigungsverbot stellt keinen konkreten Sollenssatz auf, der sich aus Tatbestand und Rechtsfolge zusammensetzt, ${ }^{667}$ sondern erfasst allgemein Handlungen, die grenzüberschreitende Schäden verursachen und daher grundsätzlich regelungsbedürftig sind. ${ }^{668}$ Dieses Bedürfnis wird sowohl durch die Staatenverantwortlichkeit als auch die Staatenhaftung gestillt. So ist auch zu erklären, dass die Konkretisierung des Schädigungsverbotes einerseits die Gestalt einer Verhinderungspflicht im Sinne der Korfu Kanal-Entscheidung und andererseits die Gestalt einer Schadensvermeidungs- und Kompensationspflicht im Sinne der Trail Smelter-Entscheidungen annimmt. ${ }^{669}$ Das Schädigungsverbot ist nicht nur Gegenstand der internationalen Rechtsprechung in den angeführten Entscheidungen zu grenzüberschreitenden Schadensfällen, sondern bildet auch das Fundament der bereits besprochenen Übereinkommen. ${ }^{670}$ Es betrifft demnach

665 Vgl. J. Bäumler, Das Schädigungsverbot im Völkerrecht, 2017, S. 53; D. B. Magraw, Transboundary Harm, AJIL, 80 (1986), S. 305 (322).

$666 \mathrm{Im}$ Übrigen führt die Abwägung von gesellschaftlichen Faktoren und Billigkeitserwägungen bei der Entscheidungsfindung ohnehin dazu, dass Klagen nur in erheblichen Schadensfällen Erfolg haben. Zudem werden Marktkräfte Kläger davon abhalten, unerhebliche Schadensersatzansprüche einzuklagen. So B. A. Walton, Duties Owed, Yale LJ 126 (2017), S. 1460 (1505).

667 J. Bäumler, Das Schädigungsverbot im Völkerrecht, 2017, S. 269.

668 Id., S. 13.

669 Siehe 1. Kapitel B.

670 Siehe 1. Kapitel D. 
unterschiedliche Regelungsbereiche und kann als allgemeiner Rechtsgrundsatz Geltung beanspruchen, dem weitgehend völkergewohnheitsrechtlicher Stellenwert zugeschrieben wird. ${ }^{671}$ Dies bedeutet allerdings nicht, dass auch die Konkretisierung des Schädigungsverbotes für verschiedene Regelungsbereiche verbindlich ist, denn der Bedeutungsgehalt des Grundsatzes ist nicht statisch, sondern von dem wandelnden Verständnis der internationalen Gemeinschaft abhängig. ${ }^{672}$ Beim Schädigungsverbot handelt es sich um ein dynamisches Prinzip ${ }^{673}$ bzw. eine konkretisierungsfähige Grundnorm ${ }^{674}$. Es kann verschiedene Ausgestaltung erfahren. ${ }^{675}$ Es ist daher entscheidend, inwiefern aus dem Schädigungsverbot Rechte und Pflichten abgeleitet werden können, die wiederum selbst Allgemeingültigkeit erlangen. ${ }^{676}$

Die konkurrierenden Interessen territorialer Souveränität und territorialer Integrität geraten insbesondere bei grenzüberschreitenden Umweltschäden in Widerstreit ${ }^{677}$ und werden hier durch unterschiedliche Konkretisierung des Schädigungsverbotes in Ausgleich gebracht. ${ }^{678}$ So erfährt das Schädigungsverbot in umweltvölkerrechtlichen Verträgen und Richtlinien zum Umweltschutz seine Ausgestaltung, indem Maßnahmen zur Schadensprävention, eine angemessene Nutzung natürlicher Ressourcen und Vorsorgemaßnahmen gefordert werden. Daneben widmet sich die Rechtsprechung dem Verständnis dieser Aspekte des Schädigungsverbotes. Erst eine eingehende Analyse vermag zu klären, ob diese Konkretisierungen des Schädigungsverbotes lediglich im Bereich des spezifischen (Umwelthaftungs-) Regimes Wirkung entfalten oder sich darüber hinausgehend zu selbstständigen allgemeinen Rechtsgrundsätzen entwickelt haben und/ oder in Völkergewohnheitsrecht erwachsen sind.

671 J. Bäumler, Das Schädigungsverbot im Völkerrecht, 2017, S. 94 m.w.N.

672 Id., S. 35 f., 269 f.; vgl. auch P. Sands/J. Peel, Principles of International Environmental Law, 2018, S. 206.

673 Sonderberichterstatter R. Q. Quentin-Baxter, Second report on international liability for injurious consequences arising out of acts not prohibited by international law, YBILC 1981-II/1, UN Doc. A/CN.4/346 and Add.1 and 2, S. 107, Rn. 15.

674 A. Epiney, Das „Verbot erheblicher grenzüberschreitender Umweltbeeinträchtigungen“, AVR 33 (1995), S. 309 (359).

675 J. Bäumler, Das Schädigungsverbot im Völkerrecht, 2017, S. 35 f., 268.

676 Vgl. id., S. 35 f., $267 \mathrm{f}$.

677 C. Hinds, Das Prinzip „sic utere tuo ut alienum non laedas“ und seine Bedeutung im internationalen Umweltrecht, AVR 30 (1992), S. 298 (299).

678 Vgl.J. Bäumler, Das Schädigungsverbot im Völkerrecht, 2017, S. 94. 


\section{Grundsatz der Schadensprävention}

Prinzip 21 der Stockholmer Deklaration der Konferenz der Vereinten Nationen über die Umwelt des Menschen von 1972 (Stockholm Deklaration ${ }^{679}$ und Prinzip 2 der Rio Deklaration über Umwelt und Entwicklung von 1992 (Rio Deklaration) ${ }^{680}$ bilden die Eckpfeiler des Umweltvölkerrechts ${ }^{681}$ und gelten als Anknüpfungspunkt für den Grundsatz der Schadensprävention (principle of prevention), ${ }^{682} \mathrm{der}$ besagt, dass Umweltschäden zu vermeiden und umweltgefährdende Aktivitäten zu begrenzen, zu reduzieren und zu kontrollieren sind. ${ }^{683}$ Demnach verdichtet sich das Schädigungsverbot zu einer staatlichen Handlungspflicht zur Schadensprävention. ${ }^{64}$ Entsprechend wird Prinzip 21 der Stockholm Deklaration auch

679 UN Doc. A/CONF.48/14/Rev.1 (1973), S. 5. Die Stockholm Konferenz von 1972 gilt als Anfang des modernen Umweltvölkerrechts. Der Fokus verlagerte sich weg von dem Ausgleich souveräner Rechte und vom nachbarschaftlichen Völkerrecht hin auf das unabhängige Schutzgut Umwelt. Damit wurde das Konzept nachhaltiger Entwicklung (sustainable development) auf die Agenda der VN gesetzt. Siehe zur Stockholm Deklaration L. B. Sohn, The Stockholm Declaration on the Human Environment, Harv. ILJ 14 (1973), S. 423 (423 ff.).

680 UN Doc. A/CONF.151/26/Rev.1 (Vol. I) Annex I (1993), S.3. Siehe zur Rio Deklaration I. M. Porras, The Rio Declaration, RECIEL 1 (1992), S. 245 (245 ff.).

681 I. M. Porras, The Rio Declaration, RECIEL 1 (1992), S. 245 (245). Der Wortlaut von Prinzip 2 der Rio Deklaration stimmt mit Prinzip 21 der Deklaration von Stockholm im Wesentlichen überein. „States have, in accordance with the Charter of the United Nations and the principles of international law, the sovereign right to exploit their own resources pursuant to their own environmental [and developmental] policies, and the responsibility to ensure that activities within their jurisdiction or control do not cause damage to the environment of other States or of areas beyond the limits of national jurisdiction."

682 Siehe ausführlich hierzu L.-A. Duvic-Paoli, The Prevention Principle in International Environmental Law, 2018, Part I/2; R. Wolfrum, General International Law (Principles, Rules, and Standards), in: ders. (Hg.), MPEPIL 2010, http:// www.mpepil.com, Rn. 48. P. Sands/J. Peel hingegen unterscheiden zwischen dem Grundsatz der Schadensprävention, dessen selbstständiger Schutzzweck die Verringerung von Umweltschäden sei, und dem Anwendungsbereich von Prinzip 21, das das Schädigungsverbot spiegele. Trotzdem gestehen sie den Konzepten eine enge Beziehung zu. P. Sands/J. Peel, Principles of International Environmental Law, 2018, S. 206, $212 \mathrm{f}$.

683 P. Birnie/A. E. Boyle/C. Redgwell, International Law and the Environment, 2009, S. 137 f.; P. Sands/J. Peel, Principles of International Environmental Law, 2018, S. $211 \mathrm{ff}$.

684 P. W. Birnie/A. E. Boyle/C. Redgwell International Law and the Environment, 2009, S. 147; K. A. Brent, The Certain Activities case, Asia Pac. J. Envtl. L., 20 
als „Nachkomme“ der Trail Smelter-Entscheidungen bezeichnet. ${ }^{655}$ Zwar sind die Erklärungen von Stockholm und Rio selbst lediglich rechtlich unverbindlich, jedoch gelten sie als bedeutendste universal anerkannte Erklärungen über allgemeine Rechte und Pflichten von Staaten im Umweltkontext. ${ }^{686}$ Die darin begründeten Grundsätze gewinnen zunehmende Bedeutung im Umweltvölkerrecht. ${ }^{687}$ So erfolgt die Ausgestaltung umweltvölkerrechtlicher Übereinkommen vielfach unter Bezugnahme auf Prinzip 21 der Stockholm Deklaration und Prinzip 2 der Rio Deklaration. ${ }^{688}$ Wie gezeigt, findet sich der Grundsatz der Schadensprävention nicht nur in umweltvölkerrechtlichen Übereinkommen, sondern auch in der Judikatur des IGH. ${ }^{689}$ Der Gerichtshof bestätigte in seiner Pulp MillsEntscheidung aus dem Jahr 2010, dass Staaten nach dem Grundsatz der Schadensprävention nicht nur gehalten sind, keine grenzüberschreitenden Schäden zu verursachen, sondern auch, alle möglichen und zumutbaren Mittel zur Schadensprävention zu ergreifen und bei der Durchsetzung sowie administrativen Kontrolle über öffentliche und private Betreiber Wachsamkeit walten zu lassen. ${ }^{690}$ Es handelt sich demnach um eine verhaltensbezogene Gewährleistungspflicht (obligation to ensure) ${ }^{691}$ Sie greift,

(2017), S. 28 (36); A. Proelß, Prinzipien des internationalen Umweltrechts, in: ders. (Hg.), Internationales Umweltrecht, 2017, S. 69 (76 Rn. 10).

685 S. C. McCaffrey, Of Paradoxes, Precedents, and Progeny, in: R. M. Bratspies/R. A. Miller (Hg.), Transboundary Harm in International Law, 2006, S. 34 (41). Für einen Vergleich der Formulierung in der Trail Smelter-Entscheidung mit Prinzip 21 der Stockholm Deklaration siehe O. Kimminich, Völkerrechtliche Haftung für das Handeln Privater im Bereich des internationalen Umweltschutzes, AVR 22 (1984), S. 241 (249f.).

686 P. W. Birnie/A. E. Boyle/C. Redgwell International Law and the Environment, 2009, S. 112; A. Proelß, Prinzipien des internationalen Umweltrechts, in: ders. (Hg.), Internationales Umweltrecht, 2017, S. 69 (71 f. Rn. 1).

687 The Iron Rhine ("Ijzeren Rijn") Railway Arbitration (Belgium v. The Netherlands), Award of 24 May 2005, UNRIAA XXVII (2005), S. 35 (66f. Rn. 59, 116 Rn. 222); P. Sands/J. Peel, Principles of International Environmental Law, 2018, S. 211.

688 The Iron Rhine ("Ijzeren Rijn") Railway Arbitration (Belgium v. The Netherlands), Award of 24 May 2005, UNRIAA XXVII (2005), S. 35 (66f. Rn. 59); $P$. Sands/J. Peel, Principles of International Environmental Law, 2018, S. 209.

689 Siehe 1. Kapitel B. I.

690 IGH, Pulp Mills on the River Uruguay (Argentina v. Uruguay), Judgment of 20 April 2010, ICJ Reports 2010, S. 14 (79 Rn. 197); vgl. auch IGH, GabcíkovoNagymaros Project (Hungary v. Slovakia), Judgment of 25 September 1997, ICJ Reports 1997, S. 7 (77 f. Rn. 140).

691 A. Proelß, Prinzipien des internationalen Umweltrechts, in: ders. (Hg.), Internationales Umweltrecht, 2017, S. 69 (76 Rn. 10). 


\section{Kapitel: Staatenhaftung in der Systematik des Völkerrechts}

sobald ein bekanntes oder objektiv bestimmbares Risiko eines erheblichen grenzüberschreitenden Schadens besteht. ${ }^{692}$ Der IGH konkretisierte weiter, dass Staaten, basierend auf den Grundsätzen der Prävention und der gebotenen Sorgfalt, Benachrichtigungs-, Informations- sowie Rücksprachepflichten treffen und eine Umweltverträglichkeitsprüfung durchzuführen ist, wenn das Risiko eines grenzüberschreitenden Schadens durch ein Vorhaben besteht. ${ }^{693}$ Diese Ausführungen des IGH stimmen mit dem Verständnis der Völkerrechtskommission von der Schadensvermeidungspflicht, die aus dem Schädigungsverbot fließt, überein. ${ }^{694}$ Der Grundsatz der Schadensprävention beinhaltet eine verfahrensbezogene und eine materielle Dimension, ${ }^{695}$ deren Einhaltung am Maßstab der gebotenen Sorgfalt unter Beachtung der technischen Standards zu beurteilen ist. ${ }^{696}$ Die materielle Dimension erfasst vornehmlich die Implementierung der erforderlichen regulatorischen und politischen Maßnahmen, ${ }^{697}$ während sich die verfahrensbezogene Pflicht auf Kooperationspflichten, wie sie bereits im Grundsatz der guten Nachbarschaft verankert sind, bezieht. Als Bindeglied zwischen den beiden Dimensionen erweist sich nach Ansicht des IGH die Pflicht zur Durchführung einer Umweltverträglichkeitsprüfung. Diese Elemente der Schadensvermeidungspflicht werden in weiteren Entscheidungen des IGH thematisiert ${ }^{698}$ und direkt oder indirekt durch Richt-

692 A. Epiney/M. Scheyli, Strukturprinzipien des Umweltvölkerrechts, 1998, S. 112 m.w.N.; W. Heintschel von Heinegg, Legal Implications of Territorial Sovereignty in Cyberspace, in: C. Czosseck/R. Ottis/K. Ziolkowski (Hg.), 2012 4th International Conference on Cyber Conflict, 2012, S. 7 (16); T. Marauhn, Customary Rules of International Environmental Law, in: K. Ziolkowski (Hg.), Peacetime Regime for State Activities in Cyberspace, 2013, S. 465 (472 f.); A. Proelß, Prinzipien des internationalen Umweltrechts, in: ders. (Hg.), Internationales Umweltrecht, 2017, S. 69 (79 f. Rn. 15); K. Ziolkowski, General Principles of International Law as Applicable in Cyberspace, in: dies. (Hg.), Peacetime Regime for State Activities in Cyberspace, 2013, S. 135 (165).

693 IGH, Pulp Mills on the River Uruguay (Argentina v. Uruguay), Judgment of 20 April 2010, ICJ Reports 2010, S. 14 (55 f. Rn. 101, 72 f. Rn. 204).

694 Siehe 1. Kapitel A. II. 2. b).

695 A. Proelß, Prinzipien des internationalen Umweltrechts, in: ders. (Hg.), Internationales Umweltrecht, 2017, S. 69 ( 81 ff. Rn. 18 ff.).

696 Id., S. 69 (78 Rn. 12).

697 The Iron Rhine ("Ijzeren Rijn”) Railway Arbitration (Belgium v. The Netherlands), Award of 24 May 2005, UNRIAA XXVII (2005), S. 66 f., Rn. 59, 116, Rn. 222; vgl. auch P. Birnie/A. E. Boyle/C. Redgwell, International Law and the Environment, 2009, S. $137 \mathrm{ff}$.

Siehe 1. Kapitel B. I. 
linien oder politische Erklärungen sowie durch die zahlreichen Übereinkünfte zu diversen Umweltmedien bzw. Umweltaktivitäten bestätigt. ${ }^{699}$

Welcher völkerrechtliche Status dieser zwischenstaatlichen Pflicht zur Schadensprävention zukommt, klingt im Nuklearwaffen-Gutachten des IGH aus dem Jahr 1996 an. Hiernach ist die allgemeine Verpflichtung von Staaten, zu gewährleisten, dass Aktivitäten unter ihrer Hoheitsgewalt und Kontrolle die Umwelt anderer Staaten oder Gebiete jenseits der Grenzen des Bereichs nationaler Hoheitsbefugnisse respektieren, ein Bestandteil "of the corpus of international law relating to the environment". ${ }^{700}$ Allerdings spezifizierte der IGH in seinem Gutachten zunächst nicht, welcher Rechtsquelle die Präventionspflicht zuzuordnen ist. ${ }^{701}$ Zum größten Teil wurde und wird der Grundsatz der Schadensprävention als völkergewohnheitsrechtliche Pflicht begriffen. ${ }^{702}$ Jahre später legte der IGH in seiner Pulp Mills-Entscheidung unter Bezugnahme auf das Nuklearwaffen-Gutachten dann auch fest, dass der Grundsatz der Schadensprävention - als gewohnheitsrechtliche Regel - seinen Ursprung in der gebotenen Sorgfalt hat. ${ }^{703}$ Andererseits wird festgestellt, dass sich die Pflicht zur Schadensprävention aus internationalen Übereinkünften herauslösen lässt und auch außerhalb dieser Regime als allgemeiner Rechtsgrundsatz zur Anwendung gelangt. ${ }^{704}$ So bestimmte das Schiedsgericht im Iron Rhine-Fall aus dem Jahr 2005, dass es sich bei der Präventionspflicht um einen allgemeinen Rechtsgrundsatz handelt, der nicht nur bei unreglementierten Aktivitäten, sondern auch bei Aktivitäten zur Umsetzung von spezifischen Verträgen Anwendung findet. ${ }^{705}$ In Anbetracht der Tatsache, dass die Schadensprävention im Rahmen der internationalen Beziehungen und internationalen

699 P. Sands/J. Peel, Principles of International Environmental Law, 2018, S. 213.

700 IGH, Legality of the Threat or Use of Nuclear Weapons, Advisory Opinion of 8 July 1996, ICJ Reports 1996, S. 226 (241 f. Rn. 29).

701 Vgl. J. E. Viñuales, The Contribution of the International Court of Justice to the Development of International Environmental Law, Fordham ILJ 32 (2008), S. $232(246)$.

702 A. Epiney, Das „Verbot erheblicher grenzüberschreitender Umweltbeeinträchtigungen“, AVR 33 (1995), S. 309 (318) m.w.N.

703 IGH, Pulp Mills on the River Uruguay (Argentina v. Uruguay), Judgment of April 2010, ICJ Reports 2010, S. 14 (55 f. Rn. 101, 72 f. Rn. 204); siehe auch IGH, Gabcíkovo-Nagymaros Project (Hungary v. Slovakia), Judgment of 25 September 1997, ICJ Reports 1997, S. 7 (38 Rn. 53).

704 R. Wolfrum, General International Law (Principles, Rules, and Standards), in: ders. (Hg.), MPEPIL 2010, http://www.mpepil.com, Rn. 51, 55.

705 The Iron Rhine ("Ijzeren Rijn") Railway Arbitration (Belgium v. The Netherlands), Award of 24 May 2005, UNRIAA XXVII (2005), S. 35 (66f. Rn. 58 ff.). 
Judikatur zur Lösung umweltbezogener Konfliktlagen herangezogen wird, ist jedenfalls festzuhalten, dass der Grundsatz der Schadensprävention bindendes Völkerrecht im Umweltkontext darstellt. ${ }^{706}$

\section{Grundsatz angemessener Nutzung}

Neben der Pflicht zur Schadensprävention im zwischenstaatlichen Bereich konkretisiert der Grundsatz angemessener Nutzung (equitable use principle) das Schädigungsverbot in Bezug auf Gemeinschaftsgüter (common goods) und die dort vorhandenen Ressourcen. ${ }^{707}$ Der Begriff Gemeinschaftsgut gilt als Oberbegriff, unter den auch die Konzepte Gemeinschaftsräume (common areas), gemeinsames Erbe (common heritage) und gemeinsames Interesse (common concern) gefasst werden können. ${ }^{708}$ Während diese Konzepte unterschiedliche Nuancen aufweisen und im Kontext ihres Regelungsbereiches zu betrachten sind, folgt aus dem Gemeinschaftscharakter die staatliche Pflicht zur Vermeidung von Schäden an diesen Gemeinschaftsgütern. ${ }^{709}$ Nach dem Grundsatz der angemessenen Nutzung sind „Gemeinsame Umweltmedien (...) so zu nutzen (...), daß den anderen Staaten eine angemessene Nutzung dieser Medien verbleibt, wie immer dieses, angemessen' im Einzelfall zu bestimmen ist. " ${ }^{\text {"110 }}$ Gemeinschaftsgüter, wie etwa Weltraum, Meeresboden, Hohe See und Antarktis, befinden sich jenseits der Grenzen des Bereichs nationaler Hoheitsbefugnisse und bedürfen in Anbetracht der Tatsache, dass sie von allen Staaten genutzt aber auch geschädigt werden können, des kollektiven Schutzes. ${ }^{711}$ Dieses

706 Vgl. O. McIntryre, The Role of Customary Rules and Principles of International Environmental Law in the Protection of Shared International Freshwater Resources, Natural Resources Journal 46 (2006), S. 157 (169 f.) m.w.N.; M. V. Soto, General Principles of International Environmental Law, ILSA J. Intl. \& Comp. L. 3 (1996), S. 193 (200).

707 Eingehend zum Schädigungsverbot an Gemeinschaftsgütern J. Bäumler, Das Schädigungsverbot im Völkerrecht, 2017, Teil II, 5. Kapitel.

708 So J. Bäumler, Das Schädigungsverbot im Völkerrecht, 2017, S. 97.

709 P. Sands/J. Peel, Principles of International Environmental Law, 2018, S. 245 f.

710 L. Gündling, Verantwortlichkeit der Staaten für grenzüberschreitende Umweltbeeinträchtigungen, ZaöRV 45 (1985), S. 265 (283).

711 J. Bäumler, Das Schädigungsverbot im Völkerrecht, 2017, S. 98; J. Brunnée, Common Areas, Common Heritage, Common Concern, in: D. Bodansky/dies./E. Hey (Hg.), The Oxford Handbook of International Environmental Law, 2007, S. $550(553,557,564)$. Die Hohe See, der Weltraum und die Umwelt sind überdies als gemeinsames Erbe der Menschheit anerkannt und begründen damit 
Bedürfnis bestätigen Prinzip 21 der Stockholm Deklaration und Prinzip 2 der Rio Deklaration sowie das Nuklearwaffen-Gutachten des IGH, indem bei der Schadensvermeidungspflicht nicht nur auf das fremde Territorium, sondern auch auf alle Gebiete außerhalb des eigenen Territoriums Bezug genommen wird.712 Dieser Ansatz findet sich in zahlreichen internationalen Übereinkünften, Resolutionen der Vereinten Nationen und staatlichen Erklärungen. ${ }^{713}$ Soweit eingewandt wird, außerhalb vertraglich normierter Bereiche bestünden im Rahmen hoheitsfreier Räume keine Anknüpfungspunkte, um gemeinschaftsgutsbezogene Rechte und Pflichten einfordern zu können, ${ }^{714}$ ist dem entgegenzuhalten, dass aufgrund der souveränen Gleichheit der Staaten auch ein gleiches Recht an der angemessenen Nutzung gemeinsamer Ressourcen bestehen muss und sich dies in entsprechenden Rechten und Pflichten der Staaten verdichtet. ${ }^{715}$ Die geäußerten Bedenken vermögen jedenfalls im Bereich geteilter Ressourcen (shared resources) nicht zu überzeugen, da der Ressourcenschutz hier Umweltmedien betrifft, die der territorialen Souveränität eines Staates unterfallen und gleichzeitig grenzüberschreitender Natur sind, und damit die territoriale Integrität anderer Staaten betreffen. Der Grundsatz angemessener Nutzung erlaubt Staaten unter Beachtung ihrer souveränen Rechte und gemeinsamen Interessen, die Nutzung geteilter Ressourcen in Einklang mit ihren internationalen Präventionspflichten zu regulieren. ${ }^{716}$ Daraus ergeben sich insbesondere verfahrensbezogene Informations- und Rücksprachepflichten hinsichtlich aller Nutzungsmöglichkeiten und Nut-

eine gemeinsame Verantwortung aller Staaten. S. Kadelbach, Ethik des Völkerrechts unter Bedingungen der Globalisierung, ZaöRV 64 (2004), 1 (11).

712 J. Bäumler, Das Schädigungsverbot im Völkerrecht, 2017, S. 100.

713 K. Odendahl, Die Umweltpflichtigkeit der Souveränität, 1998, S. 231 f. m.w.N.

714 Siehe E. Wins, Weltraumhaftung im Völkerrecht, 2000, S. $202 \mathrm{ff}$. Der IGH hat indes in dem Verfahren Whaling in the Antarctic die Durchsetzung von Gemeinschaftsinteressen gestärkt, indem er seine Zuständigkeit für das von Australien gegen Japan eingeleitete Verfahren bejahte, obgleich Australien keine eigene Rechtsverletzung geltend machte, die über die Verletzung aller anderen Vertragsparteien der International Convention for the Regulation of Whaling hinausging. IGH, Whaling in the Antarctic (Australia v. Japan; New Zealand intervening), Judgment of 31 March 2014, ICJ Reports 2014, S. 226 (242 ff. Rn. 30 ff.). Siehe hierzu J. Bäumler, Das Schädigungsverbot im Völkerrecht, 2017, S. 114.

715 SoJ. Bäumler, Das Schädigungsverbot im Völkerrecht, 2017, S. 102 unter Bezugnahme auf L. Gündling, Verantwortlichkeit der Staaten für grenzüberschreitende Umweltbeeinträchtigungen, ZaöRV 45 (1985), S. 265 (265 ff.).

716 J. Brunnée/T. Meshel, Teaching an Old Law New Tricks, GYIL 58 (2015), S. 129 (154). 
zungsvorhaben. ${ }^{717}$ Der Grundsatz angemessener Nutzung ist nicht nur im Umweltvölkerrecht verankert. Art. 2 Abs. 1 VN-Charta und die „Friendly Relations-Deklaration"718 bestimmen unter Betonung der souveränen Gleichheit von Staaten, dass Staaten grundsätzlich die gleichen Rechte und Pflichten haben. Der Grundsatz der angemessenen Nutzung wird mittlerweile als allgemeiner Rechtsgrundsatz anerkannt, dem auch ein völkergewohnheitsrechtlicher Status zugeschrieben wird. ${ }^{719}$

\section{Vorsorgegrundsatz}

Der Vorsorgegrundsatz (precautionary principle) wird auf das deutsche Umweltrecht zurückgeführt ${ }^{720}$ und aus Konzepten verschiedener nationaler, insbesondere europäischer Rechtsordnungen hergeleitet. ${ }^{721}$ Der Kerngehalt des Vorsorgegrundsatzes ergibt sich aus einem Vergleich zum Grundsatz der Schadensprävention. Letzterer begründet die Pflicht, vorhersehbare Umweltschäden durch risikobehaftete Aktivitäten zu vermeiden. Der Vorsorgegrundsatz hingegen bezieht sich auf Konstellationen, in denen Gefahrenpotenzial und Auswirkungen einer fraglichen Aktivität wissenschaftlich nicht sicher prognostizierbar sind. ${ }^{722}$

717 Vgl. zu diesen Verfahrenspflichten K. Odendahl, Die Umweltpflichtigkeit der Souveränität, 1998, S. $179 \mathrm{ff}$.

718 UN Doc. A/RES/2625 (XXV) (1970).

719 L. del Castillo-Laborde, Equitable Utilization of Shared Resources, in: R. Wolfrum (Hg.), MPEPIL 2010, http://www.mpepil.com, Rn. 25; K. Ziolkowski, General Principles of International Law as Applicable in Cyberspace, in: dies. (Hg.), Peacetime Regime for State Activities in Cyberspace, 2013, S. 135 (179).

720 L. Gündling, The Status in International Law of the Principle of Precautionary Action, in: D. Freestone/T. IJlstra (Hg.), The North Sea, 1990, S. 23 (23 f.); M. Pyhälä/A. C. Brusendorff/H. Paulomäki, The precautionary principle, in: M. Fitzmaurice/D. M. Ong/P. Merkouris (Hg.), Research Handbook on International Environmental Law, 2010, S. 203 (205).

721 B. Arndt, Das Vorsorgeprinzip im EU-Recht, 2009, S. 11 ff.; M. Schröder, Precautionary Approach/Principle, in: R. Wolfrum (Hg.), MPEPIL 2014, http://www. mpepil.com, Rn. 6.

722 S. A. Atapattu, Emerging Principles of International Environmental Law, 2006, S. 206; G. Hafner/I. Buffard, Obligations of Prevention and the Precautionary Principle, in: J. Crawford/A. Pellet/S. Olleson (Hg.), The Law of International Responsibility, 2010, S. 521 (528f.); M. Pyhälä/A. C. Brusendorff/H. Paulomäki, The precautionary principle, in: M. Fitzmaurice/D. M. Ong/P. Merkouris (Hg.), Research Handbook on International Environmental Law, 2010, S. 203 (203). 
Auf der Ebene der EU genießt der Grundsatz bereits aufgrund von Art. 191 Abs. 2 des Vertrages über die Arbeitsweise der EU (AEUV) Verbindlichkeit, während die völkerrechtliche Wirkung des Grundsatzes zunächst unklar scheint. ${ }^{723}$ Der Grundsatz fand erstmals bei den Konferenzen zum Nordseeschutz in den 1980er-Jahren Beachtung und wurde in der Folge in zahlreichen umweltvölkerrechtlichen Übereinkommen und Richtlinien in Bezug genommen. ${ }^{724}$ Allerdings ist die einzelvertragliche Ausgestaltung nicht konsistent. ${ }^{725}$ In einigen Texten, etwa im Fischereirecht, ist nicht von einem Vorsorgegrundsatz, sondern von einem Vorsorgeansatz die Rede. ${ }^{726}$ Dies führt dazu, dass der Gedanke der Vorsorge in einigen Bereichen keine rechtliche Handlungsanweisung begründet, sondern als Methode zur Problemlösung interpretiert wird. ${ }^{727}$ Im Gegensatz zu diesem minimalistischen Verständnis soll aus dem Vorsorgegrundsatz eine Beweislastumkehr zulasten des Staates oder des Akteurs folgen, von dem die potenziell schadensauslösende Aktivität ausgeht. ${ }^{728}$ Diese unterschiedlichen Ansätze erschweren die Antwort auf die Frage, ob es sich bei dem Vorsorgegrundsatz um einen allgemeinen Rechtsgrundsatz und/oder Völkergewohnheitsrecht handelt. ${ }^{729}$ Wie im Rahmen der Rechtsprechungsanalyse deutlich wurde, legen allerdings bestimmte internationale Entscheidungen zu grenzüberschreitenden Schadensfällen eine völkerrechtliche Anerkennung und Geltung des Vorsorgegrundsatzes nahe. So erklärte der IGH in der Pulp Mills-Entscheidung aus dem Jahr 2010, dass der Vorsorgeansatz im Rahmen der Interpretation und Anwendung

723 E. Hey, The Precautionary Concept in Environmental Policy and Law, GILR 4 (1992), S. 303 (303).

724 A. Proelß, Prinzipien des internationalen Umweltrechts, in: ders. (Hg.), Internationales Umweltrecht, 2017, S. 69 (84 Rn. 24).

725 Id., S. 69 (87 Rn. 29).

726 A. von Arnauld, Völkerrecht, 2019, S. 628 f., Rn. 895; N. Matz-Lück, Meeresschutz, in: A. Proelß (Hg.), Internationales Umweltrecht, 2017, S. 393 (416 Rn. 53).

727 E. Hey, The Precautionary Concept in Environmental Policy and Law, GILR 4 (1992), S. 303 (304).

728 A. Proelß, Prinzipien des internationalen Umweltrechts, in: ders. (Hg.), Internationales Umweltrecht, 2017, S. 69 (87 Rn. 27).

729 J. Cameron/J. Abouchar, The Status of the Precautionary Principle in International Law, in: D. Freestone/E. Hey (Hg.), The Precautionary Principle and International Law, 1996, S. 29 (36 ff.); G. Hafner/I. Buffard, Obligations of Prevention and the Precautionary Principle, in: J. Crawford/A. Pellet/S. Olleson (Hg.), The Law of International Responsibility, 2010, S. 521 (530 ff.); M. Schröder, Precautionary Approach/Principle, in: R. Wolfrum (Hg.), MPEPIL 2014, http://www. mpepil.com, Rn. 16 ff. 


\section{Kapitel: Staatenhaftung in der Systematik des Völkerrechts}

der streitgegenständlichen Bestimmung von Relevanz sein könne, auch wenn er nicht zu einer Beweislastumkehr führe. ${ }^{730}$ Unter Bezugnahme auf diese Feststellung des IGH bestätigte die ITLOS-Meeresbodenkammer in ihrem Gutachten aus dem Jahr 2011 zu den Pflichten und Verantwortlichkeiten von befürwortenden Staaten, dass der Vorsorgeansatz in eine steigende Zahl internationaler Verträge und Instrumente einbezogen wurde, von denen viele die Formulierung des Prinzips 15 der Rio Deklaration widerspiegeln. Dies - so die Kammer - bedeute eine Entwicklung hin zum gewohnheitsrechtlichen Status des Ansatzes. ${ }^{731}$ Wie gezeigt, bekräftigte auch der IACtHR in seinem Gutachten aus dem Jahr 2017 die wegweisende Bedeutung von Prinzip 15 der Rio Deklaration. ${ }^{732}$ Das Prinzip 15 führt diese Ansätze zur Bestimmung des Bedeutungsgehaltes des Vorsorgegrundsatzes zusammen und besagt, dass wissenschaftliche Unsicherheiten bei der Beurteilung des Eintritts von Umweltgefährdungen im Falle schwerwiegender oder bleibender Schäden keinen Aufschub von kostenwirksamen Maßnahmen zur Vermeidung rechtfertigen. ${ }^{733}$ Der Vorsorgegrundsatz verzichtet auf wissenschaftliche Gewissheit und erfasst Unsicherheiten in den Vorgängen selbst, ${ }^{734}$ wenn etwa die Aktivitäten der Akteure „nicht bekannt oder nicht beobachtbar (...) oder (...) von außen durch einen Dritten nicht wahrnehmbar sind."735 Daraus folgt zwar kein konkreter Maßnahmenkatalog, ${ }^{736}$ allerdings sind frühzeitig geeignete

730 IGH, Pulp Mills on the River Uruguay (Argentina v. Uruguay), Judgment of 20 April 2010, ICJ Reports 2010, S. 14 (71 Rn. 164).

731 ITLOS, Responsibilities and obligations of states sponsoring persons and entities with respect to activities in the Area, Advisory Opinion of 1 February 2011, ITLOS Case No. 17, ITLOS Reports 2011, S. 10 (47 Rn. 135).

732 IACtHR, Advisory Opinion on Environment and Human Rights, S. 71, Rn. 177, S. 72, Rn. 180.

733 C. Erben, Das Vorsorgegebot im Völkerrecht, 2005, S.35, $41 \mathrm{f} . ;$ A. Kiss, The Rights and Interests of Future Generations and the Precautionary Principle, in: D. Freestone/E. Hey (Hg.), The Precautionary Principle and International Law, 1996, S. 19 (27); A. Proelß, Prinzipien des internationalen Umweltrechts, in: ders. (Hg.), Internationales Umweltrecht, 2017, S. 69 (87 Rn. 30).

734 H. Krieger, Krieg gegen anonymous, AVR 50 (2012), S. 1 (6).

735 I. Spiecker genannt Döhmann, Staatliche Entscheidungen unter Unsicherheit, 2000, S. 9.

736 G. Hafner/I. Buffard, Obligations of Prevention and the Precautionary Principle, in: J. Crawford/A. Pellet/S. Olleson (Hg.), The Law of International Responsibility, 2010, S. 521 (529); M. Schröder, Precautionary Approach/Principle, in: R. Wolfrum (Hg.), MPEPIL 2014, http://www.mpepil.com, Rn. 11. 
regulatorische Maßnahmen zu ergreifen. ${ }^{737}$ Dazu zählen strategische, politische, organisatorische, administrative und legislative Vorkehrungen. ${ }^{738}$ In Anknüpfung an die völkerrechtliche Praxis stellen die Durchführung von Umweltverträglichkeitsprüfungen auf verfahrensrechtlicher Ebene ${ }^{739}$ und die Anwendung der besten Umweltschutzpraktiken auf materieller Ebene die Umsetzung des Vorsorgegrundsatzes sicher. ${ }^{70}$ Dabei geht nicht nur um die beste verfügbare Technik, die bei der Schadensprävention zum Einsatz kommt, sondern um die beste Kombination von Einsatzmitteln. So zählen auch umweltbezogene Kontrollmaßnahmen und weitergehende Strategieentwicklungen zum Pflichtenkreis. ${ }^{741}$

In Anbetracht der Tatsache, dass der Vorsorgegedanke in einer beträchtlichen Zahl von internationalen Dokumenten verankert ist und im Wesentlichen als eine Weiterentwicklung des völkergewohnheitsrechtlichen Grundsatzes der Schadensprävention gilt, ${ }^{742}$ stellen die Diskussionen um dessen völkerrechtliche Geltung in der Tat „Spiegelfechtereien“743 dar. $^{744}$ Indessen kann der Grundsatz in seinen unterschiedlichen Formulierungen Anknüpfungspunkte zur Begründung von staatlichen Pflichten in Bezug

737 A. Proelß, Prinzipien des internationalen Umweltrechts, in: ders. (Hg.), Internationales Umweltrecht, 2017, S. 69 (87 Rn. 30); K. Ziolkowski, General Principles of International Law as Applicable in Cyberspace, in: dies. (Hg.), Peacetime Regime for State Activities in Cyberspace, 2013, S. 135 (169).

738 K. Ziolkowski, General Principles of International Law as Applicable in Cyberspace, in: dies. (Hg.), Peacetime Regime for State Activities in Cyberspace, 2013, S. 135 (169).

739 U. Beyerlin/T. Marauhn, International Environmental Law, 2011, S. 54; A. Epiney/M. Scheyli, Strukturprinzipien des Umweltvölkerrechts, 1998, S. 93 f.

740 A. Epiney/M. Scheyli, Strukturprinzipien des Umweltvölkerrechts, 1998, S. 94; H. Jessen, Staatenverantwortlichkeit und seevölkerrechtliche Haftungsgrundsätze für Umweltschäden durch Tiefseebodenbergbau, ZUR 2 (2012), S. 71 (77).

741 H. Jessen, Staatenverantwortlichkeit und seevölkerrechtliche Haftungsgrundsätze für Umweltschäden durch Tiefseebodenbergbau, ZUR 2 (2012), S. 71 (77 f.).

742 C. Erben, Das Vorsorgegebot im Völkerrecht, 2005, S. 211; J. Cameron/J. Abouchar, The Status of the Precautionary Principle in International Law, in: D. Freestone/E. Hey (Hg.), The Precautionary Principle and International Law, 1996, S. 29 (46); A. Kiss, The Rights and Interests of Future Generations and the Precautionary Principle, in: D. Freestone/E. Hey (Hg.), The Precautionary Principle and International Law, 1996, S. 19 (27).

743 A. von Arnauld, Völkerrecht, 2019, S. 629, Rn. 895.

744 Der Vorsorgegrundsatz ist, wenn nicht schon als bestehendes, dann zumindest als in der Entstehung befindliches Umweltvölkergewohnheitsrecht anzuerkennen. K. Ziolkowski, General Principles of International Law as Applicable in Cyberspace, in: dies. (Hg.), Peacetime Regime for State Activities in Cyberspace, 2013, S. 135 (167) m.w.N. 
auf potenziell gefährliche Aktivitäten bieten und eine Konkretisierung in anderen Regelungsbereichen leiten. ${ }^{745}$

\section{Verursachergrundsatz}

Während die zuvor beleuchteten Umweltgrundsätze vorwiegend die Komponente der Schadensvermeidung im Konzept der Staatenhaftung untermauern, bietet ein Blick auf den Verursachergrundsatz (polluter pays principle) Aufschluss über die Rechtsgeltung der Komponente der Schadenskompensation. Hiernach werden dem Urheber, das heißt dem Verursacher die Kosten der Umweltverschmutzung zugerechnet. ${ }^{76}$ Der Verursachergrundsatz ist in zahlreiche nationale Rechtsysteme sowie in die Umweltpolitik der Europäischen Union inkorporiert und ist auf völkerrechtlicher Ebene durch eine Reihe an Empfehlungen der Organisation für wirtschaftliche Zusammenarbeit und Entwicklung umschrieben worden. ${ }^{77}$ Seither hat er Eingang in zahlreiche internationalen Instrumente zum Umweltschutz gefunden. ${ }^{748}$ Im Vergleich zu den zuvor besprochenen umweltvölkerrechtlichen Grundsätzen fehlt es allerdings an einer konsistenten Verbreitung und Umsetzung des Verursachergrundsatzes, ${ }^{749}$ so dass zu zeigen ist, inwiefern dieser überhaupt einer normativen Verdichtung, hin zu einem allgemeinen Rechtsgrundsatz, zugänglich ist. ${ }^{750}$ Prinzip 16 Rio Deklaration spiegelt den Kern des Verursachergrundsatzes im Wesentlichen wider, indem er Staaten auffordert „die Internalisierung von Umweltkosten und den Einsatz wirtschaftlicher Instrumente zu fördern, wobei sie unter gebührender Berücksichtigung des öffentlichen Interesses und unter

745 H. Krieger, Krieg gegen anonymous, AVR 50 (2012), S. 1 (5); T. Marauhn, Customary Rules of International Environmental Law, in: K. Ziolkowski (Hg.), Peacetime Regime for State Activities in Cyberspace, 2013, S. 465 (475 f., 484).

746 U. Beyerlin/T. Marauhn, International Environmental Law, 2011, S. 58; A. Epiney/M. Scheyli, Strukturprinzipien des Umweltvölkerrechts, 1998, S. 152 m.w.N., S. 95 f.; T. Maraubn, Customary Rules of International Environmental Law, in: K. Ziolkowski (Hg.), Peacetime Regime for State Activities in Cyberspace, 2013, S. 465 (476f.).

747 A. E. Boyle, Polluter Pays, in: R. Wolfrum (Hg.), MPEPIL 2009, http://www.mp epil.com, Rn. 1, 6; R. Chemain, The 'Polluter Pays' Principle, in: J. Crawford/A. Pellet/S. Olleson (Hg.), The Law of International Responsibility, 2010, S. 877 (878, 881); N. de Sadeleer, Environmental Principles, 2002, S. $26 \mathrm{ff}$.

748 A. Epiney/M. Scheyli, Strukturprinzipien des Umweltvölkerrechts, 1998, S. 153.

749 Id., S. $153 \mathrm{f}$.

750 Id., S. 156. 
Vermeidung von Verzerrungen im Welthandel und bei den internationalen Investitionen den Ansatz verfolgen sollten, dass grundsätzlich der Verursacher die Kosten der Verschmutzung zu tragen hat." Die Bestimmung verdeutlicht, dass der Verursachergrundsatz seinen Ursprung in ökonomischen Konzepten der Kosteninternalisierung, wirtschaftlichen Gerechtigkeit und Effizienz hat ${ }^{751}$ und damit die Frage der Kostenzurechnung nicht allein durch eine rechtliche Betrachtung, sondern unter Bezugnahme auf ökonomische Gesichtspunkte beantwortet werden muss. ${ }^{752}$ Nach dem Verursachergrundsatz erfordert ein effizientes Umweltmanagement, dass externe Effekte der Umweltbelastung in die Geschäftskosten jedes Schadensverursachers selbst internalisiert werden. ${ }^{753}$ Diese Kosteninternalisierung ist ferner unter Gesichtspunkten der wirtschaftlichen Gerechtigkeit einheitlich zu gestalten, damit keine öffentlichen Gelder genutzt werden, um private Maßnahmen zur Schadensprävention zu subventionieren und den betroffenen Unternehmen damit einen bedeutenden Preisvorteil auf dem internationalen Markt zu verschaffen. ${ }^{754}$

Die Kostentragungspflicht ist nicht eindeutig bestimmt. ${ }^{755}$ Die Pflicht beschränkte sich ursprünglich allein auf präventive Maßnahmen der Vermeidung, Kontrolle und Verringerung von Verschmutzungen, ${ }^{756}$ wurde im Folgenden aber auch im Sinne einer Kompensationspflicht konkretisiert. ${ }^{77}$ Erwägungen der wirtschaftlichen Gerechtigkeit sprechen für ein umfassendes Verständnis. Der Betreiber einer gefährlichen Aktivität erzielt zum einen wirtschaftliche Vorteile durch diese Aktivität und ruft gleichzeitig ein Risiko hervor, für welches er im Schadensfall einzustehen hat.

751 Id., S. 155; S. E. Gaines, The Polluter-Pays Principle, Tex. ILJ 26 (1991), S. 463 $(469,477,481)$.

752 S. E. Gaines, The Polluter-Pays Principle, Tex. ILJ 26 (1991), S. 463 (469).

753 A. E. Boyle, Polluter Pays, in: R. Wolfrum (Hg.), MPEPIL 2009, http://www.m pepil.com, Rn. 1; S. E. Gaines, The Polluter-Pays Principle, Tex. ILJ 26 (1991), S. $463(469)$.

754 A. Epiney/M. Scheyli, Strukturprinzipien des Umweltvölkerrechts, 1998, S. 155; S. E. Gaines, The Polluter-Pays Principle, Tex. ILJ 26 (1991), S. 463 (466, 470).

755 A. Epiney/M. Scheyli, Strukturprinzipien des Umweltvölkerrechts, 1998, S. 156 f.

756 S. E. Gaines, The Polluter-Pays Principle, Tex. ILJ 26 (1991), S. 463 (467 f.) unter Hinweis auf OECD Council, Guiding Principles Concerning International Economic Aspects of Environmental Policies, Recommendation C, 26 May 1972.

757 R. Chemain, The 'Polluter Pays' Principle, in: J. Crawford/A. Pellet/S. Olleson (Hg.), The Law of International Responsibility, 2010, S. 877 (881 f.); S. E. Gaines, The Polluter-Pays Principle, Tex. ILJ 26 (1991), S. 463 (482 f.); N. de Sadeleer, Les principes du pollueur-payeur, de prévention et de précaution, Essai sur la genèse et la portée juridique de quelques principes du droit de l'environnement, 1999, S. 69. 


\section{Kapitel: Staatenhaftung in der Systematik des Völkerrechts}

Die Verpflichtung zur Entschädigung ist eine Konsequenz der Risikoverursachung. Andernfalls müsste der unbeteiligte Geschädigte die Risikolast tragen. ${ }^{758}$ Wirtschaftlich betrachtet würde damit der Betreiber der gefährlichen Aktivität einen Teil der Kosten auf den Geschädigten abwälzen und damit externalisieren können. ${ }^{759}$ Dies würde zu einer ungerechtfertigten Bereicherung des Betreibers führen, der zugleich die wirtschaftlichen Vorteile aus der schadensauslösenden Aktivität zieht. ${ }^{760}$ Der Grundsatz der ungerechtfertigten Bereicherung wiederum ist in vielen nationalen Rechtsordnungen verankert ${ }^{761}$ und stützt damit ein weites Verständnis der Kostenzurechnung nach dem Verursachergrundsatz. In diesem Sinne spricht auch Prinzip 16 der Rio Deklaration allgemein von „Umweltkosten“ und „Kosten der Verschmutzung“. Im Ergebnis sind daher die Kosten der Vermeidung, Kontrolle und Verringerung sowie Beseitigung bzw. Kompensation von Umweltschäden vom Verursacher zu tragen. ${ }^{762}$

758 Sonderberichterstatter P. S. Rao, Third Report on the Legal Regime for Allocation of Loss in Case of Transboundary Harm Arising out of Hazardous Activities, YBILC 2006-II/1, UN Doc. A/CN.4/566, S. 82, Rn. 27; L. A. de la Fayette, The ILC and International Liability, RECIEL 6 (1997), S. 322 (324 f.); vgl. auch J. Barboza, Liability, Chinese JIL 1 (2002), S. 499 (521); L. F. E. Goldie, Concepts of Strict and Absolute Liability and the Ranking of Liability in terms of Relative Exposure to Risk, NYIL 16 (1985), S. 175 (189); G. Handl, Liability as an obligation established by a primary rule of international law, NYIL 16 (1985), S. 49 (77 f.); J. M. Kelson, State Responsibility and the Abnormally Dangerous Activity, Harv. ILJ 13 (1972), S. 197 (228).

759 So L. A. de la Fayette, The ILC and International Liability, RECIEL 6 (1997), S. $322(325)$.

760 L. F. E. Goldie sieht in der Verwirklichung eines Risikos eine Form der Enteignung, für die ein Bereicherungsausgleich erforderlich sei. L. F. E Goldie, Liability for Damage and the Progressive Development of International Law, ICLQ 14 (1965), S. 1189 (1212 ff.); ders., Concepts of Strict and Absolute Liability and the Ranking of Liability in terms of Relative Exposure to Risk, NYIL 16 (1985), S. 175 (212 f., 247 f.); vgl. auch J. Barboza, der hervorhebt, dass Art. 5 AIL eine ungerechtfertigte Bereicherung verhindert. J. Barboza, The Environment, Risk and Liability in International Law, 2011, S. 121.

761 C. Binder/C. Schreuer, Unjust Enrichment, in: R. Wolfrum (Hg.), MPEPIL 2017, http://www.mpepil.com, Rn. 2, $10 \mathrm{f}$.

762 A. Epiney/M. Scheyli, Strukturprinzipien des Umweltvölkerrechts, 1998, S. 152; L. A. de la Fayette, International liability for damage to the environment, in: M. Fitzmaurice/D. M. Ong/P. Merkouris (Hg.), Research Handbook on International Environmental Law, 2010, S. 320 (328); T. Marauhn, Customary Rules of International Environmental Law, in: K. Ziolkowski (Hg.), Peacetime Regime for State Activities in Cyberspace, 2013, S. 465 (476) unter Bezugnahme auf Art. 2 Abs. 2 lit. b Convention for the Protection of the Marine Environment of the North-East Atlantic 1992. 
Der Verursachergrundsatz entfaltet zunächst im innerstaatlichen Bereich Wirkung. Die nationalen Behörden sollen den Grundsatz umsetzen, indem die Verursacher von Umweltbelastungen in die Pflicht genommen werden. ${ }^{763}$ Dieses Verständnis entspricht auch den Haftungsprinzipien der Völkerrechtskommission, die ebenfalls eine Kosteninternalisierung im Sinne des Verursachergrundsatzes anstreben. ${ }^{764}$ Neben der Geltung des Verursachergrundsatzes auf nationaler Ebene wird auch eine zwischenstaatliche Anwendung auf grenzüberschreitende Schadensfälle diskutiert. ${ }^{765}$

Die Schwäche des Verursachergrundsatzes liegt jedoch darin begründet, dass es häufig schwierig ist, einen bestimmten Schadensverursacher zu identifizieren, etwa weil ein Schaden aus diversen Schadensquellen entspringt oder die Schadensquelle nicht eindeutig bestimmt werden kann. ${ }^{766}$ Nur selten können allein vom Betreiber der gefährlichen Aktivität Kompensationsleistung erlangt werden, da die Risiken von Unfällen größeren Ausmaßes kaum versicherbar sind und regelmäßig zur Insolvenz der privaten Betreiber führen. Will man den Opferschutz, der dem Haftungsrecht zugrunde liegt, nicht durch ein zu enges Verständnis des Verursachergrundsatzes aushöhlen, muss eine andere Lösung als eine komplette Kosteninternalisierung gefunden werden. ${ }^{767}$ Staaten müssen eine angemessene und unverzügliche Entschädigung sicherstellen. Um seiner Rolle im internationalen Gefüge gerecht zu werden, muss der Staat, von dessen Hoheitsgebiet die Schädigung ausgeht, daher zumindest als Garant für

763 T. Marauhn, Customary Rules of International Environmental Law, in: K. Ziolkowski (Hg.), Peacetime Regime for State Activities in Cyberspace, 2013, S. 465 (476).

764 Sonderberichterstatter Rao bekräftigt, dass „[t] he overall objective is however to achieve ,cost internalization', which is closely related to the ,polluter pays' principle“. P. S. Rao, Second report on the legal regime for the allocation of loss in case of transboundary harm arising out of hazardous activities, YBILC 2004-II/1, UN Doc. A/CN.4/540, Rn.38, S. 77 Abs. (a), Entwurfsvorschlag Prinzip 3. Der Bericht nimmt dabei auf die Ausführungen von L. Bergkamp zu den Zielen einer Haftung Bezug. Siehe L. Bergkamp, Liability and Environment, 2001, S. 70, 73.

765 A. Epiney/M. Scheyli, Strukturprinzipien des Umweltvölkerrechts, 1998, S. 158; S. E. Gaines, The Polluter-Pays Principle, Tex. ILJ 26 (1991), S. 463 (492).

766 Vgl. S. E. Gaines, The Polluter-Pays Principle, Tex. ILJ 26 (1991), S. 463 (481).

767 A. E. Boyle, Polluter Pays, in: R. Wolfrum (Hg.), MPEPIL 2009, http://www.mp epil.com, Rn. 13 unter Bezugnahme auf H. Smets, The Polluter Pays Principle in the Early 1990s, in: L. Campiglio/L. Pineschi/D. Siniscalco/T. Treves (Hg.), The Environment After Rio, 1994, S. 131 (131). 
Entschädigungsleistungen einstehen. ${ }^{768}$ Dies wird gleichsam durch die Haftungsprinzipien der Völkerrechtskommission erkannt und bestätigt, indem diese den Schutz des unbeteiligten Opfers durch eine subsidiäre Staatenhaftung - eine Art Sicherheitsnetz zur Betreiberhaftung - sicherstellen (Haftungsprinzip 4 Abs. 5). ${ }^{769}$ Der Entwurf der Völkerrechtskommission ist entsprechend gleichzeitig als "an acknowledgement of the limitations on the polluter-pays principle as commonly understood and as an appropriate implementation of the principle" zu verstehen. ${ }^{770}$

Darüber hinaus ist gerade mit Blick auf grenzüberschreitende Schadensfälle eine extensive Interpretation des Verursachergrundsatzes angezeigt. ${ }^{771}$ Denn Schadensverursacher ist, „wer die Umwelt direkt oder indirekt belastet oder eine Bedingung für die Umweltbelastung setzt." "772 Staaten, die gefährliche Aktivitäten auf ihrem Hoheitsgebiet gestatten, begründen auf diese Weise ein Risiko und müssen konsequenterweise für die Verwirklichung dieses Risikos einstehen. Sofern ein Staat also eine gefährliche Aktivität genehmigt oder nicht verbietet und damit einen anderen Staat entsprechenden Risiken aussetzt, trifft diesen aufgrund der Entscheidung auch eine Einstandspflicht im Falle der Risikorealisierung. ${ }^{773}$ Der Staat ist Verursacher im weiteren Sinne. ${ }^{774}$ Staaten gewähren derartige Aktivitä-

768 C. Foster, The ILC Draft Principles on the Allocation of Loss in the Case of Transboundary Harm Arising out of Hazardous Activities, RECIEL 14 (2005), S. 265 (277); R. Lefeber, Transboundary Environmental Interference and the Origin of State Liability, 1996, S.3, 280, 301; vgl. auch A. Epiney, "Gerechtigkeit“ im Umweltvölkerrecht, APuZ 24 (2007), S. 31 (35).

769 C. Foster, The ILC Draft Principles on the Allocation of Loss in the Case of Transboundary Harm Arising out of Hazardous Activities, RECIEL 14 (2005), S. 265 (277).

770 Ibid.

771 Vgl. E. Louka, International Environmental Law, 2006, S. 448 f.; P. Schwartz, The polluter-pays principle, in: M. Fitzmaurice/D. M. Ong/P. Merkouris (Hg.), Research Handbook on International Environmental Law, 2010, S. 243 (252).

772 Rat der Europäischen Gemeinschaften, Empfehlung vom 3. März 1975 über die Kostenzurechnung und die Intervention der öffentlichen Hand bei Umweltschutzmaßnahmen (75/436/Euratom, EGKS, EWG), Amtsblatt der Europäischen Gemeinschaften Nr. L 194/1 (25.07.1975), Anhang, Abs. 3.

773 L. A. de la Fayette, The ILC and International Liability, RECIEL 6 (1997), S. 322 (325); vgl. auch L. Gündling, Verantwortlichkeit der Staaten für grenzüberschreitende Umweltbeeinträchtigungen, ZaöRV 45 (1985), S. 265 (289).

774 S. E. Gaines, The Polluter-Pays Principle, Tex. ILJ 26 (1991), S. 463 (492 Fn. 138); J. Healey/H. Pitts schlussfolgern aus der Trail Smelter-Entscheidung: „A more accurate statement of the principle would seem to be the ,polluter's nation pays،." J. Healey/H. Pitts, Applying International Environmental Legal Norms to Cyber Statecraft, I/S: A Journal of Law and Policy for the Information 
ten wohl auch in der Erwartung eines wirtschaftlichen Vorteils, den sie etwa durch Einnahme von Gebühren für entsprechende Lizenzen und mithin durch Gewinnbeteiligung erlangen. Unter Gerechtigkeitserwägungen dürfen demnach diese profitierenden Staaten Schadenskosten nicht auf andere Staaten externalisieren. ${ }^{775}$ In diesem Sinne stützt der Grundsatz der ungerechtfertigten Bereicherung auch auf zwischenstaatlicher Ebene ein weites Verständnis des Verursachergrundsatzes, ${ }^{776}$ denn ,the creation of risk involves an expropriation of equal rights of amenities and, in the international arena, of equality and sovereign autonomy. " 777

Außerdem trifft Staaten, wie gezeigt, die Pflicht, gefährliche Aktivitäten in ihrem Hoheitsgebiet oder unter ihrer Hoheitsgewalt zu regulieren und zu kontrollieren, die möglicherweise grenzüberschreitende Schäden zur Folge haben. Denklogisch sollte der zur Prävention verpflichtete Staat auch auf Kompensationsseite eine zentrale Rolle spielen. Die Kostenzurechnung nach dem Verursachergrundsatz hat nämlich zum Ziel, einen wirtschaftlichen Anreiz zu schaffen, damit effektive Maßnahmen zur Schadensvermeidung ergriffen werden. ${ }^{778}$ Der Staat hat aufgrund seiner Regulierungsmacht die wirksamsten Kontrollmöglichkeiten auf seinem Hoheitsgebiet bzw. unter seiner Hoheitsgewalt, und eine entsprechende

Society 8 (2012), S. 356 (379) unter Bezugnahme auf M. Anderson, Derivative versus Direct Liability as a Basis for State Liability for Transboundary Harms, in: R. M. Bratspies/R. A. Miller (Hg.), Transboundary Harm in International Law, 2006, S. 99 (99); R. Lefeber, Transboundary Environmental Interference and the Origin of State Liability, 1996, S. 3, 301.

775 So L. A. de la Fayette, The ILC and International Liability, RECIEL 6 (1997), S. 322 (325); siehe auch G. Handl, International Accountability for Transboundary Environmental Harm Revisited, Environmental Policy and Law 37 (2007), S. 116 (119); J. G. Lammers, International Responsibility and Liability for Damage Caused by Environmental Interferences, Environmental Policy and Law 31 (2001), S. 42 (47); K. Zemanek, State Responsibility and Liability, in: W. Lang/H. Neuhold/ders. (Hg.), Environmental Protection and International Law, 1991, S. 187 (195).

776 L. A. de la Fayette, The ILC and International Liability, RECIEL 6 (1997), S. 322 (325).

777 L. F. E. Goldie, Concepts of Strict and Absolute Liability and the Ranking of Liability in terms of Relative Exposure to Risk, NYIL 16 (1985), S. 175 (248).

778 L. A. de la Fayette, The ILC and International Liability, RECIEL 6 (1997), S. 322 (325). Siehe auch Sonderberichterstatter P. S. Rao, First report on the legal regime for allocation of loss in case of transboundary harm arising out of hazardous activities, YBILC 2003-II/1, UN Doc. A/CN.4/531, S. 84, Rn. 45 unter Bezugnahme auf L. A. de la Fayette, The Concept of Environmental Damage in International Liability Regimes, in: M. Bowman/A. E. Boyle (Hg.), Environmental Damage in International and Comparative Law, 2002, S. 149 (179). 


\section{Kapitel: Staatenhaftung in der Systematik des Völkerrechts}

Kostenzuteilung motiviert die Staaten, ihren internationalen Schadensvermeidungspflichten in effizienter Weise nachzukommen. ${ }^{779}$ Zudem ist der Staat in der besten Position, eine Kosten-Nutzen-Analyse zwischen den Kosten der Schadensvermeidung und den Kosten im internationalen Schadensfall in die Reglementierung gefährlicher Aktivitäten miteinzubeziehen. ${ }^{780}$ Sofern der Staat, der auf zwischenstaatlicher Ebene die Kosten trägt, den privaten Betreiber soweit wie möglich in Regress nimmt, ist keine Subventionierung des privaten Verursachers gegeben und auch dessen Anreiz zur Schadensprävention wird nicht gedämmt. ${ }^{781}$

Ungeachtet seiner Rezeption in den besprochenen Umweltabkommen befindet sich der Verursachergrundsatz sowohl auf nationaler als auch zwischenstaatlicher Ebene an der Grenze zur erforderlichen normativen Verdichtung, um die Rechtsgeltung als allgemeiner Rechtsgrundsatz oder Völkergewohnheitsrecht zu beanspruchen. ${ }^{782}$ Immerhin kann das Verständnis die Ausgestaltung der staatlichen Einstandspflicht in grenzüberschreitenden Schadensfällen leiten. ${ }^{783}$

\section{Haftungsgrundsätze in den nationalen Rechtsordnungen}

Eine Haftung für rechtmäßiges Verhalten findet sich schließlich in den innerstaatlichen Rechtsordnungen. Im vorliegenden Kontext sind insbesondere zivilrechtliche Grundsätze der Haftung, der ungerechtfertigten Bereicherung und der Geschäftsführung ohne Auftrag relevant.

Zunächst ist ein Rückgriff auf die Rechtsprechung der EU sinnvoll, da diese im Wege einer wertenden Rechtsvergleichung die in Art. 340 Abs. 2 AEUV angesprochenen allgemeinen Rechtsgrundsätze zur Begründung

779 G. Handl, International Accountability for Transboundary Environmental Harm Revisited, Environmental Policy and Law 37 (2007), S. 116 (118).

780 Ibid.

781 Vgl. C. Foster, The ILC Draft Principles on the Allocation of Loss in the Case of Transboundary Harm Arising out of Hazardous Activities, RECIEL 14 (2005), S. 265 (277); R. Lefeber, Transboundary Environmental Interference and the Origin of State Liability, 1996 S. 310, 322 f.; N. de Sadeleer, Environmental Principles, 2002, S. $24 \mathrm{f}$.

782 X. Hanqin, Transboundary Damage in International Law, 2003, S. 272; R. Wolfrum, General International Law (Principles, Rules, and Standards), in: ders. (Hg.), MPEPIL 2010, http://www.mpepil.com, Rn. 51.

783 P. Schwartz, The polluter-pays principle, in: M. Fitzmaurice/D. M. Ong/P. Merkouris (Hg.), Research Handbook on International Environmental Law, 2010, S. $243(256)$. 
einer außervertraglichen Unionshaftung entwickelt. Zudem rechtfertigt die zunehmende Interdependenz von internationaler und europäischer Standardsetzung ${ }^{784}$ die Einbeziehung des europäischen Rechtsverständnisses.

Im Fall Dorsch Consult machte die Klägerin Schadensersatz gegen den Rat und die Kommission geltend, weil die EU durch Erlass der EmbargoVerordnung (EWG) Nr. 2340/90 ${ }^{785}$ den irakischen Staat dazu veranlasst habe, ein Gesetz zu erlassen, welches es einem Unternehmen eines Mitgliedstaates der Gemeinschaft unmöglich mache, die Bezahlung einer Rechnung für im Irak erbrachte Dienstleistungen zu erlangen. Dabei berief sich die klagende Dorsch Consult auf eine außervertragliche Haftung der Gemeinschaft für rechtmäßige Aktivitäten und alternativ auf die Rechtswidrigkeit der Verordnung, die nicht ohne entsprechende Kompensationsleistungen für betroffene Wirtschaftsakteure hätte erlassen werden dürfen. In der Berufungsinstanz urteilte der Europäische Gerichtshof (EuGH) im Jahr 2000, dass eine außervertragliche Haftung der Gemeinschaft für rechtmäßige Aktivitäten von Gemeinschaftsorganen in Betracht kommt, wenn drei Voraussetzungen kumulativ erfüllt sind, namentlich das tatsächliche Vorliegen des Schadens, das Vorliegen des Kausalzusammenhangs zwischen ihm und dem Verhalten der Gemeinschaftsorgane sowie der Außergewöhnlichkeit und Besonderheit des fraglichen Schadens. ${ }^{786}$ Die Entscheidung in der Rechtssache Dorsch Consult wurde teilweise als implizite Anerkennung einer Haftung für rechtmäßige Aktivitäten in der Rechtslandschaft der EU interpretiert, denn die Ausführungen des EuGH zu den Voraussetzungen einer Haftung für erlaubte Aktivitäten wären sinnlos, wenn der Gerichtshof ein solches Konzept überhaupt nicht anerkennen würde. ${ }^{787}$ Es scheint aber zumindest fraglich, ob diese Erörte-

784 Vgl. etwa in Bezug auf den Umweltkontext D. Kelemen/T. Knievel, The United States, the European Union, and international environmental law, ICON 13 (2015), S. 945 (954 ff.).

785 Verordnung (EWG) Nr. 2340/90 des Rates vom 8. August 1990 zur Verhinderung des Irak und Kuwait betreffenden Handelsverkehrs der Gemeinschaft, ABl. 1990, Nr. L 213, 1.

786 EuGH, Rs. C-237/98 P, Slg. 2000, I-4549, Rn. 53 - Dorsch Consult III; so schon die Vorinstanz EuG, Rs. T-184/95, Slg. 1998, II-667, Rn. 59 - Dorsch Consult II.

787 Außerdem „kann eine Prüfung konkreter Tatbestandsvoraussetzungen (...) erst erfolgen, wenn Klarheit über das zugrundeliegende Haftungsprinzip bzw. -institut besteht, aus dem heraus der Tatbestand im einzelnen zu entwickeln ist." S. Haack, Grundsätzliche Anerkennung der außervertraglichen Haftung der EG für rechtmäßiges Verhalten nach Art. 288 Abs. 2 EG - Anmerkungen zum Urteil des Gerichts erster Instanz (EuG) vom 14.12.2005, Rs. T-69/00, EuR 5 


\section{Kapitel: Staatenhaftung in der Systematik des Völkerrechts}

rungen des EuGH ausreichen, um ein Haftungskonzept für rechtmäßige Aktivitäten zu etablieren, da eine Entschädigung im Ergebnis aufgrund der Umstände des Einzelfalls abgelehnt wurde. ${ }^{788}$ So stellte das Gericht Erster Instanz (GEI - seit dem Vertrag von Lissabon Gericht der Europäischen Union (EuG)) in Folgeentscheidungen eine Haftung der Gemeinschaft für rechtmäßige Aktivitäten wiederholt infrage, indem er beispielsweise im Förde-Reederei-Fall aus dem Jahr 2002 betonte, dass die Ausführungen zu den Haftungsvoraussetzungen nur zur Anwendung gelängen, „sofern der Grundsatz einer solchen Haftung im Gemeinschaftsrecht anzuerkennen sein sollte“. .789

Der FIAMM-Fall aus dem Jahr 2005 verdeutlicht die ambivalente Rechtsprechung zum Bestand einer Gemeinschaftshaftung ohne Fehlverhalten der Gemeinschaft. In dem Fall ging es um Strafzölle, die die USA im Streit um die Bananenmarkt-Verordnung ${ }^{790}$ erhoben hatte. FIAMM beantragte,

(2006), S. 696 (699); ders., Die außervertragliche Haftung der EG für rechtmäßiges Verhalten - Zum Urteil des Gerichts erster Instanz (EuG) vom 28.4.1998, Rs. T-184/95, Dorsch Consult Ingenieurgesellschaft mbH/Rat der Europäischen Union und Kommission der Europäischen Gemeinschaften, EuR 3 (1999), S. 395 (398); siehe auch A. Thies, International Trade Disputes and EU Liability, 2013, S. 150. Zur erstinstanzlichen Entscheidung des EuG in der Sache Dorsch Consult siehe C. van Dam, European Tort Law, 2013, S. 567; S. Hobe, Europarecht, 2017, S. 164, Rn. 628. Vgl. auch O. Dörr, Staatshaftung in Europa, in: ders. (Hg.) Staatshaftung in Europa, 2014, S. 1 (20 Fn. 135); B. J. Hartmann, Haftung in der Europäischen Union, in: O. Dörr (Hg.) Staatshaftung in Europa, 2014, S. 31 (37).

788 Vgl. O. Dörr, Staatshaftung in Europa, in: ders. (Hg.) Staatshaftung in Europa, 2014, S. 1 (20 Fn. 135); E. Engelhard/B. van den Broek/F. de Jong/A. Keirse/E. de Kezel, Let's Think Twice before We Revise!, ULR 10 (2014), S. 55 (72).

789 EuG, T-170/00, Slg. 2002, II-515, Rn. 56 - Förde-Reederei; zu der hypothetischen Haftung für rechtmäßige Aktivitäten siehe auch EuG, T-196/99, Slg. 2001, II-3597, Rn. 171-180 - Area Cova; EuG, T-195/00, Slg. 2003, II-1677, Rn. 161 ff. - Travelex; EuG, T-64/01 und T-65/01, Slg. 2004, II-521, Rn. 150 ff. - Afrikanische Frucht-Companie; EuG, T-383/00, Slg. 2005, II-5459, Rn. 171 ff. - Beamglow Ltd.

790 Verordnung (EWG) Nr. 404/93 des Rates vom 13. Februar 1993 über die gemeinsame Marktorganisation für Bananen (ABl. L 47, S. 1). Das Streitbeilegungsorgan der WTO erklärte, dass die Verordnung nicht mit WTO-Recht vereinbar sei, da sie Präferenzregelungen für Bananen mit Ursprung in bestimmten Staaten enthalte, und berechtigte daraufhin die USA Strafzölle auf Einfuhren bestimmter Waren der Gemeinschaft zu erheben. Die Rechtmäßigkeit von Handlungen der Gemeinschaftsorgane ist allerdings nicht am WTO-Recht zu messen, so dass die Gemeinschaftsrichter auch nicht die vorgeworfene Rechtswidrigkeit des Verhaltens des Rats und der Kommission überprüfen konnten. Siehe S. Haack, Grundsätzliche Anerkennung der außervertraglichen Haftung 
die Kommission und den Rat zum Ersatz des ihr infolge der amerikanischen Zölle entstandenen Schadens zu verurteilen. Zunächst hatte das GEI in seiner Entscheidung zur Rechtssache FIAMM erstmals ausdrücklich eine Haftung für rechtmäßige Aktivitäten im Gemeinschaftsrecht anerkannt. ${ }^{791}$ Das Gericht erläuterte, dass eine Einstandspflicht der Gemeinschaft nicht nur aufgrund unrechtmäßiger Aktivitäten begründet werden kann, da gemäß Art. 288 Abs. 2 EGV auch allgemeine Rechtsgrundsätze, die den Rechtsordnungen der Mitgliedstaaten gemeinsam sind, Berücksichtigung finden. ${ }^{792}$ Das GEI führte weiter aus: „Nach den nationalen Rechtsvorschriften über die außervertragliche Haftung kann der Einzelne aber wenn auch in unterschiedlichem Umfang, in speziellen Bereichen und nach verschiedenen Modalitäten - vor Gericht bestimmte Schäden auch ohne rechtswidrige Handlung des Schadensverursachers ersetzt bekom-

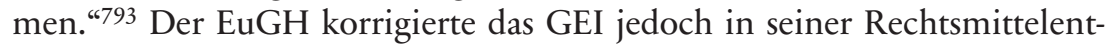
scheidung aus dem Jahr 2008, indem er darauf hinwies, aus seiner bisherigen Rechtsprechung könne nicht geschlossen werden, dass der Gerichtshof den Grundsatz der Haftung für rechtmäßiges Handeln oder Unterlassen der öffentlichen Gewalt anerkannt habe. Vielmehr stellte er fest, „dass beim derzeitigen Stand des Gemeinschaftsrechts" keine Haftungsregelung für rechtmäßiges Verhalten bestehe. ${ }^{794}$ Der EuGH räumte aber ein, dass er in seiner Dorsch Consult-Entscheidung die Voraussetzungen einer möglichen Unionshaftung für rechtmäßige Aktivitäten spezifiziert und damit eine Basis für ein solches Regime geschaffen hatte. ${ }^{795}$ In der Rechtssache FIAMM sah sich der Gerichtshof nun gezwungen, zu der Frage nach dem Bestand einer solchen Gemeinschaftshaftung für normative Handlungen explizit Stellung zu nehmen. ${ }^{796}$ Auch wenn der Gerichtshof das Konzept

der EG für rechtmäßiges Verhalten nach Art. 288 Abs. 2 EG - Anmerkungen zum Urteil des Gerichts erster Instanz (EuG) vom 14.12.2005, Rs. T-69/00, EuR 5 (2006), S. 696 (697 f.).

791 EuG, T-69/00, Slg. 2005, II-5393, Rn. 157 ff. - FIAMM; siehe auch S. Haack, Grundsätzliche Anerkennung der außervertraglichen Haftung der EG für rechtmäßiges Verhalten nach Art. 288 Abs. 2 EG - Anmerkungen zum Urteil des Gerichts erster Instanz (EuG) vom 14.12.2005, Rs. T-69/00, EuR 5 (2006), S. 696 (705).

792 EuG, T-69/00, Slg. 2005, II-5393, Rn. 158 - FIAMM.

793 Id., Rn. 159 - FIAMM.

$794 \mathrm{EuGH}$ (GK), C-120/06 und C-121/06, Slg. 2008, I-6513, Rn. 168 ff. - FIAMM.

795 Id., Rn. 169 - FIAMM.

796 Id., Rn. 171 ff. - FIAMM. 


\section{Kapitel: Staatenhaftung in der Systematik des Völkerrechts}

nicht gänzlich verwarf, ${ }^{797}$ kam er zumindest mit Blick auf die rechtsetzende Tätigkeit der Gemeinschaft zu dem Ergebnis, dass eine Haftung für rechtmäßiges Verhalten des Gemeinschaftsgesetzgebers derzeit nicht im Gemeinschaftsrecht verankert sei. ${ }^{798}$ Die Auswirkungen dieser Entscheidung sind aber noch nicht abschließend geklärt, da eine Haftung der Gemeinschaft für rechtmäßiges (nicht legislatives) Verhalten oder eine Gefährdungshaftung nicht ausdrücklich ausgeschlossen wurden, ${ }^{799}$ vielmehr dem „Wertungsspielraum“ des Gemeinschaftsgesetzgebers überantwortet wurde. ${ }^{800}$ Der Rechtsprechung des GEI und auch des EuGH lassen sich jedenfalls grundlegende Voraussetzungen für eine derartige Haftung entnehmen. ${ }^{801}$

Bemerkenswert sind ferner die rechtlichen Erwägungen von GEI und EuGH im Fall Masdar, in welchem die Klägerin behauptete, Schäden durch die Nichtbezahlung von Leistungen im Zusammenhang mit Gemeinschaftshilfeprojekten erlitten zu haben. Die beiden Gerichte bedeuteten übereinstimmend, dass eine Haftung ohne Fehlverhalten sich aus zwei weiteren Rechtsgrundsätzen ergibt. Die Konzepte der ungerechtfertigten Bereicherung (de in rem verso) und der Geschäftsführung ohne Auftrag (negotiorum gestio) setzen weder rechtswidriges noch rechtmäßiges Verhalten voraus und sind in den meisten Rechtsordnungen der Mitglied-staaten an-

797 Die ablehnende Begründung zur Haftung für rechtmäßiges Verhalten bezieht sich ausschließlich auf die Rechtsetzungstätigkeit der Gemeinschaft. Zudem werden Ausnahmekonstellationen besprochen. Vgl. id., Rn. 174, $180 \mathrm{ff}$. FIAMM.

$798 I d .$, Rn. 176 ff. - FIAMM.

799 M. Almhofer, Die Haftung der Europäischen Union für rechtswidrige Bankenaufsicht, 2018, S.220; W. Frenz/V. Götzkes, Anmerkung zur Entscheidung FIAMM des EuGH (Große Kammer) vom 9. September 2008 - C-120/06 P und C-121/06 P, DVBl. 16 (2009), S. 1052 (1053); A. Haratsch/C. Koenig/M. Pechstein, Europarecht, 2018, S. 280, Rn. 629.

800 Der EuGH weist darauf hin, dass seine Beanstandung des Rechtsfehlers, das heißt der Anerkennung einer Haftung der Gemeinschaft für die rechtmäßige Ausübung ihrer rechtsetzenden Tätigkeiten durch das GEI, „unbeschadet des weiten Wertungsspielraums erfolgt, über den der Gemeinschaftsgesetzgeber gegebenenfalls verfügt, um zu beurteilen, ob bei Erlass eines bestimmten Rechtsetzungsakts die Berücksichtigung bestimmter, sich aus diesem Erlass ergebender nachteiliger Auswirkungen es rechtfertigt, bestimmte Arten von Entschädigungen vorzusehen." EuGH (GK), C-120/06 und C-121/06, Slg. 2008, I-6513, Rn. 181 - FIAMM. Unter diesem Blickwinkel ist auch die Einschränkung in Rn. 176 des Urteils auf den "derzeitigen Stand des Gemeinschaftsrechts“ zu betrachten.

801 A. Haratsch/C. Koenig/M. Pechstein, Europarecht, 2018, S. 280, Rn. 629. 
erkannt. ${ }^{802}$ Beide Rechtsgebilde können grundsätzlich herangezogen werden, um eine Entschädigung - unabhängig von der Rechtmäßigkeit bzw. Rechtswidrigkeit des Verhaltens der EU-Institutionen - zu begründen. ${ }^{803}$

Schließlich sehen zahlreiche nationale Sondergesetze weltweit eine verschuldensunabhängige Haftung für (besonders gefährliche) rechtmäßige Aktivitäten vor. ${ }^{804}$ Allerdings ist die verschuldensunabhängige Haftung in den nationalen Rechtsordnungen für strukturell unterschiedlich gelagerte Gefahrenlagen in differenzierten Regeln normiert. Die unterschiedlichen Ausprägungen sprechen gegen die Annahme eines allgemeinen Rechtsgrundsatzes. ${ }^{805}$ Indessen ist nicht von der Hand zu weisen, dass die Haftung für rechtmäßige Aktivitäten in den meisten Rechtsordnungen bekannt ist ${ }^{806}$ und die darin verkörperten Ideen und Werte maßgeblich

802 EuG, T-333/03, Slg. 2006, II-4377, Rn. 69, 91 f. - Masdar; EuGH (GK), C-47/07 P, Slg. 2008, I-9761, Rn. 50 f., 66 ff. - Masdar.

803 EuG, T-333/03, Slg. 2006, II-4377, Rn. 91 - Masdar; EuGH (GK), C-47/07 P, Slg. 2008, I-9761, Rn. 49 - Masdar.

804 Siehe ILC Secretariat, Survey of liability regimes relevant to the topic of international liability for injurious consequences arising out of acts not prohibited by international law, (international liability in case of loss from transboundary harm arising out of hazardous activities), YBILC 1995-II/1, UN Doc. A/ CN.4/471, S.73, Rn.60; Experts Group on Environmental Law of the World Commission on Environment and Development, R. D. Munro (Chairman)/J. G. Lammers (Rapporteur), Environmental Protection and Sustainable Development: Legal Principles and Recommendations, 1987, S. 83 f.; IBA, Section on Business Law, Committee F (international environmental law), Patricia Thomas (Chairman), Environmental Liability: 7th Residential Seminar on Environmental Law, 9-13 June 1990 Montreux Switzerland, 1991, S. 111-210; siehe auch mit Blick auf Europa O. Dörr, Staatshaftung in Europa, in: ders. (Hg.) Staatshaftung in Europa, 2014, S. 1 (18f.).

805 O. Dörr, Staatshaftung in Europa, in: ders. (Hg.) Staatshaftung in Europa, 2014, S. 1 (19f.); S. Förster, Internationale Haftungsregeln für schädliche Folgewirkungen gentechnisch veränderter Organismen, 2007, S. 200; B. Saxler/J. Siegfried/A. Proelss, International liability for transboundary damage arising from stratospheric aerosol injections, Law, Innovation and Technology (7) 2015, S. 112 (127).

806 So bemerkt $O$. Schachter beispielsweise: „[D]omestic law rules applicable to such matters as (...) liability for extra-hazardous activities (...) have (...) become pertinent for recruitment into international law." O. Schachter, International Law in Theory and Practice, 1991, S. 53; siehe auch ILC Secretariat, Survey of liability regimes relevant to the topic of international liability for injurious consequences arising out of acts not prohibited by international law, (international liability in case of loss from transboundary harm arising out of hazardous activities), YBILC 1995-II/1, UN Doc. A/CN.4/471, S. 73, Rn. 60; Sonderberichterstatter P. S. Rao, Third report on the legal regime for allocation of loss in case of transboundary harm arising out of hazardous activities, YBILC 2006-II/1, UN 


\section{Kapitel: Staatenhaftung in der Systematik des Völkerrechts}

sind ${ }^{807}$ Die Expertengruppe zum Umweltrecht der Weltkommission für Umwelt und Entwicklung bestätigt in ihrem Bericht aus dem Jahr 1986: „The increasing acceptance of strict liability for ultrahazardous activities at the national level is evidence of an emerging principle of (national) law recognized by civilized nations. As known, according to Article 38(1)(c) of the Statute of the International Court of Justice, such a principle may also govern the relationship between sovereign States when there is no treaty or rule of customary international law calling for the application of a different principle or rule. ${ }^{* 808}$

\section{Ergebnis}

Die völkerrechtliche Haftung für grenzüberschreitende Schäden durch gefährliche völkerrechtlich nicht verbotene Aktivitäten ist die logische Folge des Grundsatzes der guten Nachbarschaft, des Schädigungsverbotes - in seiner Ausformung als Grundsatz der Schadensprävention, Grundsatz der angemessenen Nutzung und Vorsorgegrundsatz - sowie des Verursachergrundsatzes. Sie hat ihren Ursprung in der souveränen Gleichheit von Staaten, die in den Grundsätzen der territorialen Souveränität und territorialen Integrität zum Ausdruck kommt. ${ }^{809}$ Schließlich findet sich die Haftung für rechtmäßiges (besonders gefährliches) Verhalten in den nationalen Rechtsordnungen und belegt die zunehmende Akzeptanz eines entsprechenden Grundsatzes.

Barboza stellt zutreffend fest, dass es sich bei der Haftung für völkerrechtlich nicht verbotene Aktivitäten nicht nur um ein in der Entstehung befindliches Konzept handelt, sondern um einen allgemeinen Rechts-

Doc. A/CN.4/566, S. 82 f., Rn. 27 f. Zum Streitstand bezüglich der Existenz einer Haftung für rechtmäßige Aktivitäten siehe J. Barboza, The Environment, Risk and Liability in International Law, 2011, S. 65 ff. m.w.N.

807 Vgl. T. Kleinlein, Konstitutionalisierung im Völkerrecht, 2012, S. 632.

808 Experts Group on Environmental Law of the World Commission on Environment and Development, R. D. Munro (Chairman)/J. G. Lammers (Rapporteur), Environmental Protection and Sustainable Development: Legal Principles and Recommendations, 1987, S. 84.

809 J. Barboza, The Environment, Risk and Liability in International Law, 2011, S. $153 \mathrm{f}$. 
grundsatz, dem derzeit noch seine „offizielle Geburtsurkunde“ durch die Staatengemeinschaft verwehrt wird. ${ }^{810}$

Die besprochenen Umweltgrundsätze haben jedenfalls aufgezeigt, dass die einzelnen Elemente der Staatenhaftung völkerrechtlich verankert sind und eine gewisse Konkretisierung erfahren haben. Sie bestätigen die Erkenntnisse zu den staatlichen Schadensvermeidungs- und Kompensationspflichten aus der Rechtsprechungsanalyse. Rechtsnatur, Reichweite und Bindungswirkung dieser Grundsätze können zwar häufig nicht eindeutig bestimmt werden, ${ }^{811}$ denn zum einen verbleibt den Staaten bei ihrer Umsetzung ein gewisser Beurteilungsspielraum ${ }^{812}$ und zum anderen überschneidet sich die Anerkennung von allgemeinen Umweltgrundsätzen mit der völkergewohnheitsrechtlichen Verfestigung umweltschutzspezifischer Verhaltensregeln. ${ }^{813}$ Daraus folgt aber keine Relativierung der Rechtspflicht der Staaten, diese Grundsätze zu beachten. ${ }^{814} \mathrm{Im}$ Gegenteil wird den Umweltgrundsätzen ein Prinzipiencharakter zugeschrieben ${ }^{815}$ und

810 Id., S. 154. Vgl. auch B. A. Walton, Duties Owed, Yale LJ 126 (2017), S. 1460 (1464). Für eine Staatenhaftung de lege ferenda siehe beispielsweise J. S. Charme, Transnational Injury and Ultra-Hazardous Activity, Journal of Law and Technology 4 (1989), S. 75 (75 ff.); G. Handl, International Accountability for Transboundary Environmental Harm Revisited, Environmental Policy and Law 37 (2007), S. 116 (120); D. M. Schmitt, Staatenverantwortlichkeit für Schäden an der biologischen Vielfalt, 2018, S. $461 \mathrm{ff}$.

811 M. V. Soto resümiert zutreffend: „General principles of international environmental law may refer to rules of customary international law, to rules derived from treaties, to general principles of law as stated in article $38(\mathrm{I})(\mathrm{c})$ of the Statute of the International Court of Justice or to logical propositions resulting from judicial reasoning." M. V. Soto, General Principles of International Environmental Law, ILSA J. Intl. \& Comp. L. 3 (1996), S. 193 (193 Fn. 1); siehe auch A. Proelß, Prinzipien des internationalen Umweltrechts, in: ders. (Hg.), Internationales Umweltrecht, 2017, S. 69 (71 Rn. 1).

812 A. Epiney/M. Scheyli, Strukturprinzipien des Umweltvölkerrechts, 1998, S. 126.

813 A. Proelß, Prinzipien des internationalen Umweltrechts, in: ders. (Hg.), Internationales Umweltrecht, 2017, S. 69 (71 Rn. 1); vgl. K. Ziolkowski, General Principles of International Law as Applicable in Cyberspace, in: dies. (Hg.), Peacetime Regime for State Activities in Cyberspace, 2013, S. 135 (145 f., 187).

814 A. Epiney/M. Scheyli, Strukturprinzipien des Umweltvölkerrechts, 1998, S. 169.

815 Ibid.; A. Proelß, Prinzipien des internationalen Umweltrechts, in: ders. (Hg.), Internationales Umweltrecht, 2017, S. 69 (69 ff.); zum Schädigungsverbot J. Bäumler, Das Schädigungsverbot im Völkerrecht, 2017, S. 265 ff. Prinzipien können vereinfacht als sehr wichtige allgemeine Regeln bezeichnet werden. So A. Jakab, Prinzipien, Rechtstheorie 37 (2006), S. 49 (56). Während teilweise zwischen Regeln und Prinzipien differenziert wird, versteht der IGH die Begriffe sogar synonym. Siehe hierzu ausführlich T. Kleinlein, Konstitutionalisierung im 


\section{Kapitel: Staatenhaftung in der Systematik des Völkerrechts}

den grundlegenden Umweltschutzpflichten ein (möglicher) ius cogens-Status eingeräumt. ${ }^{816}$ Unabhängig davon, ob die Umweltgrundsätze aufgrund ihres fundamentalen verfassungsähnlichen Charakters stets als allgemeine Rechtsgrundsätze im Sinne des Art. 38 Abs. 1 lit.c IGH-Statut zu betrachten sind ${ }^{817}$ oder aber hinter den völkergewohnheitsrechtlichen Stellenwert der in den Grundsätzen zum Ausdruck kommenden Verhaltensregeln zurücktreten, ${ }^{818}$ entfalten diese regelmäßig eine weitreichende Rechtsgeltung im Umweltkontext. ${ }^{819}$ Zudem haben viele Grundsätze, unabhängig von ihrer Normgenese, eine bedeutende rechtspolitische Wirkung und bieten mithin Anknüpfungspunkte für völkervertragliche oder völkerge-

Völkerrecht, 2012, S. 663 ff. In seinem Sondervotum zum Urteil des IGH im Pulp Mills-Fall hält Richter Cançado Trindade zu der Rolle von Prinzipien in der Völkerrechtsordnung sogar fest: „those principles stand ineluctably at a superior level than the norms or rules of positive international law" IGH, Pulp Mills on the River Uruguay (Argentina v. Uruguay), Judgment of 20 April 2010, ICJ Reports 2010, Separate opinion of Judge Cançado Trindade, S. 135 (151 Rn. 39). Zur Bedeutung von Prinzipien mit Blick auf die Konstitutionalisierung des Völkerrechts siehe S. Kadelbach/T. Kleinlein, International Law - a Constitution of Mankind?, GYIL 50 (2007), S. 303 (337 ff.).

816 J. Brunnée, "Common Interest" - Echoes from an Empty Shell?, ZaöRV 49 (1989) S. 791 (800 ff.); H. Hohmann, Präventive Rechtspflichten und -prinzipien des modernen Umweltvölkerrechts, 1992, S. 255; S. Kadelbach, Ethik des Völkerrechts unter Bedingungen der Globalisierung, ZaöRV 64 (2004), 1 (12); ders., Zwingendes Völkerrecht, 1992, S. 318 ff., 323 in Bezug auf das Verbot mutwilliger und massiver Schädigungen der Umwelt.

817 D. Maurmann, Rechtsgrundsätze im Völkerrecht am Beispiel des Vorsorgeprinzips, 2008, S. 33 ff. in Bezug auf das Vorsorgeprinzip; vgl. auch IGH, Pulp Mills on the River Uruguay (Argentina v. Uruguay), Judgment of 20 April 2010, ICJ Reports 2010, Separate opinion of Judge Cançado Trindade, S. 135 (212 Rn. 210, 214 Rn. 217, 215 Rn. 220).

818 Vgl. A. Proelß, Prinzipien des internationalen Umweltrechts, in: ders. (Hg.), Internationales Umweltrecht, 2017, S. 69 (74 Rn. 6).

819 Siehe M. V. Soto, General Principles of International Environmental Law, ILSA J. Intl. \& Comp. L. 3 (1996), S. 193 (193 ff.). 
wohnheitsrechtliche Entwicklungen. ${ }^{820}$ Die Grundsätze sind demnach geeignet, das Konzept der Staatenhaftung argumentativ zu untermauern. ${ }^{821}$

\section{F. Zusammenfassung}

Die Untersuchung der völkerrechtlichen Grundlagen staatlicher Einstandspflichten hat gezeigt, dass der Staatenverantwortlichkeit und der Staatenhaftung unterschiedliche Erwägungen zugrunde liegen. Bei der Staatenverantwortlichkeit geht es - anders als bei der Staatenhaftung - um den Vorwurf völkerrechtswidrigen Verhaltens. Wie zu zeigen sein wird, bestehen aber gerade bei neuen Gefahren - etwa durch Informationstechnologien Unsicherheiten bei der Anwendung völkerrechtlicher Verbotstatbestände. Damit kann das Problem schadensträchtiger Aktivitäten, die gesellschaftliche und/oder wirtschaftliche Vorteile bieten, nicht bzw. nur unzureichend über die Staatenverantwortlichkeit gelöst werden. ${ }^{822}$ Die Staatenhaftung ermöglicht hier einen praktischen Regelungsansatz, der die Vorhersehbarkeit von Schäden durch gefährliche Aktivitäten sowie eine gerechte Verteilung von Nutzen und Kosten zum Gegenstand hat. ${ }^{823}$

Die Staatenhaftung für Schäden durch völkerrechtlich nicht verbotene Aktivitäten besteht aus einem Konglomerat von kodifiziertem Völkergewohnheitsrecht, sich herausbildenden Rechtsprinzipien und einer fortschrittlichen Weiterentwicklung des Rechts in Bereichen, in denen Staa-

820 Vgl. D. Bodansky, Customary (And Not So Customary) International Environmental Law, Ind. GLSJ 3 (1995), S. 105 (119); A. Epiney, Gegenstand, Entwicklung, Quellen und Akteure des internationalen Umweltrechts, in: A. Proelß (Hg.), Internationales Umweltrecht, 2017, S. 1 (25 ff. Rn. 69 ff.); A. Proelß, Prinzipien des internationalen Umweltrechts, in: ders. (Hg.), Internationales Umweltrecht, 2017, S. 69 (71 Rn. 1); S.-H. Schulze, Cyber-,War“ - Testfall der Staatenverantwortlichkeit, 2015, $214 \mathrm{f}$.

821 Vgl. S. Kadelbach/T. Kleinlein, die zutreffend feststellen: „If we take Article 38 para. 1 lit. c of the Statute as one possible way of legal reasoning besides reasoning based on treaty law or on custom, it is possible to regard Article 38 para. 1 lit. $\mathrm{c}$ as a positive norm regulating a certain technique of reaching solutions to legal problems on the basis of principles." S. Kadelbach/T. Kleinlein, International Law - a Constitution of Mankind?, GYIL 50 (2007), S. 303 (341). Vgl. auch S. Hobe, Die Zukunft des Völkerrechts im Zeitalter der Globalisierung, AVR 37 (1999) S. 253 (267 f.).

822 Sonderberichterstatter R. Q. Quentin-Baxter, Third report on international liability for injurious consequences arising out of acts not prohibited by international law, YBILC 1982-II/1, UN Doc. A/CN.4/360, S. 59 f., Rn. 36 ff.

823 Ibid. 
tenpraxis und Rechtsprechung mangelhaft oder unschlüssig ausfallen. Die Staatenhaftung im Gesamten kann als ein in der Entstehung befindlicher allgemeiner Rechtsgrundsatz des internationalen Rechts begriffen werden. Vergleichbar mit der Entwicklung des Rechts der Staatenverantwortlichkeit hängt die Konsolidierung des Konzepts lediglich von der politischen Bereitschaft und der Zustimmung durch die internationale Gemeinschaft ab. ${ }^{824}$

Sehr deutlich ist jedoch, dass die Staatenhaftung bereits im Umweltvölkerrecht verankert ist und zur Lösung für Schäden durch umweltgefährdende Aktivitäten, die völkerrechtlich nicht verboten sind, herangezogen wird. Ob die Staatenhaftung auch in anderen Bereichen der Völkerrechtsordnung zu Anwendung gelangen kann und muss, wird am Beispiel der Gefährdungslage in der virtuellen Welt zu zeigen sein.

Da die systematischen Voraussetzungen der staatlichen Einstandspflichten umstritten sind, müssen diese aber zunächst auf Grundlage der gewonnenen Erkenntnisse herausgestellt werden.

824 J. Barboza, Liability, Chinese JIL 1 (2002), S. 499 (516f.); A. E. Boyle, Globalising Environmental Liability, J. Envtl. L. 17 (2005), S. 3 (8). 


\section{Kapitel: Konstitutive Elemente der staatlichen Einstandspflichten}

Wie die bisherigen Ausführungen gezeigt haben, ist eine konzeptionelle Differenzierung zwischen der Staatenverantwortlichkeit und der Staatenhaftung schwierig. Im Folgenden werden die konstitutiven Elemente beider Regime konkretisiert, mithin Abgrenzungskriterien bestimmt. Erst wenn die Parameter der Staatenverantwortlichkeit (A.) einerseits und der Staatenhaftung (B.) andererseits klar definiert sind, kann in einem nächsten Schritt veranschaulicht werden, dass die Staatenhaftung neben der Staatenverantwortlichkeit notwendig ist (3. Kapitel) und wie ihre Anwendung auf neue Gefährdungen - etwa durch Informationstechnologien funktioniert (4. Kapitel).

\section{A. Konstitutive Elemente der Staatenverantwortlichkeit}

Die konstitutiven Elemente der Staatenverantwortlichkeit ergeben sich im Wesentlichen aus den völkergewohnheitsrechtlich anerkannten ASR. Art. 1 ASR bestimmt, dass jede völkerrechtswidrige Handlung eines Staates dessen völkerrechtliche Verantwortlichkeit zur Folge hat.

\section{Völkerrechtswidrigkeit}

Das Regime der Staatenverantwortlichkeit setzt einen Verstoß gegen einen anerkannten Rechtssatz des internationalen Rechts voraus. Für die Staatenhaftung hingegen bedarf es keiner Normverletzung; sie greift, wenn die Folgen einer Handlung zu einem rechtserheblichen Schaden führen. Das Kriterium der Völkerrechtswidrigkeit staatlichen Verhaltens ist mithin das entscheidende Abgrenzungskriterium. Gemäß Art. 2 ASR ist die Völkerrechtswidrigkeit des staatlichen Verhaltens zu bejahen, wenn eine Handlung oder Unterlassung die Verletzung einer völkerrechtlichen Verpflichtung des Staates begründet und dieses Verhalten dem Staat zurechenbar ist. 


\section{Kapitel: Konstitutive Elemente der staatlichen Einstandspflichten}

\section{Verletzung negativer Verpflichtungen}

Zunächst sind Staaten gehalten, die völkerrechtlichen Regeln, an die sie gebunden sind, nicht zu verletzen (Art. 12 und 13 ASR). Die Zurechnungsmechanismen in Kapitel II des Ersten Teils der ASR helfen zu bestimmen, inwiefern ein Verhalten als Verletzung dieser negativen Verpflichtung von Staaten zu qualifizieren ist. Sofern natürliche oder juristische Personen nach innerstaatlichem Recht als Staatsorgane qualifiziert werden und in amtlicher Eigenschaft agieren, ist deren völkerrechtswidriges Tun und Unterlassen dem Staat zurechenbar und begründet die Verantwortlichkeit des betreffenden Staates (Art. 4 ASR). Außerdem können private natürliche oder juristische Personen vom Staat ermächtigt sein, staatliche Funktionen wahrzunehmen (Art. 5 ASR). Zurechenbar ist zudem das Verhalten fremdstaatlicher Organe, die dem Staat zur Verfügung gestellt sind und dessen hoheitliche Befugnisse sie ausüben (Art. 6 ASR). Das Verhalten seiner Organe oder Personen, die zur Ausübung hoheitlicher Gewalt ermächtigt sind, ist einem Staat auch dann zurechenbar, wenn diese ultra vires handeln (Art. 7 ASR). ${ }^{1}$ Eine Zurechnung ist außerdem dann möglich, wenn Private faktisch im Auftrag oder unter Kontrolle oder Leitung des Staates agieren (Art. 8 ASR). Ferner begründet die faktische Ausübung hoheitlicher Gewalt bei Abwesenheit oder Ausfall der staatlichen Behörden einen zurechnungsrelevanten Zusammenhang, sofern diese Ausübung den Umständen nach erforderlich war (Art.9 ASR). Ebenso kann das Verhalten einer aufständischen oder anderen Bewegung einem Staat unter Umständen zugerechnet werden (Art.10 ASR). Schließlich ist die staatliche Anerkenntnis und Annahme privaten Verhaltens als eigenes staatliches Verhalten von Bedeutung (Art.11 ASR). Darüber hinaus kann eine Verantwortlichkeit auch in Verbindung mit fremdstaatlichem Handeln entstehen, wenn ein Staat einem anderen Staat Hilfe oder Unterstützung bietet, Leitung oder Kontrolle über diesen ausübt oder den fremden Staat nötigt (Art. 16, 17 und 18 ASR).

Die konkreten Voraussetzungen dieser Fallgruppen sind bereits Gegenstand zahlreicher Diskussionen in der Völkerrechtsliteratur. ${ }^{2}$ In jedem

1 Siehe auch IACtHR, Velásquez Rodríguez v. Honduras, Judgment of 29 July 1988, IACtHR Series C, No. 4 (1988), S. 32, Rn. 170.

2 Siehe beispielsweise G. Arangio-Ruiz, State Fault and the Forms and Degrees of International Responsibility, in: Le Droit International au Service de la Paix, de la Justice et du Développement, Mélanges Michel Virally, 1991, S. 25 ff.; J. Wolf, Die Haftung der Staaten für Privatpersonen nach Völkerrecht, 1997, S. 142 ff. 
Fall sind Staaten nicht für reines Privathandeln verantwortlich. ${ }^{3}$ Es muss eine ausreichend starke Verbindung zwischen den privaten Akteuren und den Staaten bestehen, um eine Staatenverantwortlichkeit für völkerrechtswidriges Verhalten bejahen zu können. ${ }^{4}$ Aufgrund des technologischen Fortschritts wird es aber zum einen immer schwieriger, den Nachweis zu erbringen, ${ }^{5}$ dass ein Staat hinter einer Rechtsverletzung steht, und zum anderen greifen die eng gefassten Zurechnungszusammenhänge im Rahmen gefährlicher Aktivitäten durch Private selten. ${ }^{6}$ Die völkerrechtlichen Ansätze, private Akteure selbst zur Verantwortung zu ziehen, beschränken sich auf einzelne völkerrechtliche Verstöße, und entsprechende internationale Standards befinden sich noch in der Entwicklung. ${ }^{7}$ Im Sinne eines effektiven Rechtsgüterschutzes stellt sich daher die Frage, unter welchen Voraussetzungen die zunehmenden Gefahren durch private Akteure von Staaten zu tragen sind. ${ }^{8}$

3 S. Kadelbach, The Concept of State Responsibility for Private Acts under General International Law, in: S. Hobe/S. Freeland/B. Schmidt-Tedd (Hg.), In Heaven as on Earth?, 2013, S. 105 (106); J. Wolf, Die gegenwärtige Entwicklung der Lehre über die völkerrechtliche Verantwortlichkeit der Staaten, ZaöRV 31 (1983), S. 481 (529).

4 S. Kadelbach, The Concept of State Responsibility for Private Acts under General International Law, in: S. Hobe/S. Freeland/B. Schmidt-Tedd (Hg.), In Heaven as on Earth?, 2013, S. 105 (106); A. Seibert-Fohr, Die völkerrechtliche Verantwortung des Staats für das Handeln von Privaten, ZaöRV 73 (2013), S. 37 (42).

5 Die ASR selbst enthalten indes keine Bestimmungen zu den Beweisanforderungen. Vgl. ASR, Kommentar zu Teil 1, Kapitel III, S. 54, Abs. 4. Nur aus der internationalen Rechtsprechung lassen sich allgemeine Kriterien für den Nachweis eines Zurechnungszusammenhangs entnehmen. J. D. Jolley, Attribution, State Responsibility, and the Duty to Prevent Malicious Cyber-Attacks in International Law, 2017, abrufbar unter: http://theses.gla.ac.uk/id/eprint/8452 (geprüft am 15.05.2020), S. 85; N. Tsagourias, Cyber-attacks, self-defence and the problem of attribution, JCSL 17 (2012), S. 229 (234f.).

6 Vgl. W. Heintschel von Heinegg, Cyberspace - ein völkerrechtliches Niemandsland, in: R. Schmidt-Radefeldt/C. Meissler (Hg.), Automatisierung und Digitalisierung des Krieges, 2012, S. 159-174 (172); H. Krieger, Krieg gegen anonymous, AVR 50 (2012), S. 1 (3 ff.); A. Seibert-Fohr, Die völkerrechtliche Verantwortung des Staats für das Handeln von Privaten, ZaöRV 73 (2013), S. 37 (38 ff.).

7 A. Seibert-Fohr, Die völkerrechtliche Verantwortung des Staats für das Handeln von Privaten, ZaöRV 73 (2013), S. 37 (38f.).

8 Siehe A. Epiney, Die völkerrechtliche Verantwortlichkeit von Staaten für rechtswidriges Verhalten im Zusammenhang mit Aktionen Privater, 1992. Im Rahmen der Untersuchung zur Übertragbarkeit der Zurechnungsregeln im Cyberkontext (siehe infra 3. Kapitel D.) werden die Schwierigkeiten im Zusammenhang mit etablierten Zurechnungsmechanismen deutlich und Diskussionen um Zurechnungsansätze de lege ferenda beleuchtet. Siehe hierzu auch O. Diggelmann/N. Hadorn, Gewalt- und Interventionsverbot bei Cyberangriffen, in: Fakultät für Vergleichen- 


\section{Kapitel: Konstitutive Elemente der staatlichen Einstandspflichten}

\section{Verletzung positiver Verpflichtungen}

Reines Privatverhalten kann einem Staat zwar nicht zugerechnet werden, dies bedeutet aber nicht zugleich, dass Staaten aus ihrer Verantwortung entlassen sind. Staaten können auch aufgrund von unterlassener Erfüllung positiver Verpflichtungen verantwortlich sein (vgl. Art. 2 ASR). Die Rechtsprechungsanalyse hat gezeigt, dass Staaten unter bestimmten Voraussetzungen die Pflicht trifft, völkerrechtlich verbotenes Verhalten Dritter/Privater zu verhindern und vor Verbrechen beziehungsweise Menschenrechtsverletzungen zu schützen. ${ }^{9}$ Maßgeblich für die Verantwortlichkeit des Staates ist dabei nicht etwa eine Zurechnung der privaten Verhaltensweisen oder die Annahme einer „Komplizenschaft“, sondern das völkerrechtswidrige Unterlassen seitens des Staates selbst. ${ }^{10}$ Der Menschrechtsschutz ist maßgeblich für die Entwicklung derartiger Handlungspflichten und die Globalisierung bedingt eine Ausweitung von Handlungspflichten zum Schutz internationaler Rechtsgüter auf andere völkerrechtliche Bereiche. ${ }^{11}$ Neben der normativen Verankerung positiver Pflichten durch entsprechende Übereinkommen können diese Pflichten auch dem allgemeinen Völkerrecht entstammen. ${ }^{12}$

Im Rahmen dieser Betrachtung ist entscheidend, ob ein Schaden mit der Verletzung einer (positiven) völkerrechtlichen Verpflichtung einhergeht und damit im Recht der Staatenverantwortlichkeit zu verorten ist oder ob ein Schaden ohne Völkerrechtsverletzung allein aus einem gefährlichen Verhalten resultiert und mithin die Staatenhaftung greift. ${ }^{13}$

Die Ausführungen des IGH in seiner Korfu Kanal-Entscheidung sind in diesem Zusammenhang wegweisend. Die Entscheidung setzt das Schädigungsverbot um und begründet konkrete und justiziable Rechte und

de Staats- und Rechtswissenschaften der Andrássy Gyula Universität Budapest/C. Schubel/S. Kirste/P.-C. Müller-Graff/ders./U. Hufeld (Hg.), Jahrbuch für Vergleichende Staats- und Rechtswissenschaften, 2017, S. 255 (269 ff.).

9 Siehe 2. Kapitel B.

10 S.-H. Schulze, Cyber-„War“ - Testfall der Staatenverantwortlichkeit, 2015, S. 58; A. Seibert-Fohr, Die völkerrechtliche Verantwortung des Staats für das Handeln von Privaten, ZaöRV 73 (2013), S. 37 (44).

11 Vgl. A. Seibert-Fohr, Die völkerrechtliche Verantwortung des Staats für das Handeln von Privaten, ZaöRV 73 (2013), S. 37 (37 ff.).

12 Siehe Rechtsquellenanalyse im 1 . Kapitel.

13 J. Barboza differenziert zwischen gefährlichen (hazardous) und schädlichen (noxious) Aktivitäten und verortet die Aktivitäten entsprechend in unterschiedlichen Regelungsbereichen. J. Barboza, The Environment, Risk and Liability in International Law, 2011, S. 46 ff., $51 \mathrm{f}$. 
Pflichten, deren Verletzung zur Staatenverantwortlichkeit führt. ${ }^{14}$ Die Feststellung des IGH, dass jeden Staat die Pflicht trifft, „not to allow knowingly its territory to be used for acts contrary to the rights of other States", ${ }^{15}$ bietet Anknüpfungspunkte, um die Tatbestandsvorrausetzungen für positive Handlungspflichten näher zu definieren.

\section{a) Territorium bzw. Hoheitsgewalt}

Auch wenn die Verantwortlichkeit eines Staates nicht schon dadurch entsteht, dass ein rechtsverletzendes Verhalten auf dem nationalen Territorium stattfindet, ist der Ort der Rechtsverletzung dennoch ein Indikator dafür, bei wem die Verantwortlichkeit liegen könnte. ${ }^{16}$ Wie Schiedsrichter Max Huber im Island of Palmas-Fall betonte, bringt die Souveränität über ein Territorium Pflichten mit sich. ${ }^{17}$ So sind Staaten verpflichtet, Völkerrechtsverletzungen oder nicht-staatliche Verbrechen auf staatseigenem Territorium zu verhindern bzw. zu verfolgen und zu ahnden. ${ }^{18} \mathrm{Im}$ Rahmen des Menschrechtsschutzes bildet die Hoheitsgewalt den Anknüpfungspunkt für staatliche Schutzpflichten. ${ }^{19}$ Hoheitsgewalt ist zunächst territorialbezogen und mithin auf dem eigenen Staatsgebiet anzunehmen. ${ }^{20}$ Die extraterritoriale Geltung der Menschenrechte wird indes unter Berück-

14 Vgl.J. Bäumler, Das Schädigungsverbot im Völkerrecht, 2017, S. 62 f., 266, 272 f.

15 IGH, Corfu Channel Case (United Kingdom of Great Britain and Northern Ireland v. Albania), Judgment of 9 April 1949, ICJ Reports 1949, S. 4 (22).

16 S. Heathcote, State omissions and due diligence, in: K. M. Bannelier/T. Christakis/dies. (Hg.), The ICJ and the Evolution of International Law, 2012, S. 295 (300).

17 Island of Palmas Case (or Miangas) (Netherlands vs. United States of America), Award of 4 April 1928, 2 UNRIAA (1928), S. 829 (839).

18 Siehe 1. Kapitel B. II.; siehe auch E. Suy, La protection des diplomates, in: J. Tittel (Hg.), Festschrift für Wilhelm Wengler, Band 1 Allgemeine Rechtslehre und Völkerrecht, 1973, S. 591 (609 ff.).

19 Die Frage, ob der Menschenrechtsschutz jenseits der Hoheitsgewalt extraterritoriale Schutzpflichten gebietet, wird kontrovers diskutiert, wobei derartige Pflichten sich allenfalls in der Entwicklung befinden. Siehe hierzu I. Kanalan, Extraterritoriale Staatenpflichten jenseits der Hoheitsgewalt, AVR 52 (2014), S. 495 (495 ff.).

20 W. Kälin/J. Künzli, Universeller Menschenrechtsschutz, 2019, S. 148 f. 


\section{Kapitel: Konstitutive Elemente der staatlichen Einstandspflichten}

sichtigung der einzelnen Menschenrechtskonventionen unterschiedlich bewertet. $^{21}$

Es besteht Einigkeit dahingehend, dass Hoheitsgewalt auch außerhalb des eigenen Staatsgebiets in bestimmten Konstellationen gegeben ist, etwa mit Blick auf eigene Staatsangehörige, bei Handlungen von diplomatischen bzw. konsularischen Vertretern im Ausland, bei Geschehnissen an Bord von Flugzeugen oder Schiffen, die in den jeweiligen Staaten eingetragen sind bzw. unter ihrer Flagge verkehren sowie bei der Besetzung eines fremden Staatsgebiets oder wenn eine vergleichbare effektive Staatsgewalt über ein Territorium ausgeübt wird. ${ }^{22}$

\section{b) Reale Handlungsmöglichkeit}

Staatenverantwortlichkeit wird grundsätzlich allein durch ein pflichtwidriges zurechenbares Verhalten begründet. ${ }^{23}$ Ein zusätzliches subjektives Element kann aber zur Bejahung der Unrechtmäßigkeit eines Verhaltens erforderlich sein. Die Völkerrechtkommission erklärte in diesem Zusammenhang: „Whether responsibility is 'objective' or 'subjective' in this sense depends on the circumstances, including the content of the primary obligation in question. The articles lay down no general rule in that regard. The same is true of other standards, whether they involve some degree of fault, culpability, negligence or want of due diligence. ${ }^{24}$ Demnach

21 Siehe O. A. Hathaway/E. Nielsen/A. Nowlan/W. Perdue/C. Purvis/S. Solow/J. Spiegel, Human Rights Abroad, ASLJ 43 (2011), S. 389 (398 ff.); I. Kanalan, Extraterritoriale Staatenpflichten jenseits der Hoheitsgewalt, AVR 52 (2014), S. 495 (495 ff.).

22 W. Kälin/J. Künzli, Universeller Menschenrechtsschutz, 2019, S. 156 ff.; I. Kanalan, Extraterritoriale Staatenpflichten jenseits der Hoheitsgewalt, AVR 52 (2014), S. 495 (501); N. Wenzel, Human Rights, Treaties, Extraterritorial Application and Effects, in: R. Wolfrum (Hg.), MPEPIL 2008, http://www.mpepil.com, Rn. 6 ff.

23 L. Gündling, Verantwortlichkeit der Staaten für grenzüberschreitende Umweltbeeinträchtigungen, ZaöRV 45 (1985), S. 265 (283); R. Pisillo-Mazzeschi, Forms of International Responsibility for Environmental Harm, in: F. Francioni/T. Scovazzi (Hg.), International Responsibility for Environmental Harm, 1991, S. 15 (16). Nach anderer Auffassung hat der Staat für einen ihm zurechenbaren Pflichtenverstoß nur dann einzustehen, wenn er diesen schuldhaft, das heißt vorsätzlich oder zumindest fahrlässig verursacht hat. $R$. Ago, Das Verschulden im völkerrechtlichen Unrecht, ZöR 20 (1940), S. 449 (484); H. Lauterpacht, Private Law Sources and Analogies of International Law, 1927, S. $141 \mathrm{ff}$., $\$ 62$ mit der Einschränkung, dass in Ausnahmekonstellationen eine absolute Haftung greifen könne.

24 ASR, Kommentar zu Art. 2 ASR, S. 34, Abs. 3. 
bestimmen nicht die Sekundärnormen der Staatenverantwortlichkeit sondern die primären Pflichten, ob ein subjektives Element zur Begründung der Völkerrechtswidrigkeit einer Aktivität erforderlich ist. ${ }^{25}$

Die internationale Rechtsprechung nimmt, wie gezeigt, auch auf subjektive Aspekte Bezug. So konstatierte der IGH in der Entscheidung zum Bosnischen Völkermord-Fall, dass die Schutzpflicht verhaltensbezogen und auf die dem Staat zur Verfügung stehenden Mittel beschränkt ist. ${ }^{26}$ Dieser Ansatz findet sich bereits in der Nicaragua-Entscheidung des IGH, in der er betonte, dass die gebotene Sorgfalt, die ein Staat auf seinem Hoheitsgebiet zur Verhinderung privater illegaler Aktivitäten walten lassen muss, unter Berücksichtigung dessen personeller und materieller Ressourcen zu beurteilen ist. ${ }^{27}$ Danach ist kein bestimmter Erfolg geschuldet, sondern die subjektiv besten Bemühungen um den Erfolg. Zwar wird entgegengehalten, dass der IGH im Korfu Kanal-Fall Albanien für die unterlassene Verhinderung der Beeinträchtigung fremdstaatlicher Interessen verantwortlich gemacht habe, ohne Aspekte der gebotenen Sorgfalt in seine Erwägungen miteinzubeziehen. ${ }^{28}$ Demnach sei die Verhinderungspflicht ergebnisbezogen und es käme eben nicht auf die gebotene Sorgfalt an. ${ }^{29}$ Dies lässt jedoch außer Acht, dass der IGH explizit darauf hinwies, dass in zeitlicher Hinsicht eine Benachrichtigung der Schifffahrt aller Staaten über die Existenz von Minen vor dem Zeitpunkt der Explosion schwierig, wenn nicht unmöglich gewesen wäre und daher lediglich gefordert war, dass die albanischen Behörden alle notwendigen Schritte unternehmen, um die Schiffe, die sich der Gefahrenzone nähern, zu warnen. ${ }^{30}$ Die Verantwortlichkeit Albaniens ergibt sich für den Gerichtshof schließlich aus der Tatsache, dass die albanischen Behörden nicht einmal versucht haben, das Geschehen zu verhindern. ${ }^{31}$

25 Vgl. H. P. Aust, Complicity and the Law of State Responsibility, 2011, S. 235.

26 IGH, Application of the Convention on the Prevention and Punishment of the Crime of Genocide (Bosnia and Herzegovina v. Serbia and Montenegro), Judgment of 26 February 2007, ICJ Reports 2007, S. 14 (221 Rn. 430).

27 IGH, Military and Paramilitary Activities in and against Nicaragua (Nicaragua v. United States of America), Merits, Judgment of 27 June 1986, ICJ Reports 1986, S. 14 (85 Rn. 157).

28 T. Payne, Teaching Old Law New Tricks, LCLR 20 (2016), S. 683 (709); B. A. Walton, Duties Owed, Yale LJ 126 (2017), S. 1460 (1492).

29 B. A. Walton, Duties Owed, Yale LJ 126 (2017), S. 1460 (1492).

30 IGH, Corfu Channel Case (United Kingdom of Great Britain and Northern Ireland v. Albania), Judgment of 9 April 1949, ICJ Reports 1949, S. 4 (22 f.).

31 Id., S. 4 (23). 
Allgemein ist mit Blick auf primäre Handlungspflichten ein zusätzliches Element sinnvoll, um den völkerrechtlichen Pflichtenkreis zum Tätigwerden auf ein angemessenes Maß zu begrenzen. ${ }^{32}$ Folglich kommt es entscheidend auf die reale Handlungsmöglichkeit des pflichtigen Staates zur Verhinderung bzw. zum Schutz an. ${ }^{33}$ Dabei sind die Umstände des konkreten Einzelfalls zu berücksichtigen. ${ }^{34}$

\section{c) Kenntnis}

Eng verbunden mit dem Element einer realen Handlungsmöglichkeit ist die Frage, inwiefern Kenntnis des Staates von der rechtsverletzenden Aktivität erforderlich ist, damit eine Verletzung der Verhinderungs- bzw. Schutzpflicht angenommen werden kann. Die Korfu Kanal-Entscheidung verdeutlicht, dass die Kenntnis eine grundlegende Voraussetzung für die Verhinderungspflicht ist. ${ }^{35}$ Aus den Ausführungen des IGH ergibt sich, dass nicht nur eine tatsächliche, sondern auch eine mutmaßliche bzw. hypothetische Kenntnis des Staates von der fraglichen Aktivität ausreicht, damit die Verhinderungspflicht greift. ${ }^{36}$ Eine Kenntnis ist mutmaßlich, wenn sie aufgrund der Umstände des Einzelfalles zu unterstellen ist, und hypothetische Kenntnis liegt vor, wenn der Staat bei Beachtung der gebotenen Sorgfalt Kenntnis hätte haben müssen. ${ }^{37}$ Auch in der Bosnischer Völkermord-Entscheidung bestätigte der IGH, dass die Verhinderungspflicht

32 Vgl. International Law Association (ILA), Study Group on Due Diligence in International Law, Tim Stephens (Rapporteur)/Duncan French (Chair), Second Report, July 2016, abrufbar unter: http://www.ila-hq.org/index.php/study-groups? study-groupsID=63 (geprüft am 15.05.2020), S. 2 ff.

33 J. Wolf, Die gegenwärtige Entwicklung der Lehre über die völkerrechtliche Verantwortlichkeit der Staaten, ZaöRV 31 (1983), S. 481 (505).

34 Die Anforderungen an den Staat sind grundsätzlich umso höher, je gravierender die (drohende) Rechtsverletzung ist. Je sensibler das betroffene Recht bzw. Rechtsgut ist und je höher die Wahrscheinlichkeit einer Verletzung ist, desto dringlicher ist die Handlungspflicht des Staates. Vgl. M. Hakimi, State Bystander Responsibility, EJIL 21 (2010), S. 341 (371 ff.).

35 IGH, Corfu Channel Case (United Kingdom of Great Britain and Northern Ireland v. Albania), Judgment of 9 April 1949, ICJ Reports 1949, S. 4 (22).

36 Id., S. 4 (18).

37 J. D. Jolley, Attribution, State Responsibility, and the Duty to Prevent Malicious Cyber-Attacks in International Law, 2017, abrufbar unter: http://theses.gla.ac.uk/i d/eprint/8452 (geprüft am 15.05.2020), S. 195 ff. 
greift, sobald "the State learns of, or should normally have learned of, the existence of a serious risk that genocide will be committed". 38

Entscheidend ist in diesem Zusammenhang, unter welchen Bedingungen mutmaßliche bzw. hypothetische Kenntnis anzunehmen ist. Der IGH betonte in der Korfu Kanal-Entscheidung: ,it cannot be concluded from the mere fact of the control exercised by a State over its territory and waters that the State necessarily knew, or ought to have known, of any unlawful act perpetrated therein ". ${ }^{39}$ Demnach greift keine (widerlegbare) Vermutung hinsichtlich der Kenntnis eines Staates, sobald illegale Aktivitäten auf dessen Territorium stattfinden. ${ }^{40}$ Allerdings bewirkt die exklusive territoriale Kontrolle des pflichtigen Staates innerhalb seines Hoheitsgebietes eine erleichterte Beweisführung für den von der Völkerrechtsverletzung betroffenen Opferstaat. ${ }^{41}$ Der IGH geht davon aus, dass es dem Opferstaat in der Regel nicht möglich sein wird, direkte Beweise zu liefern, so dass auch Indizienbeweise zulässig sein müssen..$^{42}$ Sofern kein Raum für begründete Zweifel verbleibt, sind logische Schlussfolgerungen aus einer Reihe von Fakten ausreichend, um den erforderlichen Beweis zu erbringen. ${ }^{43}$ So ist die zwischenstaatliche Konfliktlage im Allgemeinen zu beleuchten, und die Haltung des pflichtigen Staates vor und nach dem fraglichen Verhalten im Besonderen hat entscheidendes Gewicht bei der Beweisführung. ${ }^{44}$

\section{Konsequenzen der Staatenverantwortlichkeit}

Der StIGH konstatierte im Chorzów Factory-Fall: „It is a principle of international law that the breach of an engagement involves an obligation

38 IGH, Application of the Convention on the Prevention and Punishment of the Crime of Genocide (Bosnia and Herzegovina v. Serbia and Montenegro), Judgment of 26 February 2007, ICJ Reports 2007, S. 43 (222 Rn. 431).

39 IGH, Corfu Channel Case (United Kingdom of Great Britain and Northern Ireland v. Albania), Judgment of 9 April 1949, ICJ Reports 1949, S. 4 (18).

40 Ibid.; so auch I. Y. Liu, State Responsibility and Cyberattacks, IJICL 4 (2017), S. 191 (238).

41 IGH, Corfu Channel Case (United Kingdom of Great Britain and Northern Ireland v. Albania), Judgment of 9 April 1949, ICJ Reports 1949, S. 4 (18).

42 Ibid.

43 Ibid.

44 Id., S. 4 (18 ff.); so auch J. D. Jolley, Attribution, State Responsibility, and the Duty to Prevent Malicious Cyber-Attacks in International Law, 2017, abrufbar unter: http://theses.gla.ac.uk/id/eprint/8452 (geprüft am 15.05.2020), S. 194. 


\section{Kapitel: Konstitutive Elemente der staatlichen Einstandspflichten}

to make reparation in an adequate form. " 45 Wie gezeigt, unterscheidet der StIGH für die Bestimmung des adäquaten Umfangs der Wiedergutmachung zwischen rechtswidrigem und rechtmäßigem Verhalten. ${ }^{46}$ Die Pflicht zur Wiedergutmachung ist im Rahmen der Staatenverantwortlichkeit entsprechend umfassender als im System der Staatenhaftung. Dies liegt daran, dass die Staatenhaftung primär die Kompensation von Schäden sowie einen Interessenausgleich zwischen gleichberechtigten souveränen Staaten und eine gerechte Risikoverteilung zum Ziel hat. Das Recht der Staatenverantwortlichkeit verfolgt hingegen den Schutz der internationalen Rechtsordnung und die Durchsetzung internationaler Verpflichtungen. ${ }^{47}$

Während im Rahmen der Staatenhaftung neben den Abhilfemaßnahmen nach Schadenseintritt lediglich Geldersatz zu leisten ist, ${ }^{48}$ umfasst die Wiedergutmachung im Rahmen der Staatenverantwortlichkeit die Wiederherstellung des status quo ante (Art.35 ASR) und erst wenn die Wiederherstellung unmöglich oder unverhältnismäßig ist, muss Schadensersatz geleistet werden, der auch einen etwaigen entgangenen Gewinn umfasst (Art. 36 ASR). Sofern keine Naturalrestitution möglich ist oder der Verlust sich nicht in Vermögenswerten bemessen lässt, ist Genugtuung, etwa durch ausdrückliche Übernahme der Verantwortung, Erklärung des Bedauerns oder formale Entschuldigung zu gewähren (Art. 37 ASR).

Außerdem ist zu berücksichtigen, dass Staatenverantwortlichkeit einschneidende Konsequenzen für das zwischenstaatliche Miteinander hat, denn völkerrechtswidriges Verhalten berechtigt den Opferstaat zu Gegenmaßnahmen, die an sich völkerrechtwidrig wären und nur erlaubt sind, weil sie als Reaktion auf einen Völkerrechtsbruch durch einen anderen Staat ergriffen werden (Art. 22 ASR). ${ }^{49}$ Im Ergebnis ist daher bei der Be-

45 StIGH, Factory at Chorzów (Germany v. Poland), Judgment of 26 July 1927 (Jurisdiction), PCIJ Series A, No. 9, S. 21.

46 Siehe die Ausführungen im 1. Kapitel D. I. 4. c).

47 Vgl. Sonderberichterstatter P. S. Rao, Third report on international liability for injurious consequences arising out of acts not prohibited by international law (prevention of transboundary damage from hazardous activities), YBILC 2000II/1, UN Doc. A/CN.4/510, S. 121, Rn. 27; vgl. auch A. E. Boyle, State Responsibility and International Liability for Injurious Consequences of Acts Not Prohibited by International Law, Int'l \& Comp. L.Q. 39 (1990) S. 1 (13).

48 P. Stubbe, State Accountability for Space Debris, 2018, S. 424; A. Tanzi, Liability for Lawful Acts, in: R. Wolfrum (Hg.), MPEPIL 2013, http://www.mpepil.com, Rn. 11.

49 Vgl. R. Crootof, International Cybertorts, CLR 103 (2018), S. 565 (630); A. Tanzi, Liability for Lawful Acts, in: R. Wolfrum (Hg.), MPEPIL 2013, 
urteilung der völkerrechtswidrigen Qualität einer bestimmten Aktivität grundsätzlich ein restriktiver Maßstab anzulegen, um nicht zu einer Erosion des Völkerrechts beizutragen. ${ }^{50}$

\section{B. Konstitutive Elemente der Staatenhaftung}

Aus der Rechtsquellenanalyse lassen sich Voraussetzungen für die Staatenhaftung abstrahieren. Die Feststellung des Schiedsgerichtes in der Trail Smelter-Entscheidung: ,under the principles of international law, (...), no State has the right to use or permit the use of its territory in such a manner as to cause injury (...) in or to the territory of another or the properties or persons therein, when the case is of serious consequence and the injury is established by clear and convincing evidence ${ }^{" 51}$ dient dabei als Leitsatz.

\section{Anwendungsbereich}

Das Konzept der Staatenhaftung bezieht sich auf einen Schaden (1.), der aus einer (hoch-)gefährlichen Aktivität resultiert (2.), wobei die Kausalität zwischen der Aktivität und dem Schaden nachweisbar sein muss (3.).

\section{Schaden}

Der Schaden bildet den Anknüpfungspunkt für die Staatenhaftung, da hier keine Rechtsverletzung vorliegt und nur der Schaden einen Ausgleich konkurrierender Interessen im Rahmen völkerrechtlich nicht verbotener

http://www.mpepil.com, Rn.11. Zu den Rechtsfolgen nach dem Regime der Staatenverantwortlichkeit siehe S.-H. Schulze, Cyber-„,War“ - Testfall der Staatenverantwortlichkeit, 2015, S. $67 \mathrm{ff}$.

50 Vgl. E. T. Jensen/S. Watts, A Cyber Duty of Due Diligence, Tex. LR 95 (2017), S. 1555 (1568 ff.).

51 Trail Smelter Arbitration (United States v. Canada), Award of 16 April 1938 and 11 March 1941, 3 UNRIAA (1941), S. 1905 (1965). 
Aktivitäten erforderlich macht. ${ }^{52}$ Doch nicht jeder Schaden eröffnet den Anwendungsbereich der Staatenhaftung. ${ }^{53}$

In den völkervertraglich erfassten Konstellationen ist der Schadensbegriff in aller Regel definiert. Die Vertragsparteien einigen sich auf ein Schutzgut, dem eine Interessenabwägung vorausgeht. ${ }^{54}$ Dies veranschaulichen etwa die wirtschaftsvölkerrechtlichen Regeln, denen eine Abwägung zwischen wirtschaftlichem Interesse und zulässigen Handelspolitiken zugrunde liegt. ${ }^{55}$ Die Mitglieder der WTO haben sich darauf geeinigt, dass die Zugeständnisse, die aus den WTO-Abkommen folgen, eine geschützte Rechtsposition darstellen, deren Beeinträchtigung oder Zunichtemachen als Schaden zu werten ist. ${ }^{56}$ Außerhalb vertraglicher Bestimmungen lassen sich mithilfe der übrigen Rechtsquellen und Rechtserkenntnisquellen ebenfalls Kriterien festmachen, die das Schadensmerkmal ausfüllen. Dabei ist eine Abwägung zwischen territorialer Souveränität und territorialer Integrität maßgebend. ${ }^{57}$

Es besteht weitestgehend Einigkeit darüber, dass Mensch, Eigentum und Umwelt geschützte Rechtspositionen sind und physische Auswirkungen auf diese gegeben sein müssen, mithin nachteilige wirtschafts-, finanz- und haushaltspolitische Folgen grundsätzlich aus dem Anwendungsbereich der Staatenhaftung fallen. ${ }^{58}$

52 Im Rahmen der Diskussionen um die Staatenverantwortlichkeit finden sich ebenfalls Stimmen, die ein Schadenserfordernis proklamieren. Allerdings wird in diesem Zusammenhang jede Beeinträchtigung einer völkerrechtlichen Rechtsposition - ob materieller oder immaterieller Art - als Schaden angesehen. Dementsprechend erübrigt sich die Diskussion um das Schadenserfordernis im Rahmen der Staatenverantwortlichkeit, da nach diesem Verständnis deliktisches Handeln in aller Regel mit einem Schaden verbunden ist. Siehe ASR, Kommentar zu Art. 31, S.92, Abs.7; Sonderberichterstatter J. Crawford, Fourth report on State Responsibility, YBILC 2001-II/1, UN Doc. A/CN.4/517 and Add. 1, Rn. 34, S. 9 f.; S. Sucharitkul, State Responsibility and International Liability under International Law, Loy. L.A. ICLR 18 (1996), S. 821 (834).

53 Vgl. A. Epiney, Das „Verbot erheblicher grenzüberschreitender Umweltbeeinträchtigungen“, AVR 33 (1995), S. 309 (329 ff.).

54 M. Kumm, The Cosmopolitan Turn in Constitutionalism, Ind. GLSJ 20 (2013), S. 605 (623).

55 J. Bäumler, Das Schädigungsverbot im Völkerrecht, 2017, S. 274 f., 277.

$56 I d$., S. $274 \mathrm{f}$.

57 Vgl. id., S. 276 ff.; M. Ronzoni, The Global Order, Philosophy \& Public Affairs 37 (2009), S. 229 (249); T. Stein/T. Marauhn, Völkerrechtliche Aspekte von Informationsoperationen, ZaöRV 60 (2000), S. 1 (21); R. Verheyen, Climate Change and Damage in International Law, 2005, S. 151.

58 Präventionsartikel, Kommentar zu Art. 1, S. 151, Abs. 16. 
Außerdem ist eine Grenzüberschreitung erforderlich. Eine Grenzüberschreitung ist bereits dann gegeben, wenn das Territorium eines anderen Staates bzw. Territorium unter fremdstaatlicher Hoheitsgewalt betroffen ist, unabhängig davon, ob der einwirkende und der belastete Staat Gebietsnachbarn sind oder nicht. ${ }^{59}$ Demnach unterfallen zwischenstaatliche Schäden dem Anwendungsbereich der Staatenhaftung.

Eine Staatenhaftung kommt schließlich nur in Betracht, wenn der Schaden eine Intensität erreicht, die herkömmlicherweise nicht geduldet wird. ${ }^{60}$ Diese Erheblichkeit eines Schadens lässt sich anhand des Rangs der betroffenen (Rechts-)Güter, der Zahl der betroffenen Personen, Sachen und Umweltelemente sowie der räumlichen und zeitlichen Schadensdimension bestimmen. ${ }^{61}$ Dabei sind unter dem Blickwinkel eines gerechten Interessenausgleiches immer auch Zumutbarkeitsgesichtspunkte und Verhältnismäßigkeitsaspekte in die Bewertung mit einzubeziehen. ${ }^{62} \mathrm{Im}$ Ergebnis kann nur eine Einzelfallbetrachtung zeigen, ob die Kriterien des Schadensmerkmals erfüllt sind und ein Interessenausgleich in Gestalt der Staatenhaftung erforderlich wird. ${ }^{63}$

Das Element des grenzüberschreitenden erheblichen Schadens ist indes dynamisch und muss dem Wandel der Zeit entsprechend interpretiert werden. ${ }^{64}$ So lösen mittlerweile nicht nur zwischenstaatliche Schäden, sondern auch grenzübergreifende Schäden an anerkannten Gemeinschafts-

59 Nach Präventionsartikel 2 lit. c ist ohne Belang, ob die betroffenen Staaten aneinandergrenzen. Auch der IGH beschränkt das Schädigungsverbot nicht auf das Nachbarverhältnis. Vgl. IGH, Legality of the Threat or Use of Nuclear Weapons, Advisory Opinion of 8 July 1996, ICJ Reports 1996, S. 226 (241 f. Rn. 29).

$60 \mathrm{~J}$. Barboza, The Environment, Risk and Liability in International Law, 2011, S. $10 \mathrm{f} .$, 71. Teilweise wird weiter zwischen „serious“ und „significant" differenziert. Siehe hierzu M. N. Schmitt, Grey Zones in the International Law of Cyberspace, YIL Online 42 (2017), abrufbar unter: https://cpb-us-w2.wpmucdn.com/ca mpuspress.yale.edu/dist/8/1581/files/2017/08/Schmitt_Grey-Areas-in-the-Internati onal-Law-of-Cyberspace-1cab8kj.pdf (geprüft am 15.05.2020), S. 1 (11 f.).

61 H.-G. Dederer, Staatenverantwortlichkeit ("State responsibility") und Haftung ("liability") im Bereich der "ultrahazardous activities", in: B. Hecker/R. Hendler/A. Proelß/P. Reiff (Hg.), Verantwortlichkeit und Haftung für Umweltschäden, 2013, S. 13 (16 Fn. 5).

62 J. Bäumler, Das Schädigungsverbot im Völkerrecht, 2017, S. 278; A. Epiney, Das „Verbot erheblicher grenzüberschreitender Umweltbeeinträchtigungen“, AVR 33 (1995), S. 309 (335); R. Verheyen, Climate Change and Damage in International Law, 2005, S. $151 \mathrm{f}$.

63 Vgl. Präventionsartikel, Kommentar zu Art. 2, S. 152, Abs. 4.

64 Vgl. Präventionsartikel, Kommentar zu Art. 1, S. 149 f., Abs. 4. 
gütern in hoheitsfreien Räumen staatliche Einstandspflichten aus. ${ }^{65}$ Auch wirtschaftliche Externalitäten rücken in den Fokus der Staatenhaftung, da diese einschneidende Eingriffe in fremdstaatliche Souveränität bedeuten können und mithin das Erfordernis physischer Auswirkungen relativieren. ${ }^{66}$

\section{2. (Hoch-)gefährliche Aktivität}

Das Konzept der Staatenhaftung bezieht sich auf Aktivitäten, denen ein bestimmtes Risiko inhärent ist. Während im Rahmen der Staatenverantwortlichkeit ein spezifisches Verhalten zugerechnet wird, geht es hier also um die Zuordnung von Risikosphären. ${ }^{67}$

Der Begriff Risiko beschreibt das Produkt von Schadenswahrscheinlichkeit und Schadensumfang. ${ }^{68}$ Die hohe Wahrscheinlichkeit der Verursachung eines erheblichen Schadens, welche gefährliche Aktivitäten kennzeichnet, oder die - auch noch so geringe - Wahrscheinlichkeit der Verursachung eines katastrophalen Schadens, welche hochgefährliche Aktivitäten charakterisiert, eröffnen den Anwendungsbereich der Staatenhaftung. ${ }^{69}$ Zur Beurteilung der Schadensintensität (erheblich bzw. katastrophal) sind die Anzahl der geschädigten Personen und Sachen, die Umwelt-

65 J. Bäumler, Das Schädigungsverbot im Völkerrecht, 2017, S. 273.

66 Vgl. id., S. 276; D. Bodansky, The Art and Craft of International Environmental Law, 2010, S. 11.

67 S. Townley stellt zutreffend fest: „Risk-based norms, of course, better account for the potential harms caused by non-state actors than do state attribution rules“. $S$. Townley, The Rise and Risk in International Law, Chi. JIL 18 (2018), S. 594 (639).

68 Präventionsartikel, Kommentar zu Art. 2, S. 152, Abs. 2; J. Barboza, The Environment, Risk and Liability in International Law, 2011, S. 10; H.-G. Dederer, Staatenverantwortlichkeit ("State responsibility") und Haftung ("liability") im Bereich der "ultrahazardous activities", in: B. Hecker/R. Hendler/A. Proelß/P. Reiff (Hg.), Verantwortlichkeit und Haftung für Umweltschäden, 2013, S. 13 (15 Fn. 4).

69 Vgl. Präventionsartikel Art. 2 lit. a; H.-G. Dederer, Staatenverantwortlichkeit ("State responsibility") und Haftung ("liability") im Bereich der "ultrahazardous activities”, in: B. Hecker/R. Hendler/A. Proelß/P. Reiff (Hg.), Verantwortlichkeit und Haftung für Umweltschäden, 2013, S. 13 (15f.); G. Handl/B. Simma, Grenzüberschreitende Auswirkungen von Kernkraftanlagen und Völkerrecht, ÖZöRV 39 (1988), S. 1 (3); T. Stephens, International Courts and Environmental Protection, 2009, S. 157; C. W. Jenks, Liability for Ultrahazardous Activities in International Law, RdC 177 (1966), S. 99 (107) in Bezug auf hochgefährliche Aktivitäten. 
auswirkungen sowie die Dauer und territoriale Reichweite der Schädigung maßgeblich. ${ }^{70}$

Das Risiko-Kriterium ist für das Konzept der Staatenhaftung konstitutiv, weil es Maßnahmen zur Schadensprävention bedingt und mithin eine Verbindung zwischen den wesentlichen Komponenten der Schadensvermeidungspflicht und der Kompensationspflicht im Konzept der Staatenhaftung schafft. ${ }^{71}$ Der Anwendungsbereich der Staatenhaftung wird zugleich durch das Element des Risikos begrenzt, so dass ein gerechter Interessenausgleich erst möglich wird. ${ }^{72}$ Die Begrenzung wirkt aber nur dann, wenn nach vernünftigen Erwägungen ex ante keine Anzeichen dafür bestanden, dass Aktivitäten zu Schäden führen können. ${ }^{73}$ Diese Vorhersehbarkeit unterliegt einer weiten Auslegung, weil Staaten bereits Pflichten zur Risikoidentifizierung, Überwachung und Kontrolle treffen. ${ }^{74}$ Außerdem relativiert der Vorsorgegrundsatz das Element der Vorhersehbarkeit noch weiter, indem dieser bereits Indizien ausreichen lässt. ${ }^{75}$ Während bei positiven Handlungspflichten, deren Verletzung zur Staatenverantwortlichkeit führt, (hypothetische bzw. mutmaßliche) Kenntnis vom rechtsverletzenden Verhalten vorausgesetzt wird, ist der Anwendungsbereich der Staatenhaftung durch das Kriterium der Vorhersehbarkeit nur bedingt begrenzt. ${ }^{76}$

70 H.-G. Dederer, Staatenverantwortlichkeit ("State responsibility") und Haftung ("liability") im Bereich der "ultrahazardous activities", in: B. Hecker/R. Hendler/A. Proelß/P. Reiff (Hg.), Verantwortlichkeit und Haftung für Umweltschäden, 2013, S. 13 (16 Fn. 5).

$71 \mathrm{~J}$. Barboza, The Environment, Risk and Liability in International Law, 2011, S. 14.

72 Präventionsartikel, Kommentar zu Art. 2, S. 152, Abs. 2.

73 U. Beyerlin/T. Marauhn, International Environmental Law, 2011, S. 43; B. Saxler/J. Siegfried/A. Proelss, International liability for transboundary damage arising from stratospheric aerosol injections, Law, Innovation and Technology 7 (2015), S. 112 (124); D. M. Schmitt, Staatenverantwortlichkeit für Schäden an der biologischen Vielfalt, 2018, S. 298.

74 D. M. Schmitt, Staatenverantwortlichkeit für Schäden an der biologischen Vielfalt, 2018, S. 298.

75 Id., S. $298 \mathrm{f}$.

76 Es wurde im Rahmen der Haftungsstudie der Völkerrechtskommission diskutiert, ob Kenntnis zu fordern sei, um Entwicklungsländer zu schützen, da diese nur über beschränkte Mittel zur Identifizierung von Risikoquellen auf ihrem Territorium verfügten. Sonderberichterstatter J. Barboza, Fourth report on international liability for injurious consequences arising out of acts not prohibited by international law, YBILC 1988-II/1, UN Doc. A/CN.4/413, S. 262, Rn. 69. Dieses Erfordernis wurde im Ergebnis abgelehnt. S. Sucharitkul schlägt darüber hinaus vor, gänzlich auf diese Einschränkung zu verzichten. „On the contrary, the liability regime should cover foreseen, unforeseen, accidental as well as incidental damage arising out of the activity in question." S. Sucharitkul, Responsibility 
Das Merkmal der Vorhersehbarkeit verdeutlicht allerdings, dass die Staatenhaftung keine schädigenden Einzelakte im Laufe einer Aktivität betrifft. Denn nur bei Aktivitäten im Gesamten, wie etwa beim Betrieb einer Zink- und Bleischmelze, beim Bau und Betrieb eines Staudammes, beim Betrieb einer Nuklearanlage oder bei der Erforschung und Nutzung von Gemeinschaftsgütern bzw.- räumen, ist ein bestimmtes Risiko und damit der Regelungsbedarf von vornherein erkennbar. Die vorhersehbare Gefährdung entsteht nicht durch einen konkreten Unfall oder durch ein sonstiges schädigendes Ereignis, sondern durch die Aktivität im Gesamten. 77

Schließlich erfasst die Staatenhaftung gefährliche Aktivität nur insoweit, als sie primär wirtschaftliche und/oder gemeinnützige Vorteile bieten. Denn nur dann besteht auch ein legitimes Interesse der Staaten daran, diese Gefährdung nicht zu verbieten. Der Schadenseintritt ist hier eine ungewollte Nebenwirkung. ${ }^{78}$

Der Eintritt eines Schadens allein impliziert indes nicht, dass Risiken bestanden haben. Aus diesem Grund wird neben einer Haftung für (hoch-)gefährliche Aktivitäten eine Haftung für einfache zwischenstaatliche Schädigungen diskutiert. ${ }^{79}$ Im Rahmen des AIL-Entwurfs der Völkerrechtskommission aus dem Jahr 1996 war dementsprechend eine Haftung für Aktivitäten, denen kein Risiko immanent ist, angedacht. Allerdings stand bereits zu Beginn fest, dass diese - wenn überhaupt - eine untergeordnete Rolle bei der Ausgestaltung der Staatenhaftung spielt, und sie wurde schließlich gänzlich aus dem Anwendungsbereich der Staatenhaftung gestrichen. Eine Haftungsbeschränkung auf (hoch-)gefährliche Akti-

and Liability for Environmental Damage Under International Law, Golden Gate University Law Digital Commons 664 (1996), abrufbar unter: https://digitalcom mons.law.ggu.edu/pubs/664 (geprüft am 15.05.2020), S. 1 (18).

77 Siehe insbesondere 1. Kapitel A. II. 1. a).

78 Sonderberichterstatter R. Q. Quentin-Baxter, Third report on international liability for injurious consequences arising out of acts not prohibited by international law, YBILC 1982-II/1, UN Doc. A/CN.4/360, S. 60, Rn. 39.

79 Es wird in diesem Zusammenhang zwischen einerseits schädlichen Aktivitäten, welche definitiv negative Auswirkungen haben und zur Staatenverantwortlichkeit führen, und andererseits risikobehafteten Aktivitäten, welche potenziell zu negativen Auswirkungen führen und Staatenhaftung begründen, unterschieden. J. Barboza, The Environment, Risk and Liability in International Law, 2011, S. 13 unter Bezugnahme auf Experts Group on Environmental Law of the World Commission on Environment and Development, R. D. Munro (Chairman)/J. G. Lammers (Rapporteur), Environmental Protection and Sustainable Development: Legal Principles and Recommendations, 1987, S. 86. 
vitäten ist zwar nicht zwingend. ${ }^{80}$ Haftungsregeln für sonstige schädliche Aktivitäten sollten aber im Rahmen von Übereinkommen normiert werden, da hier in der Regel genaue Haftungsvoraussetzungen bestimmt und einer Ausuferung der Staatenhaftung entgegengewirkt werden kann. Die Analyse der wirtschaftsvölkerrechtlichen Bestimmungen hat veranschaulicht, dass Staaten eine staatliche Einstandspflicht für erlaubte Maßnahmen anerkannt haben, ohne dass es auf Prävention ankäme. Hier ist auch kein Risiko gefordert, um den Anwendungsbereich der staatlichen Einstandspflicht zu begrenzen.

\section{Kausalität}

Sowohl bei der Staatenverantwortlichkeit für die Verletzung positiver Handlungspflichten als auch im Konzept der Staatenhaftung bilden Territorium oder Hoheitsgewalt Anknüpfungspunkte für die staatliche Einstandspflicht. Die Zurechnung völkerrechtswidrigen Verhaltens auf staatlichem Hoheitsgebiet oder unter staatlicher Hoheitsgewalt basiert auf einer kausalen Beziehung zwischen dem staatlichen Unterlassen und der Rechtsverletzung, so dass die Verantwortlichkeit im Zusammenhang mit privaten Verhaltensweisen nur unter eng gefassten Voraussetzungen gegeben ist. ${ }^{81} \mathrm{Im}$ Rahmen der Haftung hingegen ist lediglich eine Ursächlichkeit zwischen der (hoch-)gefährlichen Aktivität auf staatlichem Territorium oder unter staatlicher Kontrolle und dem eingetretenen Schaden nachzuweisen und kann daher zu gerechteren Ergebnissen führen. ${ }^{82}$

Dabei wird nicht verkannt, dass bei Langzeitfolgen, wissenschaftlich unzureichend nachweisbaren oder unschlüssigen Ursachenzusammenhängen sowie bei multikausalen Verursachungsbeiträgen die Feststellung eines kausalen Zusammenhangs zwischen Aktivität und Schaden Schwierigkeiten bereiten kann. ${ }^{83}$ Erschwerend kommt hinzu, dass nach den völkerrechtlichen Beweislastregeln grundsätzlich jeder Staat die für ihn günsti-

80 D. M. Schmitt, Staatenverantwortlichkeit für Schäden an der biologischen Vielfalt, 2018, S. 298.

81 ASR, Kommentar zu Art. 31, S. 92 f., Abs. 10.

82 Vgl. S. Sucharitkul, State Responsibility and International Liability under International Law, Loy. L.A. ICLR 18 (1996), S. 821 (834f.); B. A. Walton, Duties Owed, Yale LJ 126 (2017), S. 1460 (1518).

83 J. Barboza, The Environment, Risk and Liability in International Law, 2011, S. 11; D. M. Schmitt, Staatenverantwortlichkeit für Schäden an der biologischen Vielfalt, 2018, S. 399. 


\section{Kapitel: Konstitutive Elemente der staatlichen Einstandspflichten}

gen Tatsachen nachweisen muss. ${ }^{84}$ Während aber der Nachweis der kausalen Beziehung zwischen der Verletzung einer positiven Handlungspflicht, bezogen auf privates Verhalten, und einer Rechtsverletzung strengen Anforderungen unterliegt, um nicht zu einer Umgehung des Zurechnungserfordernisses zu führen, ${ }^{85}$ ist ein erleichterter Nachweis der Kausalität einer völkerrechtlich nicht verbotenen gefährlichen Aktivität für einen Schaden wegen des umweltvölkerrechtlichen Verursacher- und Vorsorgegrundsatzes zulässig. ${ }^{86}$ Dem Verursachergrundsatz liegt der Gedanke zugrunde, dass die Kosten im Schadensfall nicht auf unbeteiligte Opfer externalisiert werden dürfen und die Risikolast von denjenigen zu tragen ist, die das Risiko direkt oder indirekt schaffen. Sofern also mehrere Verursacher in Betracht kommen und nicht klar abgrenzbar ist, welcher Beitrag für welchen Schaden ursächlich ist, oder einzelne Beiträge erst kumulativ zu einem erheblichen Schaden führen, erlaubt der Verursachergrundsatz die Annahme, dass jede der Aktivitäten den Kausalitätsanforderungen genügt. ${ }^{87}$ Außerdem sind auf der Grundlage der Risikozuteilung nach dem Verursachergrundsatz konkurrierende Beiträge in ein Verursachungsbündel zusammenzufassen, so dass das Verhalten einer Gefahrengemeinschaft im Gesamten als ursächlich gilt. ${ }^{88}$ Aufgrund des Vorsorgegrundsatzes können die Anforderungen an einen wissenschaftlich zureichenden oder schlüssigen Nachweis des Ursachenzusammenhanges erleichtert werden, indem bereits die Möglichkeit der Schadensverursachung durch eine Aktivität das Kausalitätskriterium erfüllt. ${ }^{89}$ Schließlich wird insbesondere mit

84 M. Benzing, Das Beweisrecht vor internationalen Gerichten und Schiedsgerichten in zwischenstaatlichen Streitigkeiten, 2010, S. $595 \mathrm{f}$.

85 Vgl. ITLOS, Responsibilities and obligations of states sponsoring persons and entities with respect to activities in the Area, Advisory Opinion of 1 February 2011, ITLOS Case No. 17, ITLOS Reports 2011, S. 10 (60 Rn. 182, Rn. 184); IGH, Pulp Mills on the River Uruguay (Argentina v. Uruguay), Judgment of 20 April 2010, ICJ Reports 2010, S. 14 (98 f. Rn. 257).

86 Vgl. Art. 7 IDI-Resolution; S. Förster, Internationale Haftungsregeln für schädliche Folgewirkungen gentechnisch veränderter Organismen, 2007, S. 281 f.; D. M. Schmitt, Staatenverantwortlichkeit für Schäden an der biologischen Vielfalt, 2018, S. 403.

87 D. M. Schmitt, Staatenverantwortlichkeit für Schäden an der biologischen Vielfalt, 2018, S. 401.

88 Vgl. J. Köndgen, Überlegungen zur Fortbildung des Umwelthaftpflichtrechts, UPR 11-12 (1983), S. 345 (353).

89 Id., S. 345 (346 ff., 353, 355); D. M. Schmitt, Staatenverantwortlichkeit für Schäden an der biologischen Vielfalt, 2018, S. 400 ff.; T. Stephens, International Courts and Environmental Protection, 2009, S. 134. Diese Beweiserleichterung steht indes nicht im Widerspruch zur Pulp Mills-Entscheidung, in der der IGH eine Beweis- 
Blick auf hochgefährliche Aktivitäten eine Kausalität durch die akute Gefahrenlage indiziert. ${ }^{90}$

\section{Schadensvermeidung und Schadenskompensation}

Schadensvermeidung und Schadenskompensation bilden die Grundpflichten der Staatenhaftung. ${ }^{91}$

Schadensvermeidung beinhaltet Pflichten zur Schadensvorsorge, Schadensprävention und Schadensminderung. ${ }^{92}$ Die Schadensvorsorge erfordert, dass selbst bei wissenschaftlichen Unsicherheiten bei der Bewertung von Ursachenzusammenhängen und möglichen Auswirkungen potenziell gefährlicher Aktivitäten allgemeine Vorsorgemaßnahmen zur Vermeidung ernsthafter oder unwiderruflicher Schäden zu ergreifen sind. ${ }^{93}$ So müssen administrative und legislative Strukturen zur Regulierung vorhanden sein, die besten Umweltschutzpraktiken sind anzuwenden, und strategische, politische sowie organisatorische Vorkehrungen sind zu treffen. ${ }^{94}$ Schadensprävention und -minderung bedeuten, dass alle möglichen und zumutbaren Maßnahmen zu ergreifen sind, um Schäden durch bestimmte gefährliche Aktivitäten zuvorzukommen oder jedenfalls deren konkretes Risiko zu minimieren sowie schädigende Auswirkungen möglichst zu beseitigen bzw. zu begrenzen. ${ }^{95}$ (Hoch-)gefährliche Aktivitäten müssen

lastumkehr auf Grundlage des Vorsorgegebotes ablehnte und das Vorliegen eines Schadens durch den anspruchsstellenden Staat nachgewiesen haben wollte. IGH, Pulp Mills on the River Uruguay (Argentina v. Uruguay), Judgment of 20 April 2010, ICJ Reports 2010, S. 14 (98 f. Rn. 257).

90 Vgl. D. M. Schmitt, Staatenverantwortlichkeit für Schäden an der biologischen Vielfalt, 2018, S. 403.

91 B. A. Walton, Duties Owed, Yale LJ 126 (2017), S. 1460 (1465); vgl. auch R. Lefeber, Transboundary Environmental Interference and the Origin of State Liability, 1996, S. $34 \mathrm{ff}$.

92 Siehe hierzu insbesondere die Ausführungen im 1. Kapitel B. I. und im 1. Kapitel E. II.

93 J. M. Van Dyke, Liability And Compensation For Harm Caused By Nuclear Activities, Denver JILP 35 (2006), S. 13 (18f.); A. Proelß, Prinzipien des internationalen Umweltrechts, in: ders. (Hg.), Internationales Umweltrecht, 2017, S. 69 (87 Rn. 30).

94 J. Barboza, The Environment, Risk and Liability in International Law, 2011, S. 15.

95 Id., S. 19. 
reguliert, kontrolliert und beaufsichtigt werden. ${ }^{96}$ Risiko- und Schadensmanagement müssen dem Stand der Technik entsprechen. ${ }^{97}$

Schadensvermeidung begründet zudem verfahrensbezogene Pflichten. ${ }^{98}$ Dazu zählen die Pflicht, eine Risikoanalyse, die eine Umweltverträglichkeitsprüfung beinhalten muss, durchzuführen, Notfallpläne aufzustellen, effektive Kontrollmechanismen einzusetzen sowie diverse Kooperationspflichten, etwa die Pflicht (potenziell) betroffene Staaten zu benachrichtigen, zu informieren, zu warnen und zu konsultieren. ${ }^{99}$

Die durch den Ursprungsstaat ergriffenen Maßnahmen zur Schadensvermeidung sind sodann bei der Bemessung der zu leistenden unverzüglichen, angemessenen und effektiven Wiedergutmachung zu berücksichtigen. ${ }^{100} \mathrm{Da}$ der gerechte Interessenausgleich zwischen Ursprungs- und Opferstaat das Wesen der Staatenhaftung bildet, muss aber auch der Opferstaat effektive Maßnahmen zur Schadensvermeidung, insbesondere zur Schadensminderung ergreifen. Dieser Beitrag des Opferstaates zur Schadensvermeidung beeinflusst wiederum die zwischenstaatlichen Verhandlungen zur Schadenskompensation. ${ }^{101}$

Der sekundärnormähnliche Charakter der Pflicht zur Schadenskompensation erschwert die Abgrenzung zur Staatenverantwortlichkeit. Daher ist zu betonen, dass Schadensausgleich im Rahmen der Staatenhaftung nicht die Konsequenz der Verletzung einer Primärnorm ist, sondern einer selbstständigen völkerrechtlichen Pflicht entspringt und auf Primärnormen beruht. ${ }^{102}$ Sekundärnormen sind in diesem Zusammenhang nur relevant, wenn die zusammengesetzte Primärnorm bestehend aus Schadensvermei-

96 A. E. Boyle, Nuclear Energy and International Law, BYBIL 60 (1989), S. 257 (269); A. Seibert-Fohr, Die völkerrechtliche Verantwortung des Staats für das Handeln von Privaten, ZaöRV 73 (2013), S. 37 (56 f.).

97 Präventionsartikel, Kommentar zu Art. 3, S. 155, Abs. 17.

98 Siehe 1. Kapitel B. I. 2.

99 J. Brunnée, Procedure and Substance in International Environmental Law, ESIL Reflections 5 (2016), abrufbar unter: https://esil-sedi.eu/post_name-123/ (geprüft am 15.05.2020).

100 Vgl. Art. 22 lit. a, b und j AIL.

101 Vgl. Art. 19 lit. e AIL; AIL, Kommentar zu Art. 22, S. 132, Abs. 9.

102 J. Barboza stellt zutreffend fest: „Remedies in liability are a part of primary rules, therefore they are neither sanctions nor secondary obligations. The contents of primary rules determine the amounts of and limits to compensation; those rules admit of more flexibility than the secondary rules of a responsibility regime. (...) Other remedies - such as cessation, satisfaction and securities and guarantees of non-repetition - are linked to the breach of an obligation and therefore are not applicable as part of sine delicto liability. "J. Barboza, The Environment, Risk and Liability in International Law, 2011, S. 6. 
dung und Schadenskompensation verletzt wird. Mit anderen Worten: die Verletzung der Staatenhaftung führt zu den sekundären Rechtsfolgen der Staatenverantwortlichkeit. ${ }^{103}$

Betrachtet man die Pflichten der Staatenhaftung genauer, wird ein weiterer Unterschied zur Staatenverantwortlichkeit deutlich. Die Staatenverantwortlichkeit betrifft die rechtlichen Konsequenzen nach der Verletzung einer völkerrechtlichen Pflicht, während der Fokus der Staatenhaftung auf Maßnahmen vor dem Schadenseintritt liegt. Auch wenn das Schadensmanagement Maßnahmen betrifft, die ex post zu ergreifen sind, können diese mit Blick auf die Pflicht zur Risikoidentifizierung und (relativen) Vorhersehbarkeit von Risiken bereits im Vorhinein festgelegt werden. ${ }^{104}$ Darüber hinaus wird der ex ante Perspektive des Konzepts der Staatenhaftung dadurch Rechnung getragen, dass zwischenstaatliche Verhandlungen über Kompensationsleistungen vor einem möglichen Schadenseintritt erfolgen und damit eine rechtfertigende Wirkung für die gefährliche Aktivität und mithin deren Rechtmäßigkeit entfalten. ${ }^{105}$

\section{Gebotene Sorgfalt}

Im Konzept der Staatenhaftung ist die gebotene Sorgfalt für die verhaltensbezogene Schadensvermeidungspflicht - nicht hingegen für die ergebnisbezogene Pflicht, keinen grenzüberschreitenden Schaden zu verursachen - von Bedeutung. Der geläufige Begriff der völkerrechtlichen Sorgfaltspflicht ist in diesem Zusammenhang irreführend, denn die gebotene Sorgfalt für sich stellt keine eigenständige Pflicht dar. ${ }^{106}$ Es handelt sich

103 Vgl. R. Crootof, International Cybertorts, CLR103 (2018), S. 565 (603); D. B. Magraw, Transboundary Harm, AJIL 80 (1986), S. 305 (313); A. Tanzi, Liability for Lawful Acts, in: R. Wolfrum (Hg.), MPEPIL 2013, http://www.mpepil.com, Rn. 14; B. A. Walton, Duties Owed, Yale LJ 126 (2017), S. 1460 (1488 f.).

104 A. Tanzi, Liability for Lawful Acts, in: R. Wolfrum (Hg.), MPEPIL 2013, http:// www.mpepil.com, Rn. 11.

105 Vgl. Experts Group on Environmental Law of the World Commission on Environment and Development, R. D. Munro (Chairman)/J. G. Lammers (Rapporteur), Environmental Protection and Sustainable Development: Legal Principles and Recommendations, 1987, S. 85 ff.; A. Tanzi, Liability for Lawful Acts, in: R. Wolfrum (Hg.), MPEPIL 2013, http://www.mpepil.com, Rn. 11.

106 Zur Bedeutung der gebotenen Sorgfalt im Völkerrecht siehe ILA, Study Group on Due Diligence in International Law, Tim Stephens (Rapporteur)/Duncan French (Chair), Second Report, July 2016, abrufbar unter: http://www.ila -hq.org/index.php/study-groups?study-groupsID=63 (geprüft am 15.05.2020); 
vielmehr um Sorgfaltsstandards, die als Annex zu bestimmten völkerrechtlichen Pflichten fungieren und nach der jeweiligen Sachgrundlage zu bestimmen sind. ${ }^{107}$ Die gebotene Sorgfalt bezeichnet allgemein das Bestreben zur Erreichung des in einer Verpflichtung festgelegten Ziels und variiert mit Blick auf Kontext, Sinn und Zweck der jeweiligen Regel. ${ }^{108}$ Entsprechend gibt es Konstellationen, in denen striktere oder mildere Sorgfaltsstandards gefordert werden. ${ }^{109}$ Das Fehlen von allgemeingültigen Sorgfaltsstandards führt zwar bei neuen Entwicklungen, wie sie sich im Rahmen des technologischen Fortschritts abzeichnen, zu Rechtsunsicherheit. ${ }^{110}$ Haftungssysteme, die verhaltensbezogene Schadensvermeidungspflichten etablieren und das Ausmaß der Haftung entsprechend an die Beachtung bzw. Missachtung der gebührenden Sorgfalt anpassen, gewähren aber die notwendige Flexibilität, um einen vernünftigen Ausgleich zwischen internationalen Schutzinteressen und staatlichen Einstandspflichten zu ermöglichen. Hier bestärkt das Konzept der gebotenen Sorgfalt die völkerrechtliche Akzeptanz von Einstandspflichten durch die Staatengemeinschaft und hilft bei der Ausformung von Staatenpraxis und opinio iuris in diesem Bereich. ${ }^{111}$ Dies hat oftmals zur Folge, dass variable Sorgfaltsstan-

J. Kulesza, Due Diligence in International Law, 2016. Andere Stimmen in der Völkerrechtsliteratur bezeichnen die gebotene Sorgfalt als eigenständige Primärpflicht, einen allgemeinen Rechtsgrundsatz oder auch als einen aus dem Rechtsgrundsatz der Souveränität fließenden Grundsatz. Vgl. I. Y. Liu, State Responsibility and Cyberattacks, IJICL 4 (2017), S. 191 (199ff.); M. N. Schmitt, In Defense of Due Diligence in Cyberspace, Yale LJ F 125 (2015), S. 68 (71).

107 J. Bäumler, Das Schädigungsverbot im Völkerrecht, 2017, S. 293; A. Seibert-Fohr, Die völkerrechtliche Verantwortung des Staats für das Handeln von Privaten, ZaöRV 73 (2013), S. 37 (51).

108 ILA, Study Group on Due Diligence in International Law, Tim Stephens (Rapporteur)/Duncan French (Chair), Second Report, July 2016, abrufbar unter: http://www.ila-hq.org/index.php/study-groups?study-groupsID=63 (geprüft am 15.05.2020), S. 6.

109 B. A. Walton, Duties Owed, Yale LJ 126 (2017), S. 1460 (1490).

110 Die ITLOS-Meeresbodenkammer stellt zutreffend fest, der Sorgfaltsstandard „may change over time as measures considered sufficiently diligent at a certain moment may become not diligent enough in light, for instance, of new scientific or technological knowledge. It may also change in relation to the risks involved in the activity. "ITLOS, Responsibilities and obligations of states sponsoring persons and entities with respect to activities in the Area, Advisory Opinion of 1 February 2011, ITLOS Case No. 17, ITLOS Reports 2011, S. 10 (43 Rn. 117).

111 Vgl. P.-M. Dupuy, Reviewing the Difficulties of Codification, EJIL 10 (1999), S. $371(375)$. 
dards im Laufe der Zeit zu verschuldensunabhängigen Haftungssystemen reifen. ${ }^{112}$

Es besteht Einigkeit dahingehend, dass Sorgfaltsstandards zwar nur mit Blick auf die jeweilige Situation und das jeweilige Fachgebiet spezifiziert werden können, gleichwohl haben sich für die Schadensvermeidungspflicht allgemeingültige Parameter herauskristallisiert, die zur Bestimmung des Sorgfaltsrahmens herangezogen werden können. ${ }^{113}$

Zunächst spielt das Gefährdungspotenzial einer Aktivität bei der Bestimmung der gebotenen Sorgfalt eine entscheidende Rolle. ${ }^{114}$ Je höher die Wahrscheinlichkeit eines Schadenseintritts ist oder je weitreichender bzw. empfindlicher die Schadensfolge ist, desto höher ist das Maß an Sorgfalt. ${ }^{115}$ Dies impliziert, dass die Sorgfalt mit Blick auf potenziell katastrophale Schadensfolgen eigentlich das Verbot der hochgefährlichen Aktivität selbst gebieten müsste. Allerdings erschöpft sich die gebotene Sorgfalt auch hier im Wesentlichen in Maßnahmen zur Risikominimierung. ${ }^{116}$ Denn insbesondere bei Aktivitäten, die der Gesellschaft nutzen, wird das besonders hohe Gefährdungspotenzial oftmals in Kauf genommen. Daraus folgt, dass zur Bestimmung des Sorgfaltsstandards auch die potenziell beeinträchtigten Rechtsgüter bzw. Interessen einerseits und die aus der Aktivität erwachsenden Vorteile andererseits ins Verhältnis gesetzt werden müssen. ${ }^{117}$ Trotz der Vorteile, die aus hochgefährlichen Aktivitäten erwachsen, gilt hier - wenn schon kein Verbot - aber ein besonders strenger Sorgfaltsstandard. ${ }^{118}$

112 Vgl. G. Handl, State Liability for Accidental Transnational Environmental Damage by Private Persons, AJIL 74 (1980), S. 525 ( 550 ff.).

113 A. Seibert-Fohr, Die völkerrechtliche Verantwortung des Staats für das Handeln von Privaten, ZaöRV 73 (2013), S. 37 (49 f.).

114 Präventionsartikel, Kommentar zu Art. 3, S. 154, Rn. 11.

115 ILA, Study Group on Due Diligence in International Law, Tim Stephens (Rapporteur)/Duncan French (Chair), Second Report, July 2016, abrufbar unter: http://www.ila-hq.org/index.php/study-groups?study-groupsID=63 (geprüft am 15.05.2020), S. 20 ff.; G. Handl, State Liability for Accidental Transnational Environmental Damage by Private Persons, AJIL 74 (1980), S. 525 (550).

116 J. M. Kelson, State Responsibility and the Abnormally Dangerous Activity, Harv. ILJ 13 (1972) S. 197 (242); T. Koivurova, Due Diligence, in: R. Wolfrum (Hg.), MPEPIL 2013, http://www.mpepil.com, Rn. 17.

117 Vgl. IGH, Nuclear Tests (Australia v. France), Interim Protection, Order of 22 June 1973, ICJ Reports 1973, S. 99 (140 Rn. 28); A. E. Boyle, State Responsibility and International Liability for Injurious Consequences of Acts Not Prohibited by International Law, ICLQ 39 (1990), S. 1 (12 f.).

118 Vgl. G. Handl, State Liability for Accidental Transnational Environmental Damage by Private Persons, AJIL 74 (1980), S. 525 (550). 


\section{Kapitel: Konstitutive Elemente der staatlichen Einstandspflichten}

Ferner bemisst sich die gebotene Sorgfalt am Element „guter Regierungsführung“, wonach alle angemessenen und geeigneten Maßnahmen zu ergreifen sind, die eine vernünftige Regierung in der konkreten Situation zum Schutze eines Rechtsguts ergreifen würde. ${ }^{119}$ Die Staaten haben zwar ein weites Ermessen bei der Auswahl angemessener und geeigneter Maßnahmen, jedoch gilt für die Mittelauswahl nicht die Sorgfalt in eigenen Dingen, sondern die Sorgfalt entsprechend internationalen Standards, die sich an der Bedeutung des geschützten Rechtsguts und dem Ausmaß des zu erwartenden Schadens orientieren. ${ }^{120}$ Dabei gelten logischerweise höhere Anforderungen, wenn der Staat selbst durch seine de facto bzw. de jure Organe eine (hoch-)gefährliche Aktivität durchführt; schließlich kann er eigene Aktivitäten - anders als diejenigen privater Akteure - (besser) kontrollieren. ${ }^{121}$

Kontrovers diskutiert wird, ob auch die Kapazitäten des jeweiligen Staates eine Rolle spielen und mithin die jeweiligen ökonomischen, gesellschaftlichen und örtlichen Aspekte zu berücksichtigen sind. ${ }^{122}$ Nach einem derartigen subjektiven Maßstab hängt die gebotene Sorgfalt bei der Kontrolle von (hoch-)gefährlichen Aktivitäten auch von der verfügbaren Technik sowie von den jeweiligen administrativen und legislativen Strukturen eines Staates ab. ${ }^{123}$ Nur so kann dem Umstand Rechnung getragen werden, dass die Möglichkeiten zur Erfüllung völkerrechtlicher Verpflichtungen zwischen Staaten stark variieren. Der subjektive Maßstab gewährleistet die notwendige Flexibilität, damit auch Staaten mit limitierten Möglichkeiten am internationalen Rechtssystem mitwirken können, ohne vor unangemessene normative Anforderungen gestellt zu werden. ${ }^{124} \mathrm{Ge}$ gen einen subjektiven Sorgfaltsmaßstab wird angeführt, dass dieser einem

119 ILA, Study Group on Due Diligence in International Law, Tim Stephens (Rapporteur)/Duncan French (Chair), Second Report, July 2016, abrufbar unter: http://www.ila-hq.org/index.php/study-groups?study-groupsID=63 (geprüft am 15.05.2020), S. 7 ff.

120 A. Seibert-Fohr, Die völkerrechtliche Verantwortung des Staats für das Handeln von Privaten, ZaöRV 73 (2013), S. 37 (54).

121 B. A. Walton, Duties Owed, Yale LJ 126 (2017), S. 1460 (1499).

122 J. Brunnée/T. Meshel, Teaching an Old Law New Tricks, GYIL 58 (2015), S. 129 (136).

123 ILA, Study Group on Due Diligence in International Law, Tim Stephens (Rapporteur)/Duncan French (Chair), Second Report, July 2016, abrufbar unter: http://www.ila-hq.org/index.php/study-groups?study-groupsID=63 (geprüft am 15.05.2020), S. 13 ff.; Präventionsartikel, Kommentar zu Art. 3, S. 155, Abs. 13, 17.

124 I. Y. Liu, State Responsibility and Cyberattacks, IJICL 4 (2017), S. 191 (202). 
effektiven Rechtsgüterschutz zuwiderlaufe. Es ist dem zuzustimmen, dass in Konstellationen, in denen ein subjektiver Standard zur Erosion der Schadensvermeidungspflicht beitragen würde, keine Ausnahmen oder flexible Standards gewährt werden dürfen. ${ }^{125}$ So hat die ITLOS-Meeresbodenkammer in ihrem Gutachten zu den Verantwortlichkeiten und Pflichten der Staaten, die Personen und andere Rechtsträger in Bezug auf Tätigkeiten im internationalen Meeresbodengebiet befürworten, zu Recht einen objektiven Sorgfaltsmaßstab aufgestellt, um zu verhindern, dass Unternehmen einen befürwortenden Staat mit den geringsten Haftungsrisiken wählen. ${ }^{126}$ Doch dem Gutachten lässt sich auch entnehmen, dass bei einer nicht eindeutig nachgewiesenen Gefahr die Anforderungen an Industriestaaten wegen ihrer wissenschaftlichen und technischen Kapazitäten höher sein müssen als an Entwicklungsländer. ${ }^{127}$ Der konkrete Sorgfaltsmaßstab muss demnach mit Blick auf die zu regulierenden Vorgänge aufgestellt werden. Hier liegt ein weiterer Unterschied zur gebotenen Sorgfalt im Rahmen der realen Handlungsmöglichkeit bei staatlichen Verhinderungsbzw. Schutzpflichten. Die Konsequenzen nach dem Recht der Staatenverantwortlichkeit gebieten nämlich in jedem Fall einen subjektiven Sorgfaltsmaßstab. ${ }^{128}$

Im Ergebnis verpflichtet die gebotene Sorgfalt die Staaten dazu, alle notwendigen und geeigneten Maßnahmen zu ergreifen, um dem jeweiligen Normbefehl gerecht zu werden. ${ }^{129}$ Dabei hilft die gebotene Sorgfalt, neuen globalen Herausforderungen zu begegnen und stellt einen in der Entwicklung befindlichen völkerrechtlichen Rahmen basierend auf dem Gedanken, „that States are expected to strive to achieve certain common goals and, in the spirit of good neighbourliness, to prevent problems especially of transboundary and global harm (be it environmental, econo-

125 ILA, Study Group on Due Diligence in International Law, Tim Stephens (Rapporteur)/Duncan French (Chair), Second Report, July 2016, abrufbar unter: http://www.ila-hq.org/index.php/study-groups?study-groupsID=63 (geprüft am 15.05.2020), S. 18.

126 ITLOS, Responsibilities and obligations of states sponsoring persons and entities with respect to activities in the Area, Advisory Opinion of 1 February 2011, ITLOS Case No. 17, ITLOS Reports 2011, S. 10 (53 f. Rn. 158 f.).

127 Id., S. 10 (54 Rn. 161 f.); ILA, Study Group on Due Diligence in International Law, Tim Stephens (Rapporteur)/Duncan French (Chair), Second Report, July 2016, abrufbar unter: http://www.ila-hq.org/index.php/study-groups?study-grou psID=63 (geprüft am 15.05.2020), S. 18 ff.

128 Siehe 2. Kapitel A. I. 2. b).

129 Vgl. S. Förster, Internationale Haftungsregeln für schädliche Folgewirkungen gentechnisch veränderter Organismen, 2007, S. 169. 
mic, military (including non-State terrorism), kinetic, or other) “. ${ }^{130}$ Die Arbeit wird diesen Sorgfaltsrahmen für die Schadensvermeidungspflicht im Zusammenhang mit informationstechnischen Systemen ausfüllen.

\section{Haftungsart und -standard}

In der internationalen Rechtsprechung finden sich durchaus Fälle, die eine primäre staatliche Haftung für grenzüberschreitende Schäden durch (nicht-staatliche) gefährliche Aktivitäten belegen. Doch insbesondere Haftungsübereinkommen und Gewohnheitsrecht zeigen, dass die Staaten eine primäre Staatenhaftung für völkerrechtlich nicht verbotene Aktivitäten (durch nicht-staatliche Akteure) nur selten akzeptieren und vorzugsweise den Betreiber der gefährlichen Aktivität bzw. den Schadensverursacher im engeren Sinne zur Kompensation verpflichten. Gleichzeitig verdeutlicht die Rechtsquellenanalyse aber, dass die Staaten ihrer zentralen Rolle in grenzüberschreitenden Konfliktsituationen nachkommen, indem sie zumindest gewillt sind, ergänzend oder subsidiär Entschädigung für grenzüberschreitende Schäden zu leisten und ihnen eine Art Garantenstellung für internationale Gefährdungslagen zukommt. Es bleibt im konkreten Einzelfall zu bestimmen, welche Rolle dem Staat in internationalen Schadensfällen zukommt. Dabei kann sich aus den Allgemeinen Rechtsgrundsätzen, welche den Haftungssystemen zugrunde liegen, ergeben, ob Staaten primär, ergänzend oder nur subsidiär haften. ${ }^{131}$

Den Staat, auf dessen Territorium oder unter dessen Kontrolle die fragliche Aktivität stattfindet, trifft jedenfalls die Pflicht zur Schadensvermeidung und Schadenskompensation. Die beiden Komponenten sind Bestandteil einer einzigen komplexen Pflicht, wobei Schadensvermeidung und Schadenskompensation auf zwei Arten in Verhältnis zueinanderstehen können. Es gibt zum einen verschuldensabhängige Haftungssysteme, im Rahmen derer die Kompensationspflicht nur greift, wenn die Schadensvermeidungspflicht verletzt wurde, und zum anderen verschuldensunabhängige Haftungssysteme, im Rahmen derer die Kompensationspflicht sowohl bei Beachtung als auch bei Verletzung der Schadensvermeidungs-

130 ILA, Study Group on Due Diligence in International Law, Tim Stephens (Rapporteur)/Duncan French (Chair), Second Report, July 2016, abrufbar unter: http://www.ila-hq.org/index.php/study-groups?study-groupsID=63 (geprüft am 15.05.2020), S. 47.

131 Siehe 1. Kapitel. 
pflicht greift. Der Sinn einer verschuldensabhängigen Haftung für völkerrechtlich nicht verbotene Aktivitäten liegt darin begründet, dass sowohl Schädiger als auch Geschädigter zur Gefährdungslage beigetragen haben, so dass der Geschädigte nicht den Schutz einer verschuldensunabhängigen Haftung benötigt. In allen anderen Konstellationen führt eine verschuldensunabhängige Haftung zu gerechten Ergebnissen. ${ }^{132}$ Jedenfalls verdichten sich im Rahmen hochgefährlicher Aktivitäten die Sorgfaltsanforderungen zu einem verschuldensunabhängigen Haftungsstandard, da hier selbst ohne jegliches Fehlverhalten Unfälle mit verheerenden Schadensfolgen nicht ausgeschlossen werden können. Die wirtschaftlichen bzw. gesellschaftlichen Vorteile verhindern zumeist ein Verbot der hochgefährlichen Aktivität. ${ }^{133}$ Das Konzept der Staatenhaftung mit einer verhaltensbezogenen Schadensvermeidungspflicht und einer ergebnisbezogenen Kompensationspflicht verhindert hier, dass die Schadenslast auf den unbeteiligten Geschädigten abgewälzt wird. ${ }^{134}$

Im allgemeinen Konzept der Staatenhaftung sind schließlich keine Haftungsbefreiungen und Haftungsausschlussgründe angezeigt. Diese Haftungsbeschränkungen sind vornehmlich durch völkervertragliche Bestimmungen zu normieren, so dass sie nur unter festgelegten Voraussetzungen zur Entlastung des direkten (privaten) Schadensverursachers führen. Im Rahmen der Staatenhaftung geht es hingegen um die Garantenstellung des Staates, so dass hier eine unbeschränkte Einstandspflicht angebracht ist. ${ }^{135}$

132 Siehe insbesondere die Ausführungen im 1. Kapitel D. I. 1. und im 1. Kapitel B. I. 3. a).

133 Vgl. Art. 4 Abs. 1 IDI-Resolution; J. Barboza, Liability, Chinese JIL 1 (2002), S. 499 (515 f.); G. Handl, State Liability for Accidental Transnational Environmental Damage by Private Persons, AJIL 74 (1980), S. 525 (550).

134 Vgl. J. Barboza, Liability, Chinese JIL 1 (2002), S. 499 (515 f.); T. A. Berwick, Responsibility and Liability for Environmental Damage, GIELR 10 (1997-1998), S. 257 (264); C. Foster, The ILC Draft Principles on the Allocation of Loss in the Case of Transboundary Harm Arising out of Hazardous Activities, RECIEL 14 (2005), S. 265 (266, 268, 274).

135 Vgl. D. M. Schmitt, Staatenverantwortlichkeit für Schäden an der biologischen Vielfalt, 2018, S. 464 f., die darauf hinweist, dass unverhältnismäßigen Belastungen durch eine unbeschränkte Umwelt-Staatenhaftung de lege ferenda über Solidarpflichten der Staatengemeinschaft und Umwelthilfe begegnet werden können. 


\section{Zusammenfassung}

Die konstitutiven Elemente der Staatenverantwortlichkeit und der Staatenhaftung verdeutlichen ihre unterschiedlichen Anwendungsbereiche.

Während die Staatenverantwortlichkeit unbestritten in verschiedenen völkerrechtlichen Regelungsbereichen zur Anwendung gelangt, ist dies für das Recht der Staatenhaftung noch nicht ausreichend bewiesen. Es muss gezeigt werden, dass die gefundenen Paramater der Staatenhaftung flexibel genug sind, um auch neuartige Gefährdungslagen zu regulieren.

Bei der Anwendung der beiden Systeme auf Konfliktlagen in der virtuellen Welt, wird sichtbar werden, dass die Kriterien der Staatenverantwortlichkeit zur Lösung neuer Problemstellungen schwer handhabbar sind und Lücken lassen (3. Kapitel), während die Staatenhaftung eine Regulierung von Schäden durch gefährliche völkerrechtlich nicht verbotene Aktivitäten gewährleistet (4. Kapitel). 


\section{Kapitel: Staatenverantwortlichkeit für informationstechnische Systeme}

Die negativen Auswirkungen von informationstechnischen Vorgängen können staatliche Einstandspflichten, wie sie in Kapitel 2 definiert wurden, notwendig machen. Zunächst müssen die Charakteristika dieser Vorgänge näher beschrieben werden, um sie völkerrechtlich einordnen zu können (A.). Beispiele aus der Vergangenheit verdeutlichen die Dimension der völkerrechtlichen Problemstellung (B.) und belegen zugleich die Schwächen der existenten Regelungsansätze einer Staatenverantwortlichkeit für informationstechnische Systeme. Die Völkerrechtswidrigkeit von Verhalten innerhalb informationstechnischer Systeme ist zunächst anhand vertrauter völkerrechtlicher Bestimmungen zu beurteilen (C.). Da die Handlungen nur dann für das Recht der Staatenverantwortlichkeit relevant sind, wenn ein Staatenbezug hergestellt werden kann, wird sich zeigen, dass im Regelungsfeld informationstechnischer Systeme eine Zurechnung nicht ohne Weiteres möglich ist (D.) und dass zur Begründung von staatlichen Handlungspflichten die konstitutiven Elemente der Kenntnis sowie der realen Handlungsmöglichkeit hohe Hürden darstellen und die gebotene Sorgfalt nur bedingt zur Verhinderung von bzw. zum Schutz vor rechtsverletzenden Handlungen beitragen kann (E.). Die Frage nach einer Schadenskompensation bleibt durch das Recht der Staatenverantwortlichkeit unbeantwortet (F.), so dass das Konzept der Staatenhaftung für informationstechnische Systeme in den Fokus der völkerrechtlichen Reglementierung zu rücken ist.

\section{A. Informationsoperationen}

Informationstechnische Systeme bilden eine abgeschlossene Funktionseinheit zur Informationsverarbeitung. Diese Systeme werden durch das universelle und frei zugängliche Internet auf Datenebene miteinander zu einer virtuellen Welt verbunden. ${ }^{1}$ Diese Vernetzung bedingt eine globa-

1 Bundesministerium des Innern (Hg.), Cyber-Sicherheitsstrategie für Deutschland, 2016, abrufbar unter: https://www.bmi.bund.de/DE/themen/it-und-digitalpolitik/it 
le Gefährdungslage durch sogenannte Informationsoperationen, die zum Ziel haben, fremde informationstechnische Systeme auszuforschen, zu stören, zu verfälschen oder gar zu zerstören, um sich Vorteile zu verschaffen. ${ }^{2}$ Aufgrund eines divergierenden Problemverständnisses sowie technischer und juristischer Feinheiten fehlt es derzeit zwar an international gültigen Begrifflichkeiten und Definitionen für die unterschiedlichen Informationsoperationen, ${ }^{3}$ ungeachtet dessen erlauben spezifische Merkmale von Vorgängen innerhalb informationstechnischer Systeme eine Kategorisierung in Informationsangriffe und Informationsausbeutung. ${ }^{4}$

\section{Informationsangriffe}

Informationsangriffe sind vorsätzliche Handlungen, deren Ziel es ist, ein informationstechnisches System oder ein Netzwerk oder die darauf gespeicherten Daten zu verändern, zu stören, zu manipulieren, zu schwächen, zu beeinträchtigen oder zu zerstören. ${ }^{5}$ Dabei sind im Wesentlichen zwei

-und-cybersicherheit/cyber-sicherheitsstrategie/cyber-sicherheitsstrategie-node.html (geprüft am 15.05.2020), S. 46.

2 D. B. Hollis, Why States Need an International Law for Information Operations, LCLR 11 (2007), S. 1023 (1030 ff.); T. Stein/T. Marauhn, Völkerrechtliche Aspekte von Informationsoperationen, ZaöRV 60 (2000), S. 1 (1); T. Theuerkauf, Informationsoperationen, Europäische Sicherheit 2 (2000), S. 14 (14ff.). In diesem Zusammenhang wird häufig auch von Cyberkrieg(sführung) und Informationskrieg(sführung) gesprochen. Siehe S. Gaycken, Cyberwar, 2011, S. 21 ff.; W. Heintschel von Heinegg, Informationskrieg und Völkerrecht, in: V. Epping/H. Fischer/ders. (Hg.), Brücken bauen und begehen, Festschrift für Knut Ipsen, 2000, S. 129 (129ff.); T. A. Morth, Considering Our Position, Case Western Reserve JIL 30 (1998), S. 567 (567 ff.); D. Ventre, Cyberwar and Information Warfare, 2011, S. 1 ff. Diese Terminologien weisen aber - im Gegensatz zum Begriff der Informationsoperation eine Nähe zum Kriegsbegriff auf, so dass ihnen die notwendige Offenheit fehlt, um als Oberbegriff für Handlungen im Rahmen informationstechnischer Systeme dienen zu können.

3 S.-H. Schulze, Cyber-,,War“ - Testfall der Staatenverantwortlichkeit, 2015, S. 8.

4 Vgl. die vom US-Militär entwickelte Terminologie, welche regelmäßig als Grundlage für wissenschaftliche Diskussionen über Informationsoperationen genutzt wird. Joint Chiefs of Staff, Cyberspace Operations, Joint Pub 3-12, 8 June 2018, abrufbar unter: https://fas.org/irp/doddir/dod/jp3_12.pdf (geprüft am 15.05.2020), Kapitel 2, S. 3, 6 f.; vgl. auch W. A. Owens/K. W. Dam/H. S. Lin (Hg.), Technology, Policy, Law, and Ethics Regarding U.S. Acquisition and Use of Cyberattack Capabilities, 2009, S. 1.

5 Joint Chiefs of Staff, Cyberspace Operations, Joint Pub 3-12, 8 June 2018, abrufbar unter: https://fas.org/irp/doddir/dod/jp3_12.pdf (geprüft am 15.05.2020), Glossary, 
Angriffsmethoden zu unterscheiden, welche darauf gerichtet sind, die Sicherheit von informationstechnischen Systemen zu brechen. ${ }^{6}$ Zum einen kann die Funktionsfähigkeit der Systeme von außen beeinträchtigt oder gänzlich unterbrochen werden und zum anderen können intrusive Angriffe den Abfluss und die Vernichtung von Informationen bewirken sowie Fehlfunktionen mit sekundärer Schadwirkung verursachen. ${ }^{7}$

In den meisten Staaten sind wesentliche Bereiche der Daseinsfürsorge und der staatlichen Infrastruktur an informationstechnische Systeme gebunden, die gegenüber Informationsoperationen äußerst verwundbar sind. So können Netzwerke instrumentalisiert werden, um kritische Infrastrukturen, wie etwa Kommunikations-, Verkehrs- und Energieversorgungssysteme, zu beeinträchtigen oder zu zerstören. ${ }^{8}$ Neben staatlichen Netzwerken können auch private Unternehmen und internationale Institutionen betroffen sein. Informationstechnologien sind für das Funktionieren von Wirtschaft und Gesellschaft mittlerweile unentbehrlich. Dementsprechend kann deren Ausfall enorme Schäden verursachen. ${ }^{9}$

Ferner können virtuelle Sabotage und Manipulationen die politische Landschaft und Geschicke eines Staates beeinflussen. ${ }^{10}$ So können beispielsweise Wahlergebnisse manipuliert, falsche Informationen verbreitet und das öffentliche Bewusstsein der Bevölkerung eines fremden Staates in eine bestimmte Richtung gelenkt werden. Dies kann zu Unruhen und bis hin zum Umsturz der Regierung eines Landes führen. ${ }^{11}$

S. 4; W. A. Owens/K. W. Dam/H. S. Lin (Hg.), Technology, Policy, Law, and Ethics Regarding U.S. Acquisition and Use of Cyberattack Capabilities, 2009, S. 1.

$6 \mathrm{Zu}$ den verschiedenen Angriffsmethoden siehe S. Gaycken, Cyberwar, 2011, S. $215 \mathrm{ff}$.

7 A. Bendiek, Umstrittene Partnerschaft, SWP-Studie 26/2013, S.10, Fn. 25; T. O. Keber/P. N. Roguski, Ius ad bellum electronicum?, AVR 49 (2011), S. 399 (405 f.).

8 A. Bendiek, Kritische Infrastrukturen, Cybersicherheit, Datenschutz, SWP-Aktuell 35/2013, S. 2; N. Melzer, Cyberwarfare and International Law, 2011, S. 14 f.; C. Schaller, Internationale Sicherheit und Völkerrecht im Cyberspace, SWP-Studie 18/2014, S. 18.

9 A. Bendiek, Umstrittene Partnerschaft, SWP-Studie 26/2013, S. 10 f.; J. A. Lewis/S. Baker, The Economic Impact of Cybercrime and Cyber Espionage, Center for Strategic and International Studies, Report of 22 July 2013, abrufbar unter: https://csis-prod.s3.amazonaws.com/s3fs-public/legacy_files/files/publication/ 60396rpt_cybercrime-cost_0713_ph4_0.pdf (geprüft am 15.05.2020), S. 3 ff.

$10 \mathrm{Vgl}$. T. Stein/T. Marauhn, Völkerrechtliche Aspekte von Informationsoperationen, ZaöRV 60 (2000), S. 1 (33 f.).

11 C. Schaller, Internationale Sicherheit und Völkerrecht im Cyberspace, SWP-Studie 18/2014, S. 15. 
II. Informationsausbeutung

Informationsausbeutung zielt weniger auf Destruktion als vielmehr auf Informationsgewinnung ab. ${ }^{12}$ Sie hat also das Ausspionieren informationstechnischer Systeme und die Informationsabschöpfung zum Gegenstand und kennt verschiedene Methoden, um die Vertraulichkeit dieser Systeme zu stören. ${ }^{13}$ Im Rahmen der Informationsausbeutung kommen oftmals Informationsangriffe zur Informationsbeschaffung zum Einsatz und begründen damit eine zweifache Bedrohung. ${ }^{14}$

Im Kontext zwischenstaatlicher Spionage geht es in der Regel um die Beschaffung als geheim eingestufter oder anderweitig geschützter Informationen, etwa über den Stand und die Entwicklung der Volkswirtschaft eines fremden Staates oder über Militärgeheimnisse. ${ }^{15} \mathrm{Im}$ Interesse der nationalen Sicherheit werden überdies auch persönliche Informationen über ausländische Personen gesammelt und ausgewertet. ${ }^{16}$ Unternehmen oder Einzelpersonen können Gegenstand fremdstaatlicher oder nicht-staatlicher Wirtschaftsspionage sein, die auf private Geschäfts- bzw. Betriebsgeheimnisse abzielt. ${ }^{17}$

Die Informationsausbeutung hat weitreichende negative Auswirkungen. In den Bereichen Forschung und Entwicklung führt sie zu erheblichen volkswirtschaftlichen Schäden. Unternehmen erleiden enorme wirtschaftliche Einbußen in Form von entgangenen Geschäften, wertlosen Ausgaben

12 US Joint Chiefs of Staff, Cyberspace Operations, Joint Pub 3-12, 8 June 2018, abrufbar unter: https://fas.org/irp/doddir/dod/jp3_12.pdf (geprüft am 15.05.2020), Glossary, S. 4; W. A. Owens/K. W. Dam/H. S. Lin (Hg.), Technology, Policy, Law, and Ethics Regarding U.S. Acquisition and Use of Cyberattack Capabilities, 2009, S. 2.

13 A. Bendiek, Umstrittene Partnerschaft, SWP-Studie 26/2013, S. 10, Fn. 25; A. Tsolkas/F. Wimmer, Wirtschaftsspionage und Intelligence Gathering, 2012, S. $101 \mathrm{ff}$.

14 Vgl. A. Bendiek, Umstrittene Partnerschaft, SWP-Studie 26/2013, S. 12.

15 R. Crootof, International Cybertorts, CLR 103 (2018), S. 565 (597); O. A. Hathaway/R. Crootof/P. Levitz/H. Nix/A. Nowlan/W. Perdue/J. Spiegel, The Law of CyberAttack, Cal. LR 100 (2012), S. 817 (829); C. Schaller, Internationale Sicherheit und Völkerrecht im Cyberspace, SWP-Studie 18/2014, S. 11.

16 C. Schaller, Internationale Sicherheit und Völkerrecht im Cyberspace, SWP-Studie 18/2014, S. $13 \mathrm{f}$.

17 Id., S. 11; H. W. Többens, Wirtschaftsspionage und Konkurrenzausspähung in Deutschland, NStZ 10 (2000), S. 505 (505). 
für Innovationen sowie Reputationsschäden. ${ }^{18}$ Das Ausspionieren von Individuen geht regelmäßig mit Eingriffen in die Privatheit einher. ${ }^{19}$

\section{B. Informationsoperationen in der internationalen Praxis}

Informationstechnische Systeme sind aufgrund der wachsenden globalen Vernetzung und der erhöhten Abhängigkeit von komplexen Technologien besonders anfällig für Informationsoperationen, die vermehrt zu internationalen Konflikten führen. ${ }^{20}$ Dabei handelt es sich nicht um ein neuartiges Phänomen. Bereits im Jahr 1982 kam es im Kontext des Kalten Krieges zu einer schadensverursachenden Informationsoperation. Agenten des sowjetischen Geheimdienstes entwendeten eine kanadische Software zur Kontrolle von Gaspipelines. Der US-Geheimdienst wusste von diesem Vorhaben der Sowjetunion und manipulierte in Zusammenarbeit mit dem kanadischen Softwareunternehmen die Software mittels einer Logikbombe, welche schließlich nach ihrem Einsatz in einer sibirischen Pipeline zu einer massiven Gasexplosion führte. ${ }^{21}$

Die unterschiedlichen Erscheinungsformen von Informationsoperationen werden im Folgenden anhand ausgewählter Beispiele illustriert. Dabei sind insbesondere staatliche und staatlich motivierten Informationsoperationen von Interesse, die eine negative grenzüberschreitende Wirkung haben. Informationsoperationen, die auf nicht-staatliche Akteure zurück-

18 Siehe J. Lewis, The Economic Impact of Cybercrime - No Slowing Down, Center for Strategic and International Studies, Report of 21 February 2018, abrufbar unter: https://www.csis.org/analysis/economic-impact-cybercrime (geprüft am 15.05.2020).

19 Der Begriff der Privatheit wird in diesem Zusammenhang „als Inbegriff der auf der Achtung der Menschenwürde und damit freien (informationellen) Selbstbestimmung des Individuums beruhenden Persönlichkeitsrechte, Schutz von Daten und Privatsphäre des Individuums verstanden“. I. Pernice, Vom Völkerrecht des Netzes zur Verfassung des Internets, HIIG Discussion Paper Series, Discussion Paper No. 2017-02, abrufbar unter: https://papers.ssrn.com/sol3/papers.cfm?abstr act_id=2959257 (geprüft am 15.05.2020), S. 1 (6 Fn. 23).

$20 \mathrm{Vgl}$. Bundesministerium des Innern (Hg.), Cyber-Sicherheitsstrategie für Deutschland, 2016, abrufbar unter: https://www.bmi.bund.de/cybersicherheit sstrategie/BMI_CyberSicherheitsStrategie.pdf (geprüft am 15.05.2020), S. 4 ff.; $D$. Efrony/Y. Shany, A Rule Book on the Shelf?, AJIL 112 (2018), S. 583 (594ff.).

21 S. J. Shackelford/R. B. Andres, State Responsibility for Cyber Attacks, Geo. JIL 42 (2010-2011), S. 971 (972). 
gehen und vornehmlich durch nationales Recht zu sanktionieren sind, werden im Rahmen dieser Arbeit nur am Rande zu erörtern sein. ${ }^{22}$

\section{Informationsangriffe auf Estland und Georgien}

Die Gefahr, welche die Ausweitung staatlicher Informationsinfrastrukturen mit sich bringt, zeigt sich am Beispiel der internetgestützten Angriffe auf informationstechnische Systeme in Estland im Jahr 2007 und in Georgien im Jahr 2008. Bei den Angriffen wurden informationstechnische Systeme der Länder mit einer großen Anzahl von Befehlen durch mit einer Schadsoftware infiltrierte Computer überlastet. ${ }^{23}$ Infiltrierte Computer (Bots) sind von außen fernsteuerbar und können mit anderen Bots weltweit zusammen zum Einsatz kommen (Botnet), um eine Überbeanspruchung der Rechenleistung und Netzwerkbandbreite von informationstechnischen Systemen zu bewirken. ${ }^{24}$ Derartige sogenannte Denial of Service-Angriffe führten auch in Estland und in Georgien zum Absturz wichtiger informationstechnischer Systeme. ${ }^{25}$ Daneben manipulierten Hacker durch sogenanntes Defacement Webseiten und verbreiteten Inhalte, die den Interessen der estnischen bzw. georgischen Regierung zuwiderliefen. ${ }^{26}$

22 Insoweit erfasst das nationale und internationale Strafrecht private Handlungsformen in der virtuellen Welt, wie etwa Identitätsdiebstahl, Phishing, Spams und Computerviren. Der Umgang mit derartigen Straftaten ist auch Gegenstand des Europäischen Übereinkommens über Computerkriminalität vom 23. November 2001 (BGBl. 2008 II. S. 1242, 1243; 2010 II S. 218).

23 T. O. Keber/P. N. Roguski, Ius ad bellum electronicum?, AVR 49 (2011), S. 399 (401 f.); S. J. Shackelford, From Nuclear War to Net War, BJIL 27 (2009), S. 192 (207).

24 C. Wilson, Botnets, Cybercrime, and Cyberterrorism: Vulnerabilities and Policy Issues for Congress, US Congressional Research Report of 29 January 2008, No RL32114, abrufbar unter: https://fas.org/sgp/crs/terror/RL32114.pdf (geprüft am 15.05.2020), S. 5 .

25 T. O. Keber/P. N. Roguski, Ius ad bellum electronicum?, AVR 49 (2011), S. 399 (401 f.); S. J. Shackelford, From Nuclear War to Net War, BJIL 27 (2009), S. 192 (207). Sofern mehrere Systeme betroffen sind, spricht man von Distributed Denial of Service-Angriff. Siehe S. Gaycken, Cyberwar, 2011, S. 233.

26 In Estland wurden auf diese Weise pro-russische Parolen verbreitet und in Georgien ersetzten Hacker auf Regierungswebseiten Bilder des Staatspräsidenten Mikheil Saakashvili durch Bilder von Adolf Hitler. T. O. Keber/P. N. Roguski, Ius ad bellum electronicum?, AVR 49 (2011), S. 399 (401 f.). 
In Estland wurden im Jahr 2007 informationstechnische Systeme von Regierungs- und Verwaltungsstellen, der größten estnischen Bank sowie von wichtigen Nachrichtenportalen außer Dienst gestellt. Außerdem kam es zu negativen Auswirkungen auf Krankenhäuser, Energieversorgungssysteme und Notrufnummern. Um weitere Schäden zu vermeiden, sahen sich zahlreiche Serverbetreiber gezwungen, die Verbindung zu externen Netzwerken zu unterbrechen, so dass Estland von der Außenwelt abgeschottet war. In Anbetracht der Tatsache, dass Estland bereits $2007 \mathrm{zu}$ den höchst technisierten Ländern weltweit zählte und alle wesentlichen Verwaltungsangelegenheiten und Vorgänge des öffentlichen Lebens über die gut ausgebaute elektronische Verwaltung des Landes liefen, waren die Auswirkungen der Angriffe umso einschneidender. ${ }^{27}$ Die Angriffe starteten einen Tag nach Verlegung der Statue eines Soldaten der Roten Armee von einem zentralen Platz in der Hauptstadt Tallinn auf den Militärfriedhof am Stadtrand. Die Entscheidung der estnischen Regierung über die Verlegung erzeugte Unmut bei der russischen Minderheit in Estland sowie bei außerhalb Estlands lebenden Russen, weil die Statue den Sieg der Sowjetunion im Zweiten Weltkrieg symbolisiert und ihrer gefallenen Opfer gedenkt. Dies verstärkte die Spannungen zwischen Estland und Russland, die schließlich als Anlass für die Angriffe gewertet werden. ${ }^{28}$ Während die estnische Regierung Russland für die Störung der estnischen Informationsinfrastrukturen öffentlich anprangerte, bestritt die russische Regierung aber jegliche Beteiligung an den Angriffen und verortete die Angriffsquelle bei patriotischen Hackern. ${ }^{29}$

Während die Angriffe auf Estland vornehmlich für das gesellschaftliche Leben notwendige Infrastrukturen betrafen, ging es bei den Angriffen auf informationstechnische Systeme in Georgien im Jahr 2008 vor allem darum, die nationalen und internationalen Kommunikations- sowie Infor-

27 S. Herzog, Revisiting the Estonian Cyber Attacks, JSS 4 (2011), S. 49 (50 ff.); T. O. Keber/P. N. Roguski, Ius ad bellum electronicum?, AVR 49 (2011), S. 399 (402); J. Shackelford, From Nuclear War to Net War, BJIL 27 (2009), S. 192 (202 f. Fn. 57, 207); E. Tikk/K. Kadri/L. Vibul, International Cyber Incidents, 2010, S. $18 \mathrm{ff.}$

28 S.-H. Schulze, Cyber-,,War“ - Testfall der Staatenverantwortlichkeit, 2015, S. 27 f. Auf russischsprachigen Webseiten fanden sich zudem Aufrufe und Anleitungen, um sich an den Inforationsangriffen gegen Estland zu beteiligen. E. Tikk/K. Kadri/L. Vihul, International Cyber Incidents, 2010, S. 18.

29 S. Herzog, Revisiting the Estonian Cyber Attacks, JSS 4 (2011), S. 49 (50 ff.); J. Shackelford, From Nuclear War to Net War, BJIL 27 (2009), S. 192 (204f.);

E. Tikk/K. Kadri/L. Vibul, International Cyber Incidents, 2010, S. 15 f. 
mationskanäle des Landes zu unterbrechen. ${ }^{30}$ Diese Beeinträchtigungen waren besonders gewichtig, weil sie zu einem Zeitpunkt eintraten, an dem Georgien in besonderem Maße von diesen Kanälen abhängig war. Die Angriffe standen nämlich im Zusammenhang mit dem politisch-militärischen Konflikt zwischen Russland und Georgien bezüglich des formal zu Georgien gehörenden, aber abtrünnigen Gebiets Südossetien. ${ }^{31}$ Die Angriffe auf georgische Informationsinfrastrukturen starteten vor einer diesbezüglichen Militärintervention Russlands gegen Georgien und nahmen während deren Verlauf immer weitreichendere und komplexere Ausmaße an. ${ }^{32}$ Georgien wurde mithin die Möglichkeit genommen, die eigene Bevölkerung über den Konflikt zu informieren und international hierzu Stellung zu beziehen. ${ }^{33}$ Die Urheber der gut koordinierten Angriffe konnten nicht zweifelsfrei bestimmt und lokalisiert werden. ${ }^{34}$ Auch hier bestritt die russische Regierung jegliche Beteiligung unter dem Hinweis auf patriotische Hacker. Dieses Argumentationsmuster wird in Anbetracht des zeitlichen Verlaufs, der Komplexität und der Koordinierung der Angriffe auf die staatlichen und zivilen Informationsinfrastrukturen allerdings als fragwürdig bewertet. ${ }^{35}$

30 E. Tikk/K. Kaska/K. Rünnimeri/M. Kert/A.-M. Talihärm/L. Vihul, Cyber Attacks Against Georgia, 2008, S. 15 f.

31 T. O. Keber/P. N. Roguski, Ius ad bellum electronicum?, AVR 49 (2011), S. 399 (402 mit Fn. 15); S.-H. Schulze, Cyber-,,War“ - Testfall der Staatenverantwortlichkeit, 2015, S. 28; E. Tikk/K. Kaska/K. Rünnimeri/M. Kert/A.-M. Talihärm/L. Vihul, Cyber Attacks Against Georgia, 2008, S. 4.

32 E. Tikk/K. Kaska/K. Rünnimeri/M. Kert/A.-M. Talihärm/L. Vihul, Cyber Attacks Against Georgia, 2008, S. 4.

33 W. C. Ashmore, Impact of Alleged Russian Cyber Attacks, BSDR 11 (2009), S. 4 (10); D. Hollis, Cyberwar Case Study: Georgia 2008, Small Wars Journal (2011), abrufbar unter: https://smallwarsjournal.com/blog/journal/docs-temp/639-hollis. pdf (geprüft am 15.05.2020), S. 1 (6); E. Tikk/K. Kaska/K. Rünnimeri/M. Kert/A.-M. Talihärm/L. Vihul, Cyber Attacks Against Georgia, 2008, S. 16.

34 Ähnlich wie bei den Angriffen gegen Estland wurden auf russischsprachigen Webseiten Aufrufe und Anleitungen zur Beteiligung an den Inforationsangriffen gegen Georgien verbreitet. W. C. Ashmore, Impact of Alleged Russian Cyber Attacks, BSDR 11 (2009), S. 4 (26); T. O. Keber/P. N. Roguski, Ius ad bellum electronicum?, AVR 49 (2011), S. 399 (402 f.).

35 E. Tikk/K. Kaska/K. Rünnimeri/M. Kert/A.-M. Talibärm/L. Vihul, Cyber Attacks Against Georgia, 2008, S. 12 ff. 


\section{Informationsangriff durch Schadprogramm „Stuxnet“}

Das Risiko, das informationstechnischen Systemen immanent ist, wurde in besonderem Maße durch das Schadprogramm Stuxnet deutlich, welches im Jahr 2009 über verseuchte USB-Speichersticks in Steuerungssysteme iranischer Atomanlagen eindrang und diese umprogrammierte. Stuxnet nutzte bis zu diesem Zeitpunkt unbekannte Sicherheitslücken des Betriebssystems der Anlagen, um die Rotationsfrequenz der Nuklearzentrifugen zu verändern und gleichzeitig die Kontrollsysteme mit falschen Messwerten zu überlisten. So blieb das Umprogrammieren zunächst unentdeckt und führte schließlich zur physischen Zerstörung der Zentrifugen. ${ }^{36}$

Die eingehenden Kenntnisse über die Zielsysteme, das Angriffsziel selbst und die umfassende technische Expertise, die Stuxnet zugrunde lag, deuteten auf einen staatlichen Akteur hin. ${ }^{37}$ Zudem erklärten Vertreter der US-Administration, dass Stuxnet durch die USA in Zusammenarbeit mit Israel eingesetzt wurde, mit dem Ziel iranische Atomanlagen zu manipulieren und die dortige Urananreicherung zu sabotieren. ${ }^{38}$ Die israelische und die US-amerikanische Regierung selbst hingegen bestreiten, in die weitreichende Umsetzung des Angriffs involviert gewesen zu sein, ${ }^{39}$ und auch die iranische Regierung hat trotz der eindeutigen Hinweise keinen Staat völkerrechtlich zur Verantwortung gezogen. ${ }^{40}$

36 T. O. Keber/P. N. Roguski, Ius ad bellum electronicum?, AVR 49 (2011), S. 399 (403 f.); R. Langner, Der Langner-Report: Stuxnet und Die Folgen, Was die Schöpfer von Stuxnet erreichen wollten, was sie erreicht haben, und was das für uns alle bedeutet, 2017, abrufbar unter: https://www.langner.com/wp-content /uploads/2017/08/Stuxnet-und-die-Folgen.pdf (geprüft am 15.05.2020).

37 Vgl. S. Gaycken, Cyberwar, 2011, S. 175; M. Schulze, Hacking back? Technische und politische Implikationen digitaler Gegenschläge, SWP-Aktuell 59/2017, S. 3; S.-H. Schulze, Cyber-„,War“ - Testfall der Staatenverantwortlichkeit, 2015, S. 17.

38 Siehe D. E. Sanger, Obama Ordered Sped Up Wave of Cyberattacks Against Iran, The New York Times, 1 June 2012, abrufbar unter: http://www.nytimes.com/201 2/06/01/world/middleeast/obama-ordered-wave-of-cyberattacks-against-iran.html (geprüft am 15.05.2020).

39 S. P. McGurk, (Acting Director, National Cybersecurity and Communications Integration Center, Office of Cybersecurity and Communications, National Protection and Programs Directorate, Department of Homeland Security), Statement for the Record Before the United States Senate Homeland Security and Governmental Affairs Committee, Washington DC, 17 November 2010, abrufbar unter: http://www.hsgac.senate.gov/download/2010-11-17-mcgurk-testimony-revised (geprüft am 15.05.2020).

40 D. Efrony/Y. Shany, A Rule Book on the Shelf?, AJIL 112 (2018), S. 583 (638). 
III. Informationsausbeutung durch Schadprogramme „Duqu“ und
„Flame“

Im Jahr 2011 wurde das Schadprogramm Duqu und im Jahr 2012 das Schadprogramm Flame entdeckt. Die Programme waren auf das Sammeln und Löschen großer Mengen von Informationen spezialisiert. ${ }^{41} D u q u$ und Flame sind vom Arbeitsaufwand und der dahinterstehenden Intelligenz mit Stuxnet vergleichbar. Doch im Gegensatz zu Letzterem zielten die beiden Programme nicht darauf ab, bestimmte Prozesse zu manipulieren und physischen Schaden zu verursachen, sondern wurden eingesetzt, um informationstechnische Systeme staatlicher Behörden, Unternehmen und Industrieanlagen abzuhören und auszuspionieren. ${ }^{42}$ Entweder sollten die Informationsausbeutungen Schwachstellen in den befallenen Systemen identifizieren, um später für Informationsangriffe genutzt zu werden oder sie zielten lediglich auf die Stärkung wirtschaftlicher Wettbewerbsfähigkeit. ${ }^{43}$

Während Flame lokal auf den Nahen Osten ausgerichtet war und insbesondere Computer im Iran befiel, ließen sich bei Duqu keine geografischen Präferenzen erkennen..$^{44}$ Codeanalysen zeigen, dass Duqu und Flame wohl derselben Quelle wie Stuxnet entstammen. ${ }^{45}$ Auch das Entwicklungsniveau der Programme und die Angriffsziele deuten darauf hin, dass staatliche Akteure, etwa Geheimdienste der USA und Israel, für die Entwicklung

41 B. Bencsáth/G. Pék/L. Buttyán/M. Félegyházi, The Cousins of Stuxnet: Duqu, Flame, and Gauss, Future Internet 4 (2012), S. 971 (971).

42 Id., S. $979 \mathrm{f}$.

43 David P. Fidler, Tinker, Tailor, Soldier, Duqu, IJCIP 5 (2012), S. 28 (28); M. Hauck/J. Kubn, Computervirus Duqu entdeckt, Wie gefährlich ist der StuxnetBruder?, Süddeutsche Zeitung, 19. Oktober 2011, abrufbar unter: https://www.su eddeutsche.de/digital/computervirus-duqu-entdeckt-wie-gefaehrlich-ist-der-stuxne t-bruder-1.1168324 (geprüft am 15.05.2020).

44 M. Hauck/J. Kuhn, Computervirus Duqu entdeckt, Wie gefährlich ist der StuxnetBruder?, Süddeutsche Zeitung, 19. Oktober 2011, abrufbar unter: https://www.s ueddeutsche.de/digital/computervirus-duqu-entdeckt-wie-gefaehrlich-ist-der-stu xnet-bruder-1.1168324 (geprüft am 15.05.2020); D. McElroy/C. Williams, Flame: world's most complex computer virus exposed, The Telegraph, 28 May 2012, abrufbar unter: http://www.telegraph.co.uk/news/worldnews/middleeast/iran/9 295938/Flame-worlds-most-complex-computer-virus-exposed.html (geprüft am 15.05.2020).

45 B. Bencsáth/G. Pék/L. Buttyán/M. Félegyházi, The Cousins of Stuxnet: Duqu, Flame, and Gauss, Future Internet 4 (2012), S. 971 (971); A. Gostev, Cyber weapons, Kaspersky Security Bulletin 2012, abrufbar unter: https://securelist.com/kaspersky -security-bulletin-2012-cyber-weapons/36762/ (geprüft am 15.05.2020). 
und den Einsatz der Programme verantwortlich sind. ${ }^{46}$ Diese Mutmaßungen genügten aber nicht, um völkerrechtliche Reaktionen der betroffenen Länder auszulösen. ${ }^{47}$

IV. Informationsausbeutung durch Geheimdienste „Five Eyes“

Staatliche Nachrichtendienste sind in immer größerem Umfang damit beschäftigt, private Daten von Politikern und anderen Bürgern im Inund Ausland auszuspähen, indem sie die Telekommunikation, den E-MailVerkehr und die Internetaktivitäten systematisch überwachen. Die Informationsgewinnung steht im fundamentalen Widerspruch zum Recht auf Privatheit. 48

Im Jahr 2013 begannen der britische Guardian und die amerikanische Washington Post, geheime Dokumente zu veröffentlichen, die sie vom früheren Mitarbeiter des amerikanischen Geheimdienstes der National Security Agency (NSA), Edward Snowden, bekommen hatten. Diese Dokumente enthüllten ein weltweites Netz von Spionagesystemen der NSA und deren engster Partner, den Nachrichtendiensten Großbritanniens, Neuseelands, Kanadas und Australiens - den sogenannten Five Eyes. Eine unzählige Liste an Schadprogrammen, wie beispielsweise PRISM, Tempora und Boundless Informant, wurden eingesetzt, um private Kommunikation abzuhören, aber auch Regierungsstellen, insbesondere von Mitgliedstaaten der Europäischen Union und ihren Institutionen, auszuspionieren. ${ }^{49}$ Dies führte zwar zu politischen Spannungen, allerdings folgten keine völkerrechtlichen Konsequenzen. Im Gegenteil forderten die Five Eyes zuletzt in einem gemeinsamen Statement von Anbietern von Informations- und

46 D. Weissbrodt, Cyber-Conflict, Cyber-Crime, and Cyber-Espionage, Minn. JIL 22 (2013), S. 347 (353 f.).

47 J. D. Jolley, Attribution, State Responsibility, and the Duty to Prevent Malicious Cyber-Attacks in International Law, 2017, abrufbar unter: http://theses.gla.ac.uk/i d/eprint/8452 (geprüft am 15.05.2020), S. 24.

48 I. Pernice, Vom Völkerrecht des Netzes zur Verfassung des Internets, HIIG Discussion Paper Series, Discussion Paper No. 2017-02, abrufbar unter: https://papers.ss rn.com/sol3/papers.cfm?abstract_id=2959257 (geprüft am 15.05.2020), S. 1 (5 ff.).

49 Norddeutscher Rundfunk/Das Erste, "Snowden exklusiv": der Wortlaut des Interviews von NDR Autor Hubert Seipel, 26 Januar 2014, abrufbar unter: https://ww w.presseportal.de/pm/69086/2648795 (geprüft am 15.05.2020). 
Kommunikationstechnologie sogar ihre Verschlüsselungsmethoden derart abzuschwächen, dass ein staatlicher Zugriff möglich wird. ${ }^{50}$

\section{Informationsausbeutung durch Hackergruppe „Guardians of Peace“}

Im Jahr 2014 kam es zu einem elektronischen Einbruch in die Server des US-amerikanischen Unternehmens Sony Pictures Entertainment. ${ }^{51}$ Eine Gruppe, die sich selbst als Guardians of Peace bezeichnete, erbeutete mehrere Terabyte an Daten und veröffentlichte vertrauliche Informationen, wie etwa bis dahin unveröffentlichte Filme, interne E-Mails und Sozialversicherungsnummern von Mitarbeitern. Darüber hinaus wurden alle Daten auf den betroffenen Computern und Servern gelöscht. ${ }^{52}$ Hintergrund des Angriffs war der Film „The Interview“, in dem es um einen fiktiven Mordanschlag auf den nordkoreanischen Machthaber Kim Jong-Un ging. Die Hackergruppe drohte weitere sensible Daten zu veröffentlichen, sofern die Veröffentlichung des Filmes durch Sony Pictures nicht gestoppt werde. ${ }^{53}$ Der Schaden durch die beschädigten Computersysteme, die Einnahmeausfälle und die Veröffentlichung von Betriebsgeheimnissen beläuft sich auf Millionenbeträge. ${ }^{54}$

50 Five Country Ministerial and Quintet Meeting of Attorneys General, Statement of Principles on Access to Evidence and Encryption, Australia 2018, abrufbar unter: https://www.ag.gov.au/About/CommitteesandCouncils/Documents/joint-s tatement-principles-access-evidence.pdf (geprüft am 15.05.2020).

51 D. Efrony/Y. Shany, A Rule Book on the Shelf?, AJIL 112 (2018), S. 583 (605 f.); R. Crootof, International Cybertorts, CLR 103 (2018), S. 565 (567 f.); B. A. Walton, Duties Owed, Yale LJ 126 (2017), S. 1460 (1462 f.).

52 D. Robb, Sony Hack: A Timeline, DEADLINE, 22 December 2014, abrufbar unter: http://deadline.com/2014/12/sony-hack-timeline-any-pascal-the-interview-n orth-korea-1201325501 (geprüft am 15.05.2020).

53 C. Shoard, Sony Hack: the plot to kill The Interview - a timeline so far, The Guardian, 18 December 2014, abrufbar unter: http://www.theguardian.com/film/ 2014/dec/18/sony-hack-the-interview-timeline (geprüft am 15.05.2020).

54 L. Brinded, The Interview tipped to cost Sony Pictures $\$ 200 \mathrm{~m}$ following hack and cancellation, International Business Times, 18 December 2014, abrufbar unter: http://www.ibtimes.co.uk/interview-tipped-cost-sony-pictures-200m-total-followi nghack-cancellation-1480157 (geprüft am 15.05.2020); D. Efrony/Y. Shany, A Rule Book on the Shelf?, AJIL 112 (2018), S. 583 (606); C. Kang, Sony Pictures Hack cost the movie studio at least $\$ 15$ million, The Washington Post, 4 February 2015, abrufbar unter: https:/www.washingtonpost.com/news/business/wp/2015/ 02/04/sony-pictures-hack-cost-the-movie-studio-at-least-15-million/?utm_term=.db 5b8a3b9915 (geprüft am 15.05.2020). 
Die US-Regierung reagierte mit Sanktionen gegen Nordkorea, weil sie das dortige Regime hinter dem Angriff vermutete. ${ }^{55}$ Diese Anschuldigung gründete allerdings nicht auf eindeutigen Beweisen. Im Gegenteil fanden sich ebenso Anzeichen dafür, dass es sich um einen Angriff aus den Reihen des Unternehmens selbst handeln könnte. ${ }^{56}$

\section{Informationsausbeutung und -angriff auf das „Democratic National Committee“}

In den Jahren 2015 und 2016 sorgten Zugriffe auf Datenbanken der Dachorganisation der Demokratischen Partei der USA, das Democratic National Committee (DNC), für weitreichende Konsequenzen. Die Veröffentlichung der entwendeten vertraulichen Informationen durch Wikileaks und andere Plattformen schädigte die Demokratische Partei mit Blick auf die anstehenden Kongresswahlen und war auch im Zusammenhang mit den Präsidentschaftswahlen von Bedeutung. Daneben sollen US-Wahlsysteme ein Ziel von Informationsangriffen gewesen sein, wobei aber eine erfolgreiche Manipulation dieser Systeme nicht nachgewiesen wurde. ${ }^{57}$

Die US-Geheimdienste bewerteten diese Informationsoperation als Kampagne zur Einflussnahme auf die US-Wahl durch Russland, welche die Erfolgsaussichten der Präsidentschaftskandidatin Hillary Clinton im Rahmen der Präsidentschaftswahl schmälern und Zweifel am demokratischen Wahlprozess der Vereinigten Staaten im Allgemeinen begründen

55 U.S. Department of the Treasury, Press Center, Treasury Imposes Sanctions Against the Government of the Democratic People's Republic Of Korea, 2 January 2015, abrufbar unter: https:/www.treasury.gov/press-center/press-releases/P ages/j19733.aspx (geprüft am 15.05.2020); D. Efrony/Y. Shany, A Rule Book on the Shelf?, AJIL 112 (2018), S. 583 (607 f.).

56 D. Efrony/Y. Shany, A Rule Book on the Shelf?, AJIL 112 (2018), S. 583 (607) m.w.N.; J. Goldsmith, The Sony Hack: Attribution Problems, and the Connection to Domestic Surveillance, LAWFARE, 19 December 2014, abrufbar unter: https:/ /www.lawfareblog.com/sony-hack-attribution-problems-and-connection-dom estic-surveillance (geprüft am 15.05.2020); K. Zetter, Critics Say New Evidence Linking North Korea to the Sony Hack Is Still Flimsy, WIRED, 8 January 2015, abrufbar unter: http://www.wired.com/2015/01/critics-say-new-north-korea-evide nce-sony-still-flimsy (geprüft am 15.05.2020).

57 W. Banks, State Responsibility and Attribution of Cyber Intrusions After Tallinn 2.0, Tex. LR 95 (2017), S. 1487 (1487 ff.); D. Efrony/Y. Shany, A Rule Book on the Shelf?, AJIL 112 (2018), S. 583 (609 ff.). 
sollte. ${ }^{58}$ Die US-Regierung erklärte, dass aufgrund des Ausmaßes dieser Bestrebungen nur Russlands ranghöchste Beamte diese Informationsoperation genehmigt haben könnten und reagierte infolgedessen mit Sanktionen gegen Russland und der Ausweisung russischer Diplomaten. ${ }^{59}$ Neben den wirtschaftlichen Schäden hatte diese Informationsoperation enorme wenn auch nicht quantifizierbare - negative politische Auswirkungen. ${ }^{60}$

\section{Völkerrechtswidrigkeit}

In den vergangenen Jahren diskutierten Völkerrechtler umfassend Rechtsfragen zum Themenkomplex der Informationskriegsführung. Unter der Schirmherrschaft des North Atlantic Treaty Organization Cooperative Cyber Defence Centre of Excellence (NATO CCD COE) etwa beschäftigten sich Experten seit 2009 mit völkerrechtlichen Normen, die auf virtuelle Kriegsführung Anwendung finden. Sie erstellten ein entsprechendes Handbuch mit dem Titel „Tallinn Manual on the International Law Applicable to Cyber Warfare" (Tallinn Manual), in dem ausführlich die Anwendung militärischer bzw. bewaffneter Gewalt sowie Aspekte des humanitären Völkerrechts im Zusammenhang mit Informationsoperationen thematisiert werden. ${ }^{61}$ Zunehmend werden auch völkerrechtliche Normen, die Informationsoperationen zu Friedenszeiten reglementieren,

58 D. Efrony/Y. Shany, A Rule Book on the Shelf?, AJIL 112 (2018), S. 583 (609 ff.); T. Hamburger/K. Tumulty, WikiLeaks releases thousands of documents about Clinton and internal deliberations, The Washington Post, 22 July 2016, abrufbar unter: https://www.washingtonpost.com/news/post-politics/wp/2016/07/22/on -eve-of-democratic-convention-wikileaks-releases-thousands-of-documents-abou t-clinton-the-campaign-and-internal-deliberations/ (geprüft am 15.05.2020); $D$. E. Sanger/C. Savage, U.S. Says Russia Directed Hacks to Influence Elections, The New York Times, 7 October 2016, abrufbar unter: http://www.nytimes.com/2016 /10/08/us/politics/us-formally-accuses-russia-of-stealing-dnc-emails.html (geprüft am 15.05.2020).

59 The White House, Office of the Press Secretary, Statement by the President on Actions in Response to Russian Malicious Cyber Activity and Harassment, 29 December 2016, abrufbar unter: https:/obamawhitehouse.archives.gov/the-press -office/2016/12/29/statement-president-actions-response-russian-malicious-cyber -activity (geprüft am 15.05.2020); D. Efrony/Y. Shany, A Rule Book on the Shelf?, AJIL 112 (2018), S. 583 (611 ff.).

60 R. Crootof, International Cybertorts, CLR 103 (2018), S. 565 (568).

61 M. N. Schmitt (Hg.), Tallinn Manual on the International Law Applicable to Cyber Warfare, Prepared by the International Groups of Experts at the Invitation of the NATO CCD COE, 2013. 
näher beleuchtet. Beispielsweise veröffentlichte das NATO CCD COE eine multidisziplinäre Untersuchung zum Themenkomplex Informationsoperationen unter der Überschrift „Peacetime Regime for State Activities in Cyberspace. International Law, International Relations and Diplomacy“62 sowie das Folgeprojekt zum Tallinn Manual mit dem Titel „Tallinn Manual 2.0 on the International Law Applicable to Cyber Operations" (Tallinn Manual 2.0 ${ }^{63}$. Diese Handbücher beschreiben Rechte und Pflichten von Staaten im virtuellen Raum, wie sie sich aus speziellen Bereichen des Völkerrechts - etwa dem internationalen Telekommunikationsrecht, Seerecht, Weltraumrecht, Luftfahrtrecht, Gesandtschaftsrecht oder den Menschenrechten - entnehmen lassen. ${ }^{64}$ Dabei konzentrieren sich die Werke auf Aspekte der Völkerrechtswidrigkeit von Informationsoperationen. Der Analyse der Völkerrechtler ist gemein, dass sie in der Regel zu dem Ergebnis kommen, im Völkerrecht bestehe mit Blick auf Informationsoperationen eine "Grauzone“65.

Die Schwierigkeiten bei der völkerrechtlichen Reglementierung von Informationsoperationen verdeutlichen auch die Entwicklungen im Rahmen der Arbeiten der „Groups of Governmental Experts on Developments in the Field of Information and Telecommunications in the Context of International Security“ (GGE). Sie wurden von den Vereinten Nationen eingesetzt, um sich mit der Anwendbarkeit völkerrechtlicher Bestimmungen auf den virtuellen Raum und den bestehenden Grenzen auseinanderzusetzen und unterschiedliche Lösungen im zwischenstaatlichen Austausch zu diskutieren. ${ }^{66}$ Während die Expertengruppe im Jahr 2013 noch für die Klarstellung gerühmt wurde, dass bestehendes Völkerrecht, allen voran

62 K. Ziolkowski (Hg.), Peacetime Regime for State Activities in Cyberspace, 2013.

63 M. N. Schmitt (Hg.), Tallinn Manual 2.0 on the International Law Applicable to Cyber Operations, Prepared by the International Groups of Experts at the Invitation of the NATO CCD COE, 2017.

64 Tallinn Manual 2.0, Teil II; K. Ziolkowski (Hg.), Peacetime Regime for State Activities in Cyberspace, 2013, Teil II.

65 M. N. Schmitt, Grey Zones in the International Law of Cyberspace, YIL Online 42 (2017), abrufbar unter: https://cpb-us-w2.wpmucdn.com/campuspress.yale .edu/dist/8/1581/files/2017/08/Schmitt_Grey-Areas-in-the-International-Law -of-Cyberspace-1cab8kj.pdf (geprüft am 15.05.2020), S. 1 (1 ff.); S.-H. Schulze, Cyber-„War" - Testfall der Staatenverantwortlichkeit, 2015, S. 98. R. Crootof weist daraufhin, dass die Grenze zwischen rechtmäßigen und unrechtmäßigen Informationsoperationen zusehends verwischt. $R$. Crootof, International Cybertorts, CLR 103 (2018), S. 565 (620).

66 GV Resolution 66/24 vom 02.12.2011, Rn. 4 
die VN-Charta, auch im virtuellen Raum anwendbar ist, ${ }^{67}$ konnte sich die Expertengruppe im Jahr 2017 nicht auf einen abschließenden Konsensbericht zu anwendbaren Normen, Regeln und Prinzipien im virtuellen Raum einigen. ${ }^{68}$ Diese Zäsur in den multilateralen Bemühungen ist unter anderem dem Umstand geschuldet, dass Informationsoperationen nicht ohne Weiteres in die völkerrechtlichen Verbotskategorien passen. Die dargebotenen Lösungsansätze beschränken sich vornehmlich auf Bestrebungen die "Grauzone“ entweder durch eine extensive Interpretation von Voraussetzungen für Völkerrechtsverstöße oder durch die Schaffung neuer Verbotstatbestände einzudämmen.

\section{Informationskrieg und Informationskriegsführung}

In Ermangelung spezifischer Regeln für die virtuelle Welt ist die Völkerrechtswidrigkeit von Informationskrieg und Informationskriegsführung anhand der auf konventionelle Angriffe zugeschnittenen Vorgaben der VN-Charta und des humanitären Völkerrechts zu beurteilen.

\section{Informationsangriff als Gewalt im Sinne der VN-Charta}

Zunächst sind die Unsicherheiten bei der Anwendbarkeit der wesentlichen Elemente des Rechts zum Krieg (ius ad bellum) auf Informationsoperationen zu rekapitulieren. Die Frage, ob Informationsangriffe die Voraussetzungen des Gewaltbegriffs im Sinne des Art. 2 Abs. 4 VN-Charta erfüllen oder ob diese gar einen das Selbstverteidigungsrecht auslösenden bewaffneten Angriff im Sinne des Art. 51 VN-Charta darstellen, wurde

67 Vgl. GGE, Report of the Group of Governmental Experts on Developments in the Field of Information and Telecommunications in the Context of International Security, 24 June 2013, UN Doc. A/68/98, S. 8, Rn. 19.

68 United Nations Office for Disarmament Affairs, Developments in the field of information and telecommunications in the context of international security, Fact Sheet, abrufbar unter: https://s3.amazonaws.com/unoda-web/wp-content /uploads/2018/07/Information-Security-Fact-Sheet-July2018.pdf (geprüft am 15.05.2020); siehe auch S. Soesanto/F. D'Incau, The UN GGE is dead: Time to fall forward, European Council on Foreign Relations, 15 August 2017, abrufbar unter: https://www.ecfr.eu/article/commentary_time_to_fall_forward_on_cyber_ governance (geprüft am 15.05.2020). 
im Schrifttum bereits ausgiebig diskutiert. ${ }^{69}$ Die Völkerrechtler sind sich grundsätzlich einig darüber, dass beide Kategorien bewaffnete Gewalt voraussetzen. ${ }^{70}$ Damit sind allerdings nicht (mehr) nur herkömmliche (militärische) Waffen, die kinetische Energie freisetzen, gemeint. Mittlerweile werden auch biologische und chemische Waffen unter den Begriff subsumiert, so dass weitergedacht auch Angriffe mittels Informationstechnologie in den Anwendungsbereich der VN-Charta fallen können. ${ }^{71}$ Entgegen Stimmen in der Völkerrechtsliteratur, die jegliche Art von Informationsoperation mit destruktiven Absichten und Folgen von gewisser Intensität ausreichen lassen wollen, ${ }^{72}$ müssen die Auswirkungen aber auch qualitativ und quantitativ mit denen von kinetischer Gewaltanwendung vergleichbar sein. ${ }^{73}$ Eine Vergleichbarkeit ist nur dann gegeben, wenn der Informationsangriff unmittelbar zu physischen Schäden an Menschen oder Sachwerten führt. ${ }^{74}$ Wirtschaftlicher oder politscher Zwang fallen hingegen

69 Siehe zum Beispiel H. H. Dinniss, Cyber Warfare and the Laws of War, 2012, S. 37 ff.; T. O. Keber/P. N. Roguski, Ius ad bellum electronicum?, AVR 49 (2011), S. 399 (399 ff.); R. Nguyen, Navigating Jus Ad Bellum in the Age of Cyber Warfare, Cal. LR 101 (2013), S. 1079 (1079 ff.); M. Roscini, Cyber Operations and the Use of Force in International Law, 2014, S. 43 ff.; S.-H. Schulze, Cyber-„War“ - Testfall der Staatenverantwortlichkeit, 2015, S. 95 ff.; J.-H. Woltag, Cyber Warfare, 2014, S. $111 \mathrm{ff}$.

70 H. H. Dinniss, Cyber Warfare and the Laws of War, 2012, S. $40 \mathrm{ff} . ;$ M. Roscini, Cyber Operations and the Use of Force in International Law, 2014, S. 45; S.-H. Schulze, Cyber-,WWar“ - Testfall der Staatenverantwortlichkeit, 2015, S. 95; T. Stein/T. Marauhn, Völkerrechtliche Aspekte von Informationsoperationen, ZaöRV 60 (2000), S. 1 (5).

71 Vgl. Tallinn Manual 2.0, Teil III, Allgemeiner Kommentar zu Abschnitt 14, S. 328, Rn. 1; H. Krieger, Krieg gegen anonymous, AVR 50 (2012), S. 1 (10); M. $N$. Schmitt, Computer Network Attack and the Use of Force in International Law, Col. JTL 37 (1999), S. 885 (916); S.-H. Schulze, Cyber-,War“ - Testfall der Staatenverantwortlichkeit, 2015, S. $96 \mathrm{f}$.

72 W. G. Sharp, Cyberspace and the Use of Force, 1999, S. 133, 140; N. Tsagourias, Cyber-attacks, self-defence and the problem of attribution, JCSL 17 (2012), S. 229 (231).

73 F. Dittmar, Angriffe auf Computernetzwerke, 2005, S. 154; T. O. Keber/P. N. Roguski, Ius ad bellum electronicum?, AVR 49 (2011), S. 399 (406 ff.); H. Krieger, Krieg gegen anonymous, AVR 50 (2012), S. 1 (10); T. Stein/T. Marauhn, Völkerrechtliche Aspekte von Informationsoperationen, ZaöRV 60 (2000), S. 1 (6f.).

74 F. Dittmar, Angriffe auf Computernetzwerke, 2005, S. 100; H. Krieger, Krieg gegen anonymous, AVR 50 (2012), S. 1 (10); M. N. Schmitt, Computer Network Attack and the Use of Force in International Law, Col. JTL 37 (1999), S. 885 (916f.); K. Zemanek, Armed Attack, in: R. Wolfrum (Hg.), MPEPIL 2013, http:// www.mpepil.com, Rn. 13. 
nicht unter den Gewaltbegriff. ${ }^{75}$ Zudem müssen die Auswirkungen ein gewisse Intensität erreichen; ${ }^{76}$ weder unerhebliche Auswirkungen noch eine Abfolge kumulativer Angriffe geringer Intensität reichen in diesem Zusammenhang aus. ${ }^{77}$

Zweifelsfrei können Informationsangriffe zerstörerische Folgen haben, die ebenso gravierend sind wie die Wirkungen konventioneller Angriffe. So können Informationsoperationen in Steuerungs- und Kontrollsystemen kritischer Infrastrukturen, etwa von Chemiefabriken, Atomkraftwerken oder in der Flugsicherung, Funktionsstörungen hervorrufen und auf diese Weise Todesopfer, Verletzte oder erhebliche Sachschäden verursachen. ${ }^{78}$ Bei Informationsoperationen fehlt es aber häufig am Kriterium der Unmittelbarkeit, da diese erst in einem gewissen zeitlichen Abstand zum ursächlichen Angriff oder erst zusammen mit weiteren Angriffen zu merklichen Schäden führen. ${ }^{79}$ Außerdem sind im Rahmen vieler Informationsoperationen, beispielsweise bei der Störung von Kommunikations- und Informationskanälen oder der Entwendung und/oder Zerstörung von Daten,

75 C. Greenwood, Self-Defence, in: R. Wolfrum (Hg.), MPEPIL 2011, http://www.mp epil.com, Rn. 9; H. Krieger, Krieg gegen anonymous, AVR 50 (2012), S. 1 (11).

76 Der IGH hat in seiner Nicaragua-Entscheidung einen „scale and effects"-Standard aufgestellt, um zwischen Gewaltanwendung und schwerster Gewaltanwendung zu differenzieren. IGH, Military and Paramilitary Activities in and against Nicaragua (Nicaragua v. United States of America), Merits, Judgment of 27 June 1986, ICJ Reports 1986, S. 14 (101 Rn. 191, 103 Rn. 195). Zur damit einhergehenden Diskussion, ob und inwiefern Art. 2 Abs. 4 VN-Charta einerseits und Art. 51 VN-Charta andererseits unterschiedliche Intensitätsschwellen voraussetzen, siehe Tallinn Manual 2.0, Kommentar zu Regel 69, S. 332 f., Rn. 6 f.; C. Lotrionte, Reconsidering the Consequences for State-Sponsored Hostile Cyber Operations Under International Law, CDR 3, (2018), S. 73 (87 ff.).

77 Vgl. IGH, Oil Platforms (Islamic Republic of Iran v. United States of America), Judgment of 6 November 2003, ICJ Reports 2003, S. 161 (187 Rn. 51, $191 \mathrm{f}$. Rn. 64); vgl. auch I. Brownlie, International Law and the Use of Force by States, 1963, S. 278 f.; H. Krieger, Krieg gegen anonymous, AVR 50 (2012), S. 1 (10 f.). Nach anderer Auffassung können auch eine Reihe von weniger intensiven Angriffen gegen einen Staat, die denselben Ursprung haben und zusammenhängen, als Gewaltanwendung qualifiziert werden. Tallinn Manual 2.0, Kommentar zu Regel 71, S. 342, Rn.11; T. Ruys, 'Armed Attack' and Article 51 of the UN Charter, 2010, S. 168 f.

78 C. Schaller, Internationale Sicherheit und Völkerrecht im Cyberspace, SWP-Studie 18/2014, S. 18.

79 F. Dittmar, Angriffe auf Computernetzwerke, 2005, S. 156; S. Gaycken, Die vielen Plagen des Cyberwar, in: R. Schmidt-Radefeldt/C. Meissler (Hg.), Automatisierung und Digitalisierung des Krieges, 2012, S. 89 (95); H. Krieger, Krieg gegen anonymous, AVR 50 (2012), S. 1 (11). 
keine direkten physischen Auswirkungen gegeben. ${ }^{80}$ Gleichwohl kamen die Experten des Tallinn Manual zu dem Ergebnis, dass auch derartige Informationsoperationen unerlaubte Gewalt im Sinne der VN-Charta darstellen können. ${ }^{81}$ Allerdings konnten sich die Experten nicht auf eine schlüssige Begriffsbestimmung einigen und stellten lediglich exemplarisch kontextbezogene Kriterien auf, die als Indikatoren für das Überschreiten der Schwelle zur unerlaubten Gewalt dienen. ${ }^{82}$ Für die Qualifizierung einer Informationsoperation als Verstoß gegen das Gewaltverbot sprächen der Schweregrad der Auswirkungen, die zeitliche Unmittelbarkeit der Folgen, die direkte Ursächlichkeit der Maßnahme, der Grad des Eindringens in den fremdstaatlichen Bereich, die Messbarkeit der Effekte, ein militärischer Charakter, das Ausmaß staatlicher Beteiligung sowie prima facie einschlägige Verbotskategorien. ${ }^{83}$ Dieser Kriterienkatalog trägt aber zur Unschärfe des Gewaltbegriffs bei, da dessen Evaluierung im konkreten Einzelfall von der einzelstaatlichen Interpretation abhängig ist. ${ }^{84}$ Die Subjektivierung begünstigt ein opportunistisches Begriffsverständnis und führt zur Rechtsunsicherheit. ${ }^{85}$ Außerdem birgt eine weite Interpretation des Gewaltbegriffs auch ein entsprechendes Eskalationspotenzial, indem die Voraussetzungen für nach Art. 49 Abs. 1 ASR erlaubte Gegenmaßnah-

80 S.-H. Schulze, Cyber-,,War“ - Testfall der Staatenverantwortlichkeit, 2015, S. 98 f.

81 Tallinn Manual 2.0, Kommentar zu Regel 69, S. 333 ff., Rn. 9 f. So auch M. N. Schmitt, demzufolge "[armed attacks] also include those that seriously impair the functionality of critical infrastructure or that otherwise have devastating non-physical effects, such as crippling a State's economic system“. M. N. Schmitt, Cyber Responses "By The Numbers" in International Law, EJIL:Talk!, 4 August 2015, abrufbar unter: https://www.ejiltalk.org/cyber-responses-by-the-numbers -in-international-law/ (geprüft am 15.05.2020); siehe auch N. Tsagourias, Cyber-attacks, self-defence and the problem of attribution, JCSL 17 (2012), S. 229 (231).

82 M. Benatar, The Use of Cyber Force, GOJIL 1 (2009), S. 375 (391 f.); M. Roscini, Cyber Operations and the Use of Force in International Law, 2014, S. 48; M. N. Schmitt, The 'Use of Force' in Cyberspace, in: C. Czosseck/R. Ottis/K. Ziolkowski (Hg.), 2012 4th International Conference on Cyber Conflict, 2012, S. 311 (314).

83 Tallinn Manual 2.0, Kommentar zu Regel 69, S. 333 ff., Rn. 8 f. Diese Kriterien basieren auf einem Vorschlag, der von M. N. Schmitt schon im Jahr 1999 ausgearbeitet wurde. M. N. Schmitt, Computer Network Attack and the Use of Force in International Law, Col. JTL 37 (1999) S. 885 (912 ff.).

84 M. Benatar, The Use of Cyber Force, GOJIL 1 (2009), S. 375 (391); T. O. Keber/P. N. Roguski, Ius ad bellum electronicum?, AVR 49 (2011), S. 399 (409); S.-H. Schulze, Cyber-„War“ - Testfall der Staatenverantwortlichkeit, 2015, S. 99; C. Stelter, Gewaltanwendung unter und neben der UN-Charta, 2007, S. 76.

85 S.-H. Schulze, Cyber-„War“ - Testfall der Staatenverantwortlichkeit, 2015, S. 99;

C. Stelter, Gewaltanwendung unter und neben der UN-Charta, 2007, S. 76. 
men auf Verletzungen des Gewaltverbotes aus Art. 2 Abs. 4 VN-Charta oder für bewaffnete Gewaltanwendung als Selbstverteidigung nach Art. 51 VN-Charta herabgesetzt werden. ${ }^{86}$ Die Experten des Tallinn Manual 2.0 geben in diesem Zusammenhang selbst zu bedenken, dass insbesondere im virtuellen Raum das Risiko überstürzter Vergeltungsmaßnahmen besteht, die wenig Zeit für Überlegungen über mögliche Konsequenzen lassen. ${ }^{87}$ Daher sollte an dem konventionellen Begriffsverständnis der Gewaltanwendung festgehalten werden und nur solche Informationsangriffe unter die Kategorie des ius ad bellum fallen, die unmittelbar zu Todesopfern, Verletzten oder erheblichen Sachschäden führen. ${ }^{88}$

\section{Informationsangriff als Angriff im Sinne des humanitären Völkerrechts}

Bei der Anwendbarkeit des Rechts im Krieg (ius in bello) auf den virtuellen Raum ist der Schutz von Zivilisten und zivilen Objekten während eines bewaffneten Konfliktes diskussionswürdig. Die Schutzvorschriften greifen, wenn die Informationsoperation als Angriff im Sinne des humanitären Völkerrechts zu qualifizieren ist. ${ }^{89}$ Auch in diesem Zusammenhang sind zunächst die destruktiven physischen Auswirkungen auf Menschen oder Gegenstände maßgeblich. ${ }^{90}$ Zudem suggeriert der Sinn und Zweck des humanitären Völkerrechts, dass es kein Unterschied sein darf, ob eine Informationsinfrastruktur physischen Schaden nimmt oder ob die Funktionalität derselbigen beeinträchtigt wird. Dementsprechend ist auch das Stören

86 Es finden sich unterdessen Stimmen, die nicht nur im Rahmen des Rechts auf Selbstverteidigung nach Art. 51 VN-Charta, sondern auch als Reaktion auf Gewalt im Sinne des Art. 2 Abs. 4 VN-Charta bewaffnete Gewalt als Reaktion völkerrechtlich zulassen wollen. Vgl. Tallinn Manual 2.0, Kommentar zu Regel 22, S. 125 f., Rn. 12.

87 Tallinn Manual 2.0, Kommentar zu Regel 21, S. 117, Rn. 2.

88 So auch S.-H. Schulze, der ausführt, dass nach Sinn und Zweck des Gewaltverbotes auch keine weite Interpretation des Gewaltverbotes angezeigt ist, weil betroffene Staaten in diesen Konstellationen, in denen es nicht (unmittelbar) zu Verletzungen oder dem Tod von Menschen kommt und auch keine Sachwerte zerstört werden, nicht unter demselben Handlungsdruck stehen. S.-H. Schulze, Cyber-„War“ - Testfall der Staatenverantwortlichkeit, 2015, S. 99.

89 M. N. Schmitt, Grey Zones in the International Law of Cyberspace, YIL Online 42 (2017), abrufbar unter: https://cpb-us-w2.wpmucdn.com/campuspress.yale.edu/di st/8/1581/files/2017/08/Schmitt_Grey-Areas-in-the-International-Law-of-Cyberspa ce-1cab8kj.pdf (geprüft am 15.05.2020), S. 1 (17).

Ibid. 
der Funktionsfähigkeit eines Objektes als Angriff zu werten. ${ }^{91}$ Unsicherheiten bestehen allerdings bei der Bestimmung der Intensitätsschwelle, die eine nicht-physische Störung erreichen muss, um als Angriff zu gelten. ${ }^{92}$ Entscheidend ist, ob die Störung mehr als eine „Unannehmlichkeit" verursacht. ${ }^{93}$ So dürfte das zeitweise Aussetzen der Funktionsfähigkeit einer Informationsinfrastruktur nicht ausreichen, wenn keine physischen Schäden oder Verletzungen dadurch hervorgerufen werden. ${ }^{94}$ Diese Vagheit des Angriffsbegriffs führt dazu, dass nicht eindeutig bestimmt werden kann, ob eine Informationsoperation darunter zu subsumieren ist und steht damit einem effektiven Schutz nach dem ius in bello entgegen. ${ }^{95}$ Anlass für Bedenken bietet zudem die Tatsache, dass der durch das humanitäre Völkerrecht gewährte Schutz ziviler Objekte nicht dahingehend ausgeweitet werden kann, dass auch Daten per se unter den Schutzbereich fallen. So sind zwar Daten, die essenziell für das Funktionieren der Gesellschaft sind, zunehmend Zielscheibe von Informationsoperationen, deren Erlangung, Zerstörung, Schädigung oder Verfälschung im Rahmen bewaffneter Konflikte ist aber völkerrechtlich nicht geächtet. ${ }^{96}$

91 Tallinn Manual 2.0, Kommentar zu Regel 92, S. 417, Rn. 10; M. N. Schmitt, Grey Zones in the International Law of Cyberspace, YIL Online 42 (2017), abrufbar unter: https://cpb-us-w2.wpmucdn.com/campuspress.yale.edu/dist/8/1581/files/2 017/08/Schmitt_Grey-Areas-in-the-International-Law-of-Cyberspace-1cab8kj.pdf (geprüft am 15.05.2020), S. 1 (17).

92 M. N. Schmitt, Grey Zones in the International Law of Cyberspace, YIL Online 42 (2017), abrufbar unter: https://cpb-us-w2.wpmucdn.com/campuspress.yale.edu/di st/8/1581/files/2017/08/Schmitt_Grey-Areas-in-the-International-Law-of-Cyberspa ce-1cab8kj.pdf (geprüft am 15.05.2020), S. 1 (18).

93 International Committee of the Red Cross (ICRC), International humanitarian law and the challenges of contemporary armed conflicts, Report on the 32nd International Conference of the Red Cross and Red Crescent (32IC/15/11), 2015, abrufbar unter: http://rcrcconference.org/app//uploads/2015/10/32IC-Report -on-IHL-and-challenges-of-armed-conflicts.pdf (geprüft am 15.05.2020), S. 42. Das ICRC weist in diesem Zusammenhang zugleich daraufhin, dass der Begriff „Unannehmlichkeit“ nicht definiert ist und im humanitären Völkerrecht nicht angewandt wird.

94 Vgl. Tallinn Manual 2.0, Kommentar zu Regel 92, S. 417, Rn. 10.

95 Vgl. M. N. Schmitt, Grey Zones in the International Law of Cyberspace, YIL Online 42 (2017), abrufbar unter: https://cpb-us-w2.wpmucdn.com/campuspress. yale.edu/dist/8/1581/files/2017/08/Schmitt_Grey-Areas-in-the-International-Law-o f-Cyberspace-1cab8kj.pdf (geprüft am 15.05.2020), S. 1 (18).

96 Ibid. 
3. Informationskrieg und Informationskriegsführung in der internationalen Praxis

Bei dem mehrwöchigen Informationsangriff auf Estland wurden gezielt informationstechnische Systeme von Regierungs- und Unternehmenswebseiten sowie anderer Onlinedienste gestört und es kam zum Zusammenbruch von estnischen Infrastrukturen. Der Angriff hatte, aufgrund des hohen Technisierungsgrades Estlands, zwar weitreichende Folgen für das wirtschaftliche und gesellschaftliche Leben, es kam dadurch aber weder zu Verletzungen oder Tod von Menschen noch zur Zerstörung bedeutender Sachwerte. ${ }^{97}$ Eine Vergleichbarkeit mit Waffengewalt ist damit evident zu verneinen, so dass der Angriff nicht unter die völkerrechtliche Kategorie des Informationskrieges zu fassen ist. ${ }^{98}$

Der Informationsangriff im Kontext der militärischen Auseinandersetzung zwischen Russland und Georgien, bei dem staatliche und private informationstechnische Systeme außer Betrieb gesetzt wurden, beeinträchtigte zwar Kommunikations- und Informationskanäle und behinderte mithin Kommando- und Kontrollstrukturen des georgischen Militärs. ${ }^{99}$ Gleichwohl erreichten die Auswirkungen nicht die erforderliche Intensität, um mit denjenigen von Waffengewalt vergleichbar zu sein oder als Angriff im Sinne des humanitären Völkerrechts gelten zu können. Daher ist auch in diesem Fall kein Informationskrieg gegeben. ${ }^{100}$

Von den bereits beschriebenen Informationsoperationen liefert einzig der Angriff durch das Schadprogramm Stuxnet auf das Überwachungsund Steuerungssystem iranischer Atomanlagen Anhaltspunkte für Diskussionen darüber, inwiefern der Eingriff in das Industriesystem, die Manipulation von Daten und die Kontrolle über die Rotationsgeschwindigkeit der Zentrifugen der Kategorie des Informationskrieges zuzuordnen sind. ${ }^{101}$ Der Fall gilt als Paradebeispiel für die Unsicherheiten, welche die unbe-

97 R. Buchan, Cyber Attacks, JCSL 17 (2012), S. 212 (219); K. C. Hinkle, Countermeasures in the Cyber Context, YIL Online 37 (2011), abrufbar unter: http:// www.yjil.org/docs/pub/o-37-hinkle-countermeasures-in-the-cyber-context.pdf (geprüft am 15.05.2020), S. 11 (13 f.).

98 S.-H. Schulze, Cyber-,,War“ - Testfall der Staatenverantwortlichkeit, 2015, S. 123.

99 A. Hagen, The Russo-Georgian War 2008, in: J. Healey (Hg.), A Fierce Domain, 2013, S. 194 (194 ff.); D. Hollis, Cyberwar Case Study: Georgia 2008, Small Wars Journal (2011), abrufbar unter: https://smallwarsjournal.com/blog/journal/docs -temp/639-hollis.pdf (geprüft am 15.05.2020), S. 1 (6, 8).

100 S.-H. Schulze, Cyber-,War“ - Testfall der Staatenverantwortlichkeit, 2015, S. 125.

101 R. Crootof, International Cybertorts, CLR 103 (2018), S. 565 (591). 
stimmten Schwellenvoraussetzungen der kriegsrechtlichen Kategorien in der virtuellen Welt begründen. ${ }^{102}$ Die veränderte Rotationsgeschwindigkeit und damit einhergehende Behinderung der iranischen Urananreicherung für sich stellt keine Beschädigung oder Zerstörung von Sachwerten dar und würde für sich genommen aus dem Anwendungsbereich des Informationskrieges fallen. Allerdings führte der Informationsangriff zugleich zur Zerstörung von etwa eintausend Zentrifugen. ${ }^{103}$ Diese physischen Auswirkungen überschreiten die Schwelle der Gewalt im Sinne des Art. 2 Abs. 4 VN-Charta. ${ }^{104}$ Während zum Teil schon die vom Gewaltverbot geforderte Quantität und Qualität der Schädigung infrage gestellt wird, ${ }^{105}$ ist jedenfalls entgegen anderer Auffassung ${ }^{106}$ nicht davon auszugehen, dass der Vorfall den geforderten Schweregrad erreicht, um als ein das Selbstverteidigungsrecht auslösender bewaffneter Angriff im Sinne des Art. 51 VN-Charta zu gelten. ${ }^{107}$ Auch wenn der Kriegsbegriff dem Wandel der Zeit entsprechend ausgelegt werden muss, ${ }^{108}$ können letztlich nur Informationsoperationen, die mit herkömmlichen militärischen Schädigungshandlungen vergleichbar sind, als unerlaubte Gewalt im Sinne der VN-Charta gelten. ${ }^{109}$ Einer extensiven Interpretation ist Einhalt zu gebie-

102 Tallin Manual 2.0, Kommentar zu Regel 71, S.342, Rn. 10; D. Efrony/Y. Shany, A Rule Book on the Shelf?, AJIL 112 (2018), S. 583 (638); D. P. Fidler, Was Stuxnet an Act of War? Decoding a Cyberattack, IEEE Security and Privacy Magazine 9 (2011), S. 56 (57 ff.).

103 D. Albright/P. Brannan/C. Walrond, Stuxnet Malware and Natanz: Update of ISIS December 22, 2010 Report, Institute for Science and International Security Report of 15 February 2011, abrufbar unter: http://isis-online.org/uploads/isis-re ports/documents/stuxnet_update_15Feb2011.pdf (geprüft am 15.05.2020), S. 1, 3, 6, 8 f.; S. J. Shackelford,/R. B. Andres, State Responsibility for Cyber Attacks, Geo. JIL 42 (2010-2011), S. 971 (973).

104 Tallin Manual 2.0, Kommentar zu Regel 71, S.342, Rn. 10; S.-H. Schulze, Cyber-,,War“ - Testfall der Staatenverantwortlichkeit, 2015, S. 127; D. Weissbrodt, Cyber-Conflict, Cyber-Crime, and Cyber-Espionage, Minn. JIL 22 (2013), S. 347 (376ff.).

105 J.-C. Woltag, Computer Network Operations Below the Level of Armed Force, ESIL Conference Paper Series 1 (2011), S. 1 (10).

106 D. Weissbrodt, Cyber-Conflict, Cyber-Crime, and Cyber-Espionage, Minn. JIL 22 (2013), S. 347 (378).

107 C. Lotrionte, Cyber Operations: Conflict Under International Law, Georgetown Journal of International Affairs, (2012), S. 15 (20); M. E. O'Connell, Cyber Security without Cyber War, JCSL 17 (2012), S. 187 (201 f.).

108 Vgl. Tallinn Manual 2.0, Teil III, Allgemeiner Kommentar zu Abschnitt 14, S. 328 f., Rn. 3 .

109 Selbst wenn man also auf das Unmittelbarkeitskriterium verzichten wollte, dürfen die Parallelen zu den Auswirkungen konventioneller bewaffneter Angriff 
ten, um eine Ausuferung von Gegenmaßnahmen und Gewaltanwendung in der virtuellen Welt zu verhindern.

In den überwiegenden Konstellationen erfüllen Informationsangriffe nicht die bezeichneten Schwellenvoraussetzungen und sind im Ergebnis als militärisch irrelevant einzuordnen. ${ }^{110}$ Treffenderweise werden derartige Informationsoperationen auch als „Weapons of Mass Annoyance" ${ }^{111}$ oder „Cyberkrawall“"112 bezeichnet.

\section{Informationsintervention}

Aus dem Prinzip der souveränen Gleichheit von Staaten ergibt sich das völkergewohnheitsrechtliche Interventionsverbot. ${ }^{113}$ Eine Informati-

nicht völlig verloren gehen. Maßgeblich ist nach T. Stein/T. Maraubn beispielsweise „ob nach der durch Computer bewirkten Störung der Elektronik eines Kernkraftwerkes der Kern durchzuschmelzen droht, ob nach dem Ausfall der Stromversorgung im Winter Menschen zu erfrieren drohen, oder ob die Ausschaltung jeglicher Flugsicherung den Absturz von Passagierflugzeugen zur unvermeidlichen Folge hätte. Das alles hätte auch eine quantitative Komponente; der Ausfall einzelner Beatmungsgeräte in Krankenhäusern würde einen massiven Gegenschlag kaum rechtfertigen." T. Stein/T. Maraubn, Völkerrechtliche Aspekte von Informationsoperationen, ZaöRV 60 (2000), S. 1 (8).

110 Vgl. S. Gaycken, Die vielen Plagen des Cyberwar, in: R. Schmidt-Radefeldt/C. Meissler (Hg.), Automatisierung und Digitalisierung des Krieges, 2012, S. 89 (93 ff.); H. Krieger, Krieg gegen anonymous, AVR 50 (2012), S. 1 (11).

111 J. A. Lewis, Assessing the Risks of Cyber Terrorism, Cyber War and Other Cyber Threats, Center for Strategic and International Studies, 2002, abrufbar unter: http://csis.org/files/media/csis/pubs/021101_risks_of_cyberterror.pdf (geprüft am 15.05.2020), S. 4, (unter Bezugnahme auf Stewart Baker).

112 F. Rötzer, DDoS-Angriffe auf estnische Server waren kein "Cyberwar", heise online, 12. Juni 2007, abrufbar unter: https://www.heise.de/newsticker/meldung /DDoS-Angriffe-auf-estnische-Server-waren-kein-Cyberwar-138918.html (geprüft am 15.05.2020), (unter Bezugnahme auf James Hendler).

113 IGH, Military and Paramilitary Activities in and against Nicaragua (Nicaragua v. United States of America), Merits, Judgment of 27 June 1986, ICJ Reports 1986, S. 14 (106 Rn. 202, 107 f. Rn. 205, 128 Rn. 251); Tallinn Manual 2.0, Kommentar zu Regel 66, S.312f., Rn. 1 m.w.N.; T. O. Keber/P. N. Roguski, Ius ad bellum electronicum?, AVR 49 (2011), S. 399 (409f.). Bemerkenswert ist unterdessen, dass in dem Eingriff in die freie und unabhängige Ausübung souveräner Rechte gleichzeitig auch der Schaden zu erblicken ist, der damit nicht kausal auf dem schädigenden Verhalten beruht, sondern in dem Verhalten selbst liegt. Siehe hierzu J. D. Jolley, Attribution, State Responsibility, and the Duty to Prevent Malicious Cyber-Attacks in International Law, 2017, abrufbar unter: http://theses.gla.ac.uk/id/eprint/8452 (geprüft am 15.05.2020), S. $176 \mathrm{ff}$. 
onsoperation verstößt gegen das Interventionsverbot, wenn sie in die inneren oder äußeren Angelegenheiten (domaine réservé) eines anderen Staates eingreift und dabei als Zwang zu qualifizieren ist. ${ }^{114}$ Die Unbestimmtheit dieser zwei Voraussetzungen führt zu Unsicherheiten bei der Reglementierung von Informationsoperationen. ${ }^{115}$

\section{Informationstechnische Systeme als domaine réservé}

Der domaine réservé betrifft nur solche Staatsangelegenheiten, die völkerrechtlich nicht erfasst sind. ${ }^{116}$ So überlässt es das Völkerrecht grundsätzlich jedem Staat, über sein politisches, wirtschaftliches, soziales und kulturelles System ohne Einmischung von außen frei zu entscheiden und seine AuBenpolitik zu gestalten. ${ }^{117}$

Die wachsenden internationalen Interdependenzen und zunehmenden transnationalen Kooperationen schränken die Bereiche, die der alleinigen Zuständigkeit eines Staates vorbehalten sind, aber immer weiter ein. ${ }^{118} \mathrm{Ge}-$ rade mit Blick auf informationstechnische Systeme verwischt die Grenze zwischen völkerrechtlichen und nationalen Angelegenheiten zusehends. Staaten obliegt beispielsweise das alleinige souveräne Recht, die Onlinekommunikation ihres Landes zu reglementieren. Gleichzeitig können aber internationale Regeln zum menschenrechtlichen Schutz der Meinungsfrei-

114 Tallinn Manual 2.0, Kommentar zu Regel 66, S.314, Rn. 6; P. Kunig, Prohibition of Intervention, in: R. Wolfrum (Hg.), MPEPIL 2008, http://www.mpepil.co m, Rn. 3, 5 .

115 M. N. Schmitt, Grey Zones in the International Law of Cyberspace, YIL Online 42 (2017), abrufbar unter: https://cpb-us-w2.wpmucdn.com/campuspress.yale.ed u/dist/8/1581/files/2017/08/Schmitt_Grey-Areas-in-the-International-Law-of-Cyb erspace-1cab8kj.pdf (geprüft am 15.05.2020), S. 1 (7).

116 Tallinn Manual 2.0, Kommentar zu Regel 66, S. 314, Rn. 7; K. S. Ziegler, Domaine Réservé, in: R. Wolfrum (Hg.), MPEPIL 2013, http://www.mpepil.com, Rn. $1 \mathrm{ff}$.

117 IGH, Military and Paramilitary Activities in and against Nicaragua (Nicaragua v. United States of America), Merits, Judgment of 27 June 1986, ICJ Reports 1986, S. 14 (107 f. Rn. 205).

118 Tallinn 2.0, Kommentar zu Regel 66, S.314, Rn.6; L. F. Damrosch, Politics Across Borders, AJIL 83 (1989), S. 1 (10 f.); K. S. Ziegler, Domaine Réservé, in: R. Wolfrum (Hg.), MPEPIL 2013, http://www.mpepil.com, Rn. 3, 9 ff.; vgl. auch $R$. Hofmann, Modernes Investitionsschutzrecht, in: S. Kadelbach/K. Günther (Hg.), Recht ohne Staat?, 2011, S. 119 (125). 
heit oder Privatsphäre die Interventionsfestigkeit dieses Bereichs durchbrechen. ${ }^{119}$

\section{Informationsangriff als unerlaubter Zwang}

Informationsoperationen, die einen Staat dazu zwingen, sich einem anderen Staat bei der Ausübung seiner souveränen Rechte unterzuordnen, sind als völkerrechtswidrig zu qualifizieren. ${ }^{120}$ Das Zwangselement grenzt mithin die völkerrechtswidrige Informationsintervention von der völkerrechtlich nicht verbotenen Informationseinmischung ab. ${ }^{121}$ Es fehlt dem Element allerdings an festen Konturen, so dass Fallgruppen aus der Staatenpraxis herangezogen werden, um die völkerrechtliche Qualität einer Informationsoperation zu bewerten. ${ }^{122}$

Zunächst gilt die Androhung oder Anwendung bewaffneter Gewalt (Art. 2 Abs. 4 VN-Charta) als Zwang. ${ }^{123}$ Das virtuelle Eindringen in informationstechnische Systeme für sich genommen genügt dabei nicht, da es - auch wenn virtuelle Schutzmechanismen, wie eine Firewall oder Passwortbarrieren, durchbrochen werden - keiner Gewaltandrohung bzw. Gewaltanwendung gleicht. ${ }^{124}$ Andererseits soll aber die finanzielle, logistische

119 M. N. Schmitt, Grey Zones in the International Law of Cyberspace, YIL Online 42 (2017), abrufbar unter: https://cpb-us-w2.wpmucdn.com/campuspress.yale.ed u/dist/8/1581/files/2017/08/Schmitt_Grey-Areas-in-the-International-Law-of-Cyb erspace-1cab8kj.pdf (geprüft am 15.05.2020), S. 1 (7); S.-H. Schulze, Cyber-„War“ - Testfall der Staatenverantwortlichkeit, 2015, S. $103 \mathrm{f}$.

120 T. O. Keber/P. N. Roguski, Ius ad bellum electronicum?, AVR 49 (2011), S. 399 (409f.).

121 I. Kilovaty, Doxfare, Harv. NSJ 9 (2018), S. 146 (167 ff.); M. N. Schmitt, Grey Zones in the International Law of Cyberspace, YIL Online 42 (2017), abrufbar unter: https://cpb-us-w2.wpmucdn.com/campuspress.yale.edu/dist/8/1581/files /2017/08/Schmitt_Grey-Areas-in-the-International-Law-of-Cyberspace-1cab8kj .pdf (geprüft am 15.05.2020), S. 1 (8); S.-H. Schulze, Cyber-,WWar“ - Testfall der Staatenverantwortlichkeit, 2015, S. 104.

122 S.-H. Schulze, Cyber-,War“ - Testfall der Staatenverantwortlichkeit, 2015, S. 105.

$123 \mathrm{IGH}$, Military and Paramilitary Activities in and against Nicaragua (Nicaragua v. United States of America), Merits, Judgment of 27 June 1986, ICJ Reports 1986, S. 14 (107 f. Rn. 205); P. Kunig, Prohibition of Intervention, in: R. Wolfrum (Hg.), MPEPIL 2008, http://www.mpepil.com, Rn. 5.

124 Tallin Manual 2.0, Kommentar zu Regel 66, S. 323, Rn. 33. Nach anderer Auffassung wird eine Souveränitätsverletzung angenommen, da das virtuelle Eindringen als Ausübung von Hoheitsgewalt und Anmaßung fremder Jurisdiktion zu werten sei. Vgl. W. Heintschel von Heinegg, Legal Implications of Territorial 
oder ähnliche Unterstützung von Hackergruppen, die schädigende Informationsoperationen ausführen, unerlaubten Zwang darstellen können. ${ }^{125}$ Des Weiteren können auch unterhalb der Gewaltschwelle verbleibende Maßnahmen als Zwang gelten. Dazu zählen wirtschaftliche, politische und sonstige Druckmittel, die eine willensbeugende Wirkung entfalten. ${ }^{126} \mathrm{Im}$ Rahmen von Informationsoperationen stellen sich in diesem Zusammenhang besondere Abgrenzungsschwierigkeiten, weil diese eine ganz neue Vielfalt an nicht-physischen, aber nichtsdestoweniger durchdringenden Mitteln der Einmischung kennen. ${ }^{127}$ Während etwa die umsturzorientierte Rundfunk- oder Fernsehpropaganda unerlaubten Zwang darstellen kann, ist das Verbreiten von fundierter Kritik an der Politik eines Landes nicht mehr von dem Begriff erfasst. ${ }^{128}$

\section{Informationseinmischung als Souveränitätsverletzung}

Zur Regulierung von Informationsoperationen, denen es am interventionstypischen Zwangscharakter fehlt, möchten Völkerrechtler eine souveränitätsspezifische Primärnorm gelten lassen. ${ }^{129}$ Diese völkerrechtliche

Sovereignty in Cyberspace, in: C. Czosseck/R. Ottis/K. Ziolkowski (Hg.), 2012 4th International Conference on Cyber Conflict, 2012, S. 7 (11 f.).

125 Tallinn Manual 2.0, Kommentar zu Regel 66, S.319, Rn.22, unter Bezugnahme auf IGH, Military and Paramilitary Activities in and against Nicaragua (Nicaragua v. United States of America), Merits, Judgment of 27 June 1986, ICJ Reports 1986, S. 14 (124 Rn. 242).

126 D. C. Dicke, Die Intervention mit wirtschaftlichen Mitteln im Völkerrecht, 1978, S. 176 ff., 198 f. m.w.N.; P. Kunig, Prohibition of Intervention, in: R. Wolfrum (Hg.), MPEPIL 2008, http://www.mpepil.com, Rn. 6.

127 R. Crootof, International Cybertorts, CLR 103 (2018), S. 565 (625 ff.).

128 P. Kunig, Prohibition of Intervention, in: R. Wolfrum (Hg.), MPEPIL 2008, http://www.mpepil.com, Rn. 24; S.-H. Schulze, Cyber-,War“ - Testfall der Staatenverantwortlichkeit, 2015, S. 107 m.w.N.

129 Tallinn Manual 2.0, Regel 4; M. N. Schmitt, Grey Zones in the International Law of Cyberspace, YIL Online 42 (2017), abrufbar unter: https://cpb-us-w2. wpmucdn.com/campuspress.yale.edu/dist/8/1581/files/2017/08/Schmitt_G rey-Areas-in-the-International-Law-of-Cyberspace-1cab8kj.pdf (geprüft am 15.05.2020), S. 1 (5). Gegen die Geltung einer Souveränitätsregel sprechen sich etwa Gary P. Corn (Staff Judge Advocate of the U.S. Cyber Command) und Robert Taylor (former Principal Deputy General Counsel of the U.S. Department of Defense) aus. G. P. Corn/R. Taylor, Symposium on Sovereignty, Cyberspace, and Tallinn Manual 2.0, Sovereignty in the Age of Cyber, AJIL Unbound 111 (2017), S. $207 \mathrm{ff}$. 
Souveränitätsregel sei mit dem Interventionsverbot zwar verwandt, überschneide sich aber nicht vollkommen mit Letzterem. ${ }^{130}$ Demnach sollen auch zwangsfreie Informationsoperationen untersagt sein, die auf fremdstaatlichem Territorium ausgeführt werden ${ }^{131}$ oder deren Folgen sich dort manifestieren. ${ }^{132}$ Dabei muss die Verletzung der fremdstaatlichen territorialen Integrität eine gewisse Intensität erreichen oder einen Eingriff bzw. eine Anmaßung von regierungseigenen Funktionen bedeuten. ${ }^{133}$ Zum einen besteht aber keine Einigkeit darüber, unter welchen Bedingungen diese Voraussetzungen erfüllt sein sollen. ${ }^{134}$ Zum anderen führt eine derartige Souveränitätsregel dazu, dass Kategorien wie das Gewaltverbot und das Interventionsverbot obsolet werden. ${ }^{135}$ Wollte man nämlich neben physischen Auswirkungen auch nicht-physische Auswirkungen, etwa in Gestalt von Funktionsausfällen informationstechnischer Systeme, ausreichen lassen, um eine Informationsoperation als völkerrechtswidrig zu qualifizieren, ${ }^{136}$ würde man die speziellen Voraussetzungen des Gewaltverbotes umgehen, und wenn schon jede Form der Einmischung in regierungseigene Funktionen untersagt wäre, ${ }^{137}$ hätte es keinen Sinn, speziell Interventionen mittels Zwang zu verbieten. Das Interventionsverbot ist

130 M. N. Schmitt/L. Vihul, Respect for Sovereignty in Cyberspace, Tex. LR 95 (2017), S. 1639 (1653 f.).

131 In diesem Sinne wird auch bei Informationsoperationen die physische Anwesenheit auf fremdem Hoheitsgebiet - beispielsweise zu Spionagezwecken - als Verletzung staatlicher Souveränität gewertet. In der weit verbreiteten entsprechenden Spionagepraxis sehen einige Experten einen völkergewohnheitsrechtlichen Ausnahmetatbestand zu diesem völkerrechtlichen Verbotstatbestand. Tallinn Manual 2.0, Kommentar zu Regel 4, S. 19, Rn. 7 f.

132 Tallinn Manual 2.0, Kommentar zu Regel 4, S. 19 f., Rn. 9.

133 Tallinn Manual 2.0, Kommentar zu Regel 4, S. 20, Rn. 10.

134 Tallinn Manual, Kommentar zu Regel 4, S. 20 f. Rn. 13 f.; S. 22 f., Rn. 16 ff.; $M$. N. Schmitt, Grey Zones in the International Law of Cyberspace, YIL Online 42 (2017), abrufbar unter: https://cpb-us-w2.wpmucdn.com/campuspress.yale.edu/ dist/8/1581/files/2017/08/Schmitt_Grey-Areas-in-the-International-Law-of-Cyber space-1cab8kj.pdf (geprüft am 15.05.2020), S. 1 (6f.).

135 Vgl. B. A. Walton, Duties Owed, Yale LJ 126 (2017), S. 1460 (1475).

136 W. Heintschel von Heinegg, Legal Implications of Territorial Sovereignty in Cyberspace, in: C. Czosseck/R. Ottis/K. Ziolkowski (Hg.), 2012 4th International Conference on Cyber Conflict, 2012, S. 7 (11 ff.); C. Joyner/C. Lotrionte, Information Warfare as International Coercion, EJIL 12 (2001), S. 825 (843 f.); K. Ziolkowski, General Principles of International Law as Applicable in Cyberspace, in: dies. (Hg.), Peacetime Regime for State Activities in Cyberspace, 2013, S. 135 (163).

137 Tallinn Manual 2.0, Kommentar zu Regel 4, S. 23, Rn. 19. 
bereits Ausfluss staatlicher Souveränität ${ }^{138}$ und kann durch dessen Voraussetzungen das Eskalationspotenzial durch mögliche Gegenmaßnahmen auf solche Informationsoperationen eingrenzen. ${ }^{139}$ Zudem würde ein zu weites Verständnis den Staaten unrealistische positive Handlungspflichten zur Verhinderung von nicht-staatlichen Souveränitätsverletzungen aufbürden ${ }^{140}$ und die Regulierung von Informationsoperationen behindern. ${ }^{141}$

\section{Informationsintervention in der internationalen Praxis}

Die Schwierigkeiten einer Abgrenzung von völkerrechtswidriger Informationsintervention und völkerrechtlich nicht verbotener Informationseinmischung werden am dargestellten Zugriff auf die Server des DNC deutlich. Die Informationsoperation betraf nationale politische Prozesse und damit die Ausübung souveräner Rechte, die den jeweiligen Staaten vorbehalten sind. Die Einmischung in den freien Wahlprozess unterläuft das nationale System demokratischer Willensbildung und schadet damit dem Staat und seinem Volk. ${ }^{142}$ Nach einem weiten Verständnis von Zwang

138 J. Crawford, Brownlie's Principles of Public International Law, 2019, S.431; P. Kunig, Prohibition of Intervention, in: R. Wolfrum (Hg.), MPEPIL 2008, http://www.mpepil.com, Rn. 9.

139 Vgl. R. Crootof, International Cybertorts, CLR 103 (2018), S. 565 (630 f.); B. A. Walton, Duties Owed, Yale LJ 126 (2017), S. 1460 (1477).

140 Vgl. E. T. Jensen/S. Watts, A Cyber Duty of Due Diligence, Tex. LR 95 (2017), S. 1555 (1568 ff.).

141 W. Banks gibt gegen die Anerkennung einer derartigen Souveränitätsregel zu bedenken, dass damit von transnationalen terroristischen Informationsangriffen betroffenen Staaten die Möglichkeit genommen wird, völkerrechtmäßig in die informationstechnischen Systeme des Ursprungsstaates einzugreifen, um diese Angriffe zu stoppen. W. Banks, State Responsibility and Attribution of Cyber Intrusions After Tallinn 2.0, Tex. LR 95 (2017), S. 1487 (1512 f.). Nach O. A. Hathaway würde ein zu weites Verständnis staatliche Unterstützung für humanitäre Hilfsmaßnahmen negativ beeinflussen. O. A. Hathaway, The Drawbacks and Dangers of Active Defense, in: P. Brangetto/M. Maybaum/J. Stinissen, 2014 6th International Conference on Cyber Conflict, 2014, S. 39 (49). B. A. Walton weist daraufhin, dass in der Staatengemeinschaft keine Einigkeit über das Verständnis von Territorialität und Souveränität in der virtuellen Welt besteht, so dass diese keine geeigneten Anknüpfungspunkte für eine international anerkannte Verbotsnorm im Cyberkontext bieten. B. A. Walton, Duties Owed, Yale LJ 126 (2017), S. 1460 (1475).

142 J. D. Jolley, Attribution, State Responsibility, and the Duty to Prevent Malicious Cyber-Attacks in International Law, 2017, abrufbar unter: http://theses.gla.ac.uk /id/eprint/8452 (geprüft am 15.05.2020), S. 177. 
bzw. bei Verzicht auf das Zwangselement, wäre der Informationsangriff als völkerrechtswidrige Intervention in den domaine réservé der USA zu werten. ${ }^{143}$ Die Tatsache, dass die US-Regierung auf den Informationsangriff lediglich mit unfreundlichen Akten, sogenannten Retorsionen, und nicht mit Gegenmaßnahmen reagierte, ${ }^{144}$ lässt den Schluss zu, dass sie selbst darin eben keine völkerrechtswidrige Intervention sah. ${ }^{145}$ Ein extensiver Ansatz ist demnach nicht nur in der Wissenschaft, sondern ebenso in der internationalen Praxis umstritten. Der Informationsangriff könnte allenfalls dann als völkerrechtswidrige Intervention qualifiziert werden, wenn die Sicherheitslücken in elektronischen Abstimmungsgeräten genutzt worden wären, um Wahlergebnisse zu verfälschen, da derartige Maßnahmen mit subversiver umsturzorientierter Propaganda vergleichbar sind. ${ }^{146}$ Der zwangsfreie Zugriff auf die informationstechnischen Systeme des DNC und die Verbreitung privater Informationen hingegen bewegt sich in einer Grauzone des internationalen Rechts. ${ }^{147}$

Anders ist jedoch der Informationsangriff auf informationstechnische Systeme Estlands zu bewerten. Die Entscheidung der estnischen Regierung über die Standortverlagerung der Statue eines Soldaten der Roten Armee stand in einem politischen Kontext, da sie als ideologische Annäherung

143 D. B. Hollis, Russia and the DNC Hack: What Future for a Duty of Non-Intervention?, OpinoJuris, 25 July 2016, abrufbar unter: http://opiniojuris.org/201 6/07/25/russia-and-the-dnc-hack-a-violation-of-the-duty-of-non-intervention/ (geprüft am 15.05.2020); J. D. Jolley, Attribution, State Responsibility, and the Duty to Prevent Malicious Cyber-Attacks in International Law, 2017, abrufbar unter: http://theses.gla.ac.uk/id/eprint/8452 (geprüft am 15.05.2020), S. 177.

144 D. Roberts, Obama imposes new sanctions against North Korea in response to Sony Hack, The Guardian, 2 January 2015, abrufbar unter: http://www.theguard ian.com/us-news/2015/jan/02/obama-imposes-sanctions-north-korea-sony-hack-t he-interview (geprüft am 15.05.2020).

145 W. Banks, State Responsibility and Attribution of Cyber Intrusions After Tallinn 2.0, Tex. LR 95 (2017), S. 1487 (1512); D. Efrony/Y. Shany, A Rule Book on the Shelf?, AJIL 112 (2018), S. 583 (643).

146 Tallinn Manual 2.0, Kommentar zu Regel 66, S. 313, Rn. 2; B. J. Egan, International Law and Stability in Cyberspace, BJIL 35 (2017), S. 169 (175).

147 M. N. Schmitt stellt in diesem Zusammenhang zutreffend fest: „By acting within legal grey zones, Russia makes it difficult for other States to definitively name and shame the country as having committed an internationally wrongful act." M. N. Schmitt, Grey Zones in the International Law of Cyberspace, YIL Online 42 (2017), abrufbar unter: https://cpb-us-w2.wpmucdn.com/campuspress.yale .edu/dist/8/1581/files/2017/08/Schmitt_Grey-Areas-in-the-International-Law -of-Cyberspace-1cab8kj.pdf (geprüft am 15.05.2020), S. 1 (2); W. Banks, State Responsibility and Attribution of Cyber Intrusions After Tallinn 2.0, Tex. LR 95 (2017), S. 1487 (1501). 
Estlands an den Westen und die NATO verstanden wird. ${ }^{148}$ Überdies steht es jedem Staat grundsätzlich frei, den Standort bedeutender Statuen zu bestimmen. Die Entscheidung über die Verlegung fällt mithin in den domaine réservé Estlands. ${ }^{149}$ Während der Informationsangriff nicht die Voraussetzungen erfüllt, um Gewalt darzustellen, sprechen mehrere Aspekte dafür, dass er willensbeugende Wirkung entfalten sollte und daher als unzulässige Ausübung von Zwang zu qualifizieren ist. Die weitereichende Störung der informationstechnischen Systeme diente nämlich als Druckmittel, um die estnische Regierung zur Rücknahme ihrer Entscheidung zu bewegen. ${ }^{150}$

Unter dem Blickwinkel politischer Einflussnahme dürften auch die weitreichenden Angriffe gegen informationstechnische Systeme in Georgien erfolgt sein. Südossetien war und blieb integraler Bestandteil Georgiens und betraf damit innere Angelegenheiten des Staates. ${ }^{151}$ Der Informationsangriff unterstützte mit seinen einschneidenden Auswirkungen südossetische Sezessionsbestrebungen und verhinderte die politische Interaktion zwischen Regierung und Bürgern sowie Alliierten. Demnach kann der Informationsangriff durchaus als unzulässige Ausübung von Zwang auf den domaine réservé Georgiens gewertet werden. ${ }^{152}$

Der Informationsangriff durch das Schadprogramm Stuxnet auf informationstechnische Systeme iranischer Atomanlagen, ist - wie gezeigt als Gewalt im Sinne des Art. 2 Abs. 4 VN-Charta zu qualifizieren. Gewalt stellt die stärkste Form von unerlaubtem Zwang dar. ${ }^{153}$ In dem Fall wurde

148 Vgl. W. C. Ashmore, Impact of Alleged Russian Cyber Attacks, BSDR 11 (2009), S. 4 (6); S. Herzog, Revisiting the Estonian Cyber Attacks, JSS 4 (2011), S. 50 f.; A. Schmidt, The Estonian Cyber Attacks, in: J. Healey (Hg.), A Fierce Domain, 2013, S. $174 \mathrm{ff}$.

149 S.-H. Schulze, Cyber-„War“ - Testfall der Staatenverantwortlichkeit, 2015, S. $123 \mathrm{f}$.

150 R. Buchan, Russell, Cyber Attacks, JCSL 17 (2012), S. 212 (225 f.); J. D. Jolley, Attribution, State Responsibility, and the Duty to Prevent Malicious Cyber-Attacks in International Law, 2017, abrufbar unter: http://theses.gla.ac.uk/id/eprin t/8452 (geprüft am 15.05.2020), S. 126; S.-H. Schulze, Cyber-„,War“ - Testfall der Staatenverantwortlichkeit, 2015, S. $124 \mathrm{f}$.

151 S.-H. Schulze, Cyber-,War“ - Testfall der Staatenverantwortlichkeit, 2015, S. $125 \mathrm{f}$.

152 J. D. Jolley, Attribution, State Responsibility, and the Duty to Prevent Malicious Cyber-Attacks in International Law, 2017, abrufbar unter: http://theses.gla.ac.u k/id/eprint/8452 (geprüft am 15.05.2020), S. 126; S.-H. Schulze, Cyber-,War“ Testfall der Staatenverantwortlichkeit, 2015, S. 126.

153 S.-H. Schulze, Cyber-,,War“ - Testfall der Staatenverantwortlichkeit, 2015, S. 127. 
Zwang ausgeübt, um die Urananreicherung des Iran zu beeinträchtigen, so dass zugleich der domaine réservé des Staates betroffen war. ${ }^{154}$

\section{Informationsspionage}

In aller Regel steht Spionage in nationalen Rechtsordnungen unter Strafe. ${ }^{155}$ Dies hindert Staaten aber nicht daran, ausländische Regierungen, Wirtschaftsunternehmen und Privatpersonen auszuspähen. Daher stellt sich die Frage, ob Spionage auf internationaler Ebene einem Verbot unterliegt und mithin die Völkerrechtswidrigkeit von Informationsoperationen begründen kann.

\section{Völkerrechtliche Verbote zur Informationsspionage}

Zunächst können die Methoden der Informationsgewinnung gegen das Gewaltverbot oder Interventionsverbot verstoßen, ohne dass die Informationsausbeutung als solche völkerrechtswidrig ist. ${ }^{156}$

Des Weiteren existieren für spezifische völkerrechtliche Regelungsbereiche, wie das Diplomaten- und Konsularrecht, das Seerecht oder das Recht internationaler bewaffneter Konflikte, Normen, die sich mit Spionage auseinandersetzen und begrenzt Schutz vor entsprechenden Handlungen bieten. ${ }^{157}$

Auch im Wirtschaftsvölkerrecht gibt es völkervertragliche Bestimmungen, die Rechte am geistigen Eigentum und Urheberrechte unter den Schutz der Vertragsstaaten innerhalb ihres Hoheitsgebietes stellen und

154 Daran ändern auch der Atomwaffensperrvertrag, das geltende Inspektionsregime und die einschlägigen Resolutionen des Sicherheitsrates nichts. Es wird indes diskutiert, ob es sich bei Stuxnet um eine völkerrechtlich legale Gegenmaßnahme in Reaktion auf das völkerrechtswidrige Verhalten Irans im Atomstreit gehandelt haben könnte. Siehe S.-H. Schulze, Cyber-„War“ - Testfall der Staatenverantwortlichkeit, 2015, S. $127 \mathrm{f}$.

155 Es ist zu beachten, dass sich aus der weitverbreiteten Kriminalisierung von Spionage in nationalen Rechtsordnungen kein allgemeiner Rechtsgrundsatz herleiten lässt. Siehe hierzu K. Ziolkowski, Peacetime Cyber Espionage, in: dies. (Hg.), Peacetime Regime for State Activities in Cyberspace, 2013, S. 425 (431 f.).

156 Tallinn Manual 2.0 Kommentar zu Regel, S. 170 f., Rn. 8.

157 C. Schaller, Internationale Sicherheit und Völkerrecht im Cyberspace, SWP-Studie 18/2014, S. 11, Fn. 19 ff.; K. Ziolkowski, Peacetime Cyber Espionage, in: dies. (Hg.), Peacetime Regime for State Activities in Cyberspace, 2013, S. 443 ff. 
somit Informationsausbeutung bis zu einem gewissen Grad beschränken. Diese Bestimmungen gehen aber nicht so weit, Wirtschaftsspionage durch fremde Staaten auf internationaler Ebene zu verbieten. ${ }^{158}$ Die Mitgliedstaaten der WTO tolerieren fremdstaatliche Wirtschaftsspionage sogar vermutlich, um sich selbst diese Möglichkeit zu erhalten. ${ }^{159}$

Schließlich sind menschenrechtliche Normen bei der Ausspähung von Individuen zu berücksichtigen. ${ }^{160}$ Der Schutz der Privatsphäre und Vertraulichkeit der Kommunikation ist in internationalen und regionalen Menschenrechtsinstrumenten verankert. ${ }^{161}$ Staaten, die Personen auf dem eigenen Hoheitsgebiet überwachen, oder auf informationstechnische Systeme zugreifen, die sich auf eigenem staatlichen Territorium befinden, können diese Rechte verletzen. ${ }^{162}$ Dabei ist zu beachten, dass die Bestimmungen kein allgemeines Spionageverbot begründen. Eingriffe in die Rechte auf Privatsphäre und Vertraulichkeit der Kommunikation können nämlich durch nationale Vorschriften zum Schutz der öffentlichen Sicherheit erlaubt sein. ${ }^{163}$ Der menschenrechtliche Schutz der Privatheit und Vertraulichkeit der Kommunikation bezieht sich zudem nur auf Individuen auf eigenem staatlichen Territorium und unter eigener staatlicher Hoheitsgewalt. Angehörige anderer Staaten im Ausland oder Daten, die auf Informationsinfrastrukturen in fremdem Hoheitsgebiet gespeichert sind oder diese durchlaufen, sind grundsätzlich nicht vom Schutzbereich erfasst. ${ }^{164}$ Allerdings gibt es Bestrebungen, den Schutz auf extraterritoriale

158 K. Ziolkowski, Peacetime Cyber Espionage, in: dies. (Hg.), Peacetime Regime for State Activities in Cyberspace, 2013, S. 435 f.

159 Id., S. 436.

160 D. Korff, First Do No Harm, in: B. Wagner/M. C. Kettemann/K. Vieth (Hg.), Research Handbook on Human Rights and Digital Technology, 2019, S. 129 ff.; I. Pernice, Vom Völkerrecht des Netzes zur Verfassung des Internets, HIIG Discussion Paper Series, Discussion Paper No. 2017-02, abrufbar unter: https:// papers.ssrn.com/sol3/papers.cfm?abstract_id=2959257 (geprüft am 15.05.2020), S. 1 (6ff.).

161 Siehe K. Ziolkowski, Peacetime Cyber Espionage, in: dies. (Hg.), Peacetime Regime for State Activities in Cyberspace, 2013, S. 43.

162 Siehe C. Schaller, Internationale Sicherheit und Völkerrecht im Cyberspace, SWP-Studie 18/2014, S. 13.

163 K. Ziolkowski, Peacetime Cyber Espionage, in: dies. (Hg.), Peacetime Regime for State Activities in Cyberspace, 2013, S. 435, Fn. 51.

164 C. Forcese, Spies Without Borders, JNSLP 5 (2011), S. 179 (208); R. D. Williams, (Spy) Game Change, GWLR 79 (2011), S. 1162 (1176ff.); K. Ziolkowski, Peacetime Cyber Espionage, in: dies. (Hg.), Peacetime Regime for State Activities in Cyberspace, 2013, S. 434. 


\section{Kapitel: Staatenverantwortlichkeit für informationstechnische Systeme}

Informationsausbeutung auszuweiten. ${ }^{165}$ Die Speicherung und Weiterverarbeitung von Daten auf staatseigenem Territorium ${ }^{166}$ oder die virtuelle Kontrolle durch einen Staat ${ }^{167}$ etwa werden als Anknüpfungspunkte für die menschenrechtlichen Schutzpflichten vorgeschlagen. Hoheitsgewalt im Sinne der Konventionen zum Menschenrechtsschutz unterliegt allerdings einem physischen Begriffsverständnis. Sie kann grundsätzlich nicht durch außerhalb des eigenen Staatsgebiets stattfindende digitale Überwachungsmaßnahmen ausgeübt werden. ${ }^{168}$ Zudem sind die juristischen Differenzen in der Staatengemeinschaft zum extraterritorialen Schutz der Menschenrechte derzeit zu groß, um extraterritoriale Informationsausbeutung völkerrechtlich zu ahnden. ${ }^{169}$

Zusammenfassend lässt sich festhalten, dass keine allgemeingültige Völkerrechtsnorm existiert, die Spionage per se verbietet. ${ }^{170}$ Die durchdringenden und schädigenden Dimensionen der Ausbeutung informationstechnischer Systeme, die nicht mit denen der traditionellen Spionage vergleichbar sind, bieten aber Anlass zur Diskussion über ein neues völkergewohnheitsrechtliches Verbot der Informationsspionage. ${ }^{171}$ Ein solches Verbot lässt sich jedoch nicht durch entsprechende Staatenpraxis und opinio iuris belegen. Insgesamt ist das Interesse an entsprechenden völkerrechtlichen

$165 \mathrm{Ob}$ sich der Interpretationsansatz des IACtHR, wonach Staaten es anderen Staaten nicht erschweren dürfen, den Menschenrechtsschutz im staatseigenen Hoheitsgebiet zu gewährleisten (siehe 1. Kapitel B. II. 3.), insbesondere auch außerhalb umweltrechtlicher Problemkreise durchsetzt, bleibt zu zeigen.

166 H. P. Aust, Stellungnahme zur Sachverständigenanhörung am 5. Juni 2014 im 1. Untersuchungsausschuss der 18. Wahlperiode des Deutschen Bundestages, abrufbar unter: https://www.bundestag.de/resource/blob/282870/fc52462f2ffd2 54849bce19d25f72fa2/mat_a_sv-4-1_aust-pdf-data.pdf (geprüft am 15.05.2020), S. 13 f., Rn. 35 .

167 A. Peters, Surveillance without Borders, EJIL: Talk! 4 November 2013, abrufbar unter: www.ejil talk.org/surveillance-without-borders-the-unlawfulness-ofthe-nsa-panopticon-part-ii/ (geprüft am 15.05.2020).

168 Tallinn Manual 2.0, Kommentar zu Regel 34, S. 185, Rn. 9; C. Schaller, Internationale Sicherheit und Völkerrecht im Cyberspace, SWP-Studie 18/2014, S. 13 f.

169 C. Schaller, Internationale Sicherheit und Völkerrecht im Cyberspace, SWP-Studie 18/2014, S. 14.

170 Das Völkerrecht schweigt zu der Frage der Recht- oder Unrechtmäßigkeit von Spionage zu Friedenszeiten. H. P. Aust, Stellungnahme zur Sachverständigenanhörung am 5. Juni 2014 im 1. Untersuchungsausschuss der 18. Wahlperiode des Deutschen Bundestages, abrufbar unter: https://www.bundestag.de/resource/bl ob/282870/fc52462f2ffd254849bce19d25f72fa2/mat_a_sv-4-1_aust-pdf-data.pdf (geprüft am 15.05.2020), S. 14, Rn. 39.

171 K. Ziolkowski, Peacetime Cyber Espionage, in: dies. (Hg.), Peacetime Regime for State Activities in Cyberspace, 2013, S. $446 \mathrm{ff}$. 
Reglementierungen in der Staatengemeinschaft gering. ${ }^{172}$ Das fehlende Verbot der Informationsspionage bietet Staaten vielmehr Anreiz, ihre Kapazitäten zur Informationsausbeutung weiterzuentwickeln. Zugleich wird das Misstrauen zwischen den Staaten stärker. Dies führt wiederum zu einer verminderten internationalen Kooperationsbereitschaft zur Bekämpfung und Reglementierung von Informationsoperationen, die die internationale Sicherheit bedrohen. ${ }^{173}$

2. Informationsspionage in der internationalen Praxis

Der Informationsangriff auf die informationstechnischen Systeme des DNC unterliegt keinem völkerrechtlichen Verbot. Auch die Informationsausbeutung im Rahmen dieser Informationsoperation ist als völkerrechtlich nicht verbotene Spionage einzustufen, auf die Opferstaaten nur begrenzt mit Mitteln der Selbsthilfe reagieren können. ${ }^{174}$

Duqu und Flame demonstrieren, wie informationstechnische Systeme in großem Stil zur grundsätzlich erlaubten Militär- oder Wirtschaftsspionage genutzt werden. ${ }^{175}$ Die Ausspähung durch die Five Eyes zeigt, wie es in der virtuellen Welt zur legalen Aushöhlung des Menschenrechtsschutzes kommt. ${ }^{176}$

Die Informationsausbeutung durch die Guardians of Peace veranschaulicht zum einen die immensen wirtschaftlichen Schäden, die durch Informationsausbeutung verursacht werden können. ${ }^{177}$ Zum anderen zeigt die Reaktion der USA in diesem Fall, dass auch völkerrechtlich nicht verbotene Informationsoperationen zwischenstaatliche Spannungen begründen. Ohne benennen zu können, inwiefern die Informationsoperation gegen Völkerrecht verstößt, machte die US-Regierung Nordkorea für den „cyber-

172 Tallinn Manual 2.0, Kommentar zu Regel 32, S. 169, Rn. 5; vgl. K. Ziolkowski, Peacetime Cyber Espionage, in: dies. (Hg.), Peacetime Regime for State Activities in Cyberspace, 2013, S. $437 \mathrm{ff}$.

173 D. P. Fidler, Tinker, Tailor, Soldier, Duqu, IJCIP 5 (2012), S. 28 (29).

174 Vgl. R. Crootof, International Cybertorts, CLR 103 (2018), S. 565 (570, 597 f.); M. N. Schmitt, Grey Zones in the International Law of Cyberspace, YIL Online 42 (2017), abrufbar unter: https://cpb-us-w2.wpmucdn.com/campuspress.yale.ed u/dist/8/1581/files/2017/08/Schmitt_Grey-Areas-in-the-International-Law-of-Cyb erspace-1cab8kj.pdf (geprüft am 15.05.2020), S. $1(2,8)$.

175 D. P. Fidler, Tinker, Tailor, Soldier, Duqu, 5 (2012), S. 28 (28 f.).

176 Vgl. M. N. Schmitt/L. Vibul, The Nature of International Law Cyber Norms, in: A.-M. Osula/H. Rõigas, International Cyber Norms, 2016, S. 23 (44).

177 R. Crootof, International Cybertorts, CLR 103 (2018), S. 565 (588, 592). 
vandalism" 178 durch die Guardians of Peace verantwortlich und belegte den Staat mit Sanktionen. ${ }^{179}$

\section{Zurechnung}

Staaten sind für völkerrechtswidrige Informationsoperationen verantwortlich, wenn diese ihnen zurechenbar sind.

\section{Zurechnungsregeln}

In Ermangelung spezifischer Zurechnungsregeln für die virtuelle Welt haben die für die Staatenverantwortlichkeit etablierten Zurechnungsmechanismen zu gelten. ${ }^{180}$ Dementsprechend beleuchtet auch das Tallinn Manual 2.0 deren Anwendbarkeit auf Informationsoperationen. ${ }^{181}$

Eine Zurechnung erfolgt zunächst bei Informationsoperationen, die durch staatliche Organe in amtlicher Eigenschaft ausgeführt werden (Regel 15 Alt. 1 Tallinn Manual 2.0). Zu diesen Organen zählen unter anderem das Militär, Geheimdienste, aber auch staatliche Cyber-Sicherheitszentren, Computersicherheitsereignis- und Reaktionsteams und Cyber-Einheiten, die auf Informationsoperationen spezialisiert sind und zunehmend von Staaten errichtet werden, um die Informationstechnik zum staatlichen Vorteil zu nutzen. ${ }^{182} \mathrm{Um}$ das Verhalten von Privaten mit dem von staat-

178 E. Bradner, Obama: North Korea's Hack not war, but 'cybervandalism', CNN, 24 December 2014, abrufbar unter: http://www.cnn.com/2014/12/21/politics/ob ama-north-koreas-hack-not-war-but-cyber-vandalism (geprüft am 15.05.2020).

179 Die Reaktion der US-Regierung steht exemplarisch für die wachsende Zahl an Konstellationen, in denen Staaten andere Staaten für Informationsoperationen verantwortlich machen, ohne aber genau darlegen zu können, welche völkerrechtliche Norm verletzt wurde. B. A. Walton, Duties Owed, Yale LJ 126 (2017), S. 1460 (1463).

180 J. D. Jolley, Attribution, State Responsibility, and the Duty to Prevent Malicious Cyber-Attacks in International Law, 2017, abrufbar unter: http://theses.gla.ac.u k/id/eprint/8452 (geprüft am 15.05.2020), S. 89 ff.; S.-H. Schulze, Cyber-,War“Testfall der Staatenverantwortlichkeit, 2015, S. $129 \mathrm{ff}$.

181 Tallinn Manual 2.0, Teil I, Kapitel 4, Abschnitt 1, S. 79 ff.

182 J. D. Jolley, Attribution, State Responsibility, and the Duty to Prevent Malicious Cyber-Attacks in International Law, 2017, abrufbar unter: http://theses.gla.ac. uk/id/eprint/8452 (geprüft am 15.05.2020), S. 95; S.-H. Schulze, Cyber-„War“ Testfall der Staatenverantwortlichkeit, 2015, S. $129 \mathrm{f}$. 
lichen Organen geleichstellen zu können, wird eine komplette Abhängigkeit und Kontrolle der privaten Akteure vom und durch den jeweiligen Staat gefordert. ${ }^{183}$ Eine Zurechnung in diesem Sinne kann schon in der materialen Welt kaum festgestellt werden und ist in der virtuellen Welt umso schwieriger darzulegen. ${ }^{184}$

Eine Zurechnung ist ferner möglich, wenn private Personen oder Einrichtungen zur Ausübung hoheitlicher Befugnisse ermächtigt sind (Regel 15 Alt. 2 Tallinn Manual 2.0 $)^{185}$ oder in Ausübung eines konkreten staatlichen Auftrages handeln (Regel 17 lit. a Alt. 1 Tallinn Manual 2.0) ${ }^{186}$. So können private Unternehmen beispielsweise durch Rechtsakte zur defensiven und offensiven Verteidigung staatlicher Informationsinfrastrukturen ermächtigt sein ${ }^{187}$ oder sonst mit der Ausführung von bestimmten Informationsoperationen betraut sein. ${ }^{188}$ Sofern diese Akteure im Rahmen der übertragenen Befugnisse und Aufgaben völkerrechtswidrig agieren, entsteht eine Verantwortlichkeit des jeweiligen Staates. ${ }^{189}$ Der geheime Charakter von Informationsoperationen verhindert in aller Regel, dass eine derartige Verbindung offengelegt werden kann. ${ }^{190}$

Daneben können Informationsoperationen durch staatliche Organe, etwa von Geheimdiensten, die einem anderen Staat zur Verfügung gestellt

183 IGH, Application of the Convention on the Prevention and Punishment of the Crime of Genocide (Bosnia and Herzegovina v. Serbia and Montenegro), Judgment of 26 February 2007, ICJ Reports 2007, S. 43 (205 Rn. 393); siehe auch Tallinn Manual 2.0, Kommentar zu Regel 15, S. 88, Rn.4; J. Maddocks, Outsourcing of Governmental Functions in Contemporary Conflict, Va JIL 59 (2019), S. 47 (57).

184 J. D. Jolley, Attribution, State Responsibility, and the Duty to Prevent Malicious Cyber-Attacks in International Law, 2017, abrufbar unter: http://theses.gla.ac.uk /id/eprint/8452 (geprüft am 15.05.2020), S. 93.

185 Zur Zurechnung nach Art. 5 ASR siehe J. Maddocks, Outsourcing of Governmental Functions in Contemporary Conflict, Va JIL 59 (2019), S. 47 (47 ff.).

186 Zur Zurechnung nach Art. 8, 1. Alt ASR siehe A. Epiney, Zur Rechtsfigur des de facto-Organs im Recht der Staatenverantwortlichkeit, in: A. Fischer-Lescano/ H.-P. Gasser/T. Marauhn/N. Ronzitti (Hg.), Frieden in Freiheit: Festschrift für Michael Bothe, 2008, S. 883 (885 ff.).

187 Tallinn Manual 2.0, Kommentar zu Regel 15, S. 89, Rn. 8.

188 Tallinn Manual 2.0, Kommentar zu Regel 17, S. 95 f., Rn. 4.

189 Eine Zurechnung im Sinne des Art. 15 Tallinn Manual 2.0 erfolgt auch bei ultra vires Handlungen. Tallinn Manual 2.0, S. 90 f., Rn. 12.

190 J. D. Jolley, Attribution, State Responsibility, and the Duty to Prevent Malicious Cyber-Attacks in International Law, 2017, abrufbar unter: http://theses.gla.ac .uk/id/eprint/8452 (geprüft am 15.05.2020), S.94; J. Maddocks, Outsourcing of Governmental Functions in Contemporary Conflict, Va JIL 59 (2019), S. 47 (83). 
werden, die Verantwortlichkeit letzteren Staates begründen (Regel 16 Tallinn Manual 2.0). Auch die Hilfe oder Unterstützung, Leitung oder Kontrolle eines anderen Staates bei oder die Nötigung eines anderen Staates zur Begehung einer völkerrechtswidrigen Informationsoperation können einen verantwortungsbegründenden Zusammenhang herstellen (Regel 18 Tallinn Manual 2.0). Eine Verantwortlichkeit für Informationsoperationen, bei denen mehrere Staaten zu einem bestimmten Grad involviert sind, ist aber überaus schwierig zu begründen. Es lässt sich beispielsweise schon kaum nachweisen, ob ein Geheimdienst Informationen für einen fremden Staat sammelt, um dessen völkerrechtswidrige Informationsoperation zu ermöglichen. ${ }^{191}$

Schließlich ist die Zurechnung aufgrund staatlicher Kontrolle oder Leitung von besonderem Interesse (Regel 17 lit. a Alt. 2 Tallinn Manual 2.0), ${ }^{192}$ denn Informationsoperationen erfolgen regelmäßig durch Akteure, die zwar in keiner Weise in den Staatsapparat eingegliedert sind, aber gleichzeitig in gewisser Verbindung zum Staat stehen. ${ }^{193}$ In vielen Konstellationen finden sich Anzeichen, die auf eine staatliche Förderung der nicht-staatlichen Akteure hinweisen. ${ }^{194}$ Zudem agieren sogenannte patriotische Hacker (patriotic hacktivists) oder staatliche Stellvertreter (proxies) oftmals im Einklang mit der politischen Agenda eines Staates. ${ }^{195}$ Hier führen die unbestimmten Voraussetzungen für eine Zurechnung aufgrund staatli-

191 Vgl. J. D. Jolley, Attribution, State Responsibility, and the Duty to Prevent Malicious Cyber-Attacks in International Law, 2017, abrufbar unter: http://these s.gla.ac.uk/id/eprint/8452 (geprüft am 15.05.2020), S. 96.

192 Der Völkerrechtskommission zufolge sind die Elemente der Leitung und der Kontrolle zwar separate Fallgruppen, sie werden in der Rechtsprechung aber regelmäßig zusammen besprochen. Tallinn Manual 2.0, Kommentar zu Regel 17, S. 96, Rn. 5.

193 J. Maddocks, Outsourcing of Governmental Functions in Contemporary Conflict, Va JIL 59 (2019), S. 47 (82 f.).

194 Vgl. D. Garrie/S. R. Reeves, So You're Telling Me There's A Chance, Harv. NSJ Online Features, 17 December 2015, abrufbar unter: https://harvardnsj.org/ wp-content/uploads/sites/13/2016/01/Garrie-and-Reeves-Non-State-Actor-a nd-Self-Defense.pdf, (geprüft am 15.05.2020), S. 1 ff.; M. Kahn, FBI Director Christopher Wray's Remarks on Encryption to the International Conference on Cyber Security, LAWFARE 9 January 2018, abrufbar unter: https:/www.lawfare blog.com/fbi-director-christopher-wrays-remarks-encryption-international-confe rence-cyber-security (geprüft am 15.05.2020).

195 M. Gervais, Cyber Attacks and the Laws of War, BJIL 30 (2012), S. 525 (546); J. D. Jolley, Attribution, State Responsibility, and the Duty to Prevent Malicious Cyber-Attacks in International Law, 2017, abrufbar unter: http://theses.gla.ac.uk /id/eprint/8452 (geprüft am 15.05.2020), S. 27, 84, 110. 
cher Kontrolle oder Leitung in der virtuellen Welt zu Unsicherheiten. ${ }^{196}$ Das für eine Zurechnung erforderliche $\mathrm{Ma}$ an staatlicher Einflussnahme auf Private erfordert nach der Rechtsprechung des IGH eine sogenannte effektive Kontrolle. ${ }^{197}$ Demnach muss der Staat jederzeit über Ziel, Durchführungsmethode und Beendigung des fraglichen privaten Verhaltens bestimmen können. ${ }^{198}$ Diese auf kinetische Operationen zugeschnittenen Anforderungen werden mit Blick auf die anonyme und ubiquitäre virtuelle Welt als zu eng gewertet. ${ }^{199}$ So gibt es Tendenzen zur Herabsetzung der Zurechnungsschwelle. Unter Bezugnahme auf die Rechtsprechung des Internationalen Strafgerichtshofs für das ehemalige Jugoslawien (IStGHJ) soll eine sogenannte staatliche Gesamtkontrolle über die Handlungen einer nicht-staatlichen Gruppe ausreichen. ${ }^{200}$ Während der strenge Maßstab des IGH also die effektive Kontrolle über die konkrete Verletzungshandlung voraussetzt, genügt nach dem weniger strengen Maßstab des IStGHJ die allgemeine Kontrolle über das Tätigkeitsfeld der privaten Akteure, um einen zurechnungsrelevanten Zusammenhang zu begründen. Doch auch die Gesamtkontrolle erfordert staatliche Planung und Überwachung der fraglichen Operation. ${ }^{201}$ Dementsprechend reicht das Ausrüsten mit Schadsoftware oder das Finanzieren der privaten Akteure nach keinem

196 Zur Zurechnung nach Art. 8, 2. Alt ASR siehe A. Epiney, Zur Rechtsfigur des de facto-Organs im Recht der Staatenverantwortlichkeit, in: A. Fischer-Lescano/ H.-P. Gasser/T. Marauhn/N. Ronzitti (Hg.), Frieden in Freiheit: Festschrift für Michael Bothe, 2008, S. 883 (888 ff.).

197 IGH, Military and Paramilitary Activities in and against Nicaragua, (Nicaragua v. United States of America), Merits, Judgment of 27 June 1986, ICJ Reports 1986, S. 14 (64 f. Rn. 115).

198 A. Epiney, Zur Rechtsfigur des de facto-Organs im Recht der Staatenverantwortlichkeit, in: A. Fischer-Lescano/H.-P. Gasser/T. Marauhn/N. Ronzitti (Hg.), Frieden in Freiheit: Festschrift für Michael Bothe, 2008, S. 883 (890).

199 S.-H. Schulze, Cyber-,War“ - Testfall der Staatenverantwortlichkeit, 2015, S. $131 \mathrm{f}$.

200 IStGHJ, Appeals Chamber, Prosecutor v. Duško Tadić, Judgment of 15 July 1999, No. IT-94-1-A, ILM 38 (1999), S. 1518 (1541 Rn. 120, 1544 Rn. 131, 1544f. Rn. 137, 1546 Rn. 145). Es finden sich Stimmen, die schon die bloße Unterstützung und Beherbergung von nicht-staatlichen Akteuren ausreichen lassen wollen, um einen zurechnungsrelevanten Zusammenhang zu begründen. Siehe hierzu D. Jinks, State Responsibility for the Acts of Private Armed Groups, Chi. JIL 4 (2003), S. 83 (83 ff.).

201 IStGHJ, Appeals Chamber, Prosecutor v. Duško Tadić, Judgment of 15 July 1999, No. IT-94-1-A, ILM 38 (1999), S. 1518 (1541 Rn. 120); A. Cassese, The Nicaragua and Tadić Tests Revisited in Light of the ICJ Judgment on Genocide in Bosnia, EJIL 18 (2007), S. 649 (655 ff.). 
der beiden Zurechnungsstandards aus, um Staaten eine nicht-staatliche Informationsoperation zuzurechnen. ${ }^{202}$ Genauso wenig liegt eine staatliche Kontrolle vor, wenn private Hacker ungefragt Informationsoperationen zur Unterstützung staatlicher Politik ausführen. ${ }^{203}$ Eine Verantwortlichkeit kann in diesem Zusammenhang allenfalls durch die staatliche Anerkenntnis und Annahme eines nicht-staatlichen Verhaltens angenommen werden (Regel 17 lit. b Tallinn Manual 2.0). Dabei reicht das bloße Befürworten von patriotischen Informationsoperationen allein nicht aus. Der Staat muss diese vielmehr für sich vereinnahmen, indem er beispielsweise die nicht-staatlichen Akteure vor gegnerischen Informationsoperationen schützt und damit die Fortführung der patriotischen Informationsoperation ermöglicht. ${ }^{204}$

Der IGH verdeutlicht indes durch seine Rechtsprechung, dass der allgemeine Grundsatz, nach dem Staaten nur für Handlungen ihrer eigenen Organe verantwortlich sind, nicht zu sehr ausgedehnt werden soll. ${ }^{205} \mathrm{Ge}$ gen eine Lockerung der Zurechnungsmaßstäbe im Kontext von Informationsoperationen spricht zudem die Gefahr, dass Staaten vorschnell zu Unrecht für völkerrechtswidrige Handlungen verantwortlich gemacht werden könnten. Denn die Möglichkeit, den wahren Urheber eines Angriffs zu verschleiern oder gar falsche Spuren zu legen, ist für Informationsoperationen kennzeichnend. ${ }^{206}$ Durch die irrtümliche Ausübung von Gegenmaßnahmen können dann zwischenstaatliche Spannungen provoziert oder gar unbeteiligte Staaten in einen Konflikt hineingezogen werden. Zu beachten ist überdies, dass die Auseinandersetzungen im virtuellen Raum schnell in militärische Konfrontation umschlagen können. ${ }^{207}$

202 Tallinn Manual 2.0, Kommentar zu Regel 17, S.97, Rn. 8. Eine Verantwortlichkeit kann in dieser Konstellation aber dann entstehen, wenn diese staatliche Unterstützung für sich genommen das Völkerrecht verletzt. Tallinn Manual 2.0, Kommentar zu Regel 17, S. 92, Rn.9.

203 M. N. Schmitt, Grey Zones in the International Law of Cyberspace, YIL Online 42 (2017), abrufbar unter: https://cpb-us-w2.wpmucdn.com/campuspress.yale.ed u/dist/8/1581/files/2017/08/Schmitt_Grey-Areas-in-the-International-Law-of-Cyb erspace-1cab8kj.pdf (geprüft am 15.05.2020), S. 1 (10).

204 Tallinn Manual 2.0, Kommentar zu Regel 17, S. 99, Rn. 16.

205 Vgl. J. Kranz, Die völkerrechtliche Verantwortlichkeit für die Anwendung militärischer Gewalt, AVR 48 (2010), S. 281 (289ff.).

206 Siehe ausführlich hierzu S.-H. Schulze, Cyber-,War“ - Testfall der Staatenverantwortlichkeit, 2015, S. $36 \mathrm{ff} ., 149 \mathrm{ff}$.

207 H. Krieger, Krieg gegen anonymous, AVR 50 (2012), S. 1 (13); C. Schaller, Internationale Sicherheit und Völkerrecht im Cyberspace, SWP-Studie 18/2014, S. 22. 
Schließlich erübrigen sich Diskussionen zu Fragen der Übertragbarkeit der etablierten Zurechnungskriterien und der Herabsetzung der traditionellen Zurechnungsschwelle, wenn schon Ursprung und Urheber der Informationsoperation nicht festgestellt werden können. ${ }^{208}$ Die IT-Forensik ist nicht ausreichend entwickelt, um die vielfältigen Rückverfolgungsbzw. Identifizierungsprobleme zu lösen. ${ }^{209}$ Selbst in Konstellationen, in denen ein Informationsangriff die Informationsinfrastruktur eines Staates durchläuft oder sogar von regierungseigenen informationstechnischen Systemen ausgeht, kann daraus nicht zweifelsfrei auf eine aktive Beteiligung des betreffenden Staates geschlossen werden. Die geografische Herkunft der Informationsoperation oder ein bestimmter Programmierstil können allenfalls Vermutungen begründen. ${ }^{210}$

Selbst wenn eine technische Zurechnung erfolgreich ist, bestehen immer noch die Nachweisschwierigkeiten hinsichtlich der rechtlichen Beziehung zwischen dem Urheber der Informationsoperation und dem beschuldigten Staat. ${ }^{211}$ So lassen sich Zurechnungszusammenhänge in der komplexen virtuellen Welt grundsätzlich nur aus einem Zusammenspiel aus technischen Daten und spezifischen Informationen, etwa über politische Konfliktlagen und staatliche Interessen, konstruieren. ${ }^{212}$ Aufgrund dieser rechtlichen und technischen Hürden im Rahmen der Zurechnung von Informationsoperationen sind die geltenden Beweisanforderungen zu hinterfragen. Im Völkerrecht trifft grundsätzlich den beeinträchtigten Staat die Pflicht, zugrunde liegende Sachverhalte aufzuklären und die

208 S.-H. Schulze weist richtigerweise daraufhin, dass die Frage nach der Zurechnungsschwelle sich nur stellt, wenn der Akteur zweifelsfrei identifiziert ist und der betroffene Staat sich allenfalls Beweisschwierigkeiten - nicht aber Identifizierungs- bzw. Rückverfolgungshürden - ausgesetzt sieht. S.-H. Schulze, Cyber„War" - Testfall der Staatenverantwortlichkeit, 2015, S. 134. J. D. Jolley befasst sich ebenfalls ausführlich mit den technischen Hürden bei der Zurechnung von Informationsoperationen und kommt zu dem Ergebnis, dass die rechtliche $\mathrm{Zu}$ rechnung schon aufgrund der Schwierigkeiten bei der technischen Zurechnung nicht möglich ist. J. D. Jolley, Attribution, State Responsibility, and the Duty to Prevent Malicious Cyber-Attacks in International Law, 2017, abrufbar unter: http://theses.gla.ac.uk/id/eprint/8452 (geprüft am 15.05.2020), Kapitel 4.

209 J. D. Jolley, Attribution, State Responsibility, and the Duty to Prevent Malicious Cyber-Attacks in International Law, 2017, abrufbar unter: http://theses.gla.ac.uk /id/eprint/8452 (geprüft am 15.05.2020), Kapitel 4; S.-H. Schulze, Cyber-„War“ Testfall der Staatenverantwortlichkeit, 2015, S. $36 \mathrm{ff}$.

210 Tallinn Manual 2.0, Kommentar zu Regel 15, S. 91, Rn. 13 f.

211 D. Efrony/Y. Shany, A Rule Book on the Shelf?, AJIL 112 (2018), S. 583 (632 f.).

212 Vgl. Tallinn Manual 2.0, Allgemeiner Kommentar zu Teil I, Kapitel 4, Abschnitt 1, S. 81 f., Rn. 10. 
nötigen Nachweise zu führen. Diese Beweispflicht unterliegt allerdings keinen definierten Standards. ${ }^{213}$ Dem IGH zufolge sind die Beweisanforderungen umso höher, je schwerwiegender die Anschuldigungen und je weitreichender die Konsequenzen sind. ${ }^{214}$ Die Experten des Tallinn Manual 2.0 bestätigen diesen allgemeinen Grundsatz zunächst, da entsprechend der Schwere des vorgeworfenen Völkerrechtsverstoßes auch die Schwere der zulässigen Reaktion wächst. Allerdings weisen die Experten zugleich darauf hin, dass es bei weniger schweren Angriffen einfacher sein dürfte, mehr Beweise für eine Zurechnung zu sammeln, als bei besonders verheerenden Angriffen, die eine sofortige Reaktion zu deren Beendigung erfordern. Demnach soll der Beweisstandard anhand des konkreten Einzelfalles bestimmt werden. ${ }^{215}$ In Rechtsprechung und Literatur findet sich jedenfalls kein einheitliches Bild zu den Beweisanforderungen. ${ }^{216}$ So werden unter anderem ein Nachweis „ohne jeden Zweifel“217, „mit hinreichender Sicherheit“"218 bzw. „ohne begründete Zweifel“219 oder auch eine „klare

213 W. Banks, State Responsibility and Attribution of Cyber Intrusions After Tallinn 2.0, Tex. LR 95 (2017), S. 1487 (1504 f.).

214 IGH, Corfu Channel Case (United Kingdom of Great Britain and Northern Ireland v. Albania), Judgment of 9 April 1949, ICJ Reports 1949, S. 4 (17); IGH, Oil Platforms (Islamic Republic of Iran v. United States of America), Judgment of 6 November 2003, ICJ Reports 2003, Separate Opinion of Judge Higgins, S. 225 (234 Rn.33); IGH, Application of the Convention on the Prevention and Punishment of the Crime of Genocide (Bosnia and Herzegovina v. Serbia and Montenegro), Judgment of 26 February 2007, ICJ Reports 2007, S. 43 (119 Rn. 181, 129 Rn. 208 f., 130 Rn. 210); IGH, Application of the Convention on the Prevention and Punishment of the Crime of Genocide (Croatia v. Serbia), Judgment of 3 February 2015, ICJ Reports 2015, S. 3 (74 Rn. 178).

215 Tallinn Manual 2.0, Allgemeiner Kommentar zu Teil I, Kapitel 4, Abschnitt 1, S. 82, Rn. 11.

216 M. Roscini, Evidentiary Issues in International Disputes Related to State Responsibility for Cyber Operations, in: J. D. Ohlin/K. Govern/C. Finkelstein (Hg.), Cyberwar, 2015, S. 215 (222 ff.).

217 IGH, Application of the Convention on the Prevention and Punishment of the Crime of Genocide (Bosnia and Herzegovina v. Serbia and Montenegro), Judgment of 26 February 2007, ICJ Reports 2007, S. 43 (218 Rn. 422); T. Stein/T. Marauhn, Völkerrechtliche Aspekte von Informationsoperationen, ZaöRV 60 (2000), S. 1 (35).

218 Iran - US Claims Tribunal, Yeager v. The Islamic Republic of Iran, Award of 2 November 1987 (Award No. 324-10199-1), Iran-USCTR 17 (1987), S. 92 (101 f.).

219 R. Wolfrum/M. Möldner, International Courts and Tribunals, Evidence, in: R. Wolfrum (Hg.), MPEPIL 2013, http://www.mpepil.com, Rn. 75, 77. 
und überzeugende Beweislage ${ }^{\text {“220 }}$ gefordert. Diese Beweisanforderungen wurden jedenfalls mit Blick auf kinetische Angriffe aufgestellt und können wegen der allenfalls indiziellen Beweiskraft der zur Verfügung stehenden Beweismittel in der virtuellen Welt kaum erfüllt werden. ${ }^{221}$ Außerdem ist zu beachten, dass die Aufklärung von Informationsoperationen im Vergleich zu traditionellen Angriffsmethoden mehr Zeit erfordert. ${ }^{222}$ Dies hat zur Folge, dass Gegenmaßnahmen durch beeinträchtigte Staaten regelmäßig aufgrund des Zeitablaufs völkerrechtswidrig sein werden. Denn wartet der beeinträchtigte Staat bis eine den Beweisanforderungen genügende Aufklärung der Zurechnungszusammenhänge möglich ist, unterfallen Gegenmaßnahmen aufgrund ihrer zeitlichen Begrenzung regelmäßig der Kategorie der völkerrechtswidrigen Bestrafung. ${ }^{223}$ Reagiert der beeinträchtigte Staat hingegen vorschnell und ergibt sich, dass die Beweisanforderungen für eine Zurechnung nicht erfüllt sind, dann ist die Gegenmaßnahme ebenfalls als völkerrechtswidrig zu qualifizieren. ${ }^{224}$

Dementsprechend wird zuweilen die bloße Vermutung für eine Zurechnung von Informationsoperationen als ausreichend erachtet. ${ }^{225}$ Maßgeblich sei der Kontext der Informationsoperation. Die Vermutung der Urhe-

220 R. Geiß/H. Lahmann, Freedom and Security in Cyberspace, in: K. Ziolkowski (Hg.), Peacetime Regime for State Activities in Cyberspace, 2013, S. 621 (624); J. D. Jolley, Attribution, State Responsibility, and the Duty to Prevent Malicious Cyber-Attacks in International Law, 2017, abrufbar unter: http://theses.gla.ac.uk /id/eprint/8452 (geprüft am 15.05.2020), S. 130.

221 J. D. Jolley, Attribution, State Responsibility, and the Duty to Prevent Malicious Cyber-Attacks in International Law, 2017, abrufbar unter: http://theses.gla.ac.u k/id/eprint/8452 (geprüft am 15.05.2020), S. 122, 129; C. Schaller, Internationale Sicherheit und Völkerrecht im Cyberspace, SWP-Studie 18/2014, S. 22; S. J. Shackelford/R. B. Andres, State Responsibility for Cyber Attacks, Geo. JIL 42 (2010-2011), S. 971 (986).

222 Tallinn Manual 2.0, Kommentar zu Teil I, Kapitel 4, Abschnitt 1, S. 81, Rn. 9; D. Efrony/Y. Shany, A Rule Book on the Shelf?, AJIL 112 (2018), S. 583 (632); C. Schaller, Internationale Sicherheit und Völkerrecht im Cyberspace, SWP-Studie 18/2014, S. 22.

223 C. Schaller, Internationale Sicherheit und Völkerrecht im Cyberspace, SWP-Studie 18/2014, S. 22; S.-H. Schulze, Cyber-„War“ - Testfall der Staatenverantwortlichkeit, 2015, S. $80 \mathrm{f}$.

224 Tallinn 2.0, Kommentar zu Teil I, Kapitel 4, Abschnitt 1, S. 82 f., Rn. 12; E. T. Jensen/S. Watts, A Cyber Duty of Due Diligence, Tex. LR 95 (2017), S. 1555 (1564f.).

225 W. Heintschel von Heinegg, Cyberspace, in: R. Schmidt-Radefeldt/C. Meissler (Hg.), Automatisierung und Digitalisierung des Krieges, 2012, S. 159 (172). S.-H. Schulze schlägt zudem eine Umkehr der Beweislast vor. S.-H. Schulze, Cyber-„War“ - Testfall der Staatenverantwortlichkeit, 2015, S. $149 \mathrm{ff}$. 
berschaft treffe etwa denjenigen Staat, dem die Informationsoperation von Nutzen ist ${ }^{226}$ oder der nicht bereit ist, bei der Aufklärung mitzuwirken ${ }^{227}$. Sofern kritische Infrastrukturen betroffen sind, wollen einige Stimmen in der Literatur gänzlich auf das Nachweiserfordernis für eine Zurechnung verzichten. ${ }^{228}$ Diese Ansätze könnten abermals aufgrund der erwartbaren Gegenmaßnahmen auf völkerrechtswidriges Verhalten eskalatorische Wirkung entfalten. Der Sinn und Zweck der völkerkriegsrechtlichen Bestimmungen, zwischenstaatliche Gewaltanwendung so weit wie möglich zu begrenzen, wird konterkariert, wenn schon Vermutungen ausreichen, um mit Selbstverteidigung zu reagieren.229 Im Ergebnis muss daher zumindest mit klaren und überzeugenden Beweisen belegt werden, dass ein Staat hinter einem Informationsangriff steht, um dessen Verantwortlichkeit zu begründen. ${ }^{230}$

\section{Zurechnung in der internationalen Praxis}

Betrachtet man aber die bereits dargestellten Beispiele von Informationsoperationen unter diesen theoretischen Aspekten, wird deutlich, dass eine Zurechnung völkerrechtswidriger Informationsoperationen nahezu unmöglich ist. ${ }^{231}$

226 Vgl. M. C. Libicki, Cyberdeterrence and Cyberwar, 2009, S. 43 ff.

227 R. K. Knake, Untangling Attribution: Moving to Accountability in Cyberspace, Statement Before the Subcommittee on Technology and Innovation, Committee on Science and Technology, United States House of Representatives 2nd Session, 111th Congress, 2010, abrufbar unter: https://www.cfr.org/sites/default/ files/pdf/2010/07/Knake\%20-Testimony\%20071510.pdf (geprüft am 15.05.2020), S. $8 \mathrm{f}$.

228 S. M. Condron, Getting it Right, Harv. JOLT 20 (2007), S. 403 (416); siehe auch T. O. Keber/P. N. Roguski, Ius ad bellum electronicum?, AVR 49 (2011), S. 399 (416).

229 Vgl. E. T. Jensen/S. Watts, A Cyber Duty of Due Diligence, Tex. LR 95 (2017), S. 1555 (1568); H. Krieger spricht - unter Verweis auf M. Bothe, Terrorism and the Legality of Pre-emptive Force, EJIL 14 (2003), S. 227 (232) - von einer unzulässigen Verbindung des ius ad bellum mit dem Vorsorgegebot. H. Krieger, Krieg gegen anonymous, AVR 50 (2012), S. 1 (12 f.)

230 J. D. Jolley, Attribution, State Responsibility, and the Duty to Prevent Malicious Cyber-Attacks in International Law, 2017, abrufbar unter: http://theses.gla.ac .uk/id/eprint/8452 (geprüft am 15.05.2020), S. 130; C. Schaller, Internationale Sicherheit und Völkerrecht im Cyberspace, SWP-Studie 18/2014, S. 22.

231 Vgl. auch J. S. Davis II/B. Boudreaux/J. W. Welburn/J. Aguirre/C. Ogletree/G. McGovern/M. S. Chase, Stateless Attribution, 2017, S. 9 ff.; D. Efrony/Y. Shany, A Rule 
Die Informationsoperation, von der Estland betroffen war, veranschaulicht die Schwierigkeiten bei der Zurechnung von Informationsoperationen durch patriotische Hackergruppen. ${ }^{232}$ Die Angriffe wurden zu Internetadressen in Russland zurückverfolgt ${ }^{233}$ und teilweise der regierungsnahen russischen Jugendorganisation Nashi zugeschrieben. ${ }^{234}$ Auch wenn die Informationsoperation als Reaktion auf die Verbringung des Kriegsgefallenendenkmals in Einklang mit den Interessen der russischen Regierung stand, wurde keine Ermächtigung oder Beauftragung durch Russland festgestellt. ${ }^{235}$ Zudem verneinte die russische Regierung jedwede Beteiligung an den Angriffen. ${ }^{236}$ Allerdings finanziert und unterstützt Russland die Jugendorganisation, so dass durchaus eine gewisse Verbindung zum Staat besteht. ${ }^{237}$ Eine Leitung oder Kontrolle der Organisation durch die russische Regierung ist hingegen nicht gegeben, so dass kein zurechnungsrelevanter Zusammenhang hergestellt werden kann.238

Die Informationsangriffe auf Georgien wurden mit Russland in Verbindung gebracht, weil diese sich zeitlich im Vorlauf und Parallel zum kinetischen Konflikt mit Russland ereigneten und von russischen Internet-

Book on the Shelf?, AJIL 112 (2018), S. 583 (632); J. D. Jolley, Attribution, State Responsibility, and the Duty to Prevent Malicious Cyber-Attacks in International Law, 2017, abrufbar unter: http://theses.gla.ac.uk/id/eprint/8452 (geprüft am 15.05.2020), S. 81 f., 100, 216.

232 T. Payne, Teaching Old Law New Tricks, LCLR 20 (2016), S. 683 (706f.).

233 N. Anderson, Massive DDOS attacks target Estonia; Russia accused, ars Technica, 14 May 2007, abrufbar unter: https://arstechnica.com/information-technolo gy/2007/05/massive-ddos-attacks-target-estonia-russia-accused/ (geprüft am 15.05.2020); K. Flook, Russia and the Cyber Threat, Critical Threats, 13 May 2009, abrufbar unter: http://www.criticalthreats.org/russia/russia-and-cyber-thre at (geprüft am 15.05.2020). Der Angriff erfolgte indes über infizierte Computer weltweit, so dass die Beweiskraft der Rückverfolgung relativiert wird. S. Herzog, Revisiting the Estonian Cyber Attacks, JSS 4 (2011), S. 49 (52).

234 E. Tikk/K. Kadri/L. Vihul, International Cyber Incidents, 2010, S. 23.

235 S. Herzog, Revisiting the Estonian Cyber Attacks, JSS 4 (2011), S. 49 (53); E. Tikk/K. Kadri/L. Vihul, International Cyber Incidents, 2010, S. $23 \mathrm{f}$.

236 K. Flook, Russia and the Cyber Threat, Critical Threats, 13 May 2009, abrufbar unter: http://www.criticalthreats.org/russia/russia-and-cyber-threat (geprüft am 15.05.2020); S. Herzog, Revisiting the Estonian Cyber Attacks, JSS 4 (2011), S. 49 (53).

237 S. L. Myers, Youth Groups Created by Kremlin Serve Putin's Cause, The New York Times, 8 July 2007, abrufbar unter: https://www.nytimes.com/2007/07/08 /world/europe/08moscow.html?mtrref=www.google.com\&gwh=A2CDBA353 18FF4A9402F71EECFBA0E21\&gwt=pay\&assetType=REGIWALL (geprüft am 15.05.2020).

238 T. Payne, Teaching Old Law New Tricks, LCLR 20 (2016), S. 683 (707). 
adressen stammten. ${ }^{239}$ In Anbetracht des militärischen Konflikts um die Region Südossetien wird diskutiert, ob eine faktische Ausübung hoheitlicher Gewalt durch staatliche Stellvertreter zu bejahen ist, indem diese ohne explizite Ermächtigung durch die eigene Regierung, Angriffe gegen fremde Staaten starten, um einen Konflikt zu unterstützen oder ihrer Missbilligung gegenüber dem fremdstaatlichen Verhalten im Rahmen des zwischenstaatlichen Konflikts Ausdruck zu verleihen. ${ }^{240}$ Für eine Zurechnung unter diesem Gesichtspunkt ist aber unter anderem eine Aufforderung durch den Staat erforderlich. ${ }^{241}$ Der russischen Regierung könnte hier allenfalls der Vorwurf einer stillschweigenden Zustimmung durch ein bewusstes Wegschauen gemacht werden. ${ }^{242}$ Dies genügt allerdings nicht, um eine Zurechnung zu begründen.

Ebenso wenig wurde ein Staat für Stuxnet - oder dessen Abwandlungen Duqu und Flame - verantwortlich gemacht. Allerdings deuten Programmierstil, erforderlicher Aufwand und technische Expertise auf einen staatlichen Akteur hin und die mögliche Motivation für eine derartige Informationsoperation indiziert eine Urheberschaft der USA und Israel. ${ }^{243}$ Darüber hinaus bezeugen Aussagen von Vertretern der US-Administration, dass die US-Regierung hinter Stuxnet steht. ${ }^{244}$ Dies kann zwar durchaus als klare und überzeugende Beweislage gelten. Allerdings erfolgte durch den Iran keine Zurechnung der verbotenen Gewaltanwendung zu den USA oder Israel. ${ }^{245}$ Dies verdeutlicht den Widerwillen der Staatengemeinschaft einen Präzedenzfall für die Zurechnung von völkerrechtswidrigen Informationsoperationen zu schaffen, vermutlich um einer potenziellen ei-

239 J. D. Jolley, Attribution, State Responsibility, and the Duty to Prevent Malicious Cyber-Attacks in International Law, 2017, abrufbar unter: http://theses.gla.ac.uk /id/eprint/8452 (geprüft am 15.05.2020), S. 22.

240 Id., S. 110.

241 Vgl. ASR, Kommentar zu Artikel 9, S. 49, Abs. 6.

242 J. D. Jolley, Attribution, State Responsibility, and the Duty to Prevent Malicious Cyber-Attacks in International Law, 2017, abrufbar unter: http://theses.gla.ac.uk /id/eprint/8452 (geprüft am 15.05.2020), S. 111.

243 C. Morton, Stuxnet, Flame, and Duqu, in: J. Healey (Hg.), A Fierce Domain, 2013, S. 212 (212 ff.); S. J. Shackelford, Scott J./R. B. Andres, State Responsibility for Cyber Attacks, Geo. JIL 42 (2010-2011), S. 971 (991).

244 D. E. Sanger, Obama Ordered Sped Up Wave of Cyberattacks Against Iran, The New York Times, 1 June 2012, abrufbar unter: http://www.nytimes.com/2012/ 06/01/world/middleeast/obama-ordered-wave-of-cyberattacks-against-iran.html (geprüft am 15.05.2020).

245 J. D. Jolley, Attribution, State Responsibility, and the Duty to Prevent Malicious Cyber-Attacks in International Law, 2017, abrufbar unter: http://theses.gla.ac.uk /id/eprint/8452 (geprüft am 15.05.2020), S. 24. 
genen Staatenverantwortlichkeit für derartige Operationen zu entgehen. ${ }^{246}$ Im Zusammenhang mit völkerrechtlich nicht verbotenen Informationsoperation hingegen erfolgt eine Zurechnung durch betroffene Staaten schon eher.

Die durch die US-Regierung erfolgte Zurechnung der - wenngleich völkerrechtmäßigen - Informationsoperation gegen das DNC zu Russland verdeutlicht dabei, wie langwierig und aufwendig die Beweisführung im Kontext von Informationsoperationen ist. ${ }^{247}$ Zudem erfolgte die Zurechnung, ohne dass entsprechende Beweise dargetan wurden. ${ }^{248}$ Die Zurechnungsanalyse betraf nämlich eine als geheim eingestufte Informationsgewinnung und Staaten trifft keine Pflicht, ihre Beweisführung offenzulegen. ${ }^{249}$ Die Zurechnung begründete nicht nur politische Spannungen, sondern bezeugt auch, dass die unbestimmten Beweisstandards einen für das Recht der Staatenverantwortlichkeit zu großen Spielraum lassen..$^{250}$ Letztlich kann selbst eine umfassende Zusammenschau von technischen Indizien und nachrichtendienstlichen Erkenntnissen Zweifel an der Urheberschaft nicht beseitigen. ${ }^{251}$ So sieht sich auch die von der US-Regierung erfolgte Zurechnung der völkerrechtlich nicht verbotenen Informationsoperation durch die Guardians of Peace zu Nordkorea der Kritik ausgesetzt,

246 Vgl. D. Efrony/Y. Shany, A Rule Book on the Shelf?, AJIL 112 (2018), S. 583 (635).

247 Ausführlich zur Beweisführung bei der Informationsoperation gegen das DNC siehe W. Banks, State Responsibility and Attribution of Cyber Intrusions After Tallinn 2.0, Tex. LR 95 (2017), S. 1487 (1487 ff.).

248 W. Banks, State Responsibility and Attribution of Cyber Intrusions After Tallinn 2.0, Tex. LR 95 (2017), S. 1487 (1489 ff.).

249 Vgl. Tallinn Manual 2.0, Kommentar zu Teil I, Kapitel 4, Abschnitt 1, S. 83, Rn. 13; D. Efrony/Y. Shany, A Rule Book on the Shelf?, AJIL 112 (2018), S. 583 (633).

250 Vgl. W. Banks, State Responsibility and Attribution of Cyber Intrusions After Tallinn 2.0, Tex. LR 95 (2017), S. 1487 (1491 f., 1510 f.); D. Efrony/Y. Shany, A Rule Book on the Shelf?, AJIL 112 (2018), S. 583 (633); J. Goldsmith, Yet More Thoughts on the DNC Hack: Attribution and Precedent, LAWFARE, 27 July 2016, abrufbar unter: https://www.lawfareblog.com/yet-more-thoughts-dnc -hack-attribution-and-precedent (geprüft am 15.05.2020); J. D. Jolley, Attribution, State Responsibility, and the Duty to Prevent Malicious Cyber-Attacks in International Law, 2017, abrufbar unter: http://theses.gla.ac.uk/id/eprint/8452 (geprüft am 15.05.2020), S. $82 \mathrm{f}$.

251 W. Banks, State Responsibility and Attribution of Cyber Intrusions After Tallinn 2.0, Tex. LR 95 (2017), S. 1487 (1507 f.); J. D. Jolley, Attribution, State Responsibility, and the Duty to Prevent Malicious Cyber-Attacks in International Law, 2017, abrufbar unter: http://theses.gla.ac.uk/id/eprint/8452 (geprüft am 15.05.2020), S. $82 \mathrm{f}$. 
da nunmehr unternehmensinterne Urheber hinter den Angriffen vermutet werden. ${ }^{252}$ Schließlich verdeutlichen die Enthüllungen über die Informationsausbeutung durch die Five Eyes, wie umfassend die Beweislage sein muss, um eine Zurechnung unstreitig vornehmen zu können. Im Ergebnis blieb aber auch dieser Fall wegen der fehlenden Völkerrechtswidrigkeit ohne völkerrechtliche Konsequenzen. ${ }^{253}$

Im Ergebnis bleibt wegen der unsicheren Beweislage im virtuellen Raum lediglich die Erwartung, dass beeinträchtigte Staaten „vernünftig“ auf Informationsoperationen reagieren. ${ }^{254}$

\section{E. Verletzung positiver Verpflichtungen}

Die aufgezeigte Zurechnungslücke zwischen schadhaften Informationsoperationen und der Verantwortlichkeit von Staaten möchten Völkerrechtler durch eine allgemeine Sorgfaltsregel schließen. ${ }^{255}$ Dabei wird jedoch verkannt, dass es sich bei der gebotenen Sorgfalt um einen Annex zu primären Pflichten und nicht gleichzeitig um eine selbstständige Norm handelt. Entsprechend unübersichtlich sind die Begründungsansätze für diese allgemeine Sorgfaltsregel und deren Elemente. So erklären die Experten des Tallinn Manual 2.0 zirkelschlüssig: „Properly understood, due diligence is the standard of conduct expected of States when complying

252 J. Goldsmith, The Sony Hack: Attribution Problems, and the Connection to Domestic Surveillance, LAWFARE, 19 December 2014, abrufbar unter: https:/ /www.lawfareblog.com/sony-hack-attribution-problems-and-connection-dom estic-surveillance (geprüft am 15.05.2020); K. Zetter, Critics Say New Evidence Linking North Korea to the Sony Hack Is Still Flimsy, WIRED, 8 January 2015, abrufbar unter: http://www.wired.com/2015/01/critics-say-new-north-korea-evid ence-sony-still-flimsy (geprüft am 15.05.2020).

253 Vgl. M. N. Schmitt/L. Vihul, The Nature of International Law Cyber Norms, in: A.-M. Osula/H. Rõigas, International Cyber Norms, 2016, S. 23 (44).

254 Tallinn Manual 2.0, Kommentar zu Teil I, Kapitel 4, Abschnitt 1, S. 81 f., Rn. 10; D. Efrony/Y. Shany, A Rule Book on the Shelf?, AJIL 112 (2018), S. 583 (633).

255 Tallinn Manual 2.0, Regel 6 und 7; K. Bannelier-Christakis, Cyber Diligence, Baltic YBIL 14 (2014), S. 23 (27 ff.); E. T. Jensen/S. Watts, A Cyber Duty of Due Diligence, Tex. LR 95 (2017), S. 1555 (1565); I. Y. Liu, State Responsibility and Cyberattacks, IJICL 4 (2017), S. 191 (199 ff.); M. N. Schmitt, In Defense of Due Diligence in Cyberspace, The Yale LJ Forum 125 (2015), S. 68 (79f.); S. J. Shackelford/S. Russell/A. Kuehn, Unpacking the International Law on Cybersecurity Due Diligence, Chi. JIL 17 (2016), S. 1 (1 ff.); K. Ziolkowski, General Principles of International Law as Applicable in Cyberspace, in: dies. (Hg.), Peacetime Regime for State Activities in Cyberspace, 2013, S. 135 (168). 
with this [due diligence] principle.“ ${ }^{256}$ Außerdem verwischt dieser Ansatz die Grenze zwischen dem Konzept der Staatenhaftung und demjenigen der Staatenverantwortlichkeit. Denn eine Verletzung der Sorgfaltsregel sei danach gegeben, wenn durch die Informationsoperation Rechte anderer Staaten betroffen seien und zugleich erhebliche negative Auswirkungen für andere Staaten entstünden. ${ }^{257}$ Die im Trail Smelter-Schiedsspruch aufgestellte haftungsbegründende Voraussetzung „serious consequence“ 258 einerseits und die im Sinne der Korfu Kanal-Entscheidung für die Verantwortlichkeit erforderlichen ,acts contrary to the rights of other States“259 andererseits werden folgewidrig in einer Sorgfaltsregel für den virtuellen Raum vermischt. ${ }^{260}$ Dies verhindert eine klare Abgrenzung zwischen der Schadensvermeidungspflicht und der Verhinderungspflicht. ${ }^{261}$

Sinnvoll ist zwar, dass die Sorgfaltsregel in Bezug auf nicht-staatliche Informationsoperationen nur dann greift, wenn die Operation, hätte der Staat diese selbst vorgenommen, völkerrechtswidrig wäre. ${ }^{262}$ Allerdings wird selbst diese Voraussetzung nicht konsequent beibehalten. Demnach erstreckt sich die Sorgfaltsregel auch auf nicht-staatliche Informationsoperationen, die nicht per se völkerrechtswidrig sind, aber dennoch erhebliche

256 Tallinn Manual 2.0, Kommentar zu Regel 6, S. 30, Rn. 1.

257 Tallinn Manual 2.0, Regel 6; siehe auch I. Y. Liu, The due diligence doctrine under Tallinn Manual 2.0, CLSR 33 (2017), S. 390 (392).

258 Trail Smelter Arbitration (United States v. Canada), Award of 16 April 1938 and 11 March 1941, 3 UNRIAA (1941), S. 1905 (1965).

259 IGH, Corfu Channel Case (United Kingdom of Great Britain and Northern Ireland v. Albania), Judgment of 9 April 1949, ICJ Reports 1949, S. 4 (22).

260 Vgl. Tallinn Manual 2.0, Kommentar zu Regel 6, S.34, Rn. 15, S. 36 f., Rn. 25; $R$. Buchan, Cyberspace, Non-State Actors and the Obligation to Prevent Transboundary Harm, JCSL 21 (2016), S. 429 (449 f.); E. T. Jensen/S. Watts, A Cyber Duty of Due Diligence, Tex. LR 95 (2017), S. 1555 (1565 ff.); M. N. Schmitt, Grey Zones in the International Law of Cyberspace, YIL Online 42 (2017), abrufbar unter: https://cpb-us-w2.wpmucdn.com/campuspress.yale.edu/dist/8/1581/files/2 017/08/Schmitt_Grey-Areas-in-the-International-Law-of-Cyberspace-1cab8kj.pdf (geprüft am 15.05.2020), S. 1 (11 f.).

261 J. D. Jolley erklärt, dass "the duty to prevent and due diligence obligation are closely intertwined. This chapter sets them apart as two distinct theories within international law“, ohne jedoch eine genaue Abgrenzung vorzunehmen. J. D. Jolley, Attribution, State Responsibility, and the Duty to Prevent Malicious Cyber-Attacks in International Law, 2017, abrufbar unter: http://theses.gla.ac.u k/id/eprint/8452 (geprüft am 15.05.2020), S. 236 f. Siehe auch S.-H. Schulze, der betont, dass die allgemeine Verhinderungspflicht nicht mit dem völkergewohnheitsrechtlich geltenden (Umwelt-)Schädigungsverbot zu verwechseln ist. S.-H. Schulze, Cyber-,War“ - Testfall der Staatenverantwortlichkeit, 2015, S. 117.

262 Tallinn Manual 2.0, Kommentar zu Regel 6, S. 35, Rn. 18. 
negative Auswirkungen auf fremde Staaten haben. ${ }^{263}$ Dies führt zu dem paradoxen Ergebnis, dass die Staaten selbst derartige Informationsoperationen ausüben dürfen, aber dazu verpflichtet sind, nicht-staatliche Akteure davon abzuhalten. ${ }^{264}$ In diesem Zusammenhang sind auch die Anleihen aus dem Umweltvölkerrecht und die Diskussionen hinsichtlich der Voraussetzungen der erheblichen Schädigung deplatziert. ${ }^{265}$ Diese gehören vielmehr zur Schadensvermeidungspflicht aus dem Konzept der Staatenhaftung. Denn im Rahmen staatlicher Verantwortlichkeit wird allenfalls der Eintritt eines immateriellen Schadens gefordert, der aber bereits durch die verantwortlichkeitsbegründende Rechtsverletzung, das heißt „acts contrary to the rights of other States" gegeben ist. ${ }^{266}$ Die Bezugnahme auf das Umweltvölkerrecht geht ferner deswegen fehl, weil die für die virtuelle Welt postulierte Sorgfaltsregel keine staatlichen Präventionspflichten, wie etwa die Sicherung der Informationsinfrastruktur und die Überwachung verdächtiger Netzwerkaktivitäten, begründet. ${ }^{267}$ Das Umweltvölkerrecht ist aber gerade von dem Gedanken der Prävention gekennzeichnet, da Umweltschäden regelmäßig unumkehrbar sind. ${ }^{268}$

In Anbetracht dieser Unzulänglichkeiten überzeugt die Sorgfaltsregel für den virtuellen Raum nicht.

263 Tallinn Manual 2.0, Kommentar zu Regel 6, S. 35 f., Rn. 21.

264 B. A. Walton, Duties Owed, Yale LJ 126 (2017), S. 1460 (1498).

265 Vgl. Tallinn Manual 2.0, Kommentar zu Regel 6, S. 36 f., Rn. 25.

266 Siehe 2. Kapitel B. I. 1. Vgl. hingegen R. Buchan, der unter Bezugnahme auf die Korfu Kanal-Entscheidung und das Nuklearwaffen-Gutachten zwar ausführt, dass für die Verletzung der völkergewohnheitsrechtlichen Verhinderungspflicht grundsätzlich kein zusätzliches Schadenselement gefordert wird, dann aber den Trail Smelter-Schiedsspruch heranzieht, um zu zeigen, dass Schadensverursachung die Staatenverantwortlichkeit für Informationsoperationen bedingt. R. Buchan, Cyberspace, Non-State Actors and the Obligation to Prevent Transboundary Harm, JCSL 21 (2016), S. 429 (449f.).

267 Tallinn Manual 2.0, Kommentar zu Regel 6, S. 41 f., Rn. 42, Kommentar zu Regel 7, S. 44 f. Rn. 7 ff.; zustimmend I. Y. Liu, The due diligence doctrine under Tallinn Manual 2.0, CLSR 33 (2017), S. 390 (395). Nach anderer Auffassung soll die Sorgfaltsregel auch präventive Maßnahmen umfassen. S. J. Shackelford/S. Russell/A. Kuehn, Unpacking the International Law on Cybersecurity Due Diligence, Chi. JIL 17 (2016), S. 1 (35 ff.); K. Ziolkowski, General Principles of International Law as Applicable in Cyberspace, in: dies. (Hg.), Peacetime Regime for State Activities in Cyberspace, 2013, S. 135 (165 ff.).

268 Vgl. A. Proelß, Raum und Umwelt im Völkerrecht, in: W. Graf Vitzthum/ders. (Hg.), Völkerrecht, S. 361 (427 Rn. 112); M. V. Soto, General Principles of International Environmental Law, ILSA J. Intl. \& Comp. L. 3 (1996), S. 193 (199f.). 
Ein anderer Lösungsansatz für die Zurechnungsprobleme in der virtuellen Welt wird aus den Reaktionen auf die Anschläge des 11. September 2001 hergeleitet. Bekanntermaßen befand die US-Regierung, dass sie das Recht hatte, Selbstverteidigungsmaßnahmen gegen Afghanistan zu ergreifen, weil die Taliban-Regierung Mitglieder des terroristischen Netzwerks Al-Qaida beherbergte. Während einige darin eine Herabsetzung der $\mathrm{Zu}$ rechnungsschwelle erblicken und damit eine Verantwortlichkeit der Taliban-Regierung für die Handlungen von Al-Qaida konstruieren, ${ }^{269}$ sehen andere in der Reaktion der Staatengemeinschaft auf die Anschläge eine neue Ausnahme von den völkergewohnheitsrechtlichen Zurechnungsregeln der Staatenverantwortlichkeit. Demnach sei ein Staat grundsätzlich auch für nicht-staatliche Völkerrechtsverletzungen verantwortlich, wenn er vorherige Kenntnis von deren Begehung erlangt hatte und nichts dagegen unternommen hat. ${ }^{270}$ Eine Verantwortlichkeit für nicht-staatliches Verhalten verstößt aber gegen den Grundsatz der Nicht-Zurechnung reinen Privathandelns. ${ }^{271}$ Außerdem wird aufgrund der Entwicklungen nach den terroristischen Anschlägen eine spezifische Verhinderungspflicht mit Blick auf terroristische Handlungen vertreten. Ein Staat habe nunmehr die negative Pflicht, (Cyber-)Terroristen nicht wissentlich sicheren Unterschlupf auf eigenem Staatsgebiet zu gewähren. ${ }^{272}$

Es ist richtig, dass die staatliche Untätigkeit in Kenntnis von nicht-staatlichen, an sich völkerrechtswidrigen Handlungen ausschlaggebend für staatliche Verantwortlichkeit sein muss. Allerdings entsteht diese Verant-

269 D. Jinks, State Responsibility for the Acts of Private Armed Groups, Chi. JIL 4 (2003), S. 83 (83 ff.).

270 J. D. Jolley, Attribution, State Responsibility, and the Duty to Prevent Malicious Cyber-Attacks in International Law, 2017, abrufbar unter: http://theses.gla.ac.uk /id/eprint/8452 (geprüft am 15.05.2020), S. 270 ff.

271 In Bezug auf mögliche Menschenrechtsverletzungen scheinen diese Ansätze auf den ersten Blick zwar begrüßenswert, allerdings dürfen andere rechtliche Konsequenzen nicht außer Acht gelassen werden. Die Umgehung der Zurechnungsvoraussetzungen wäre insbesondere mit Blick auf den ius ad bellum Kontext problematisch. Wenn die bloße Duldung von völkerrechtswidrigen Maßnahmen für eine Zurechnung ausreichte, dann könnte dem duldenden Staat ohne Weiteres ein Angriff auf einen anderen Staat zugerechnet werden. Dies würde wiederum Selbstverteidigungsmaßnahmen gegen den duldenden Staat rechtfertigen. Daher ist eine Zurechnung nur möglich, wenn eine Ausübung staatlicher Aufgaben, eine staatliche Kontrolle oder eine nachträgliche Anerkennung gegeben ist. A. Seibert-Fohr, Die völkerrechtliche Verantwortung des Staats für das Handeln von Privaten, ZaöRV 73 (2013), S. 37 (42).

272 J. Bäumler, Das Schädigungsverbot im Völkerrecht, 2017, S. 296; S.-H. Schulze, Cyber-,,War“ - Testfall der Staatenverantwortlichkeit, 2015, S. 153 ff., 157 ff. 
wortlichkeit für staatliches Fehlverhalten und nicht für nicht-staatliche Aktivitäten. Dabei handelt es sich nicht um eine neue Ausnahmeregel der Staatenverantwortlichkeit bzw. um eine spezifische Pflicht in Bezug auf nicht-staatliches Verhalten, sondern lediglich um die altbewährte Regel des internationalen Rechts, dass Staaten nicht nur für völkerrechtswidriges Handeln, sondern auch für völkerrechtswidriges Unterlassen verantwortlich sind. 273

Es ist im Ergebnis also gar nicht notwendig, dem Konzept der gebotenen Sorgfalt den Status einer selbstständigen Primärpflicht zu verleihen oder eine neue Regel der Staatenverantwortlichkeit zu konstruieren. Wie im Rahmen der Rechtsprechungsanalyse deutlich wurde, haben Staaten positive Handlungspflichten, dafür zu sorgen, dass das nationale Territorium nicht für völkerrechtswidrige Handlungen missbraucht wird und dass unter ihrer staatlichen Hoheitsgewalt keine Verbrechen oder Menschenrechtsverletzungen verübt werden (I.). Die gebotene Sorgfalt spielt als Annex zu dieser positiven Handlungspflicht bei den Elementen „reale Handlungsmöglichkeit“ (II.) und „Kenntnis“ (III.) eine Rolle. ${ }^{274}$ Im Folgenden ist zu zeigen, inwiefern die völkerrechtliche Verhinderungs- bzw. Schutzpflicht in der virtuellen Welt zur Anwendung gelangt (IV.).

\section{Territorium bzw. Hoheitsgewalt}

Die virtuelle Welt wird teilweise als Gemeinschaftsgut betrachtet, welches sich der einzelstaatlichen Souveränität entzieht. ${ }^{275}$ Die virtuelle Welt kann aber nicht losgelöst von informationstechnischen Infrastrukturen (Com-

273 Siehe 2. Kapitel A. I. 2.

274 Siehe 1. Kapitel B. II.

275 Stellungnahme Deutschlands in: Report of the Secretary-General, Developments in the field of information and telecommunications in the context of international security, Replies received from Governments, UN Doc. A/68/156/ Add.1 vom 09.09.2013, S. 7; M. Aaltola/J. Sipilä/V. Vuorisalo, Securing Global Commons, A Small State Perspective, The Finnish Institute of International Affairs, Working Paper 71 (2011), abrufbar unter: https://www.fiia.fi/wp-con tent/uploads/2017/01/wp71.pdf (geprüft am 15.05.2020), S. 9; J. P. Barlow, A Declaration of the Independence of Cyberspace, Electronic Frontier Foundation, 1996, abrufbar unter: https://www.eff.org/de/cyberspace-independence (geprüft am 15.05.2020); D. Mandsager (Hg.), Sanremo Handbook on Rules of Engagement, 2009, S. 15; C. Schaller, Internationale Sicherheit und Völkerrecht im Cyberspace, SWP-Studie 18/2014, S. 7; T. Stein/T. Maraubn, Völkerrechtliche Aspekte von Informationsoperationen, ZaöRV 60 (2000), S. 1 (21 f.). 
putern, Sendeanlagen, Kabelnetzen und anderen Einrichtungen) bestehen und auch die Nutzer der informationstechnischen Systeme sind zu berücksichtigen. Diese physischen Komponenten befinden sich nämlich innerhalb staatlicher Territorien und unterliegen mithin der auf der Souveränität beruhenden Hoheitsgewalt der jeweiligen Staaten. ${ }^{276}$ Zur Begründung extraterritorialer Hoheitsgewalt ist die Ausübung tatsächlicher Kontrolle über Einrichtungen der Informationsinfrastruktur maßgeblich. ${ }^{277}$ Im Ergebnis bilden Territorium und Hoheitsgewalt damit auch in der virtuellen Welt Anknüpfungspunkte für staatliche Verhinderungs- bzw. Schutzpflichten. So sind Staaten verpflichtet, völkerrechtswidrige Informationsoperationen, die ihren Ursprung auf ihrem Territorium oder unter ihrer Hoheitsgewalt haben, zu verhindern ${ }^{278}$ und Personen unter ihrer Hoheitsgewalt vor menschenrechtsverletzenden Informationsoperationen zu schützen. ${ }^{279}$

Die grenzüberschreitende Vernetzung von informationstechnischen Systemen, die dezentrale Speicherung von Daten und der globale Datenverkehr über Internet-Knoten führen zugegebenermaßen zu Schwierigkeiten bei der Erfüllung der staatlichen Handlungspflichten. ${ }^{280}$ In der Regel sind aber sensible Daten, wie nachrichtendienstliche und sonstige streng geheime Informationen, auf lokalen Datenbanken gespeichert und digitale Steuerungs- und Kontrollsysteme für kritische Infrastrukturen, wie etwa Atommeiler, in auf staatlichem Territorium befindlichen Netzwerken eingegliedert, so dass sich die Problematik der territorialen Zuordnung in Grenzen hält. ${ }^{281}$ Die Tatsache, dass Staaten die Nutzung der virtuellen Welt entsprechend völkerrechtlich anerkannter Jurisdiktionstitel reglementieren, beweist zudem, dass eine einzelstaatliche Hoheitsgewalt auch hier möglich ist. ${ }^{282}$ Problematisch ist aber, dass viele Informationsopera-

276 W. Heintschel von Heinegg, Legal Implications of Territorial Sovereignty in Cyberspace, in: C. Czosseck/R. Ottis/K. Ziolkowski (Hg.), 2012 4th International Conference on Cyber Conflict, 2012, S. 7 (10, 13 f.); S.-H. Schulze, Cyber-„War“ - Testfall der Staatenverantwortlichkeit, 2015, S. 112; K. Ziolkowski, General Principles of International Law as Applicable in Cyberspace, in: dies. (Hg.), Peacetime Regime for State Activities in Cyberspace, 2013, S. 135 (162).

277 Tallinn Manual 2.0, Kommentar zu Regel 6, S. 33, Rn. 11.

278 J. Brunnée/T. Meshel, Teaching an Old Law New Tricks, GYIL 58 (2015), S. 129 (137f.).

279 Tallinn Manual 2.0, Kommentar zu Regel 36, S. 198, Rn. 6.

280 S.-H. Schulze, Cyber-„,War“ - Testfall der Staatenverantwortlichkeit, 2015, S. 111.

281 Ibid.

282 Ausführlich zur Anwendbarkeit der völkerrechtlichen Jurisdiktionstitel im virtuellen Raum siehe Tallinn Manual 2.0, Teil I, Abschnitt 3. 
tionen über Botnets erfolgen und die einzelnen Bots, aus denen sich das Botnet zusammensetzt, in verschiedenen Staaten lokalisiert sein können. ${ }^{283}$ Auch einzelne Datenpakete können durch die Infrastruktur mehrerer Staaten weitergeleitet werden und erst am Zielstaat zusammengefasst erhebliche Auswirkungen haben. ${ }^{284}$ Die staatliche Verhinderungspflicht greift aber nur dann, wenn die jeweilige Maßnahme auf staatlichem Territorium bzw. unter staatlicher Hoheitsgewalt für sich völkerrechtswidrig ist. Die völkerrechtswidrige Qualität einer Informationsoperation kann in den meisten Konstellationen hingegen nicht durch die einzelnen Bots in verschiedenen Staaten, sondern erst durch das gesamte Botnet begründet werden. ${ }^{285}$ Auch Datenpakete, die durch einen bestimmten Staat fließen, betreffen nur dann dessen Verhinderungspflicht, wenn sie für sich die Intensität der Völkerrechtswidrigkeit erreichen und nicht schon dann, wenn erst das gesamte Datenwerk, welches durch mehrere Staaten fließt, am Endziel eine völkerrechtswidrige Informationsoperation verkörpert. ${ }^{286}$

\section{Reale Handlungsmöglichkeit}

Auch wenn Staaten lediglich alle möglichen und zumutbaren Maßnahmen zur Verhinderung völkerrechtswidriger Informationsoperationen ergreifen müssen, stellt diese verhaltensbezogene Pflicht Ursprungs- bzw. Transitstaaten im virtuellen Raum vor Herausforderungen. In Anbetracht der Tatsache, dass die Verhinderungspflicht keine präventiven Maßnah-

283 Tallinn Manual 2.0, Kommentar zu Regel 6, S. 38, Rn. 29; M. N. Schmitt, Grey Zones in the International Law of Cyberspace, YIL Online 42 (2017), abrufbar unter: https://cpb-us-w2.wpmucdn.com/campuspress.yale.edu/dist/8/1581/files/2 017/08/Schmitt_Grey-Areas-in-the-International-Law-of-Cyberspace-1cab8kj.pdf (geprüft am 15.05.2020), S. 1 (12).

284 W. Heintschel von Heinegg, Legal Implications of Territorial Sovereignty in Cyberspace, in: C. Czosseck/R. Ottis/K. Ziolkowski (Hg.), 2012 4th International Conference on Cyber Conflict, 2012, S. 7 (17).

285 Tallinn Manual 2.0, Kommentar zu Regel 6, S. 38 f., Rn. 29, 31.

286 W. Heintschel von Heinegg, Legal Implications of Territorial Sovereignty in Cyberspace, in: C. Czosseck/R. Ottis/K. Ziolkowski (Hg.), 2012 4th International Conference on Cyber Conflict, 2012, S. 7 (17). Die Experten des Tallinn Manual 2.0 hingegen sehen Transitstaaten in der Pflicht, wenn diese Kenntnis von der im Ergebnis völkerrechtswidrigen Informationsoperation haben und die Möglichkeit zu deren Beendigung besitzen. Tallinn Manual 2.0, Kommentar zu Regel 6, S. 33, Rn. 13. 
men umfasst, ${ }^{287}$ bleiben nämlich in der virtuellen Welt insbesondere aufgrund der Zeit-Raum-Kompression, also der durch die Virtualisierung und Vernetzung bedingten Verkürzung von Distanzen zwischen Zeit und Raum, ${ }^{288}$ kaum wirksame Maßnahmen zur Verhinderung von Informationsoperationen. ${ }^{289}$ Erschwerend kommt hinzu, dass Daten und Datenpfade, die für völkerrechtswidrige Informationsoperationen genutzt werden, nicht ohne Weiteres lesbar bzw. lenkbar sind. So wird es Staaten in aller Regel schwerfallen, unverzüglich wirksame Verhinderungsmaßnahmen zu ergreifen. ${ }^{290}$ Informationsoperationen können unmittelbar von auf staatlichem Territorium befindlichen Infrastrukturen ausgehen, ohne dass der Staat überhaupt die Möglichkeit hat, rechtzeitig zu reagieren. ${ }^{291}$

Allerdings umfasst die positive Handlungspflicht nicht nur die Verhinderung des Eintritts der völkerrechtswidrigen Informationsoperation, sondern auch das Unterbinden von andauernden völkerrechtswidrigen Vorgängen und die Verfolgung sowie Bestrafung von Verursachern. ${ }^{292}$ Aber selbst wenn potenziell völkerrechtswidrige Informationsoperationen entdeckt werden, ist es dem Ursprungs- bzw. Transitstaat oftmals nicht möglich, diese zu unterbinden, ohne gleichzeitig erhebliche negative Auswirkungen auf nationale Netzwerke in Kauf nehmen zu müssen. So ist unter Umständen die komplette Isolation des eigenen Netzwerks notwendig, um Informationsoperationen gegen fremde Staaten zu verhindern bzw. zu unterbinden. Dies beeinträchtigt wiederum die Nutzung informationstechnischer Systeme im Ursprungs- bzw. Transitsaat selbst. ${ }^{293}$ Die Interessen des Ursprungs- bzw. Transitstaates einerseits und des Opferstaates andererseits müssen in einen vernünftigen Ausgleich gebracht werden, ${ }^{294}$ mit der Fol-

287 Wie sich aus den Ausführungen im 1. Kapitel ergibt, ist die Schadensprävention im Konzept der Staatenhaftung zu verorten. Vgl. auch in Bezug auf die postulierte Sorgfaltsregel Tallinn Manual 2.0, Kommentar zu Regel 6, S. 41 f., Rn. 42, S. 46, Rn. 12 f.; I. Y. Liu, The due diligence doctrine under Tallinn Manual 2.0, CLSR 33 (2017), S. 390 (394 f.).

288 S. Böschen/K. Weis, Die Gegenwart der Zukunft, 2007, S. 83.

289 Vgl. Tallinn Manual 2.0, Kommentar zu Regel 7, S. 49, Rn. 24 f.; J. Brunnée/T. Meshel, Teaching an Old Law New Tricks, GYIL 58 (2015), S. 129 (141).

290 Tallinn Manual 2.0, Kommentar zu Regel 6, S. 34, Rn. 14.

291 I. Y. Liu, State Responsibility and Cyberattacks, IJICL 4 (2017), S. 191 (232).

292 Vgl. IGH, United States Diplomatic and Consular Staff in Tehran (USA v. Iran), Judgment of 24 May 1980, ICJ Reports 1980, S. 3 (3 ff.); M. J. Sklerov, Solving the Dilemma of State Responses to Cyberattacks, Military Law Review 201 (2009), S. 1 (62).

293 Tallinn Manual 2.0, Kommentar zu Regel 7, S. 49 f., Rn. 25.

294 Ibid. 
ge, dass angesichts der Konsequenzen für den Ursprungs- bzw. Transitstaat regelmäßig wirksame Gegenmaßnahmen gerade nicht erwartet werden können.

Zudem bestehen in der virtuellen Welt besondere Hürden bei der Wahrnehmung staatlicher Schutzpflichten. Staaten können nämlich ausländische Informationsoperationen, die Rechte von Personen unter ihrer Hoheitsgewalt verletzen, kaum abwehren, verfolgen oder ahnden, da die Verursacher regelmäßig der Jurisdiktion eines anderen Staates unterfallen. Die Staaten können allenfalls Auslieferungsbegehren stellen oder den Ursprungsstaat auffordern, Untersuchungen anzustellen und Täter strafrechtlich zu verfolgen. ${ }^{295} \mathrm{Im}$ Ergebnis können die Staaten ihren Schutzpflichten damit nicht effektiv nachkommen.

Schließlich bestimmt sich die reale Handlungsmöglichkeit des pflichtigen Staates nach dessen technischen Kapazitäten und Ressourcen zur Abwehr der jeweiligen Informationsoperation. ${ }^{296}$ In der Praxis ist es selbst entwickelten und technisierten Staaten nur selten möglich, die Quelle komplexer Informationsoperationen aufzuspüren und diese zu unterbinden, so dass eine reale Handlungsmöglichkeit zur Verhinderung von nichtstaatlichen bzw. fremdstaatlichen Rechtsverletzungen im virtuellen Raum nur in begrenzten Fällen gegeben sein wird. ${ }^{297}$

295 Die Hürden können durch das Übereinkommen über Computerkriminalität des Europarats vom 23.11.2001, (European Treaty Series - No. 185; BGBl. 2008 II S. 1242) für Vertragsstaaten zwar eingedämmt, aber nicht komplett beseitigt werden. Vgl. E. T. Jensen, Cyber Sovereignty, Tex. ILJ 50 (2014) S. 275 (278, 301); M. F. Miquelon-Weismann, The Convention on Cybercrime, J. Marshall J. Computer \& Info. L. 23 (2005), S. 329 (335 f.).

296 J. D. Jolley, Attribution, State Responsibility, and the Duty to Prevent Malicious Cyber-Attacks in International Law, 2017, abrufbar unter: http://theses.gla.ac. uk/id/eprint/8452 (geprüft am 15.05.2020), S. 230; M. N. Schmitt, Grey Zones in the International Law of Cyberspace, YIL Online 42 (2017), abrufbar unter: https://cpb-us-w2.wpmucdn.com/campuspress.yale.edu/dist/8/1581/files/2017 /08/Schmitt_Grey-Areas-in-the-International-Law-of-Cyberspace-1cab8kj.pdf (geprüft am 15.05.2020), S. 1 (12).

297 S.-H. Schulze, Cyber-„War“ - Testfall der Staatenverantwortlichkeit, 2015, S. $36 \mathrm{ff}$. 


\section{Kenntnis}

In der virtuellen Welt sind außerdem die Voraussetzungen, unter welchen das Kenntniserfordernis erfüllt ist, unbestimmt. ${ }^{298}$

Während der pflichtige Staat eine vorherige tatsächliche Kenntnis von den nicht-staatlichen bzw. fremdstaatlichen Informationsoperationen in aller Regel bestreiten wird und diese damit nicht ohne Weiteres nachweisbar ist, ${ }^{299}$ wird tatsächliche Kenntnis jedenfalls ab dem Zeitpunkt bestehen, ab dem der Opferstaat den Ursprungs- bzw. Transitstaat von der Informationsoperation unterrichtet. ${ }^{300}$ Dies spiegelt die Entscheidung des IGH im Teheraner Geiselnahme-Fall wieder, in der der Gerichtshof konstatierte, dass dem Iran die dringliche Notwendigkeit zur Ergreifung von Maßnahmen in Anbetracht der wiederholten Hilferufe an das iranische Außenministerium vollkommen bewusst war. ${ }^{301}$ Schwieriger ist die Beantwortung der Frage, unter welchen Voraussetzungen mutmaßliche bzw. hypothetische Kenntnis in der virtuellen Welt angenommen werden kann. Dies soll insbesondere dann der Fall sein, wenn sich die Akteure der regierungseigenen Infrastruktur des Ursprungs- bzw. Transitstaates bedienen, wenn die Infrastruktur direkter staatlicher Kontrolle unterliegt oder ausschließlich öffentlichen Zwecken dient. ${ }^{302}$ Es ist aber zu berücksichtigen, dass die Verschleierung der wahren Identität der Nutzer informati-

298 J. Brunnée/T. Meshel, Teaching an Old Law New Tricks, GYIL 58 (2015), S. 129 (142); J. D. Jolley, Attribution, State Responsibility, and the Duty to Prevent Malicious Cyber-Attacks in International Law, 2017, abrufbar unter: http://the ses.gla.ac.uk/id/eprint/8452 (geprüft am 15.05.2020), S. 195 ff.; I. Y. Liu, State Responsibility and Cyberattacks, IJICL 4 (2017), S. 191 (232 ff.).

299 Tallinn Manual 2.0, Kommentar zu Regel 6, S.40, Rn. 38; I. Y. Liu, State Responsibility and Cyberattacks, IJICL 4 (2017), S. 191 (233).

300 Tallinn Manual 2.0, Kommentar zu Regel 6, S. 40, Rn.37; C. Antonopoulos, State Responsibility in Cyberspace, in: N. Tsagourias/R. Buchan (Hg.), Research Handbook on International Law and Cyberspace, 2015, S. 55 (69); J. D. Jolley, Attribution, State Responsibility, and the Duty to Prevent Malicious Cyber-Attacks in International Law, 2017, abrufbar unter: http://theses.gla.ac.uk/id/eprin t/8452 (geprüft am 15.05.2020), S. 195.

301 IGH, United States Diplomatic and Consular Staff in Tehran (USA v. Iran), Judgment of 24 May 1980, ICJ Reports 1980, S. 3 (32 f. Rn. 68).

302 Tallinn Manual 2.0, Kommentar zu Regel 6, S.41, Rn. 40; W. Heintschel von Heinegg, Legal Implications of Territorial Sovereignty in Cyberspace, in: C. Czosseck/R. Ottis/K. Ziolkowski (Hg.), 2012 4th International Conference on Cyber Conflict, 2012, S. 7 (17); B. Pirker, Territorial Sovereignty and Integrity and the Challenges of Cyberspace, in: K. Ziolkowski (Hg.), Peacetime Regime for State Activities in Cyberspace, 2013, S. 189 (205 f.). 
onstechnischer Systeme (Spoofing) oder die Verschlüsselung von Transferund Verbindungsdaten und Anonymisierung der Internetnutzung (Onion Routing) in der virtuellen Welt Usus sind und Regierungsserver sogar manipuliert werden, um Konflikte zu provozieren. ${ }^{303}$

Dementsprechend stellen sich - ähnlich wie im Kontext der Zurechnung völkerrechtswidriger Informationsoperationen - auch im Zusammenhang mit der Kenntnis von derartigen Operationen Beweisschwierigkeiten im virtuellen Raum. Der Nachweis durch den Opferstaat kann oftmals nicht geführt werden, weil Informationsoperationen sich durch ihre Unsichtbarkeit und Heimlichkeit auszeichnen, netzinterne Vorgänge des Ursprungs- bzw. Transitstaates betreffen und gleichzeitig von ubiquitärem Charakter mit weitreichender Fernwirkung sind. Auch hier wird diskutiert, ob eine Umkehr der Beweislast angezeigt ist. ${ }^{304}$ Während einige Stimmen in der Literatur eine widerlegliche Vermutung der Kenntnis bzw. des Kennenmüssens auf Informationsoperationen mittels staatlicher bzw. zu staatlichen Zwecken genutzter Infrastrukturen beschränken wollen, ${ }^{305}$ möchten andere die Reichweite der Vermutung auf alle im staatlichen Hoheitsbereich befindliche Infrastrukturen ausweiten. ${ }^{306}$ Der IGH lehnte in seiner Korfu Kanal-Entscheidung aber eine widerlegliche Vermutung in

303 I. Y. Liu, State Responsibility and Cyberattacks, IJICL 4 (2017), S. 191 (237); M. Pihelgas, Back-Tracing and Anonymity in Cyberspace, in: K. Ziolkowski (Hg.), Peacetime Regime for State Activities in Cyberspace, 2013, S. 31 (45); B. Pirker, Territorial Sovereignty and Integrity and the Challenges of Cyberspace, in: $K$. Ziolkowski (Hg.), Peacetime Regime for State Activities in Cyberspace, 2013, S. 189 (212).

304 S.-H. Schulze, Cyber-,War“ - Testfall der Staatenverantwortlichkeit, 2015, S. $134 \mathrm{f}$. mit Fn. 441, $151 \mathrm{ff}$. Es finden sich Vorschläge, die gänzlich auf das Element der Kenntnis verzichten wollen. In Anlehnung an das Umweltvölkerrecht sollen die Maßnahmen, die ein Staat allgemein zur Vermeidung von schadhaften Informationsoperationen unternimmt, maßgeblich sein. M. J. Sklerov, Solving the Dilemma of State Responses to Cyberattacks, Military Law Review 201 (2009), S. 1 (71). Der Verzicht auf das Kenntniserfordernis geht aber für das Regime der Staatenverantwortlichkeit zu weit. B. Pirker, Territorial Sovereignty and Integrity and the Challenges of Cyberspace, in: K. Ziolkowski (Hg.), Peacetime Regime for State Activities in Cyberspace, 2013, S. 189 (205).

305 W. Heintschel von Heinegg, Legal Implications of Territorial Sovereignty in Cyberspace, in: C. Czosseck/R. Ottis/K. Ziolkowski (Hg.), 2012 4th International Conference on Cyber Conflict, 2012, S. 7 (17); B. Pirker, Territorial Sovereignty and Integrity and the Challenges of Cyberspace, in: K. Ziolkowski (Hg.), Peacetime Regime for State Activities in Cyberspace, 2013, S. 189 (205 f.); S.-H. Schulze, Cyber-,War“ - Testfall der Staatenverantwortlichkeit, 2015, S. 152.

306 C. Antonopoulos, State Responsibility in Cyberspace, in: N. Tsagourias/R. Buchan (Hg.), Research Handbook on International Law and Cyberspace, 2015, 
Bezug auf die Kenntnis des Staates, den die Verhinderungspflicht trifft, eindeutig ab. ${ }^{307}$ Die staatliche Kontrolle über ein Territorium führt nicht zu einer Beweislastumkehr. ${ }^{308}$ Dem Opferstaat wird lediglich eine erleichterte Beweisführung zugestanden, ${ }^{309}$ so dass Aspekte, wie die Haltung des beschuldigten Staates vor und nach der Informationsoperation und der Umstand, dass eine Reihe von gleichgelagerten Informationsoperationen ihren Ursprung in einem Staat haben, hinzugezogen werden können, um staatliche Kenntnis zu belegen. ${ }^{310}$ Allerdings ist der geforderte Beweis, nach dem kein Raum für begründete Zweifel bleiben darf,311 im Kontext von Informationsoperationen nur in den wenigsten Fällen führbar. ${ }^{312}$ Trotzdem sollten die Anforderungen nicht herabgesetzt werden, da speziell in der virtuellen Welt die Gefahr besteht, dass Ursprungs- bzw. Transitstaaten zu Unrecht für ein Unterlassen verantwortlich gemacht werden. ${ }^{313}$

S. 55 (64); D. J. Ryan/M. Dion/E. Tikk/J. J. C. H. Ryan, International Cyberlaw, Geo. JIL 42 (2011), S. 1161 (1185, 1188 f.).

307 IGH, Corfu Channel Case (United Kingdom of Great Britain and Northern Ireland v. Albania), Judgment of 9 April 1949, ICJ Reports 1949, S. 4 (18).

308 Ibid.

309 Ibid.

310 J. D. Jolley, Attribution, State Responsibility, and the Duty to Prevent Malicious Cyber-Attacks in International Law, 2017, abrufbar unter: http://theses.gla.ac.u k/id/eprint/8452 (geprüft am 15.05.2020), S. 196; I. Y. Liu, State Responsibility and Cyberattacks, IJICL 4 (2017), S. 191 (241 f.).

311 IGH, Corfu Channel Case (United Kingdom of Great Britain and Northern Ireland v. Albania), Judgment of 9 April 1949, ICJ Reports 1949, S. 4 (18).

312 J. D. Jolley, Attribution, State Responsibility, and the Duty to Prevent Malicious Cyber-Attacks in International Law, 2017, abrufbar unter: http://theses.gla.ac.u k/id/eprint/8452 (geprüft am 15.05.2020), S. 122, 129; C. Schaller, Internationale Sicherheit und Völkerrecht im Cyberspace, SWP-Studie 18/2014, S. 22; S. J. Shackelford/R. B. Andres, State Responsibility for Cyber Attacks, Geo. JIL 42 (2010-2011), S. 971 (986).

313 I. Y. Liu, State Responsibility and Cyberattacks, IJICL 4 (2017), S. 191 (237); vgl. auch J. Stubbs/C. Bing, Hacking the hackers: Russian group hijacked Iranian spying operation, officials say, Reuters, 21 October 2019, abrufbar unter: https:/ /www.reuters.com/article/us-russia-cyber/hacking-the-hackers-russian-group-h ijacked-iranian-spying-operation-officials-say-idUSKBN1X00AK (geprüft am 15.05.2020). 
IV. Positive Verpflichtungen in der internationalen Praxis

Steht also lediglich eine fehlende Zurechenbarkeit von an sich völkerrechtswidrigen Informationsoperationen einer Staatenverantwortlichkeit wegen der Verletzung negativer Pflichten entgegen, kann die Verletzung einer positiven Verhinderungs- bzw. Schutzpflicht eine entsprechende Verantwortlichkeit begründen.

In diesem Zusammenhang sind daher die völkerrechtswidrigen Informationsangriffe gegen Estland und Georgien näher zu betrachten. ${ }^{314}$ Bei der Informationsintervention gegen Estland nutzten die Verursacher ein Botnet, welches Computer weltweit betraf. ${ }^{315}$ Einige betroffene Staaten neutralisierten die auf ihrem Territorium infiltrierten Systeme freiwillig, das heißt ohne einer entsprechenden Rechtspflicht nachzukommen. ${ }^{316}$ Der Großteil der Bots ist auf russische Internetadressen zurückzuführen, so dass die vom russischen Territorium ausgehenden Angriffe wohl schon für sich die Intensität der Völkerrechtswidrigkeit erreichten. ${ }^{317}$ Estland unterrichtete Russland zwar nicht von den Geschehnissen, ${ }^{318}$ so dass eine tatsächliche Kenntnis vor und während der Informationsoperation nicht bejaht werden kann. Jedoch stand die Informationsoperation zumindest im zeitlichen Zusammenhang mit den politischen Spannungen zwischen den Staaten. ${ }^{319}$ Zudem weigerte sich Russland, bei der Identifizierung

314 Wie gezeigt, lässt die Beweislage im Fall Stuxnet eine Zurechnung der völkerrechtswidrigen Informationsoperation zur US-Regierung zu (siehe 3. Kapitel D. II.). Das Schadprogramm infiltrierte über verseuchte USB-Speichersticks Steuerungssysteme von Atomanlagen auf iranischem Hoheitsgebiet. Eine positive Verhinderungspflicht des Iran selbst ist allerdings mangels Kenntnis zu verneinen. Nach Kenntniserlangung wurde der Angriff überdies gestoppt und investigiert (siehe 3. Kapitel B. II.).

315 E. Tikk/K. Kadri/L. Vibul, International Cyber Incidents, 2010, S. 23 m.w.N.

316 Id., S. 24.

317 N. Anderson, Massive DDOS attacks target Estonia; Russia accused, ars Technica, 14 May 2007, abrufbar unter: https://arstechnica.com/information-technolo gy/2007/05/massive-ddos-attacks-target-estonia-russia-accused/ (geprüft am 15.05.2020); K. Flook, Russia and the Cyber Threat, Critical Threats, 13 May 2009, abrufbar unter: http://www.criticalthreats.org/russia/russia-and-cyber-thre at (geprüft am 15.05.2020); M. N. Schmitt, Grey Zones in the International Law of Cyberspace, YIL Online 42 (2017), abrufbar unter: https://cpb-us-w2.wpmuc dn.com/campuspress.yale.edu/dist/8/1581/files/2017/08/Schmitt_Grey-Areas-in -the-International-Law-of-Cyberspace-1cab8kj.pdf (geprüft am 15.05.2020), S. 1 (11).

318 I. Y. Liu, State Responsibility and Cyberattacks, IJICL 4 (2017), S. 191 (234).

319 S. Herzog, Revisiting the Estonian Cyber Attacks, JSS 4 (2011), S. 49 (50 f.). 
der russischen Hacker behilflich zu sein oder diese strafrechtlich zu belangen. ${ }^{320}$ Aufgrund der Verbindung zwischen der russischen Regierung und der Hackergruppe Nashi, der Instruktionen zur Beteiligung an den Angriffen auf russischsprachigen Internetseiten und der Dauer der Angriffe bleiben keine vernünftigen Zweifel daran, dass Russland mutmaßliche oder zumindest hypothetische Kenntnis von der Informationsoperation hatte. ${ }^{321}$ Die Informationsoperation gegen Georgien glich derjenigen gegen Estland. Auch hier haben Sicherheitsexperten die Angriffe auf russische Internetadressen zurückverfolgt. Zudem werden die Angriffe im Kontext des kinetischen Konflikts zwischen Russland und Georgien beurteilt und als Vorbereitung und Unterstützung der militärischen Bodenoperation durch Russland gewertet. ${ }^{322}$ Während das bewusste Wegschauen der russischen Regierung in diesem Fall für eine Zurechnung nicht ausreicht, begründet es zumindest eine mutmaßliche oder hypothetische Kenntnis von den Informationsangriffen. Dementsprechend kann eine Verantwortlichkeit Russlands wegen der Verletzung seiner Handlungspflichten angenommen werden. Russland hätte die Informationsinterventionen gegen Estland bzw. Georgien, wenn schon nicht im Vorhinein verhindern, zumindest die andauernden Operationen unterbinden oder im Nachhinein ahnden müssen. ${ }^{323}$ Gleichwohl wurde die Verantwortlichkeit Russlands in der realen Welt in keinem der beiden Fälle völkerrechtlich geltend gemacht. ${ }^{324}$ Dies liegt wohl daran, dass sich die Staaten noch unsicher bei der

320 E. Tikk/K. Kadri/L. Vibul, International Cyber Incidents, 2010, S. 27 f.

321 Id., S. $18 \mathrm{ff}$.

322 S. Blank, Cyber War and Information War à la Russe, in: G. Perkovich/A. E. Levite, Understanding Cyber Conflict, 2017, S. 81 (88 ff.). A. Hagen, The RussoGeorgian War 2008, in: J. Healey (Hg.), A Fierce Domain, 2013, S. 194 (194 ff.); J. D. Jolley, Attribution, State Responsibility, and the Duty to Prevent Malicious Cyber-Attacks in International Law, 2017, abrufbar unter: http://theses.gla.ac.uk /id/eprint/8452 (geprüft am 15.05.2020), S. 22.

$323 \mathrm{~J}$. Kulesza, State responsibility for acts of cyber-terrorism, Global Internet Governance Academic Network, Annual Symposium, Vilnius 2010, S.12; M. N. Schmitt, Grey Zones in the International Law of Cyberspace, YIL Online 42 (2017), abrufbar unter: https://cpb-us-w2.wpmucdn.com/campuspress.yale.edu/ dist/8/1581/files/2017/08/Schmitt_Grey-Areas-in-the-International-Law-of-Cyber space-1cab8kj.pdf (geprüft am 15.05.2020), S. 1 (11).

324 J. D. Jolley, Attribution, State Responsibility, and the Duty to Prevent Malicious Cyber-Attacks in International Law, 2017, abrufbar unter: http://theses.gla.ac.uk /id/eprint/8452 (geprüft am 15.05.2020), S. 22. 
Anwendbarkeit herkömmlicher Rechte und Pflichten auf den virtuellen Bereich sind. ${ }^{325}$

Die Fälle der Informationsspionage verstoßen für sich genommen nicht gegen das Völkerrecht und begründen mithin keine Verhinderungspflicht der Ursprungsstaaten. Auch ist eine extraterritoriale Schutzpflicht der Ursprungsstaaten für die Eingriffe in die Privatheit derzeit nicht völkerrechtlich etabliert. ${ }^{326}$ Dem Grunde nach besteht zwar eine Schutzpflicht der Zielstaaten gegenüber Personen, die unter ihrer Hoheitsgewalt stehen. ${ }^{327}$ Allerdings fehlt es in Konstellationen wie Five Eyes an der Kenntnis und der realen Handlungsmöglichkeit der betroffenen Staaten zum Schutz vor der fremdstaatlichen Missachtung der Menschenrechte. ${ }^{328}$

\section{F. Zusammenfassung}

Das Recht der Staatenverantwortlichkeit ist zur Reglementierung von Informationsoperationen ungeeignet. Dies liegt zum einen daran, dass schon die besprochenen völkerrechtlichen Verbotstatbestände klar definierte rechtliche Umrisse vermissen lassen, und zum anderen daran, dass die spezifischen technischen Aspekte von Informationsoperationen die Übertragbarkeit dieser Verbote und entsprechender Handlungspflichten auf den virtuellen Raum erschweren.

Informationsoperationen unterfallen nur selten den herkömmlichen völkerrechtlichen Verbotskategorien, da deren Auswirkungen grundsätzlich nicht mit denen von unerlaubter Gewalt oder unerlaubtem Zwang vergleichbar sind. Der Schutz durch das humanitäre Völkerrecht kann

325 P. von Wussow stellt beispielsweise fest: „Der ursprüngliche Völkerrechtsenthusiasmus, wie er noch im sogenannten Tallin Manual (2013/2017) zum Ausdruck kam, weicht dabei immer mehr einem komplexeren Verständnis der Prozesse, in denen sich Normen für den Cyberkrieg erst herausbilden." $P$. von Wussow, „Cyberkrieg“, Ethik und Militär 1 (2019), abrufbar unter: http://www.ethiku ndmilitaer.de/fileadmin/ethik_und_militaer/Ethik-und-Militaer-2019-1.pdf (geprüft am 15.05.2020), S. 11 (16).

326 Ausführlich zum Diskussionsstand über die extraterritoriale Geltung der Menschenrechte siehe I. Kanalan, Extraterritoriale Staatenpflichten jenseits der Hoheitsgewalt, AVR 52 (2014), S. 495 (495 ff.).

327 W. Kälin/J. Künzli, Universeller Menschenrechtsschutz, 2019, S. 148 f.

328 Kritisch in Bezug auf die behauptete Unkenntnis Deutschlands K. von Notz, Der demokratische Rechtsstaat und das Geheimnis der Dienste, Die Friedens-Warte 90 (2015), S. 17 (17 ff.). 
ebenso wenig greifen, weil Informationsoperationen nicht ohne Weiteres unter den Angriffsbegriff subsumiert werden können.

Neben diesen Unsicherheiten sind bei der Staatenverantwortlichkeit für informationstechnische Systeme auch stets die wegen völkerrechtswidriger Informationsoperationen möglichen Gegenmaßnahmen zu berücksichtigen, so dass gerade in der virtuellen Welt ein restriktiver Ansatz geboten ist. Bestrebungen, neue Verbote für die virtuelle Welt aufzustellen bzw. bestehende Vorschriften extensiv auszulegen, um die Völkerrechtswidrigkeit von Informationsoperationen begründen zu können, sind aufgrund der fehlenden Grundlage in der Staatenpraxis sowie der Bedrohung für den zwischenstaatlichen Frieden wenig sinnvoll.

Jedenfalls scheitert die Zurechnung von völkerrechtswidrigen Informationsoperationen regelmäßig an den Beweisschwierigkeiten in der virtuellen Welt, denen - wegen des beschriebenen Eskalationspotenzials - nicht mit einer Absenkung der Anforderungen begegnet werden kann.

Schließlich ist eine Verletzung staatlicher Handlungspflichten in der virtuellen Welt regelmäßig mangels realer Handlungsmöglichkeit und/oder fehlenden Nachweises von staatlicher Kenntnis zu verneinen.

Die Fälle aus der internationalen Praxis belegen außerdem, dass die Staaten mit Blick auf das Recht der Staatenverantwortlichkeit und die entsprechenden Regeln des Tallinn Manuals eine Strategie der „silence and ambiguity" verfolgen. ${ }^{329}$ Gründe dafür sind geo(-politische) Erwägungen, Geheimhaltungsinteressen hinsichtlich eigener nachrichtendienstlicher Erkenntnisquellen sowie technologischer Kompetenzen und nicht zuletzt Bestrebungen, eigene Informationsoperationen durchzuführen. ${ }^{330}$ Insgesamt befinden sich die Staaten noch in einer Reflexionsphase zu der Anwendbarkeit völkerrechtlicher Pflichten in der virtuellen Welt. Aufgrund dieser abwartenden Haltung ist das Völkerrecht in der virtuellen Welt „chronically underenforced“. 331 Dies bedeutet aber nicht, dass eine Möglichkeit zur Reglementierung von Informationsoperationen im Völkerrecht fehlt ${ }^{332}$; die Reaktionen der Staaten beweisen lediglich, dass das Regime der Staatenverantwortlichkeit nicht das richtige Instrument zur Regulierung neuer durch die Globalisierung bedingter Gefährdungslagen

329 So D. Efrony/Y. Shany, A Rule Book on the Shelf?, AJIL 112 (2018), S. 583 ( 583 ff.); vgl. auch R. Hofmann, Modernes Investitionsschutzrecht, in: S. Kadelbach/K. Günther (Hg.), Recht ohne Staat?, 2011, S. 119 (129 f.).

330 D. Efrony/Y. Shany, A Rule Book on the Shelf?, AJIL 112 (2018), S. 583 (634 f.).

331 Id., S. 583 (647).

332 So aber $i d$., S. 583 (648). 
3. Kapitel: Staatenverantwortlichkeit für informationstechnische Systeme

ist. Im Folgenden wird veranschaulicht, dass die Staatenhaftung die völkerrechtliche Lösung für diese Problematik bietet. 


\section{Kapitel: Staatenhaftung für informationstechnische Systeme}

Die Rechtsquellenanalyse hat bewiesen, dass eine völkerrechtliche Staatenhaftung, wie sie von der Völkerrechtskommission ursprünglich konzipiert wurde, keineswegs eine deplatzierte Schwerpunktsetzung im völkerrechtlichen Diskurs um staatliche Einstandspflichten darstellt, sondern eine Lösung für die Regulierung von transnationalen Gefährdungslagen bietet. Eine Gesamtschau der Rechtsquellen verdeutlicht allerdings, dass das Konzept der Staatenhaftung im Umweltvölkerrecht zu verorten ist. Daher ist zunächst die Möglichkeit einer analogen Anwendung der bestehenden umweltvölkerrechtlichen Staatenhaftung auf die virtuelle Welt zu untersuchen (A.), um sodann die konkrete Umsetzung der Staatenhaftung für informationstechnische Systeme beschreiben zu können (B.).

\section{A. Anwendbarkeit von Umweltvölkerrecht in der virtuellen Welt}

Die Analogie ist Ausdruck grundlegender Gerechtigkeitserwägungen, die ein elementarer Bestandteil der Völkerrechtsordnung sind. ${ }^{1}$ Sie beruht auf dem Postulat, dass Gleichartiges rechtlich gleich zu behandeln ist. ${ }^{2}$ In diesem Sinne ist die Analogie besonders geeignet, die Defizite bei

1 Ausführlich zur (umstrittenen) Zulässigkeit der Analogie im Völkerrecht A. Bleckmann, Analogie im Völkerrecht, AVR 17 (1977), S. 161 (161 ff.); ders., Grundprobleme und Methoden des Völkerrechts, 1982, S. 198 ff., 227 ff.; G. Dahm/J. Delbrück/R. Wolfrum, Völkerrecht, Band I/1 Die Grundlagen. Die Völkerrechtssubjekte, 1989, S. 80 ff.; U. Fastenrath, Lücken im Völkerrecht, 1991, S.136; C. L. Riemer, Staatengemeinschaftliche Solidarität in der Völkerrechtsordnung, 2003, S. 294 f.; A. Verdross, Die Verfassung der Völkerrechtsgemeinschaft, 1926, S. 71; S. Vöneky, Analogy in International Law, in: R. Wolfrum (Hg.), MPEPIL 2008, http://www.mpepil.com, Rn. 7 ff. Die Analogie wird unterdessen nicht nur als allgemeiner Rechtsgrundsatz, sondern auch als Rechtsquelle qualifiziert. So $A$. Bleckmann, Analogie im Völkerrecht, AVR 17 (1977), S. 161 (169); ders., Zur Feststellung und Auslegung von Völkergewohnheitsrecht, ZaöRV 37 (1977), S. 504 (516); ders., Die Aufgaben einer Methodenlehre des Völkerrechts, 1978, S. 43; ders., Grundprobleme und Methoden des Völkerrechts, 1982, S. 250.

2 U. Fastenrath, Lücken im Völkerrecht, 1991, S. 134; K. Larenz/C.-W. Canaris, Methodenlehre der Rechtswissenschaft, 1995, S. 202. 
der völkerrechtlichen Regulierung von völkerrechtlich nicht verbotenen Informationsoperationen auszugleichen. Analogiebasis, Analogiebedürfnis und eine ausreichende Vergleichbarkeit zwischen umweltspezifischer und informationstechnischer Problemlage zeigen, dass die Staatenhaftung die rechtliche Lücke in der virtuellen Welt im Wege der Analogie schließt. ${ }^{3}$

\section{Analogiebasis}

Das Völkerrecht kennt zwei Analogiearten, die sich nach der Breite der Analogiebasis unterscheiden. Bei der Einzelanalogie steht eine einzelne Bestimmung zur Verfügung, die als Ausdruck eines allgemeineren Prinzips angesehen wird und sich damit zur Übertragung auf einen bislang ungeregelten Sachverhalt eignet, während bei der Gesamtanalogie aus mehreren Bestimmungen ein übergreifender Rechtsgedanke gewonnen wird, der sich dann auf andere ungeregelte Fälle erstrecken kann. ${ }^{4}$ Die Analogiebasis ergibt sich vorliegend aus den in Kapitel 1 (Staatenhaftung in der Systematik des Völkerrechts) beleuchteten umweltspezifischen Bestimmungen, die sich - wie in Kapitel 2 B. (Konstitutive Elemente der Staatenhaftung) gezeigt - zu einem Konzept der Staatenhaftung für erhebliche grenzüberschreitende Schäden durch völkerrechtlich nicht verbotene Aktivitäten zusammenfassen lassen. Da das Umweltvölkerrecht spezielle Problemlagen erfasst, ist nicht ohne Weiteres evident, dass diese Basis für eine Gesamtanalogie zur Lösung virtueller Konflikte geeignet ist. ${ }^{5}$ Dies ergibt sich aber aus der Erwägung, dass das Umweltvölkerrecht eine ähnliche Entwicklung durchlief, wie sie das Völkerrecht der virtuellen Welt nun durchläuft. ${ }^{6}$ Die ersten Umweltbelastungen verursachten internationale Problemlagen, ohne dass darauf zugeschnittene völkerrechtliche Regeln bereitstanden. Im Gegenteil war ein Rückgriff auf allgemeine Rechtsgrundsätze des Völker-

$3 \mathrm{Zu}$ den Voraussetzungen einer Analogie im Völkerrecht siehe C. L. Riemer, Staatengemeinschaftliche Solidarität in der Völkerrechtsordnung, 2003, S. 295 f.; S. Vöneky, Analogy in International Law, in: R. Wolfrum (Hg.), MPEPIL 2008, http:// www.mpepil.com, Rn. 4 f., 12 ff.

4 A. Bleckmann, Analogie im Völkerrecht, AVR 17 (1977), S. 161 (176); U. Fastenrath, Lücken im Völkerrecht, 1991, S. 134.

5 Vgl. T. Maraubn, Customary Rules of International Environmental Law, in: K. Ziolkowski (Hg.), Peacetime Regime for State Activities in Cyberspace, 2013, S. 465 (480 f.).

6 J. Brunnée/T. Meshel, Teaching an Old Law New Tricks, GYIL 58 (2015), S. 129 (130 f.). 
rechts notwendig. Gleichzeitig erforderten und erfordern internationale Umweltprobleme Reaktionsmaßnahmen, die dem technologischen und gesellschaftlichen Wandel gerecht werden. ${ }^{7}$ Dementsprechend besteht das Umweltvölkerrecht aus einem Konglomerat von völkervertraglichen und völkergewohnheitsrechtlichen Regeln sowie allgemeinen Rechtsgrundsätzen, das den Staaten durch ausreichende Rechtssicherheit ermöglicht, das nationale Regelungsumfeld so zu gestalten, dass Wirtschaft und Industrie funktionsfähig bleiben, und das gleichzeitig flexibel genug ist, um Schutz vor internationalen Umweltbelastungen zu bieten. ${ }^{8}$ Die virtuelle Welt steht ebenso vor unwägbaren und sich stetig weiterentwickelnden Konfliktlagen, deren Reglementierung mit der rasanten technologischen Entwicklung mithalten und gleichzeitig genügend Rechtssicherheit für ihre ökonomische Nutzung bieten muss. ${ }^{9}$ Folglich kann der Regelungsansatz des Umweltvölkerrechts perspektivisch eine passende Regulierung von informationstechnischen Systemen bieten. ${ }^{10}$ An dieser Stelle dienen insbesondere die Umweltgrundsätze als Basis für eine mögliche Analogie, denn diese erweitern aufgrund ihres Prinzipiencharakters ${ }^{11}$ das Regelungspotential des Völkerrechts. ${ }^{12}$ Die Bedeutung der Umweltgrundsätze für eine Analogie wird zudem durch den Umstand gestützt, dass diese eine Art Renaissance erleben, ${ }^{13}$ die mit einer schwindenden Bedeutung von völkervertraglichen Übereinkünften zur Reglementierung neuartiger Umwelt-

7 Id., S. 129 (131); T. Marauhn, Customary Rules of International Environmental Law, in: K. Ziolkowski (Hg.), Peacetime Regime for State Activities in Cyberspace, 2013, S. 465 (481 f.).

8 T. Marauhn, Customary Rules of International Environmental Law, in: K. Ziolkowski (Hg.), Peacetime Regime for State Activities in Cyberspace, 2013, S. 465 (481 f.).

9 Id., S. 465 (481).

10 J. Brunnée/T. Meshel, Teaching an Old Law New Tricks, GYIL 58 (2015), S. 129 (134f.); J. D. Jolley, Attribution, State Responsibility, and the Duty to Prevent Malicious Cyber-Attacks in International Law, 2017, abrufbar unter: http://theses .gla.ac.uk/id/eprint/8452 (geprüft am 15.05.2020), S. 210; T. Maraubn, Customary Rules of International Environmental Law, in: K. Ziolkowski (Hg.), Peacetime Regime for State Activities in Cyberspace, 2013, S. 465 (481). Auch die Experten des Tallinn Manual 2.0 bestimmen die Sorgfaltspflicht im virtuellen Raum explizit in Analogie zum Umweltrecht. Tallinn Manual 2.0, Kommentar zu Regel 6, S. 36 f., Rn. 25.

11 Siehe 1. Kapitel E. V.

12 Vgl. U. Fastenrath, Lücken im Völkerrecht, 1991, S. 125.

13 So J. Brunnée/T. Meshel, Teaching an Old Law New Tricks, GYIL 58 (2015), S. 129 (131). 
probleme einhergeht. ${ }^{14}$ Lösungen für neuartige informationstechnische Konfliktlagen sind ebenso auf der Grundlage von allgemeinen Grundsätzen zu eruieren, denn auch in der virtuellen Welt sind Bestrebungen, völkervertragliche Übereinkünfte zur Reglementierung von Informationsoperationen zu etablieren, aufgrund der divergierenden Interessen der Staaten wenig aussichtsreich. ${ }^{15}$ In diesem Sinne nimmt auch die vom Europarat aufgestellte Arbeitsgruppe zu grenzüberschreitenden Fragen des Internet (Ad-hoc Advisory Group on Cross-border Internet) Bezug auf die Präventionsartikel der Völkerrechtskommission und auf das Schädigungsverbot im Umweltkontext, um staatliche Pflichten in der virtuellen Welt auszubuchstabieren. ${ }^{16}$

Es wird zwar zum Teil bemängelt, dass keine klare Linie zwischen Analogien und allgemeinen Rechtsgrundsätzen gezogen werden könne. ${ }^{17}$ Dies ist aber jedenfalls bei der Frage nach der Übertragbarkeit von rechtlich anerkannten Regelungen auf ungeregelte Bereiche nicht hinderlich. Die beiden völkerrechtlichen Regelinstrumente können sich vielmehr bei der Übertragbarkeit von bestehenden Regeln auf ungeregelte Bereiche argumentativ stützen. Die besprochenen allgemeinen Rechtsgrundsätze untermauern wie gezeigt in ihrer umweltspezifischen Konkretisierung das Gesamtkonzept der Staatenhaftung und dienen hier als Basis für eine Übertragbarkeit des Konzepts auf die virtuelle Welt. ${ }^{18}$

14 Ibid.

15 Vgl. S. Hobe, Die Zukunft des Völkerrechts im Zeitalter der Globalisierung, AVR 37 (1999) S. 253 (267 f.); Ausführlich zu den Gründen, aus denen ein umfassendes Vertragswerk für die virtuelle Welt unwahrscheinlich ist siehe Jack Goldsmith, Cybersecurity Treaties, A Skeptical View, Hoover Institution Task Force on National Security and Law, Future Challenges Essay 9 March 2011, abrufbar unter: http://media.hoover.org/sites/default/files/documents/Future Challenges_Goldsmith.pdf (geprüft am 15.05.2020). R. Crootof spricht nicht nur dem Völkervertragsrecht, sondern auch dem Völkergewohnheitsrecht die Möglichkeit zur umfassenden Regulierung von Aktivitäten in der virtuellen Welt ab. R. Crootof, International Cybertorts, CLR 103 (2018), S. 565 (640 ff.; 642 f.).

16 Council of Europe, Ad-hoc Advisory Group on Cross-border Internet, Interim Report to the Steering Committee on the Media and New Communication Services incorporating Analysis of Proposals for International and Multi-stakeholder Cooperation on Cross-border Internet, H/Inf (2010) 10, Rn. 60 ff.

17 G. Beaucamp/L. Treder, Methoden und Technik der Rechtsanwendung, 2015, S. 67, Rn. 248; N. MacCormick, Legal Reasoning and Legal Theory, 1978, S. 161, 186.

18 Vgl. auch die Ausführungen von C. L. Riemer, der in Bezug auf eine staatengemeinschaftliche Solidarität in der Völkerrechtsordnung in vergleichbarer Weise davon spricht, „daß sich die einzelnen solidartragenden Umweltregime in ihrer 


\section{Bedürfnis der Übertragbarkeit}

Wie den Betrachtungen in Kapitel 3 (Staatenverantwortlichkeit für informationstechnische Systeme) zu entnehmen ist, bestehen eine regelungssystematische Lücke des Völkerrechts mit Blick auf informationstechnische Problemlagen und das Bedürfnis zu ihrer Schließung. Der in der völkerrechtlichen Literatur erfolgte Perspektivenwechsel von der Bedeutung des ius ad bellum und des ius in bello im virtuellen Raum hin zu einer möglichen Reglementierung von Informationsoperationen zu Friedenszeiten, verdeutlicht das Regelungsbedürfnis von Informationsoperationen, die unterhalb der Schwellenvoraussetzungen von Gewaltanwendung bzw. Intervention bleiben und mithin nicht ohne Weiteres als völkerrechtswidrig qualifiziert werden können. Im Schrifttum werden derartige Informationsoperationen als „,below the threshold" cyber operations"19, „malicious

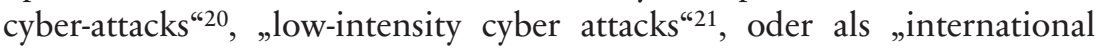
cybertorts" ${ }^{22}$ bezeichnet und dabei mit unterschiedlichen Nuancen von "cyberwarefare and cybercrime“ abgegrenzt. ${ }^{23}$ Diese Arbeit subsumiert derartige Vorgänge unter die Kategorie der völkerrechtlich nicht verbotenen Informationsoperationen. Deren Regelungsbedürfnis ergibt sich, wie so oft im Völkerrecht, aus dem Entwicklungsrückstand der Rechtsordnung gegenüber den modernen Anforderungen einer globalen und vernetzten Welt. ${ }^{24}$ Wie dargelegt, resultieren aus dem heimlichen und ubiquitären Charakter von Informationsoperationen Unsicherheiten mit Blick auf Verursacher und Ablauf von Informationsoperationen. Daher ist eine Rege-

Funktion und Geltung wie die Teilstücke eines Kuchens zu einer ganzen Solidaritäts-,Torte“ ergänzen“ können und mithin grundsätzliche geeignet sind, „eine in Sachbereiche aufgegliederte multiple Basis für eine Analogie“ zu bieten. C. L. Riemer, Staatengemeinschaftliche Solidarität in der Völkerrechtsordnung, 2003, S. 300 .

19 M. N. Schmitt, "Below the Threshold" Cyber Operations, Va JIL 54 (2014), S. 697 (697 ff.).

20 J. D. Jolley, Attribution, State Responsibility, and the Duty to Prevent Malicious Cyber-Attacks in International Law, 2017, abrufbar unter: http://theses.gla.ac.uk/i d/eprint/8452 (geprüft am 15.05.2020), S. 32 f.

21 B. A. Walton, Duties Owed, Yale LJ 126 (2017), S. 1460 (1460 ff.).

22 R. Crootof, International Cybertorts, CLR 103 (2018), S. 565 (565 ff.).

23 Id., S. 565 (592 ff.), mit dem Hinweis, es käme zu Überschneidungen der Kategorien (auf S. 593 f.).

24 Vgl. J. Barboza, The Environment, Risk and Liability in International Law, 2011, S. 153; S. Hobe, Die Zukunft des Völkerrechts im Zeitalter der Globalisierung, AVR 37 (1999) S. 253 (280). 
lungskonzept vorzugswürdig, das nicht die Zurechnung einer konkreten Handlung erfordert, sondern auf einer Risikozuweisung basiert. ${ }^{25}$ Eine staatliche Haftung ist somit grundsätzlich geeignet, den Herausforderungen für die Völkerrechtsordnung durch völkerrechtlich nicht verbotene Informationsoperationen zu begegnen.

Während die meisten Autoren völkerrechtliche Verbotskategorien ausweiten bzw. ergänzen oder umweltrechtliche Grundsätze auf die virtuelle Welt übertragen wollen, deren Verletzung zur Staatenverantwortlichkeit führt, ${ }^{26}$ taucht die Idee der Staatenhaftung für Informationsoperationen nur vereinzelt im Schrifttum auf. ${ }^{27}$ Die völkerrechtliche Bestandskraft der Staatenhaftung ${ }^{28}$ bzw. deren Anwendbarkeit auf die virtuelle Welt ${ }^{29}$ wird dabei vorausgesetzt. Alle erheblich schädigenden Informationsoperationen, die keine Verletzung des Gewaltverbotes oder Interventionsverbotes darstellen, ${ }^{30}$ seien danach dem Anwendungsbereich der Staatenhaftung zu unterstellen. ${ }^{31}$ Die Staatenhaftung sei zwar vornehmlich im Umweltvöl-

25 Vgl. H. Krieger, Krieg gegen anonymous, AVR 50 (2012), S. 1 (6) in Bezug auf das Vorsorgeprinzip.

26 Vgl. hierzu schon die Ausführungen im 3. Kapitel.

27 So etwa bei R. Crootof, International Cybertorts, CLR 103 (2018), S. 565 (565 ff.); J. D. Jolley, Attribution, State Responsibility, and the Duty to Prevent Malicious Cyber-Attacks in International Law, 2017, abrufbar unter: http://theses.gla.ac.uk/i d/eprint/8452 (geprüft am 15.05.2020), S. 204 ff.; B. A. Walton, Duties Owed, Yale LJ 126 (2017), S. 1460 (1460 ff.).

28 J. D. Jolley spricht von einer völkergewohnheitsrechtlich anerkannten Theorie der verschuldensunabhängigen Haftung für hochgefährliche Aktivitäten. J. D. Jolley, Attribution, State Responsibility, and the Duty to Prevent Malicious Cyber-Attacks in International Law, 2017, abrufbar unter: http://theses.gla.ac.uk/id/eprint/ 8452 (geprüft am 15.05.2020), S. 206.

29 R. Crootof, International Cybertorts, CLR 103 (2018), S. 565 (588, 604); B. A. Walton, Duties Owed, Yale LJ 126 (2017), S. 1460 (1499ff.).

30 J. D. Jolley, Attribution, State Responsibility, and the Duty to Prevent Malicious Cyber-Attacks in International Law, 2017, abrufbar unter: http://theses.gla.ac.uk /id/eprint/8452 (geprüft am 15.05.2020), S. 17; B. A. Walton, Duties Owed, Yale LJ 126 (2017), S. 1460 (1466). Insbesondere bei den Ausführungen von R. Crootof verschwimmt aber die Grenze zwischen der staatlichen Einstandspflicht für rechtmäßige und unrechtmäßige Aktivitäten. Vgl. R. Crootof, International Cybertorts, CLR 103 (2018), S. 565 (593).

31 R. Crootof, International Cybertorts, CLR 103 (2018), S. 565 (588 ff., 604); J. D. Jolley, Attribution, State Responsibility, and the Duty to Prevent Malicious Cyber-Attacks in International Law, 2017, abrufbar unter: http://theses.gla.ac.uk/i d/eprint/8452 (geprüft am 15.05.2020), S. 212 ff.; B. A. Walton, Duties Owed, Yale LJ 126 (2017), S. 1460 (1499). 
kerrecht zu verorten, ${ }^{32}$ allerdings bestärke insbesondere die Korfu KanalEntscheidung die Annahme, dass die Staatenhaftung grundsätzlich auch auf andere Bereiche des Völkerrechts anwendbar sei. ${ }^{33}$ Diese Schlussfolgerung geht jedoch aufgrund der gebotenen Differenzierung zwischen Verhinderungs- und Schadensvermeidungspflichten fehl. ${ }^{34}$ Vielmehr ist eine Übertragbarkeit des umweltvölkerrechtlichen Konzepts der Staatenhaftung auf die virtuelle Welt nur dann möglich, wenn sich die virtuelle Welt und die natürliche Umwelt ausreichend gleichen, entsprechende rechtliche Herausforderungen bedingen und vergleichbare Interessen bzw. Werte betreffen. ${ }^{35}$

\section{Vergleichbarkeit}

Die virtuelle Welt und die natürliche Umwelt gleichen sich dadurch, dass sich Cyber- bzw. Umweltschäden, die durch Handlungen in einem Staat begründet sind, in einem anderen Staat verwirklichen können. ${ }^{36}$ Zugleich sind beide Welten internationale Räume ohne physische Staatsgrenzen. ${ }^{37}$

32 J. D. Jolley, Attribution, State Responsibility, and the Duty to Prevent Malicious Cyber-Attacks in International Law, 2017, abrufbar unter: http://theses.gla.ac.uk/ id/eprint/8452 (geprüft am 15.05.2020), S. 204 f.; B. A. Walton, Duties Owed, Yale LJ 126 (2017), S. 1460 (1480); zustimmend R. Crootof, International Cybertorts, CLR 103 (2018), S. 565 (603).

33 B. A. Walton, Duties Owed, Yale LJ 126 (2017), S. 1460 (1483 f.); zustimmend R. Crootof, International Cybertorts, CLR 103 (2018), S. 565 (603 f.); vgl. auch J. D. Jolley, Attribution, State Responsibility, and the Duty to Prevent Malicious Cyber-Attacks in International Law, 2017, abrufbar unter: http://theses.gla.ac.uk/i d/eprint/8452 (geprüft am 15.05.2020), S. 212.

34 Siehe 1. Kapitel B.

35 J. D. Jolley diskutiert ebenfalls eine Vergleichbarkeit zwischen natürlicher Umwelt und virtueller Welt, um eine analoge Anwendung der verschuldensunabhängigen Haftung für hochgefährliche Aktivitäten auf „malicious cyber-attacks“ zu begründen. J. D. Jolley, Attribution, State Responsibility, and the Duty to Prevent Malicious Cyber-Attacks in International Law, 2017, abrufbar unter: http://t heses.gla.ac.uk/id/eprint/8452 (geprüft am 15.05.2020), S. $206 \mathrm{ff}$.

36 J. Brunnée/T. Meshel, Teaching an Old Law New Tricks, GYIL 58 2015), S. 129 (131); J. D. Jolley, Attribution, State Responsibility, and the Duty to Prevent Malicious Cyber-Attacks in International Law, 2017, abrufbar unter: http://these s.gla.ac.uk/id/eprint/8452 (geprüft am 15.05.2020), S. 207; T. Stein/T. Maraubn, Völkerrechtliche Aspekte von Informationsoperationen, ZaöRV 60 (2000), S. 1 (21).

37 J. Bäumler, Das Schädigungsverbot im Völkerrecht, 2017, S. 299; J. D. Jolley, Attribution, State Responsibility, and the Duty to Prevent Malicious Cyber-Attacks in 
Dementsprechend ist der Schutz von Gemeinschaftsgütern, für deren Erhalt Staaten eine gemeinsame, geteilte Verantwortung tragen, sowohl im Kontext der Umwelt, etwa mit Blick auf den Weltraum, die Hohe See, die Antarktis, als auch hinsichtlich der virtuellen Welt von Bedeutung. ${ }^{38}$ Staaten haben nicht nur ein Interesse am Erhalt natürlicher Ressourcen, sondern ebenso an der Funktionalität des virtuellen Raums. ${ }^{39}$ So ist das Internet als gemeinsame bzw. geteilte Ressource zu qualifizieren, die eine nachhaltige und gerechte Nutzung gebietet. ${ }^{40}$ Ähnlich wie natürliche Ressourcen haben technische Ressourcen einen essenziellen Wert und Nutzen für die Menschheit. Kommunikationsinfrastrukturen sind notwendig für sozialwirtschaftliche Beziehungen und sonstige ökonomische Betätigungen. ${ }^{41}$ Diese sind, wie im Umweltkontext etwa das Klima oder die Biodi-

International Law, 2017, abrufbar unter: http://theses.gla.ac.uk/id/eprint/8452 (geprüft am 15.05.2020), S. 207.

$38 \mathrm{~J}$. Bäumler spricht von einer möglichen Analogie zu Gemeinschaftsgütern. J. Bäumler, Das Schädigungsverbot im Völkerrecht, 2017, S. 299; siehe auch $P$. W. Franzese, Sovereignty in Cyberspace, AF Law Review 64 (2009), S. 1 (14 ff.); J. D. Jolley, Attribution, State Responsibility, and the Duty to Prevent Malicious Cyber-Attacks in International Law, 2017, abrufbar unter: http://theses.gla.ac.uk /id/eprint/8452 (geprüft am 15.05.2020), S. 209 f.; M. C. Kettemann, Das Internet als internationales Schutzgut, ZaöRV 72 (2012), S. 469 (476f.); K. Ziolkowski, General Principles of International Law as Applicable in Cyberspace, in: dies. (Hg.), Peacetime Regime for State Activities in Cyberspace, 2013, S. 135 (167). Es gibt zwar Stimmen, die sich gegen die Sinnhaftigkeit dieses Rechtsinstituts im Cyberkontext aussprechen. So J. Brunnée/T. Meshel, Teaching an Old Law New Tricks, GYIL 58 2015), S. 129 (133). Allerdings sollen an dieser Stelle zunächst die Parallelen zwischen der virtuellen und natürlichen Welt dargelegt werden, die sich anhand der diskutierten Lösungsansätze herauskristallisieren lassen.

39 Das Interesse am Bestehen der Funktionalitäten des Internets korrespondiere mit einer erga omnes-Pflicht des potenziellen Störer-Staates hinsichtlich der Stabilität, Integrität und Funktionalität des Internets. So M. C. Kettemann, Das Internet als internationales Schutzgut, ZaöRV 72 (2012), S. 469 (478) unter Hinweis auf Prinzip 3 und 5 der Erklärung des Ministerkomitees des Europarates über die Grundsätze der Internet Governance, angenommen bei der 1121. Sitzung der Stellvertreter der Minister am 21.09.2011.

40 Id., S. 469 (467 f., 479 f.); T. Stein/T. Maraubn, Völkerrechtliche Aspekte von Informationsoperationen, ZaöRV 60 (2000), S. 1 (21 f.); K. Ziolkowski, General Principles of International Law as Applicable in Cyberspace, in: dies. (Hg.), Peacetime Regime for State Activities in Cyberspace, 2013, S. 135 (180).

41 So T. Marauhn, Customary Rules of International Environmental Law, in: K. Ziolkowski (Hg.), Peacetime Regime for State Activities in Cyberspace, 2013, S. 465 (467), siehe auch J. D. Jolley, Attribution, State Responsibility, and the Duty to Prevent Malicious Cyber-Attacks in International Law, 2017, abrufbar unter: http://theses.gla.ac.uk/id/eprint/8452 (geprüft am 15.05.2020), S. 207. 
versität, zu schützen. ${ }^{42}$ Die Vergleichbarkeit von virtueller und natürlicher Welt wird durch den Umstand bestärkt, dass sowohl das Internet als auch Umweltbestandteile wie die Luft allgegenwärtig und gleichzeitig nicht greifbar sind. ${ }^{43}$

Darüber hinaus sind Umweltverschmutzungen, so wie „Verschmutzungen" der virtuellen Welt auch, nicht ohne Weiteres zu identifizieren und zu stoppen und machen damit staatliche Kooperations- und Einstandspflichten unumgänglich. ${ }^{44}$ Die grenzüberschreitenden Auswirkungen auf die Umwelt und auf die virtuelle Welt erfordern dabei gleichermaßen einen Ausgleichsmechanismus zwischen der Wahrung von Souveränitätsrechten einerseits und der Sicherstellung zwischenstaatlicher Verpflichtungen andererseits. ${ }^{45}$

Ferner entstehen sowohl informationstechnische als auch umweltbezogene Problemlagen primär durch nicht-staatliche und weniger durch staatliche Akteure, ${ }^{46}$ so dass eine völkerrechtliche Verbindung zwischen staatlichen Pflichten und nicht-staatlichen Aktivitäten notwendig ist. Eine solche Verbindung kann weder durch wissenschaftliche noch durch technische Zurechnung erreicht werden; in der vielschichtigen natürlichen bzw. vir-

42 O. Gross, Cyber Responsibility to Protect, Cornell ILJ 48 (2015), S. 481 (495). Die Verletzung der staatlichen Schutzpflicht hinsichtlich der Funktionalität, Stabilität und Integrität des Internets könne zur Staatenverantwortlichkeit führen. So M. C. Kettemann, Das Internet als internationales Schutzgut, ZaöRV 72 (2012), S. 469 (480).

43 So J. Bäumler spricht von einer möglichen Analogie zu Gemeinschaftsgütern. J. Bäumler, Das Schädigungsverbot im Völkerrecht, 2017, S. 299.

$44 \mathrm{~J}$. Healey/H. Pitts, International Environmental Legal Norms to Cyber Statecraft, I/S: A Journal of Law and Policy for the Information Society 8 (2012), S. 356 (384 f.); vgl. auch J. Bäumler, Das Schädigungsverbot im Völkerrecht, 2017, S. 299 bezüglich der Anwendung des Schädigungsverbotes auf schädigende Handlungen im Internet.

45 J. Healey/H. Pitts, International Environmental Legal Norms to Cyber Statecraft, I/S: A Journal of Law and Policy for the Information Society 8 (2012), S. 356 (384).

46 J. Brunnée/T. Meshel, Teaching an Old Law New Tricks, GYIL 58 2015), S. 129 (131); J. D. Jolley, Attribution, State Responsibility, and the Duty to Prevent Malicious Cyber-Attacks in International Law, 2017, abrufbar unter: http://theses .gla.ac.uk/id/eprint/8452 (geprüft am 15.05.2020), S. 219; T. Maraubn, Customary Rules of International Environmental Law, in: K. Ziolkowski (Hg.), Peacetime Regime for State Activities in Cyberspace, 2013, S. 465 (482). 
tuellen Welt kann nur ein regulatorischer Ansatz in Form von Risikozuteilung zielführend sein. ${ }^{47}$

Im Ergebnis können die wissenschaftlichen und technischen Unsicherheiten und die potenziell schädigenden Auswirkungen, die sich in beiden Welten zeigen, nur durch normativ festgelegte Regulierungskriterien bewältigt werden. ${ }^{48}$

\section{Ergebnis}

Trotz der aufgezeigten Parallelen zur Umwelt gibt es durchaus Aspekte in der virtuellen Welt, die spezifische Regulierungen erfordern. Zu Bedenken sind etwa die vornehmlich nicht greifbaren Auswirkungen von Informationstechnik, die durch die technischen Möglichkeiten bedingte Zeit-Raum-Kompression sowie der anonyme und ubiquitäre Charakter der virtuellen Welt. Nichtsdestoweniger hält das konsolidierte Umweltvölkerrecht Mechanismen bereit, die auch im Kontext informationstechnischer Systeme funktionieren. ${ }^{49}$

\section{B. Umsetzung der Staatenhaftung für informationstechnische Systeme}

Die folgende Darstellung einer Staatenhaftung für informationstechnische Systeme wird zeigen, dass die informationstechnischen Hürden für das Völkerrecht durch existente normative Instrumente überwindbar und erhebliche grenzüberschreitende Schäden durch Informationsoperationen nicht etwa "lost in translation" sind. ${ }^{50}$

47 Vgl. T. Marauhn, Customary Rules of International Environmental Law, in: K. Ziolkowski (Hg.), Peacetime Regime for State Activities in Cyberspace, 2013, S. 465 (482).

48 Ibid.

49 Id., S. 465 (483 f.) mit dem Hinweis, dass beim Rekurs auf das Umweltvölkerrecht die fehlende Territorialität in der virtuellen Welt Berücksichtigung finden muss.

50 J. E. Messerschmidt, Hackback, Col. JTL 52 (2013), S. 275 (296). 
I. Anwendungsbereich

Eine Staatenhaftung für informationstechnische Systeme kommt nur dann in Betracht, wenn diesen das Risiko eines erheblichen grenzüberschreitenden Schadens immanent ist.

\section{Schaden}

Der herkömmliche Schadensbegriff setzt zunächst physische Auswirkungen auf Mensch, Eigentum oder Umwelt voraus. ${ }^{51}$ Allerdings führen Informationsoperationen wie gezeigt nicht nur zur Zerstörung von physischen Komponenten der virtuellen Welt, beispielsweise von Computern, sondern zu neuartigen digitalen, immateriellen und wirtschaftlichen Schäden, wie Zerstörung von Datensystemen, Datenverlust, Veröffentlichung privater und vertraulicher Informationen, Umsatz- und Imageverluste von Unternehmen oder Kosten für die Abwehr und Beseitigung von Informationsoperationen. ${ }^{52}$ Die Beschränkung der Staatenhaftung auf die physische Qualität einer Aktivität und deren Schadensfolgen ist indes nicht mehr zeitgemäß, ${ }^{53}$ denn in vielen Bereichen potenzieren sich die Möglichkeiten zwischenstaatlicher Beeinträchtigungen durch die fortschreitende Technisierung, aufgrund der zunehmenden wirtschaftlichen Interdependenzen und der Globalisierung im Allgemeinen. ${ }^{54}$ Passt man das Konzept der Staatenhaftung dem Zeitalter des Internets an, müssen demnach auch digitale Schäden in den Anwendungsbereich fallen. ${ }^{55}$ Denn erst eine Neudefinition des Schadensbegriffs ermöglicht es, neuartigen Gefährdungslagen zu begegnen. ${ }^{56}$ Der Begriff des Schadens ist hierfür auch entwick-

51 X. Hanqin, Transboundary Damage in International Law, 2003, S. 5.

52 Siehe 3. Kapitel A. und B.

53 S. Townley, The Rise and Risk in International Law, Chi. JIL 18 (2018), S. 594 $(627,631)$.

54 J. Bäumler, Das Schädigungsverbot im Völkerrecht, 2017, S. 5, 276.

55 So J. D. Jolley, Attribution, State Responsibility, and the Duty to Prevent Malicious Cyber-Attacks in International Law, 2017, abrufbar unter: http://theses.gla .ac.uk/id/eprint/8452 (geprüft am 15.05.2020), S. 137; vgl. auch S. Townley, The Rise and Risk in International Law, Chi. JIL 18 (2018), S. 594 (631 f.).

56 R. M. Bratspies/R. A. Miller, Introduction, in: dies. (Hg.), Transboundary Harm in International Law, 2006, S. 1 (8); S. Townley, The Rise and Risk in International Law, Chi. JIL 18 (2018), S. 594 (627). Nach B. A. Walton bestünde hingegen kein Bedürfnis für eine Neudefinition, da es schon gar nicht auf die physische Qualität ankomme, wenn eine gewisse Erheblichkeit der nicht-physischen Auswirkungen 
lungsoffen, dies zeigt die Haftungsstudie der Völkerrechtskommission. Auch wenn der Schutz der Umwelt im Vordergrund stand, beabsichtigte sie keine Begrenzung auf Fragen ökologischer Natur oder Kategorien physischer Gebietsnutzung. ${ }^{57}$ Zudem greifen Mechanismen der Staatenhaftung bereits im Wirtschaftsvölkerrecht, mithin für Beeinträchtigungen wirtschaftlicher Interessen. ${ }^{58}$ Bemerkenswert ist, dass schon in den Trail Smelter-Entscheidungen wirtschaftliche Erwägungen Gegenstand der Schadensbestimmung waren. So bezog sich die Staatenhaftung im Trail SmelterFall nicht nur auf physische Schäden an der Umwelt, sondern auch auf finanzielle Verluste in der Landwirtschaft aufgrund ausgebliebener Erträge sowie sonstiger Wertverluste der Grundstücke und auch die Kosten für die Beseitigung von Schäden spielten eine Rolle. ${ }^{59}$ Im Ergebnis sind im Sinne eines dynamischen Schadensbegriffs auch digitale, immaterielle und wirtschaftliche Posten bei der Schadensbestimmung zu berücksichtigen.

Außerdem muss der Schaden durch die fragliche Informationsoperation grenzüberschreitend sein, das heißt auf fremdstaatlichem Territorium oder in anderen Gebieten unter der Hoheitsgewalt eines fremden Staates eintreten. ${ }^{60}$ Wie bereits dargelegt, wird die virtuelle Welt, wie auch Bereiche der Umwelt, als Gemeinschaftsgut qualifiziert, welches sich außerhalb einzelstaatlicher Hoheitsgewalt befinde. Dies steht der Anwendbarkeit der Staatenhaftung nicht entgegen, da sich jedenfalls die physischen Kompo-

gegeben sei. In diesem Sinne seien bereits nicht-physische Schäden durch Strahlenbelastungen als Folge von Atomtests Gegenstand von völkerrechtlichen Streitfällen zu Kompensationsansprüchen. B. A. Walton, Duties Owed, Yale LJ 126 (2017), S. 1460 (1505) unter Bezugnahme auf ILC Secretariat, Survey of liability regimes relevant to the topic of international liability for injurious consequences arising out of acts not prohibited by international law (international liability in case of loss from transboundary harm arising out of hazardous activities), YBILC 2004-II/1, UN Doc. A/CN.4/543, S. 79, und IGH, Nuclear Tests (Australia v. France), Interim Protection, Order of 22 June 1973, ICJ Reports 1973, S. 99 (99 ff.). Nach J. D. Jolley seien die Schäden durch Informationsoperationen zwar digital, besäßen aber zugleich aufgrund der monetären Verluste physische Qualität. J. D. Jolley, Attribution, State Responsibility, and the Duty to Prevent Malicious Cyber-Attacks in International Law, 2017, abrufbar unter: http://theses.gla.a c.uk/id/eprint/8452 (geprüft am 15.05.2020), S. 138.

57 Sonderberichterstatter R. Q. Quentin-Baxter, Fourth report on international liability for injurious consequences arising out of acts not prohibited by international law, YBILC 1983-II/1, UN.Doc. A/CN.4/373, S. 205 f., Rn. 17.

58 Siehe 1. Kapitel D. I. 4.

59 Trail Smelter Arbitration (United States v. Canada), Award of 16 April 1938 and 11 March 1941, 3 UNRIAA (1941), S. 1905 (1925 f.).

60 Vgl. X. Hanqin, Transboundary Damage in International Law, 2003, S. 8. 
nenten informationstechnischer Systeme und auch die Verursacher bzw. Opfer von Informationsoperationen auf Gebieten befinden, die der Souveränität von Staaten unterliegen. ${ }^{61}$ Ferner bezieht sich die Staatenhaftung für informationstechnische Systeme mit Blick auf ihre praktische Umsetzbarkeit nicht auf diffuse allgemeine grenzübergreifende Schäden, die etwa durch die Überbeanspruchung der globalen Ressource Internet (information pollution) entstehen und das Gemeinschaftsgut "virtueller Raum" gefährden, sondern auf Informationsoperationen, die zu konkreten nachteiligen Auswirkungen auf andere Staaten führen. So qualifiziert auch die vom Europarat aufgestellte Arbeitsgruppe zu grenzüberschreitenden Fragen des Internet (Ad-hoc Advisory Group on Cross-border Internet) das Internet einerseits als Gemeinschaftsgut, ${ }^{62}$ stellt aber andererseits zur Begründung staatlicher Pflichten im virtuellen Raum auf nachteilige Auswirkungen auf andere Staaten ab, und nicht auf Schäden am virtuellen Raum im Allgemeinen. ${ }^{63} \mathrm{Im}$ Rahmen der folgenden rechtlichen Erwägungen sind also kollektive Rechte und Pflichten von Staaten hinsichtlich der Stabilität, Integrität und Funktionalität der globalen Ressourcen der virtuellen Welt von untergeordneter Bedeutung. ${ }^{64}$ Der Grundsatz angemessener Nutzung geteilter Ressourcen hingegen liefert auch in der virtuellen Welt Anknüpfungspunkte für eine Staatenhaftung für informationstechnische Systeme, da er Ressourcen betrifft, die der Hoheitsgewalt von Staaten unterliegen und gleichzeitig grenzüberschreitender Natur sind..$^{65}$

Letztlich müssen Parameter gefunden werden, die einen Schaden durch eine Informationsoperation als erheblich qualifizieren. ${ }^{66}$ Zur Abgrenzung von einer hinzunehmenden Beeinträchtigung wird ein Vergleich mit der territorialen Integrität von Staaten vorgeschlagen. Sind die betroffenen Interessen von ähnlicher Bedeutung wie die territoriale Integrität eines Staa-

61 Siehe hierzu 1. Kapitel E. I.

62 Council of Europe, Ad-hoc Advisory Group on Cross-border Internet, Interim Report to the Steering Committee on the Media and New Communication Services incorporating Analysis of Proposals for International and Multi-stakeholder Cooperation on Cross-border Internet, H/Inf (2010) 10, Rn. 14.

63 Id., Rn. 69.

64 Siehe hierzu M. C. Kettemann, Das Internet als internationales Schutzgut, ZaöRV 72 (2012), S. 469 (469 ff.).

65 Vgl. J. Brunnée/T. Meshel, Teaching an Old Law New Tricks, GYIL 58 (2015), S. 129 (154 f.); M. Herdegen, Possible Legal Framework and Regulatory Models for Cyberspace, GYIL 85 (2015), S. 169 (179).

66 Vgl. X. Hanqin, Transboundary Damage in International Law, 2003, S. 7 f. mit dem Hinweis, dass die Erheblichkeitsschwelle ziel- und kontextabhängig zu bestimmen ist. 
tes, dann ist eine Erheblichkeit anzunehmen. ${ }^{67}$ Informationsinfrastrukturen erfassen alle Bereiche des gesellschaftlichen Lebens und sind für die Staaten von essenzieller Bedeutung. Gleichzeitig bedingen sie durch die Allgegenwärtigkeit der Vernetzung eine besondere Verwundbarkeit. ${ }^{68}$ Die Beeinträchtigung von Informationsinfrastrukturen ist demnach mit Auswirkungen auf die territoriale Integrität vergleichbar. ${ }^{69}$ Schwierig gestaltet sich die Erheblichkeitsbeurteilung bei immateriellen Schäden, etwa als Konsequenz von Wahleinmischung oder Ausspähung Privater durch Informationsoperationen, da derartige Beeinträchtigungen schwer zu beziffern sind. In diesem Zusammenhang findet sich der Vorschlag, dass für die Frage nach der Erheblichkeit entscheidend sei, inwiefern die Opferstaaten selbst zur Vermeidung von vergleichbaren schädigenden Aktivitäten beitragen würden. ${ }^{70}$ Die Erheblichkeit von Schäden durch Aktivitäten, die auf internationaler Ebene regelmäßig praktiziert und geduldet würden, sei zu verneinen. Dementsprechend falle Spionage mangels Erheblichkeit der daraus resultierenden Schäden aus dem Anwendungsbereich der Staatenhaftung. ${ }^{71}$ Allerdings unterliegt herkömmliche Spionage in der physischen Welt aufgrund möglicher strafrechtlicher Verfolgung der Spione durch den Opferstaat gewissen Begrenzungen. Die virtuelle Welt hingegen bietet nicht nur eine größere Angriffsfläche für Spionage, sondern dämmt zugleich das Risiko des Entdecktwerdens sowie der Strafverfolgung für Spione ein, die von ihrem Heimatstaat aus die Informationsausspähung und Informationsausbeutung durchführen können. ${ }^{72}$ Die entsprechenden Beispiele aus der internationalen Praxis verdeutlichen, dass die Spionage in der virtuellen Welt Dimensionen erreicht, die nicht mehr hinnehmbar sind und zunehmend zu zwischenstaatlichen Spannungen führen. Die Staatenhaftung bietet mit Blick auf die erheblichen Auswirkungen von Informationsspionage in der virtuellen Welt eine geeignete Reaktionsmög-

67 J. Bäumler, Das Schädigungsverbot im Völkerrecht, 2017, S. 276.

68 C. Schaller, Internationale Sicherheit und Völkerrecht im Cyberspace, SWP-Studie 18/2014, S. 5; vgl. auch S. P. Kanuk, Information Warfare, Harv. ILJ 37 (1996), S. 272 (285).

69 T. Stein/T. Marauhn, Völkerrechtliche Aspekte von Informationsoperationen, ZaöRV 60 (2000), S. 1 (21).

70 B. A. Walton, Duties Owed, Yale LJ 126 (2017), S. 1460 (1506) unter Bezugnahme auf Präventionsartikel 10 lit. d und lit. f; vgl. auch J. D. Jolley, Attribution, State Responsibility, and the Duty to Prevent Malicious Cyber-Attacks in International Law, 2017, abrufbar unter: http://theses.gla.ac.uk/id/eprint/8452 (geprüft am 15.05.2020), S. 187.

71 B. A. Walton, Duties Owed, Yale LJ 126 (2017), S. 1460 (1506).

72 R. Crootof, International Cybertorts, CLR 103 (2018), S. 565 (607). 
lichkeit durch Opferstaaten, ohne dabei einen Teufelskreis von Retorsionen in Gang zu setzen. Hilfreich für die Erheblichkeitsbeurteilung sind auch Überlegungen, die effektive Souveränitätsausübung eines Staates als schützenswertes Rechtsgut zu kategorisieren. ${ }^{73}$ Demnach haftet ein Staat, wenn er die Souveränitätsausübung eines anderen Staates in einer Weise behindert, dass letzterem die Abwehr bzw. Beseitigung dieser Beeinträchtigung unmöglich oder nur unter erheblichen eigenen Verlusten möglich ist. ${ }^{74}$ Ein Vergleich zu nationalem Deliktsrecht zeigt schließlich, dass eine Entschädigung für immaterielle Schäden durchaus möglich ist und im Grunde die Anspruchssteller selbst entscheiden, wann ein Schaden erheblich genug ist, um seine Kompensation gerichtlich einzufordern, und wann Sachverständige zur Beurteilung der Erheblichkeit herangezogen werden..$^{75}$ So wird sich die Erheblichkeitsschwelle auf internationaler Ebene durch die Anwendung auf konkrete Fälle konkretisieren. ${ }^{76}$

\section{2. (Hoch-)Gefährliche Aktivität}

Das Konzept der Staatenhaftung bezieht sich auf (hoch-)gefährliche Aktivitäten. Die Kriterien zur Beurteilung der Gefährlichkeit einer Aktivität sind nicht statisch und müssen - sowie der Schadensbegriff auch - dem Wandel der Zeit entsprechend angepasst werden. ${ }^{77}$ Demnach können auch informationstechnische Aktivitäten dem Anwendungsbereich der Staatenhaftung unterfallen. Auch für andere neuartige Gefährdungslagen, beispielsweise im Zusammenhang mit dem Einsatz von autonomen Waffensystemen, werden entsprechende Ansätze diskutiert. ${ }^{78}$ Dabei darf der Anwendungsbereich der Staatenhaftung aber nicht zu weit gefasst werden, um ihren Sinn und Zweck nicht zu unterlaufen. Das Konzept der Staa-

73 J. Bäumler, Das Schädigungsverbot im Völkerrecht, 2017, S. 276 unter Bezugnahme auf M. Ronzoni, The Global Order, Philosophy \& Public Affairs 37 (2009), S. 229 (248 f.).

74 Vgl. id., S. 277.

75 R. Crootof, International Cybertorts, CLR 103 (2018), S. 565 (608f.).

76 Id., S. 565 (608) zum Vorschlag zur Etablierung eines Expertengremiums, das entsprechende Ansprüche evaluiert (S. $367 \mathrm{ff}$.).

77 Vgl. J. Barboza, The Environment, Risk and Liability in International Law, 2011, S. 9; S. Townley, The Rise and Risk in International Law, Chi. JIL 18 (2018), S. 594 ( 627 ff.).

78 N. Bhuta/S.-E. Pantazopoulous, Autonomy and uncertainty, in: N. Bhuta/S. Beck/ R. Geiß/H.-Y. Liu/C. Kreß (Hg.), Autonomous Weapons Systems, 2016, S. 284 (291). 
tenhaftung zielt, wie gezeigt, auf eine ex ante Regulierung von Gefahrenlagen, um daraus resultierenden Unsicherheiten zu begegnen und einen gerechten Interessenausgleich der betroffenen Staaten schon im Vorhinein zu gewährleisten. Schließlich erfasst der Anwendungsbereich der Staatenhaftung keine schädigenden Einzelakte, sondern gemeinnützige und/oder wirtschaftlich vorteilhafte Aktivitäten, bei denen der Schadenseintritt allenfalls eine ungewollte Nebenwirkung darstellt. ${ }^{79}$

Vor diesem Hintergrund können einzelne Informationsoperationen nicht als Anknüpfungspunkt für die Staatenhaftung in der virtuellen Welt dienen. Informationsoperationen sind so vielgestaltig, dass eine Kategorisierung in gefährlich bzw. hochgefährlich nicht ohne Weiteres möglich ist. So besteht zwar bei Informationsausbeutung regelmäßig die hohe Wahrscheinlichkeit erheblicher finanzieller bzw. wirtschaftlicher Schäden oder erheblicher Beeinträchtigungen des Rechts auf Privatheit, während bei Informationsangriffen grundsätzlich die Möglichkeit katastrophaler Schäden, etwa durch die Störung kritischer Infrastrukturen, gegeben ist. ${ }^{80}$ Allerdings ist eine ex post facto Analyse unabdingbar, um die Schadenswahrscheinlichkeit und den Schadensumfang einer bestimmten Informationsoperation beurteilen zu können. ${ }^{81}$ Das Risiko jeder einzelnen Informationsoperation kann nicht ex ante im Sinne eines gerechten Interessenausgleichs reguliert werden. Informationsoperationen stellen im Übrigen auch keine vorrangig gemeinnützige oder wirtschaftlich vorteilhafte Aktivität dar; Informationsoperationen sind vielmehr Einzelakte, die einen Schaden intendieren.

Zur Beurteilung der Gefährlichkeit ist daher auf informationstechnische Systeme im Allgemeinen abzustellen. Der Betrieb dieser Systeme ist der Gesellschaft und auch der Staatengemeinschaft primär von Nutzen. Durch die Erlaubnis zum Betrieb informationstechnischer Systeme - und nicht durch die Erlaubnis zu jeder einzelnen Informationsoperation - schaffen Staaten erst das Risiko, welches sie regulieren und für dessen Realisierung - in Gestalt einer Informationsoperation - sie am Ende einstehen müssen. ${ }^{82}$ Vergleichbar mit dem Betrieb (hoch-)gefährlicher Anlagen ist auch

79 Siehe 1. Kapitel A. II. 1. a) und 2. Kapitel B. I. 2.

80 Siehe 3. Kapitel A. und B.

81 Vgl. G. Handl, State Liability for Accidental Transnational Environmental Damage by Private Persons, AJIL 74 (1980), S. 525 (555).

82 H. Krieger stellt vergleichbar hinsichtlich staatlicher Sorgfalts-, Informations- und Warnpflichten fest: „Nach dem Gedanken des Vorsorgeprinzips muss der Blick weg vom Verursacher auf den Betreiber gerichtet werden, d.h. auf alle Staaten, die die Gefährdungslage für die internationale Sicherheit schaffen, weil sie das In- 
hier das Risiko im Vorhinein bestimmbar und eine ex ante Regulierung der Gefährdung auf Grundlage eines gerechten Interessenausgleichs möglich. Der Betrieb informationstechnischer Systeme ist grundsätzlich jeder Art von Informationsoperation zugänglich und ist im Ergebnis als hochgefährlich zu kategorisieren, da die auch noch so geringe Wahrscheinlichkeit katastrophaler Schäden durch diese Systeme begründet wird. ${ }^{83}$

\section{Kausalität}

Der Betrieb informationstechnischer Systeme auf staatlichem Territorium bzw. unter staatlicher Hoheitsgewalt muss ursächlich für den Schaden sein, damit die Staatenhaftung greifen kann. Im Umweltkontext ist Kausalität in der Regel durch eine physische Verbindung zwischen einer Aktivität und ihren Folgen gekennzeichnet. ${ }^{84}$ Bei einer direkten Beziehung zwischen den informationstechnischen Systemen und den schädigenden Auswirkungen kann nichts anderes gelten. ${ }^{85}$ Informationstechnische Systeme können nämlich ohne Weiteres Schäden verursachen, wenn sich das ihnen immanente Risiko - in Gestalt einer Informationsoperation - realisiert.

Während für die Zurechnung völkerrechtswidriger Informationsoperationen eine klare und überzeugende Beweislage gefordert wird, ${ }^{86}$ reicht für den Kausalitätsnachweis bei Schäden durch völkerrechtlich nicht verbotene Informationsoperationen im Sinne des Vorsorgegrundsatzes schon die Möglichkeit der Schadensverursachung aus. ${ }^{87}$ Diese unterschiedlichen Beweisanforderungen spiegeln sich auch in der Rechtsprechung des IGH, wonach eindeutigere Nachweise gefordert werden, je einschneidender der

ternet ohne hinreichende technische Sicherung nutzen.“ H. Krieger, Krieg gegen anonymous, AVR 50 (2012), S. 1 (16); vgl. auch S. Erichsen, Das Liability-Projekt der ILC, ZaöRV 51 (1991), S. 94 (109 f.).

83 Vgl. O. Gross, Cyber Responsibility to Protect, Cornell ILJ 48 (2015), S. 481 (482f.).

84 X. Hanqin, Transboundary Damage in International Law, 2003, S. 4.

85 J. D. Jolley, Attribution, State Responsibility, and the Duty to Prevent Malicious Cyber-Attacks in International Law, 2017, abrufbar unter: http://theses.gla.ac.uk /id/eprint/8452 (geprüft am 15.05.2020), S. 137 f.; vgl. auch B. A. Walton, Duties Owed, Yale LJ 126 (2017), S. 1460 (1465 Fn. 25).

86 Siehe 3. Kapitel D. I.

87 Vgl. 2. Kapitel B. I. 3. 
Eingriff ist. ${ }^{88}$ In der Regel ist die geografische Herkunft von Informationsoperationen anhand der Internetadressen ermittelbar und mithin auf die jeweiligen informationstechnischen Systeme des Ursprungsstaates zurückführbar. ${ }^{89}$ Dementsprechend wird der geforderte Kausalitätsnachweis in diesen Fällen gelingen.

Bei technischen Rückverfolgungsproblemen, etwa im Fall von Onion Routing, hilft die erleichterte Beweisführung, die auch für den Nachweis der Kenntnis des Ursprungsstaates von völkerrechtswidrigen Informationsoperationen gilt. ${ }^{90}$ Danach ist der Kontext der Informationsoperation maßgeblich. Als Indizien für die Herkunft einer Informationsoperation können beispielsweise der Nutzen einer Informationsoperation für einen

88 IGH, Corfu Channel Case (United Kingdom of Great Britain and Northern Ireland v. Albania), Judgment of 9 April 1949, ICJ Reports 1949, S. 4 (17); IGH, Oil Platforms (Islamic Republic of Iran v. United States of America), Judgment of 6 November 2003, ICJ Reports 2003, Separate Opinion of Judge Higgins, S. 225 (234 Rn.33); IGH, Application of the Convention on the Prevention and Punishment of the Crime of Genocide (Bosnia and Herzegovina v. Serbia and Montenegro), Judgment of 26 February 2007, ICJ Reports 2007, S. 43 (119 Rn. 181, 129 Rn. 208 f., 130 Rn. 210); IGH, Application of the Convention on the Prevention and Punishment of the Crime of Genocide (Croatia v. Serbia), Judgment of 3 February 2015, ICJ Reports 2015, S. 3 (74 Rn. 178).

$89 \mathrm{~J}$. Healey/H. Pitts, Applying International Environmental Legal Norms to Cyber Statecraft, I/S: A Journal of Law and Policy for the Information Society, 8 (2012), S. 356 (379).

90 Siehe 3. Kapitel E. III. Die Bezugnahme auf die erleichtere Beweisführung im Zusammenhang mit völkerrechtswidrigen Informationsoperationen ändert aber nichts daran, dass der Nachweis für die Kenntnis von völkerrechtswidrigen Informationsoperationen keine begründeten Zweifel lassen darf, während für den Kausalitätsnachweis im Kontext von völkerrechtlich nicht verbotenen Informationsoperationen die Möglichkeit der Schadensverursachung ausreicht. 
bestimmten Staat ${ }^{91}$ oder die mangelnde Kooperationsbereitschaft eines Staates bei der Aufklärung der Sachlage ${ }^{92}$ herangezogen werden. ${ }^{93}$

Ähnlich wie im Rahmen der staatlichen Pflichten zur Verhinderung von bzw. zum Schutz vor Informationsoperationen stellt sich aber bei der Kausalitätsprüfung die Problematik von Bots oder Datenpaketen, die erst im Zielstaat erhebliche Schäden verursachen. Wie beschrieben, ist im Rahmen der staatlichen Verhinderungs- bzw. Schutzpflicht eine Verantwortlichkeit des Ursprungsstaates nur zu bejahen, wenn die Bots bzw. Datenpakete auf staatlichem Hoheitsgebiet bzw. unter staatlicher Hoheitsgewalt für sich allein die notwendige Intensität erreichen, um als völkerrechtswidriges Verhalten qualifiziert werden zu können. Ist eine völkerrechtswidrige Informationsoperation nur durch das gesamte Botnet bzw. Datenwerk begründet, entsteht keine Verhinderungs- bzw. Schutzpflicht hinsichtlich der einzelnen Bots bzw. Datenpakete. ${ }^{94}$ Denn Staaten werden den Vorwurf völkerrechtswidrigen Verhaltens von sich weisen und nicht gewillt sein, eine Verantwortlichkeit für gesamte Botnets bzw. Datenwerke zu akzeptieren, wenn sich ihr eigener kausaler Beitrag lediglich auf einzelne Bots bzw. Datenpakete beschränkt, welche für sich genommen keine völkerrechtswidrige Informationsoperation darstellen. Eine andere Betrachtungsweise

91 F. Hare, The Significance of Attribution to Cyberspace Coercion, in: C. Czosseck/R. Ottis/K. Ziolkowski (Hg.), 2012 4th International Conference on Cyber Conflict, 2012, S. 125 (136 ff.).

92 J. D. Jolley, Attribution, State Responsibility, and the Duty to Prevent Malicious Cyber-Attacks in International Law, 2017, abrufbar unter: http://theses.gla.ac .uk/id/eprint/8452 (geprüft am 15.05.2020), S. 201 ff. unter Bezugnahme auf IGH, Corfu Channel Case (United Kingdom of Great Britain and Northern Ireland v. Albania), Judgment of 9 April 1949, ICJ Reports 1949, S. 4 (18 f.); vgl. R. K. Knake, Untangling Attribution: Moving to Accountability in Cyberspace, Statement Before the Subcommittee on Technology and Innovation, Committee on Science and Technology, United States House of Representatives 2nd Session, 111th Congress, 2010, abrufbar unter: https://www.cfr.org/sites/default/files/pdf/2 010/07/Knake\%20-Testimony\%20071510.pdf (geprüft am 15.05.2020), S. 8.

93 M. C. Libicki lehnt dagegen diese Anknüpfungspunkte für eine Zurechnung $\mathrm{ab}$, da diese missbraucht und mithin irreführend sein könnten. Aussagekräftiger seien Methode und Ausführung der Informationsoperation sowie das darin zum Ausdruck kommende Wissen über die Zielsysteme. M. C. Libicki, Cyberdeterrence and Cyberwar, 2009, S. 44 ff., 47 f. Die kontextbezogene Zurechnung mag für das Regime der Staatenverantwortlichkeit ungenügend sein, ist aber im Bereich der Staatenhaftung zulässig, da hier allgemein weniger strenge Anforderungen gelten. Vgl. in Bezug auf politische Zurechnung K. Ziolkowski, Confidence Building Measures for Cyberspace, in: dies. (Hg.), Peacetime Regime for State Activities in Cyberspace, 2013, S. 553 (556).

94 Siehe 3. Kapitel E. I. 
ist aber bei der Staatenhaftung gerechtfertigt, da Staaten hier kein Fehlverhalten ihrerseits eingestehen müssen, sondern lediglich für den eigenen kausalen Beitrag zur Verursachung eines Schadens durch völkerrechtskonformes Verhalten Entschädigung leisten müssen. ${ }^{95}$ Wie gezeigt, kommt hier der Verursachergrundsatz zum Tragen, nach dem schon einzelne Verursachungsbeiträge die Kausalitätsanforderungen erfüllen. ${ }^{96}$ In Haftungsübereinkommen finden sich bereits entsprechende Regeln für eine gesamtschuldnerische Haftung der beteiligten Staaten, wenn beispielsweise zwei Weltraumgegenstände zur Schädigung eines Drittstaates beitragen (Art. IV Weltraumhaftungsübereinkommen). Zwar besteht für den in Anspruch genommenen Staat die Möglichkeit zur Schadloshaltung bei den anderen mitverursachenden Staaten, so dass auch eine Gesamthaftung in Betracht käme. Allerdings dürfte dieser Ansatz außerhalb völkervertraglicher Übereinkünfte auf Ablehnung der Staaten stoßen, ${ }^{97}$ weil sie möglicherweise Hindernisse bei der Regressnahme befürchten. Daher ist in dieser Konstellation eine anteilige Haftung der jeweiligen Ursprungsstaaten interessengerecht und vorzugswürdig. ${ }^{98}$

\section{Schadensvermeidung und Schadenskompensation}

Die völkerrechtliche Diskussion über die Verantwortung von Staaten für Informationsoperationen entfernt sich sinnvollerweise von dem Erfordernis völkerrechtlicher Verbotstatbestände hin zum Erfordernis allgemei-

95 M. B. Akehurst, International liability for injurious consequences arising out of acts not prohibited by international law, NYIL 16 (1985), S. 3 (15) unter Bezugnahme auf Sonderberichterstatter R. Q. Quentin-Baxter, Preliminary report on international liability for injurious consequences of acts not prohibited by international law, YBILC 1980-II/1, UN Doc. A/CN.4/334 and Add.1 and 2, S. 263, Rn. 56; siehe auch J. E. Noyes/B. D. Smith, State Responsibility and the Principle of Joint and Several Liability, YIL 13 (1988), S. 225 (264). Selbst wenn man die notwendige Kausalität nur dann bejahen wollte, wenn Bots auf dem Hoheitsgebiet oder unter der Hoheitsgewalt eines Staates für sich genommen einen erheblichen Schaden verursachen, ist jedenfalls - im Gegensatz zur Schwelle der Völkerrechtswidrigkeit - die Schwelle zur Erheblichkeit schneller erreicht, so dass die Staatenhaftung auch hier greifen kann.

96 2. Kapitel B. I. 3.

97 Vgl.J. Bäumler, Das Schädigungsverbot im Völkerrecht, 2017, S. 282.

98 Ibid. 
ner Schadensvermeidungspflichten für die virtuelle Welt. ${ }^{99}$ Themen wie Kriegsführung und Gegenmaßnahmen in der virtuellen Welt rücken derweil in den Hintergrund. Die vom Europarat aufgestellte Arbeitsgruppe zu grenzüberschreitenden Fragen des Internet (Ad-hoc Advisory Group on Cross-border Internet) betonte überdies, dass sich die staatliche Pflicht zur Verhinderung und Regulierung grenzüberschreitender Störungen des Internets grundsätzlich von der Frage der Staatenverantwortlichkeit für völkerrechtswidriges Verhalten unterscheidet und legte den Fokus ihrer Untersuchungen auf verfahrensbezogene Schadensvermeidungspflichten und Sorgfaltsstandards in der virtuellen Welt. ${ }^{100}$ Die diskutierten Regelungsansätze bilden die Grundlage für die Konkretisierung von Schadensvermeidungspflichten in der virtuellen Welt und liefern mithin Anknüpfungspunkte für einen gerechten Schadensausgleich für Schäden durch völkerrechtlich nicht verbotene Informationsoperationen.

\section{Schadensprävention}

Die Staaten müssen alle möglichen und zumutbaren Maßnahmen ergreifen, um erheblichen grenzüberschreitenden Schäden durch die Nutzung von informationstechnischen Systemen zuvorzukommen oder zumindest deren konkretes Risiko zu minimieren. ${ }^{101}$

Die effektive Umsetzung von konkreten Präventionspflichten in der virtuellen Welt ist wiederum von der schädigenden Informationsoperation im Einzelfall abhängig.

Prävention in der virtuellen Welt ist offenkundig bei staatseigenen oder staatlich befürworteten Informationsoperationen möglich. Da es aber gerade das Ziel von Informationsoperationen ist, grenzüberschreitende

99 J. Brunnée/T. Meshel, Teaching an Old Law New Tricks, GYIL 58 (2015), S. 129 (132f.) m.w.N.

100 Council of Europe, Ad-hoc Advisory Group on Cross-border Internet, Interim Report to the Steering Committee on the Media and New Communication Services incorporating Analysis of Proposals for International and Multi-stakeholder Cooperation on Cross-border Internet, H/Inf(2010)10, Rn. 85.

101 Vgl. 2. Kapitel B. II.; Council of Europe, Ad-hoc Advisory Group on Cross-border Internet, Interim Report to the Steering Committee on the Media and New Communication Services incorporating Analysis of Proposals for International and Multi-stakeholder Cooperation on Cross-border Internet, $\mathrm{H} / \operatorname{Inf}(2010) 10$, Rn. 72, 74; R. Kolb, Reflections on Due Diligence Duties and Cyberspace, GYIL 58 (2015), S. 113 (123). 
Schäden zu verursachen, werden Ursprungsstaaten keine Maßnahmen zur Schadensprävention oder Risikominimierung ergreifen. Staaten könnten allenfalls Anlass zur Risikoanalyse, Regulierung und Kontrolle der (hoch-)gefährlichen Informationsoperation haben, um möglichen Kollateralschäden an Drittstaaten (Spillover-Effekte) zuvorzukommen oder deren Risiko zu begrenzen. Kooperationspflichten wird der Ursprungsstaat in aller Regel aufgrund von Geheimhaltungsinteressen weder mit Zielstaaten noch mit potenziell betroffenen Drittstaaten umsetzen.

Bei nicht-staatlichen Informationsoperationen besteht zwar kein staatliches Geheimhaltungsinteresse, so dass eine Kooperation mit betroffenen Staaten durch Benachrichtigung, Information, Warnung und Konsultation möglich ist. ${ }^{102} \mathrm{Maßnahmen}$ zur Schadensprävention können allerdings aufgrund der Zeit-Raum-Kompression in der virtuellen Welt einerseits und des heimlichen Charakters von Informationsoperationen andererseits in aller Regel nicht ergriffen werden, bevor eine nicht-staatliche Informationsoperation durchgeführt wird und mithin grenzüberschreitende Schäden eingetreten sind oder ein konkretes Risiko gegeben ist. ${ }^{103}$ Neben den technischen Hürden bei der Entdeckung von nicht-staatlichen Informationsoperationen ist überdies zu beachten, dass die in Betracht kommenden Maßnahmen der Schadensprävention, namentlich Überwachungsund Kontrollmechanismen, erheblichen menschenrechtlichen Bedenken begegnen. ${ }^{104}$ Denn anders als im Kontext umweltbelastender Aktivitäten können in der virtuellen Welt Präventionspflichten nicht nach Größe und Ausmaß der gefährlichen Aktivitäten begrenzt werden. Smartphones,

102 Eine Mitteilungs- und Warnpflicht bei Computerviren wird teilweise in Analogie zu den Vorgaben der Weltgesundheitsorganisation in Fällen von Infektionskrankheiten vorgeschlagen. So C. Walter, Cyber Security als Herausforderung für das Völkerrecht, Juristenzeitung 14 (2015), S. 685 (689); siehe auch R. Geiß/H. Lahmann, Freedom and Security in Cyberspace, in: K. Ziolkowski (Hg.), Peacetime Regime for State Activities in Cyberspace, 2013, S. 621 (656).

103 Die Experten des Tallinn Manual 2.0 gehen noch weiter und stellen nicht nur die effektive Umsetzbarkeit von Präventionspflichten in der virtuellen Welt in Frage, sondern verneinen schon das Bestehen von Präventionspflichten und schließen allgemeine Vorsorgemaßnahmen kategorisch aus. Tallinn Manual 2.0, Kommentar zu Regel 7, S. 44 f., Rn. 7 ff. Dies liegt daran, dass die Experten zum einen Kenntnis des Ursprungsstaates von der Informationsoperation voraussetzen (Kommentar zu Regel 7, S. 45, Rn. 9), und zum anderen daran, dass sie keine klare Trennung zwischen Verhinderungs- und Schadensvermeidungspflichten vornehmen (Kommentar zu Regel 7, S. 44 f., Rn. 7).

104 Vgl. Tallinn Manual 2.0, Kommentar zu Regel 7, S. 45, Rn. 8; O. Dörr, Obligations of the State of Origin of a Cyber Security Incident, GYIL 58 (2015), S. 87 (95). 
Computer und sonstige Geräte sind gleichermaßen geeignet, erhebliche grenzüberschreitende Schäden durch Informationsoperationen zu verursachen. ${ }^{105}$ Eine breit angelegte Überwachung aller informationstechnischen Aktivitäten auf staatlichem Territorium bzw. unter staatlicher Hoheitsgewalt wäre mit menschenrechtlichen Verpflichtungen zum Schutz der Privatheit unvereinbar. ${ }^{106}$ Folglich führen die spezifischen Charakteristika von Informationsoperationen nur zu beschränkten Möglichkeiten der Schadensprävention in der virtuellen Welt.

\section{Schadensvorsorge}

Während staatliche Präventionspflichten von der konkreten Gefahr durch spezifische Informationsoperationen abhängig sind, werden durch den Vorsorgegrundsatz die staatlichen Pflichten weit ins Vorfeld einer Gefahr verlagert. Der Vorsorgegrundsatz gelangt zur Anwendung, wenn Ursachenzusammenhänge und Auswirkungen von zu regulierenden Vorgängen ungewiss sind. Vorsorgemaßnahmen sind schon zu ergreifen, bevor ein konkretes Schadensrisiko entsteht. ${ }^{107}$ Dies ist insbesondere in der virtuellen Welt, die durch wissenschaftliche und technische Unsicherheiten bei der Regulierung informationstechnischer Aktivitäten gekennzeichnet ist, sinnvoll. ${ }^{108}$ Staaten müssen von vornherein strategische, politische, organisatorische, administrative und legislative Vorkehrungen für den Betrieb informationstechnischer Systeme treffen. ${ }^{109}$ Anleihen für konkrete Vorsorgemaßnahmen lassen sich dabei dem Umweltkontext entnehmen.

105 So C. Walter, Cyber Security als Herausforderung für das Völkerrecht, Juristenzeitung 14 (2015), S. 685 (688).

$106 \mathrm{~J}$. Healey/H. Pitts, Applying International Environmental Legal Norms to Cyber Statecraft, I/S: A Journal of Law and Policy for the Information Society 8 (2012), S. 356 (365 f.); A. Reinisch/M. Beham, Mitigating Risks, GYIL 58 (2015), S. 101 (112); C. Walter, Cyber Security als Herausforderung für das Völkerrecht, Juristenzeitung 14 (2015), S. 685 (688).

107 Siehe 1. Kapitel E. II. 3.

108 H. Krieger, Krieg gegen anonymous, AVR 50 (2012), S. 1 (5f.); T. Marauhn, Customary Rules of International Environmental Law, in: K. Ziolkowski (Hg.), Peacetime Regime for State Activities in Cyberspace, 2013, S. 465 (475); S.-H. Schulze, Cyber-„War“ - Testfall der Staatenverantwortlichkeit, 2015, S. 215.

109 K. Ziolkowski, General Principles of International Law as Applicable in Cyberspace, in: dies. (Hg.), Peacetime Regime for State Activities in Cyberspace, 2013, S. 135 (169). 


\section{a) Kooperation}

Wie im Umweltkontext bilden Kooperationspflichten auch in der virtuellen Welt einen zentralen Anknüpfungspunkt zur Schadensvorsorge. Effektive Sicherheitsvorkehrungen sind nur durch Information, Warnung, Benachrichtigung und Konsultationen möglich. ${ }^{110}$ Der Zugang zu sicherheitsrelevanten Informationen kann durch Meldepflichten über feindliche Aktivitäten und durch das Einrichten von Frühwarnverfahren gewährleistet werden. ${ }^{111}$ Die Effektivität solcher Maßnahmen zeigt sich am Beispiel der Informationsausspähungen, die oftmals für zukünftige Manipulationsund Sabotageakte genutzt werden. ${ }^{112}$ Aufgrund einer entsprechenden Information kann eine vorsorgliche Abschaltung oder Neukonfiguration kompromittierter Systeme erfolgen, um weiteren nachteiligen Auswirkungen zuvorkommen. ${ }^{113}$ Aber auch Informationsaustauch über den Umgang mit Präzedenzfällen kann zur Reifung von erprobten Reaktionsmaßnahmen auf Informationsoperationen beitragen. ${ }^{114}$ Schließlich ist die multinationale und organisationsübergreifende Koordination zur Bewältigung virtueller Bedrohungslagen zu verbessern und Dialoge mit Stakeholdern aus dem Bereich der Cybersicherheit sind zu fördern. ${ }^{115}$ Diese konkreten Pflichten sind für informationstechnische Systeme noch nicht etabliert, können aber als politisch verbindliche Richtlinien langfristig zu Gewohnheitsrecht erstarken. ${ }^{116}$ Entsprechende Forderungen finden sich bereits im internationalen Diskurs zur Cybersicherheit. ${ }^{117}$

110 J. Brunnée/T. Meshel, Teaching an Old Law New Tricks, GYIL 58 (2015), S. 129 (144 ff., 149); H. Krieger, Krieg gegen anonymous, AVR 50 (2012), S. 1 (20).

111 H. Krieger, Krieg gegen anonymous, AVR 50 (2012), S. 1 (18 f.).

112 Id., S. 1 (19).

113 S. Gaycken, Die vielen Plagen des Cyberwar, in: R. Schmidt-Radefeldt/C. Meissler (Hg.), Automatisierung und Digitalisierung des Krieges, 2012, S. 89 (110).

114 Vgl. C. Walter, Cyber Security als Herausforderung für das Völkerrecht, Juristenzeitung 14 (2015), S. 685 (689).

115 Ausführlich zu den Regulierungsansätzen internationaler und regionaler Organisationen im Bereich Cybersicherheit und den derzeitigen Schwächen L. Areng, International Cyber Crisis Management and Conflict Resolution Mechanisms, in: K. Ziolkowski (Hg.), Peacetime Regime for State Activities in Cyberspace, 2013, S. 565 (565 ff.).

116 C. Schaller, Internationale Sicherheit und Völkerrecht im Cyberspace, SWP-Studie 18/2014, S. 6; K. Ziolkowski, Confidence Building Measures for Cyberspace, in: dies. (Hg.), Peacetime Regime for State Activities in Cyberspace, 2013, S. 553 (560 f.).

117 Ausführlich hierzu siehe S.-H. Schulze, Cyber-„War“ - Testfall der Staatenverantwortlichkeit, 2015, S. 170 ff., 199 f., 219. 
b) Risikoanalyse

Eine Risikoanalyse von möglicherweise schädigenden Aktivitäten ist nicht nur im Umweltkontext sinnvoll, sondern auch in der virtuellen Welt denkbar. Eine Art Cyberumweltverträglichkeitsprüfung kann die möglichen Auswirkungen des Betriebs informationstechnischer Systeme bewerten und unter Umständen als Vorsorgemaßnahme eine Nutzungsbeschränkung oder gar ein Verbot gebieten. ${ }^{118}$ In diese Richtung gehen auch Überlegungen, die einen Vergleich zu Massenvernichtungswaffen und Atomwaffen vornehmen. ${ }^{119}$ Entsprechende Diskussionen zu einer Vorsorgeverpflichtung von Staaten, die Entwicklung, Herstellung und Anwendung von Schadprogrammen und -befehlen (sogenannte Informationswaffen) zu untersagen, wurden schon 1998 im Rahmen der Vereinten Nationen geführt. ${ }^{120}$ Im Ergebnis ist dieser Ansatz aber nicht umsetzbar, da Informationsoperationen eben mittels alltäglicher Informationstechnik erfolgen, ihren Ausgangspunkt überall haben und über unterschiedliche Pfade laufen können. ${ }^{121}$ Ein Betriebsverbot informationstechnischer Systeme ist im Ergebnis unrealistisch und auch eine Nutzungsbeschränkung ist in der virtuellen Welt kaum zu kontrollieren. ${ }^{122}$

\section{c) Beste Schutzpraktiken}

Eine Umsetzung des Vorsorgegrundsatzes in Gestalt von besten Schutzpraktiken ist in der virtuellen Welt von besonderem Interesse, da hier - wie auch im Umweltkontext - eine Kombination von Einsatzmitteln,

118 Nach C. Walter sprechen Gründe des Freiheitsschutzes gegen eine „Cyberverträglichkeitsprüfung“. C. Walter, Cyber Security als Herausforderung für das Völkerrecht, Juristenzeitung 14 (2015), S. 685 (688 f.).

119 Siehe hierzu H. Krieger, Krieg gegen anonymous, AVR 50 (2012), S. 1 (7 f.); S.H. Schulze, Cyber-„War“ - Testfall der Staatenverantwortlichkeit, 2015, S. $216 \mathrm{ff}$.

120 VN-Generalversammlung, First Committee, Fifty-third session, 30. September 1998, Role of science and technology in the context of international security, disarmament and other related fields, Appendix Developments in the field of information and telecommunications in the context of international security Russian Federation: draft resolution, UN Doc. A/C.1/53/3, S. 3 f., Abs. 7, Rn.3, lit. c.

121 H. Krieger, Krieg gegen anonymous, AVR 50 (2012), S. 1 (8); zustimmend S.-H. Schulze, Cyber-, War“ - Testfall der Staatenverantwortlichkeit, 2015, S. 217.

122 Vgl. S.-H. Schulze, Cyber-„War“ - Testfall der Staatenverantwortlichkeit, 2015, S. $217 \mathrm{f}$. 
wie Kontrollmaßnahmen und weitergehende Strategieentwicklung, zielführend ist. ${ }^{123}$ Wenngleich der Betrieb informationstechnischer Systeme im Allgemeinen aufgrund von menschenrechtlichen Bedenken keiner umfassenden Kontrolle unterstellt werden kann, ist dies für besonders schützenswerte informationstechnische Systeme denkbar. Demnach ist zunächst eine Unterscheidung zwischen kritischen Infrastrukturen, wie etwa von Krankenhäusern, Atomkraftwerken oder militärischen und staatlichen Einrichtungen, und sonstigen Informationsinfrastrukturen vorzunehmen. ${ }^{124}$ Erstere erfordern besondere Vorsorge vor Gefahren durch Informationsoperationen, da zum einen verheerende Folgen durch Angriffe drohen und zum anderen aufgrund der globalen Vernetzung Angriffe auf kritische Infrastrukturen in einem Staat zu empfindlichen Auswirkungen auf interdependente Systeme und Netzwerke in anderen Staaten führen können. ${ }^{125}$ Die Begrenzung auf militärische und kritische zivile Infrastrukturen rechtfertigt eine staatliche Überwachung und ist durch eine entsprechende Entnetzung auch technisch möglich. ${ }^{126}$ Die Entkopplung von zivilen Netzwerken und ausschließliche Nutzung von stark kontrollierten internen Netzen ist in Anbetracht der besonderen Gefährdungslage auch trotz der hohen Umstellungskosten nicht unverhältnismäßig, ${ }^{127}$ zumal die Neuorientierung schrittweise erfolgen kann.

Schließlich erfordern die besten Schutzpraktiken aber zusätzlich, den Selbstschutz informationstechnischer Systeme im Allgemeinen zu erhöhen

123 Vgl. mit Blick auf Kapazitätenaufbau im Bereich Cybersicherheit H. TiirmaaKlaar, Cyber Diplomacy, in: K. Ziolkowski (Hg.), Peacetime Regime for State Activities in Cyberspace, 2013, S. 509 (523 ff.).

124 Vgl. H. Krieger, Krieg gegen anonymous, AVR 50 (2012), S. 1 (17f.). Zum Begriff der kritischen Infrastrukturen S. Gaycken/M. Karger, Entnetzung statt Vernetzung, Paradigmenwechsel bei der IT-Sicherheit, Multimedia und Recht 1 (2011), S. 3 (5); S.-H. Schulze, Cyber-„War“ - Testfall der Staatenverantwortlichkeit, 2015, S. $19 \mathrm{ff}$.

125 S.-H. Schulze, Cyber-,War“ - Testfall der Staatenverantwortlichkeit, 2015, S. 219.

126 S. Gaycken/M. Karger, Entnetzung statt Vernetzung, Multimedia und Recht 1 (2011), S. 3 (8); H. Krieger, Krieg gegen anonymous, AVR 50 (2012), S. 1 (17 f.); S. Schmahl, Cybersecurity, in: N. Dethloff/G. Nolte/A. Reinisch (Hg.), Freiheit und Regulierung in der Cyberwelt, 2016, S. 159 (182) m.w.N.; S.-H. Schulze, Cyber-, War“ - Testfall der Staatenverantwortlichkeit, 2015, S. 203 f.

127 H. Krieger, Krieg gegen anonymous, AVR 50 (2012), S. 1 (17 f.). 
und fortwährend dem Stand der Technik anzupassen ${ }^{128}$ sowie Notfallpläne für informationstechnische Vorfälle aufzustellen. ${ }^{129}$

\section{Schadensminderung}

Auch wenn aufgrund der wissenschaftlichen und technischen Unsicherheiten selten Maßnahmen zur Schadensprävention vor Beginn einer (nichtstaatlichen) Informationsoperation ergriffen werden können, sind jedenfalls nach Eintritt eines Vorfalls und bei andauernden Geschehnissen sowohl vom Ursprungs- als auch vom Opferstaat Maßnahmen zur Schadensminderung zu ergreifen. Die Schadensminderungspflicht stellt eine Verlängerung der Schadenspräventionspflicht in die Phase der andauernden bzw. beendeten Informationsoperation dar. In dieser Phase ist eine konkrete Gefährdungslage durch eine Informationsoperation gegeben und die Auswirkungen sind in aller Regel bestimmbar. ${ }^{130}$ Darüber hinaus kann der Ursprung der Informationsoperation auf spezifische informationstechnische Systeme nun eingegrenzt oder gar zurückgeführt werden, so dass entsprechende Maßnahmen zur Beseitigung oder Begrenzung negativer Auswirkungen durchführbar sind. ${ }^{131}$

Zuvorderst sind aber auch hier menschenrechtliche Schutzgewährleistungen und staatliche Bestrebungen zur Beendigung schadhafter Informationsoperationen - durch Abwägung zwischen Eingriff und Legitimität im Einzelfall - in Einklang zu bringen. ${ }^{132}$ Wenn Staaten die eigene Infrastruktur sichern und abschalten, sind etwaige Auswirkungen auf die In-

128 S. Gaycken/M. Karger, Entnetzung statt Vernetzung, Multimedia und Recht 1 (2011), S. 3 (5); H. Krieger, Krieg gegen anonymous, AVR 50 (2012), S. 1 (19); S.-H. Schulze, Cyber-, ,War“ - Testfall der Staatenverantwortlichkeit, 2015, S. 204.

129 S. Gaycken/M. Karger, Entnetzung statt Vernetzung, Multimedia und Recht 1 (2011), S. 3 (5); O. Gross, Cyber Responsibility to Protect, Cornell ILJ 48 (2015), S. 481 (501 f.).

130 Vgl. C. Walter, Cyber Security als Herausforderung für das Völkerrecht, Juristenzeitung 14 (2015), S. 685 (689 f.) unter Hinweis auf Präventionsartikel, Kommentar zu Art. 12, S. 165, Abs. 12.

131 In diese Richtung zielt auch die Sorgfaltsregel des Tallinn Manual 2.0, wonach alle möglichen Maßnahmen zu ergreifen sind, um eine Informationsoperation zu beenden (Regel 7 Tallinn Manual 2.0).

132 Die Meinungsäußerungsfreiheit im Internet kann beispielsweise nur aufgrund des aus Menschenrechtskodifikationen bekannten dreiteiligen Tests eingeschränkt werden. VN Human Rights Council, Sonderberichterstatter Frank La Rue, Report on the Promotion and Protection of the Right to Freedom of Opin- 
formations- und Kommunikationsinfrastruktur des eigenen Staates sowie von Drittstaaten so gering wie möglich zu halten. ${ }^{133}$ Denn jeder Mensch hat „das Recht auf Meinungsfreiheit und freie Meinungsäußerung; dieses Recht schließt die Freiheit ein, Meinungen ungehindert anzuhängen sowie über Medien jeder Art und ohne Rücksicht auf Grenzen Informationen und Gedankengut zu suchen, zu empfangen und zu verbreiten“. ${ }^{134}$ Zudem hat ein vernünftiger Interessenausgleich mit Blick auf Natur, Ausmaß und Reichweite des (potenziellen) Schadens beim Ursprungsstaat einerseits und beim betroffenen Staat andererseits zu erfolgen. ${ }^{135}$ Schließlich dürfen Selbsthilfemaßnahmen, wie sogenannte Hackbacks, welche darauf gerichtet sind die Systeme des Angriffsursprungs außer Dienst zu stellen oder zu zerstören, zumeist aufgrund rechtlicher Hürden nicht ergriffen werden. Derartige Informationsoperationen verletzen nämlich unter Umständen Schutzgewährleistungen des humanitären Völkerrechts und/oder nationale Vorschriften. ${ }^{136}$ Diese Defizite können zum Teil durch verfahrensbezogene Pflichten aufgefangen werden. ${ }^{137}$ Damit wird deutlich, dass in dieser Phase zwischenstaatliche Kooperationspflichten wie Informationsaustauch, Mitteilung und Warnung sowie Absprachen zu Abhilfemaßnahmen, essenziell für eine Schadensbegrenzung sind. ${ }^{138}$

ion and Expression, UN Doc. A/HRC/17/27, 16.05.2011, S. 19, Rn. 69, S. 20, Rn. 72.

133 Tallinn Manual 2.0, Kommentar zu Regel 7, S. 49 f., Rn. 25.

134 Art. 19 Allgemeine Erklärung der Menschenrechte; siehe auch Art. 19 Internationaler Pakt über Bürgerliche und Politische Rechte; Art. 10 Europäische Menschenrechtskonvention; Art. 13 Amerikanische Menschenrechtskonvention; Art. 9 Afrikanische Menschenrechtskonvention.

135 Tallinn Manual 2.0, Kommentar zu Regel 7, S. 49 f., Rn. 25.

136 O. Gross, Cyber Responsibility to Protect, Cornell ILJ 48 (2015), S. 481 (501) m.w.N.

137 Vgl. O. Dörr, Obligations of the State of Origin of a Cyber Security Incident, GYIL 58 (2015), S. 87 (96).

138 K. Ziolkowski, General Principles of International Law as Applicable in Cyberspace, in: dies. (Hg.), Peacetime Regime for State Activities in Cyberspace, 2013, S. 135 (186). Es wird indes vorgeschlagen, dass sich diese Kooperationspflicht auch auf die Ermittlung und Verfolgung von Urhebern schadhafter Informationsoperationen erstreckt. J. Brunnée/T. Meshel, Teaching an Old Law New Tricks, GYIL 58 (2015), S. 129 (145) unter Bezugnahme auf M. J. Sklerov, Solving the Dilemma of State Responses to Cyberattacks, Military Law Review 201 (2009), S. 1 (62) und W. Heintschel von Heinegg, Territorial Sovereignty and Neutrality in Cyberspace, International Law Studies 89 (2013), S. 123 (135). Allerdings geht es im Rahmen der Staatenhaftung um Schadensvermeidungsund Kompensationspflichten und nicht um Verhinderungs- bzw. Schutzpflichten mit Blick auf kriminelle Aktivitäten, die eine strafrechtliche Verfolgung 


\section{Schadenskompensation}

Angesichts der dargelegten Schwierigkeiten bei der Schadensvermeidung in der virtuellen Welt kann es regelmäßig dennoch zu einem erheblichen grenzüberschreitenden Schaden durch völkerrechtlich nicht verbotene Informationsoperationen kommen. Daher ist der Staat, auf dessen Territorium bzw. unter dessen Hoheitsgewalt eine Informationsoperation stattfindet, die einen erheblichen Schaden in einem anderen Staat verursacht, zur Kompensation der entstandenen Schäden verpflichtet. Bei der Bemessung der Kompensationspflicht werden die ergriffenen Maßnahmen zur Schadensvermeidung, das heißt Schadensprävention, Schadensvorsorge und Schadensminderung, Berücksichtigung finden. Der Opferstaat darf nicht auf den Kosten sitzen bleiben, gleichzeitig hat ein gerechter Interessenausgleich zwischen den betroffenen Staaten zu erfolgen. ${ }^{139}$ So ist bei der Bemessung der Kompensationspflicht auch der Grad der Beteiligung des Opferstaates an der Konfliktlage in die Abwägung miteinzubeziehen. Die staatliche Einstandspflicht für reaktive völkerrechtmäßige Hackbacks ${ }^{140}$ auf feindliche Informationsoperationen ist beispielsweise im Umfang zu begrenzen, wenn deren Auswirkungen nicht außer Verhältnis stehen.

\section{Gebotene Sorgfalt}

Die gebotene Sorgfalt zur Schadensvermeidung richtet sich auch in der virtuellen Welt nach der konkreten Gefährdungslage und dem anzulegenden Sorgfaltsmaßstab. Grundsätzlich gilt, dass gefährliche Aktivitäten, denen das Risiko erheblicher Schäden immanent ist, einen flexibleren Sorgfaltsstandard erfordern als hochgefährliche Aktivitäten, die zu katastrophalen Schäden führen können. ${ }^{141}$ Informationsangriffe gebieten demnach die Beachtung eines strikten Sorgfaltsstandards, während bei Informationsausbeutungen flexible Sorgfalt gilt.

Dies entbehrt aber nicht, den Kontext, Sinn und Zweck der gebotenen Sorgfalt zur Schadensvermeidung sowie die Umstände des konkreten Einzelfalls bei der Bestimmung des Sorgfaltsstandards zu berücksichtigen. So

und Bestrafung von Tätern erfordern. Vgl. in Bezug auf die Sorgfaltspflicht des Tallinn Manual 2.0, Kommentar zu Regel 7, S. 48, Rn. 21.

139 Siehe 2. Kapitel B. II.

140 Siehe hierzu J. E. Messerschmidt, Hackback, Col. JTL 52 (2013), S. 275 (275 ff.).

141 Siehe 2. Kapitel B. III. 
sind aufgrund des absichtlichen Charakters von Informationsoperationen unterschiedliche Sorgfaltsmaßstäbe bei staatlichen und staatlich befürworteten Informationsoperationen einerseits und nicht-staatlichen Informationsoperationen andererseits anzulegen. ${ }^{142}$

Bei staatlichen oder staatlich befürworteten Informationsoperationen ist angesichts der intendierten Schäden eine Missachtung der gebotenen Sorgfalt - unabhängig vom anzulegenden Maßstab - indiziert, so dass den Staat diesbezüglich in jedem Fall eine vollumfängliche Einstandspflicht trifft. Nichts anderes gilt für Kollateralschäden. Bedingt durch die internationale Vernetzung und den ubiquitären Charakter des Internets können selbst strategisch und technisch durchdachte Informationsoperationen zu nicht in unmittelbarem Zusammenhang mit dem Ziel der Operation stehenden, aber dennoch billigend in Kauf genommen Begleitschäden führen. ${ }^{143}$ Folglich erübrigt sich hier eine Differenzierung der Sorgfaltsanforderungen nach gefährlichen und hochgefährlichen Informationsoperationen.

Bei nicht-staatlichen Informationsoperationen ist nicht die Absicht oder Fahrlässigkeit der nicht-staatlichen Akteure entscheidend, sondern die Missachtung der gebotenen Sorgfalt bei der Schadensvermeidung durch den Staat, auf dessen Hoheitsgebiet bzw. unter dessen Hoheitsgewalt informationstechnische Systeme für die Informationsoperation genutzt werden. Allerdings ist eine ex ante Bestimmung der Gefährdungslage nach Art und Ausmaß der nicht-staatlichen Informationsoperation nicht möglich und eine ex post Bewertung zur Festlegung des Sorgfaltsstandards wäre der Rechtssicherheit abträglich. ${ }^{144}$ Dementsprechend ist für die Bestimmung der Sorgfaltsanforderungen auf das Risiko, das informationstechnischen Systemen im Allgemeinen immanent ist, abzustellen. Die Staaten müssen bei deren Nutzung durch nicht-staatliche Akteure die gebotene Sorgfalt zur Schadensvermeidung walten lassen. ${ }^{145}$ Bei der nicht-staatlichen Nutzung informationstechnischer Systeme besteht die Wahrscheinlichkeit katastrophaler Schadensfolgen, da diese Systeme sowohl gefährlichen In-

142 Zwar spielt die Absicht bei der Staatenhaftung selbst keine Rolle, sie ist aber durchaus bei der Bewertung der gebotenen Sorgfalt von Interesse. B. A. Walton, Duties Owed, Yale LJ 126 (2017), S. 1460 (1503 f., 1499).

143 A. McKay/J. Neutze/P. Nicholas/K. Sullivan, International Cyber Security Norms, Reducing Conflict in an Internet- Dependent World, Microsoft Whitepaper 2014, abrufbar unter: https:/query.prod.cms.rt.microsoft.com/cms/api/am/binar y/REVroA (geprüft am 15.05.2020), S. 4.

144 Vgl. 2. Kapitel B. III.

145 Für unfallbedingte Schäden durch die Nutzung informationstechnischer Systeme kann nichts anderes gelten. 
formationsausbeutungen als auch hochgefährlichen Informationsangriffen zugänglich sind. ${ }^{146}$ Damit scheint ein strikter Sorgfaltsstandard für informationstechnische Systeme im Allgemeinen angezeigt.

Doch ein solcher Sorgfaltsstandard würde Staaten zu einer Kontrolle dieser Systeme und Beschränkung des freien Zugangs und der freien Nutzung motivieren, was aus menschenrechtlichen Gesichtspunkten bedenklich ist. ${ }^{147}$ Ein strikter Sorgfaltsstandard könnte gar ein Nutzungsverbot von informationstechnischen Systemen aufgrund der nicht auszuschließenden Möglichkeit katastrophaler Schadensfolgen erfordern. Dies ließe aber den erheblichen sozialen, ökonomischen und technischen Nutzen von informationstechnischen Systemen außer Acht. Ein Ausgleich dieser Belange kann nur mit einem nachgiebigen, das heißt flexibleren Sorgfaltsstandard erreicht werden. ${ }^{148}$

Ferner ist die gebotene Sorgfalt in der von technischen und wissenschaftlichen Unsicherheiten geprägten virtuellen Welt in hohem Maße von den zumutbaren und zur Verfügung stehenden Möglichkeiten zur Schadensvermeidung abhängig. Geeignete und angemessene Schadensvermeidungsmaßnahmen sind nicht immer verfügbar. ${ }^{149}$ So kann unter Verhältnismäßigkeitsgesichtspunkten nicht erwartet werden, dass ein Staat etwa seine gesamte Kommunikationsinfrastruktur abstellt, um negative Auswirkungen auf andere Staaten zu vermeiden. ${ }^{150}$

Schließlich müssen die Sorgfaltsanforderungen entsprechend den unterschiedlichen technischen Kapazitäten und Fähigkeiten von Staaten variabel bleiben. ${ }^{151}$ Ein subjektiver Sorgfaltsstandard, der sich am (sicherheits-)technischen Entwicklungsstand eines Staates orientiert, ist zwar problematisch, weil angesichts der globalen Vernetzung Sicherheitslücken in einem weniger entwickelten Staat genutzt werden könnten, um in verbun-

146 Siehe 3. Kapitel A und B.

147 Vgl. Tallinn Manual 2.0, Kommentar zu Regel 6, S. 31 f., Rn. 5 f., Kommentar zu Regel 7, S.45, Rn. 8; O. Dörr, Obligations of the State of Origin of a Cyber Security Incident, GYIL 58 (2015), S. 87 (95).

148 Vgl. B. A. Walton, Duties Owed, Yale LJ 126 (2017), S. 1460 (1492 ff.).

149 Tallinn Manual 2.0, Kommentar zu Regel 7, S. 47, Rn. 16f., S. 49, Rn. 24; $R$. Kolb, Reflections on Due Diligence Duties and Cyberspace, GYIL 58 (2015), S. 113 (126f.).

150 Tallinn Manual 2.0, Kommentar zu Regel 7, S. 49 f., Rn. 25.

151 R. Kolb, Reflections on Due Diligence Duties and Cyberspace, GYIL 58 (2015), S. 113 (123); A. Reinisch/M. Beham, Mitigating Risks, GYIL 58 (2015), S. 101 (105 f.). 
denen Systemen weltweit Schäden anzurichten. ${ }^{152}$ Ein objektiver Standard kommt allerdings nicht in Betracht, da einem Staat keine ihm aufgrund seines Leistungsvermögens unmögliche Pflicht auferlegt werden kann. ${ }^{153}$ Ein subjektiver Maßstab führt zu dem interessengerechten Ergebnis, dass die Anforderungen an technisch entwickelte Staaten aufgrund des ihnen zugänglichen Wissens und der technischen Möglichkeiten höher sind. ${ }^{154}$ Zudem werden die weiter entwickelten Staaten, die im Grunde nicht gewillt sind, ihre grundsätzlich als vertraulich eingestuften Möglichkeiten zur Aufdeckung und Bekämpfung von Informationsangriffen Preis zu geben, ${ }^{155}$ zur Zusammenarbeit und zum Kapazitätenaufbau sowie Technologietransfer veranlasst. Schließlich haben sie ein ureigenes Interesse am Schließen von Sicherheitslücken, die zu grenzüberschreitenden Schäden führen können. ${ }^{156}$

IV. Haftungsart und -standard

Für die Umsetzung einer Haftung für informationstechnische Systeme sind schließlich die Haftungsart und der Haftungsstandard festzulegen.

\section{Haftungsart}

Wie sich aus der Rechtsquellenanalyse im 1. Kapitel ergibt, kennt das Völkerrecht die primäre Staatenhaftung, die subsidiäre Staatenhaftung und die ergänzende Staatenhaftung. Im Folgenden wird dargetan, dass bei der Umsetzung eines Haftungssystems für informationstechnische Systeme alle drei Haftungsarten zum Tragen kommen. Ein Rückgriff auf die Ausführungen zu den Haftungsübereinkommen ${ }^{157}$ sowie zum Verursa-

152 O. Gross, Cyber Responsibility to Protect: Legal Obligations of States Directly Affected by Cyber-Incidents, Cornell ILJ 48 (2015), S. 481 (498); R. Kolb, Reflections on Due Diligence Duties and Cyberspace, GYIL 58 (2015), S. 113 (127).

153 R. Kolb, Reflections on Due Diligence Duties and Cyberspace, GYIL 58 (2015), S. 113 (123).

154 Siehe 2. Kapitel B. III.

155 M. N. Schmitt, The Law of Cyber Warfare, SLPR 25 (2014), S. 269 (278).

156 S.-H. Schulze, Cyber-„War“ - Testfall der Staatenverantwortlichkeit, 2015, S. 221 m.w.N.

157 Siehe 1. Kapitel D. 
chergrundsatz ${ }^{158}$ zeigt besonders deutlich, dass dem Haftungssystem für die virtuelle Welt dieselben Erwägungen wie den Haftungskonzeptionen im Umweltvölkerrecht zugrunde liegen: Zunächst dürfen die Schäden durch gefährliche Aktivitäten in der virtuellen Welt ebenso wenig auf das Opfer abgewälzt werden. Folgerichtig müssen die direkten Schadensverursacher primär haften (a). Des Weiteren ist auch hier die Garantenstellung der Staaten im internationalen Gefüge von Bedeutung. ${ }^{159}$ Sofern der Schadensausgleich durch den Verursacher im engeren Sinne nicht oder nur begrenzt erfolgt, ist eine subsidiäre bzw. ergänzende Haftung des Ursprungsstaates angezeigt. Vergleichbar mit der Schlüsselfunktion des Ursprungsstaates aufgrund der Erlaubnis zum Betrieb umweltgefährdender Anlagen setzt der Staat durch die Erlaubnis zum Betrieb informationstechnischer Systeme auf seinem Hoheitsgebiet indirekt die Schadensursache und ist damit in der virtuellen Welt ebenfalls als Verursacher im weiteren Sinne zu qualifizieren (b). Der Umfang der ergänzenden und der subsidiären Staatenhaftung ist schließlich - wie im Umweltvölkerrecht auch - entsprechend der staatlichen Bemühungen zur Schadensvermeidung zu begrenzen. ${ }^{160}$ Daher ist auf dritter Ebene ein Entschädigungsfonds durch die Staatengemeinschaft einzurichten, der darüber hinausgehende Schäden auffängt. Der Fonds soll aber nicht nur ergänzend, sondern insbesondere auch subsidiär greifen, wenn der Ursprungsstaat der Informationsoperation schon nicht festgestellt werden kann. Durch den Entschädigungsfonds wird so eine Kollektivierung des Schadensausgleiches möglich. Auch diese Idee stammt aus dem Umweltvölkerrecht, wo Entschädigungsfonds als Ausdruck des Interesses der Staatengemeinschaft am Ausgleich katastrophaler (Umwelt-)Schäden und am Erhalt von Gemeinschaftsgütern fungieren $(\mathrm{c})$.

a) Primäre Staatenhaftung für staatliche und staatlich befürwortete Informationsoperationen

Nach dem Verursachergrundsatz trifft die Kompensationspflicht den Urheber der grenzüberschreitenden Schädigung. Demnach muss der Staat Entschädigung leisten, wenn staatseigene Informationsoperationen grenzüberschreitende Schäden verursachen. Im Umweltkontext sind ferner Be-

158 Siehe 1. Kapitel E. III.

159 Vgl. 1. Kapitel C. II. 6.

160 Vgl. 1. Kapitel A. II. 1. c) und 1. Kapitel D. IV. 2. 
treiber (hoch-)gefährlicher Aktivitäten bzw. Eigentümer von Anlagen bzw. Schiffen, die für (hoch-)gefährliche Aktivitäten genutzt werden, als Verursacher zu qualifizieren und mithin zur Schadenskompensation verpflichtet. Übertragen auf die virtuelle Welt sind Staaten als Betreiber bzw. Eigentümer regierungseigener Informationsinfrastrukturen zu betrachten und daher für grenzüberschreitende Schäden durch die Nutzung dieser Systeme einstandspflichtig. ${ }^{161}$

Dies bedeutet jedoch nicht, dass Staaten ohne Urheber- oder Betreiberbzw. Eigentümereigenschaft per se von der Haftung freigestellt sind. Dies gilt namentlich bei staatlich befürworteten Informationsoperationen. Hier müssen unter Gerechtigkeitserwägungen die Vorteile, welche dem Staat durch derartige Informationsoperationen entstehen, Berücksichtigung finden. Denn Informationsoperationen durch patriotische Hacker und staatliche Stellvertreter bedeuten durchaus wirtschaftliche Vorteile und politisch-strategischen Machtgewinn auf internationaler Ebene für Staaten. ${ }^{162}$ Bei derartigen Informationsoperationen liegt es nahe, dass die profitierenden Staaten diese ignorieren bzw. dulden. Damit verschmelzen staatliche und nicht-staatliche Akteure zu Verursachern von pseudostaatlichen Informationsoperationen. ${ }^{163}$ Hier entgehen Staaten zwar einer zurechnungsbasierten Staatenverantwortlichkeit für staatlich befürwortete Informationsoperationen, jedoch muss der Staat als Kehrseite der erlangten Vorteile auch für Schäden einstehen.

In diesem Zusammenhang sind erneut die Beweisschwierigkeiten mit Blick auf den staatlichen Ursprung bzw. die staatliche Befürwortung einer Informationsoperation zu berücksichtigen. Während Indizienbeweise für die Zurechnung einer völkerrechtswidrigen Aktivität nicht ausreichen, ${ }^{164}$ ist im Rahmen der Staatenhaftung aber eine erleichterte Beweisführung gestattet. ${ }^{165}$ In Konstellationen, in denen die Beweise der ausschließlichen

161 Vgl. I. Y. Liu, State Responsibility and Cyberattacks, IJICL 4 (2017), S. 191 (209f.).

162 J. D. Jolley, Attribution, State Responsibility, and the Duty to Prevent Malicious Cyber-Attacks in International Law, 2017, abrufbar unter: http://theses.gla.ac.uk /id/eprint/8452 (geprüft am 15.05.2020), S. 5, 30.

163 Id., S. 30 f., 84; B. A. Walton, Duties Owed, Yale LJ 126 (2017), S. 1460 (1515).

164 J. D. Jolley, Attribution, State Responsibility, and the Duty to Prevent Malicious Cyber-Attacks in International Law, 2017, abrufbar unter: http://theses.gla.ac.u $\mathrm{k} /$ id/eprint/8452 (geprüft am 15.05.2020), S.36. In diesem Zusammenhang sei erneut darauf hingewiesen, dass Indizien nur für den Nachweis von Kenntnis und damit zur Begründung positiver Handlungspflichten ausreichen können.

165 Vgl. C. Walter, der für den Nachweis eines völkerrechtswidrigen bewaffneten Angriffs „full proof" fordert (bezugnehmend auf IGH, Oil Platforms (Islamic 
Kontrolle der beklagten Seite unterliegen, ist ein Rückgriff auf Indizien zur Begründung staatlicher Einstandspflichten für völkerrechtmäßige Aktivitäten zulässig, sofern die Indizien nicht der verfügbaren direkten Beweis- und Faktenlage widersprechen. ${ }^{166}$ Informationen über die staatliche Beziehung zu einer bestimmten Informationsoperation unterliegen in der Regel dem Ursprungsstaat, der aus Gründen der nationalen Sicherheit nicht bereit sein wird, diese Informationen zu teilen und auch nicht dazu verpflichtet ist. ${ }^{167}$ Demnach müssen kontextbezogene Vermutungen ausreichen, so dass eben die Vorteile von pseudostaatlichen Informationsoperationen für einen Staat, die staatliche Befürwortung der Informationsoperationen indizieren, ${ }^{168}$ mithin zur Begründung einer staatlichen Einstandspflicht beitragen.

b) Subsidiäre und ergänzende Haftung des Ursprungsstaates für nichtstaatliche Informationsoperationen

Im Rahmen nicht-staatlicher Informationsoperationen sind Aspekte der Risikozuweisung wegweisend, um die Art der staatlichen Haftung festzulegen. Der nicht-staatliche Urheber der Informationsoperation kann aufgrund der Hürden bei der Beweisführung in der virtuellen Welt nur selten zur Rechenschaft gezogen werden, während die privaten Betreiber bzw. Eigentümer informationstechnischer Systeme in Anbetracht der besonders unsicheren Gefährdungslage und den potenziell katastrophalen Auswir-

Republic of Iran v. United States of America), Judgment of 6 November 2013, ICJ Reports 2003, S. 161 (161 Rn. 57 ff.)) und in Bezug auf Aufklärungs- und Informationspflichten feststellt, dass "the standard of proof is lower" (bezugnehmend auf IGH, Corfu Channel Case (United Kingdom of Great Britain and Northern Ireland v. Albania), Judgment of 9 April 1949, ICJ Reports 1949, S. 4 (18)). C. Walter, Obligations of States Before, During, and After a Cyber Security Incident, GYIL 58 (2015), S. 67 (82).

166 R. Crootof, International Cybertorts, CLR 103 (2018), S. 565 (638f. Fn. 324) bezugnehmend auf die Rechtsprechung des IGH.

167 Id., S. 565 (638).

168 Vgl. D. Jolley, Attribution, State Responsibility, and the Duty to Prevent Malicious Cyber-Attacks in International Law, 2017, abrufbar unter: http://theses.gla .ac.uk/id/eprint/8452 (geprüft am 15.05.2020), S. 30 ff.; P. Pernik, A Playbook for Hybrid War in Cyberspace?, International Center for Defence and Security, 29 August 2014, abrufbar unter: https://icds.ee/a-playbook-for-hybrid-war-in-cybers pace/ (geprüft am 15.05.2020). 
kungen von Informationsoperationen kaum in der Lage sein werden, die Schadenslast zu antizipieren und zu tragen.

Die Möglichkeit von grenzüberschreitenden Schäden durch die Nutzung informationstechnischer Systeme ist vergleichbar mit möglichen Schäden durch den Betrieb von Kernkraftwerken, bei dem eine subsidiäre sowie eine ergänzende staatliche Einstandspflicht des Anlagenstaates greifen. ${ }^{169}$ Derartige Einstandspflichten sind auch für informationstechnische Systeme sinnvoll. Mit der Erlaubnis zur Nutzung informationstechnischer Systeme ohne ausreichende Sicherung vor Informationsoperationen schaffen Staaten eine Gefährdungslage und müssen daher eine adäquate und unverzügliche Entschädigung für eine schadensbegründende Nutzung dieser Systeme gewährleisten. ${ }^{170}$ Der Staat ist Verursacher im weiteren Sinne.

Die staatliche Regulierungsmacht bei der Nutzung informationstechnischer Systeme weist dem Staat eine zentrale Rolle im Bereich der Haftung für diese Systeme zu. ${ }^{171}$ Wenn also Urheber der Informationsoperation oder Betreiber bzw. Eigentümer der informationstechnischen Systeme nicht identifizierbar sind, trifft den Staat eine Ausfallhaftung. Selbst wenn die Verursacher im engeren Sinne feststehen, dürfte der Staat als solventeste Partei mit der besten Möglichkeit zur Stärkung der Sicherheit informationstechnischer Systeme nicht aus seiner Einstandspflicht entlassen werden. ${ }^{172} \mathrm{Im}$ Gegenteil müssen die Ursprungsstaaten auch dann subsidiär bzw. ergänzend haften, wenn die Verursacher im engeren Sinne die (volle) Schadensumme nicht begleichen können bzw. müssen. Der Staat muss bekannte Urheber oder Betreiber bzw. Eigentümer allerdings soweit möglich in Regress nehmen. ${ }^{173}$

Schließlich sprechen auch Erwägungen der wirtschaftlichen Gerechtigkeit für diesen Ansatz, denn den Staaten erwächst aus der Nutzung informationstechnischer Systeme durch sie selbst aber auch durch ihre

169 Vgl. auch D. Jolley, Attribution, State Responsibility, and the Duty to Prevent Malicious Cyber-Attacks in International Law, 2017, abrufbar unter: http://these s.gla.ac.uk/id/eprint/8452 (geprüft am 15.05.2020), S. 214.

170 Vgl. H. Krieger, Krieg gegen anonymous, AVR 50 (2012), S. 1 (16).

171 Vgl. F. O. Vicuña, der in Bezug auf die Umwelthaftung feststellt: „Given the complexity of many environmental regimes States cannot realistically expect that the whole burden of liability might fall upon private operators or other entities.“ F. O. Vicuña, Responsibility and Liability for Environmental Damage under International Law, GIELR 10 (1998), S. 279 (287).

172 B. A. Walton, Duties Owed, Yale LJ 126 (2017), S. 1460 (1515).

173 C. Foster, The ILC Draft Principles on the Allocation of Loss in the Case of Transboundary Harm Arising out of Hazardous Activities, RECIEL 14 (2005), S. 265 (277); N. de Sadeleer, Environmental Principles, 2002, S. $24 \mathrm{f}$. 
Staatsangehörigen ein nicht unerheblicher wirtschaftlicher Vorteil. ${ }^{174}$ Die Schaffung von Risiken durch die Genehmigung gefährlicher Aktivitäten und daraus resultierende grenzüberschreitende Schäden gehen dann auf internationaler Ebene mit der Enteignung von Gleichheit und Souveränität einher. Daher lässt sich die staatliche Einstandspflicht auch mit dem Grundsatz der ungerechtfertigten Bereicherung erklären. ${ }^{175}$

c) Errichtung eines Entschädigungsfonds für eine subsidiäre Haftung bei nicht feststellbarem Ursprung der Informationsoperation und für eine ergänzende Haftung bei begrenzter Einstandspflicht des Ursprungsstaates

Die Hauptprobleme bei Einstandspflichten für Schäden durch (hoch-)gefährliche Aktivitäten werden im Haftungsrecht durch die Errichtung von Fonds gelöst. Entschädigungsfonds sind in erster Linie dazu gedacht, denjenigen, die Schäden durch Unfälle oder durch bestimmte nicht verbotene Aktivitäten erlitten haben, unverzügliche und angemessene Abhilfe zu gewähren. ${ }^{176}$ Fonds ermöglichen dabei eine bessere Ausreichung größerer Entschädigungssummen und bieten Lösungen für Aufklärungsschwierigkeiten. ${ }^{177}$ Die kollektiv organisierten Finanzmittel können zum einen eine individuelle Entschädigungspflicht ergänzen und auf diese Weise Risiken absichern, die nicht mehr vom Haftungsadressaten getragen werden können oder müssen. Zum anderen können kollektive Entschädigungsmechanismen vollständig individuelle Entschädigungssysteme ersetzen und

174 D. Jolley, Attribution, State Responsibility, and the Duty to Prevent Malicious Cyber-Attacks in International Law, 2017, abrufbar unter: http://theses.gla.ac.uk /id/eprint/8452 (geprüft am 15.05.2020), S. 214.

175 So kommt L. F. E. Goldie anhand der Analyse von Haftungskonzepten zu dem Ergebnis, dass "the creation of risk involves an expropriation of equal rights of amenities and, in the international arena, of equality and sovereign autonomy. This international law thesis of expropriation, moreover, is measured in terms of the application, in international relations, of the equitable doctrine of unjust enrichment." L. F. E. Goldie, Concepts of Strict and Absolute Liability and the Ranking of Liability in terms of Relative Exposure to Risk, NYIL 16 (1985), S. 175 (248).

176 A. Douhan, Liability for Environmental Damage, in: R. Wolfrum (Hg.), MPEPIL 2013, http://www.mpepil.com, Rn.34; C. Kojima, Compensation Fund, in: R. Wolfrum (Hg.), MPEPIL 2009, http://www.mpepil.com, Rn. 1.

177 C. Kojima, Compensation Fund, in: R. Wolfrum (Hg.), MPEPIL 2009, http://w ww.mpepil.com, Rn.3. 
damit insbesondere bei unsicheren Verursachungszusammenhängen zum Einsatz kommen. ${ }^{178}$ So übernehmen Fonds die finanzielle Entschädigung im Fall von Nachweisproblemen, etwa bei Unmöglichkeit der Identifizierung des konkreten Verursachers, bei einer Vielzahl potenzieller Verursacher, bei Synergieschäden oder bei unbestimmter Schadensursache. ${ }^{179}$

Während auf dem Gebiet der Kernenergie Schadensausmaße, die nicht mehr vom Betreiber des Kernkraftwerkes oder dem Anlagenstaat zu tragen sind, durch kollektive Finanzierungssysteme nach dem BZÜ und ÜEE kompensiert werden, ${ }^{180}$ kommt der Internationale Entschädigungsfonds für Ölverschmutzungsschäden auch dann zum Einsatz, wenn der Verursacher nicht identifiziert werden kann und begründet mithin eine kollektive Ausfallhaftung. ${ }^{181}$ Ebenso sehen das HNS-Übereinkommen $2010^{182}$ und Anlage VI des Antarktisumweltschutzprotokolls über die Haftung bei umweltgefährdenden Notfällen ${ }^{183}$ Entschädigungsfonds vor, die auch greifen sollen, wenn die Identität des Verursachers unklar ist. Bemerkenswert sind auch die entsprechenden Bestrebungen auf Ebene der EU zur Errichtung eines Entschädigungsfonds für Umweltschäden durch industrielle Unfälle, der bei Insolvenzrisiko und Versagen der Märkte für Deckungsvorsorge sowie bei Unbekanntheit des verantwortlichen Betreibers greifen soll. ${ }^{184}$ Schließlich ist der Vorschlag der Kammer für Meeresbodenstreitigkeiten, die Einrichtung eines Treuhandfonds zur Schadensdeckung in Betracht zu ziehen, um den aufgezeigten Haftungslücken zu begegnen, nicht außer Acht zu lassen. ${ }^{185}$

Wie gezeigt, bestehen insbesondere bei Informationsoperationen aufgrund der komplexen technischen Verursachungsstrukturen und des nur selten hinreichend bestimmbaren Ursprungs der Operationen Nachweisprobleme. Zudem ist die Kompensationspflicht von bestimmbaren Ursprungsstaaten für nicht-staatliche Informationsoperationen dahingehend begrenzt, dass sich deren Ausmaß nach der Beachtung der gebotenen

178 Siehe 1. Kapitel D.

179 Art. 12 IDI-Resolution; C. Kojima, Compensation Fund, in: R. Wolfrum (Hg.), MPEPIL 2009, http://www.mpepil.com, Rn. 3; U. Ranke, Klima und Umweltpolitik, 2019, S. 102.

180 Siehe Ausführungen im 1. Kapitel D. II. 2.

181 Siehe Ausführungen im 1. Kapitel D. IV. 3. c).

182 Siehe Ausführungen im 1. Kapitel D. IV. 3. c).

183 Siehe Ausführungen im 1. Kapitel D. III. 2.

184 Siehe Ausführungen im 1. Kapitel D. IV. 3. c).

185 Siehe Ausführungen im 1. Kapitel B. I. 3. a). 
Sorgfalt bei der Schadensvermeidung bemisst. ${ }^{186}$ Darüber hinausgehende Schäden dürfen aber nicht auf das Opfer abgewälzt werden. Folglich ist für informationstechnische Systeme ebenso ein Entschädigungsfonds einzurichten, der bei Schäden greift, deren Ursprung nicht nachweisbar ist oder die ein Ausmaß erreichen, das von einzelnen Staaten nicht aufgefangen werden kann bzw. muss. ${ }^{187}$

Neben dem Aspekt des Opferschutzes spielen Erwägungen der (wirtschaftlichen) Gerechtigkeit und der Risikozuweisung eine wesentliche Rolle. ${ }^{188}$ Alle Staaten können nicht nur Opfer von Informationsoperationen werden, sondern profitieren ebenso von vernetzten informationstechnischen Systemen. So besteht ein grundsätzliches Eigeninteresse aller Staaten am Zugriff auf (vernetzte) Informationstechnologie, etwa für die nationale Sicherheit. Nicht ohne Grund werden Anbieter derartiger Technologien von Staaten aufgefordert, ihre Verschlüsselungsmethoden entsprechend abzuschwächen, ${ }^{189}$ was im Umkehrschluss zu einer gesteigerten Gefährdung für die internationale Sicherheit führt. In Anbetracht der Tatsache, dass die Technisierung und Vernetzung informationstechnischer Systeme immer weiter voranschreitet und damit die Abhängigkeit von informationstechnisch-gestützten Strukturen weltweit steigt, müssen auch entsprechend stärkere negative Auswirkungen aufgefangen werden, und zwar von der Staatengemeinschaft, die von diesen global verbundenen Systemen profitiert. Der Gerechtigkeitsgedanke gebietet mithin, dass Staaten für die informationstechnische Gefährdungslage einen Entschädigungsfonds zur Ausreichung von Entschädigungssummen einrichten. ${ }^{190}$

186 Vgl. 1. Kapitel A. II. 1. c) und 1. Kapitel D. IV. 2.

187 Vgl. J. Kulesza, Due Diligence in Cyberspace, in: I. M. Portela/F. Almeida (Hg.), Cyberspace, Organizational, Legal, and Technological Dimensions of Information System Administration, 2014, S. 76 (84 f.).

188 Vgl. 1. Kapitel E. III.

189 In einem gemeinsamen Statement „ermutigen“ die USA, Großbritannien, Kanada, Australien und Neuseeland die Anbieter von Informations- und Kommunikationstechnologie, „freiwillig rechtmäßige Zugangswege zu ihren Produkten und Diensten einzurichten“. Five Country Ministerial and Quintet Meeting of Attorneys General, Statement of Principles on Access to Evidence and Encryption, Australia 2018, abrufbar unter: https://www.ag.gov.au/About/Committees andCouncils/Documents/joint-statement-principles-access-evidence.pdf (geprüft am 15.05.2020).

190 Vgl. S. Sucharitkul, Responsibility and Liability for Environmental Damage Under International Law, Golden Gate University Law Digital Commons 664 (1996), abrufbar unter: https://digitalcommons.law.ggu.edu/pubs/664 (geprüft am 15.05.2020), S. 12. 
Darüber hinaus ist der eigene Risikobeitrag, den die Staaten durch die Erlaubniserteilung zum Betrieb der informationstechnischen Systeme oder zu deren Nutzung selbst leisten, zu berücksichtigen. Damit besteht nämlich eine Verantwortungsnähe zu Schäden durch die Risikorealisierung in Gestalt von Informationsoperationen. Wie im Umweltvölkerrecht auch, ist der Verursachergrundsatz aufgrund dieser besonderen Nähe im Sinne einer Gruppenverantwortlichkeit zu verstehen. ${ }^{191}$ Die logische Konsequenz ist die Errichtung eines Fonds, um der Haftung für die weltweit vernetzten informationstechnischen Systeme gerecht zu werden. ${ }^{192}$ Es geht dabei allerdings nicht um Freiwilligkeit, sondern um eine Pflicht der Staatengemeinschaft - als Verursachergruppe.

Anreize zur Errichtung eines Entschädigungsfonds werden durch die dem internationalen Umweltschutz inhärenten Kooperations- und Solidaritätsgedanken geboten, die im Bereich informationstechnischer Systeme entsprechende Wirkung entfalten können. ${ }^{193}$ Wie gezeigt, ist Kooperation für die Grundsätze der Schadensvorsorge, Schadensprävention und Schadensminderung essenziell. ${ }^{194}$ Sie fordert von Staaten, finanzielle Mittel bereitzustellen, um Technologien, Warnsysteme und sonstige Kapazitäten zur Schadensvermeidung aufzubauen und zu teilen, aber auch Schäden aufzufangen. ${ }^{195}$ Diese Maßnahmen zur Schadensvermeidung und Schadensabhilfe können effektiv durch Fonds verwirklicht werden. ${ }^{196}$ Entschädigungsfonds bestehen zumeist aus solidarischen Beiträgen bestimmter Verursachergruppen. ${ }^{197}$ Wenngleich die Solidarität keine völkerrechtlich festgelegte Rechtspflicht darstellt, prägt sie das Verhalten der Staatenge-

191 Vgl. W. Kahl, Umweltprinzip und Gemeinschaftsrecht, 1993, S. 24 f.; M. Schröder, Umweltschutz als Gemeinschaftsziel und Grundsätze des Umweltschutzes, in: H.-W. Rengeling (Hg.), Handbuch zum europäischen und deutschen Umweltrecht, Band I, 2003, $\$ 9$, Rn. 42.

192 Vgl. C. Calliess, Art. 191 AEUV (ex-Art. 174 EGV), in: ders./M. Ruffert (Hg.), EUV/AEUV, Kommentar, 2016, Rn. 38 (39).

193 Vgl. O. Kimminich, Völkerrechtliche Haftung für das Handeln Privater im Bereich des internationalen Umweltschutzes, AVR 22 (1984), S. 241 (266); W. Rudolf, Haftung für rechtmäßiges Verhalten im Völkerrecht, in: J. Damrau (Hg.), Festschrift für Otto Mühl, 1981, S. 535 (549 f.).

194 Siehe insbesondere 1. Kapitel E. II. und 2. Kapitel B. II.

195 Vgl. I. Bantekas, Trust Funds, in: R. Wolfrum (Hg.), MPEPIL 2010, http://ww w.mpepil.com Rn. 6; O. Gross, Cyber Responsibility to Protect, Cornell ILJ 48 (2015), S. 481 (495 f.); U. Ranke, Klima und Umweltpolitik, 2019, S. 102.

196 C. Kojima, Compensation Fund, in: R. Wolfrum (Hg.), MPEPIL 2009, http://w ww.mpepil.com, Rn. 23.

197 M. Bothe/L. Gündling/R. Hofmann/C. Rumpf, Neuere Tendenzen des Umweltrechts im internationalen Vergleich, in: Umweltbundesamt (Hg.), Umweltfor- 
meinschaft. ${ }^{198}$ So motiviert der Solidaritätsgedanke Staaten sehr anschaulich im Bereich der Naturkatastrophen zur Abhilfe in Schadensfällen. ${ }^{199}$ Bei genauer Betrachtung handelt es sich dabei jedoch nicht um eine altruistische oder gar intrinsische Motivation. Ähnlich wie Naturkatastrophen sind Sicherheitslücken in informationstechnischen Systemen derzeit - und wohl auch zukünftig - unvermeidbar und können gleichzeitig mehrere Schadensopfer betreffen. ${ }^{200}$ Alle Staaten sind von Informationsoperationen bedroht und haben ein Interesse an ausreichenden und verfügbaren Finanzmitteln für den Schadensfall. ${ }^{201}$ Bedingt durch die globale Vernetzung von informationstechnischen Systemen entsteht eine diffuse und ubiquitäre Gefährdungslage, von der Staaten weltweit betroffen sind. ${ }^{202}$ Diese Gefährdungslage wird dadurch potenziert, dass die Sicherheit informationstechnischer Systeme nur so stark wie das schwächste Glied in den weltweit vernetzten Systemen ist. Sicherheitslücken informationstechnischer Systeme in einem Staat können zu erheblichen negativen Auswirkungen in anderen Staaten führen (Spillover-Effekte). ${ }^{203}$ Die Digitalisierung aller Gesellschaftsbereiche und die Allgegenwärtigkeit der Vernetzung informationstechnischer Systeme bilden demnach einen sicherheitspolitischen Raum, der nicht nur enorme Vorteile bietet, sondern auch eine globale Gefährdung bedeutet, da nationale Sicherheitsprobleme in der virtuellen Welt schnell zu internationalen Problemen werden können. Entsprechend haben schon 121 Staaten Sicherheitsstrategien für den virtuellen Raum aufgestellt ${ }^{204}$ und auch supranationale bzw. zwischenstaatliche Institutionen wie EU und NATO haben entsprechende Vereinbarungen

schungsplan des Bundesministers für Umwelt, Naturschutz und Reaktorsicherheit, Berichte des Umweltbundesamtes, Band 2/90, 1990, S. 1 (274).

198 Siehe zur Geltung des Solidaritätsprinzips im Völkerrecht D. Campanelli, Principle of Solidarity, in: R. Wolfrum (Hg.), MPEPIL 2011, http://www.mpepil. com; C. L. Riemer, Staatengemeinschaftliche Solidarität in der Völkerrechtsordnung, 2003, insbesondere S. 308.

199 O. Gross, Cyber Responsibility to Protect, Cornell ILJ 48 (2015), S. 481 (491, $509 \mathrm{f}$.).

$200 I d .$, S. 481 (499f.).

201 Id., S. 481 (497).

202 M. Thiel, Die „Entgrenzung“ der Gefahrenabwehr, 2011, S. 117 f.

203 O. Gross, Cyber Responsibility to Protect, Cornell ILJ 48 (2015), S. 481 (493).

204 International Telecommunications Union, National Cybersecurity Strategies Repository, abrufbar unter: http://www.itu.int/en/ITU-D/Cybersecurity/Page s/National-Strategies-repository.aspx (geprüft am 15.05.2020). 
getroffen. ${ }^{205}$ Demnach besteht ein ureigenes Interesse aller Staaten an internationaler Kooperation zum Schutz des sicherheitspolitischen Raums. Eine Entschädigungslösung durch einen Fonds verdeutlicht dabei, in vergleichbarer Weise wie im Bereich der Meere und der Antarktis, die Bedeutung der virtuellen Welt als Gemeinschaftsgut. ${ }^{206}$

Schließlich dürften ökonomische Gesichtspunkte den größten Anreiz für Staaten zur Errichtung eines Fonds bieten. Wie aufgezeigt, droht in zunehmendem Maße die einzelstaatliche Einstandspflicht für Schäden durch informationstechnische Systeme. Zum einen erfordern die möglichen katastrophalen Auswirkungen eine Ausreichung größerer Schadenssummen im Sinne einer Deckungsvorsorge. Zum anderen verändern sich die informationstechnischen Risiken und Schadenszenarien schnell und fortwährend und stehen damit einer umfassenden Versicherbarkeit der Gefährdung entgegen. ${ }^{207}$ Dementsprechend werden alle Staaten an einer Kollektivierung des Schadensausgleichs interessiert sein. ${ }^{208}$

Die finanzielle Ausstattung von Entschädigungsfonds erfolgt in der Regel durch Beitragszahlungen von Regierungen oder privaten Industriegruppen, die das Risiko der Schadensverursachung schaffen. Die Beitragsleistungen variieren dabei nach der Höhe des Risikos, welches durch die jeweilige Partei verursacht wird. ${ }^{209}$ Bei Anwendung dieses Modells auf einen Fonds für Schäden durch informationstechnische Systeme müsste für eine zwingende staatliche Beitragsleistung ein Zusammenhang zwischen den einzelnen Staaten und der Erhöhung des Risikos durch diese feststellbar sein. Weniger (sicherheits-)technisch entwickelte Staaten schaf-

205 NATO, Cyber Defence Pledge, 8 July 2016, abrufbar unter: http://www.nato .int/cps/en/natohq/official_texts_133177.htm (geprüft am 15.05.2020) und European Commission, Joint Communication to the European Parliament, the Council, the European Economic and Social Committee and the Committee of the Regions, Cybersecurity Strategy of the European Union: An Open, Safe and Secure Cyberspace, 7 February 2013, abrufbar unter: http://eeas.europa.e $\mathrm{u} /$ archives/docs/policies/eu-cyber-security/cybsec_comm_en.pdf (geprüft am 15.05.2020).

206 Vgl. C. Kojima, Compensation Fund, in: R. Wolfrum (Hg.), MPEPIL 2009, http://www.mpepil.com, Rn. 2, 23.

207 Vgl. C. Wilson/T. Gaidosch/F. Adelmann/A. Morozova, Cybersecurity Risk Supervision, International Monetary Fund, Monetary and Capital Markets Departmental Paper Series No.19/15, 2019, Annex I.

208 Vgl. G. Brüggemeier, Umwelthaftungsrecht, Kritische Justiz 22 (1989), S. 209 (222).

209 S. Förster, Internationale Haftungsregeln für schädliche Folgewirkungen gentechnisch veränderter Organismen, 2007, S.366f., 370. Siehe auch Ausführungen im 1. Kapitel D. IV. 3. b). 
fen durch entsprechende Sicherheitslücken ein erhöhtes Risiko und könnten daher einer größeren Beitragspflicht unterliegen; allerdings profitieren gerade technisierte Staaten von informationstechnischen Systemen, von denen sie zugleich in größerem Umfang abhängig und damit in besonderem Maße für Informationsangriffe anfällig sind. Der spezifische Risikobeitrag eines Staates ist mithin nur schwer zu quantifizieren. Bei Entschädigungsfonds kommt es aber auch auf Gesichtspunkte der Praktikabilität an. ${ }^{210}$ Vor diesem Hintergrund kann für einen Entschädigungsfonds für informationstechnische Schadensfälle nur das Bruttosozialprodukt der jeweiligen Staaten maßgeblich für die Beitragshöhe sein. ${ }^{211}$

\section{Haftungsstandard}

Wie bereits dargelegt, trifft den Staat im Rahmen verschuldensabhängiger Haftungssysteme nur dann eine Kompensationspflicht, wenn dieser die gebotene Sorgfalt außer Acht gelassen hat, während verschuldensunabhängige Haftungssysteme eine Einstandspflicht unabhängig von einem etwaigen Fehlverhalten des haftenden Staates begründen und lediglich das Ausmaß der Kompensationspflicht entsprechend der angewandten Sorgfalt bemessen. Eine verschuldensabhängige Haftung ist sinnvoll, wenn der Ursprungsstaat und der Opferstaat gleichermaßen zur Gefährdungslage beitragen. Staaten erlauben zwar die Nutzung informationstechnischer Systeme und schaffen damit eine Gefahrenquelle. Allerdings ist die konkrete Nutzung in Gestalt einer bestimmten Informationsoperation, die zu grenzüberschreitenden Schäden führt, nicht in gleicher Weise vom Ursprungsstaat und Opferstaat verursacht. ${ }^{212}$ In diesem Zusammenhang

210 M. Bothe/L. Gündling/R. Hofmann/C. Rumpf, Neuere Tendenzen des Umweltrechts im internationalen Vergleich, in: Umweltbundesamt (Hg.), Umweltforschungsplan des Bundesministers für Umwelt, Naturschutz und Reaktorsicherheit, Berichte des Umweltbundesamtes, Band 2/90, 1990, S. 1 (274).

211 Vgl. Art. 12 BZÜ, wonach sich die Beitragshöhe je Vertragsstaat für nukleare Schäden zur Hälfte nach dem Bruttosozialprodukt der jeweiligen Staaten richtet. Alternativ könnten freiwillige Beitragsleistungen zur finanziellen Ausstattung des Fonds in Betracht gezogen werden. Der antarktische Haftungsfonds etwa soll gemäß Art. 12 Abs. 4 Anlage VI durch freiwillige Beiträge von Staaten oder Personen finanziert werden. Vgl. auch Art. 14 Abs. 1 Fondsübereinkommen 1992 und Art. 23 Abs. 1 HNS-Übereinkommen 2010, wonach Staaten die private Beitragspflicht freiwillig übernehmen können.

212 Eine Differenzierung zwischen verschuldensabhängiger Haftung bei nicht-staatlichen Informationsangriffen und verschuldensunabhängiger Haftung bei staat- 
spielt außerdem die Verflechtung von Haftungsstandard und Sorgfaltsstandard eine entscheidende Rolle: Zum einen fehlt es insbesondere bei komplexen Vorgängen im Zusammenhang mit modernen risikobehafteten Technologien an festgelegten internationalen Sorgfaltsstandards zur Schadensvermeidung, was nicht zulasten des Staates gehen darf, der weder in ein konkretes Risiko eingewilligt hat oder Kontrolle über die betreffende Aktivität ausüben kann, noch von dieser in irgendeiner Form profitiert. Dies spricht für eine verschuldensunabhängige Haftung. ${ }^{213}$ Zum anderen ist der Betrieb informationstechnischer Systeme ohnehin als hochgefährlich zu qualifizieren. Da sich der Sorgfaltsstandard bei hochgefährlichen Aktivitäten grundsätzlich zu einer verschuldensunabhängigen Haftung verdichtet, ${ }^{214}$ ist im Ergebnis in jedem Fall ein verschuldensunabhängiges Haftungssystem für informationstechnische Systeme angezeigt, so dass die Beachtung bzw. Missachtung der gebotenen Sorgfalt das Ausmaß der Kompensationspflicht bestimmt. ${ }^{215}$

lichen und staatlich geförderten Informationsangriffen - wie sie B. A. Walton, Duties Owed, Yale LJ 126 (2017), S. 1460 (1499 ff.) vornimmt - findet sich nicht in den beleuchteten Völkerrechtsquellen. Diesen zufolge spielt eine Differenzierung nach staatlich und nicht-staatlich vielmehr für die Unterscheidung zwischen originärer, ergänzender und subsidiärer staatlicher Haftung eine Rolle spielt.

213 A. E. Boyle, Globalising Environmental Liability, J. Envtl. L. 17 (2005), S. 3 (7).

214 G. Handl, State Liability for Accidental Transnational Environmental Damage by Private Persons, AJIL 74 (1980), S. 525 (550).

215 Bei der Staatenhaftung geht es im Gegensatz zur Staatenverantwortlichkeit nicht um die Wiederherstellung des status quo ante, sondern um einen gerechten Interessenausgleich, so dass sich die staatliche Kompensationspflicht bei Beachtung der gebotenen Sorgfalt zur Schadensvermeidung gar auf eine Minimalentschädigung reduziert. Nichtsdestotrotz soll das Opfer nicht die Schäden tragen. Als Lösung bieten sich, wie gezeigt, Entschädigungsfonds an, die diese Schadensposten auffangen. 


\section{Schlussbetrachtung}

Wir leben in einer "Weltrisikogesellschaft“, die von Gefahren durch moderne Technologien gekennzeichnet ist. ${ }^{1}$ Rechtssysteme müssen dem immerwährenden Fortschritt Rechnung tragen und dies zeitnah. Im Völkerrecht bietet sich dafür die Staatenhaftung an. ${ }^{2}$

Die Staatenhaftung, wie sie von der Völkerrechtskommission im Jahr 1996 konzipiert wurde, gelangt durch die internationale Rechtsprechung zur Anwendung. Zwar kann ihr völkergewohnheitsrechtlicher Status durch Staatenpraxis und opinio iuris nicht ohne Weiteres nachgewiesen werden. Die Beispiele aus der Praxis zeigen aber, dass Staaten in grenzüberschreitenden Schadensfällen zumindest eine Art Garantenstellung zukommt. Die staatliche Haftung findet überdies in einer Reihe von Übereinkommen für spezifische Bereiche des Völkerrechts unterschiedliche Ausprägung. Gleichzeitig lassen sich aus den Übereinkommen übereinstimmende Merkmale ableiten, die Anwendungsbereich und Tatbestandsvoraussetzungen eines allgemeingültigen Systems der Staatenhaftung näher zu bestimmen helfen. Schließlich belegen die der Staatenhaftung zugrunde liegenden allgemeinen Rechtsgrundsätze, dass das Konzept durchaus auf einem völkerrechtlichen Fundament steht.

Doch obwohl die Problematik von Schäden durch völkerrechtlich nicht verbotene Aktivitäten seit Jahrzehnten auf der internationalen rechtlichen Agenda steht, gibt es kaum einen Fortschritt hin zu einer allgemeingültigen Bindungswirkung der völkerrechtlichen Staatenhaftung. Gründe dafür sind die Souveränität der Staaten und deren Widerwillen, sich einem zwingenden Haftungssystem zu unterwerfen. ${ }^{3}$ Allerdings ist eine gegenläufige Entwicklung im Umweltvölkerrecht zu sehen. Das Konzept der Staatenhaftung für umweltgefährdende Aktivitäten hat eine verlässliche Grundlage in den Rechtsquellen des Völkerrechts. Dies liegt an dem Bewusstsein der Gesellschaft für umweltgefährdende Problemlagen und dem

1 Siehe U. Beck, Weltrisikogesellschaft, 2015.

2 Vgl. J. Barboza, Liability, Chinese JIL 1 (2002), S. 499 (523 f.); S. Townley, The Rise and Risk in International Law, Chi. JIL 18 (2018), S. 594 (618 f.).

3 J. Barboza, The Environment, Risk and Liability in International Law, S. 153 f.; $S$. Kadelbach, The Concept of State Responsibility for Private Acts under General International Law, in: S. Hobe/S. Freeland/B. Schmidt-Tedd (Hg.), In Heaven as on Earth?, 2013, S. 105 (118). 
entsprechenden Druck auf die internationale Politik, etwa durch Bürgerinitiativen. ${ }^{4}$

Es ist aufgezeigt worden, dass sich das Konzept der Staatenhaftung auf andere gefährliche völkerrechtlich nicht verbotene Aktivitäten sinnvoll übertragen lässt. Die Notwendigkeit ergibt sich schon daraus, dass die fehlende Kategorisierung und Kriminalisierung der neuartigen schadensverursachenden Aktivitäten sowie deren fehlende Zurechenbarkeit der Begründung einer Staatenverantwortlichkeit entgegenstehen. Staaten kommen ihren Pflichten nicht nach oder sind aufgrund der veränderten Situation in der modernen Welt daran gehindert. Oftmals erhalten die Opfer keine Schadenskompensation. Ihnen fehlt die Möglichkeit, ihre Rechte im internationalen Raum zu schützen und einzufordern. Die Staatenhaftung hilft hier weiter, indem sie gerade dann greift, wenn keine spezifischen völkerrechtlichen Regeln für neuartige Gefahrenlagen bestehen. ${ }^{5}$ Das Beispiel einer Staatenhaftung für informationstechnische Systeme bestätigt diese These.

Die Geschwindigkeit des technologischen Fortschritts in der virtuellen Welt steht nicht im Einklang mit der traditionellen Herausbildung des Völkerrechts - sei es durch multilaterale Verträge oder durch Völkergewohnheitsrecht. Ferner verhindern Zeit-Raum-Kompression sowie Rückverfolgungs- und Nachweisschwierigkeiten in der virtuellen Welt effektive Mechanismen zur Reglementierung von Informationsoperationen. Das Konzept der Staatenhaftung entfaltet eine stabilisierende Wirkung im Umgang mit informationstechnischen Systemen und daraus resultierenden grenzüberschreitenden Schäden, ohne dabei dem virtuellen Raum seine Bedeutung für eine freie und offene Gesellschaft zu nehmen.

Im Vergleich zur Staatenverantwortlichkeit ist das Konzept der Staatenhaftung auch deshalb zur Regulierung von gefährlichen Aktivitäten erfolgsversprechend, weil völkerrechtswidrigem Verhalten ein gewisses Stigma anhängt, welches Staaten davon abhält, für Schäden einzustehen. Die Staatenhaftung hingegen vereinfacht einen Schadensausgleich im Wege einer gütlichen Einigung. ${ }^{6}$ Das Regime der Staatenverantwortlichkeit auf

4 Vgl. I. Y. Liu, The due diligence doctrine under Tallinn Manual 2.0, CLSR 33 (2017), S. 390 (393).

5 B. A. Walton, Duties Owed, Yale LJ 126 (2017), S. 1460 (14634 f., 1477).

6 M. B. Akehurst, International liability for injurious consequences arising out of acts not prohibited by international law, NYIL 16 (1985), S. 3 (15) unter Bezugnahme auf Sonderberichterstatter R. Q. Quentin-Baxter, Preliminary report on international liability for injurious consequences of acts not prohibited by international law, YBILC 1980-II/1, UN Doc. A/CN.4/334 and Add.l and 2, S. 263, Rn. 56. 
neuartige Gefährdungslagen auszudehnen, würde zudem die Provokation von zwischenstaatlichen Konflikten bedeuten. Nur ein völkerrechtliches Haftungssystem ist zielführend. ${ }^{7}$

Das Konzept der Staatenhaftung vermag effektive Lösungen zu bieten. Es begründet die zusammengesetzte staatliche Pflicht der Vermeidung und der Kompensation von erheblichen grenzüberschreitenden Schäden durch völkerrechtlich nicht verbotene, aber (hoch-)gefährliche Aktivitäten.

Die konkret zu ergreifenden Maßnahmen sind dabei von der Gefährdungslage abhängig. Aufgrund der spezifischen Charakteristika von Informationsoperationen ist Schadensvermeidung durch Prävention in der virtuellen Welt kaum möglich. Der Schwerpunkt der Schadensvermeidung liegt hier vielmehr auf der Schadensvorsorge durch Kooperation im Bereich der Informationssicherheit und der Anwendung der besten Schutzpraktiken in Gestalt der Überwachung und Entnetzung kritischer Infrastrukturen, der stetigen Verbesserung des Selbstschutzes informationstechnischer Systeme sowie der Erarbeitung von Notfallplänen für informationstechnische Vorfälle. Daneben spielt für die Schadensvermeidung in der virtuellen Welt auch die Schadensminderung durch verfahrensbezogene Maßnahmen, wie Informationsaustausch, Mitteilung und Warnung sowie Absprachen über Abhilfemaßnahmen, eine wichtige Rolle. Der subjektive Sorgfaltsstandard gewährleistet indessen, dass den Staaten, etwa mit Blick auf ihre technischen und wissenschaftlichen Kapazitäten, keine unrealistischen Pflichten aufoktroyiert werden. Dem steht auch nicht der verschuldensunabhängige Haftungsstandard entgegen, da die Bemühungen zur Schadensvermeidung Einfluss auf den Umfang der Kompensationspflicht haben. Schließlich gewährleistet die Staatenhaftung die notwendige Flexibilität, um der komplexen und unsicheren Beweislage in der virtuellen Welt gerecht zu werden. Denn der Staat haftet den Gegebenheiten des konkreten Einzelfalls entsprechend entweder primär, subsidiär oder ergänzend. Dieses mehrstufige Haftungssystem garantiert einen möglichst umfassenden und angemessen Schadensausgleich.

Das Konzept der Staatenhaftung mag der modernen von einzelstaatlichen Partikularinteressen geprägten Gestaltung völkerrechtlicher Regeln für unterschiedliche Bereiche nicht entsprechen. Es bildet aber die Basis für internationale Sicherheit und Frieden im Wege eines gerechten Interessenausgleiches und bietet letztlich jedem Staat Vorteile.

7 B. A. Walton, Duties Owed, Yale LJ 126 (2017), S. 1460 (1519). 


\section{Bibliografie}

\section{A. Literatur}

Abdullah, Nuraisyah Chua/Shah, Rohani Mohd/Husin, Zaliha Hj/Rahman, Hamisah $A b d$., Antarctic Tourism: The responsibilities and liabilities of tour operators and state parties, Procedia - Social and Behavioral Sciences 202 (2015), S. 227233.

Ago, Roberto, Das Verschulden im völkerrechtlichen Unrecht, Zeitschrift für Öffentliches Recht 20 (1940), S. 449-484.

Akehurst, Michael B., International Liability for Injurious Consequences out of Acts not Prohibited by International Law, Netherlands Yearbook of International Law 16 (1985), S. 3-16.

Almhofer, Martina, Die Haftung der Europäischen Union für rechtswidrige Bankenaufsicht, Tübingen 2018.

Anderson, Mark, Derivative versus Direct Liability as a Basis for State Liability for Transboundary Harms, in: Bratspies, Rebecca M./Miller, Russell A. (Hg.), Transboundary Harm in International Law: Lessons from the Trail Smelter Arbitration, Cambridge 2006, S. 99-108.

Anton, Donald K., The Principle of Residual Liability in the Seabed Disputes Chamber of the International Tribunal for the Law of the Sea: The Advisory Opinion on Responsibility and Liability for International Seabed Mining (ITLOS Case No. 17), McGill International Journal of Sustainable Development Law and Policy 7 (2011-2012), S. 242-257.

Anton, Donald K./Makgill, Robert A./Payne, Cymie R., Seabed Mining: Advisory Opinion on Responsibility and Liability, Environmental Policy and Law 41 (2011), S. 60-65.

Antonopoulos, Constantine, State Responsibility in Cyberspace, in: Tsagourias, Nicholas/Buchan, Russell (Hg.), Research Handbook on International Law and Cyberspace, Cheltenham [u.a.] 2015, S. 55-71.

Arangio-Ruiz, Gaetano, State Fault and the Forms and Degrees of International Responsibility: Questions of Attribution and Relevance, in: Le Droit International au Service de la Paix, de la Justice et du Développement, Mélanges Michel Virally, Paris 1991, S. 25-42.

Aréchaga, Eduardo Jiménez de, International law in the past third of a century, Recueil des Cours 159 (1978-I), S. 1-344.

Areng, Liina, International Cyber Crisis Management and Conflict Resolution Mechanisms, in: Ziolkowski, Katharina (Hg.), Peacetime Regime for State Activities in Cyberspace: International Law, International Relations and Diplomacy, Tallinn 2013, S. 565-594. 


\section{Bibliografie}

Arnauld, Andreas von, Völkerrechtliche Informationspflichten bei Naturkatastrophen, Archiv des Völkerrechts, 43 (2005), S. 279-311.

Arnauld, Andreas von, Völkerrecht, 4. Auflage, Heidelberg 2019.

Arndt, Birger, Das Vorsorgeprinzip im EU-Recht, Tübingen 2009.

Ashmore, William C., Impact of Alleged Russian Cyber Attacks, Baltic Security \& Defence Review 11 (2009), S. 4-40.

Atapattu, Sumudu A., Emerging Principles of International Environmental Law, Ardsley, N.Y. 2006.

Aust, Helmut Philipp, Complicity and the Law of State Responsibility, Cambridge [u.a.] 2011.

Banks, William, State Responsibility and Attribution of Cyber Intrusions After Tallinn 2.0, Texas Law Review 95 (2017), S. 1487-1513.

Bannelier-Christakis, Karine, Cyber Diligence: A Low-Intensity Due Diligence Principle for Low-Intensity Cyberspace?, Baltic Yearbook of International Law 14 (2014), S. 23-39.

Bantekas, Ilias, Trust Funds, in: Wolfrum, Rüdiger (Hg.), Max Planck Encyclopedia of Public International Law, Online Edition www.mpepil.com, Stand: 2010.

Barboza, Julio, Liability: Can We Put Humpty-Dumpty Together Again?, Chinese Journal of International Law 1 (2002), S. 499-526.

Barboza, Julio, The Environment, Risk and Liability in International Law, Leiden [u.a.] 2011.

Bassiouni, M. Cherif, A Functional Approach to "General Principles of International Law", Michigan Journal of International Law 11 (1990), S. 768-818.

Bastmeijer, Kees, Antarctica, in: Nollkaemper, André/Plakokefalos, Ilias (Hg.), The Practice of Shared Responsibility in International Law, Cambridge 2017, S. 399425.

Bäumler, Jelena, Das Schädigungsverbot im Völkerrecht: Eine Untersuchung anhand des Umwelt-, Welthandels- und Finanzvölkerrechts, Berlin [u.a.] 2017.

Beaucamp, Guy/Treder, Lutz, Methoden und Technik der Rechtsanwendung, 3. Auflage, Heidelberg 2015.

Beck, Ulrich, Weltrisikogesellschaft: Auf der Suche nach der verlorenen Sicherheit, 3. Auflage, Frankfurt am Main 2015.

Benatar, Marco, The Use of Cyber Force: Need for Legal Justification?, Goettingen Journal of International Law 1 (2009), S. 375-396.

Bencsáth, Boldizsár/Pék, Gábor/Buttyán, Levente/Félegyházi, Márk, The Cousins of Stuxnet: Duqu, Flame, and Gauss, Future Internet 4 (2012), S. 971-1003.

Benzing, Markus, Das Beweisrecht vor internationalen Gerichten und Schiedsgerichten in zwischenstaatlichen Streitigkeiten, Berlin [u.a.] 2010.

Bergkamp, Lucas, Liability and Environment: Private and Public Law Aspects of Civil Liability for Environmental Harm in an International Context, Den Haag [u.a.] 2001. 
Berwick, Teresa A., Responsibility and Liability for Environmental Damage: A Roadmap for International Environmental Regimes, Georgetown International Environmental Law Review 10 (1997-1998), S. 257-267.

Beyerlin, Ulrich, Grenzüberschreitender Umweltschutz und allgemeines Völkerrecht, in: Hailbronner, Kay/Ress, Georg/Stein, Torsten (Hg.), Staat und Völkerrechtsordnung: Festschrift für Karl Doehring, Berlin [u.a.] 1989, S. 37-62.

Beyerlin, Ulrich/Marauhn, Thilo, International Environmental Law, Oxford 2011.

Bhuta, Nehal/Pantazopoulous, Stavros-Evdokimos, Autonomy and uncertainty: increasingly autonomous weapons systems and the international legal regulation of risk, in: Bhuta, Nehal/Beck, Susanne/Geiß, Robin/Liu, Hin-Yan/Kreß, Claus (Hg.), Autonomous Weapons Systems: Law, Ethics, Policy, Cambridge 2016, S. 284-300.

Binder, Christina, Die Grenzen der Vertragstreue im Völkerrecht: am Beispiel der nachträglichen Änderung der Umstände, Heidelberg [u.a.] 2013.

Binder, Christina/Schreuer, Christoph, Unjust Enrichment, in: Wolfrum, Rüdiger (Hg.), Max Planck Encyclopedia of Public International Law, Online Edition www.mpepil.com, Stand: 2017.

Birnie, Patricia W./Boyle, Alan E./Redgwell, Catherine, International Law and the Environment, 3. Auflage, Oxford 2009.

Blank, Stephen, Cyber War and Information War à la Russe, in: Perkovich, George/ Levite, Ariel E. (Hg.), Understanding Cyber Conflict: 14 Analogies, Washington, D.C. 2017, S. 81-98.

Bleckmann, Albert, Analogie im Völkerrecht, Archiv des Völkerrechts 17 (1977), S. 161-180.

Bleckmann, Albert, Zur Feststellung und Auslegung von Völkergewohnheitsrecht, Zeitschrift für ausländisches öffentliches Recht und Völkerrecht 37 (1977), S. 504-529.

Bleckmann, Albert, Die Aufgaben einer Methodenlehre des Völkerrechts: Probleme der Rechtsquellenlehre im Völkerrecht, Heidelberg [u.a.] 1978.

Bleckmann, Albert, Grundprobleme und Methoden des Völkerrechts, Freiburg [u.a.] 1982.

Bodansky, Daniel, Customary (And Not So Customary) International Environmental Law, Indiana Journal of Global Legal Studies 3 (1995), S. 105-119.

Bodansky, Daniel, The Art and Craft of International Environmental Law, Cambridge [u.a.] 2010.

Boisson de Chazounes, Laurence/Campanelli, Danio, Neighbour States, in: Wolfrum, Rüdiger (Hg.), Max Planck Encyclopedia of Public International Law, Online Edition www.mpepil.com, Stand: 2006.

Borda, Aldo Zammit, A Formal Approach to Article 38(1)(d) of the ICJ Statute from the Perspective of the International Criminal Courts and Tribunals, European Journal of International Law 24 (2013), S. 649-661.

Bordin, Fernando Lusa, Reflections of Customary International Law: The Authority of Codification Conventions and ILC Draft Articles in International Law, International and Comparative Law Quarterly, 63 (2014), S. 535-567. 


\section{Bibliografie}

Bornheim, Gaby, Haftung für grenzüberschreitende Umweltbeeinträchtigungen im Völkerrecht und im Internationalen Privatrecht, Frankfurt am Main [u.a.] 1995.

Bos, Maarten, Prolegomena to the Identification of Custom in International Law, in: T. M. C. Asser Instituut (Hg.), Essays on International \& Comparative Law in Honour of Judge Erades, Den Haag 1983, S. 1-13.

Böschen, Stefan/Weis, Kurt, Die Gegenwart der Zukunft: Perspektiven zeitkritischer Wissenspolitik, Wiesbaden 2007.

Bosma, Shane/Underwood, Josh, Compensation claims arising from the Montara Oil Spill, in: Handl, Günther/Svendsen, Kristoffer (Hg.), Managing the Risk of Offshore Oil and Gas Accidents: The International Legal Dimension, Cheltenham [u.a.] 2019, S. 425-446.

Bothe, Michael, Die Bedeutung der Rechtsvergleichung in der Praxis internationaler Gerichte, Zeitschrift für ausländisches öffentliches Recht und Völkerrecht 36 (1976), S. 280-299.

Boyle, Alan E., Nuclear Energy and International Law: An Environmental Perspective, British Yearbook of International Law 60 (1989), S. 257-313.

Boyle, Alan E., State Responsibility and International Liability for Injurious Consequences of Acts Not Prohibited by International Law: A Necessary Distinction?, International and Comparative Law Quarterly 39 (1990), S. 1-26.

Boyle, Alan E., Globalising Environmental Liability: The Interplay of National and International Law, Journal of Environmental Law 17 (2005), S. 3-26.

Boyle, Alan E., Polluter Pays, in: Wolfrum, Rüdiger (Hg.), Max Planck Encyclopedia of Public International Law, Online Edition www.mpepil.com, Stand: 2009.

Boyle, Alan E., Liability for injurious consequence arising out of acts not prohibited by international law, in: Crawford, James/Pellet, Alain/Olleson, Simon (Hg.), The Law of International Responsibility, Oxford 2010, S. 95-104.

Boyle, Alan E., Transboundary air pollution: a tale of two paradigms, in: Jayakumar, Shunmugam/Koh, Tommy/Beckman, Robert/Phan, Hao Duy (Hg.), Transboundary Pollution: Evolving Issues of International Law and Policy, Cheltenham [u.a.] 2015, S. 233-262.

Bratspies, Rebecca M./Miller, Russell A., Introduction, in: dies. (Hg.), Transboundary Harm in International Law: Lessons from the Trail Smelter Arbitration, Cambridge [u.a.] 2006, S. 1-10.

Brent, Kerryn Anne, The Certain Activities case: what implications for the no-harm rule?, Asia Pacific Journal of Environmental Law 20 (2017), S. 28-56.

Brownlie, Ian, International Law and the Use of Force by States, Oxford 1963.

Brunnée, Jutta, "Common Interest" - Echoes from an Empty Shell?: Some Thoughts on Common Interest and International Environmental Law, Zeitschrift für ausländisches öffentliches Recht und Völkerrecht 49 (1989) S. 791-808.

Brunnée, Jutta, Of Sense and Sensibility: Reflections on International Liability Regimes as Tools for Environmental Protection, International and Comparative Law Quarterly 53 (2004), S. 351-367. 
Brunnée, Jutta, Common Areas, Common Heritage, Common Concern, in: Bodansky, Daniel/dies./Hey, Ellen (Hg.), The Oxford Handbook of International Environmental Law, Oxford 2007, S. 550-573.

Brunnée, Jutta, Sic utere tuo ut alienum non laedas, in: Wolfrum, Rüdiger (Hg.), Max Planck Encyclopedia of Public International Law, Online Edition www.mpepil.com, Stand: 2010.

Brunnée, Jutta/Meshel, Tamar, Teaching an Old Law New Tricks: International Environmental Law Lessons for Cyberspace Governance, German Yearbook of International Law 58 (2015), S. 129-168.

Bruns, Viktor, Völkerrecht als Rechtsordnung I., Zeitschrift für ausländisches öffentliches Recht und Völkerrecht 1 (1929), S. 1-56.

Brüggemeier, Gert, Umwelthaftungsrecht: Ein Beitrag zum Recht der »Risikogesellschaft«?, Kritische Justiz 22 (1989), S. 209-230.

Bryde, Brun-Otto, Umweltschutz durch allgemeines Völkerrecht?, Archiv des Völkerrechts 31 (1993), S. 1-12.

Buchan, Russell, Cyber Attacks: Unlawful Uses of Force or Prohibited Interventions?, Journal of Conflict and Security Law, 17 (2012), S. 212-227.

Buchan, Russell, Cyberspace, Non-State Actors and the Obligation to Prevent Transboundary Harm, Journal of Conflict \& Security Law 21 (2016), S. 429-453.

Byers, Michael, Abuse of Rights: An Old Principle, A New Age, McGill Law Journal 47 (2002), S. 389-431.

Calliess, Christian, Art. 191 AEUV (ex-Art. 174 EGV): Umweltpolitische Ziele; Schutzmaßnahmen; internationale Zusammenarbeit, in: ders./Ruffert, Matthias (Hg.), EUV/AEUV: Das Verfassungsrecht der Europäischen Union mit Europäischer Grundrechtecharta, Kommentar, 5. Auflage, München 2016, Rn. 38-40.

Cameron, James/Abouchar, Juli, The Status of the Precautionary Principle in International Law, in: Freestone, David/Hey, Ellen (Hg.), The Precautionary Principle and International Law: The Challenge of Implementation, Den Haag [u.a.] 1996, S. 29-52.

Campanelli, Danio, Principle of Solidarity, in: Wolfrum, Rüdiger (Hg.), Max Planck Encyclopedia of Public International Law, Online Edition www.mpep il.com, Stand: 2011.

Carmody, Chios, A Theory of WTO Law, Journal of International Economic Law 11 (2008), S. 527-557.

Caron, David D., The ILC Articles on State Responsibility: The Paradoxical Relationship between Form and Authority, American Journal of International Law 96 (2002), S. 857-873.

Cassese, Antonio, The Nicaragua and Tadić Tests Revisited in Light of the ICJ Judgment on Genocide in Bosnia, European Journal of International Law 18 (2007), S. 649-668.

Castillo-Laborde, Lilian del, Case Law on International Watercourses, in: Dellapenna, Joseph W./Gupta, Joyeeta (Hg.), The Evolution of the Law and Politics of Water, Dordrecht 2009, S.319-336. 


\section{Bibliografie}

Castillo-Laborde, Lilian del, Equitable Utilization of Shared Resources, in: Wolfrum, Rüdiger (Hg.), Max Planck Encyclopedia of Public International Law, Online Edition www.mpepil.com, Stand: 2010.

Charme, Joni S., Transnational Injury and Ultra-Hazardous Activity: An Emerging Norm or International Strict Liability, Journal of Law and Technology 4 (1989), S. 75-92.

Chemain, Régis, The 'Polluter Pays' Principle, in: Crawford, James/Pellet, Alain/ Olleson, Simon (Hg.), The Law of International Responsibility, Oxford 2010, S. 877-885.

Cheng, Bin, General Principles of Law: As Applied by International Courts and Tribunals, Cambridge 1987.

Condorelli, Luigi, La réparation des dommages catastrophiques causés par les activités spatiales, in: Bibliothèque de la Faculté de Droit de l'Université Catholique de Louvain ( $\mathrm{Hg}$. ), La réparation des dommages catastrophiques: Les risques technologiques majeurs en droit international et en droit communautaire, Brüssel 1990, S. 263-300.

Condron, Sean M., Getting it Right: Protecting American Critical Infrastructure in Cyberspace, Harvard Journal of Law and Technology 20 (2007), S. 403-422.

Corn, Gary P./Taylor, Robert, Symposium on Sovereignty, Cyberspace, and Tallinn Manual 2.0, Sovereignty in the Age of Cyber, American Journal of International Law Unbound 111 (2017), S. 207-212.

Crawford, James, The International Law Commission's Articles on State Responsibility, 3. Auflage, Cambridge [u.a.] 2005.

Crawford, James, State Responsibility, in: Wolfrum, Rüdiger (Hg.), Max Planck Encyclopedia of Public International Law, Online Edition www.mpepil.com, Stand: 2006.

Crawford, James, Brownlie's Principles of Public International Law, 9. Auflage, Oxford 2019.

Crespo, Laura/Wanner, Bastien/Ghernaouti, Solange, Cybersecurity Capacity Building: A Swiss Approach, in: Bartsch, Michael/Frey, Stefanie (Hg.), Cybersecurity Best Practices: Lösungen zur Erhöhung der Cyberresilienz für Unternehmen und Behörden, Wiesbaden 2018, S. 525-537.

Crootof, Rebecca, International Cybertorts: Expanding State Accountability in Cyberspace, Cornell Law Review 103 (2018), S. 565-644.

D'Oliveira, Hans Ulrich Jessurun, The Sandoz Blaze: The Damage and the Public and Private Liabilities, in: Francioni, Francesco/Scovazzi, Tullio (Hg.), International Responsibility for Environmental Harm, London [u.a.] 1991, S. 429-445.

Dahm, Georg/Delbrück, Jost/Wolfrum, Rüdiger, Völkerrecht, Band I/1 Die Grundlagen. Die Völkerrechtssubjekte, 2. Auflage, Berlin [u.a.] 1989.

Dam, Cees van, European Tort Law, 2. Auflage, Oxford 2013.

Damrosch, Lori Fisler, Politics Across Borders: Nonintervention and Nonforcible Influence over Domestic Affairs, American Journal of International Law 83 (1989), S. 1-50. 
Davis, John S. II/Boudreaux, Benjamin/Welburn, Jonathan William/Aguirre, Jair/Ogletree, Cordaye/McGovern, Geoffrey/Chase, Michael S., Stateless Attribution: Toward International Accountability in Cyberspace, Santa Monica 2017.

Dederer, Hans-Georg, Staatenverantwortlichkeit ("State responsibility") und Haftung ("liability") im Bereich der "ultrahazardous activities", in: Hecker, Bernd/ Hendler, Reinhard/Proelß, Alexander/Reiff, Peter (Hg.), Verantwortlichkeit und Haftung für Umweltschäden, Berlin 2013, S. 13-50.

Dicke, Detlev Christian, Die Intervention mit wirtschaftlichen Mitteln im Völkerrecht: zugleich ein Beitrag zu den Fragen der wirtschaftlichen Souveränität, Baden-Baden, 1978.

Diekmann, Jochen, Verstärkte Haftung und Deckungsvorsorge für Schäden nuklearer Unfälle: Notwendige Schritte zur Internalisierung externer Effekte, Zeitschrift für Umweltpolitik \& Umweltrecht 34 (2001), S. 119-132.

Diggelmann, Oliver/Hadorn, Nina, Gewalt- und Interventionsverbot bei Cyberangriffen: Ausgewählte Schlüsselfragen, in: Fakultät für Vergleichende Staats- und Rechtswissenschaften der Andrássy Gyula Universität Budapest/Schubel, Christian/Kirste, Stephan/Müller-Graff, Peter-Christian/ders./Hufeld, Ulrich (Hg.), Jahrbuch für Vergleichende Staats- und Rechtswissenschaften - 2016/2017, Baden-Baden 2017, S. 255-274.

Dinniss, Heather Harrison, Cyber Warfare and the Laws of War, Cambridge [u.a.] 2012.

Dittmar, Falko, Angriffe auf Computernetzwerke: Ius ad bellum und ius in bello, Berlin 2005.

Dolzer, Rudolf, Eigentum, Enteignung und Entschädigung im geltenden Völkerrecht, Berlin [u.a.] 1985.

Dolzer, Rudolf/Schreuer, Christoph, Principles of International Investment Law, 2. Auflage, Oxford 2014.

Dörr, Oliver, Staatshaftung in Europa: Vergleichende Bestandsaufnahme, in: ders. (Hg.) Staatshaftung in Europa: Nationales und Unionsrecht, Berlin [u.a.] 2014, S. $1-30$.

Dörr, Oliver, Obligations of the State of Origin of a Cyber Security Incident, German Yearbook of International Law 58 (2015), S. 87-100.

Dörr, Oliver, Weitere Rechtsquellen des Völkerrechts, in: Ipsen, Knut, Völkerrecht, 7. Auflage München 2018, $\mathbb{1 9}$.

Dörr, Oliver, Völkerrechtliche Verantwortlichkeit, in: Ipsen, Knut, Völkerrecht, 7. Auflage München 2018, $\$ 29$.

Douhan, Alena, Liability for Environmental Damage, in: Wolfrum, Rüdiger (Hg.), Max Planck Encyclopedia of Public International Law, Online Edition www.mpepil.com, Stand: 2013.

Drumbl, Mark A., Trail Smelter and the International Law Commission`s Work on State Responsibility for Internationally Wrongful Acts and State Liability, in: Bratspies, Rebecca M./Miller, Russell A. (Hg.), Transboundary Harm in International Law: Lessons from the Trail Smelter Arbitration, Cambridge [u.a.] 2006, S. 85-98. 


\section{Bibliografie}

Dupuy, Pierre-Marie, Reviewing the Difficulties of Codification: On Ago's Classification of Obligations of Means and Obligations of Result in Relation to State Responsibility, European Journal of International Law 10 (1999), S. 371-385.

Dupuy, Pierre-Marie, Droit International public, 5. Auflage, Paris 2000.

Dupuy, Pierre-Marie/Hoss, Cristina, Trail Smelter and Terrorism: International Mechanisms to Combat Transboundary Harm, in: Bratspies, Rebecca M./Miller, Russell A. (Hg.), Transboundary Harm in International Law: Lessons from the Trail Smelter Arbitration, Cambridge [u.a.] 2006, S. 225-239.

Duvic-Paoli, Leslie-Anne, The Prevention Principle in International Environmental Law, Cambridge [u.a.] 2018.

Efrony, Dan/Shany, Yuval, A Rule Book on the Shelf? Tallinn Manual 2.0 on Cyberoperations and Subsequent State Practice, American Journal of International Law 112 (2018), S. 583-657.

Egan, Brian J., International Law and Stability in Cyberspace, Berkeley Journal of International Law 35 (2017), S. 169-180.

Ellis, Jaye, Has International Law Outgrown Trail Smelter?, in: Bratspies, Rebecca M./Miller, Russell A. (Hg.), Transboundary Harm in International Law: Lessons from the Trail Smelter Arbitration, Cambridge [u.a.] 2006, S. 56-65.

Engelhard, Esther/Broek, Berthy van den/Jong, Ferry de/Keirse, Anne/Kezel, Evelien de, Let's Think Twice before We Revise! 'Égalité' as the Foundation of Liability for Lawful Public Sector Acts, Utrecht Law Review 10 (2014), S. 55-76.

Epiney, Astrid, Die völkerrechtliche Verantwortlichkeit von Staaten für rechtswidriges Verhalten im Zusammenhang mit Aktionen Privater, Baden-Baden, 1992.

Epiney, Astrid, Das „Verbot erheblicher grenzüberschreitender Umweltbeeinträchtigungen“: Relikt oder konkretisierungsfähige Grundnorm?, Archiv des Völkerrechts 33 (1995), S. 309-360.

Epiney, Astrid, Umweltvölkerrechtliche Rahmenbedingungen für Entwicklungsprojekte, in: Meng, Werner/Magnus, Ulrich/Schlemmer-Schulte, Sabine/Cottier, Thomas/Stoll, Peter-Tobias/dies. (Hg.), Das internationale Recht im Nord-SüdVerhältnis, Heidelberg 2005, S. 329-381.

Epiney, Astrid, „Gerechtigkeit“ im Umweltvölkerrecht, Aus Politik und Zeitgeschichte 24 (2007), S. 31-38.

Epiney, Astrid, Zur Rechtsfigur des de facto-Organs im Recht der Staatenverantwortlichkeit, in: Fischer-Lescano, Andreas/Gasser, Hans-Peter/Marauhn, Thilo/Ronzitti, Natalino (Hg.), Frieden in Freiheit: Festschrift für Michael Bothe zum 70. Geburtstag, Baden-Baden 2008, S. 883-896.

Epiney, Astrid, Gegenstand, Entwicklung, Quellen und Akteure des internationalen Umweltrechts, in: Proelß, Alexander (Hg.), Internationales Umweltrecht, 2017, S. 1-36.

Epiney, Astrid/Scheyli, Martin, Strukturprinzipien des Umweltvölkerrechts, BadenBaden 1998.

Erben, Cosima, Das Vorsorgegebot im Völkerrecht, Berlin 2005. 
Erichsen, Sven, Das Liability-Projekt der ILC: Fortentwicklung des allgemeinen Umweltrechts oder Kodifizierung einer Haftung für besonders gefährliche Aktivitäten?, Zeitschrift für ausländisches öffentliches Recht und Völkerrecht 51 (1991), S. 94-144.

Erichsen, Sven, Der ökologische Schaden im internationalen Umwelthaftungsrecht: Völkerrecht und Rechtsvergleichung, Frankfurt am Main [u.a.] 1993.

Esposito, Roberto, The ICJ and the Future of Transboundary Harm Disputes: A Preliminary Analysis of the Case Concerning Aerial Herbicide Spraying (Ecuador v. Columbia), Pace International Law Review Online Companion 2 (2010), abrufbar unter: https://digitalcommons.pace.edu/cgi/viewcontent.cgi?referer $=\&$ httpsredir $=1 \&$ article $=1014 \&$ context $=$ pilronline (geprüft am 15.05.2020), S. 1-53.

Fastenrath, Ulrich, Lücken im Völkerrecht: Zu Rechtscharakter, Quellen, Systemzusammenhang, Methodenlehre und Funktionen des Völkerrechts, Berlin 1991.

Fayette, Louise A. de la, The ILC and International Liability: A Commentary, Review of European, Comparative and International Environmental Law 6 (1997), S. 322-333.

Fayette, Louise A. de la, The Concept of Environmental Damage in International Liability Regimes, in: Bowman, Michael/Boyle, Alan E. (Hg.), Environmental Damage in International and Comparative Law: Problems of Definition and Valuation, Oxford [u.a.] 2002, S. 149-190.

Fayette, Louise A. de la, International liability for damage to the environment, in: Fitzmaurice, Malgosia/Ong, David M./Merkouris, Panos (Hg.), Research Handbook on International Environmental Law, Cheltenham [u.a.] 2010, S. 320-360.

Feria-Tinta, Monica/Milnes, Simon C., The Rise of Environmental Law in International Dispute Resolution: The Inter-American Court of Human Rights Issues a Landmark Advisory Opinion on the Environment and Human Rights, Yearbook of International Environmental 27 (2016), S. 64-81.

Fidler, David P., Was Stuxnet an Act of War?: Decoding a Cyberattack, IEEE Security and Privacy Magazine 9 (2011), S. 56-59.

Fidler, David P., Tinker, Tailor, Soldier, Duqu: Why cyberespionage is more dangerous than you think, International Journal of Critical Infrastructure Protection 5 (2012), S. 28-29.

Fitzmaurice, Gerald, Some Problems Regarding the Formal Sources of International Law, in: Koskenniemi, Martti (Hg.), Sources of International Law, Aldershot [u.a.] 2000, S. 57-80.

Forcese, Craig, Spies Without Borders: International Law and Intelligence Collection, Journal of National Security Law and Policy 5 (2011), S. 179-210.

Förster, Susanne, Internationale Haftungsregeln für schädliche Folgewirkungen gentechnisch veränderter Organismen: Europäische und internationale Entwicklungen und Eckwerte für ein Haftungsregime im internationalen Recht, Berlin [u.a.] 2007. 


\section{Bibliografie}

Foster, Caroline, The ILC Draft Principles on the Allocation of Loss in the Case of Transboundary Harm Arising out of Hazardous Activities: Privatizing Risk?, Review of European, Comparative and International Environmental Law 14 (2005), S. 265-282.

Francioni, Francesco, Liability for Damage to the Antarctic Environment, in: ders./ Tullio Scovazzi (Hg.), International Law for Antarctica, The Hague [u.a.] 1996, S. 581-602.

Franzese, Patrick W., Sovereignty in Cyberspace: Can it Exist?, Air Force Law Review 64 (2009), S. 1-42.

Frenz, Walter/Götzkes, Vera, Anmerkung zur Entscheidung FIAMM des EuGH (Große Kammer) vom 9. September 2008 - C-120/06 P und C-121/06 P, Deutsches Verwaltungsblatt 16 (2009), S. 1052-1053.

Gaines, Sanford E., The Polluter-Pays Principle: From Economic Equity to Environmental Ethos, Texas International Law Journal 26 (1991), S. 463-496.

Gärditz, Klaus Ferdinand, Ungeschriebenes Völkerrecht durch Systembildung, Archiv des Völkerrechts 45 (2007), S. 1-34.

Gaycken, Sandro, Cyberwar: Das Internet als Kriegsschauplatz, München 2011.

Gaycken, Sandro, Die vielen Plagen des Cyberwar, in: Schmidt-Radefeldt, Roman/Meissler, Christine (Hg.), Automatisierung und Digitalisierung des Krieges: Drohnenkrieg und Cyberwar als Herausforderungen für Ethik, Völkerrecht und Sicherheitspolitik, Baden-Baden 2012, S. 89-116.

Gaycken, Sandro/Karger, Michael, Entnetzung statt Vernetzung: Paradigmenwechsel bei der IT-Sicherheit, Multimedia und Recht 1 (2011), S. 3-8.

Gehring, Thomas/Jachtenfuchs, Markus, Haftung und Umwelt: Interessenkonflikte im internationalen Weltraum-, Atom- und Seerecht, Frankfurt am Main [u.a.] 1988.

Gehring, Thomas/Jachtenfuchs, Markus, Liability for Transboundary Environmental Damage Towards a General Liability Regime?, European Journal of International Law 4 (1993), S. 92-106.

Geiß, Robin/Labmann, Henning, Freedom and Security in Cyberspace: Shifting the Focus away from Military Responses towards Non-forcible Countermeasures and Collective Threat-Prevention, in: Ziolkowski, Katharina (Hg.), Peacetime Regime for State Activities in Cyberspace: International Law, International Relations and Diplomacy, Tallinn 2013, S. 621-657.

Gervais, Michael, Cyber Attacks and the Laws of War, Berkeley Journal of International Law 30 (2012), S. 525-579.

Goldie, Louis Frederick Edward, Liability for Damage and the Progressive Development of International Law, International and Comparative Law Quarterly 14 (1965), S. 1189-1264.

Goldie, Louis Frederick Edward, Concepts of Strict and Absolute Liability and the Ranking of Liability in terms of Relative Exposure to Risk, Netherlands Yearbook of International Law 16 (1985), S. 175-248. 
Greenwood, Christopher, Self-Defence, in: Wolfrum, Rüdiger (Hg.), Max Planck Encyclopedia of Public International Law, Online Edition www.mpepil.com, Stand: 2011.

Gross, Oren, Cyber Responsibility to Protect: Legal Obligations of States Directly Affected by Cyber-Incidents, Cornell International Law Journal 48 (2015), S. 481-511.

Gündling, Lothar, Verantwortlichkeit der Staaten für grenzüberschreitende Umweltbeeinträchtigungen, Zeitschrift für ausländisches öffentliches Recht und Völkerrecht 45 (1985), S. 265-292.

Gündling, Lothar, The Status in International Law of the Principle of Precautionary Action, in: Freestone, David/IJlstra, Ton (Hg.), The North Sea: Perspectives on Regional Environment Co-operation, London [u.a.] 1990, S. $23-30$.

Haack, Stefan, Die außervertragliche Haftung der EG für rechtmäßiges Verhalten - Zum Urteil des Gerichts erster Instanz (EuG) vom 28.4.1998, Rs. T-184/95, Dorsch Consult Ingenieurgesellschaft $\mathrm{mbH} / \mathrm{Rat}$ der Europäischen Union und Kommission der Europäischen Gemeinschaften, Europarecht 3 (1999), S. 395403.

Haack, Stefan, Grundsätzliche Anerkennung der außervertraglichen Haftung der EG für rechtmäßiges Verhalten nach Art. 288 Abs. 2 EG - Anmerkungen zum Urteil des Gerichts erster Instanz (EuG) vom 14.12.2005, Rs. T-69/00, Europarecht 5 (2006), S. 696-705.

Hach, Renate, Völkerrechtliche Pflichten zur Verminderung grenzüberschreitender Luftverschmutzung in Europa, Berlin [u.a.] 1993.

Hafner, Gerhard/Buffard, Isabelle, Obligations of Prevention and the Precautionary Principle, in: Crawford, James/Pellet, Alain/Olleson, Simon (Hg.), The Law of International Responsibility, Oxford [u.a.] 2010, S. 521-534.

Hagen, Andreas, The Russo-Georgian War 2008, in: Healey, Jason (Hg.), A Fierce Domain: Conflict in Cyberspace, 1986-2012, Washington, D.C. 2013, S. 194204.

Hakimi, Monica, State Bystander Responsibility, European Journal of International Law 21 (2010), S. 341-385.

Handl, Günther, Territorial Sovereignty and the Problem of Transnational Pollution, American Journal of International Law 69 (1975), S. 50-76.

Handl, Günther, State Liability for Accidental Transnational Environmental Damage by Private Persons, American Journal of International Law 74 (1980), S. 525-565.

Handl, Günther, Liability as an obligation established by a primary rule of international law: Some basic reflections on the International Law Commission's work, Netherlands Yearbook of International Law 16 (1985), S. 49-79.

Handl, Günther, Trail Smelter in Contemporary International Environmental Law: Its Relevance in the Nuclear Energy Context, in: Bratspies, Rebecca M./Miller, Russell A. (Hg.), Transboundary Harm in International Law: Lessons from the Trail Smelter Arbitration, Cambridge [u.a.] 2006, S. 125-139. 


\section{Bibliografie}

Handl, Günther, International Accountability for Transboundary Environmental Harm Revisited: What Role for "State Liability"?, Environmental Policy and Law 37 (2007), S. 116-125.

Handl, Günther, International Law and the Liability for Catastrophic Environmental Damage, Remarks, American Society of International Law Proceedings 105 (2011), S. 427-430.

Handl, Günther, Transboundary Impacts, in: Bodansky, Daniel/Brunnée, Jutta/Hey, Ellen (Hg.), The Oxford Handbook of International Environmental Law, Oxford [u.a.] 2008, S. 531-549.

Handl, Günther, Allocating transboundary loss from offshore oil and gas accidents, in: ders./Svendsen, Kristoffer (Hg.), Managing the Risk of Offshore Oil and Gas Accidents: The International Legal Dimension, Cheltenham [u.a.] 2019, S. 253284.

Handl, Günther/Simma, Bruno, Grenzüberschreitende Auswirkungen von Kernkraftanlagen und Völkerrecht, Österreichische Zeitschrift für öffentliches Recht und Völkerrecht 39 (1988), S. 1-8.

Hanqin, Xue, Transboundary Damage in International Law, Cambridge [u.a.] 2003.

Haratsch, Andreas/Koenig, Christian/Pechstein, Matthias, Europarecht, 11. Auflage, Tübingen 2018.

Hardy, M. J. L., International Protection Against Nuclear Risks, International and Comparative Law Quarterly 10 (1961), S. 739-759.

Hare, Forrest, The Significance of Attribution to Cyberspace Coercion: A Political Perspective, in: Czosseck, Christian/Ottis, Rain/Ziolkowski, Katharina (Hg.), 2012 4th International Conference on Cyber Conflict, Tallinn 2012, S. 125-139.

Hartmann, Bernd J., Haftung in der Europäischen Union, in: Dörr, Oliver (Hg.) Staatshaftung in Europa: Nationales und Unionsrecht, Berlin [u.a.] 2014, S. 3160.

Hathaway, Oona A., The Drawbacks and Dangers of Active Defense, in: Brangetto, Pascal/Maybaum, Markus/Stinissen, Jan, 2014 6th International Conference on Cyber Conflict, Tallinn 2014, S. 39-50.

Hathaway, Oona A./Crootof, Rebecca/Levitz, Philip/Nix, Haley/Nowlan, Aileen/Perdue, William/Spiegel, Julia, The Law of Cyber-Attack, California Law Review 100 (2012), S. 817-885.

Hathaway, Oona A./Nielsen, Elizabeth/Nowlan, Aileen/Perdue, William/Purvis, Chelsea/Solow, Sara/Spiegel, Julia, Human Rights Abroad: When do Human Rights Treaty Obligations Apply Extraterritorially?, Arizona State Law Journal 43 (2011), S. 389-426.

Healey, Jason/Pitts, Hannah, Applying International Environmental Legal Norms to Cyber Statecraft, I/S: A Journal of Law and Policy for the Information Society 8 (2012), S. 356-387. 
Heathcote, Sarah, State omissions and due diligence: aspects of fault, damage and contribution to injury in the law of state responsibility, in: Bannelier, Karine/ Christakis, Théodore/dies. (Hg.), The ICJ and the Evolution of International Law: The enduring impact of the Corfu Channel case, London [u.a.] 2012, S. 295-314.

Heintschel von Heinegg, Wolff, Informationskrieg und Völkerrecht - Angriffe auf Computernetzwerke in der Grauzone zwischen nachweisbarem Recht und rechtspolitischer Forderung, in: Epping, Volker/Fischer, Horst/ders. (Hg.), Brücken bauen und begehen: Festschrift für Knut Ipsen zum 65. Geburtstag, München 2000, S. 129-156.

Heintschel von Heinegg, Wolff, Cyberspace - ein völkerrechtliches Niemandsland, in: Schmidt-Radefeldt, Roman/Meissler, Christine (Hg.), Automatisierung und Digitalisierung des Krieges: Drohnenkrieg und Cyberwar als Herausforderungen für Ethik, Völkerrecht und Sicherheitspolitik, Baden-Baden, 2012, S. 159-174.

Heintschel von Heinegg, Wolff, Legal Implications of Territorial Sovereignty in Cyberspace, in: Czosseck, Christian/Ottis, Rain/Ziolkowski, Katharina (Hg.), 2012 4th International Conference on Cyber Conflict, Tallinn 2012, S. 7-19.

Heintschel von Heinegg, Wolff, Territorial Sovereignty and Neutrality in Cyberspace, International Law Studies 89 (2013), S. 123-156.

Hemmings, Alan D., Liability postponed: the failure to bring Annex VI of the Madrid Protocol into force, The Polar Journal 8 (2018), S. 315-332.

Herczegh, Géza, General principles of international law and the international legal order, Budapest 1969.

Herdegen, Matthias, Possible Legal Framework and Regulatory Models for Cyberspace: Due Diligence Obligations and Institutional Models for Enhanced Inter-State Cooperation, German Yearbook of International Law 85 (2015), S. $169-185$.

Herzog, Stephen, Revisiting the Estonian Cyber Attacks: Digital Threats and Multinational Responses, Journal of Strategic Security 4 (2011), S. 49-60.

Hey, Ellen, The Precautionary Concept in Environmental Policy and Law: Institutionalizing Caution, Georgetown International Law Review, 4 (1992), S. 303318.

Hey, Ellen, The Watercourse Convention: To What Extent Does it Provide a Basis for Regulating Uses of International Watercourses?, Review of European Comparative International Environmental Law 7 (1998), S. 291-300.

Higgins, Rosalyn, Problems and Process: International Law and How We Use It, Oxford 1995.

Higgins, Rosalyn, International Law in a Changing International System, Cambridge Law Journal 58 (1999), S. 78-95.

Hinds, Caroline, Das Prinzip „sic utere tuo ut alienum non laedas“ und seine Bedeutung im internationalen Umweltrecht, Archiv des Völkerrechts 30 (1992), S. 298-235. 


\section{Bibliografie}

Hinkle, Katharine C., Countermeasures in the Cyber Context: One More Thing to Worry About, Yale Journal of International Law Online 37 (2011), abrufbar unter: http://www.yjil.org/docs/pub/o-37-hinkle-countermeasures-in-the-cyber-c ontext.pdf (geprüft am 15.05.2020), S. 11-21.

Hinteregger, Monika, Atomhaftung in Europa - Bestand und Perspektiven, in: Apathy, Peter/Bollenberger, Raimund/Bydlinski, Peter/Iro, Gert/Karner, Ernst/ Karollus, Martin (Hg.), Festschrift für Helmut Koziol zum 70. Geburtstag, Wien 2010, S. 667-680.

Hipp, Margarete, The International Sea Bed Authority, in: Ehlers, Peter/Lagoni, Rainer (Hg.), International Maritime Organisations and Their Contribution Towards a Sustainable Marine Development, Hamburg 2006, S. 135-155.

Hobe, Stephan, Die Zukunft des Völkerrechts im Zeitalter der Globalisierung, Archiv des Völkerrechts 37 (1999), S. 253-282.

Hobe, Stephan, Europarecht, 9. Auflage, München 2017.

Hofmann, Rainer, Modernes Investitionsschutzrecht: Ein Beispiel für entstaatlichte Setzung und Durchsetzung von Recht, in: Kadelbach, Stefan/Günther, Klaus (Hg.), Recht ohne Staat?: Zur Normativität nichtstaatlicher Rechtsetzung, Frankfurt [u.a.] 2011, S. 119-145.

Hofmann, Rainer, Modern International Investment Law as an Example of Extra-territorial Law-making and Law-enforcement, in: Handl, Günther/Zekoll, Joachim/Zumbaansen, Peer (Hg.), Beyond Territoriality: Transnational Legal Authority in an Age of Globalisation, Leiden 2012, S. 439-461.

Hohmann, Harald, Präventive Rechtspflichten und -prinzipien des modernen Umweltvölkerrechts: Zum Stand des Umweltvölkerrechts zwischen Umweltnutzung und Umweltschutz, Berlin, 1992.

Hollis, Duncan B., Why States Need an International Law for Information Operations, Lewis \& Clark Law Review 11 (2007), S. 1023-1061.

Hollis, Duncan B., An e-SOS for Cyberspace, Harvard International Law Journal 52 (2011), S. 373-432.

Horbach, Nathalie Louisa Johanna Theodora, Liability versus responsibility under international law: Defending strict state responsibility for transboundary damage, Leiden 1996.

Jakab, András, Prinzipien, Rechtstheorie 37 (2006), S. 49-65.

Jenks, C. Wilfred, Liability for Ultra-Hazardous Activities in International Law, Recueil des Cours 117 (1966-I), S. 99-200.

Jensen, Eric Talbot, Cyber Sovereignty: The Way Ahead, Texas International Law Journal 50 (2014) S. 275-304.

Jensen, Eric Talbot/Watts, Sean, A Cyber Duty of Due Diligence: Gentle Civilizer or Crude Destabilizer?, Texas Law Review 95 (2017), S. 1555-1577.

Jessen, Henning, Staatenverantwortlichkeit und seevölkerrechtliche Haftungsgrundsätze für Umweltschäden durch Tiefseebodenbergbau, Zeitschrift für Umweltrecht 2 (2012), S. 71-81.

Jinks, Derek, State Responsibility for the Acts of Private Armed Groups, Chicago Journal of International Law 4 (2003), S. 83-96. 
Jolley, Jason D., Attribution, State Responsibility, and the Duty to Prevent Malicious Cyber-Attacks in International Law, Ph.D. thesis, University of Glasgow, 2017, abrufbar unter: http://theses.gla.ac.uk/id/eprint/8452 (geprüft am 15.05.2020).

Joyner, Christopher C./Lotrionte, Catherine, Information Warfare as International Coercion: Elements of a Legal Framework, European Journal of International Law 12 (2001), S. 825-865.

Jung, Han-Wei, Rechtserkenntnis und Rechtsfortbildung im Völkergewohnheitsrecht: Das Verhältnis zwischen Methodik und Rechtsquellenlehre, Göttingen 2012.

Kadelbach, Stefan, Zwingendes Völkerrecht, Berlin 1992.

Kadelbach, Stefan, Ethik des Völkerrechts unter Bedingungen der Globalisierung, Zeitschrift für ausländisches öffentliches Recht und Völkerrecht 64 (2004), S. 120.

Kadelbach, Stefan, The Concept of State Responsibility for Private Acts under General International Law, in: Hobe, Stephan/Freeland, Steven/Schmidt-Tedd, Bernhard (Hg.), In Heaven as on Earth?: The Interaction of Public International Law on the Legal Regulation of Outer Space, Bonn 2013, S. 105-119.

Kadelbach, Stefan/Kleinlein, Thomas, Überstaatliches Verfassungsrecht, Zur Konstitutionalisierung im Völkerrecht, Archiv des Völkerrechts 44 (2006), S. 235-266.

Kadelbach, Stefan/Kleinlein, Thomas, International Law - a Constitution of Mankind?: An Attempt at a Re-appraisal with an Analysis of Constitutional Principles, German Yearbook of International Law 50 (2007), S. 303-347.

Kadens, Emily/Young, Ernest A., How customary is customary international law?, William \& Mary Law Review 54 (2013), S. 885-920.

Kahl, Wolfgang, Umweltprinzip und Gemeinschaftsrecht: Eine Untersuchung zur Rechtsidee des "bestmöglichen Umweltschutzes" im EWG-Vertrag, Heidelberg 1993.

Kälin, Walter/Künzli, Jörg, Universeller Menschenrechtsschutz: Der Schutz des Individuums auf globaler und regionaler Ebene, 4. Auflage, Basel [u.a.] 2019.

Kämmerer, Jörn Axel, Der Schutz des Eigentums im Völkerrecht, in: 42. Bitburger Gespräche: Eigentum - Ordnungsidee, Zustand und Entwicklungen, München, Jahrbuch 2004/I, S. 143-168.

Kammerhofer, Jörg, Uncertainty in the Formal Sources of International Law: Customary International Law and Some of Its Problems, European Journal of International Law 15 (2004), S. 523-553.

Kanalan, Ibrahim, Extraterritoriale Staatenpflichten jenseits der Hoheitsgewalt: Ein neues Konzept für umfassende extraterritoriale Staatenpflichten, Archiv des Völkerrechts 52 (2014), S. 495-521.

Kanuk, Sean P., Recent Developments: Information Warfare: New Challenges for Public International Law, Harvard International Law Journal 37 (1996), 272285. 


\section{Bibliografie}

Keber, Tobias O./Roguski, Przemystaw Nick, Ius ad bellum electronicum?: Cyberangriffe im Lichte der UN-Charta und aktueller Staatenpraxis, Archiv des Völkerrechts 49 (2011), S. 399-434.

Kelemen, Daniel/Knievel, Tim, The United States, the European Union, and international environmental law: The domestic dimensions of green diplomacy, International Journal of Constitutional Law 13 (2015), S. 945-965.

Kelson, John M., State Responsibility and the Abnormally Dangerous Activity, Harvard International Law Journal 13 (1972) S. 197-244.

Kettemann, Matthias C., Das Internet als internationales Schutzgut: Entwicklungsperspektiven des Internetvölkerrechts anlässlich des Arabischen Frühlings, Zeitschrift für ausländisches öffentliches Recht und Völkerrecht 72 (2012), S. 469482.

Kilovaty, Ido, Doxfare: Politically Motivated Leaks and the Future of the Norm on Non-Intervention in the Era of Weaponized Information, Harvard National Security Journal 9 (2018), S. 146-179.

Kim, Dae-Won, Non-violation complaints in WTO law: Theory and Practice, Bern [u.a.] 2006.

Kimminich, Otto, Völkerrechtliche Haftung für das Handeln Privater im Bereich des internationalen Umweltschutzes, Archiv des Völkerrechts 22 (1984), S. 241282.

Kiss, Alexandre, The Rights and Interests of Future Generations and the Precautionary Principle, in: Freestone, David/Hey, Ellen (Hg.), The Precautionary Principle and International Law: The Challenge of Implementation, Den Haag [u.a.] 1996, S. 19-28.

Klein, Eberhard, Umweltschutz im völkerrechtlichen Nachbarrecht, Berlin 1976.

Kleinlein, Thomas, Konstitutionalisierung im Völkerrecht: Konstruktion und Elemente einer idealistischen Völkerrechtslehre, Berlin [u.a.] 2012.

Koivurova, Timo, Due Diligence, in: Wolfrum, Rüdiger (Hg.), Max Planck Encyclopedia of Public International Law, Online Edition www.mpepil.com, Stand: 2013.

Kojima, Chie, Compensation Fund, in: Wolfrum, Rüdiger (Hg.), Max Planck Encyclopedia of Public International Law, Online Edition www.mpepil.com, Stand: 2009.

Kolb, Robert, Principles as sources of international law (with special reference to good faith), Netherlands International Law Review 53 (2006), S. 1-36.

Kolb, Robert, Reflections on Due Diligence Duties and Cyberspace, German Yearbook of International Law 58 (2015), S. 113-128.

Köndgen, Johannes, Überlegungen zur Fortbildung des Umwelthaftpflichtrechts, Umwelt- und Planungsrecht 11-12 (1983), S. 345-356.

Korff, Douwe, First Do No Harm: The Potential of Harm Caused to Fundamental Rights and Freedoms by State Cybersecurity interventions, in: Wagner, Ben/Kettemann, Matthias C./Vieth, Kilian (Hg.), Research Handbook on Human Rights and Digital Technology: Global Politics, Law and International Relations, Cheltenham 2019, S. 129-155. 
Krajewski, Markus, Wirtschaftsvölkerrecht, 4. Auflage, Heidelberg 2017.

Kranz, Jerzy, Die völkerrechtliche Verantwortlichkeit für die Anwendung militärischer Gewalt: Maßstäbe der Zurechenbarkeit, Archiv des Völkerrechts 48 (2010), S. 281-337.

Krieger, Heike, Die Verantwortlichkeit Deutschlands nach der EMRK für seine Streitkräfte im Auslandseinsatz, Zeitschrift für ausländisches öffentliches Recht und Völkerrecht 62 (2002), S. 669-702.

Krieger, Heike, Krieg gegen anonymous: Völkerrechtliche Regelungsmöglichkeiten bei unsicherer Zurechnung im Cyberwar, Archiv des Völkerrechts 50 (2012), S. 1-20.

Kulesza, Joanna, State responsibility for acts of cyber-terrorism, Global Internet Governance Academic Network, Annual Symposium, Vilnius 2010.

Kulesza, Joanna, Due Diligence in Cyberspace in: Portela, Irene Maria/Almeida, Fernando (Hg.), Cyberspace, Organizational, Legal, and Technological Dimensions of Information System Administration, Hershey PA 2014, S. 76-95.

Kulesza, Joanna, Due Diligence in International Law, Leiden [u.a.] 2016.

Kumm, Mattias, The Cosmopolitan Turn in Constitutionalism: An Integrated Conception of Public Law, Indiana Journal of Global Legal Studies 20 (2013), S. 605-628.

Kunig, Phillip, Prohibition of Intervention, in: Wolfrum, Rüdiger (Hg.), Max Planck Encyclopedia of Public International Law, Online Edition www.mpep il.com, Stand: 2008.

Kypraios, Christos/Carpanelli, Elena, Space Debris, in: Wolfrum, Rüdiger (Hg.), Max Planck Encyclopedia of Public International Law, Online Edition www.mpepil. com, Stand: 2018.

Lammers, Johan G., Pollution of the International Watercourses: A Search for Substantive Rules and Principles of Law, Boston [u.a.] 1984.

Lammers, Johan G., International Responsibility and Liability for Damage Caused by Environmental Interferences, Environmental Policy and Law 31 (2001), S. $42-50$.

Lapidoth, Ruth, International Straits, in: Wolfrum, Rüdiger (Hg.), Max Planck Encyclopedia of Public International Law, Online Edition www.mpepil.com, Stand: 2018.

Larenz, Karl/Canaris, Claus-Wilhelm, Methodenlehre der Rechtswissenschaft, 3. Auflage, Berlin [u.a.] 1995.

Lauterpacht, Hersch, Private Law Sources and Analogies of International Law: with special references to international arbitration, London [u.a.] 1927.

Lefeber, René, Transboundary Environmental Interference and the Origin of State Liability, Den Haag 1996.

Libicki, Martin C., Cyberdeterrence and Cyberwar, Santa Monica 2009.

Liu, Ian Yuying, State Responsibility and Cyberattacks: Defining Due Diligence Obligations, Indonesian Journal of International \& Comparative Law 4 (2017), S. 191-260. 


\section{Bibliografie}

Liu, Ian Yuying, The due diligence doctrine under Tallinn Manual 2.0, Computer Law \& Security Review 33 (2017), S. 390-395.

Lotrionte, Catherine, Cyber Operations: Conflict Under International Law, Georgetown Journal of International Affairs, (2012), S. 15-24.

Lotrionte, Catherine, Reconsidering the Consequences for State-Sponsored Hostile Cyber Operations Under International Law, The Cyber Defense Review 3, (2018), S. 73-114.

Louka, Elli, International Environmental Law: Fairness, Effectiveness, and World Order, Cambridge [u.a.] 2006.

Loures, Flavia Rocha/Rieu-Clarke, Alistair/Dellapenna, Joseph W./Lammers, Johan, The authority and function of the UN Watercourses Convention, in: Loures, Flavia Rocha/Rieu-Clarke, Alistair (Hg.), The UN Watercourses Convention in Force: Strengthening international law for transboundary water management, Abingdon Oxon 2013, S. 49-66.

MacCormick, Neil, Legal Reasoning and Legal Theory, Oxford 1978.

Maddocks, Jennifer, Outsourcing of Governmental Functions in Contemporary Conflict: Rethinking the Issue of Attribution, Virginia Journal of International Law 59 (2019), S. 47-95.

Maestral, Armand Lever Claude de (Hg.), Canadian Practice in International Law during 1972 as Reflected in Resolutions of the House of Commons and in Government Statements in the House of Commons, Canadian Yearbook of International Law 11 (1973), S. 314-346.

Mager, Ute, Die Entwicklung des Wasserwirtschaftsrechts - Referenzgebiet für ein materiell-rechtlich fundiertes internationales Verwaltungsrecht, Zeitschrift für ausländisches öffentliches Recht und Völkerrecht 70 (2010), S. 789-818.

Magraw, Daniel Barstow, The International Law Commission's Study of International Liability for Nonprohibited Acts as it Relates to Developing States, Washington Law Review 61 (1986), S. 1041-1060.

Magraw, Daniel Barstow, Transboundary Harm: The International Law Commission `s Study of „International Liability“, American Journal of International Law 80 (1986), S. 305-330.

Mahmoudi, Said, Gut Dam Claims, in: Wolfrum, Rüdiger (Hg.), Max Planck Encyclopedia of Public International Law, Online Edition www.mpepil.com, Stand: 2007.

Malanczuk, Peter, Akehurst's Modern Introduction to International Law, 7. Auflage, London [u.a.] 1997.

Malone, Linda A., The Chernobyl Accident: A Case Study in International Law Regulating State Responsibility for Transboundary Nuclear Pollution, Columbia Journal of Environmental Law 12 (1987), S. 203-241.

Mandsager, Dennis (Hg.), Sanremo Handbook on Rules of Engagement, Sanremo 2009. 
Marauhn, Thilo, Customary Rules of International Environmental Law - Can they Provide Guidance for Developing a Peacetime Regime for Cyberspace?, in: Ziolkowski, Katharina (Hg.), Peacetime Regime for State Activities in Cyberspace: International Law, International Relations and Diplomacy, Tallinn 2013, S. 465-484.

Marboe, Irmgard, The Importance of Guidelines and Codes of Conduct for Liability of States and Private Actors, in: dies. (Hg.), Soft Law in Outer Space, Böhlau [u.a.] 2012, S. 119-144.

Matsui, Yoshiro, The Transformation of the Law of State Responsibility, in: Provost, René (Hg.), State Responsibility in International Law, Aldershot [u.a.], 2002, S. 5-65.

Matsushita, Mitsuo/Schoenbaum, Thomas J./Mavroidis, Petros C./Hahn, Michael, The World Trade Organization: Law, Practice, and Policy, 3. Auflage, Oxford 2015.

Matz-Lück, Nele, Meeresschutz, in: Proelß, Alexander (Hg.), Internationales Umweltrecht, Berlin [u.a.] 2017, S. 393-454.

Maurmann, Dorothee, Rechtsgrundsätze im Völkerrecht am Beispiel des Vorsorgeprinzips, Baden-Baden, 2008.

McCaffrey, Stephen. C., Expediting the Provision of Compensation to Accident Victims, in: G. Handl/R. Lutz (Hg.), Transferring Hazardous Technologies and Substances: The International Legal Challenge, London [u.a.] 1989, S. 199-239.

McCaffrey, Stephen C., Of Paradoxes, Precedents, and Progeny: The Trail Smelter Arbitration 65 Years Later, in: Bratspies, Rebecca M./Miller, Russell A. (Hg.), Transboundary Harm in International Law: Lessons from the Trail Smelter Arbitration, Cambridge [u.a.] 2006, S. 34-45.

McCaffrey, Stephen C., The 1997 U.N. Watercourses Convention: Retrospect and Prospect, Global Business \& Development Law Journal 21 (2008), S. 165-173.

McCaffrey, Stephen C., International Watercourses, Environmental Protection, in: Wolfrum, Rüdiger (Hg.), Max Planck Encyclopedia of Public International Law, Online Edition www.mpepil.com, Stand: 2011.

McDougal, Myres S./Schlei, Norbert A., The Hydrogen Bomb Tests in Perspective: Lawful Measures for Security, Yale Law Journal 64 (1955), S. 648-710.

McIntryre, Owen, The Role of Customary Rules and Principles of International Environmental Law in the Protection of Shared International Freshwater Resources, Natural Resources Journal 46 (2006), S. 157-210.

Mellor, Justin, Radioactive Waste and Russia's Northern Fleet: Sinking the Principles of International Environmental Law, Denver Journal of International Law and Policy 28 (1999), S. 51-72.

Melzer, Nils, Cyberwarfare and International Law, Geneva 2011.

Menzel, Eberhard, Grundprobleme der Ermittlung, Anwendung und Geltung von völkerrechtlichen Normen, Juristische Schulung 1963, S. 41-51.

Messerschmidt, Jan E., Hackback: Permitting Retaliatory Hacking by Non-State Actors as Proportionate Countermeasures to Transboundary Cyberharm, Columbia Journal of Transnational Law 52 (2013), S. 275-324. 


\section{Bibliografie}

Mickelson, Karin, Rereading Trail Smelter, Canadian Yearbook of International Law 31 (1993), S. 219-234.

Miquelon-Weismann, Miriam F., The Convention on Cybercrime: A Harmonized Implementation of International Penal Law: What Prospects for Procedural Due Process?, The John Marshall Journal of Information Technology \& Privacy Law 23 (2005), S. 329-362.

Morth, Todd A., Considering Our Position: Viewing Information Warfare as a Use of Force Prohibited by Article 2(4) of the U.N. Charter, Case Western Reserve Journal of International Law 30 (1998), S. 567-600.

Morton, Chris, Stuxnet, Flame, and Duqu - the Olympic Games, in: Healey, Jason (Hg.), A Fierce Domain: Conflict in Cyberspace, 1986-2012, Washington, D.C. 2013, S. 212-231.

Mosler, Hermann, General Principles of Law, in: Bernhardt, Rudolf (Hg.), Encyclopedia of Public International Law, Band II, Amsterdam [u.a.] 1995, S. 511-527.

Mrozek, Marius, Europäisches Marktordnungsrecht, die WTO und die Osterweiterung: Das Steuerungsinstrumentarium des EU-Agrarrechts am Beispiel der Marktordnungen für Getreide und Zucker im Lichte der WTO und zugleich eine rechtsvergleichende Analyse der Agrarordnung Polens in der Vorbeitrittsphase, Berlin 2003.

Murphy, Sean D., Prospective Liability Regimes for the Transboundary Movement of Hazardous Wastes, American Journal of International Law 88 (1994), S. 2475.

Nanda, Ved P./Pring, George (Rock), International Environmental Law and Policy for the 21st Century, 2. Auflage, Leiden [u.a.] 2013.

Nanteuil, Arnaud de, Droit international de l'investissement, Paris 2014.

Nedzel, Nadia E., Antidumping and Cotton Subsidies: A Market-based Defense of Unfair Trade Remedies, Northwestern Journal of International Law \& Business 28 (2008), S. 215-272.

Nguyen, Reese, Navigating Jus Ad Bellum in the Age of Cyber Warfare, California Law Review 101 (2013), S. 1079-1129.

Notz, Konstantin von, Der demokratische Rechtsstaat und das Geheimnis der Dienste - Neubewertung eines Spannungsverhältnisses, Die Friedens-Warte 90 (2015), S. 17-25.

Noyes, John E./Smith, Brian D., State Responsibility and the Principle of Joint and Several Liability, Yale Journal of International Law 13 (1988), S. 225-267.

O'Connell, Mary Ellen, Cyber Security without Cyber War, Journal of Conflict \& Security Law 17 (2012), S. 187-209.

Odendabl, Kerstin, Die Umweltpflichtigkeit der Souveränität: Reichweite und Schranken territorialer Souveränitätsrechte über die Umwelt und die Notwendigkeit eines veränderten Verständnisses staatlicher Souveränität, Berlin 1998.

Okowa, Phoebe N., State Responsibility for Transboundary Air Pollution in International Law, Oxford [u.a.] 2000. 
Owens, William A./Dam, Kenneth W./Lin, Herbert S. (Hg.), Technology, Policy, Law, and Ethics Regarding U.S. Acquisition and Use of Cyberattack Capabilities, Washington, D.C. 2009.

Parrish, Austen L., Trail Smelter Déjà Vu: Extraterritoriality, International Environmental Law and the Search for Solutions to Canadian-U.S. Transboundary Water Pollution Disputes, Boston University Law Review 85 (2005), S. 363-429.

Payne, Thomas, Teaching Old Law New Tricks: Applying and Adapting State Responsibility to Cyber Operations, Lewis \& Clark Law Review 20 (2016), S. 683715.

Peavey-Joanis, Jennifer, A Pyrrhic Victory: Applying the Trail Smelter Principle to State Creation of Refugees, in: Bratspies, Rebecca M./Miller, Russell A. (Hg.), Transboundary Harm in International Law: Lessons from the Trail Smelter Arbitration, Cambridge [u.a.] 2006, S. 254-267.

Pedrazzi, Marco, Outer Space, Liability for Damage, in: Wolfrum, Rüdiger (Hg.), Max Planck Encyclopedia of Public International Law, Online Edition www.mpepil.com, Stand: 2008.

Peil, Michael, Scholarly Writings as a Source of Law: A Survey of the Use of Doctrine by the International Court of Justice, Cambridge Journal of International and Comparative Law 1 (2012), S. 136-161.

Pellet, Allain, The ILC's Articles on State Responsibility for Internationally Wrongful Acts and Related Texts, in: Crawford, James/ders./Olleson, Simon (Hg.), The Law of International Responsibility, Oxford [u.a.] 2010, S. 75-94.

Pihelgas, Mauno, Back-Tracing and Anonymity in Cyberspace, in: Ziolkowski, Katharina (Hg.), Peacetime Regime for State Activities in Cyberspace: International Law, International Relations and Diplomacy, Tallinn 2013, S. 31-60.

Pirker, Benedikt, Territorial Sovereignty and Integrity and the Challenges of Cyberspace, in: Ziolkowski, Katharina (Hg.), Peacetime Regime for State Activities in Cyberspace: International Law, International Relations and Diplomacy, Tallinn 2013, S. 189-216.

Pisillo-Mazzeschi, Riccardo, Forms of International Responsibility for Environmental Harm, in: Francioni, Francesco/Scovazzi, Tullio (Hg.), International Responsibility for Environmental Harm, London [u.a.] 1991, S. 15-35.

Porras, Ileana M., The Rio Declaration: A New Basis for International Cooperation, Review of European Community and International Environmental Law 1 (1992), S. 245-253.

Proelß, Alexander, Raum und Umwelt im Völkerrecht, in: Vitzthum, Wolfgang Graf/ders. (Hg.), Völkerrecht, 7. Auflage, Berlin [u.a.] 2016, S. 361-454.

Proelß, Alexander, Prinzipien des internationalen Umweltrechts, in: ders. (Hg.), Internationales Umweltrecht, Berlin [u.a.] 2017, S. 69-104.

Pyhälä, Minna/Brusendorff, Anne Christine/Paulomäki, Hanna, The precautionary principle, in: Fitzmaurice, Malgosia/Ong, David M./Merkouris, Panos (Hg.), Research Handbook on International Environmental Law, Cheltenham [u.a.] 2010, S. 203-226. 


\section{Bibliografie}

Randelzhofer, Albrecht, Probleme der völkerrechtlichen Gefährdungshaftung, in: Rauschning, Dietrich/ders., Staatenverantwortlichkeit: Berichte der Deutschen Gesellschaft für Völkerrecht, Heft 24, Heidelberg 1984, S. 35-78.

Randelzhofer, Albrecht/Simma, Bruno, Das Kernkraftwerk an der Grenze: Eine „ultra-hazardous activity “ im Schnittpunkt von internationalem Nachbarrecht und Umweltschutz, in: Blumenwitz, Dieter/Randelzhofer, Albrecht (Hg.), Festschrift für Friedrich Berber zum 75. Geburtstag, München 1973, S. 389-432.

Ranke, Ulrich, Klima und Umweltpolitik, Berlin [u.a.] 2019.

Ratner, Steven R., Compensation for Expropriations in a World of Investment Treaties: Beyond the Lawful/Unlawful Distinction, American Journal of International Law 111 (2017), S. 7-56.

Rau, Markus, IGH v. 9.4.1949 - Korfu-Kanal, Souveränität verpflichtet! Unterlassenshaftung und Beweiserleichterung im Recht der Staatenverantwortlichkeit, in: Menzel, Jörg/Pierlings, Tobias/Hoffmann, Jeannine (Hg.), Völkerrechtsprechung, Tübingen 2005, S. 756-760.

Rauschning, Dietrich, Allgemeine Völkerrechtsregeln zum Schutz gegen Umweltbeeinträchtigungen, in: Münch, Ingo von (Hg.), Staatsrecht, Völkerrecht, Europarecht: Festschrift für Hans-Jürgen Schlochauer zum 75. Geburtstag am 28. März 1981, Berlin [u.a.] 1981, S. 557-576.

Read, John E., The Trail Smelter Dispute, Canadian Yearbook of International Law 1 (1963), S. 213-229.

Reinisch, August/Beham, Markus, Mitigating Risks: Inter-State Due Diligence Obligations in Case of Harmful Cyber-Incidents and Malicious Cyber-Activity Obligations of the Transit State, German Yearbook of International Law 58 (2015), S. 101-112.

Rensmann, Thilo, Wertordnung und Verfassung: Das Grundgesetz im Kontext grenzüberschreitender Konstitutionalisierung, Tübingen 2007.

Riemer, Carsten Ludwig, Staatengemeinschaftliche Solidarität in der Völkerrechtsordnung: Eine normative Analyse staatengemeinschaftlicher Solidarkonzepte in Bezug auf die internationale Staatenpraxis aus den Bereichen Friedenswahrung, Entwicklungshilfe, Wirtschaft und Umweltschutz, Düsseldorf 2003.

Ripinsky, Sergey/Williams, Kevin, Damages in International Investment Law, London 2008.

Rivera, Rose, U.S. State Responsibility á la Trail Smelter: Arms Trafficking and Transboundary Harm to Mexico, Mexican Law Review 5 (2012), S. 3-39.

Roberts, Anthea Elizabeth, Traditional and Modern Approaches to Customary International Law: A Reconciliation, American Journal of International Law 95 (2001), S. 757-791.

Ronzoni, Miriam, The Global Order: A Case of Background Injustice? A PracticeDependent Account, Philosophy \& Public Affairs 37 (2009), S. 229-256.

Roscini, Marco, Cyber Operations and the Use of Force in International Law, Oxford 2014. 
Roscini, Marco, Evidentiary Issues in International Disputes Related to State Responsibility for Cyber Operations, in: Ohlin, Jens David/Govern, Kevin/Finkelstein, Claire (Hg.), Cyberwar: Law and Ethics for Virtual Conflict, Oxford 2015, S. 215-248.

Rosenstock, Robert, The ILC and State Responsibility, American Journal of International Law, 96 (2002), S. 792-797.

Rubin, Alfred P., Pollution by Analogy: The Trail Smelter Arbitration, Oregon Law Review 50 (1971), S. 259-282.

Rudolf, Walter, Haftung für rechtmäßiges Verhalten im Völkerrecht, in: Damrau, Jürgen (Hg.), Festschrift für Otto Mühl zum 70. Geburtstag, Stuttgart [u.a.] 1981, S. 535-552.

Ruys, Tom, 'Armed Attack' and Article 51 of the UN Charter: Evolutions in Customary Law and Practice, Cambridge [u.a.] 2010.

Ryan, Daniel J./Dion, Maeve/Tikk, Eneken/Ryan, Julie J. C. H., International Cyberlaw: A Normative Approach, Georgetown Journal of International Law 42 (2011), S. 1161-1199.

Sadeleer, Nicolas de, Les principes du pollueur-payeur, de prévention et de précaution, Essai sur la genèse et la portée juridique de quelques principes du droit de l'environnement, Brüssel [u.a.] 1999.

Sadeleer, Nicolas de, Environmental Principles: From Political Slogans to Legal Rules, Oxford [u.a.] 2002.

Sands, Philippe J., The Environment, Community and International Law, Harvard International Law Journal 30 (1989), S. 393-420.

Sands, Philippe/Peel, Jacqueline, Principles of International Environmental Law, 4. Auflage, Cambridge [u.a.] 2018.

Saxler, Barbara/Siegfried, Jule/Proelss, Alexander, International liability for transboundary damage arising from stratospheric aerosol injections, Law, Innovation and Technology (7) 2015, S. 112-147.

Scanlan, Kevin P., The International Law Commission's First Ten Draft Articles on the Law of the Non-Navigational Uses of International Watercourses: Do They Adequately Address All the Major Issues of Water Usage in the Middle East?, Fordham International Law Journal 19 (1996), S. 2179-2229.

Schachter, Oscar, International Law in Theory and Practice: General Course in Public International Law, Dordrecht [u.a.] 1991.

Schmahl, Stefanie, Cybersecurity, in: Dethloff, Nina/Nolte, Georg/Reinisch, August (Hg.), Freiheit und Regulierung in der Cyberwelt - Rechtsidentifikation zwischen Quelle und Gericht, Heidelberg 2016, S. 159-196.

Schmalenbach, Kirsten, Verantwortlichkeit und Haftung, in: Proelß, Alexander (Hg.), Internationales Umweltrecht, Berlin [u.a.] 2017, S. 211-242.

Schmidt, Andreas, The Estonian Cyber Attacks, in: Healey, Jason (Hg.), A Fierce Domain: Conflict in Cyberspace, 1986-2012, Washington, D.C. 2013, S. 174193.

Schmitt, Daniela M., Staatenverantwortlichkeit für Schäden an der biologischen Vielfalt, Baden-Baden 2018. 


\section{Bibliografie}

Schmitt, Michael N., Computer Network Attack and the Use of Force in International Law: Thoughts on a Normative Framework, Columbia Journal of Transnational Law 37 (1999), S. 885-937.

Schmitt, Michael N., Preemptive Strategies in International Law, Michigan Journal of International Law 24 (2003), S. 513-548.

Schmitt, Michael N., The 'Use of Force' in Cyberspace: A Reply to Dr Ziolkowski, in: Czosseck, Christian/Ottis, Rain/Ziolkowski, Katharina (Hg.), 2012 4th International Conference on Cyber Conflict, Tallinn 2012, S. 311-317.

Schmitt, Michael N. (Hg.), Tallinn Manual on the International Law Applicable to Cyber Warfare, Prepared by the International Groups of Experts at the Invitation of the NATO Cooperative Cyber Defence Centre of Excellence, Cambridge [u.a.] 2013.

Schmitt, Michael N., "Below the Threshold" Cyber Operations: The Countermeasures Response Option and International Law, Virginia Journal of International Law 54 (2014), S. 697-732.

Schmitt, Michael N., The Law of Cyber Warfare: Quo Vadis?, Stanford Law \& Policy Review 25 (2014), S. 269-299.

Schmitt, Michael N., In Defense of Due Diligence in Cyberspace, The Yale Law Journal Forum 125 (2015), S. 68-81.

Schmitt, Michael N. (Hg.), Tallinn Manual 2.0 on the International Law Applicable to Cyber Operations, Prepared by the International Groups of Experts at the Invitation of the NATO Cooperative Cyber Defence Centre of Excellence, 2. Auflage, Cambridge [u.a.] 2017.

Schmitt, Michael N., Grey Zones in the International Law of Cyberspace, The Yale Journal of International Law Online 42 (2017), abrufbar unter: https://cpb-us -w2.wpmucdn.com/campuspress.yale.edu/dist/8/1581/files/2017/08/Schmitt _Grey-Areas-in-the-International-Law-of-Cyberspace-1cab8kj.pdf (geprüft am 15.05.2020), S. 1-21.

Schmitt, Michael N./Vibul, Liis, The Nature of International Law Cyber Norms, in: Osula, Anna-Maria/Rõigas, Henry, International Cyber Norms: Legal, Policy \& Industry Perspectives, Tallinn 2016, S. 23-47.

Schmitt, Michael N./Vihul, Liis, Respect for Sovereignty in Cyberspace, Texas Law Review 95 (2017), S. 1639-1670.

Schreuer, Christoph H., The Concept of Expropriation under the ETC and other Investment Protection Treaties, Transnational Dispute Management 5 (2005), S. 1-39.

Schröder, Meinhard, Umweltschutz als Gemeinschaftsziel und Grundsätze des Umweltschutzes, in: Rengeling, Hans-Werner (Hg.), Handbuch zum europäischen und deutschen Umweltrecht: Eine systematische Darstellung des europäischen Umweltrechts mit seinen Auswirkungen auf das deutsche Recht und mit rechtspolitischen Perspektiven, Band I Allgemeines Umweltrecht, 2. Auflage, Köln [u.a.] 2003, S. 199-238. 
Schröder, Meinhard, Precautionary Approach/Principle, in: Wolfrum, Rüdiger (Hg.), Max Planck Encyclopedia of Public International Law, Online Edition www.mpepil.com, Stand: 2014.

Schulze, Sven-Hendrik, Cyber-„War“ - Testfall der Staatenverantwortlichkeit, Tübingen 2015.

Schütz, Raimund, Solidarität im Wirtschaftsvölkerrecht: Eine Bestandsaufnahme zentraler entwicklungsspezifischer Solidarrechte und Solidarpflichten im Völkerrecht, Berlin 1994.

Schwabach, Aaron, The Sandoz Spill: The Failure of International Law to Protect the Rhine from Pollution, Ecology Law Quarterly 16 (1989) S. 443-480.

Schwartz, Priscilla, The polluter-pays principle, in: Fitzmaurice, Malgosia/Ong, David M./Merkouris, Panos (Hg.), Research Handbook on International Environmental Law, Cheltenham [u.a.] 2010, S. 243-261.

Schwarzenberger, Georg, The Principles and Standards of International Economic Law, Recueil des Cours 117 (1966-I), S. 1-98.

Seibert-Fohr, Anja, Die völkerrechtliche Verantwortung des Staats für das Handeln von Privaten: Bedarf nach Neuorientierung?, Zeitschrift für ausländisches öffentliches Recht und Völkerrecht 73 (2013), S. 37-60.

Shackelford, Scott J., From Nuclear War to Net War: Analogizing Cyber Attacks in International Law, Berkeley Journal of International Law 27 (2009), S. 192-251.

Shackelford, Scott J./Andres, Richard B., State Responsibility for Cyber Attacks: Competing Standards for a Growing Problem, Georgetown Journal of International Law 42 (2010-2011), S. 971-1016.

Shackelford, Scott J./Russell, Scott/Kuehn, Andreas, Unpacking the International Law on Cybersecurity Due Diligence: Lessons from the Public and Private Sectors, Chicago Journal of International Law 17 (2016), S. 1-50.

Sharp, Walter Gary Sr., Cyberspace and the Use of Force, North Falls Church 1999.

Simma, Bruno/Alston, Philip, The Sources of Human Rights Law: Custom, Jus Cogens, and General Principles, Australian Yearbook of International Law 12 (1992), S. 82-108.

Sivakumaran, Sandesh, The Influence of Teachings of Publicists on the Development of International Law, International and Comparative Law Quarterly 66 (2017), S. 1-37.

Sklerov, Matthew J., Solving the Dilemma of State Response to Cyberattacks: A Justification for the Use of Active Defenses Against States Who Neglect Their Duty to Prevent, Military Law Review 201 (2009), S. 1-85.

Smets, Henri, The Polluter Pays Principle in the Early 1990s, in: Campiglio, Luigi/Pineschi, Laura/Siniscalco, Domenico/Treves, Tullio (Hg.), The Environment After Rio: International Law and Economics, London 1994, S. 131-144.

Sohn, Louis B., The Stockholm Declaration on the Human Environment, Harvard International Law Journal 14 (1973), S. 423-515.

Soto, Max Valverde, General Principles of International Environmental Law, ILSA Journal of International \& Comparative Law 3 (1996), S. 193-209. 


\section{Bibliografie}

Spiecker genannt Döhmann, Indra, Staatliche Entscheidungen unter Unsicherheit juristische und ökonomische Vorgaben, Bonn 2000.

Springer, Allen L., Cases of Conflict: Transboundary Disputes and the Development of International Law, 2016.

Staiger, Robert W./Sykes, Alan O., How Important Can the Non-Violation Clause Be for the GATT/WTO?, American Economic Journal: Microeconomics 9 (2017), S. $149-187$.

Stein, Torsten/Marauhn, Thilo, Völkerrechtliche Aspekte von Informationsoperationen, Zeitschrift für ausländisches öffentliches Recht und Völkerrecht 60 (2000), S. $1-40$.

Stelter, Christian, Gewaltanwendung unter und neben der UN-Charta, Berlin 2007.

Stephens, Tim, International Courts and Environmental Protection, Cambridge 2009.

Stubbe, Peter, State Accountability for Space Debris: A Legal Study of Responsibility for Polluting the Space Environment and Liability for Damage Caused by Space Debris, Leiden [u.a.] 2018.

Suchartikul, Sompong, State Responsibility and International Liability under International Law, Loyola of Los Angeles International and Comparative Law Review 18 (1996), S. 821-839.

Suy, Erik, La protection des diplomates, in: Tittel, Josef (Hg.), Festschrift für Wilhelm Wengler zu seinem 65. Geburtstag, Band 1 Allgemeine Rechtslehre und Völkerrecht, Berlin 1973, S. 591-622.

Tanzi, Attila, The UN Convention on International Watercourses as a Framework for the Avoidance and Settlement of Waterlaw Disputes, Leiden Journal of International Law 11 (1998), S. 441-472.

Tanzi, Attila, Liability for Lawful Acts, in: Wolfrum, Rüdiger (Hg.), Max Planck Encyclopedia of Public International Law, Online Edition www.mpepil.com, Stand: 2013.

Theuerkauf, Thomas, Informationsoperationen, Europäische Sicherheit 2 (2000), S. 14-18.

Thiel, Markus, Die „Entgrenzung“ der Gefahrenabwehr: Grundfragen von Freiheit und Sicherheit im Zeitalter der Globalisierung, Tübingen 2011.

Thies, Anne, International Trade Disputes and EU Liability, Cambridge [u.a.] 2013.

Tiirmaa-Klaar, Heli, Cyber Diplomacy: Agenda, Challenges and Mission, in: Ziolkowski, Katharina (Hg.), Peacetime Regime for State Activities in Cyberspace: International Law, International Relations and Diplomacy, Tallinn 2013, S. 509-532.

Tikk, Eneken/Kadri, Kaska/Vihul, Liis, International Cyber Incidents: Legal Considerations, Tallinn 2010.

Tikk, Eneken/Kaska, Kadri/Rünnimeri, Kristel/Kert, Mari/Talihärm, Anna-Maria/Vibul, Liiis, Cyber Attacks Against Georgia: Legal Lessons Identified, Tallinn 2008.

Többens, Hans W., Wirtschaftsspionage und Konkurrenzausspähung in Deutschland, Neue Zeitschrift für Strafrecht 10 (2000), S. 505-512. 
Tomuschat, Christian, The International Law Commission - An Outdated Institution?, German Yearbook of International Law 49 (2006), S. 77-105.

Townley, Stephen, The Rise and Risk in International Law, Chicago Journal of International Law 18 (2018), S. 594-646.

Tsagourias, Nicholas, Cyber-attacks, self-defence and the problem of attribution, Journal of Conflict \& Security Law 17 (2012), S. 229-244.

Tsolkas, Alexander/Wimmer, Friedrich, Wirtschaftsspionage und Intelligence Gathering: Neue Trends der wirtschaftlichen Vorteilsbeschaffung, Wiesbaden 2012.

Türk, Helmut, Water in the Contemporary World, in: Hestermeyer, Holger P./ König, Doris/Matz-Lück, Nele/Röben, Volker/Seibert-Fohr, Anja/Stoll, Peter-Tobias/Vöneky, Silja (Hg.), Coexistence, Cooperation and Solidarity: Liber Americum Rüdiger Wolfrum, Band 2, Leiden [u.a.] 2012, S. 1037-1064.

Van Dyke, Jon M., Liability and Compensation for Harm Caused by Nuclear Activities, Denver Journal of International Law and Policy35 (2006), S. 13-46.

Ventre, Daniel, Cyberwar and Information Warfare, London 2011.

Verdross, Alfred, Die Verfassung der Völkerrechtsgemeinschaft, Wien [u.a.] 1926.

Verheyen, Roda, Climate Change Damage and International Law: Prevention Duties and State Responsibility, Leiden [u.a.] 2005.

Vicuña, Francisco Orrego, Responsibility and Liability for Environmental Damage under International Law: Issues and Trends, Georgetown International Environmental Law Review 10 (1998), S. 279-308.

Vinnem, Jan-Erik/Røed, Willy, Offshore Risk Assessment Band 1: Principles, Modelling and Applications of QRA Studies, 4. Auflage, London 2020.

Viñuales, Jorge E., The Contribution of the International Court of Justice to the Development of International Environmental Law: A Contemporary Assessment, Fordham International Law Journal 32 (2008), S. 232-258.

Virally, Michel, The Sources of International Law, in: Sørensen, Max (Hg.), Manual of Public International Law, London [u.a.], 1968, S. 116-174.

Voigt, Christina, The Role of General Principles in International Law and their Relationship to Treaty Law, Retfærd Årgang 31 (2008) Nr. 2/121, S. 3-25.

Vöneky, Silja, Analogy in International Law, in: Wolfrum, Rüdiger (Hg.), Max Planck Encyclopedia of Public International Law, Online Edition www.mpepil. com, Stand: 2008.

Vöneky, Silja/Addison-Agyei, Sange, Antarctica, in: Wolfrum, Rüdiger (Hg.), Max Planck Encyclopedia of Public International Law, Online Edition www.mpepil. com, Stand: 2011.

Waibel, Michael, Corfu Channel Case, in: Wolfrum, Rüdiger (Hg.), Max Planck Encyclopedia of Public International Law, Online Edition www.mpepil.com, Stand: 2013.

Walter, Christian, Cyber Security als Herausforderung für das Völkerrecht, Juristenzeitung 14 (2015), S. 685-693.

Walter, Christian, Obligations of States Before, During, and After a Cyber Security Incident, German Yearbook of International Law 58 (2015), S. 67-86. 


\section{Bibliografie}

Walton, Beatrice A., Duties Owed: Low-Intensity Cyber Attacks and Liability for Transboundary Torts in International Law, The Yale Law Journal 126 (2017), S. 1460-1519.

Wehling, Philine, Wasserrechte am Nil: Der Einfluss des internationalen Wasserrechts auf die Entwicklung eines Vertragsregimes zur Nutzungsverteilung und gemeinsamen Wasserwirtschaft am Beispiel des Nils, Berlin [u.a.] 2018.

Weissbrodt, David, Cyber-Conflict, Cyber-Crime, and Cyber-Espionage, Minnesota Journal of International Law 22 (2013), S. 347-387.

Weiß, Wolfgang, Allgemeine Rechtsgrundsätze des Völkerrechts, Archiv des Völkerrechts 39 (2001), S. 394-431.

Wenzel, Nicola, Human Rights, Treaties, Extraterritorial Application and Effects, in: Wolfrum, Rüdiger (Hg.), Max Planck Encyclopedia of Public International Law, Online Edition www.mpepil.com, Stand: 2008.

Whiteman, Marjorie M., Digest of international law, Band 4, Washington, D.C. 1965.

Williams, Robert D., (Spy) Game Change: Cyber Networks, Intelligence Collection, and Covert Action, The George Washington Law Review 79 (2011), S.11621200.

Wins, Elmar, Weltraumhaftung im Völkerrecht, Berlin 2000.

Wolf, Joachim, Die gegenwärtige Entwicklung der Lehre über die völkerrechtliche Verantwortlichkeit der Staaten: Untersucht am Beispiel des Urteils des Internationalen Gerichtshofs in der Teheraner Geiselaffaire, Zeitschrift für ausländisches öffentliches Recht und Völkerrecht 31 (1983), S. 481-536.

Wolf, Joachim, Die Haftung der Staaten für Privatpersonen nach Völkerrecht, Berlin 1997.

Wolfrum, Rüdiger, The Principle of the Common Heritage of Mankind, Zeitschrift für ausländisches öffentliches Recht und Völkerrecht (43) 1983, S. 312-337.

Wolfrum, Rüdiger, Purposes and Principles of International Environmental Law, German Yearbook of International Law 33 (1990), S. 308-330.

Wolfrum, Rüdiger, Hohe See und Tiefseeboden (Gebiet), in: Vitzthum, Wolfgang Graf (Hg.), Handbuch des Seerechts, München 2006, S. 287-346.

Wolfrum, Rüdiger, Liability for Environmental Damage in Antarctica: Supplement to the Rules on State Responsibility or a Lost Opportunity?, in: Buffard, Isabelle/Crawford, James/Pellet, Alain/Wittich, Stephan (Hg.), International Law Between Universalism and Fragmentation: Festschrift in Honour of Gerard Hafner, Leiden 2008, S. 817-827.

Wolfrum, Rüdiger, General International Law (Principles, Rules, and Standards), in: ders. (Hg.), Max Planck Encyclopedia of Public International Law, Online Edition www.mpepil.com, Stand: 2010.

Wolfrum, Rüdiger/Langenfeld, Christine, Umweltschutz durch internationales Haftungsrecht, Heidelberg 1999.

Wolfrum, Rüdiger/Möldner, Mirka, International Courts and Tribunals, Evidence, in: Wolfrum, Rüdiger (Hg.), Max Planck Encyclopedia of Public International Law, Online Edition www.mpepil.com, Stand: 2013. 
Woltag, Johann-Christoph, Computer Network Operations Below the Level of Armed Force, ESIL Conference Paper Series 1 (2011), S. 1-18.

Woltag, Johann-Christoph, Cyber Warfare: Military Cross-Border Computer Network Operations under International Law, Cambridge [u.a.] 2014.

Wood, Michael, Teachings of the Most Highly Qualified Publicists (Art. 38 (1) ICJ Statute), in: Wolfrum, Rüdiger (Hg.), Max Planck Encyclopedia of Public International Law, Online Edition www.mpepil.com, Stand: 2017.

Wussow, Philipp von, „Cyberkrieg“: Geschichte und Gegenwart eines umkämpften Begriffs, Konfliktzone Cyberspace: Perspektiven für Sicherheit und Frieden, Ethik und Militär - Kontroversen in Militärethik \& Sicherheitspolitik 1 (2019), abrufbar unter: http://www.ethikundmilitaer.de/fileadmin/ethik_und_militaer/ Ethik-und-Militaer-2019-1.pdf (geprüft am 15.05.2020), S. 11-17.

Yasuhiro, Shigeta, Some Reflections on the Relationship between the Principle of Equitable Utilization of International Watercourses and the Obligation not to Cause Transfrontier Pollution Harm, Asian Yearbook of International Law 9 (2000), S. 147-189.

Zemanek, Karl, State Responsibility and Liability, in: Lang, Winfried/Neuhold, Hanspeter/ders. (Hg.), Environmental Protection and International Law, London [u.a.] 1991, S. 187-201.

Zemanek, Karl, Armed Attack, in: Wolfrum, Rüdiger (Hg.), Max Planck Encyclopedia of Public International Law, Online Edition www.mpepil.com, Stand: 2013.

Ziegler, Katja S., Domaine Réservé, in: Wolfrum, Rüdiger (Hg.), Max Planck Encyclopedia of Public International Law, Online Edition www.mpepil.com, Stand: 2013.

Ziolkowski, Katharina, Confidence Building Measures for Cyberspace, in: dies. (Hg.), Peacetime Regime for State Activities in Cyberspace: International Law, International Relations and Diplomacy, Tallinn 2013, S. 553-564.

Ziolkowski, Katharina, General Principles of International Law as Applicable in Cyberspace, in: dies. (Hg.), Peacetime Regime for State Activities in Cyberspace: International Law, International Relations and Diplomacy, Tallinn 2013, S. 135-188.

Ziolkowski, Katharina, Peacetime Cyber Espionage - New Tendencies in Public International Law, in: dies. (Hg.), Peacetime Regime for State Activities in Cyberspace, International Law, International Relations and Diplomacy, Tallinn 2013, S. 425-464.

Zumbansen, Peer, The Conundrum of Corporate Social Responsibility: Reflections on the Changing Nature of Firms and State, in: Bratspies, Rebecca M./Miller, Russell A. (Hg.), Transboundary Harm in International Law: Lessons from the Trail Smelter Arbitration, Cambridge [u.a.] 2006, S. 240-253. 


\section{Bibliografie}

\section{B. Berichte, Studien, Zeitungsartikel und sonstige Onlineressourcen}

Aaltola, Mika/Sipilä, Joonas/Vuorisalo, Valtteri, Securing Global Commons: A Small State Perspective, The Finnish Institute of International Affairs, Working Paper 71, June 2011, abrufbar unter: https:/www.fiia.fi/wp-content/uploads/2017/01/ wp71.pdf (geprüft am 15.05.2020).

Albright, David/Brannan, Paul/Walrond, Christina, Stuxnet Malware and Natanz: Update of ISIS December 22, 2010 Report, Institute for Science and International Security Report, 15 February 2011, abrufbar unter: http://isis-online.org/u ploads/isis-reports/documents/stuxnet_update_15Feb2011.pdf (geprüft am 15.05.2020).

Anderson, Nate, Massive DDOS attacks target Estonia; Russia accused, ars Technica, 14 May 2007, abrufbar unter: https://arstechnica.com/information-technolo gy/2007/05/massive-ddos-attacks-target-estonia-russia-accused/ (geprüft am 15.05.2020).

Arimatsu, Louise, International Law Applicable to Naval Mines, Chatham House, International Security Department, Workshop Summary, October 2014, abrufbar unter: https:/www.chathamhouse.org/sites/files/chathamhouse/field/field_d ocument/20140226NavalMines.pdf (geprüft am 15.05.2020).

Aust, Helmut Philipp, Stellungnahme zur Sachverständigenanhörung am 5. Juni 2014 im 1. Untersuchungsausschuss der 18. Wahlperiode des Deutschen Bundestages, Humboldt-Universität zu Berlin, 27. Mai 2014, abrufbar unter: https:// www.bundestag.de/resource/blob/282870/fc52462f2ffd254849bce19d25f72fa2/m at_a_sv-4-1_aust-pdf-data.pdf (geprüft am 15.05.2020).

Barboza, Julio (Special Rapporteur), Preliminary report on international liability for injurious consequences arising out of acts not prohibited by international law, YBILC 1985-II/1, UN Doc. A/CN.4/394.

Barboza, Julio (Special Rapporteur), Third report on international liability for injurious consequences arising out of acts not prohibited by international law, YBILC 1987-II/1, UN Doc. A/CN.4/405.

Barboza, Julio (Special Rapporteur), Fourth report on international liability for injurious consequences arising out of acts not prohibited by international law, YBILC 1988-II/1, UN Doc. A/CN.4/413.

Barboza, Julio (Special Rapporteur), Sixth report on international liability for injurious consequences arising out of acts not prohibited by international law, YBILC 1990-II/1, UN Doc. A/CN.4/428 and Add.1.

Barboza, Julio (Special Rapporteur), Eighth report on international liability for injurious consequences arising out of acts not prohibited by international law, YBILC 1992-II/1, UN Doc. A/CN.4/443.

Barboza, Julio (Special Rapporteur), Tenth report on international liability for injurious consequences arising out of acts not prohibited by international law, YBILC 1994-II/1, UN Doc. A/CN.4/459. 
Barlow, John Perry, A Declaration of the Independence of Cyberspace, Electronic Frontier Foundation, 8 February 1996, abrufbar unter: https://www.eff.org/de/cy berspace-independence (geprüft am 15.05.2020).

Bendiek, Annegret, Kritische Infrastrukturen, Cybersicherheit, Datenschutz: Die EU schlägt Pflöcke für digitale Standortpolitik ein, SWP-Aktuell 35/2013.

Bendiek, Annegret, Umstrittene Partnerschaft: Cybersicherheit, Internet Governance und Datenschutz in der transatlantischen Zusammenarbeit, SWP-Studie 26/2013.

Berkes, Antal, A New Extraterritorial Jurisdictional Link Recognised by the IACtHR, EJIL: Talk!, 28 March 2018, abrufbar unter: https://www.ejiltalk.or g/a-new-extraterritorial-jurisdictional-link-recognised-by-the-iacthr/ (geprüft am 15.05.2020).

Bio Intelligence Service/Stevens \& Bolton LLP and Cardiff University/Naider/TME, Study to explore the feasibility of creating a fund to cover environmental liability and losses occurring from industrial accidents, Final Report prepared for European Commission, Directorate-General for Environment, 17 April 2013, abrufbar unter: ec.europa.eu/environment/archives/liability/eld/eldfund/pdf/ Final\%20report\%20ELD\%20Fund\%20BIO\%20for\%20web2.pdf (geprüft am 15.05.2020).

Bothe, Michael/Gündling, Lothar/Hofmann, Rainer/Rumpf, Christian, Neuere Tendenzen des Umweltrechts im internationalen Vergleich, in: Umweltbundesamt (Hg.), Umweltforschungsplan des Bundesministers für Umwelt, Naturschutz und Reaktorsicherheit: Umweltplanung, Ökologie, Berichte des Umweltbundesamtes, Band 2/90, Berlin 1990, S. 1-310.

Bradner, Eric, Obama: North Korea's Hack not war, but 'cybervandalism', CNN, 24 December 2014, abrufbar unter: http://www.cnn.com/2014/12/21/politics/obam a-north-koreas-hack-not-war-but-cyber-vandalism (geprüft am 15.05.2020).

Brinded, Lianna, The Interview tipped to cost Sony Pictures $\$ 200 \mathrm{~m}$ following hack and cancellation, International Business Times, 18 December 2014, abrufbar unter: http://www.ibtimes.co.uk/interview-tipped-cost-sony-pictures-200m-total -followinghack-cancellation-1480157 (geprüft am 15.05.2020).

Brunnée, Jutta, Procedure and Substance in International Environmental Law: Confused at a Higher Level?, ESIL Reflections 5 (2016), abrufbar unter: https://esil-se di.eu/post_name-123/ (geprüft am 15.05.2020).

Bundesministerium des Innern (Hg.), Cyber-Sicherheitsstrategie für Deutschland, Berlin 2016, abrufbar unter: https://www.bmi.bund.de/DE/themen/it-und-di gitalpolitik/it-und-cybersicherheit/cyber-sicherheitsstrategie/cyber-sicherheitsstra tegie-node.html (geprüft am 15.05.2020).

Christiansen, Sabine/Jaeckel, Aline/Houghton, Katherine, Ecological safeguards for deep seabed mining, German Environment Agency, Final Report 113 (2019), abrufbar unter: https://www.umweltbundesamt.de/sites/default/files/medien/14 10/publikationen/2019-07-11_texte_113-2019_deep-seabed-mining.pdf (geprüft am 15.05.2020). 


\section{Bibliografie}

Council of Europe, Ad-hoc Advisory Group on Cross-border Internet, Interim Report to the Steering Committee on the Media and New Communication Services incorporating Analysis of Proposals for International and Multi-stakeholder Cooperation on Cross-border Internet, H/Inf(2010)10, abrufbar unter: http:// humanrightseurope.blogspot.com/2011/01/proposals-for-international-cooperati on.html (geprüft am 15.05.2020).

Crawford, James (Special Rapporteur), Second report on State responsibility, YBILC 1999-II/1, UN Doc. A/CN.4/498 and Add.1 - 4.

Crawford, James (Special Rapporteur), Fourth report on State Responsibility, YBILC 2001-II/1, UN Doc. A/CN.4/517 and Add. 1.

European Commission, Green paper on remedying environmental damage, Document COM (93) 47 final, Brussels, 14 May 1993, abrufbar unter: http://aei.pitt.e du/950/ (geprüft am 15.05.2020).

European Commission, Joint Communication to the European Parliament, the Council, the European Economic and Social Committee and the Committee of the Regions, Cybersecurity Strategy of the European Union: An Open, Safe and Secure Cyberspace, 7 February 2013, abrufbar unter: http://eeas.europa.e $\mathrm{u} /$ archives/docs/policies/eu-cyber-security/cybsec_comm_en.pdf (geprüft am 15.05.2020).

Experts Group on Environmental Law of the World Commission on Environment and Development, R. D. Munro (Chairman)/J. G. Lammers (Rapporteur), Environmental Protection and Sustainable Development: Legal Principles and Recommendations, London [u.a.] 1987.

Five Country Ministerial and Quintet Meeting of Attorneys General, Statement of Principles on Access to Evidence and Encryption, Australia 2018, abrufbar unter: https://www.ag.gov.au/About/CommitteesandCouncils/Documents/joint-statem ent-principles-access-evidence.pdf (geprüft am 15.05.2020).

Flook, Kara, Russia and the Cyber Threat, Critical Threats, 13 May 2009, abrufbar unter: http://www.criticalthreats.org/russia/russia-and-cyber-threat (geprüft am 15.05.2020).

Garrie, Daniel/Reeves, Shane R., So You're Telling Me There's A Chance: How the Articles on State Responsibility Could Empower Corporate Responses to StateSponsored Cyber Attacks, Harvard National Security Journal Online Features, 17 December 2015, abrufbar unter: https://harvardnsj.org/wp-content/upload s/sites/13/2016/01/Garrie-and-Reeves-Non-State-Actor-and-Self-Defense.pdf, (geprüft am 15.05.2020).

Gaßner, Hartmut/Buchholz, Georg, Atomhaftung in Europa und Deutschland - Defizite und Empfehlungen zur Fortentwicklung, Gutachten im Auftrag der Bundestagsfraktion Bündnis 90/Die Grünen, 14. März 2013, abrufbar unter: https:// www.zeit-lupe.com/mediapool/132/1322391/data/Gutachten_Atomhaftung_B90 _Gruene_Maerz_2013_.pdf (geprüft am 15.05.2020).

Goldsmith, Jack, Cybersecurity Treaties: A Skeptical View, Hoover Institution Task Force on National Security and Law, Future Challenges Essay, 9 March 2011, abrufbar unter: http://media.hoover.org/sites/default/files/documents/FutureCh allenges_Goldsmith.pdf (geprüft am 15.05.2020). 
Goldsmith, Jack, The Sony Hack: Attribution Problems, and the Connection to Domestic Surveillance, LAWFARE, 19 December 2014, abrufbar unter: https:// www.lawfareblog.com/sony-hack-attribution-problems-and-connection-domesti c-surveillance (geprüft am 15.05.2020).

Goldsmith, Jack, Yet More Thoughts on the DNC Hack: Attribution and Precedent, LAWFARE, 27 July 2016, abrufbar unter: https:/www.lawfareblog.com/yet-mor e-thoughts-dnc-hack-attribution-and-precedent (geprüft am 15.05.2020).

Gostev, Alexander, Cyber weapons, Kaspersky Security Bulletin 2012, abrufbar unter: https://securelist.com/kaspersky-security-bulletin-2012-cyber-weapons /36762/ (geprüft am 15.05.2020).

Hamburger, Tom/Tumulty, Karen, WikiLeaks releases thousands of documents about Clinton and internal deliberations, The Washington Post, 22 July 2016, abrufbar unter: https:/www.washingtonpost.com/news/post-politics/wp/2016/07/22/on-e ve-of-democratic-convention-wikileaks-releases-thousands-of-documents-about-cl inton-the-campaign-and-internal-deliberations/ (geprüft am 15.05.2020).

Hauck, Mirjam/Kuhn, Johannes, Computervirus Duqu entdeckt: Wie gefährlich ist der Stuxnet-Bruder?, Süddeutsche Zeitung, 19. Oktober 2011, abrufbar unter: https://www.sueddeutsche.de/digital/computervirus-duqu-entdeckt-wie-gefaehrli ch-ist-der-stuxnet-bruder-1.1168324 (geprüft am 15.05.2020).

Hollis, David, Cyberwar Case Study: Georgia 2008, Small Wars Journal, 6 January 2011, abrufbar unter: https://smallwarsjournal.com/blog/journal/docs-temp/639 -hollis.pdf (geprüft am 15.05.2020), S. 1-9.

Hollis, Duncan B., Russia and the DNC Hack: What Future for a Duty of Non-Intervention?, OpinoJuris, 25 July 2016, abrufbar unter: http://opiniojuris.org/201 6/07/25/russia-and-the-dnc-hack-a-violation-of-the-duty-of-non-intervention/ (geprüft am 15.05.2020).

Human Rights Council, Frank La Rue (Special Rapporteur), Report on the Promotion and Protection of the Right to Freedom of Opinion and Expression, 16 May 2011, UN Doc. A/HRC/17/27.

Institut de Droit International, Eighth Commission, Francisco Orrego Vicuña (Rapporteur) Session of Strasbourg, 1997 - Second Part, Responsibility and Liability under International Law for Environmental Damage, Resolution of 4 September 1997, IDI-Yearbook 1998-67/II, S. 487-513.

International Atomic Energy Agency, International Expert Group on Nuclear Liability, The 1997 Vienna Convention on Civil Liability for Nuclear Damage and the 1997 Convention on Supplementary Compensation for Nuclear Damage Explanatory Texts, IAEA International Law Series No. 3 (Revised), Wien 2017.

International Bar Association, Section on Business Law, Committee F (international environmental law), Patricia Thomas (Chairman), Environmental Liability: 7th Residential Seminar on Environmental Law, 9-13 June 1990 Montreux Switzerland, London [u.a.] 1991. 


\section{Bibliografie}

International Committee of the Red Cross, International humanitarian law and the challenges of contemporary armed conflicts, Report on the 32nd International Conference of the Red Cross and Red Crescent (32IC/15/11), October 2015, abrufbar unter: http://rcrcconference.org/app//uploads/2015/10/32IC-Report-on -IHL-and-challenges-of-armed-conflicts.pdf (geprüft am 15.05.2020).

International Law Association, Dietrich Rauschning (Rapporteur), Montreal Rules of International Law Applicable to Transfrontier Pollution, Umwelt- und Planungsrecht 1 (1983), S. 21-22.

International Law Association, Study Group on Due Diligence in International Law, Tim Stephens (Rapporteur)/Duncan French (Chair), Second Report, 20 July 2016, abrufbar unter: http://www.ila-hq.org/index.php/study-groups?study-grou psID=63 (geprüft am 15.05.2020).

International Law Commission, Report on the work of its twenty-first session, YBILC 1969-II, UN Doc. A/7610/Rev.1.

International Law Commission, Summary records of the twenty-fifth session, 1204th meeting, YBILC 1973-I, UN Doc. A/CN.4/217 and Add.1; A/CN.4/233; A/ CN.4/246 and Add.l to 3; A/CN.4/264 and Add.1.

International Law Commission, Report on the work of its twenty-fifth session, YBILC 1973-II, UN Doc. A/9010/Rev.1.

International Law Commission, Report on the work of its twenty-sixth session, YBILC 1974-II/1, UN Doc. A/9610/Rev.1.

International Law Commission, Report on the work of its thirtieth session, YBILC 1978-II/2, UN Doc. A/33/10.

International Law Commission, Report on the work of its thirty-second session, YBILC 1980-II/2, UN Doc. A/35/10.

International Law Commission, Report on the work of its thirty-eighth session, YBILC 1986-II/2, UN Doc. A/41/10.

International Law Commission, Report on the work of its forty-second session, YBILC 1990-II/2, UN Doc. A/45/10.

International Law Commission, Report on the work of its forty-fourth session, YBILC 1992-II/2, UN Doc. A/47/10.

International Law Commission, Report on the work of its forty-sixth session, YBILC 1994-II/2, UN Doc. A/49/10.

International Law Commission, Report on the work of its forty-ninth session, YBILC 1997-II/2, UN Doc. A/52/10.

International Law Commission, Report on the work of its fifty-third session, YBILC 2001-II/2, UN Doc. A 56/10 (and Corr. 1.).

International Law Commission, Report on the work of its fifty-fourth session, YBILC 2002-II/2, UN. Doc. A/57/10.

International Law Commission, Report on the work of its fifty-fifth session, YBILC 2003-II/2, UN Doc. A/58/10.

International Law Commission, Report on the work of its fifty-eighth session, YBILC 2006-II/2, UN Doc. A/61/10. 
International Law Commission, Report on the work of its sixtieth session, YBILC 2008-II/2, UN Doc. A/63/10.

International Law Commission, Report on the work of its seventieth session, UN Doc. A/73/10, 2018.

International Law Commission, Secretariat, Topical summary of the discussion on the report of the International Law Commission on the work of its thirty-second session (1980) in the Sixth Committee during the thirty-fifth session of the General Assembly, UN Doc. A/CN.4/L.326, 1981.

International Law Commission, Secretariat, Survey of practice relevant to international liability for injurious consequences arising out of acts not prohibited by international law, YBILC 1985-II/1, UN Doc. A/CN.4/384.

International Law Commission, Secretariat, Survey of liability regimes relevant to the topic of international liability for injurious consequences arising out of acts not prohibited by international law, (international liability in case of loss from trans-boundary harm arising out of hazardous activities), YBILC 1995-II/1, UN Doc. A/CN.4/471.

International Law Commission, Secretariat, Survey of liability regimes relevant to the topic of international liability for injurious consequences arising out of acts not prohibited by international law (international liability in case of loss from transboundary harm arising out of hazardous activities), YBILC 2004-II/1, UN Doc. A/CN.4/543.

International Law Commission, Working Group, Report on international liability for injurious consequence arising out of acts not prohibited by international law, UN Doc. A/CN.4/L.533 and Add.1, in: International Law Commission, Report on the work of its forty-eighth session, YBILC 1996-II/2, UN Doc. A/51/10, Annex I.

International Law Commission, Working Group, Report on international liability for injurious consequence arising out of acts not prohibited by international law, UN Doc. A/CN.4/L.284 and Corr 1., in: International Law Commission, Report on the work of its thirtieth session, YBILC 1978-II/2, UN Doc. A/33/10, Annex.

International Law Commission, Working Group, Report on international liability for injurious consequences arising out of acts not prohibited by international law, UN Doc. A/CN.4/L.536, 1997.

Janowitz, Nathaniel, Revealed: Documents Show BP Quietly Paid Just \$25 Million to Mexico After The Worst Oil Spill Of The Century, BuzzFeedNews, 28 September 2018, abrufbar unter: https:/www.buzzfeednews.com/article/nat hanieljanowitz/bp-mexico-deepwater-horizon-oil (geprüft am 15.05.2020).

Jeppesen, Helle, Haftung verschoben auf irgendwann, Deutsche Welle Online, 25. Februar 2013, abrufbar unter: https://www.dw.com/de/haftung-verschob en-auf-irgendwann/a-16623112 (geprüft am 15.05.2020).

Kahn, Matthew, FBI Director Christopher Wray's Remarks on Encryption to the International Conference on Cyber Security, LAWFARE, 9 January 2018, abrufbar unter: https:/www.lawfareblog.com/fbi-director-christopher-wrays-remarks-encr yption-international-conference-cyber-security (geprüft am 15.05.2020). 


\section{Bibliografie}

Kang, Cecilia, Sony Pictures Hack cost the movie studio at least \$15 million, The Washington Post, 4 February 2015, abrufbar unter: https://www.washingtonpost .com/news/business/wp/2015/02/04/sony-pictures-hack-cost-the-movie-studio-at-1 east-15-million/?utm_term=.db5b8a3b9915 (geprüft am 15.05.2020).

Knake, Robert K., Untangling Attribution: Moving to Accountability in Cyberspace, Statement Before the Subcommittee on Technology and Innovation, Committee on Science and Technology, United States House of Representatives 2nd Session, 111th Congress, 2010, abrufbar unter: https:/www.cfr.org/sites/ default/files/pdf/2010/07/Knake\%20-Testimony\%20071510.pdf (geprüft am 15.05.2020).

Langenfeld, Christine/Minnerop, Petra, Environmental Liability Provisions in International Law, in: Umweltbundesamt (Hg.), Environmental Liability in International Law: Towards a Coherent Conception, Berichte des Umweltbundesamtes, Band 02/05, Berlin 2005, S. 3-158.

Langner, Ralph, Der Langner-Report: Stuxnet und Die Folgen: Was die Schöpfer von Stuxnet erreichen wollten, was sie erreicht haben, und was das für uns alle bedeutet, München, August 2017, abrufbar unter: https:/www.langner.com/wp -content/uploads/2017/08/Stuxnet-und-die-Folgen.pdf (geprüft am 15.05.2020).

Lewis, James Andrew, Assessing the Risks of Cyber Terrorism, Cyber War and Other Cyber Threats, Center for Strategic and International Studies, Report of 1 November 2002, abrufbar unter: http://csis.org/files/media/csis/pubs/021101_ris ks_of_cyberterror.pdf (geprüft am 15.05.2020).

Lewis, James Andrew, The Economic Impact of Cybercrime - No Slowing Down, Center for Strategic and International Studies, Report of 21 February 2018, abrufbar unter: https://www.csis.org/analysis/economic-impact-cybercrime (geprüft am 15.05.2020).

Lewis, James Andrew/Baker, Stewart, The Economic Impact of Cybercrime and Cyber Espionage, Center for Strategic and International Studies, Report of 22 July 2013, abrufbar unter: https://csis-prod.s3.amazonaws.com/s3fs-public/legacy_f iles/files/publication/60396rpt_cybercrime-cost_0713_ph4_0.pdf (geprüft am 15.05.2020).

McElroy, Damien/Williams, Christopher, Flame: world's most complex computer virus exposed, The Telegraph, 28 May 2012, abrufbar unter: http://www.telegrap h.co.uk/news/worldnews/middleeast/iran/9295938/Flame-worlds-most-complex -computer-virus-exposed.html (geprüft am 15.05.2020).

McGurk, Seán P. (Acting Director, National Cybersecurity and Communications Integration Center Office of Cybersecurity and Communications National Protection and Programs Directorate Department of Homeland Security), Statement for the Record Before the United States Senate Homeland Security and Governmental Affairs Committee, Washington DC, 17 November 2010, abrufbar unter: http://www.hsgac.senate.gov/download/2010-11-17-mcgurk-testimony -revised (geprüft am 15.05.2020). 
McKay, Angela/Neutze, Jan/Nicholas, Paul/Sullivan, Kevin, International Cyber Security Norms: Reducing conflict in an Internet-dependent world, Microsoft Whitepaper 2014, abrufbar unter: https:/query.prod.cms.rt.microsoft.com/cms/ api/am/binary/REVroA (geprüft am 15.05.2020).

Mercado, Virginia/Soto, Luis A., Fischer gegen Ölmulti, Entwicklung und Zusammenarbeit Online, 19. März 2017, abrufbar unter: https://www.dandc.eu/de/arti cle/sieben-jahre-nach-dem-untergang-der-oelbohrplattform-deepwater-horizon-k aempft-mexiko-noch, (geprüft am 15.05.2020).

Myers, Steven Lee, Youth Groups Created by Kremlin Serve Putin's Cause, The New York Times, 8 July 2007, abrufbar unter: https://www.nytimes.com/2007/07/08 /world/europe/08moscow.html?mtrref=www.google.com\&gwh=A2CDBA353 18FF4A9402F71EECFBA0E21\&gwt=pay\&assetType=REGIWALL (geprüft am 15.05.2020).

Nikièma, Suzy H., Compensation for Expropriation, International Institute for Sustainable Development, Best Practices Series, March 2013, abrufbar unter: https:/ /www.iisd.org/pdf/2013/best_practice_compensation_expropriation_en.pdf (geprüft am 15.05.2020).

Norddeutscher Rundfunk/Das Erste, "Snowden exklusiv": der Wortlaut des Interviews von NDR Autor Hubert Seipel, 26 Januar 2014, abrufbar unter: https://www.pre sseportal.de/pm/69086/2648795 (geprüft am 15.05.2020).

North Atlantic Treaty Organization, Cyber Defence Pledge, 8 July 2016, abrufbar unter: http://www.nato.int/cps/en/natohq/official_texts_133177.htm (geprüft am 15.05.2020)

Pernice, Ingolf, Vom Völkerrecht des Netzes zur Verfassung des Internets: Privacy und Digitale Sicherheit im Zeichen eines schrittweisen Paradigmenwechsels, HIIG Discussion Paper Series, Discussion Paper No. 2017-02, abrufbar unter: https://papers.ssrn.com/sol3/papers.cfm?abstract_id=2959257 (geprüft am 15.05.2020), S. 1-27.

Pernik, Piret, A Playbook for Hybrid War in Cyberspace?, International Center for Defence and Security, 29 August 2014, abrufbar unter: https://icds.ee/a-playbook -for-hybrid-war-in-cyberspace/ (geprüft am 15.05.2020).

Peters, Anne, Surveillance without Borders: The Unlawfulness of the NSA-Panopticon, Part II, EJIL: Talk!, 4 November 2013, abrufbar unter: https://www.ejiltalk. org/surveillance-without-borders-the-unlawfulness-of-the-nsa-panopticon-part-ii/ (geprüft am 15.05.2020).

Quentin-Baxter, Robert Q. (Special Rapporteur), Preliminary report on international liability for injurious consequences of acts not prohibited by international law, YBILC 1980-II/1, UN Doc. A/CN.4/334 and Add.l and 2.

Quentin-Baxter, Robert Q. (Special Rapporteur), Second report on international liability for injurious consequences arising out of acts not prohibited by international law, YBILC 1981-II/1, UN Doc. A/CN.4/346 and Add.1 and 2.

Quentin-Baxter, Robert Q. (Special Rapporteur), Third report on international liability for injurious consequences arising out of acts not prohibited by international law, YBILC 1982-II/1, UN Doc. A/CN.4/360. 


\section{Bibliografie}

Quentin-Baxter, Robert Q. (Special Rapporteur), Fourth report on international liability for injurious consequences arising out of acts not prohibited by international law, YBILC 1983-II/1, UN Doc. A/CN.4/373.

Rao, Pemmaraju S. (Special Rapporteur), First report on prevention of transboundary damage from hazardous activities, YBILC 1998-II/1, UN Doc. A/CN.4/487 and Add.1.

Rao, Pemmaraju S. (Special Rapporteur), Third report on international liability for injurious consequences arising out of acts not prohibited by international law (prevention of transboundary damage from hazardous activities), YBILC 2000-II/1, UN Doc. A/CN.4/510.

Rao, Pemmaraju S. (Special Rapporteur), First report on the legal regime for allocation of loss in case of transboundary harm arising out of hazardous activities, YBILC 2003-II/1, UN Doc. A/CN.4/531.

Rao, Pemmaraju S. (Special Rapporteur), Second report on the legal regime for the allocation of loss in case of transboundary harm arising out of hazardous activities, YBILC 2004-II/1, UN Doc. A/CN.4/540.

Rao, Pemmaraju S. (Special Rapporteur), Third report on the legal regime for allocation of loss in case of transboundary harm arising out of hazardous activities, YBILC 2006-II/1, UN Doc. A/CN.4/566.

Robb, David, Sony Hack: A Timeline, DEADLINE, 22 December 2014, abrufbar unter: http://deadline.com/2014/12/sony-hack-timeline-any-pascal-the-interview -north-korea-1201325501 (geprüft am 15.05.2020).

Roberts, Dan, Obama imposes new sanctions against North Korea in response to Sony Hack, The Guardian, 2 January 2015, abrufbar unter: http://www.theguard ian.com/us-news/2015/jan/02/obama-imposes-sanctions-north-korea-sony-hack-t he-interview (geprüft am 15.05.2020).

Rötzer, Florian, DDoS-Angriffe auf estnische Server waren kein "Cyberwar", heise online, 12. Juni 2007, abrufbar unter: https://www.heise.de/newsticker/meldung /DDoS-Angriffe-auf-estnische-Server-waren-kein-Cyberwar-138918.html (geprüft am 15.05.2020).

Sanger, David E., Obama Ordered Sped Up Wave of Cyberattacks Against Iran, The New York Times, 1 June 2012, abrufbar unter: http://www.nytimes.com/2012/ 06/01/world/middleeast/obama-ordered-wave-of-cyberattacks-against-iran.html (geprüft am 15.05.2020).

Sanger, David E./Savage, Charlie, U.S. Says Russia Directed Hacks to Influence Elections, The New York Times, 7 October 2016, abrufbar unter: http://www.ny times.com/2016/10/08/us/politics/us-formally-accuses-russia-of-stealing-dnc-emai ls.html (geprüft am 15.05.2020).

Schaller, Christian, Internationale Sicherheit und Völkerrecht im Cyberspace: Für klarere Regeln und mehr Verantwortung, SWP-Studie 18/2014.

Schmitt, Michael N., Cyber Responses "By The Numbers" in International Law, EJIL: Talk!, 4 August 2015, abrufbar unter: https://www.ejiltalk.org/cyber-respo nses-by-the-numbers-in-international-law/ (geprüft am 15.05.2020). 
Schulze, Matthias, Hacking back? Technische und politische Implikationen digitaler Gegenschläge, SWP-Aktuell 59/2017.

Shoard, Catherine, Sony Hack: the plot to kill The Interview - a timeline so far, The Guardian, 18 December 2014, abrufbar unter: http://www.theguardian.com/fil $\mathrm{m} / 2014 / \mathrm{dec} / 18 /$ sony-hack-the-interview-timeline (geprüft am 15.05.2020).

Soesanto, Stefan/D 'Incau, Fosca, The UN GGE is dead: Time to fall forward, European Council on Foreign Relations, 15 August 2017, abrufbar unter: https://w ww.ecfr.eu/article/commentary_time_to_fall_forward_on_cyber_governance (geprüft am 15.05.2020).

Stubbs, Jack/Bing, Christopher, Hacking the hackers: Russian group hijacked Iranian spying operation, officials say, Reuters, 21 October 2019, abrufbar unter: https:/ /www.reuters.com/article/us-russia-cyber/hacking-the-hackers-russian-group-h ijacked-iranian-spying-operation-officials-say-idUSKBN1X00AK (geprüft am 15.05.2020).

Sucharitkul, Sompong, Responsibility and Liability for Environmental Damage Under International Law, Golden Gate University Law Digital Commons 664 (1996), abrufbar unter: https://digitalcommons.law.ggu.edu/pubs/664 (geprüft am 15.05.2020), S. 1-31.

The White House, Office of the Press Secretary, Statement by the President on Actions in Response to Russian Malicious Cyber Activity and Harassment, 29 December 2016, abrufbar unter: https://obamawhitehouse.archives.gov/the-pres s-office/2016/12/29/statement-president-actions-response-russian-malicious-cyber -activity (geprüft am 15.05.2020).

United Nations Environment Programme, Expert Group on Best Available Techniques and Best Environmental Practices, Development of Guidelines on Best Available Techniques and Provisional Guidance on Best Environmental Practices Relevant to Art. 5 and Annex $\mathrm{C}$ of the Stockholm Convention on Persistent Organic Pollutants: Use of the Terms Best Available Techniques, Best Environmental Practices and Related Concepts in International Environmental Instruments, Note by Secretariat, 29 January 2003, UNEP/POPS/EGB.1/INF/3, Annex, abrufbar unter: http://chm.pops.int/Portals/0/docs/from_old_website/documents/mee tings/bat_bep/1st_session/EGB1_INF3_terms3.pdf (geprüft am 15.05.2020).

United Nations Office for Disarmament Affairs, Developments in the field of information and telecommunications in the context of international security, Fact Sheet, abrufbar unter: https://s3.amazonaws.com/unoda-web/wp-content/u ploads/2018/07/Information-Security-Fact-Sheet-July2018.pdf (geprüft am 15.05.2020).

US Department of the Treasury, Press Center, Treasury Imposes Sanctions Against the Government of the Democratic People's Republic Of Korea, 2 January 2015, abrufbar unter: https:/www.treasury.gov/press-center/press-releases/Pages/j19733 .aspx (geprüft am 15.05.2020).

US Joint Chiefs of Staff, Cyberspace Operations, Joint Pub 3-12, 8 June 2018, abrufbar unter: https://fas.org/irp/doddir/dod/jp3_12.pdf (geprüft am 15.05.2020). 


\section{Bibliografie}

Vega-Barbosa, Giovanny/Aboagye, Lorraine, A Commentary on the Advisory Opinion of the Inter-American Court of Human Rights on the Environment and Human Rights, Note e commenti, DPCE Online 34 (2018), abrufbar unter: http://www .dpceonline.it/index.php/dpceonline/article/view/506 (geprüft am 15.05.2020), Rn. 291-298.

Weeramantry, Christopher G., Nukleare Reaktorkatastrophe in Japan: Ein offener Brief an die Umweltminister aller Staaten, 14. März 2011, abrufbar unter: https:/ /www.ialana.de/files/pdf/veröffentlichungen/Offener_Brief_von_C_Weeramantry_Fukushima.pdf (geprüft am 15.05.2020).

Wilson, Christopher/Gaidosch, Tamas/Adelmann, Frank/Morozova, Anastasiia, Cybersecurity Risk Supervision, International Monetary Fund, Monetary and Capital Markets Departmental Paper Series No.19/15, Washington, D.C. 2019.

Wilson, Clay, Botnets, Cybercrime, and Cyberterrorism: Vulnerabilities and Policy Issues for Congress, US Congressional Research Report of 29 January 2008, No RL32114, abrufbar unter: https://fas.org/sgp/crs/terror/RL32114.pdf (geprüft am 15.05.2020).

Zetter, Kim, Critics Say New Evidence Linking North Korea to the Sony Hack Is Still Flimsy, WIRED, 8 January 2015, abrufbar unter: http://www.wired.com /2015/01/critics-say-new-north-korea-evidence-sony-still-flimsy (geprüft am 15.05.2020). 